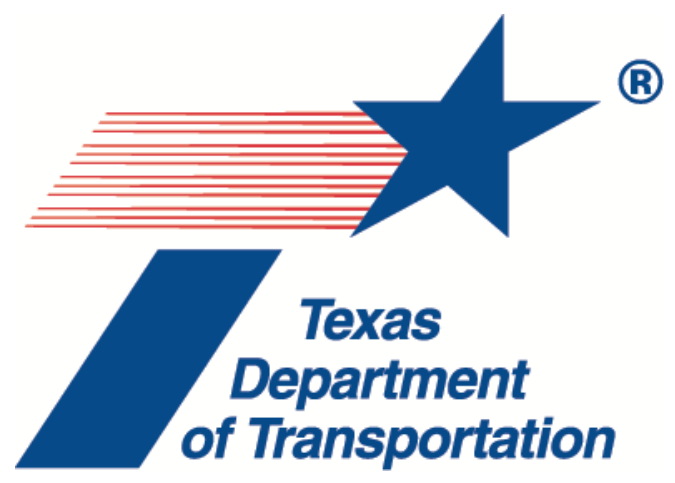

\title{
Data Recovery Investigations: Murvaul Creek Site (41PN175), Panola County, Texas (CSJ 1222-01-014, Atlanta District)
} VOLUME II: APPENDICES

By

Arlo McKee

Charles D. Frederick, Ph.D.

Timothy K. Perttula, Ph.D.

Robert Z. Selden, Ph.D.

Leslie Bush, Ph.D.

Leonard Kemp

Brittney Gregory

Chad Yost

Linda Scott Cummings, Ph.D.

Principal Investigator

Duane E. Peter
Jeffrey R. Ferguson, Ph.D.

Michael D. Glascock, Ph.D.

Steve Tomka, Ph.D.

Leslie Cecil, Ph.D.

Caroline Masiello, Ph.D.

Xiaodong Gao, Ph.D.

Christopher Goodmaster

Virgil Beasley, III, Ph.D.

Texas Department of Transportation, Environmental Affairs Division Archeological Studies Program Report Number 165

Texas Antiquities Permit Number 5879

May 2015 
for

Texas Department of Transportation, Environmental Affairs Division under

TxDOT Work Authorization Nos. 57906 SA005 and 59008 SA005 ISBN 978-1-935545-32-3 
APPENDIX A: ARTIFACT DATA TABLES 

A-1: INVENTORY OF CERAMIC VESSEL SHERDS 



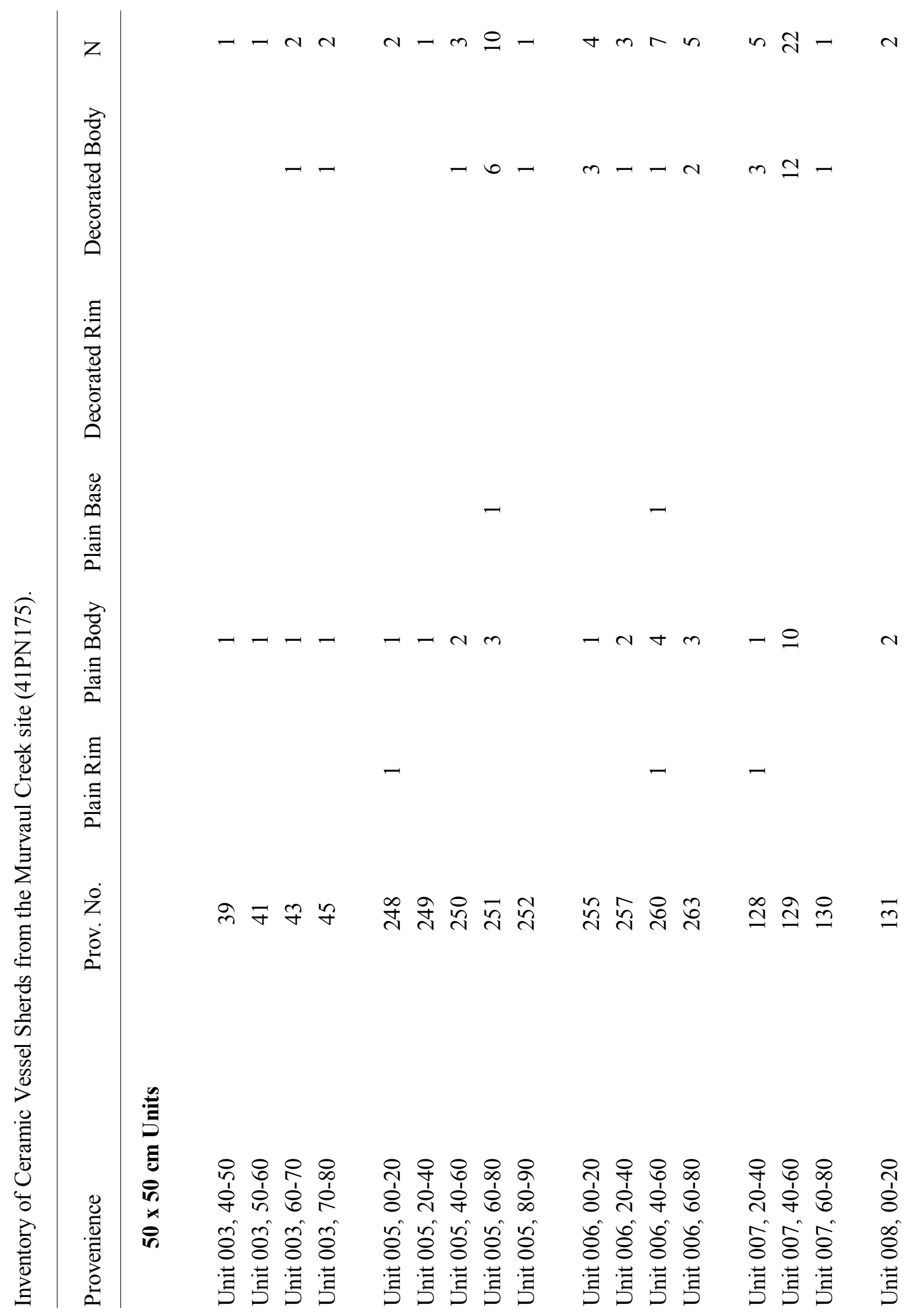




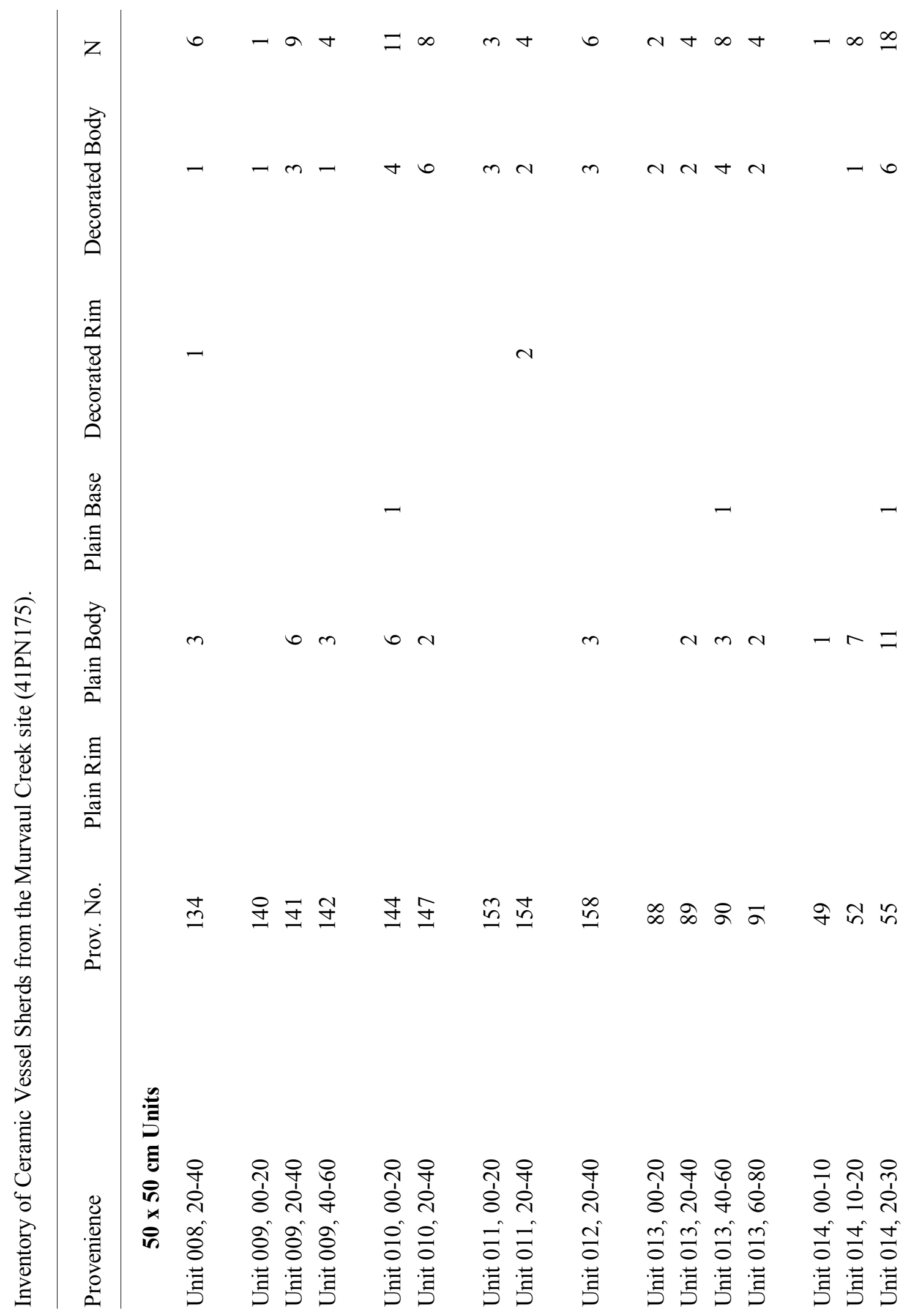




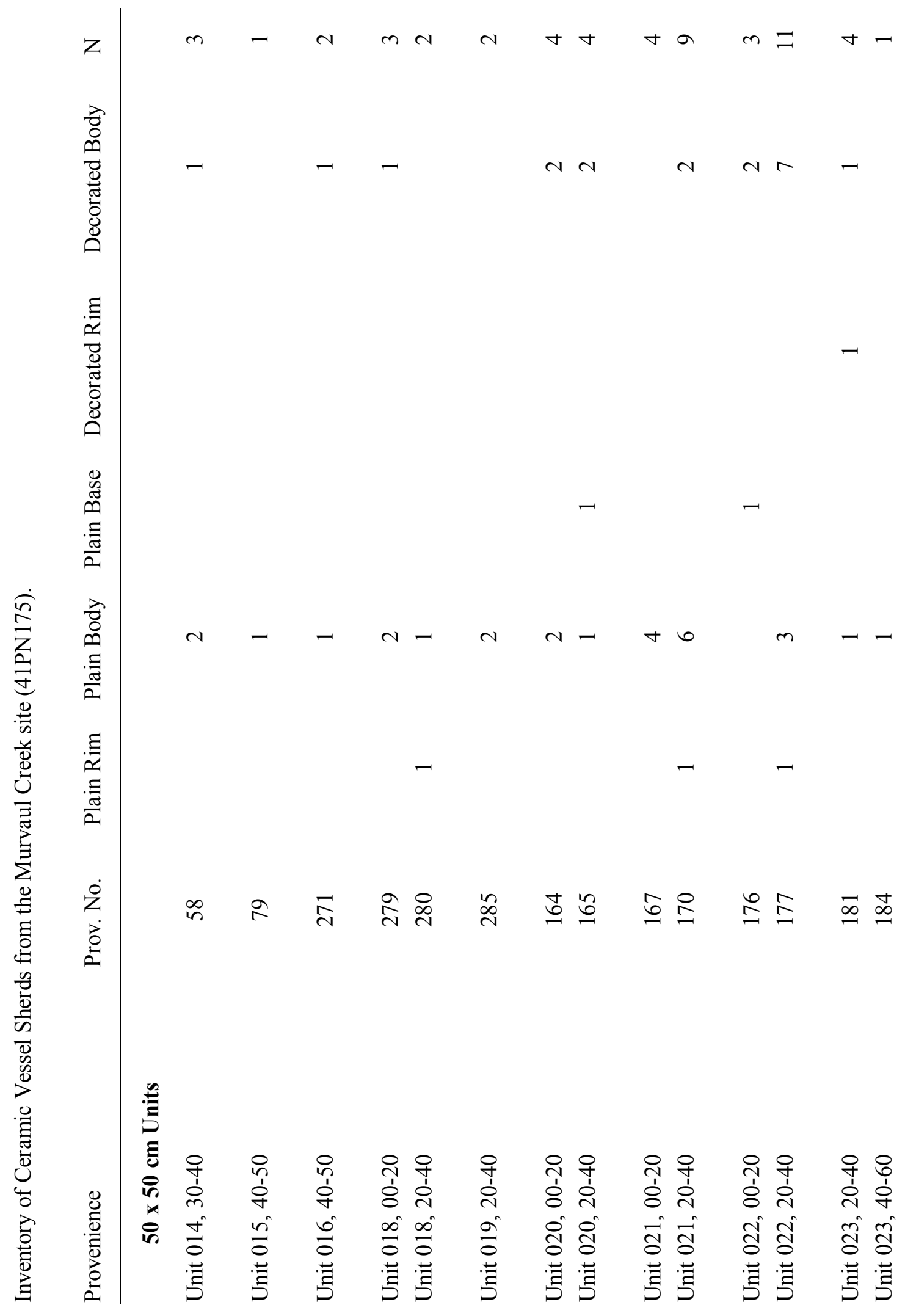




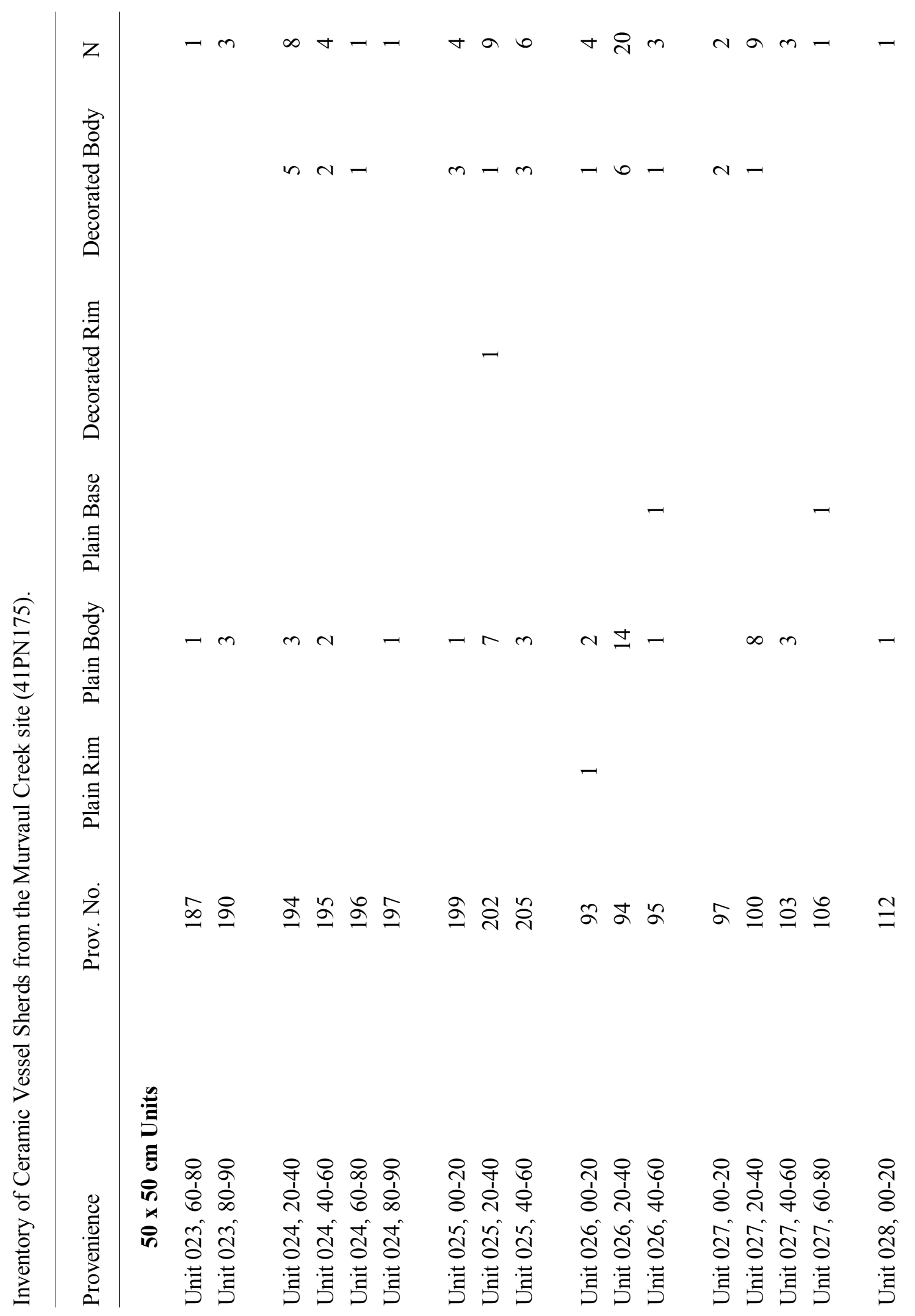




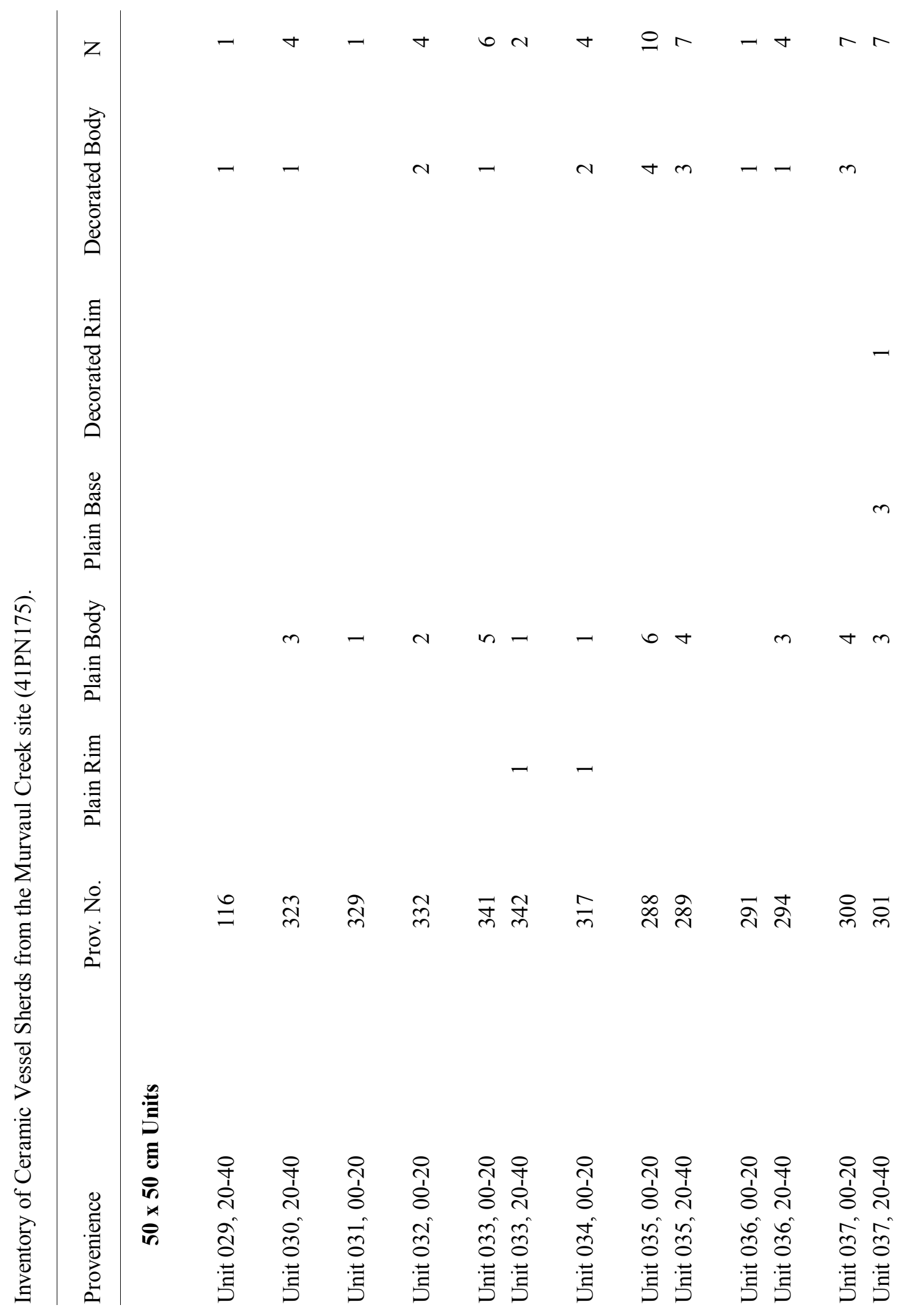




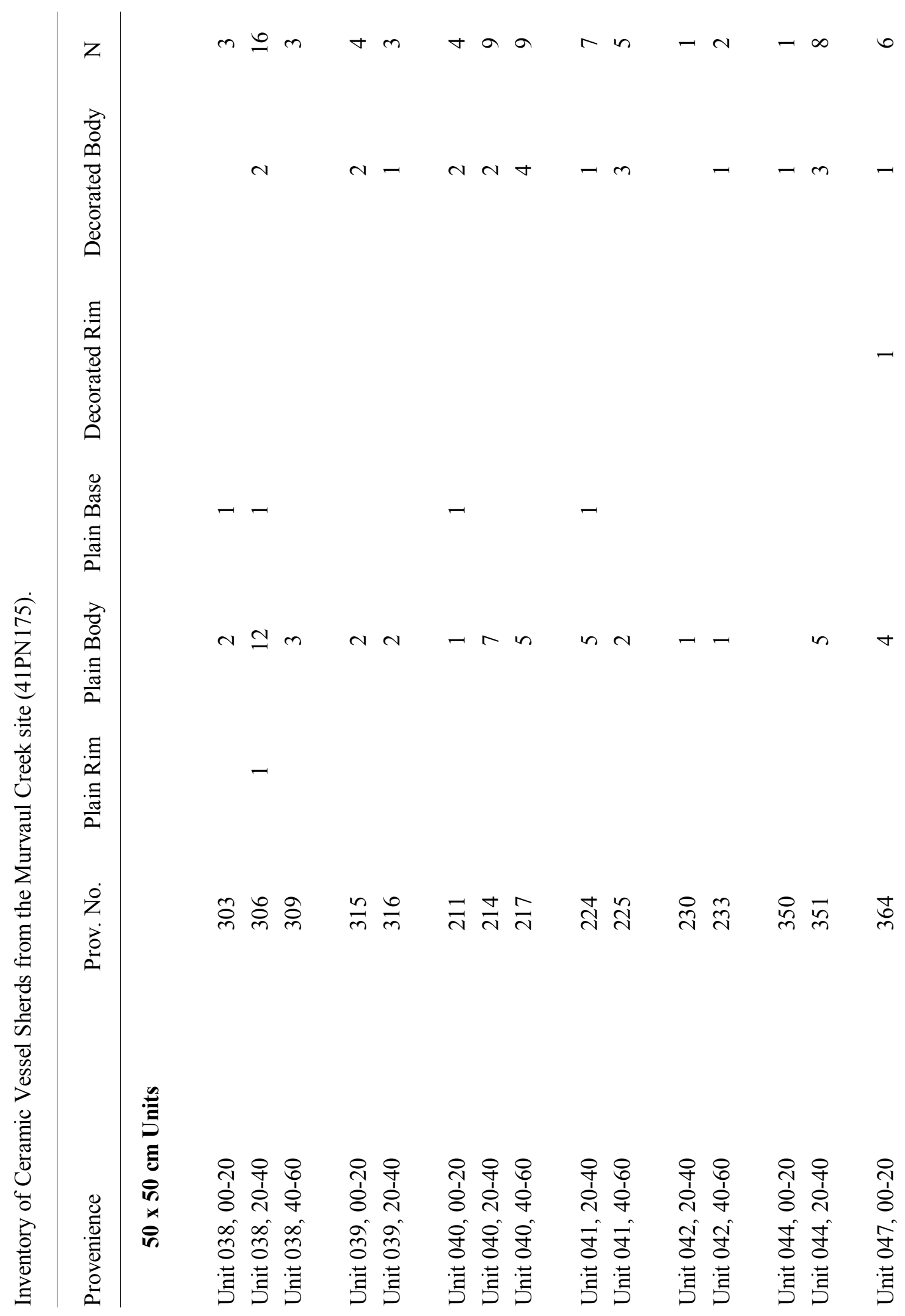




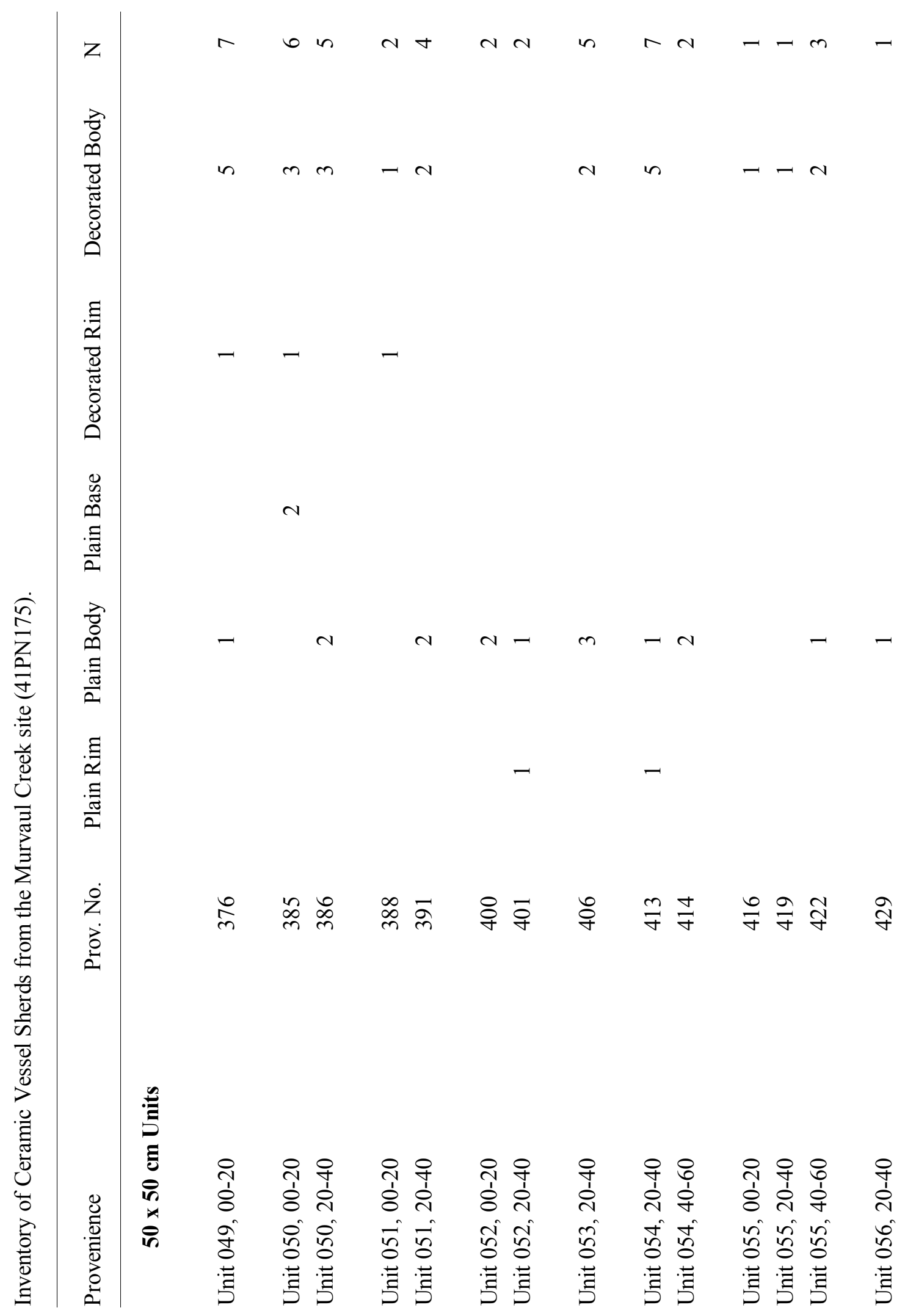




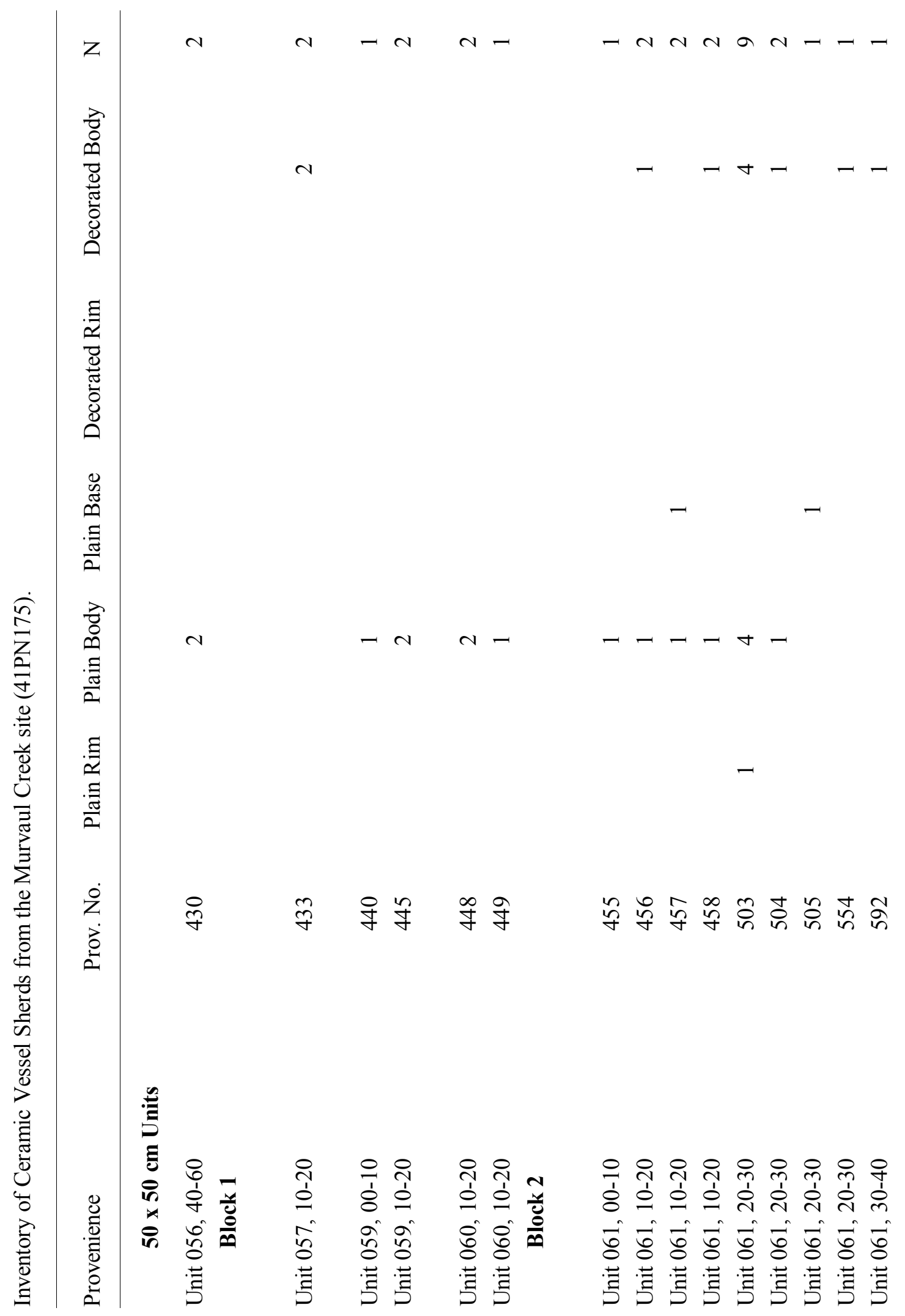




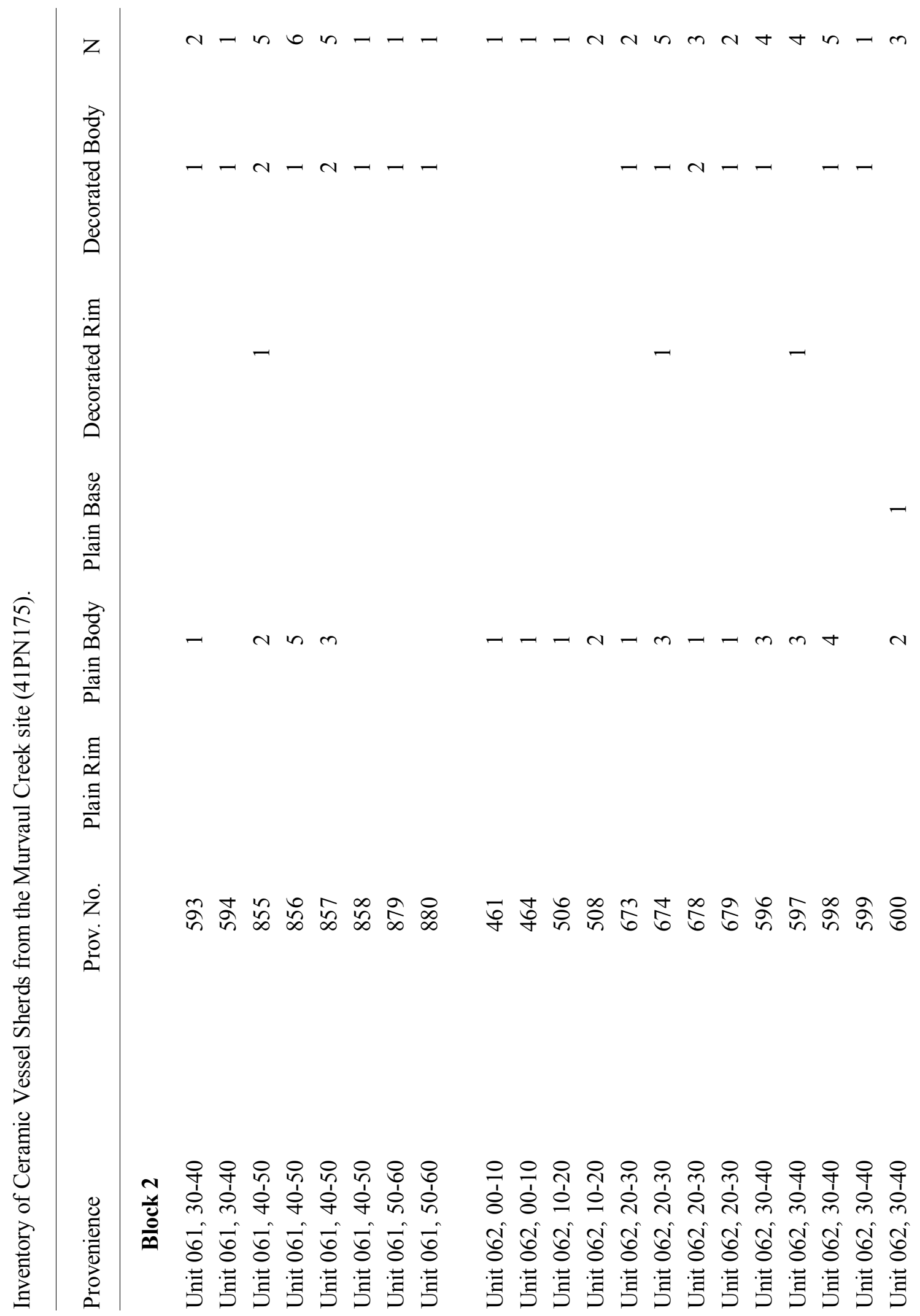




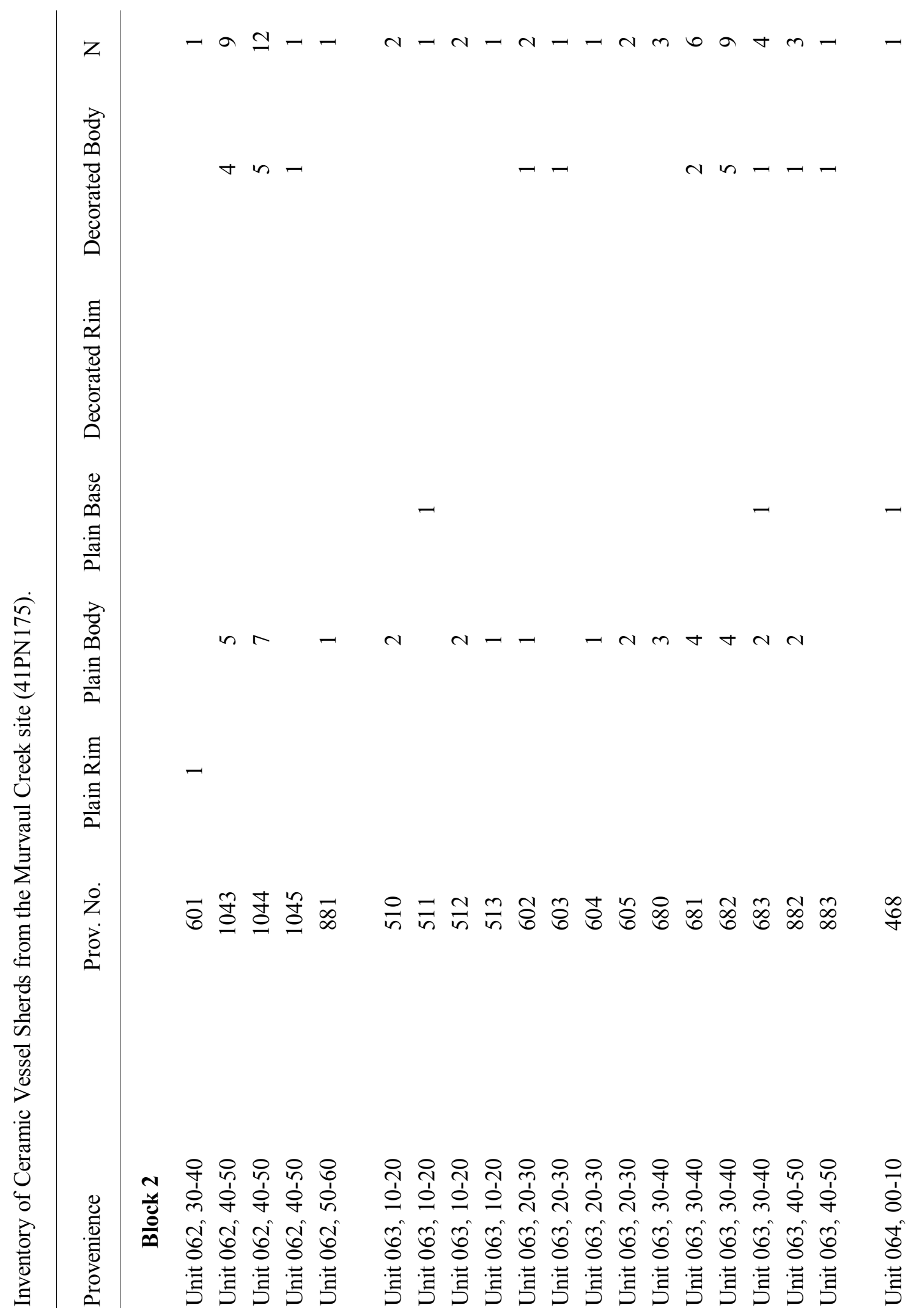




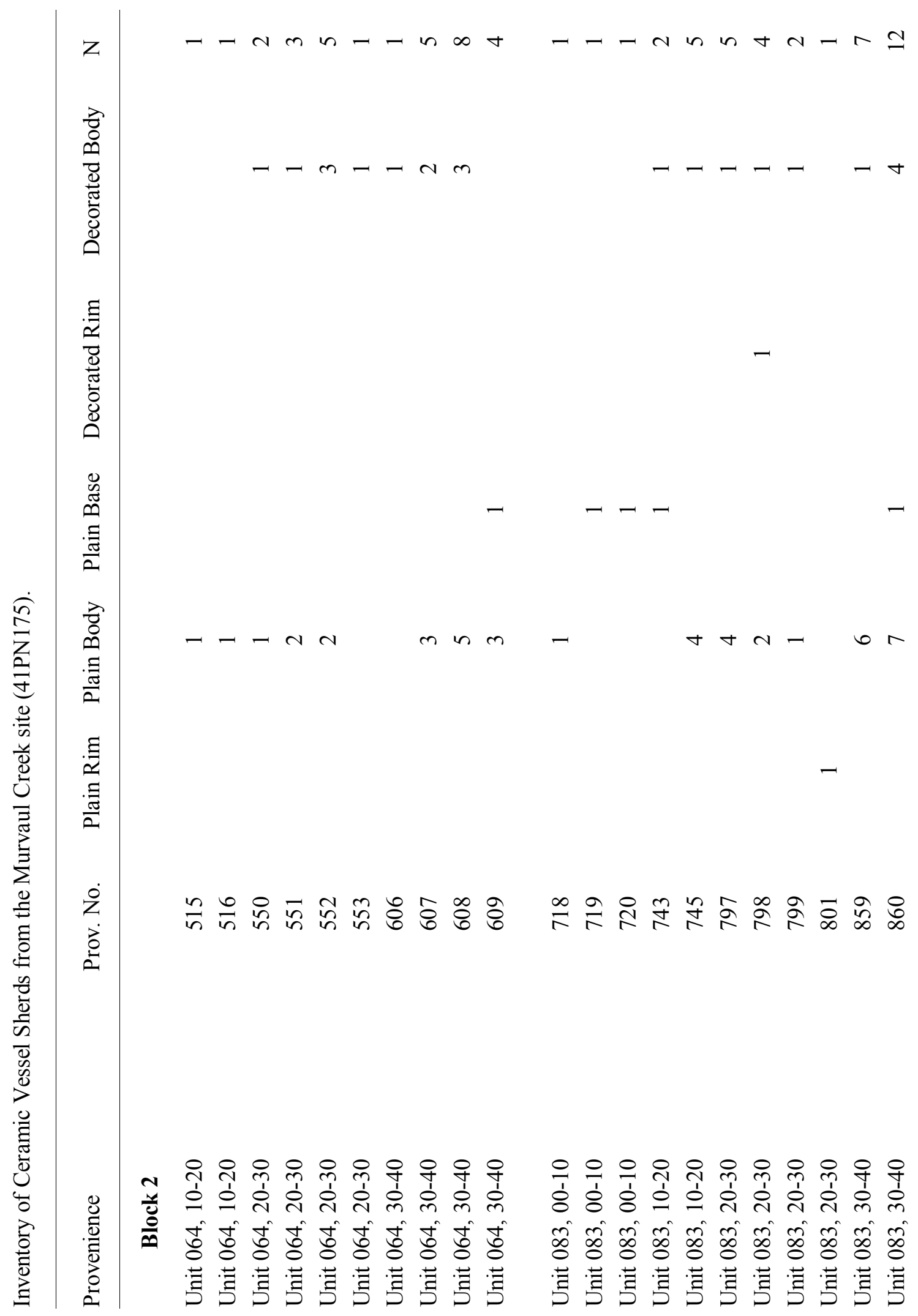




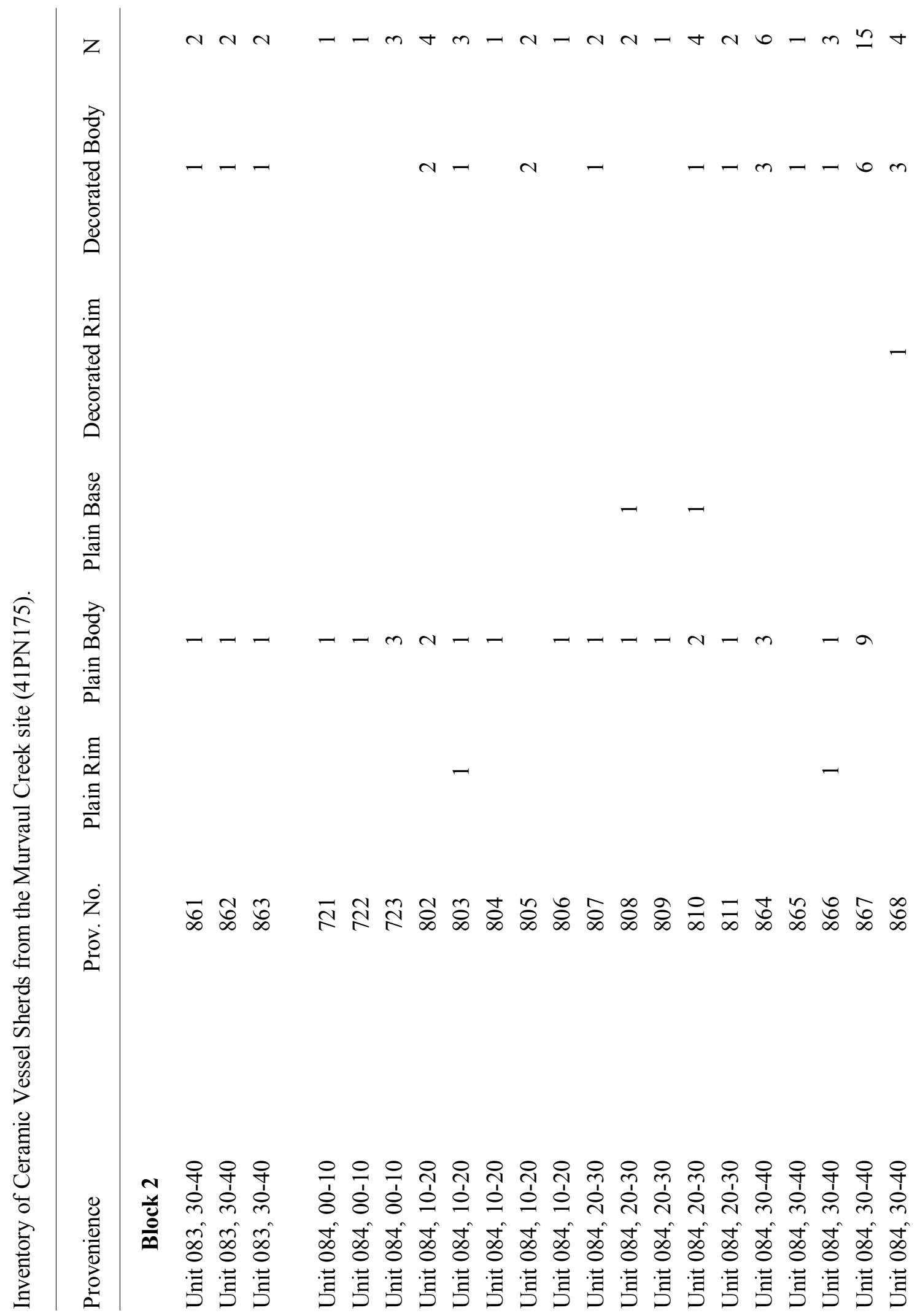




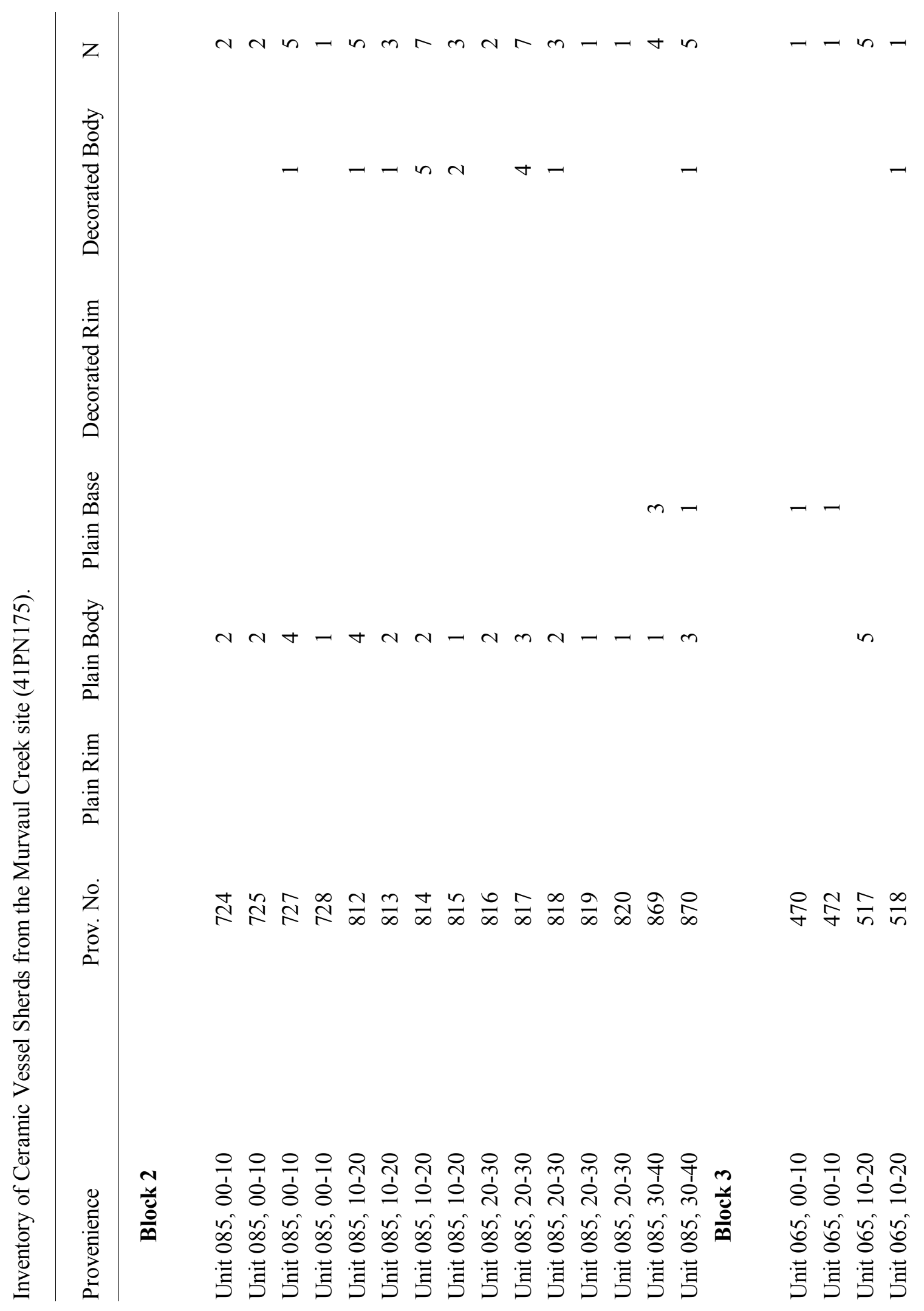




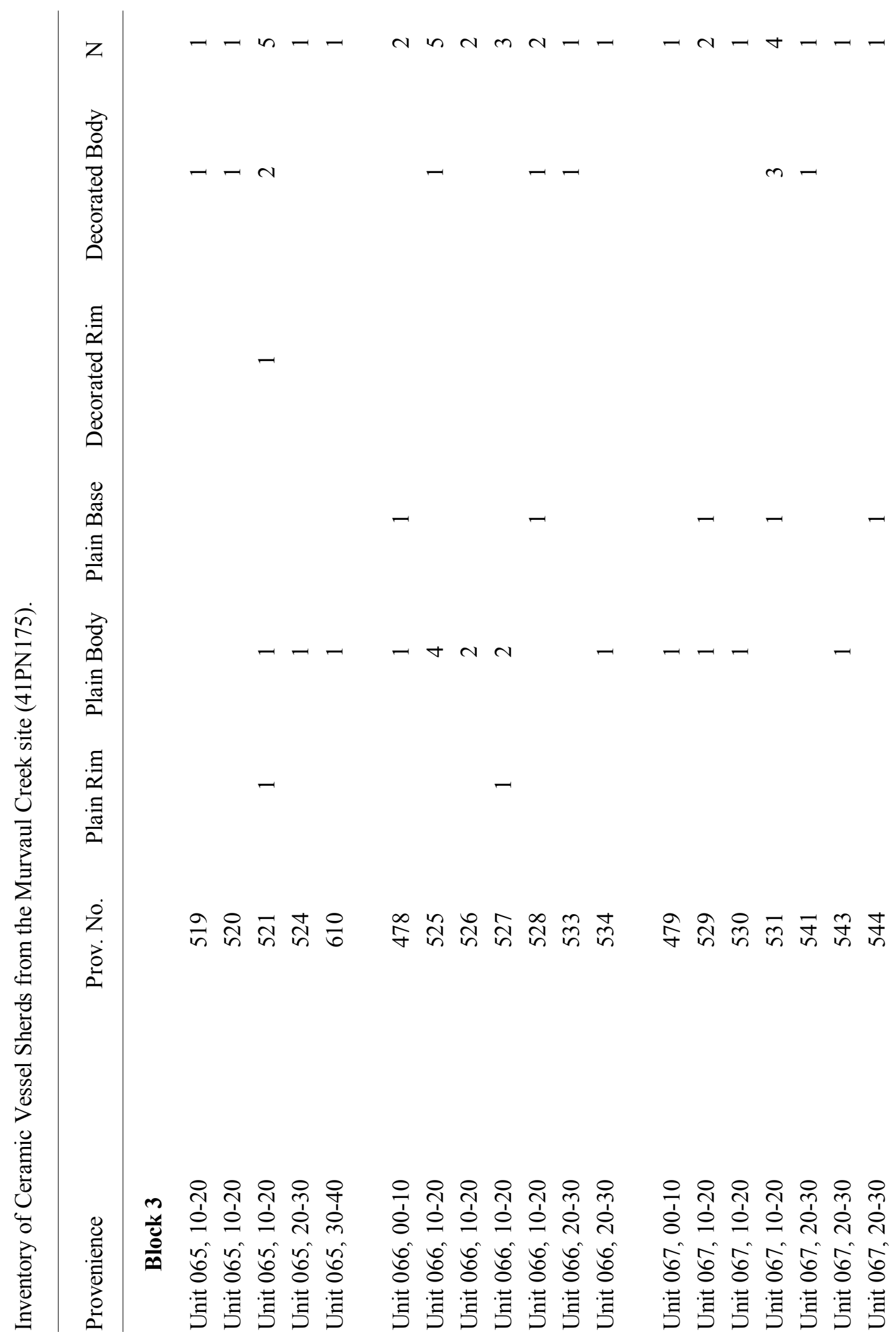




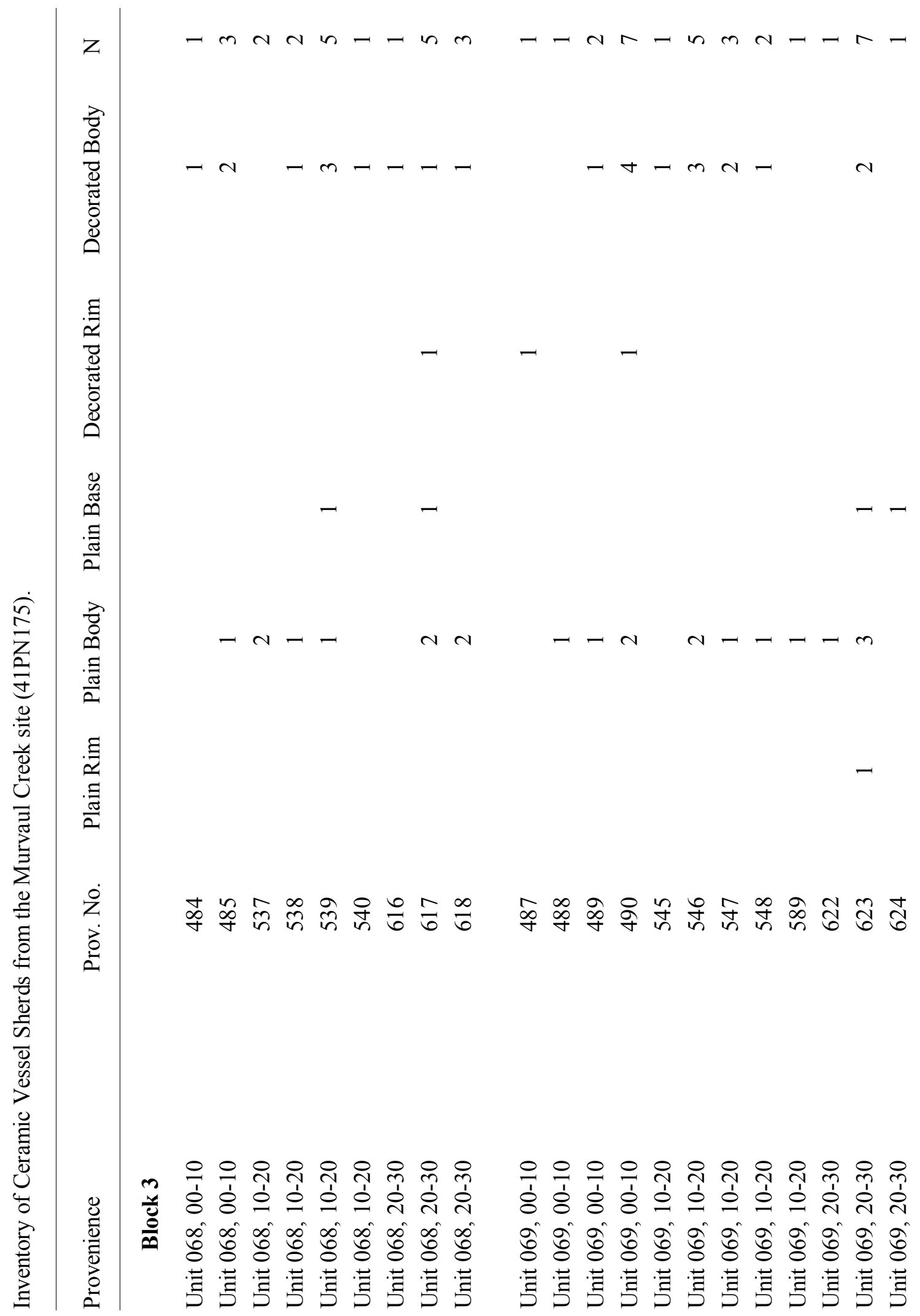









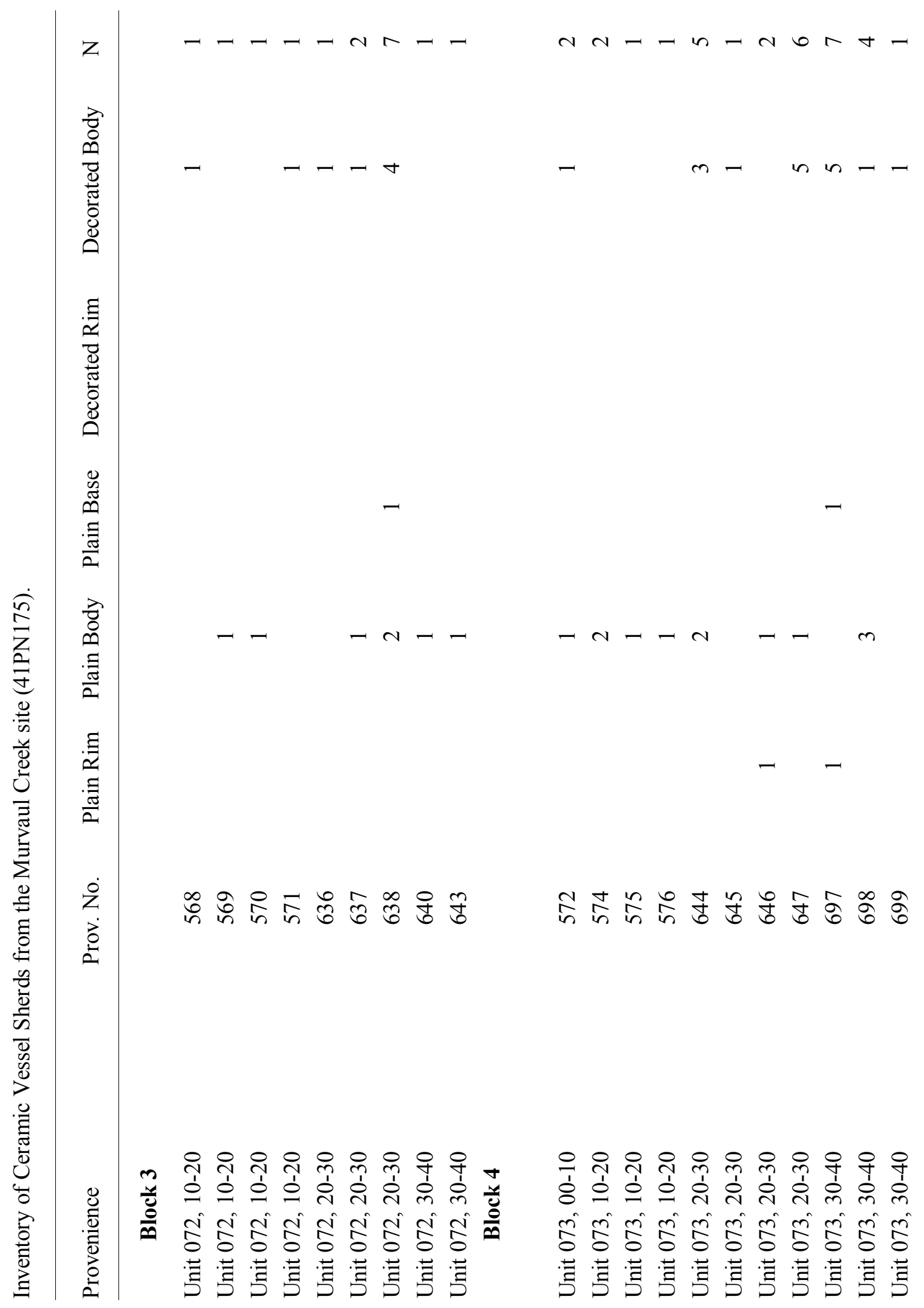




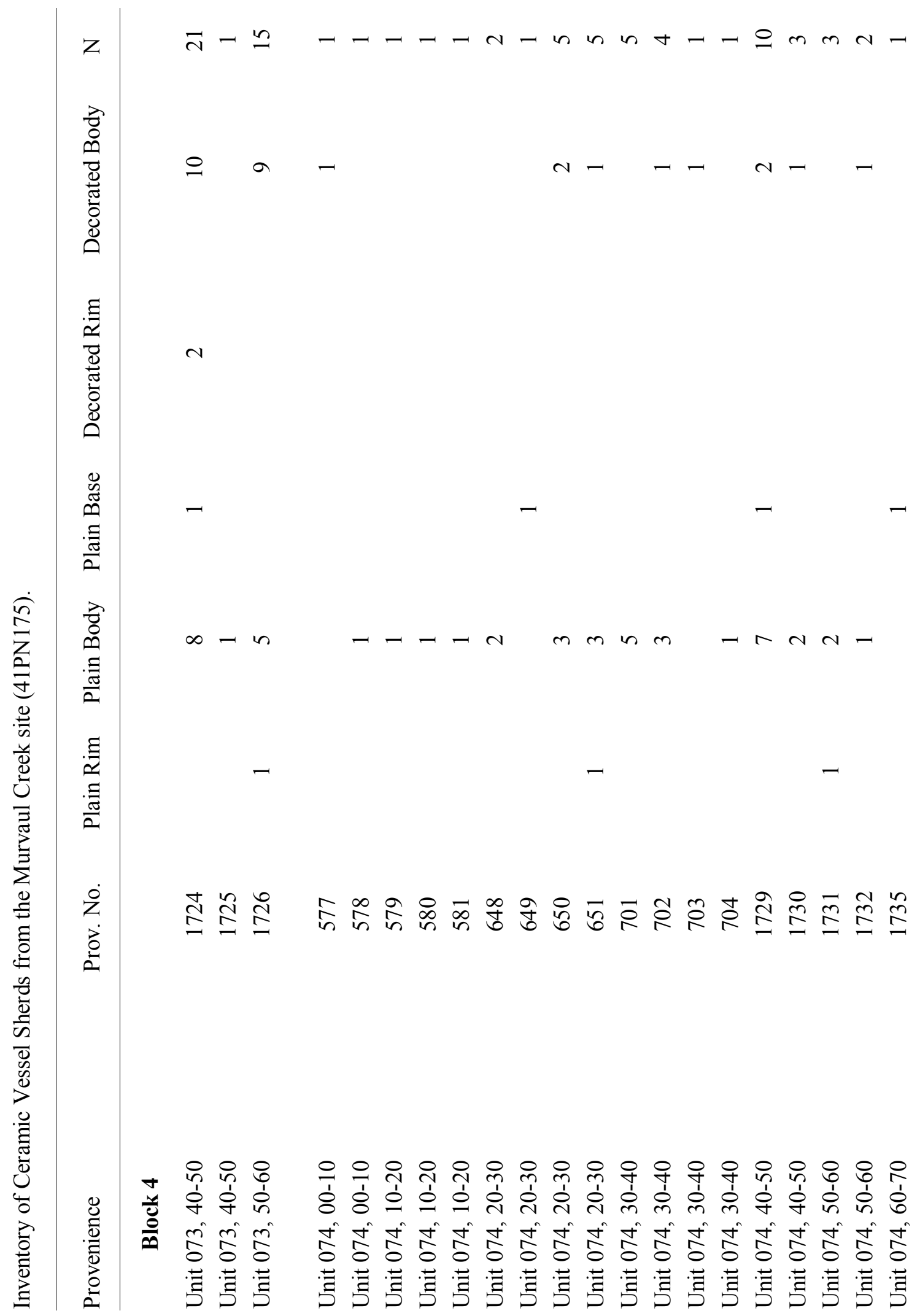




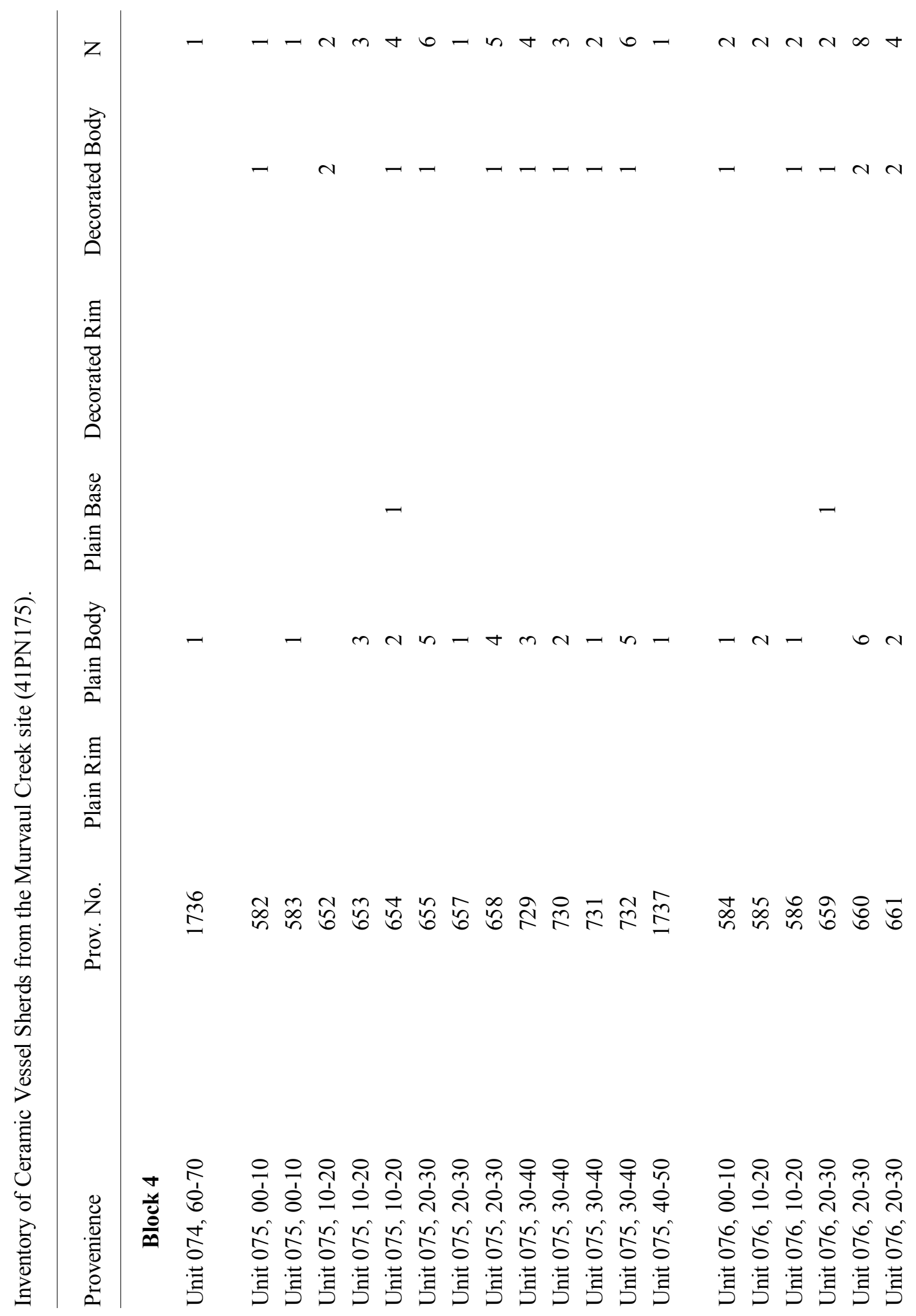




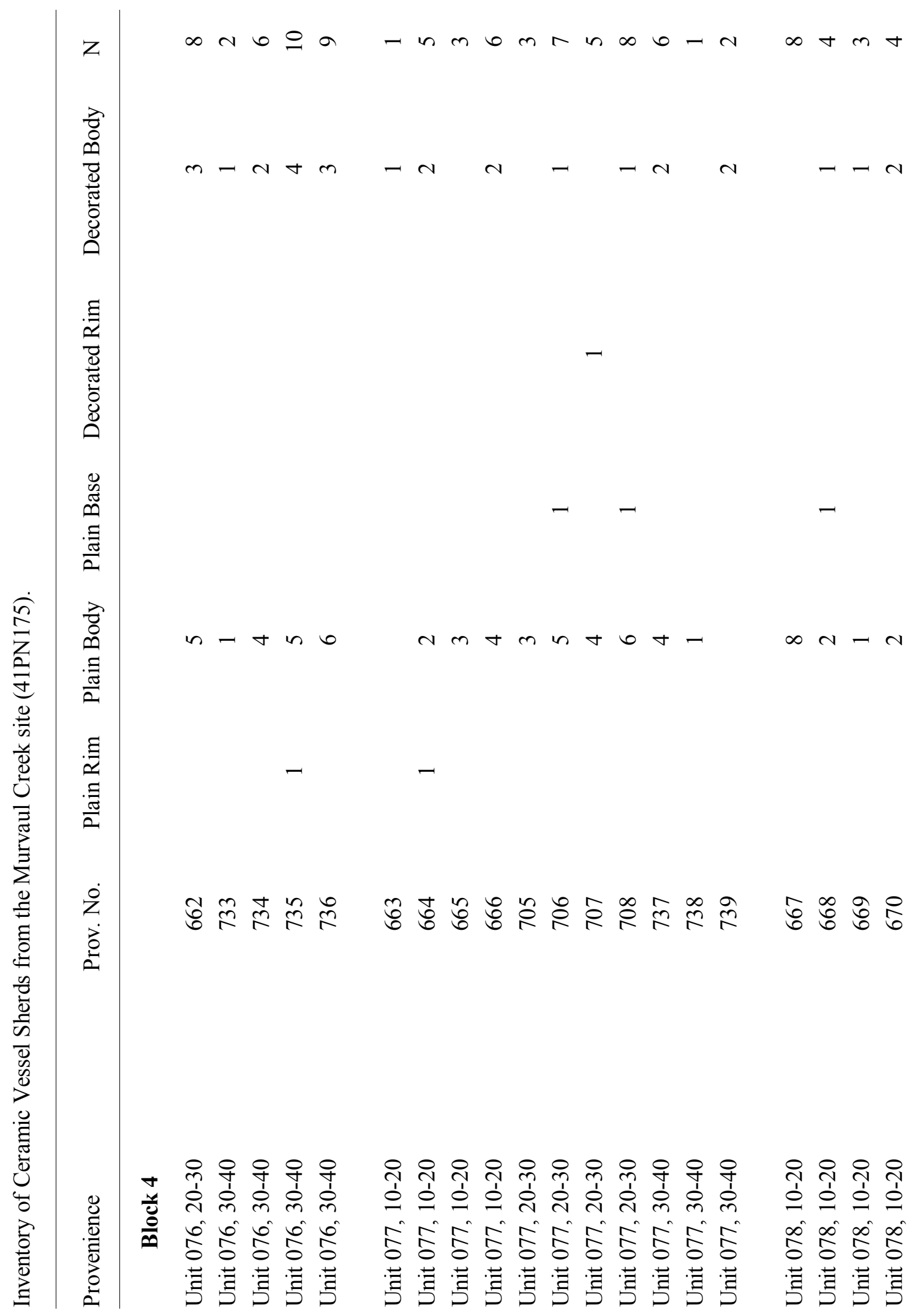




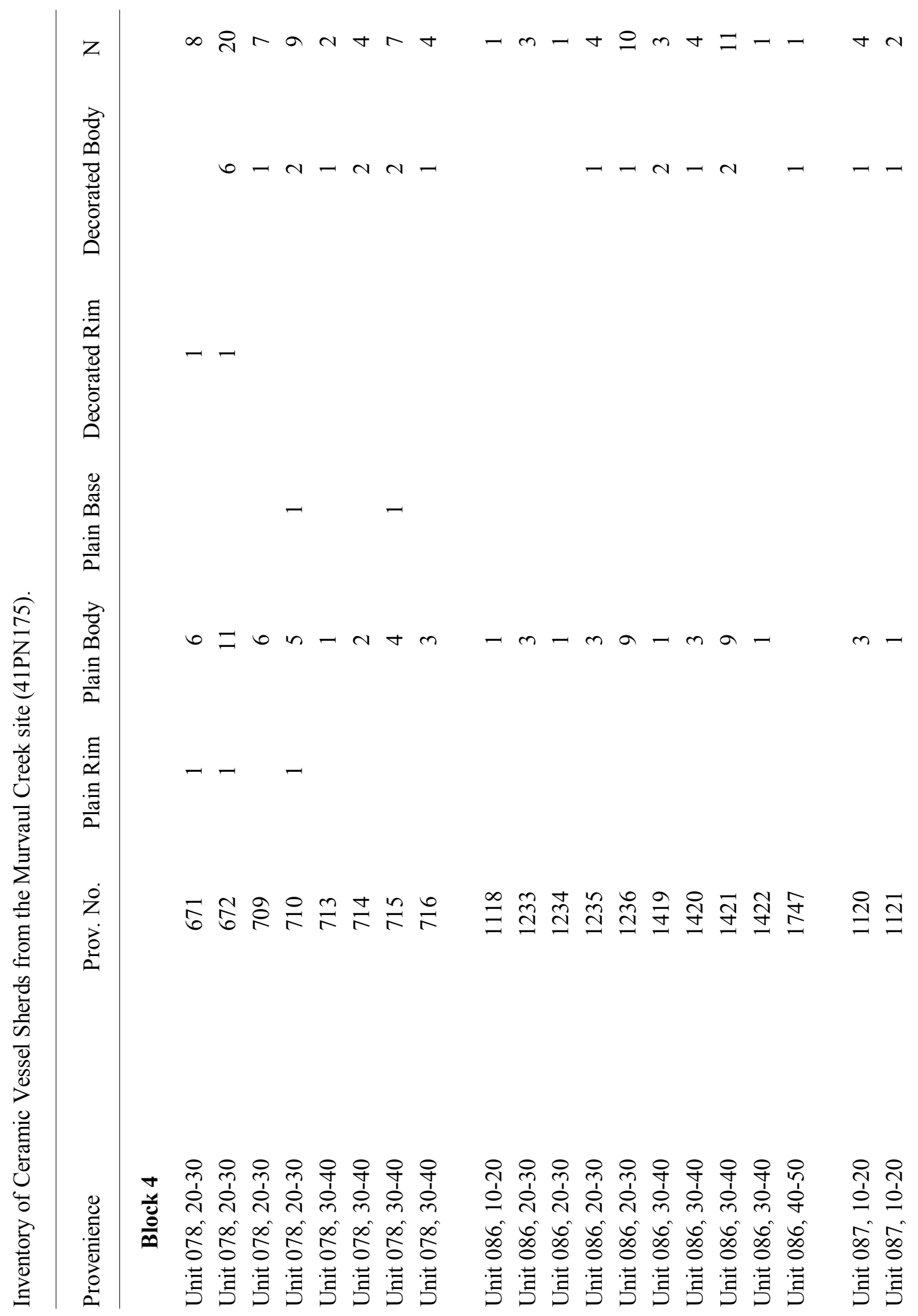




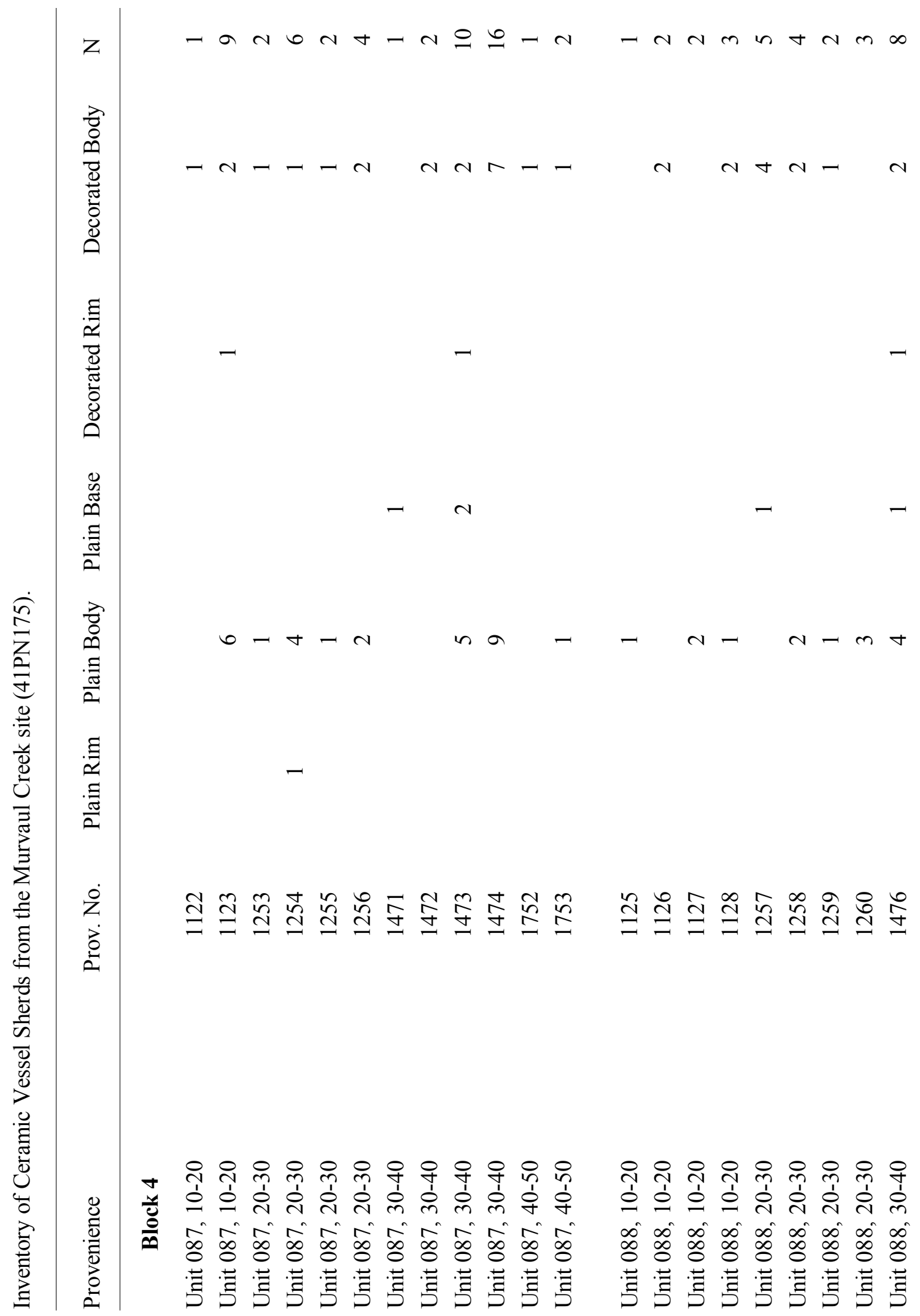




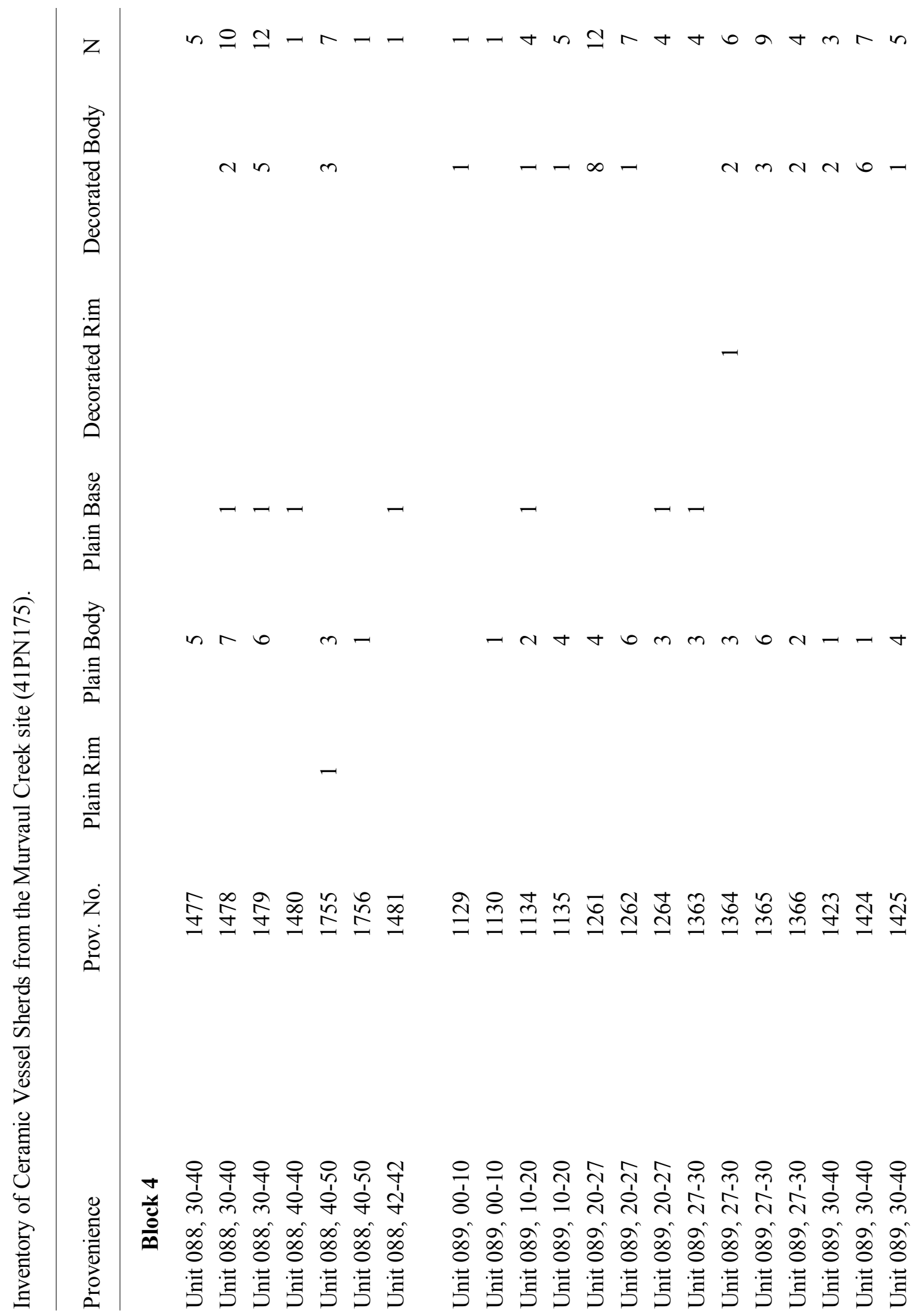




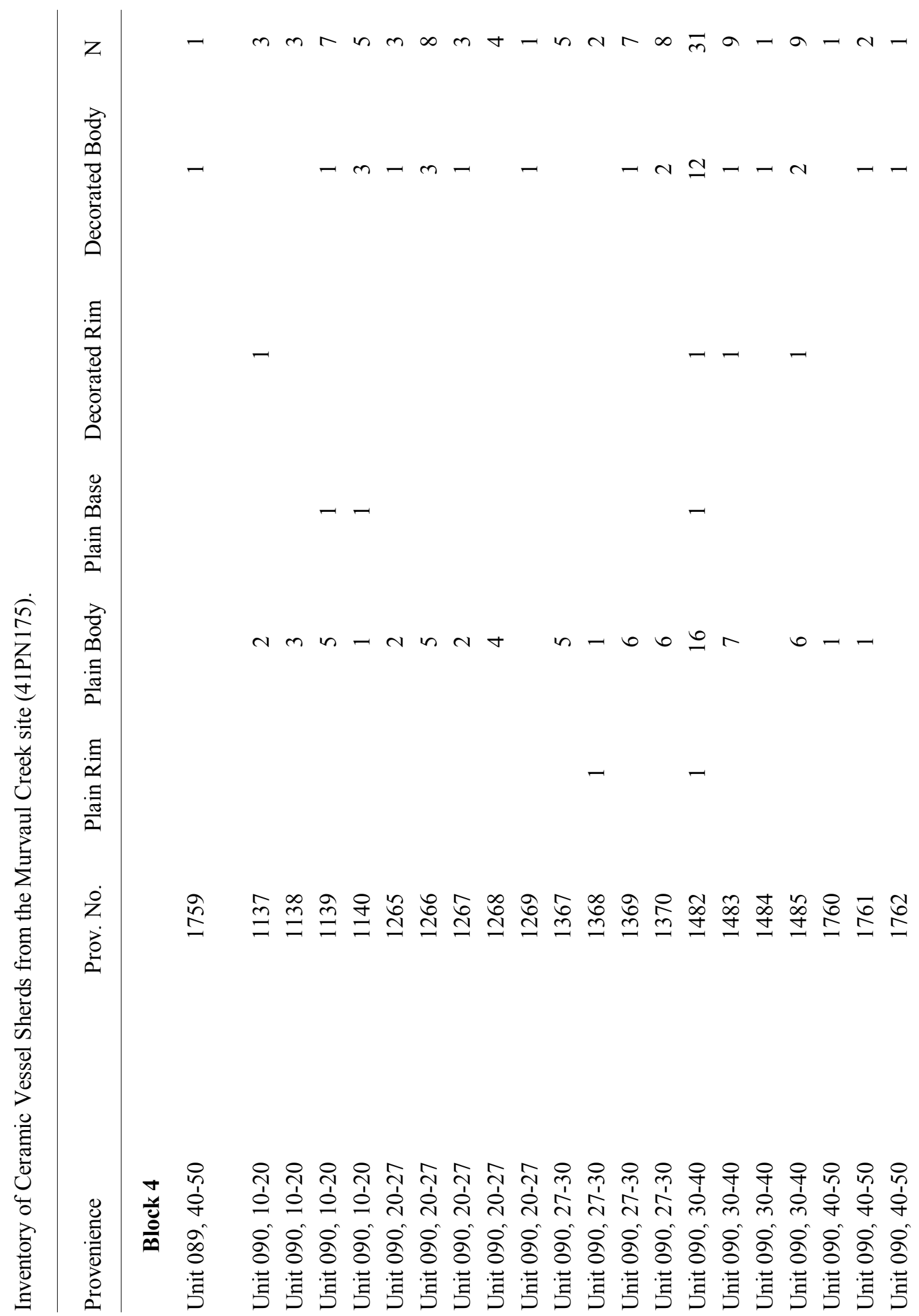




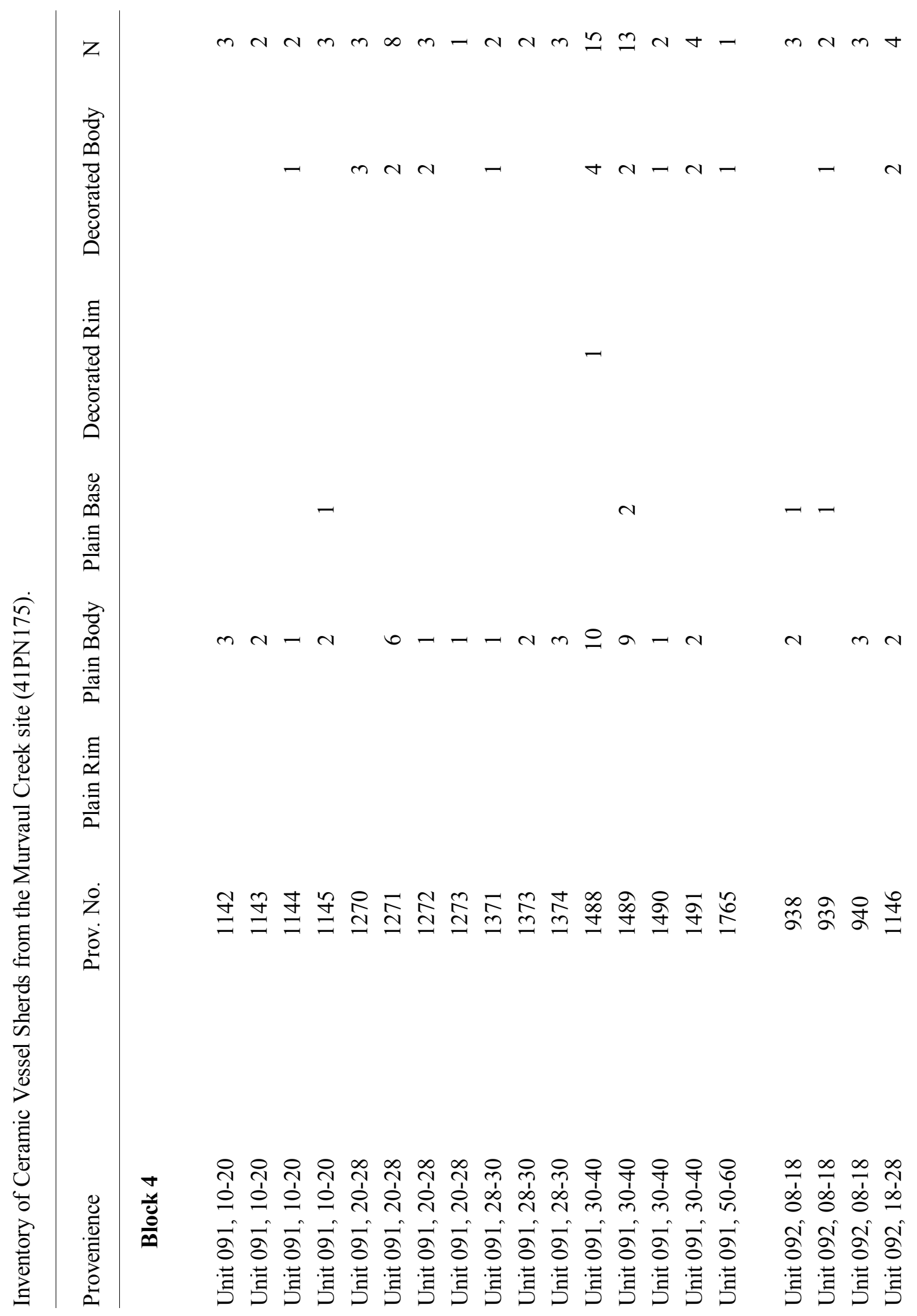




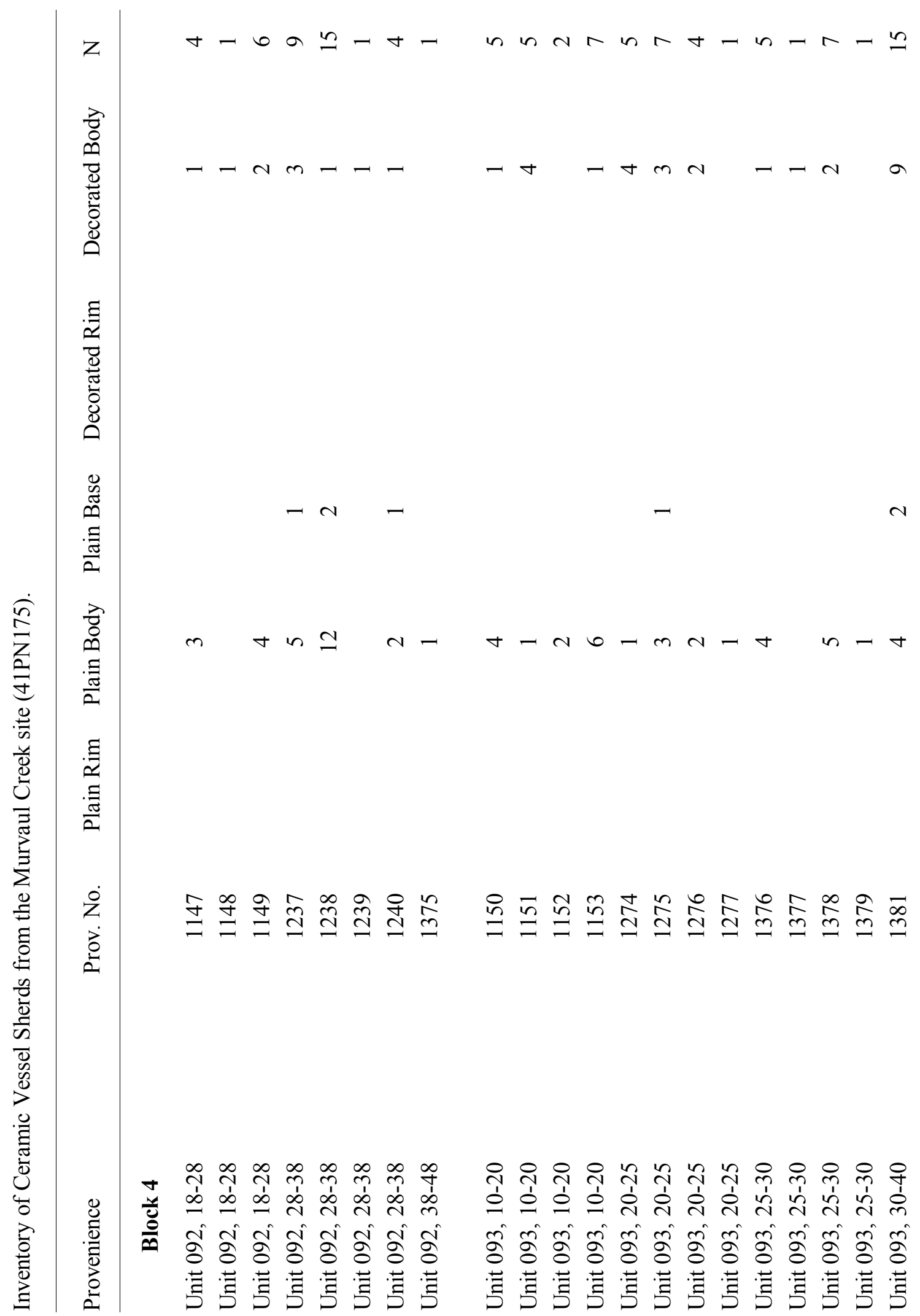




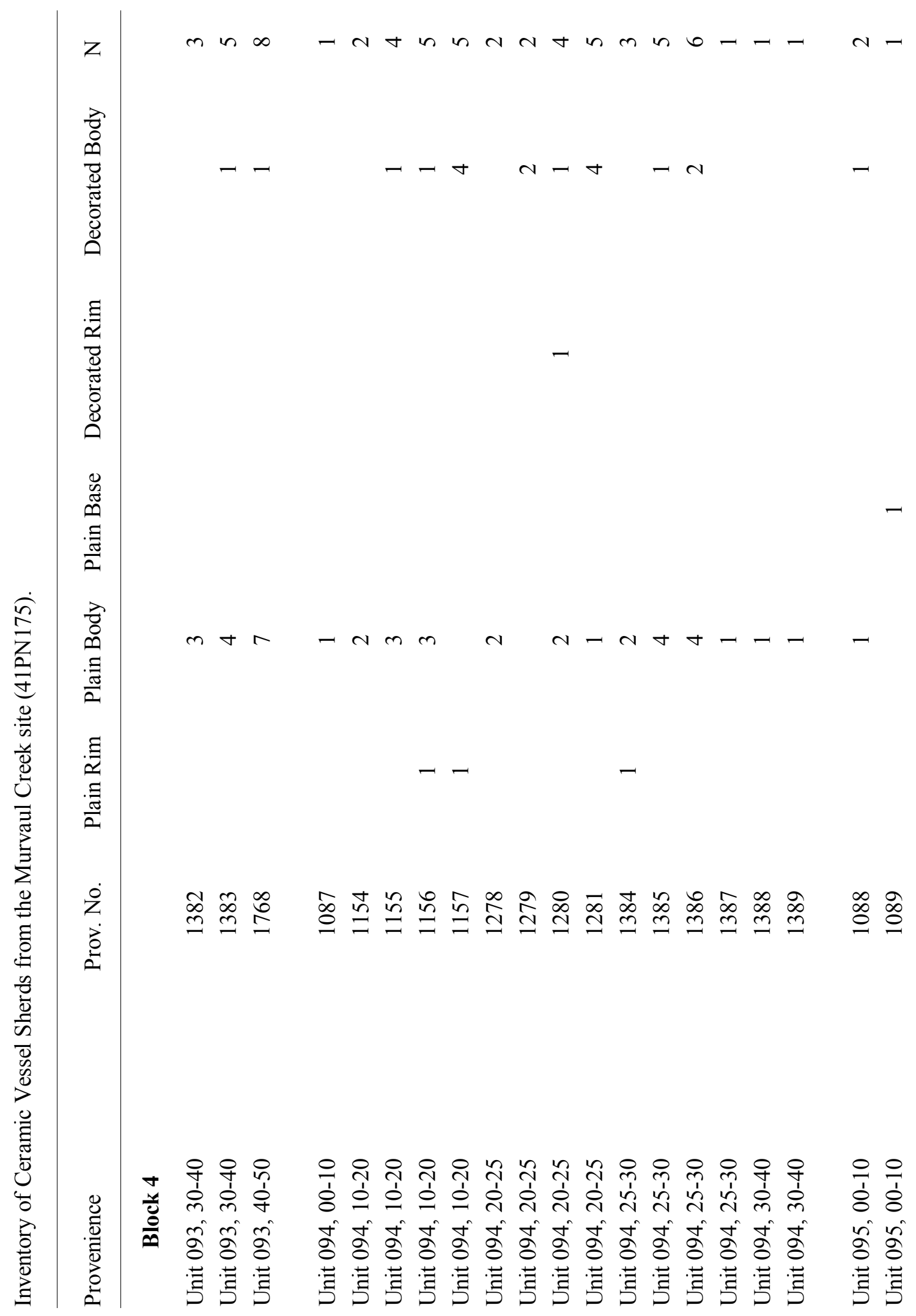




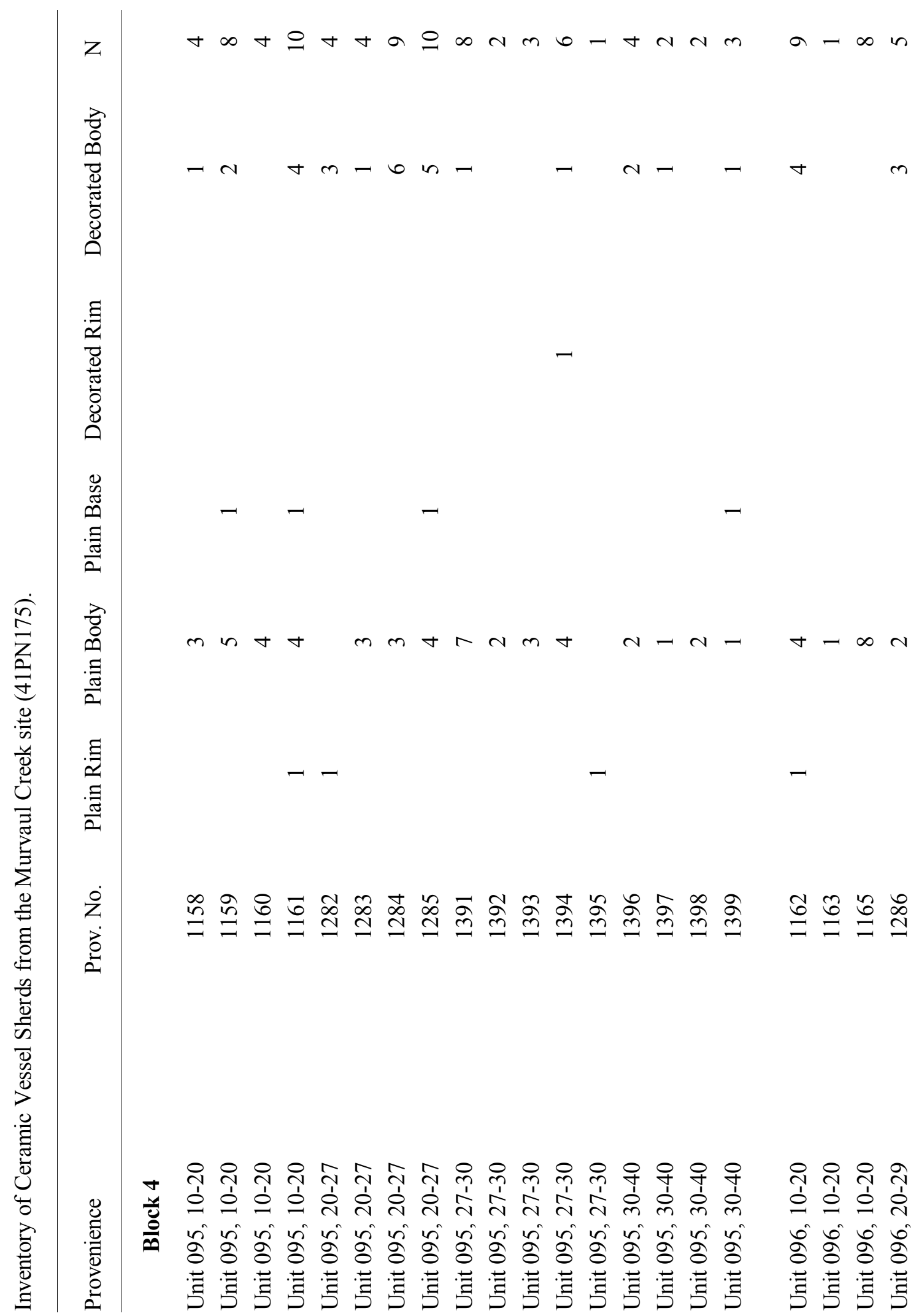




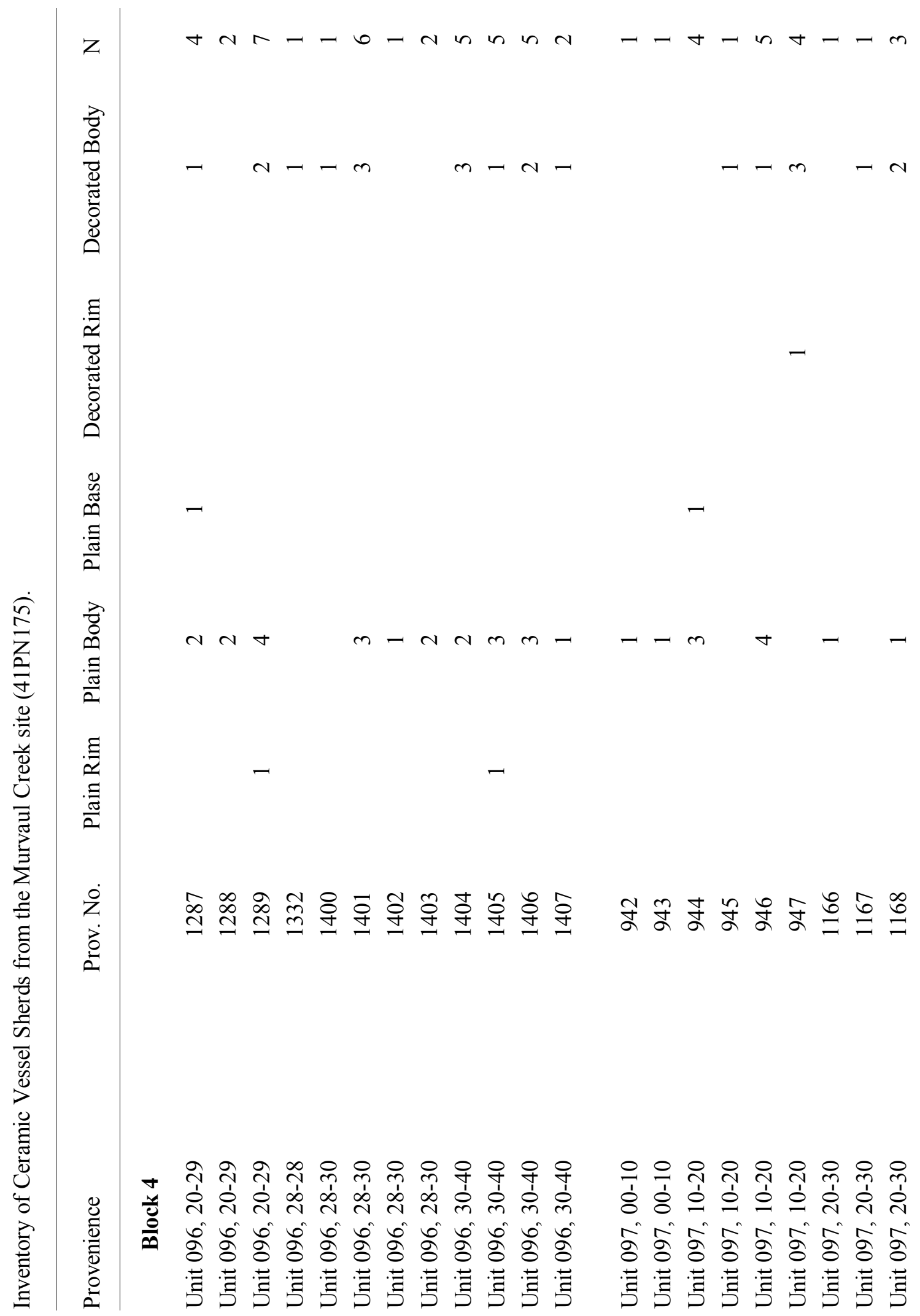




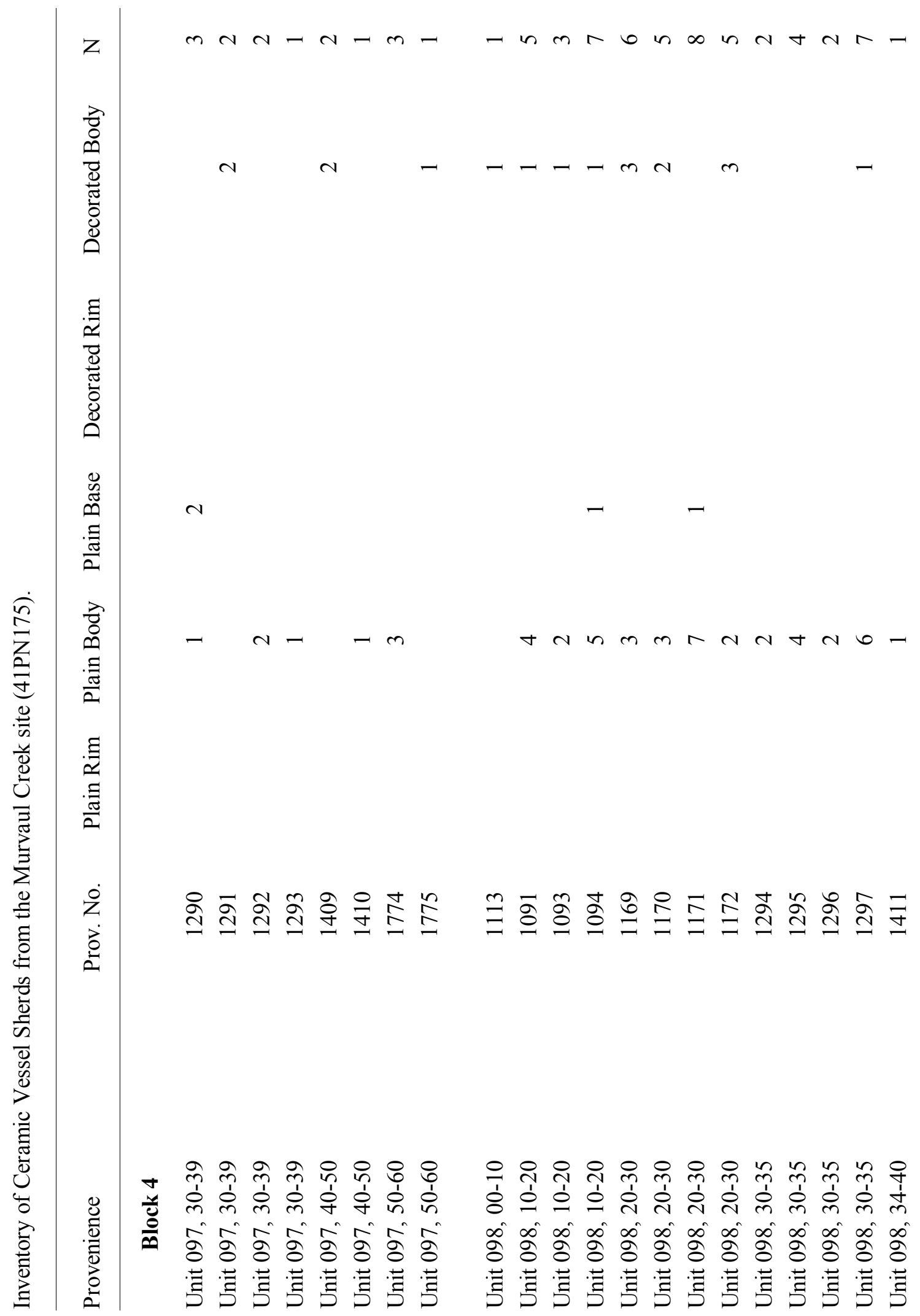




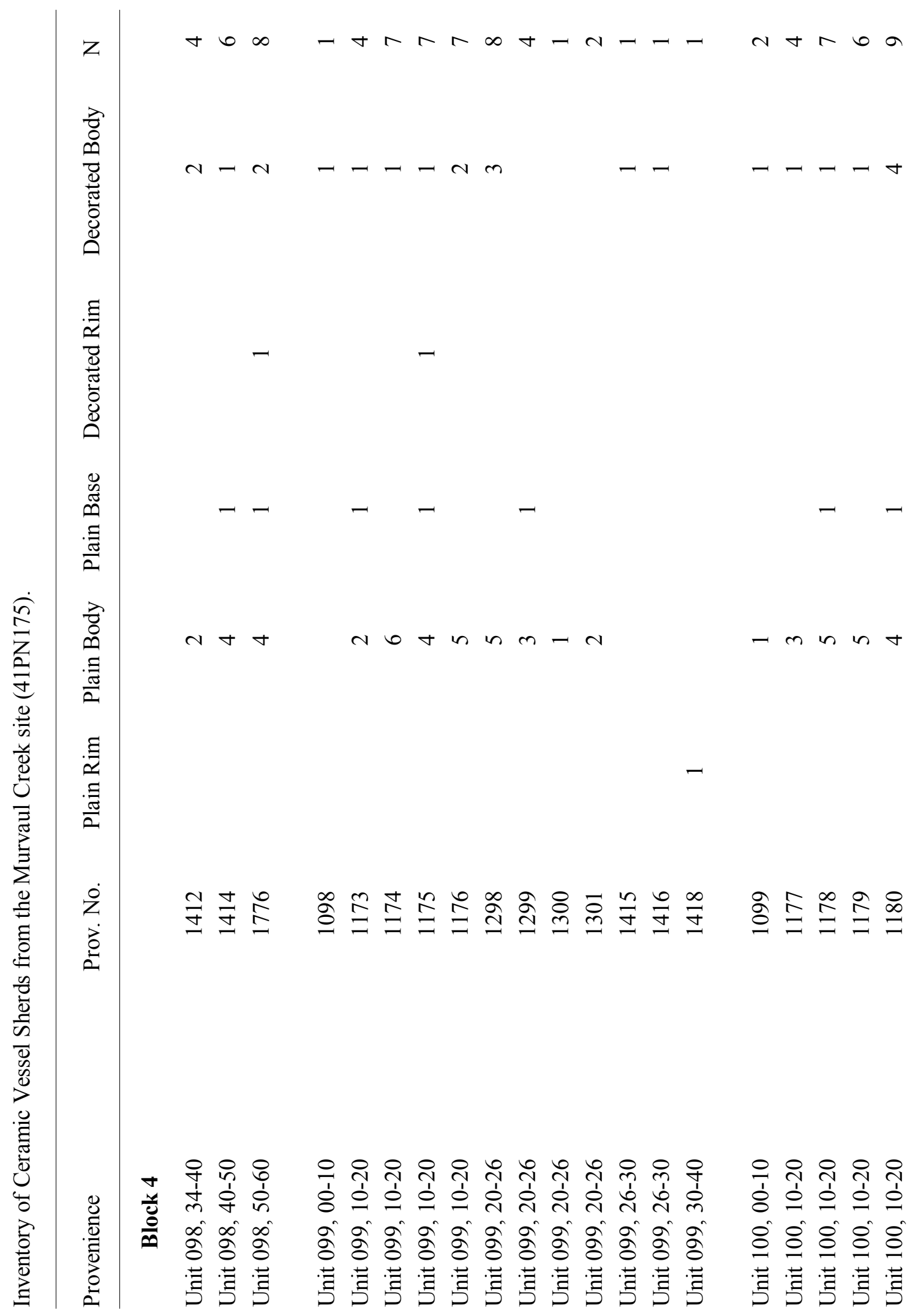




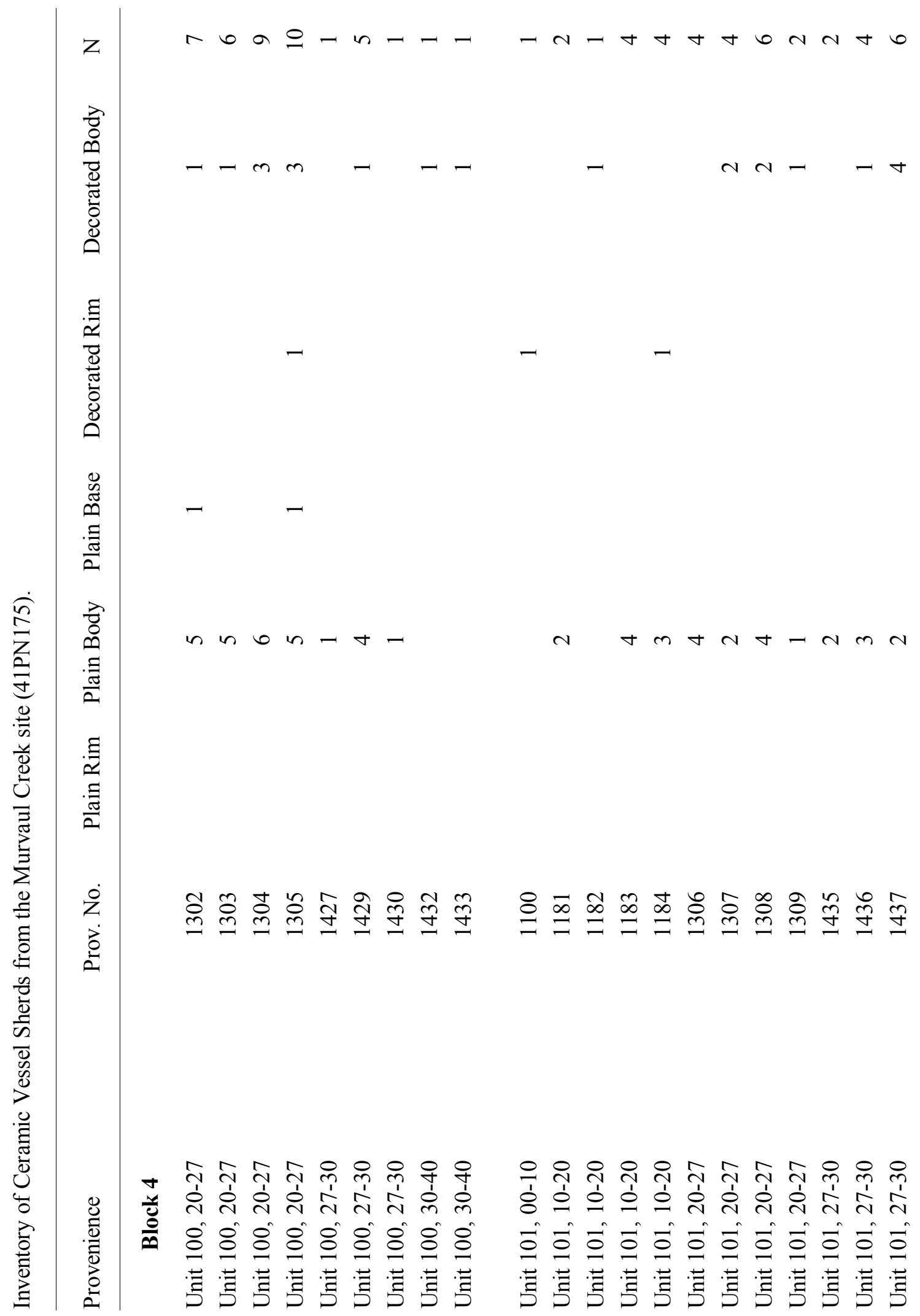




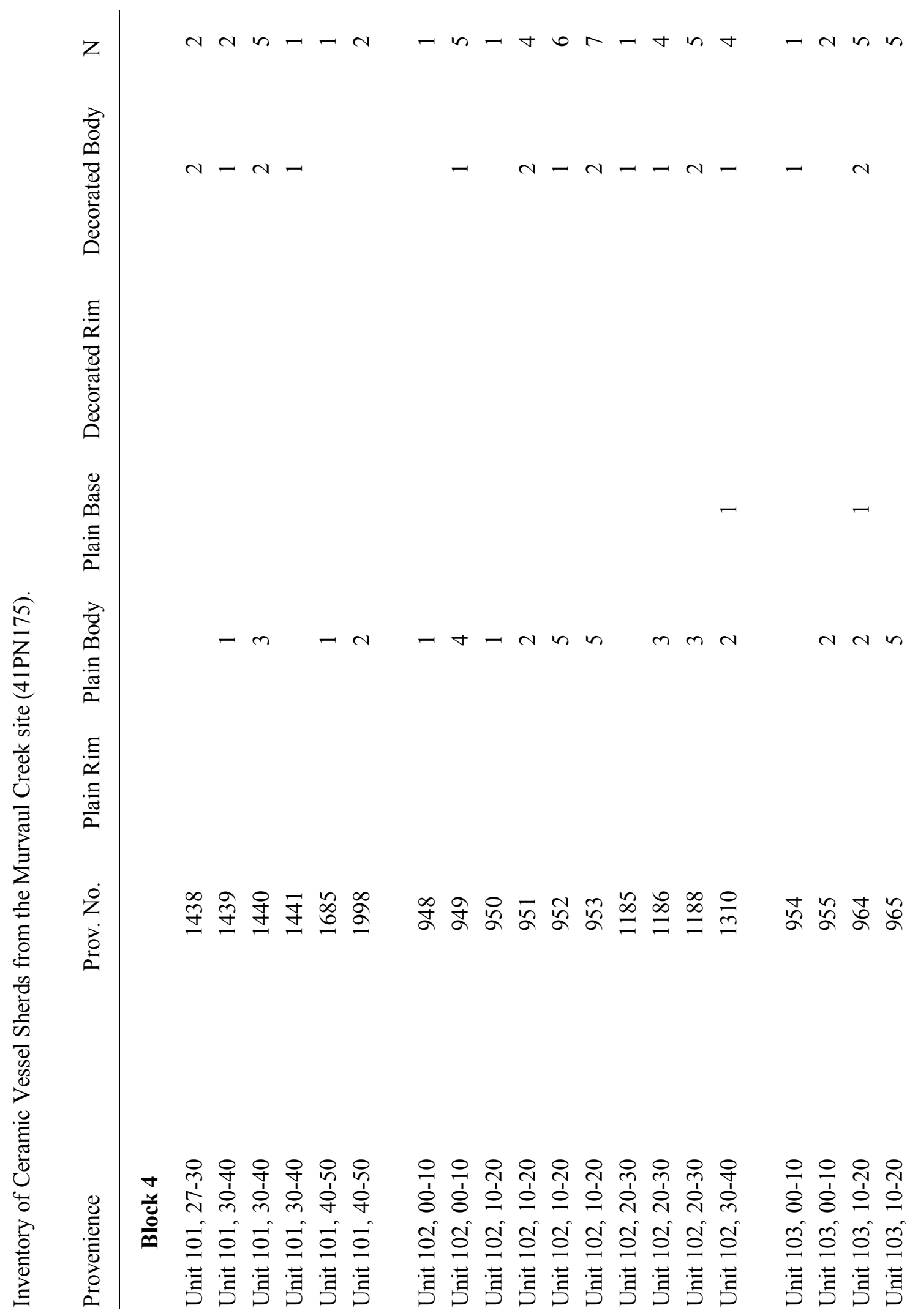




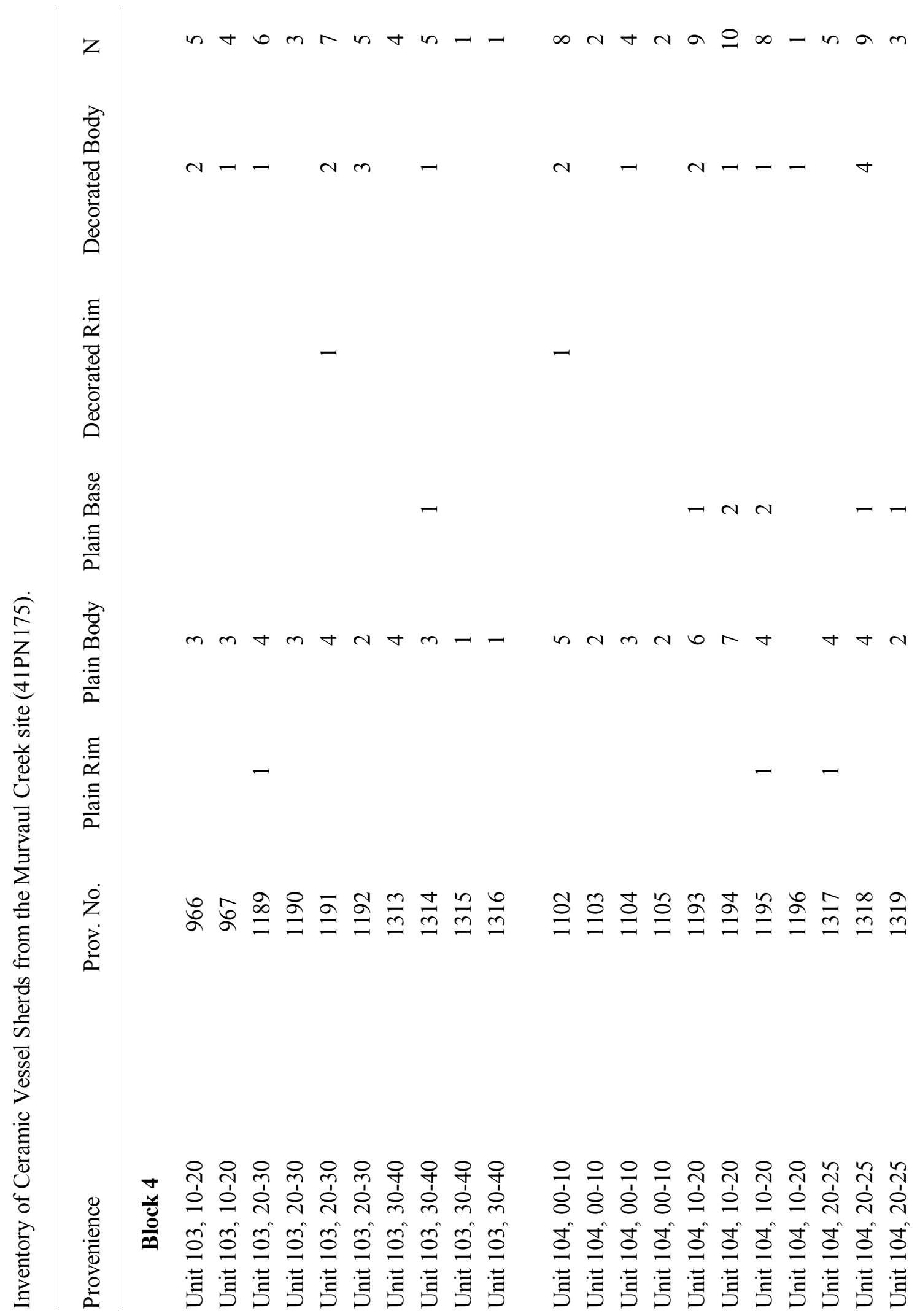




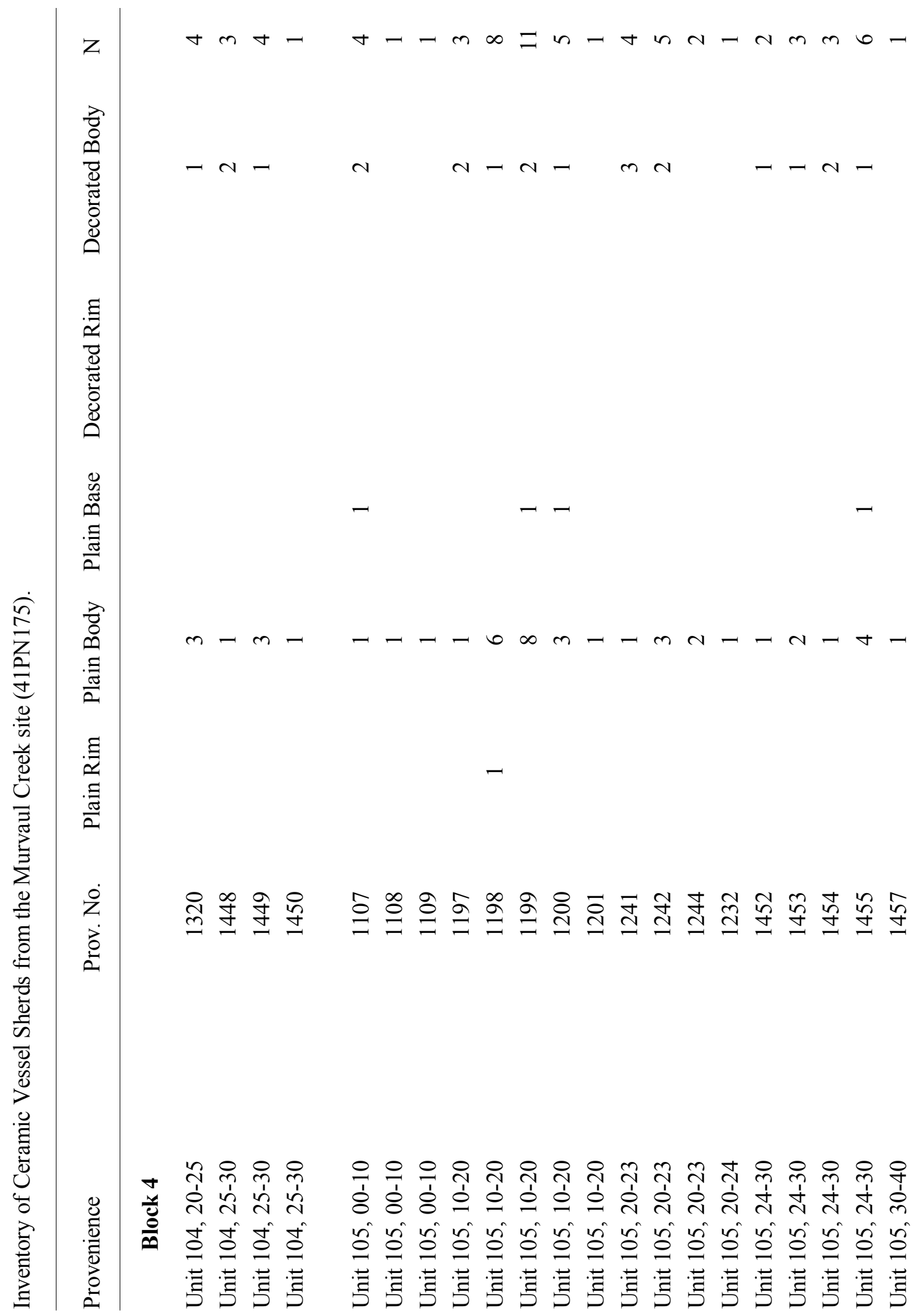




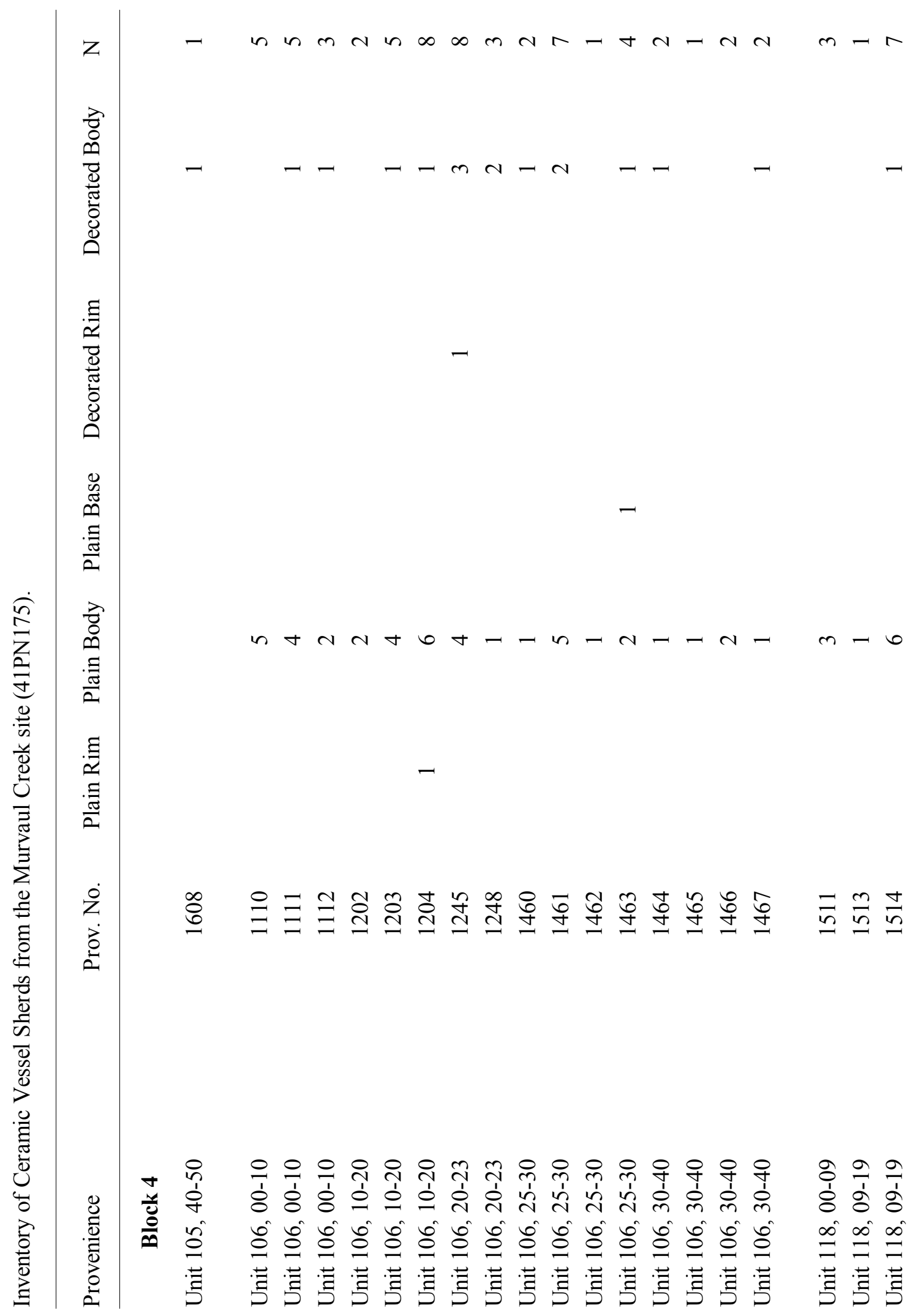




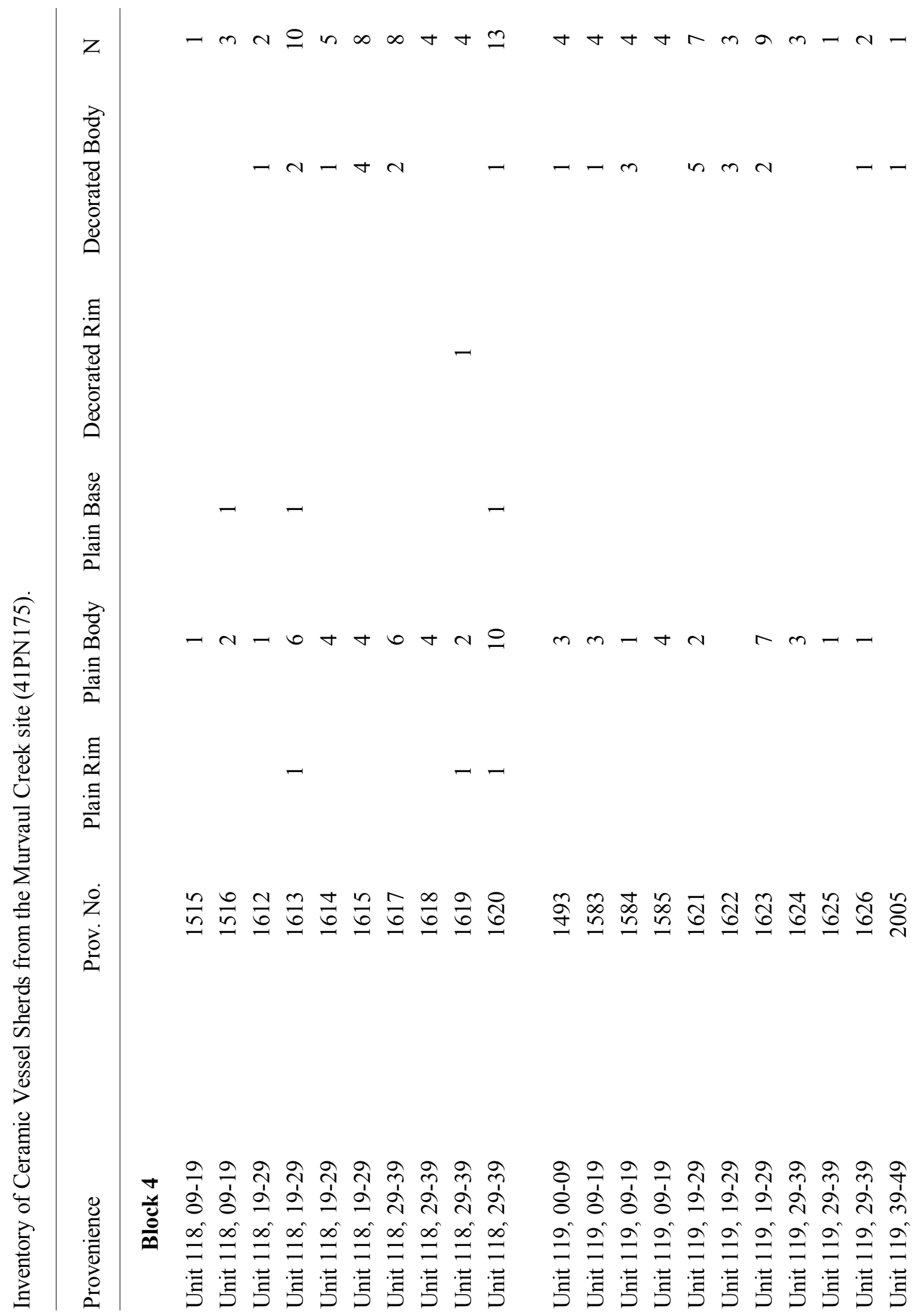




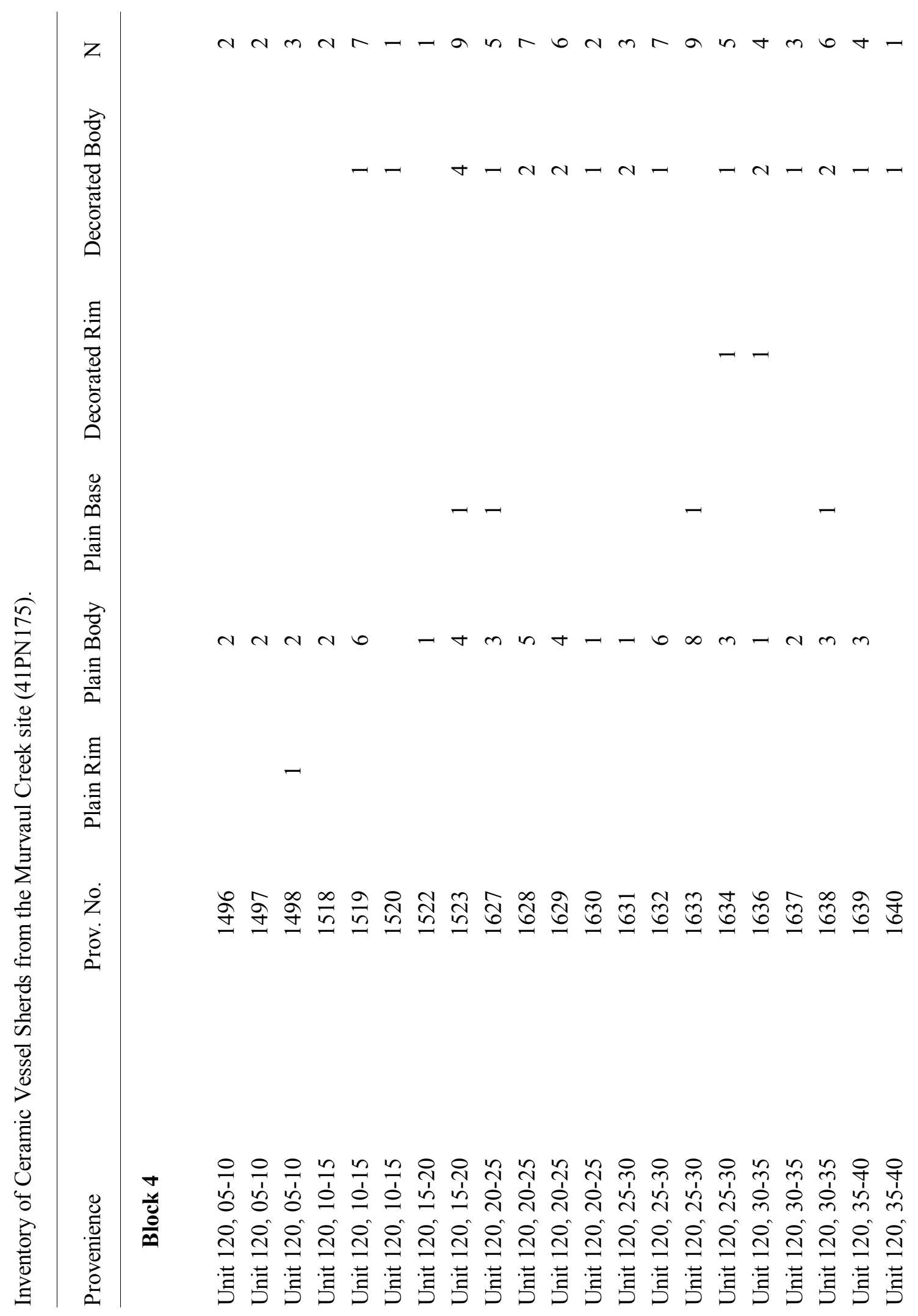




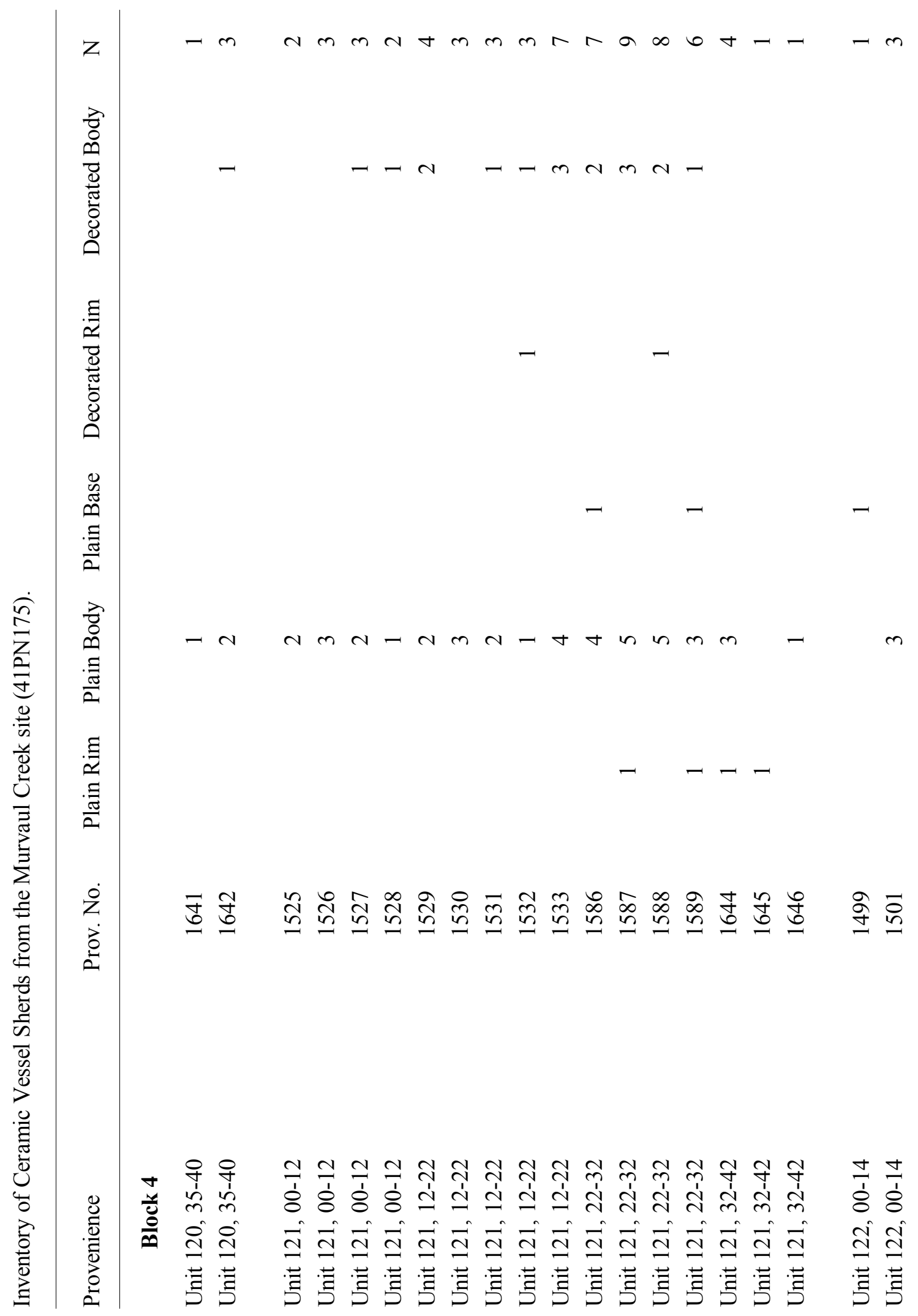




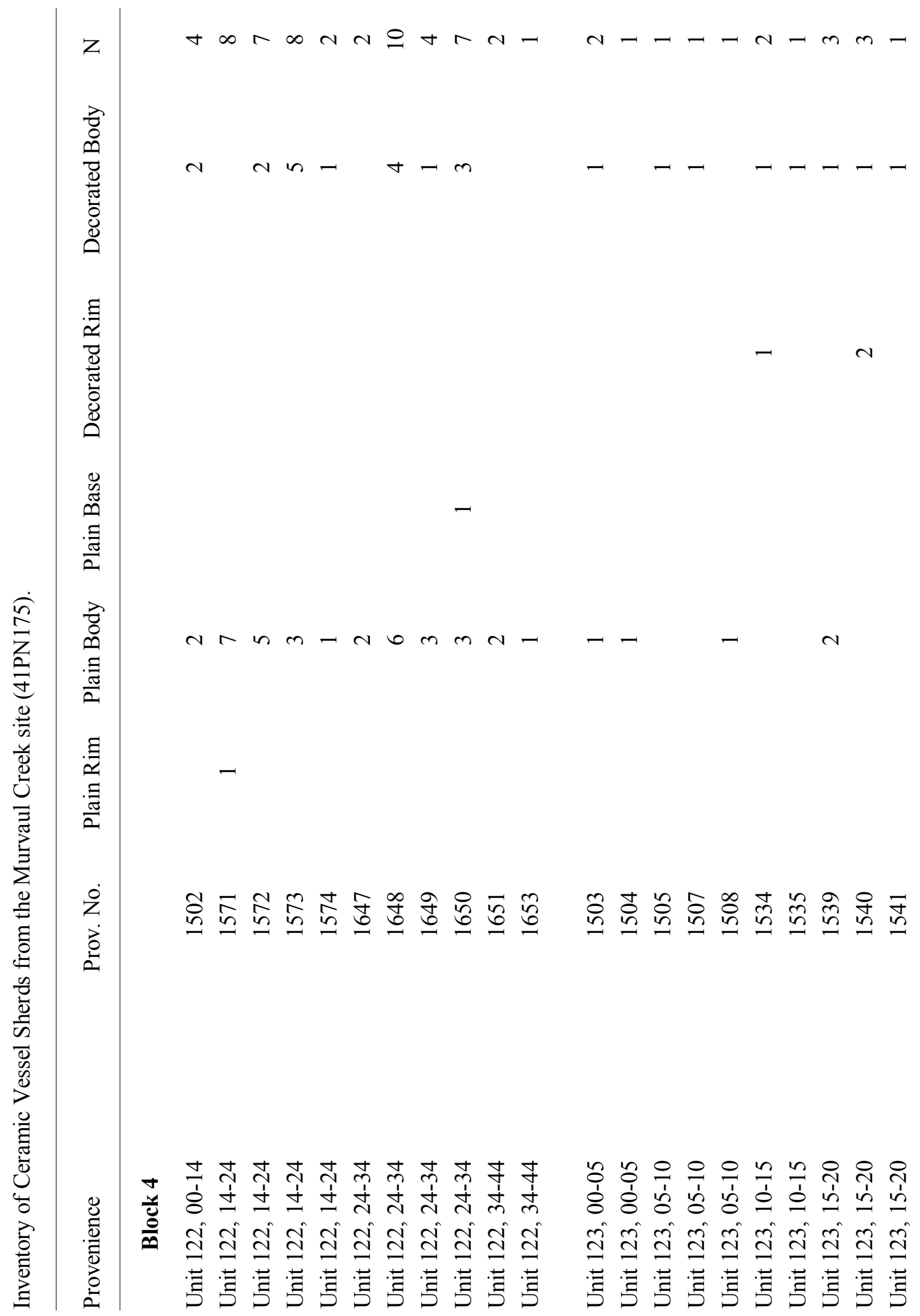




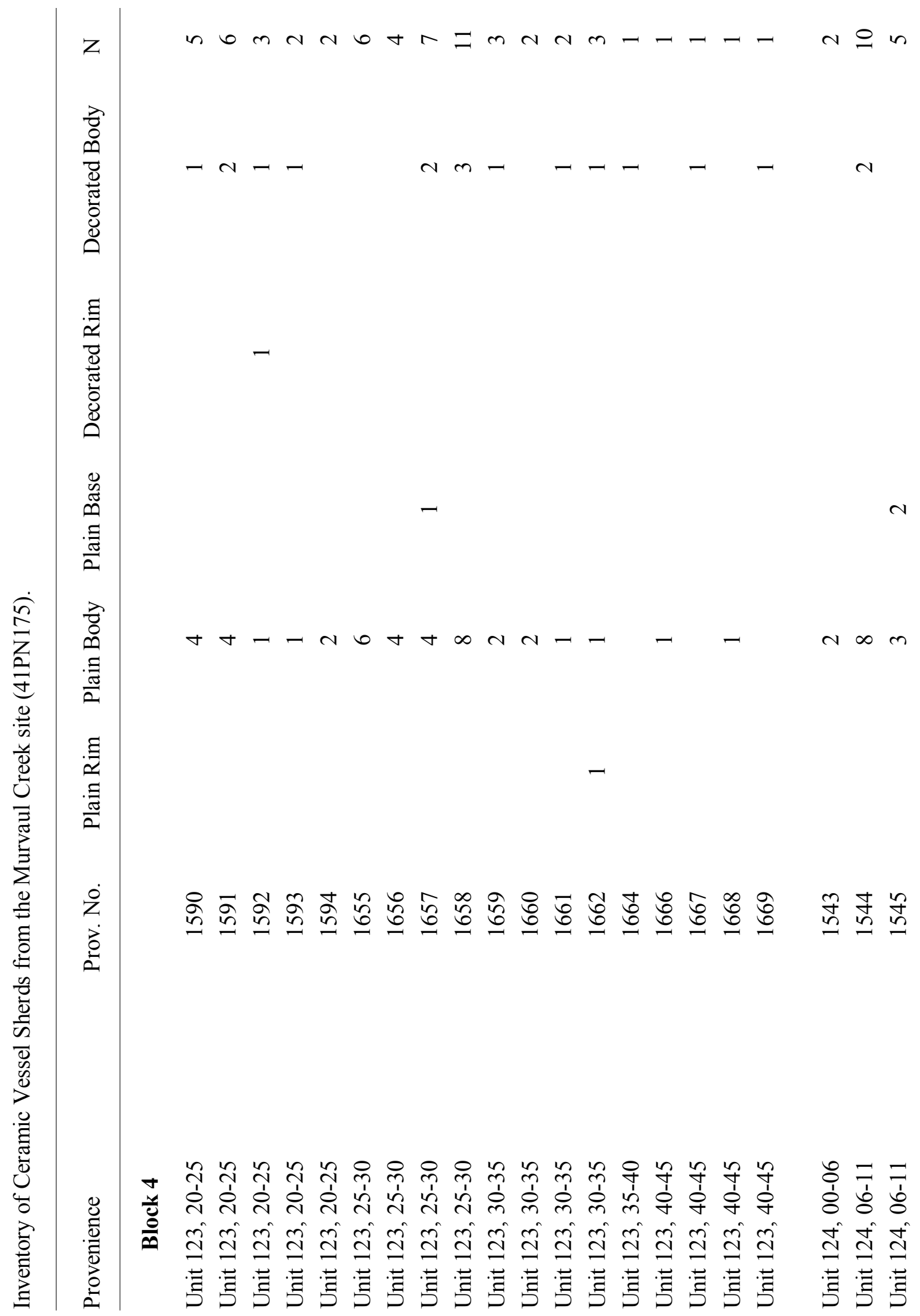




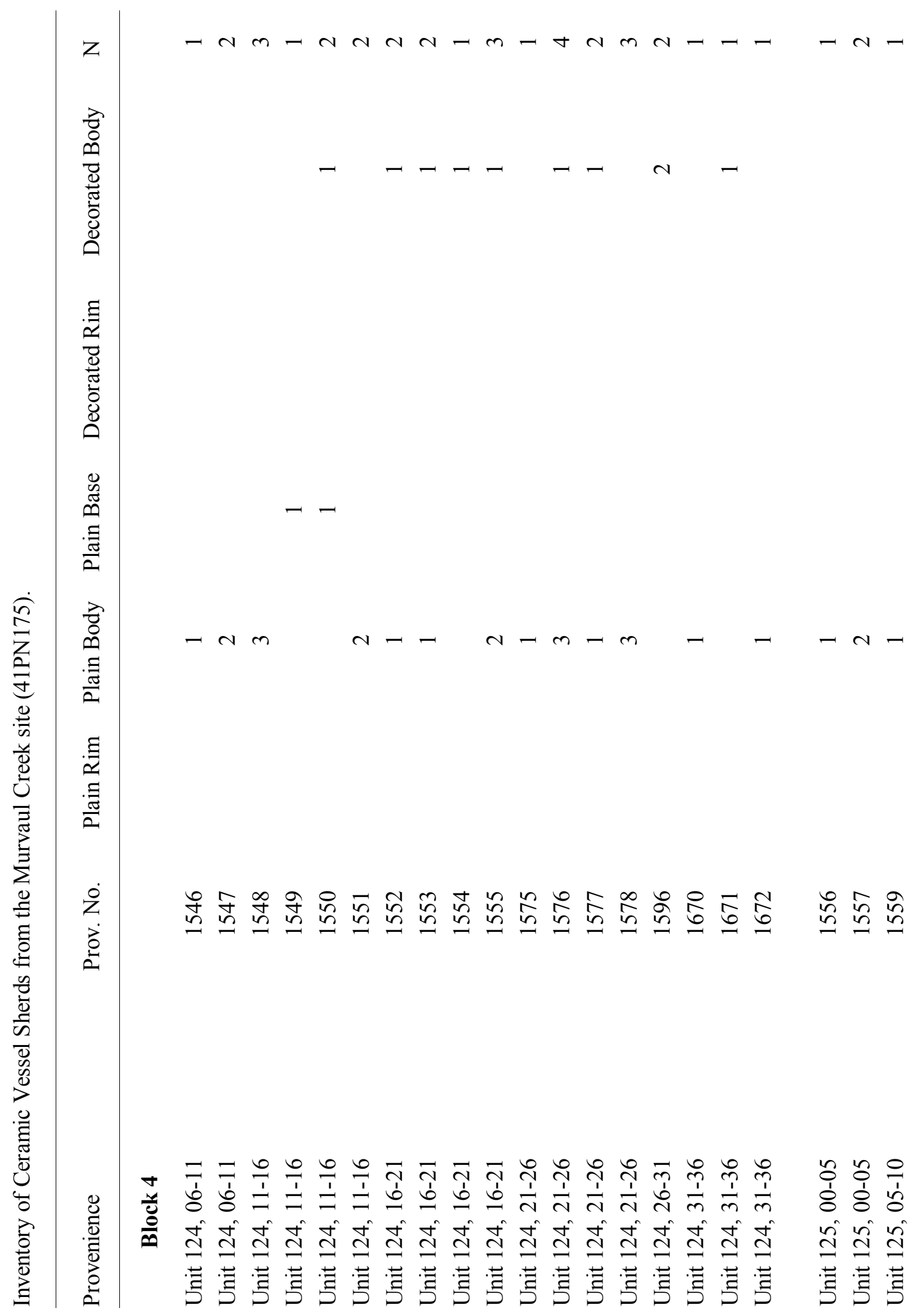




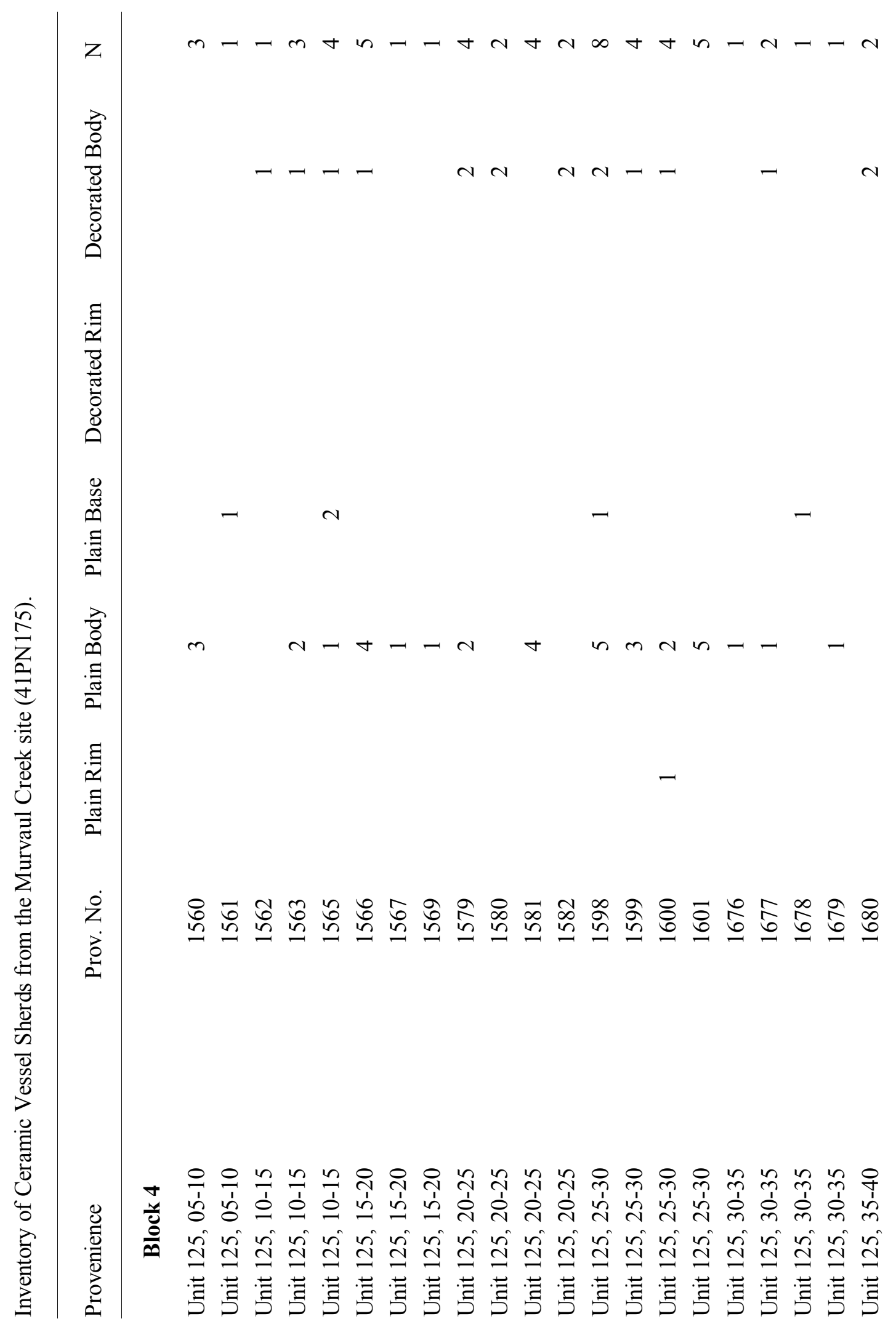




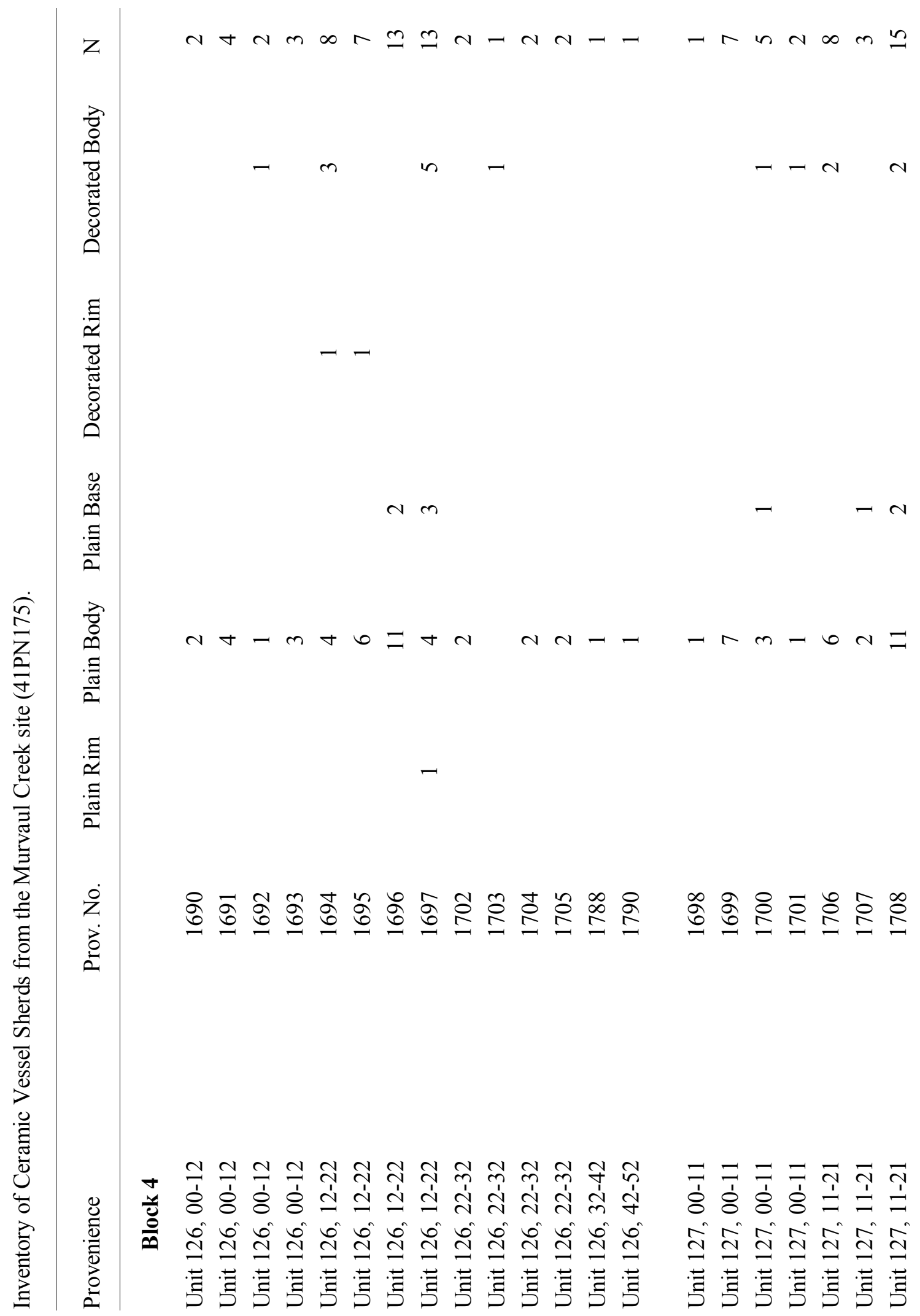




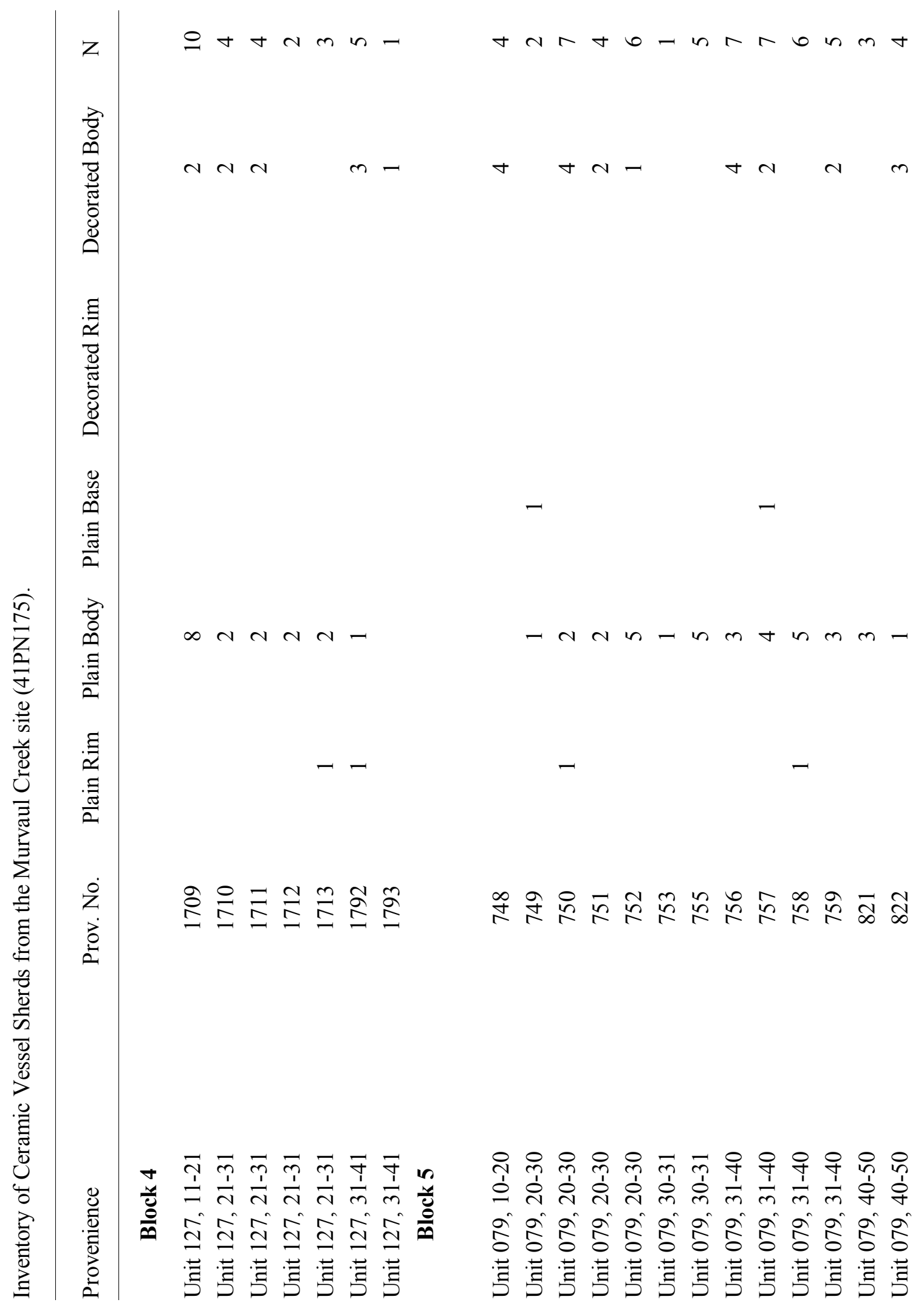




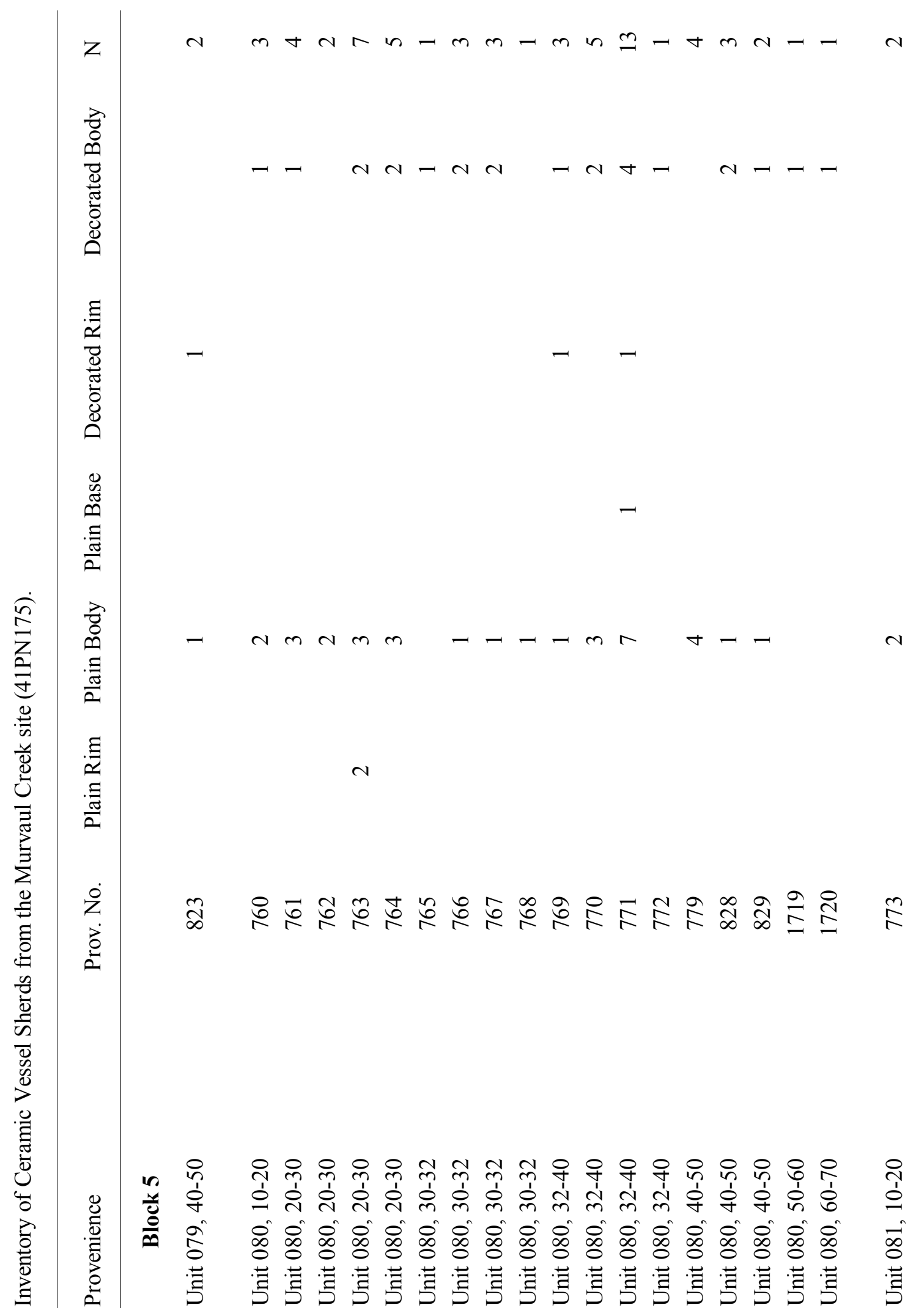




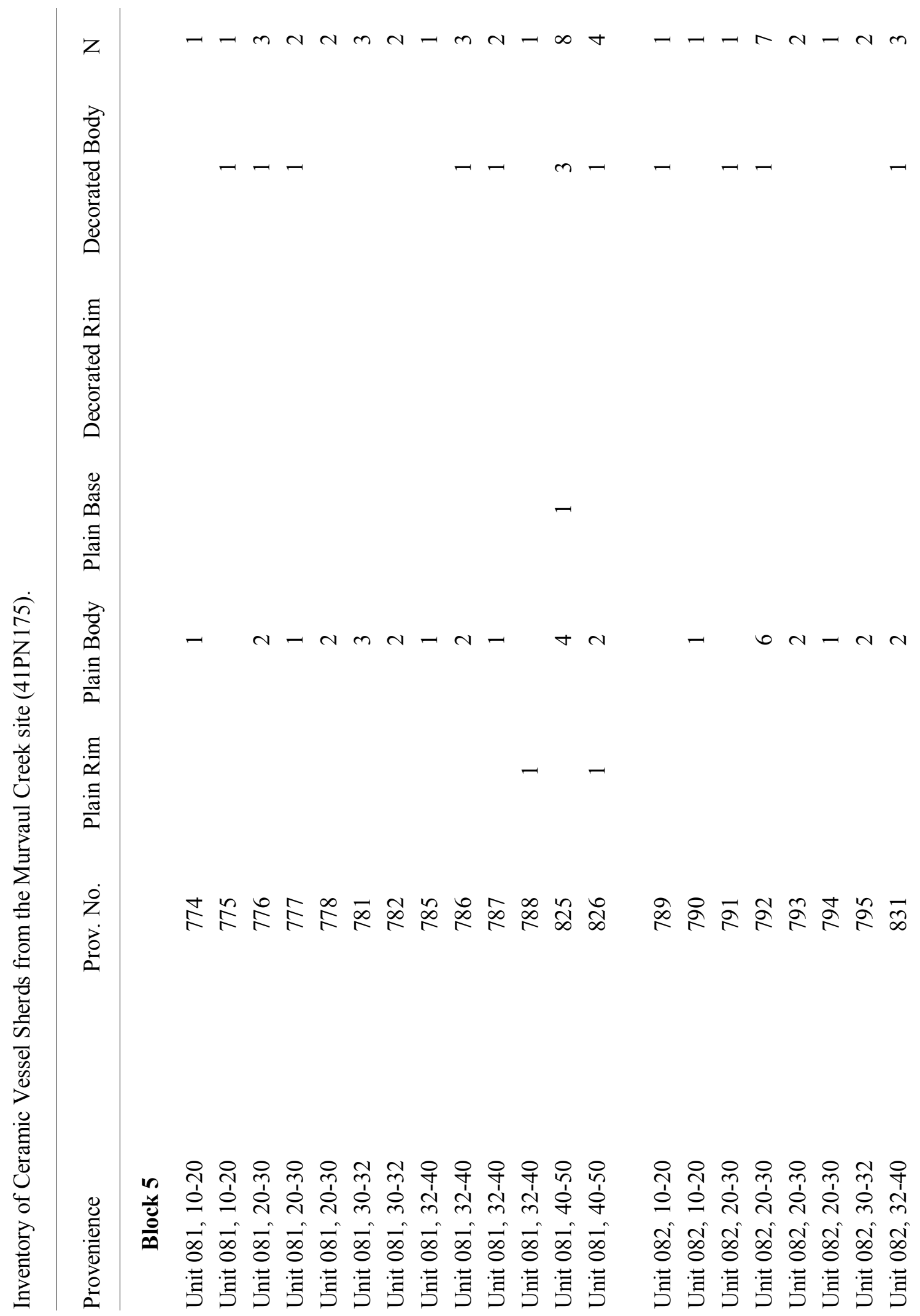




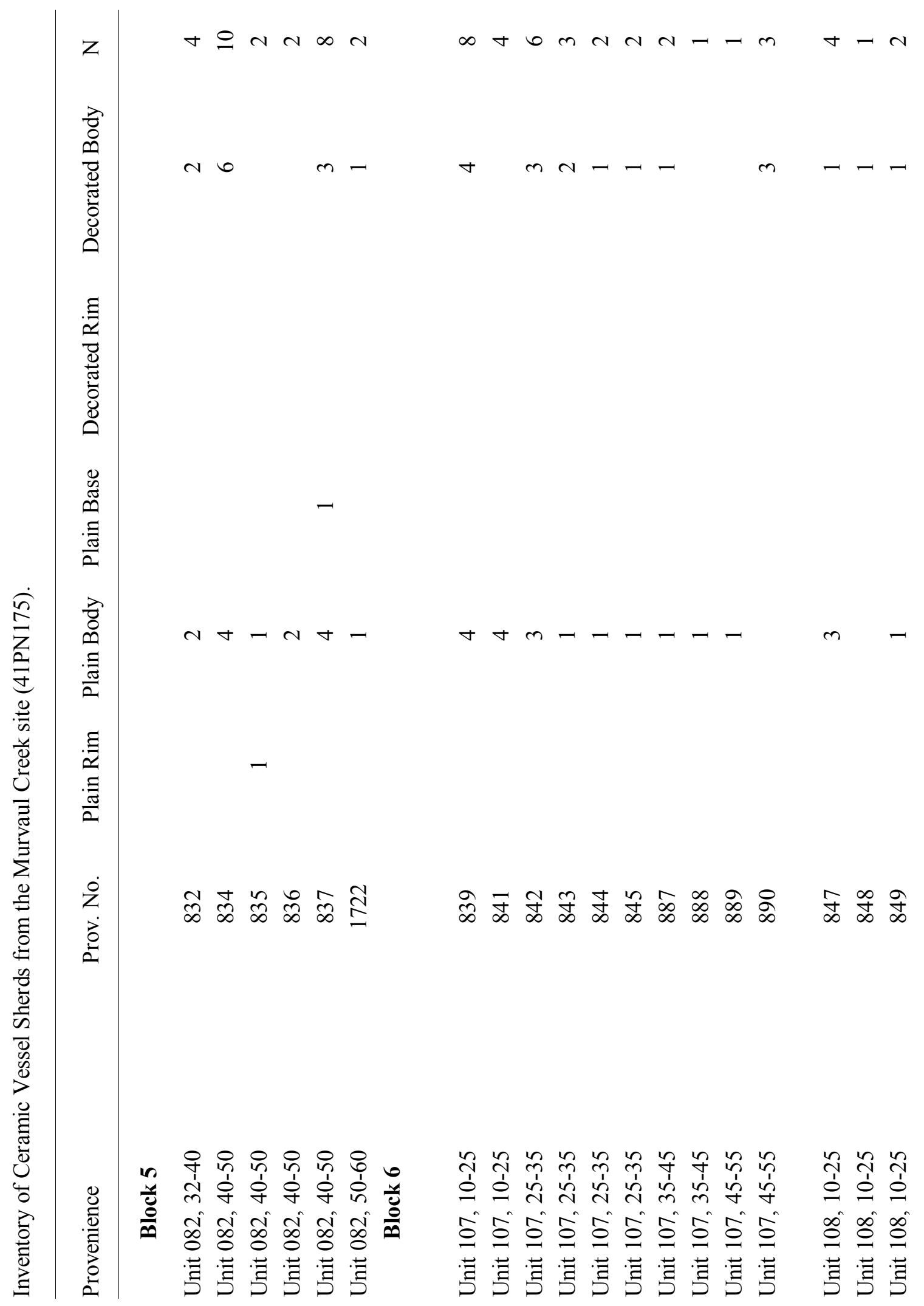




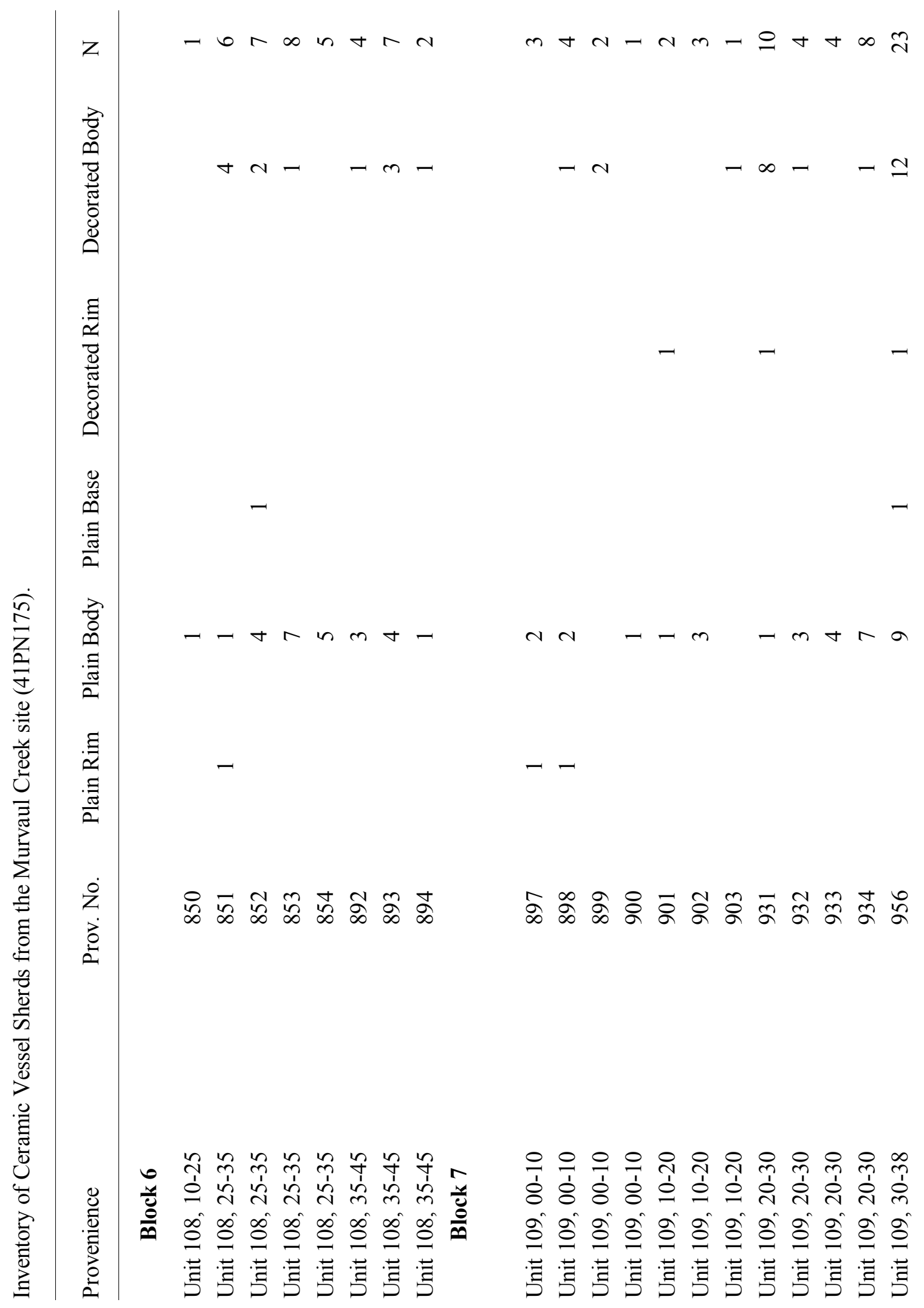




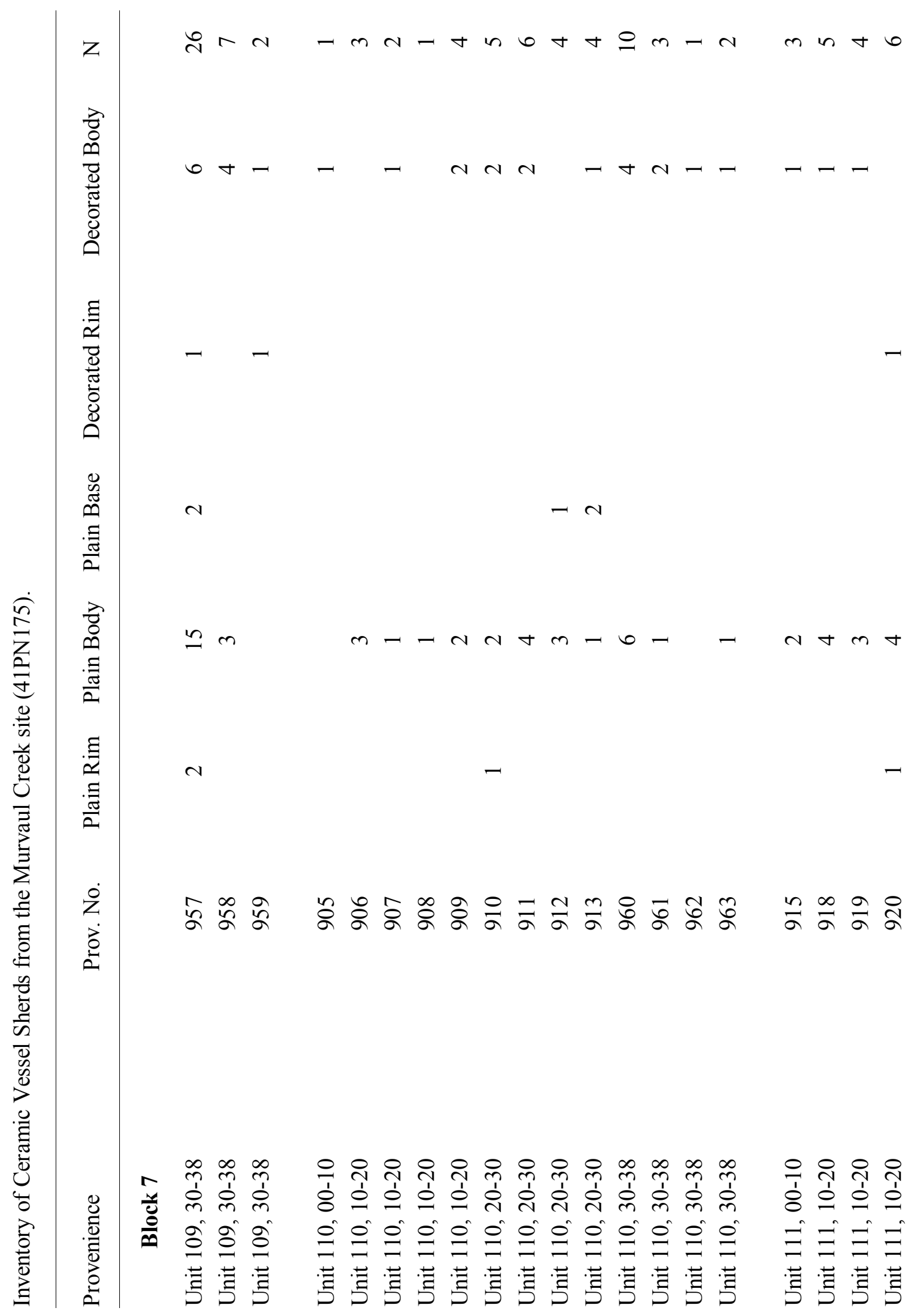




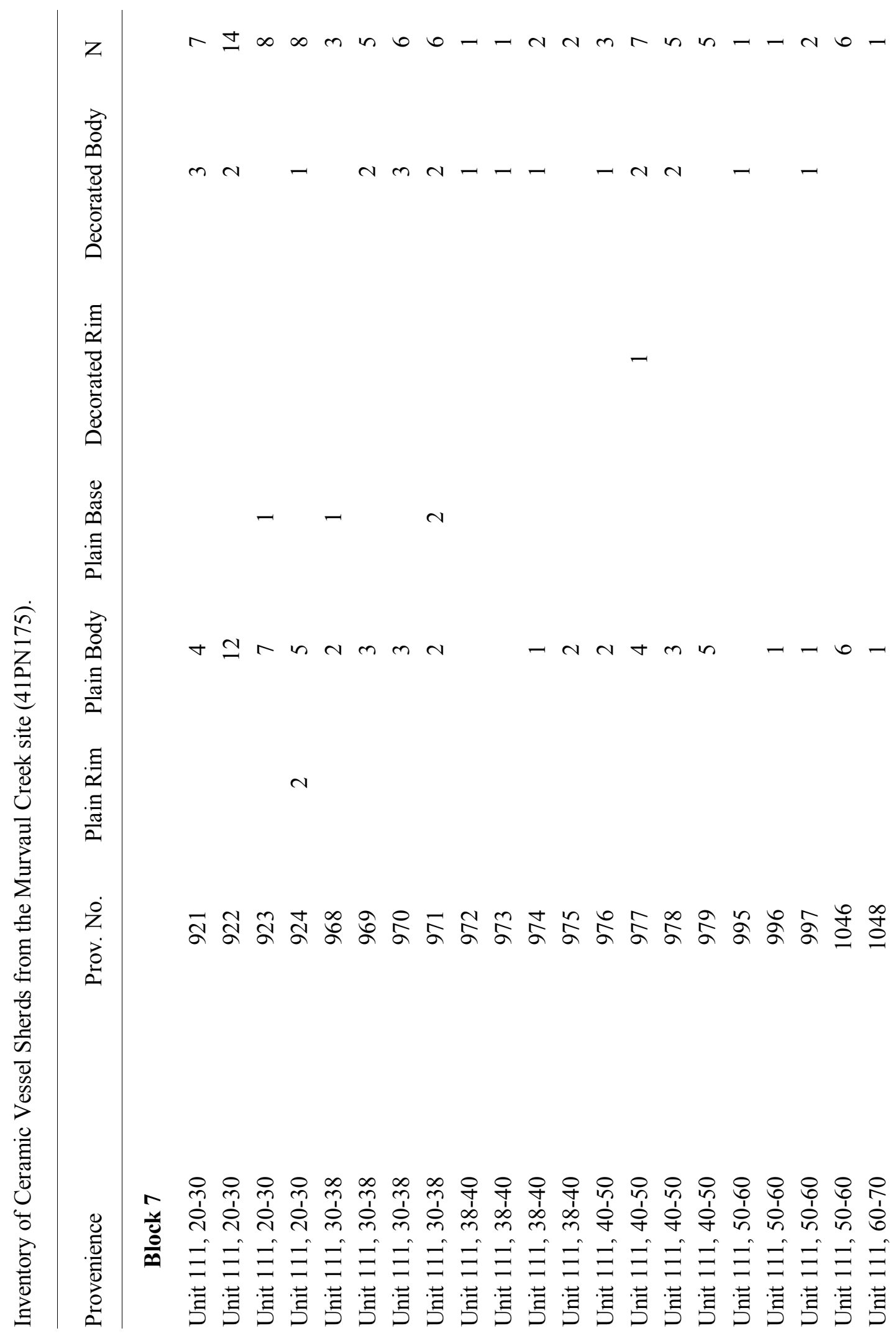




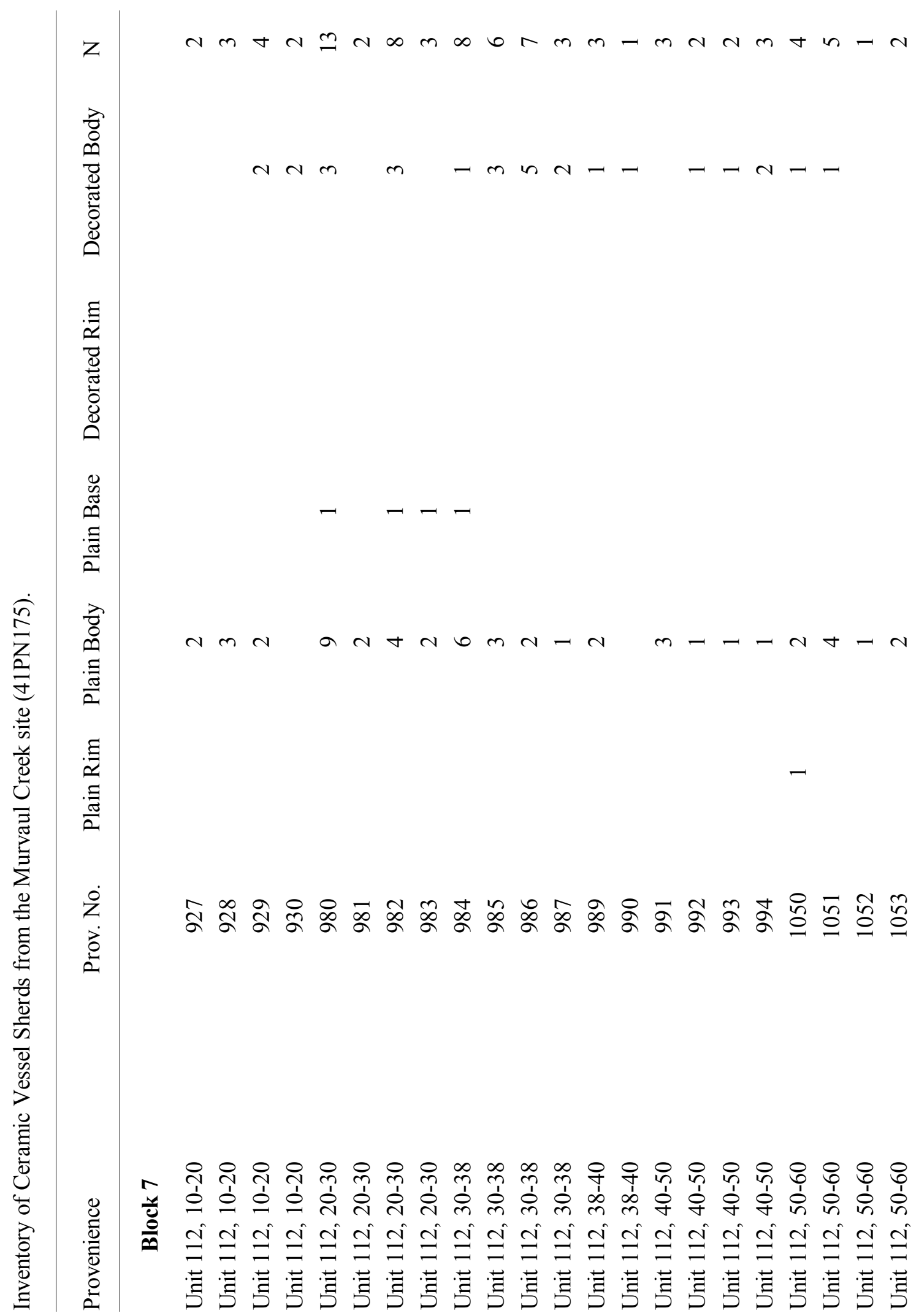




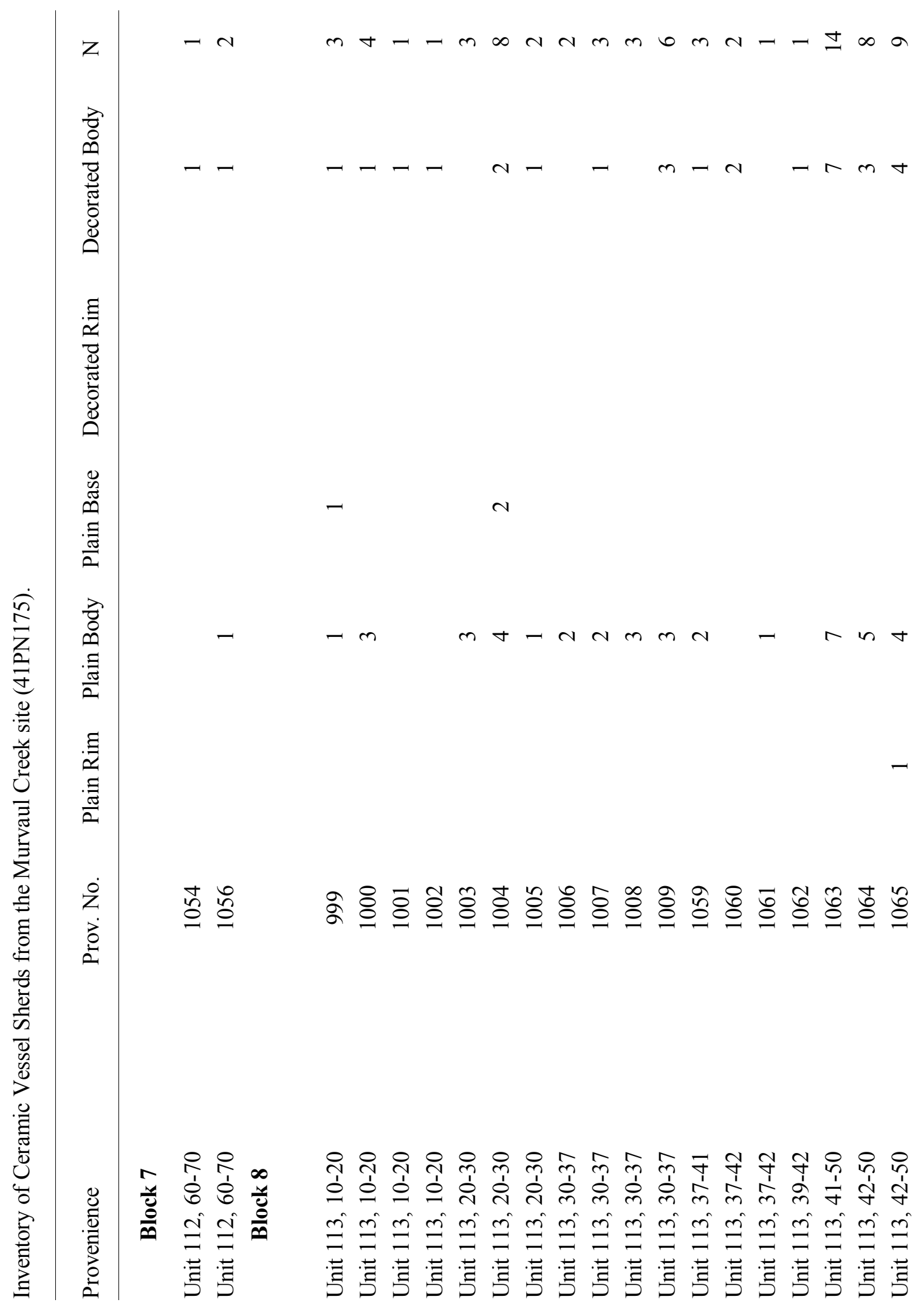




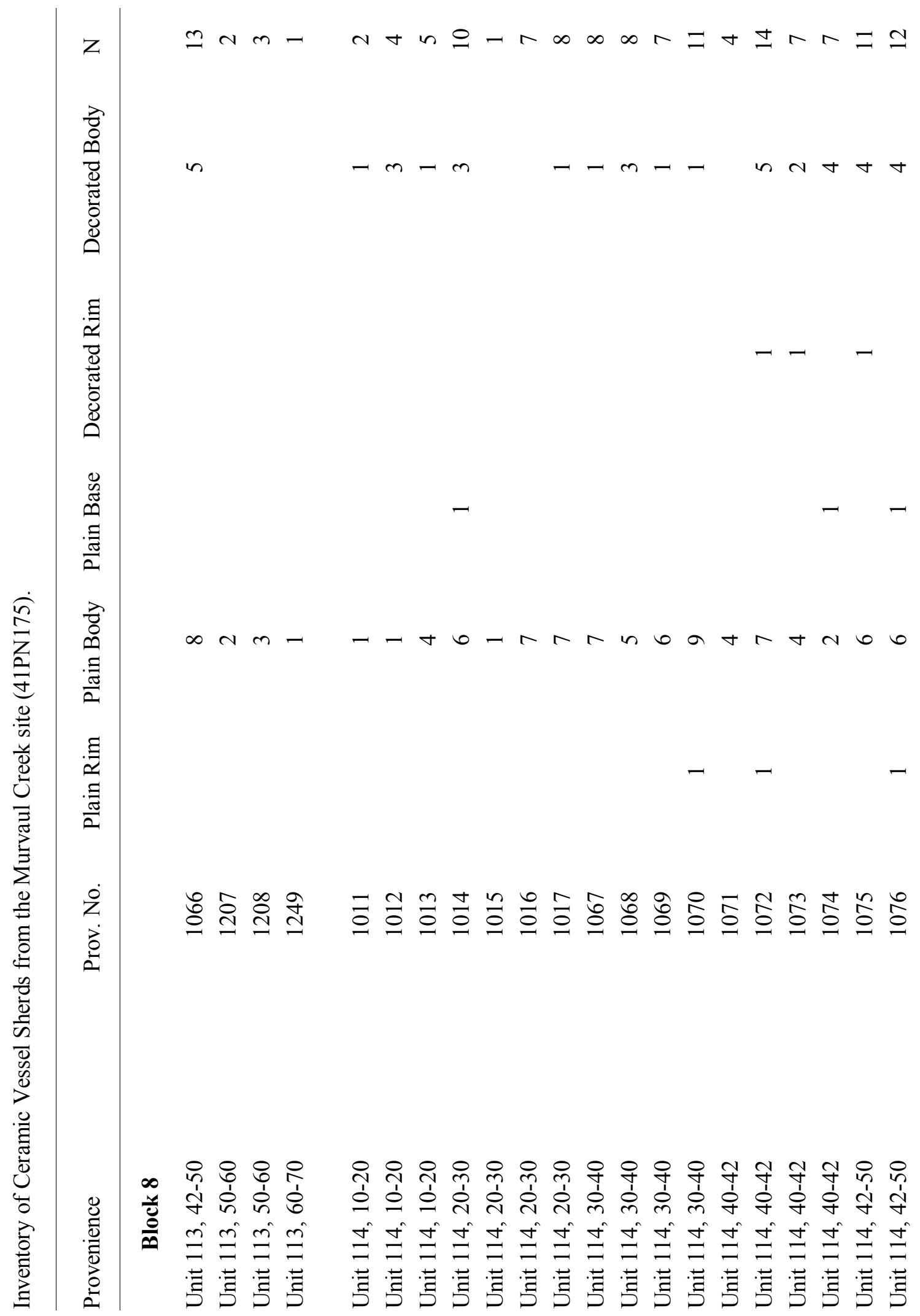




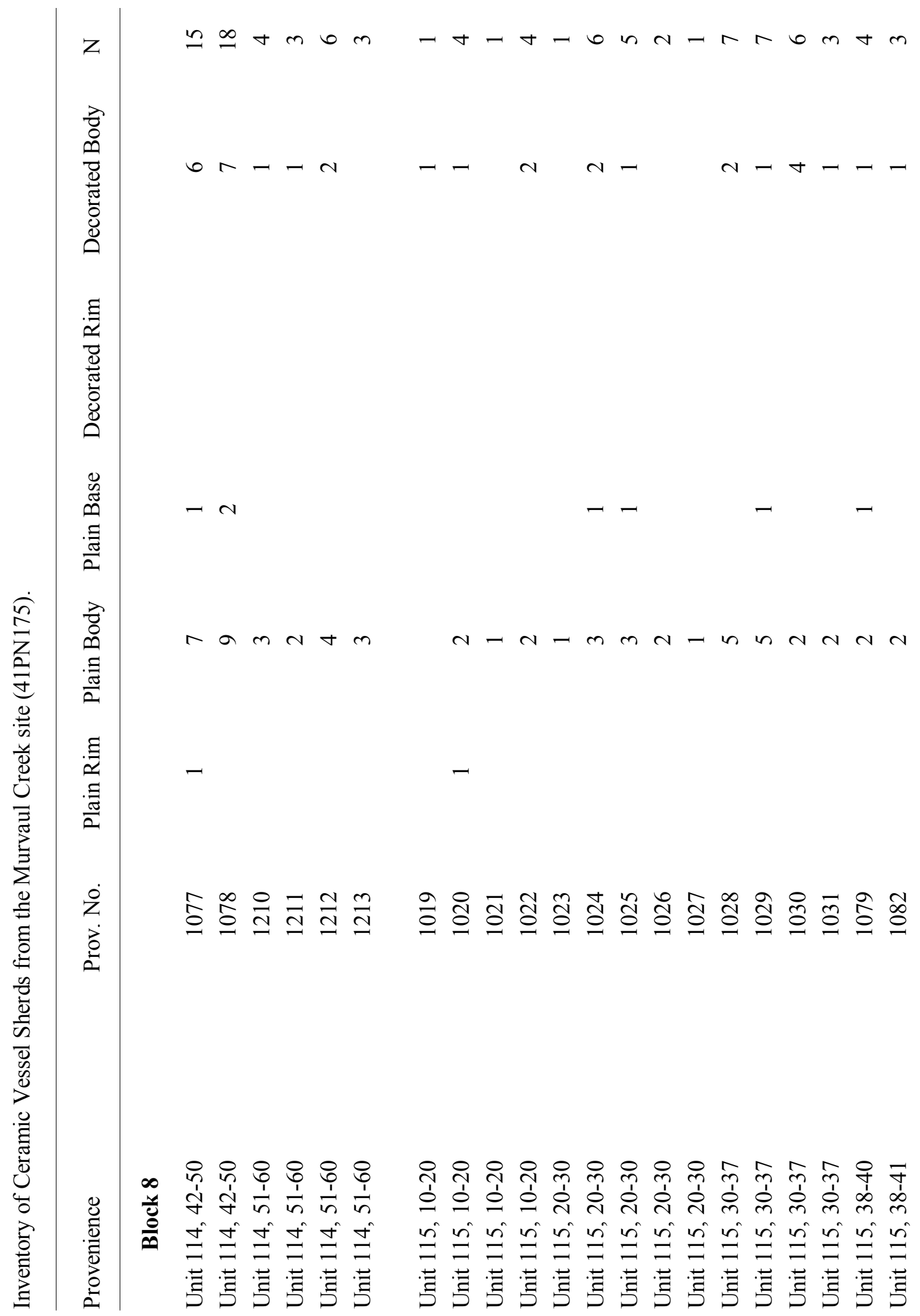




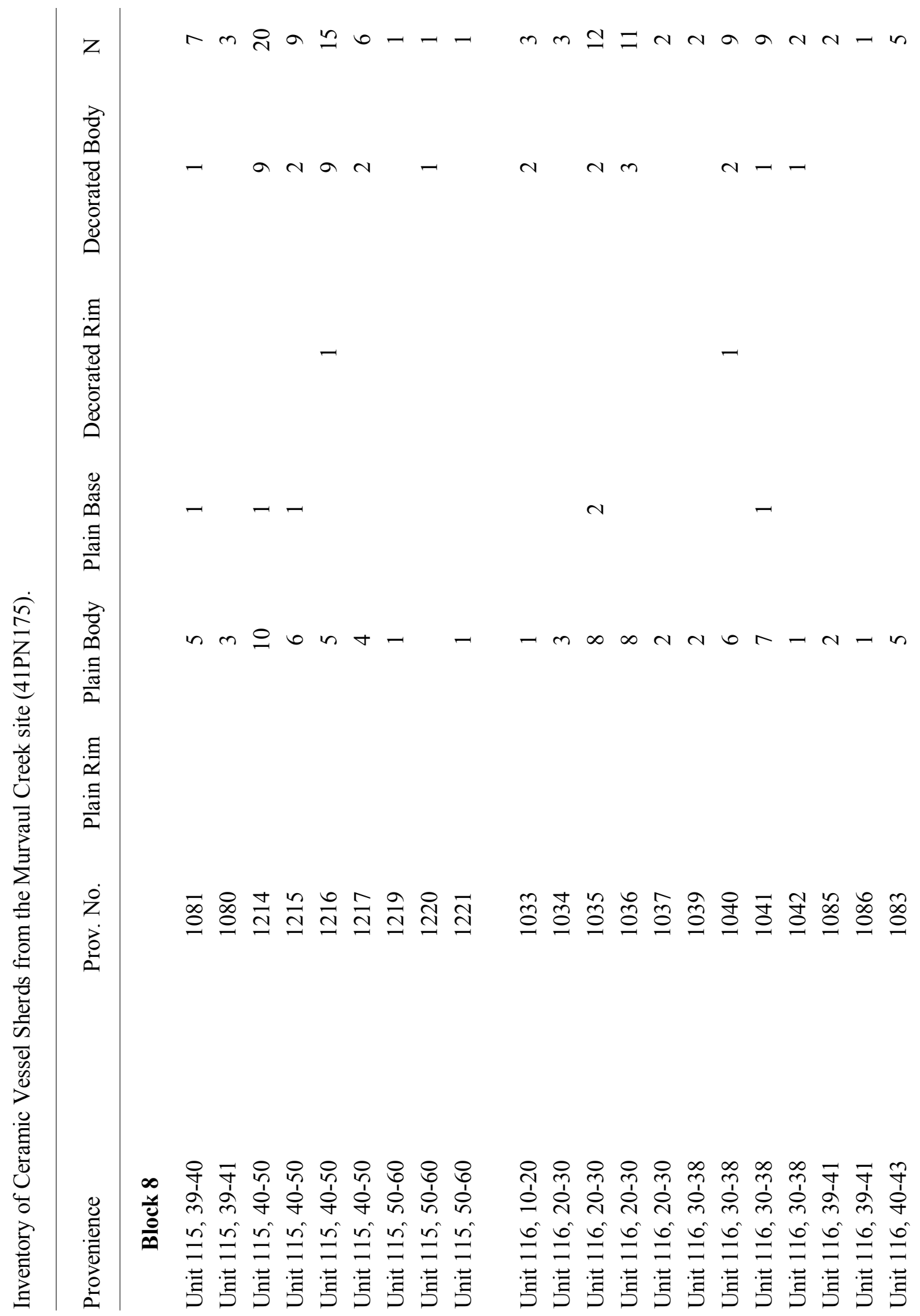




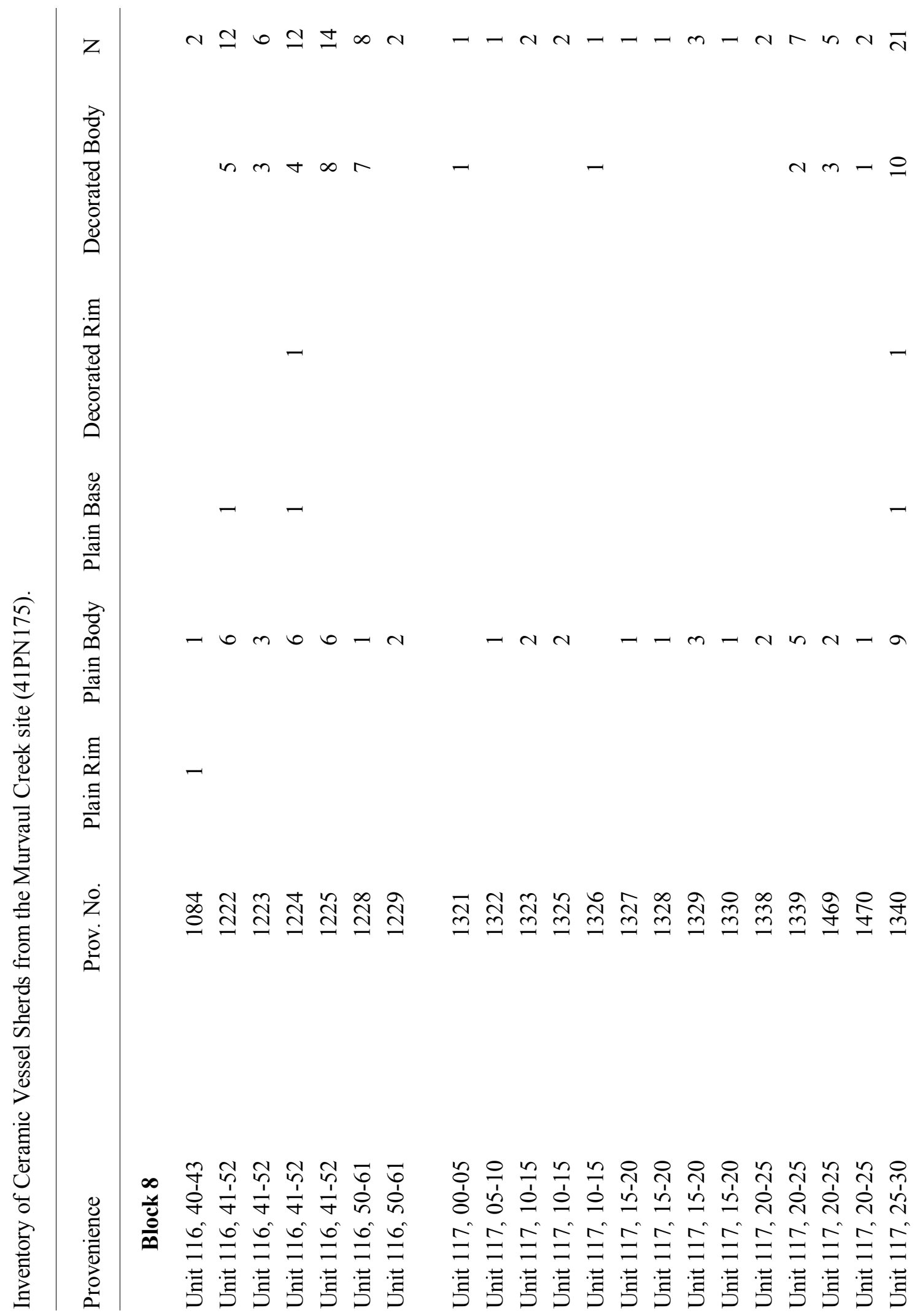




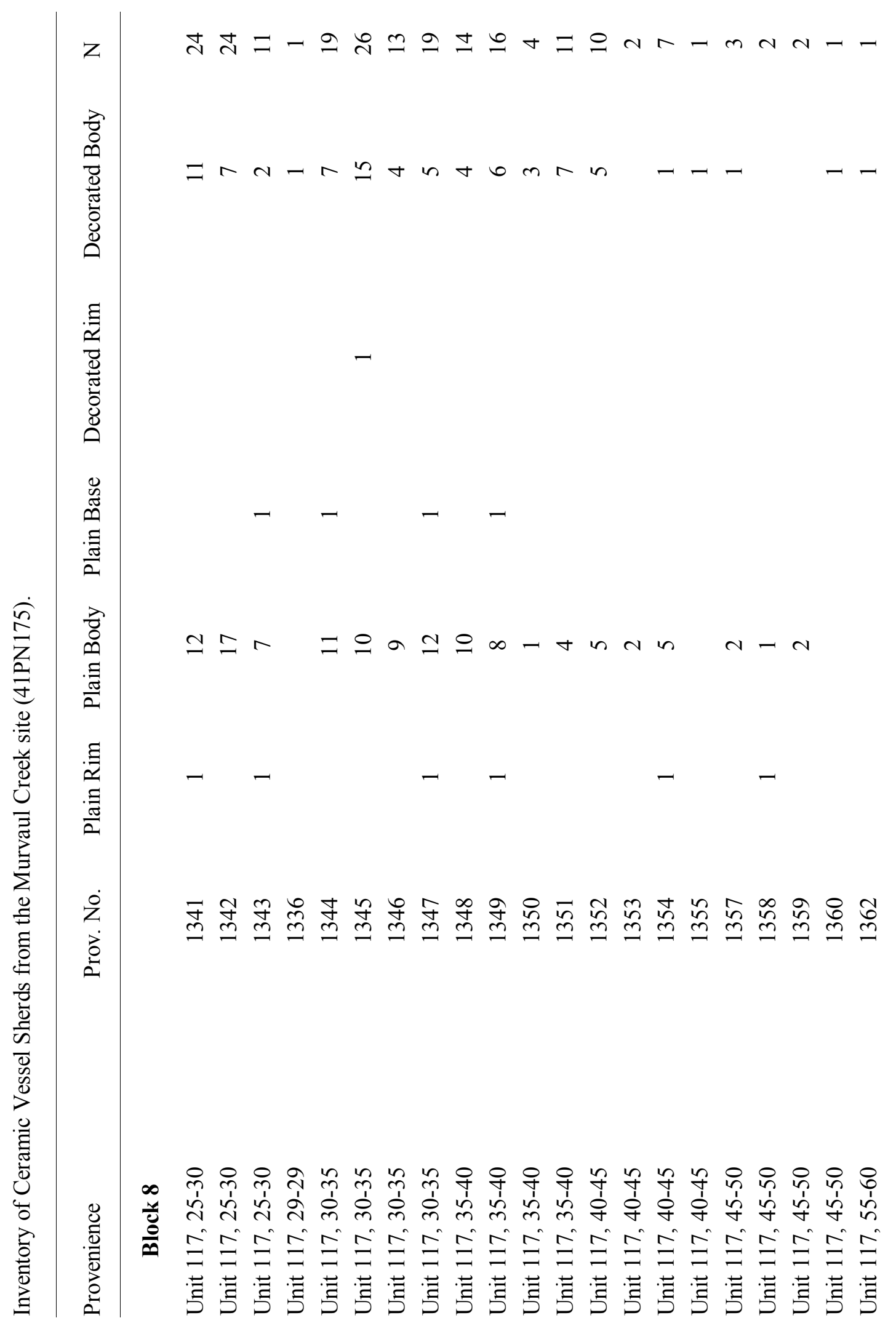




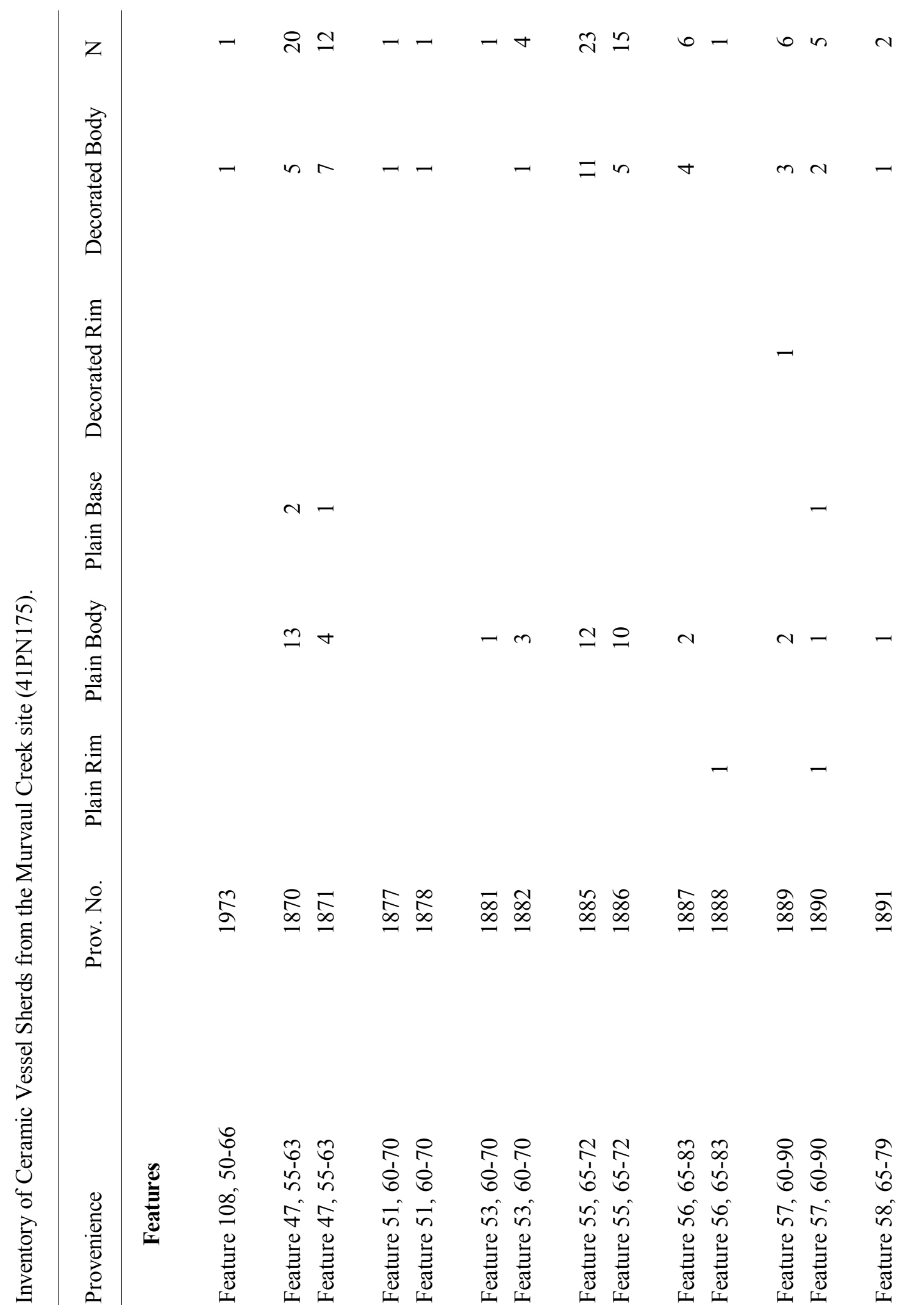




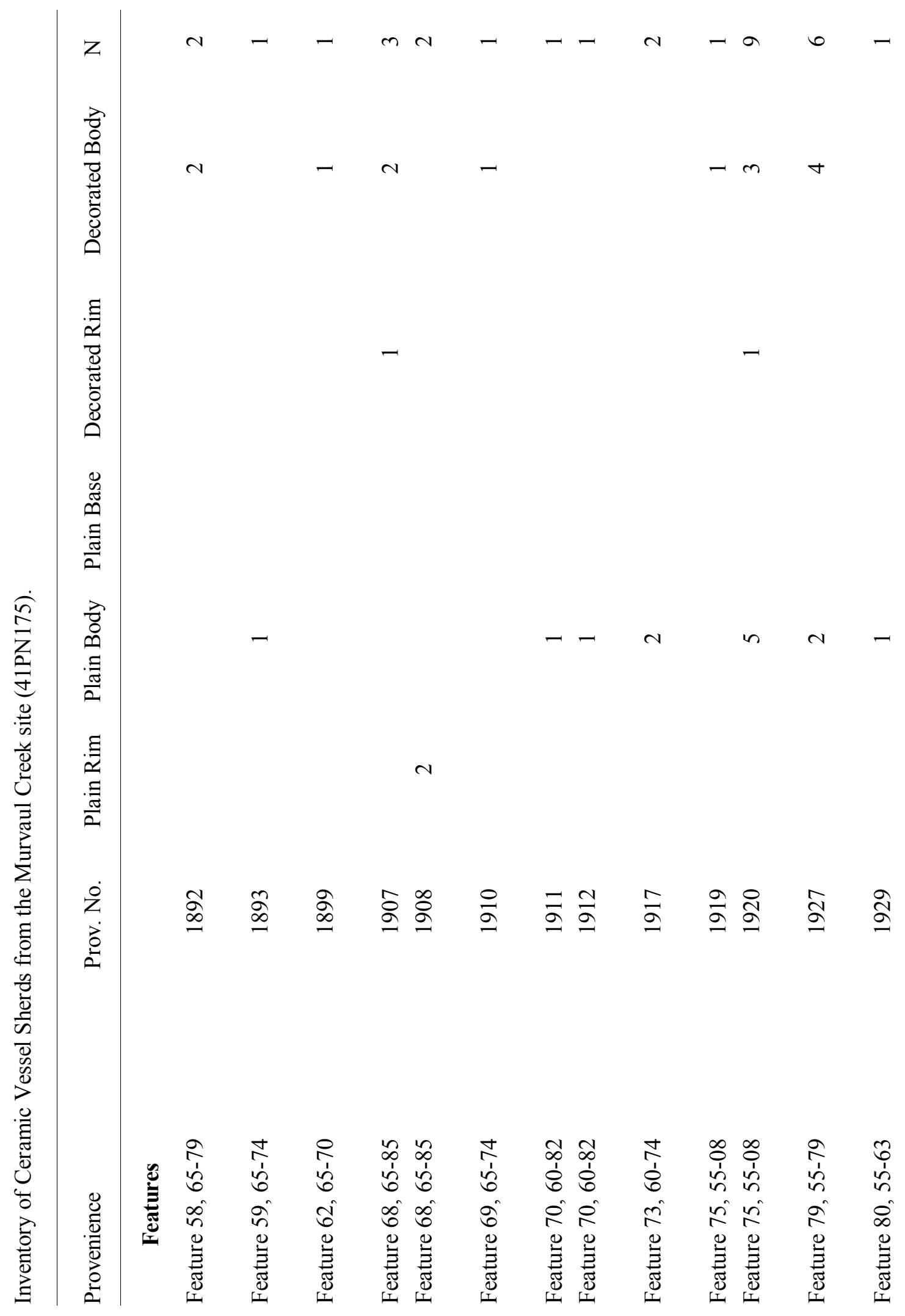




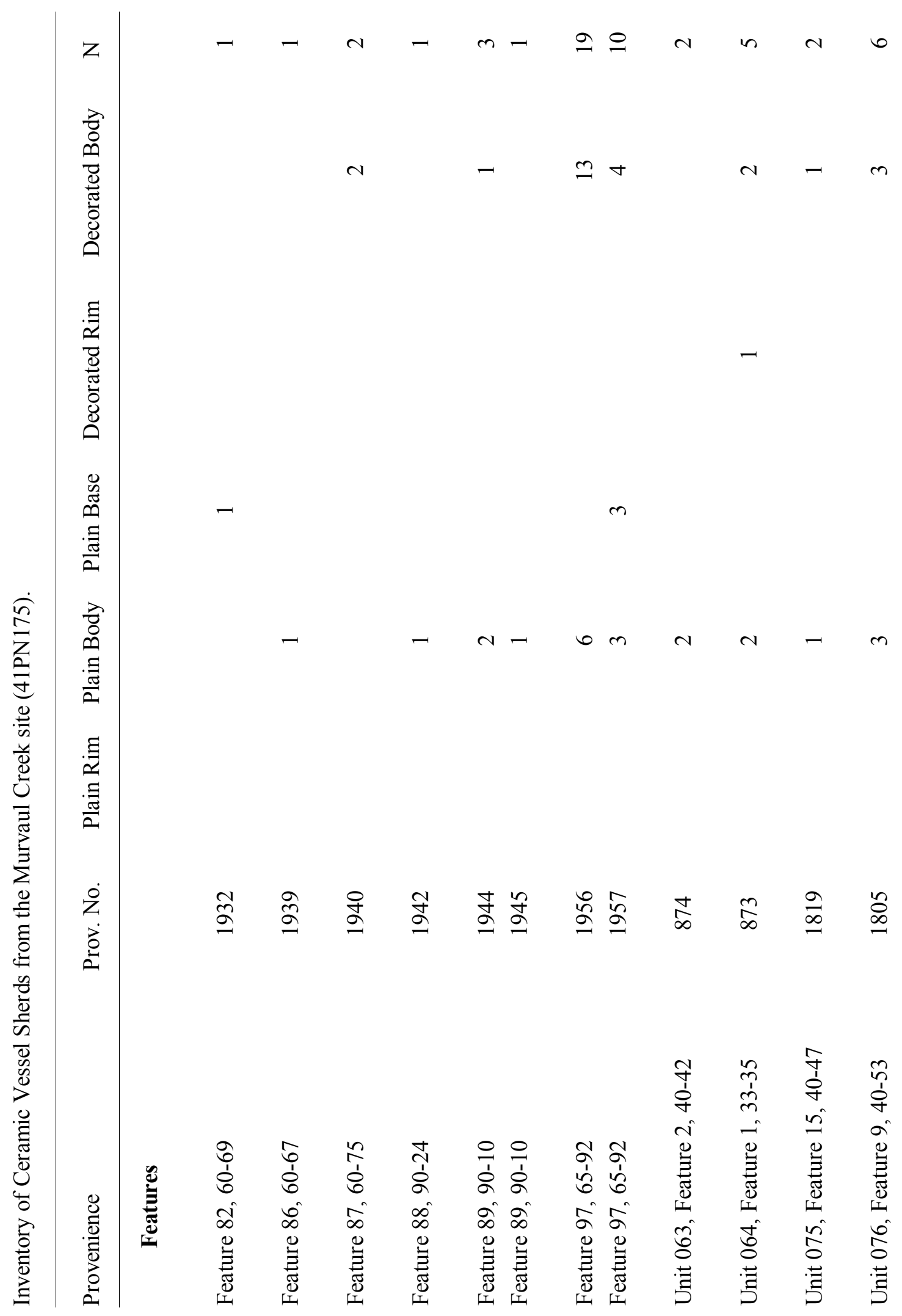









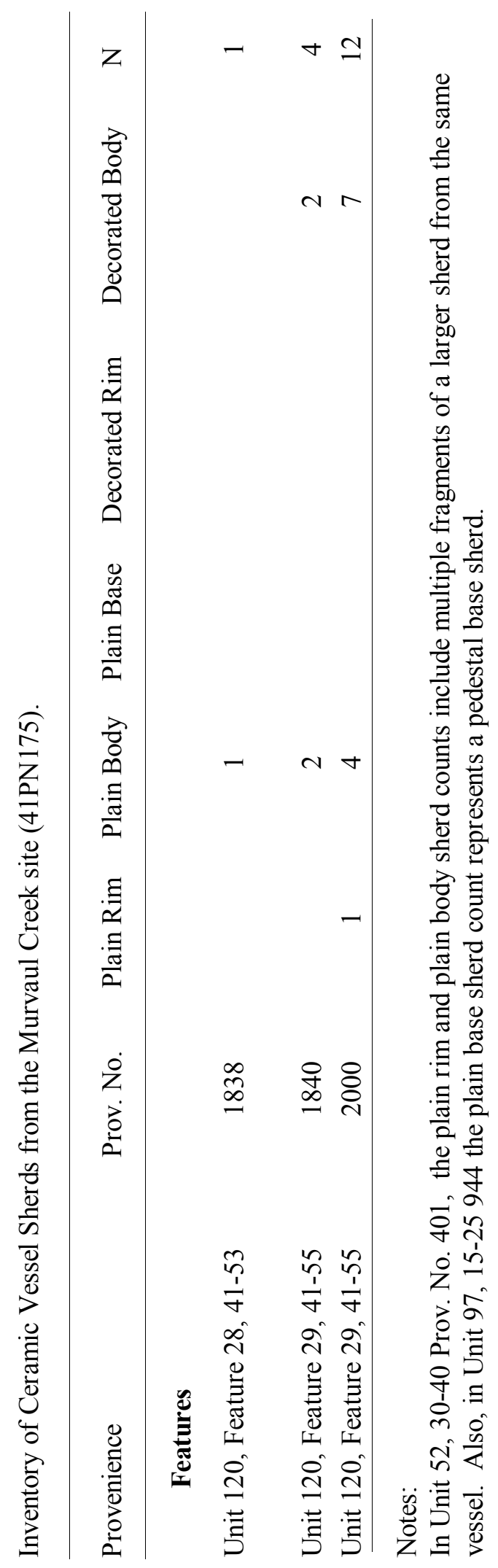



A-2: PROVENIENCE OF DECORATED SHERDS BY DECORATIVE METHOD 



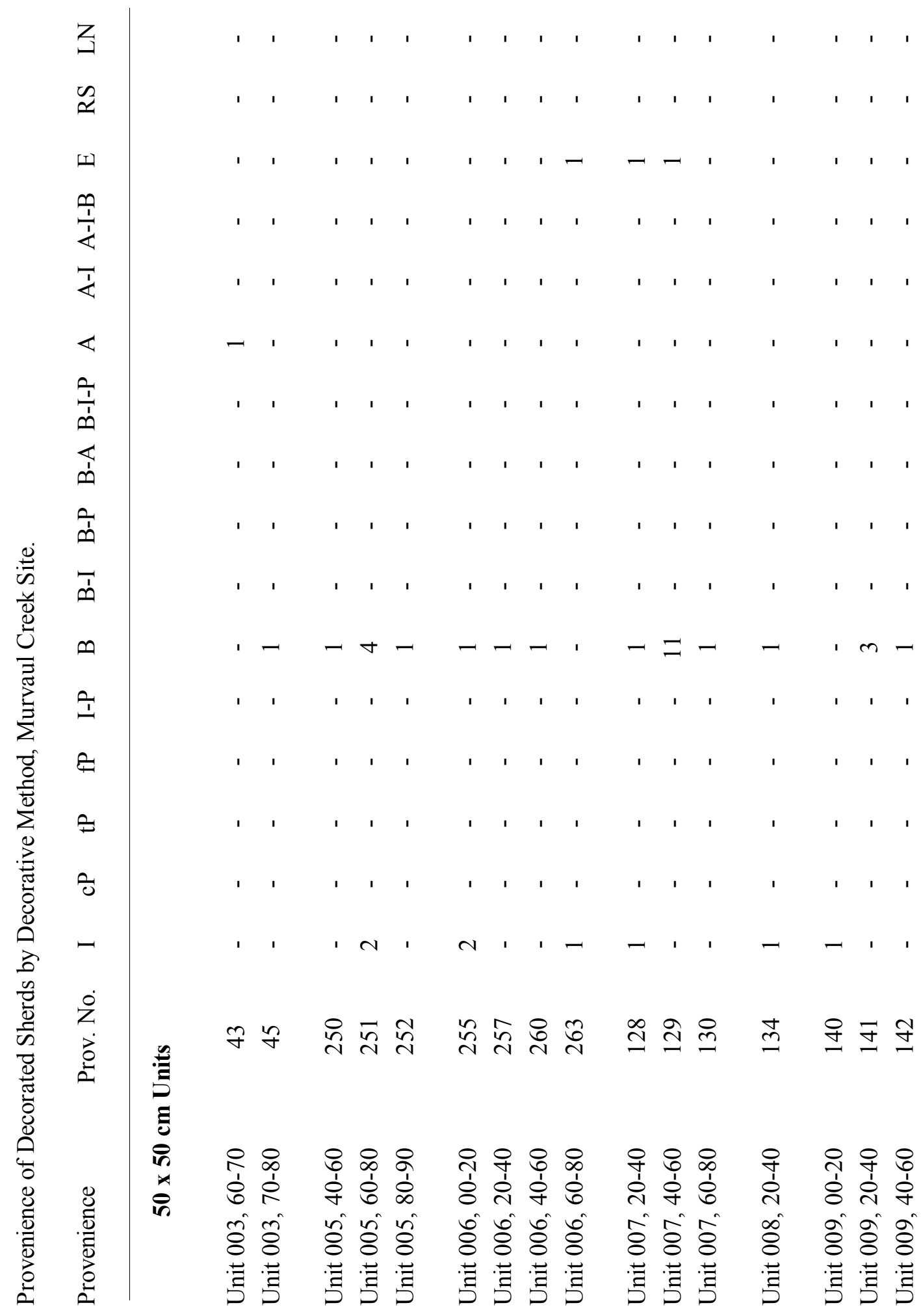




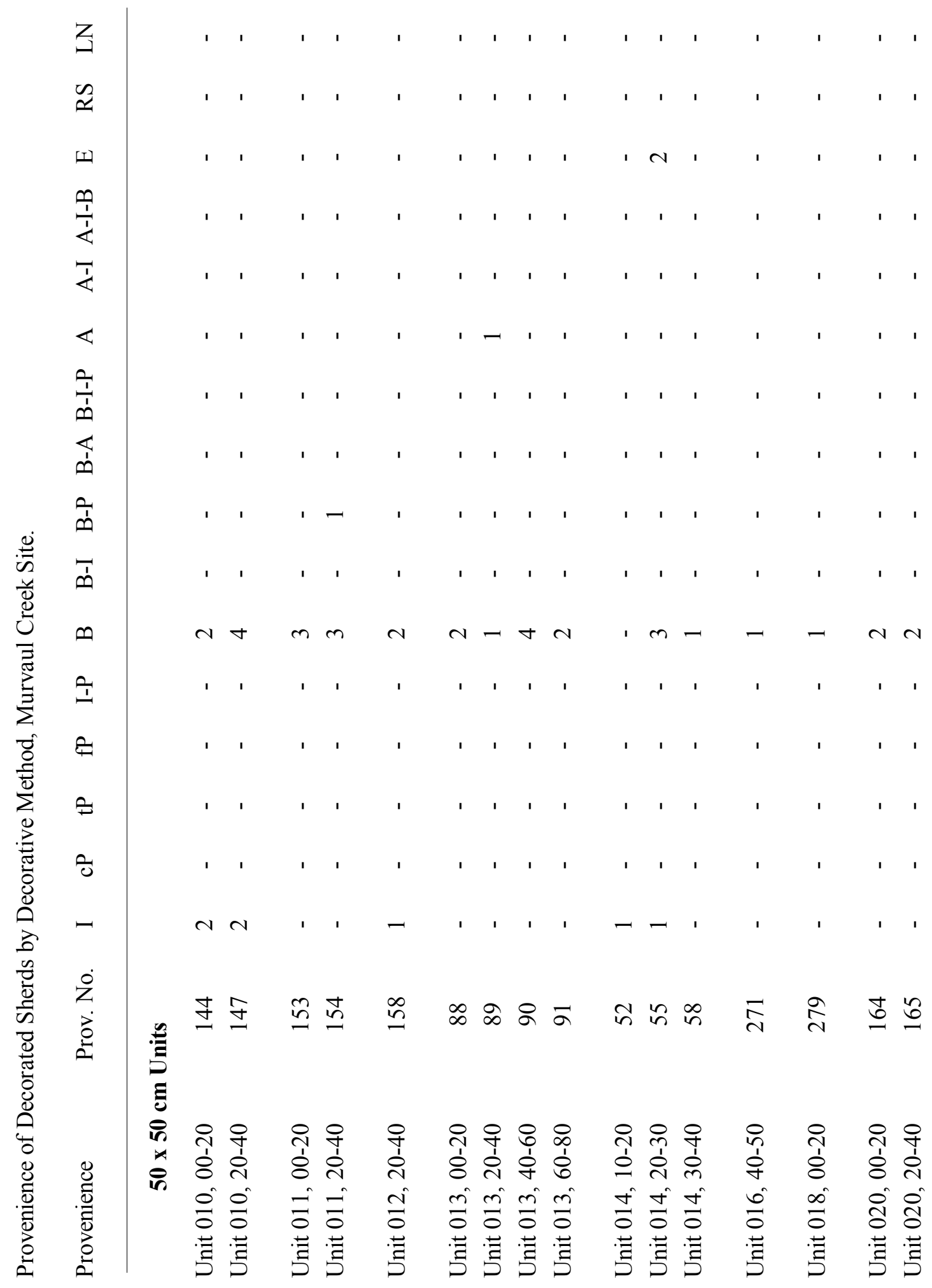




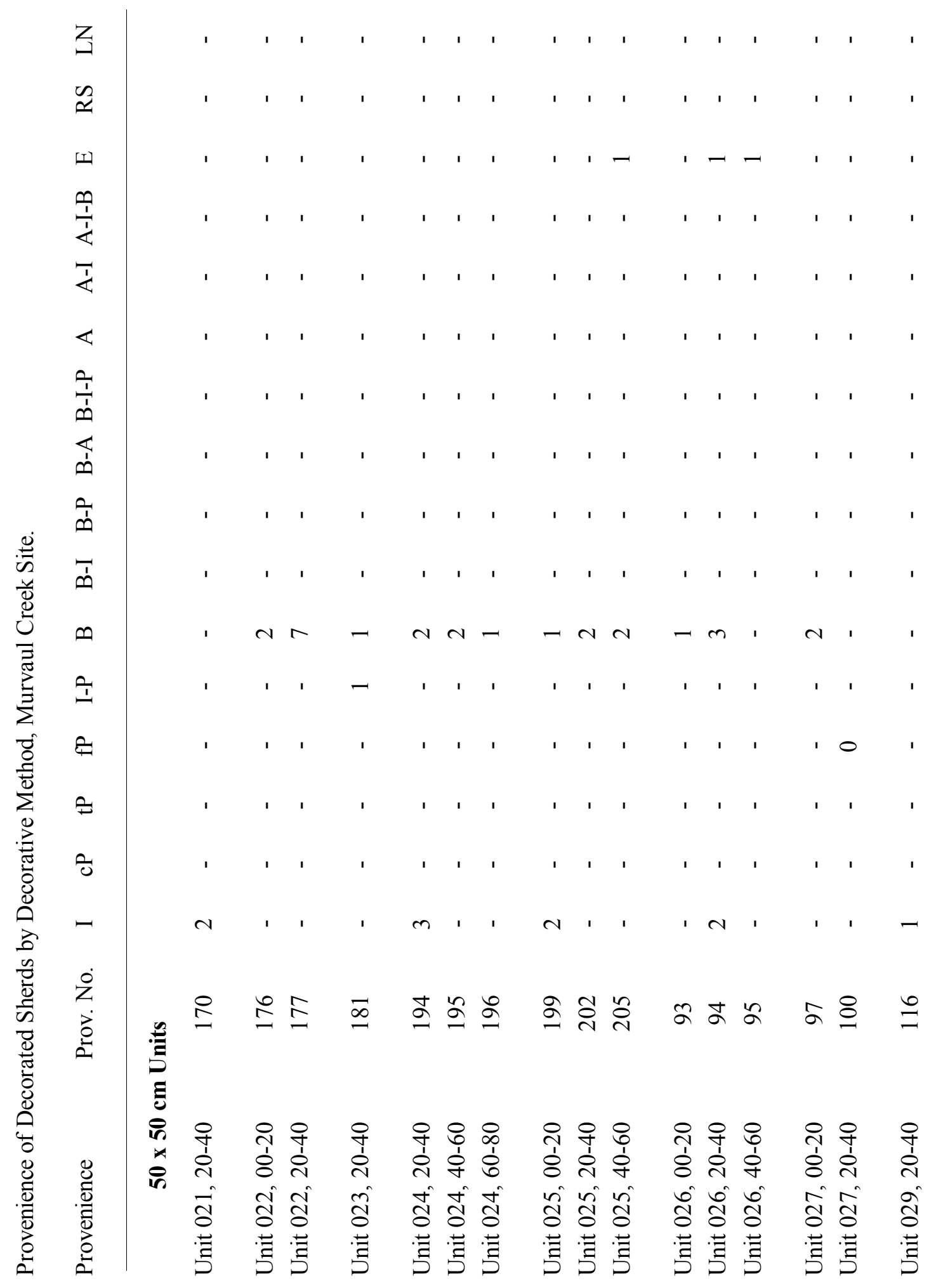




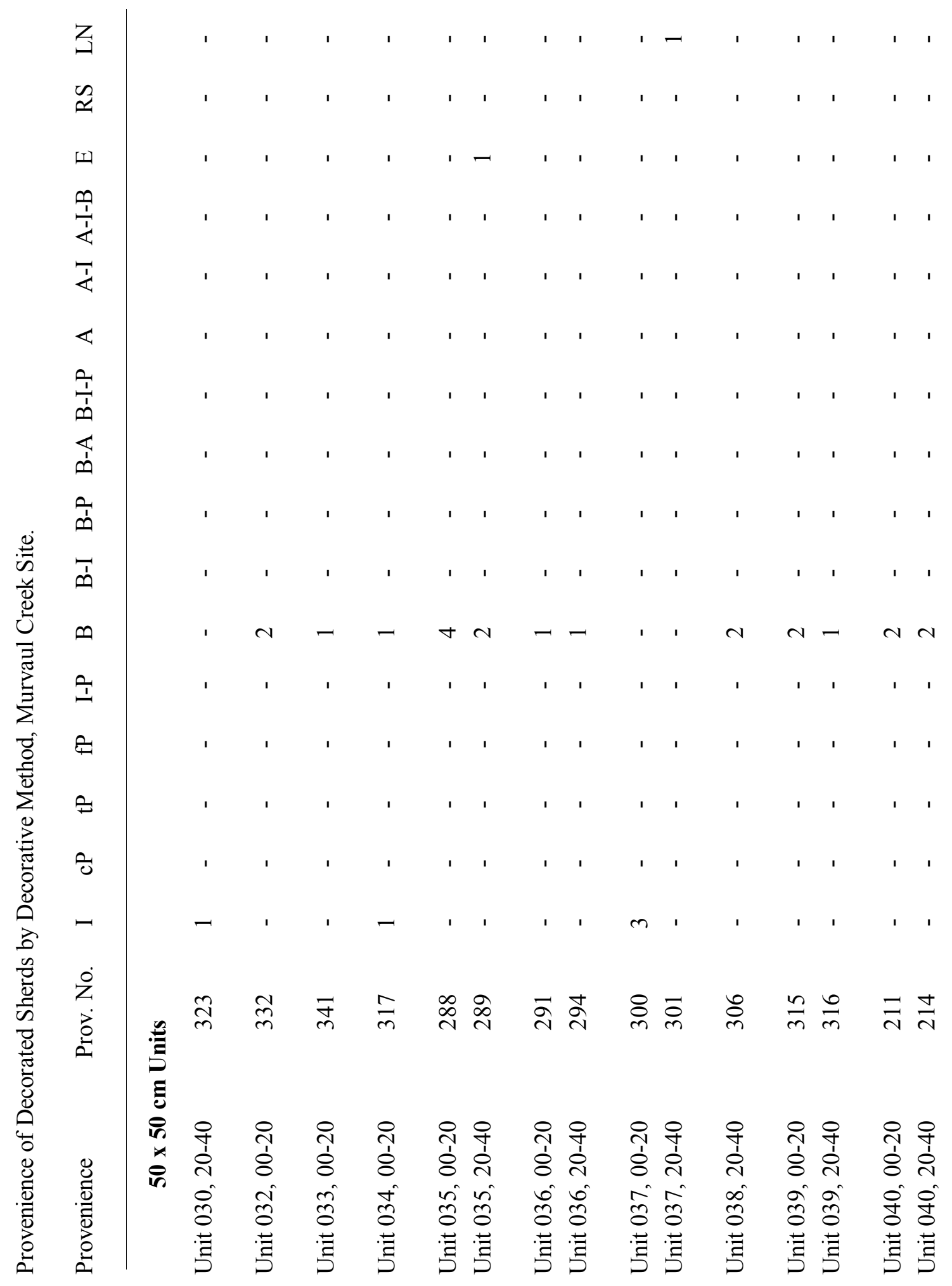




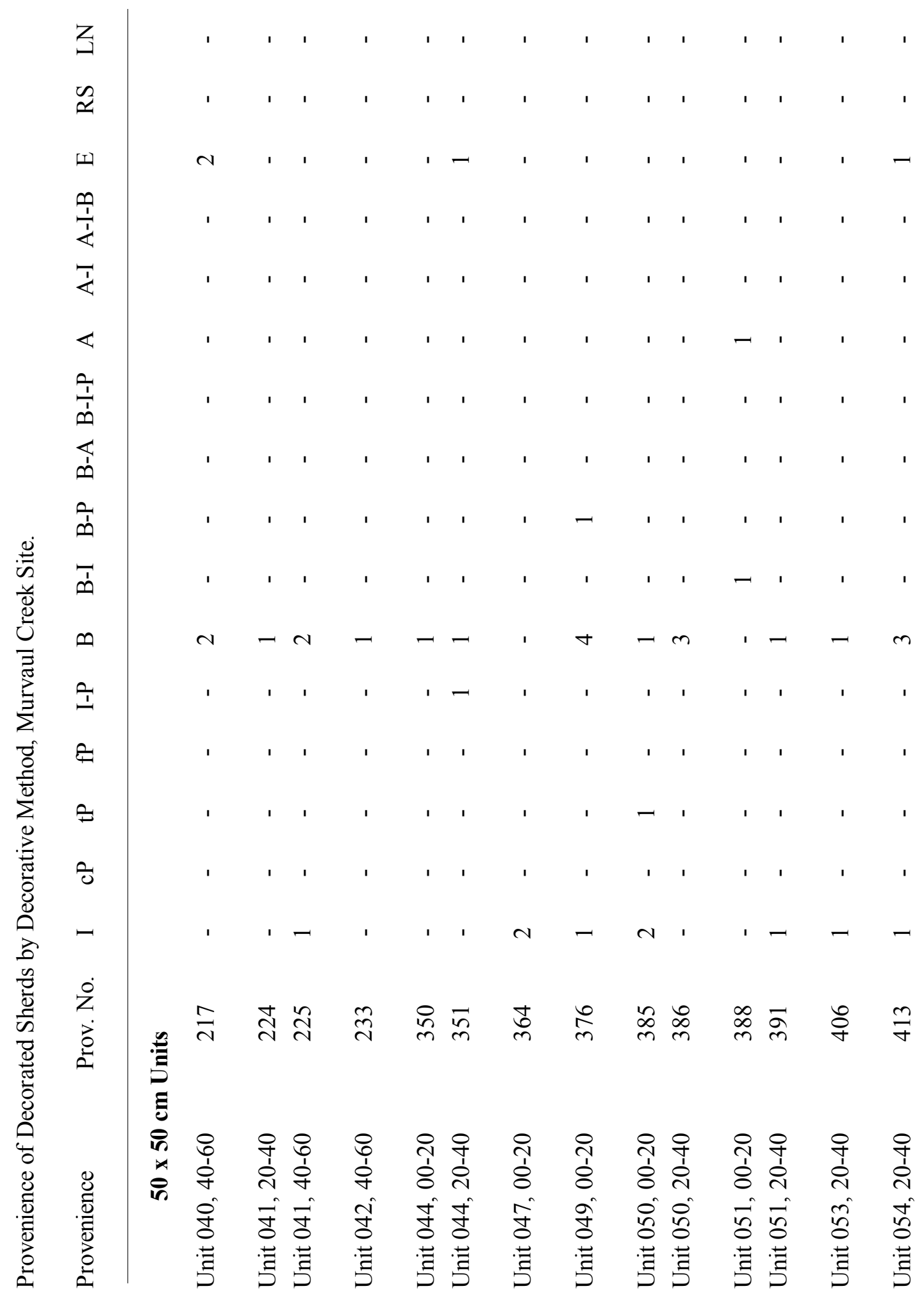




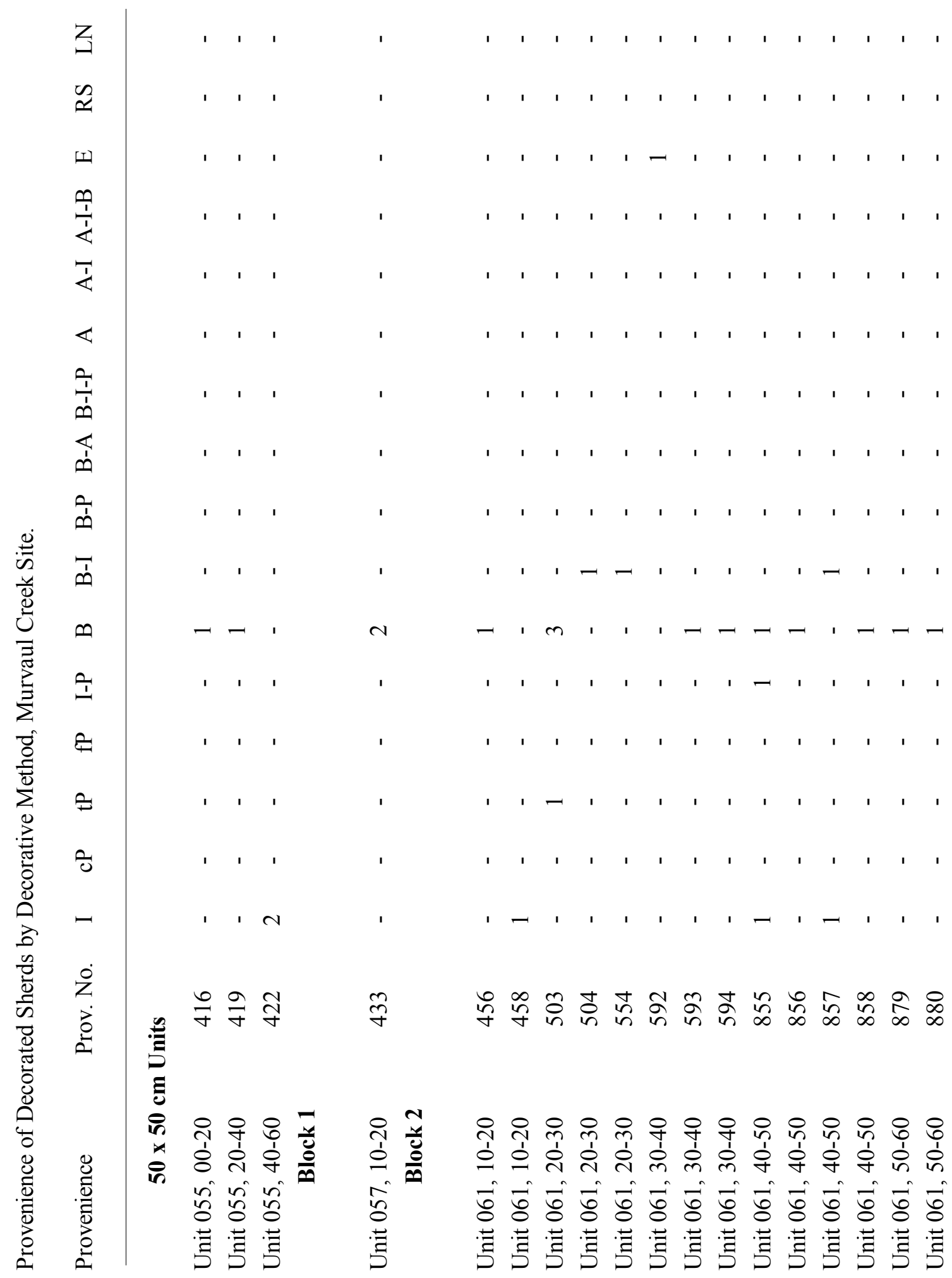




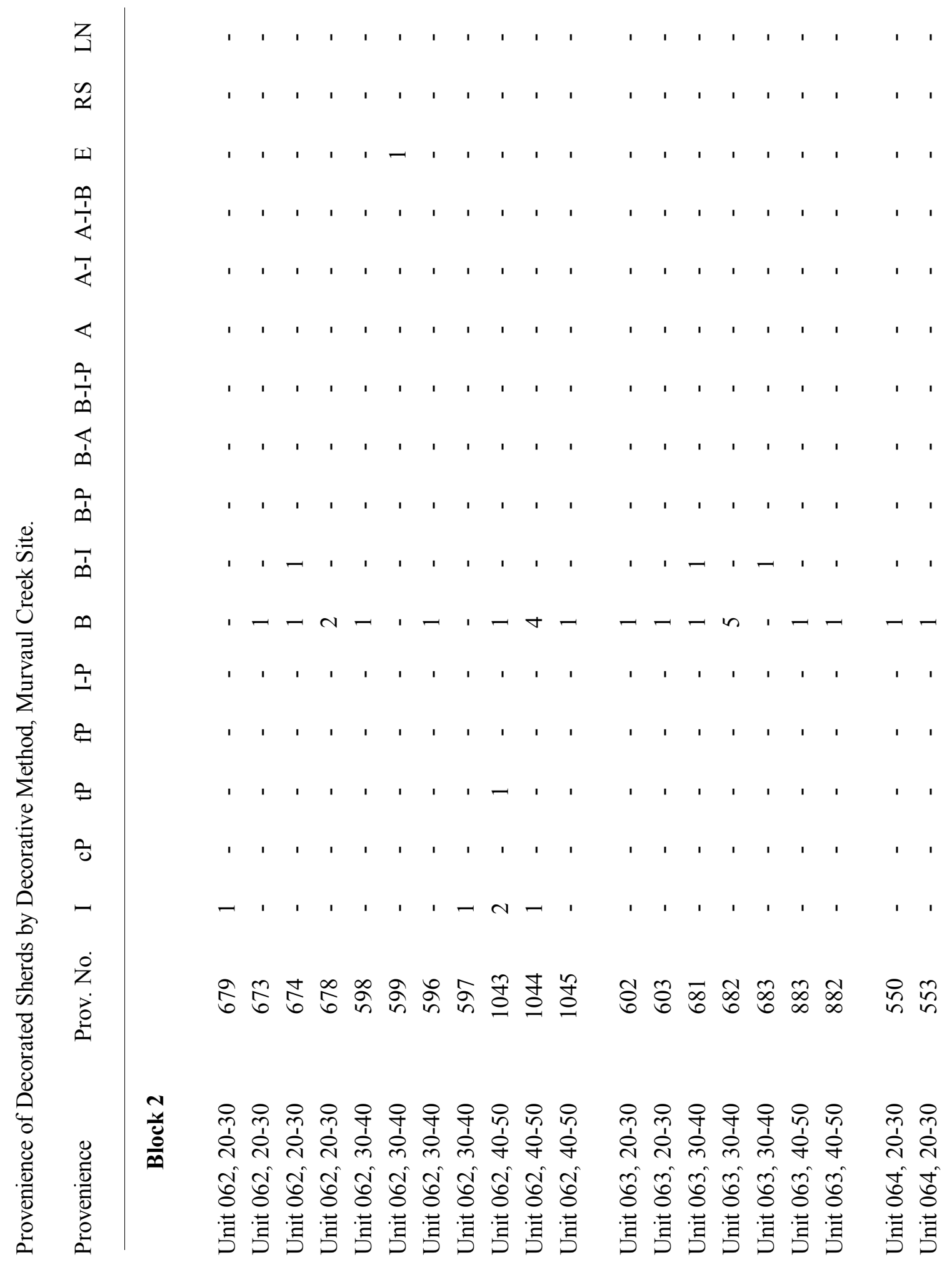




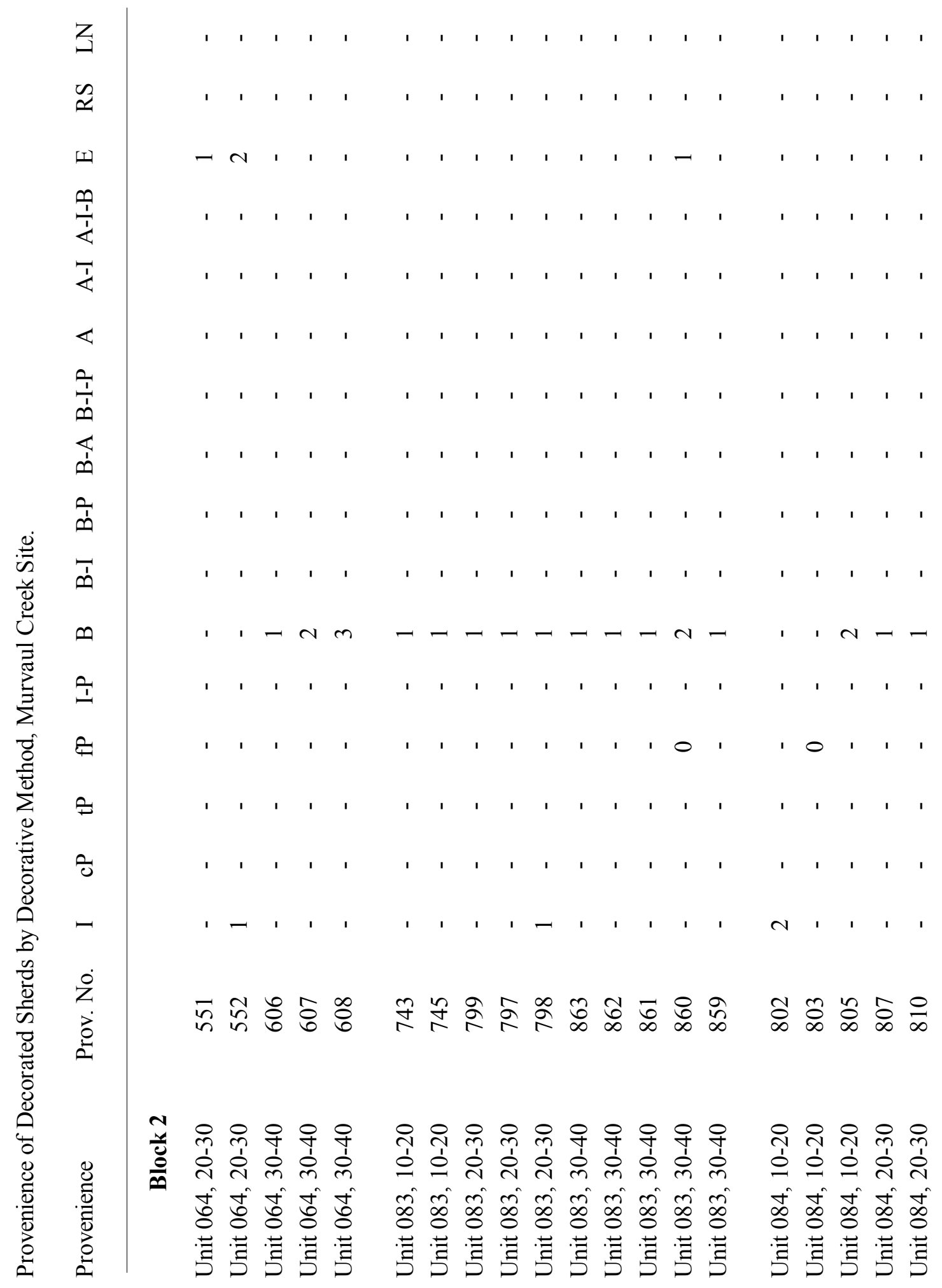




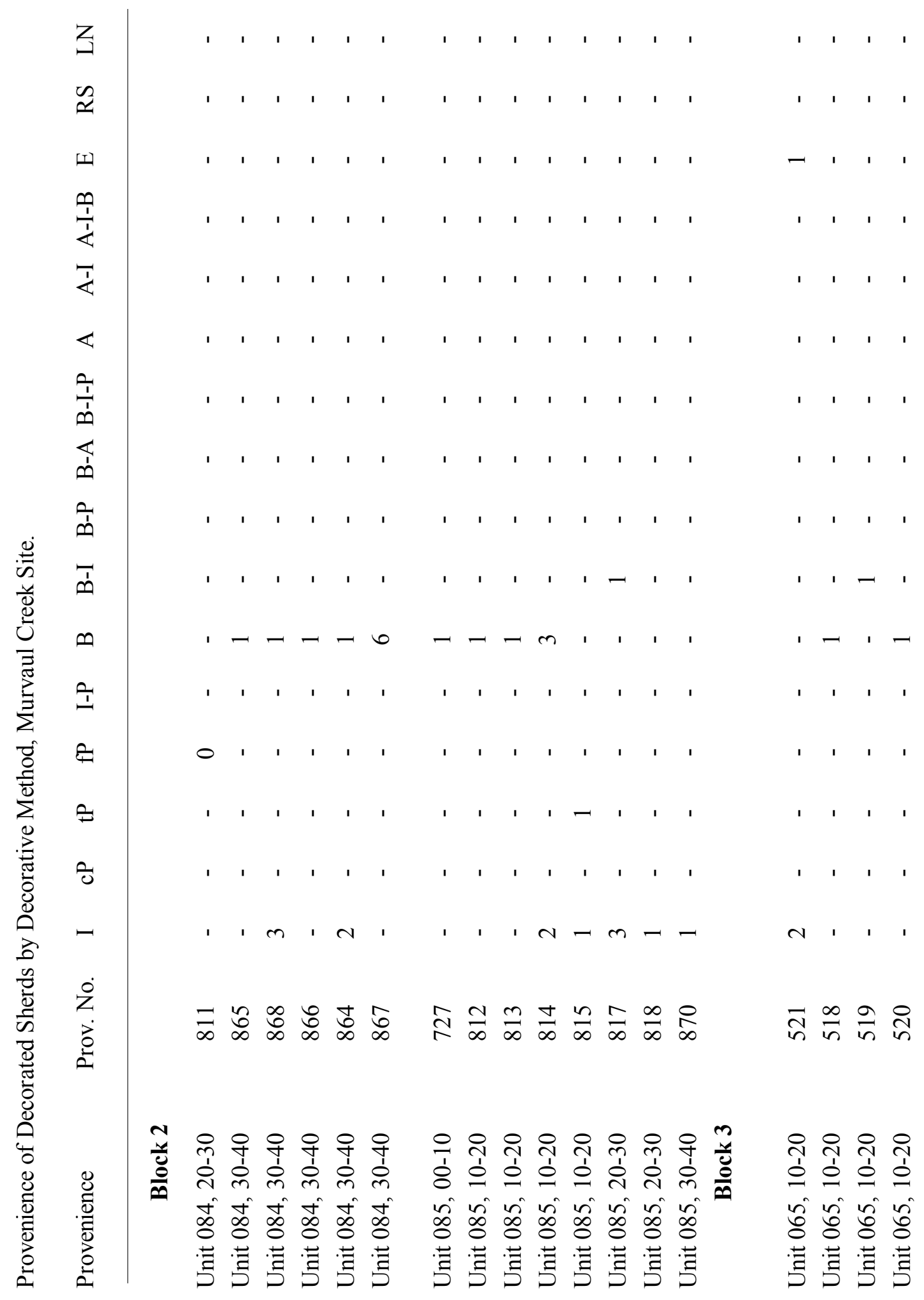




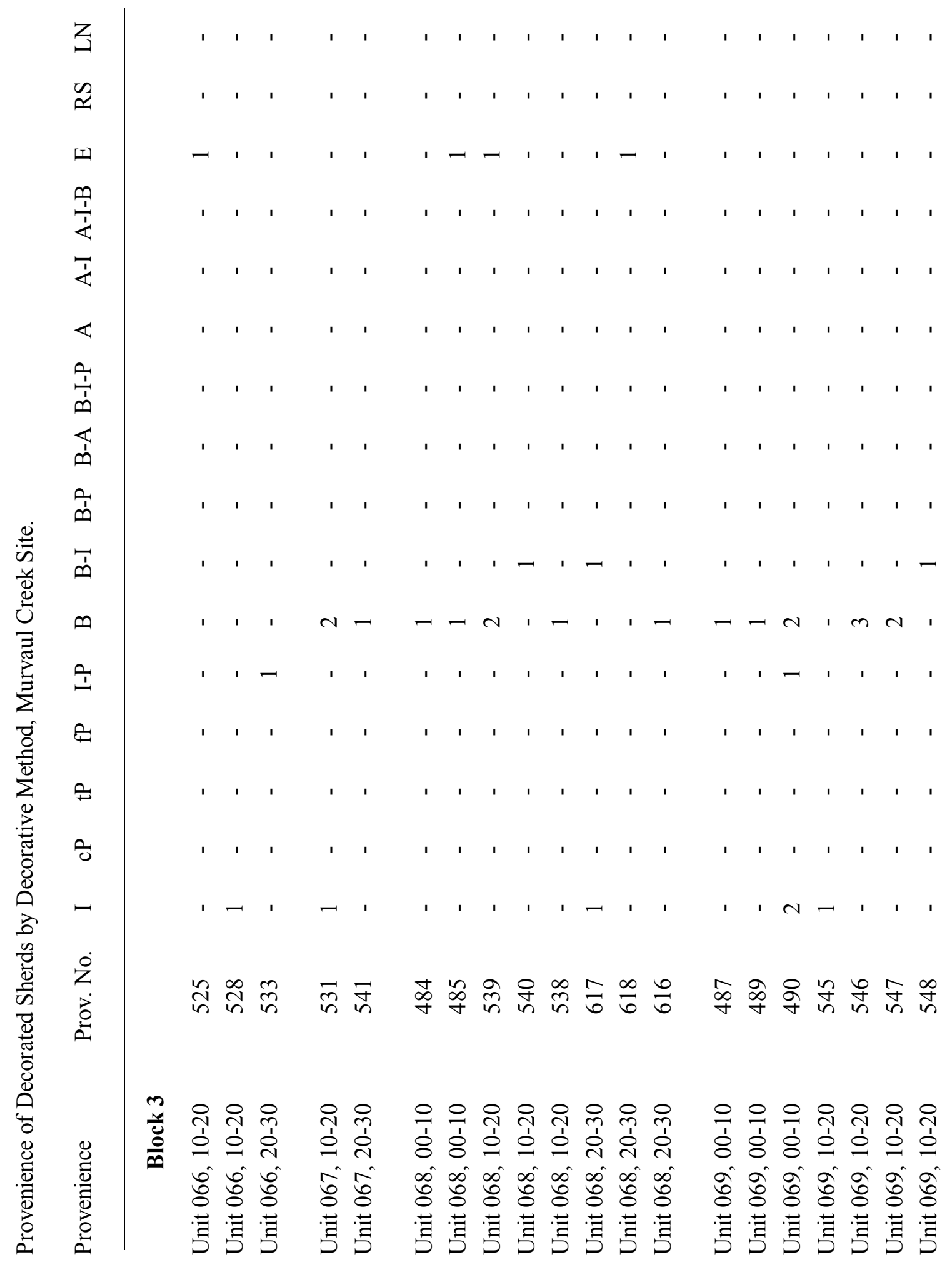




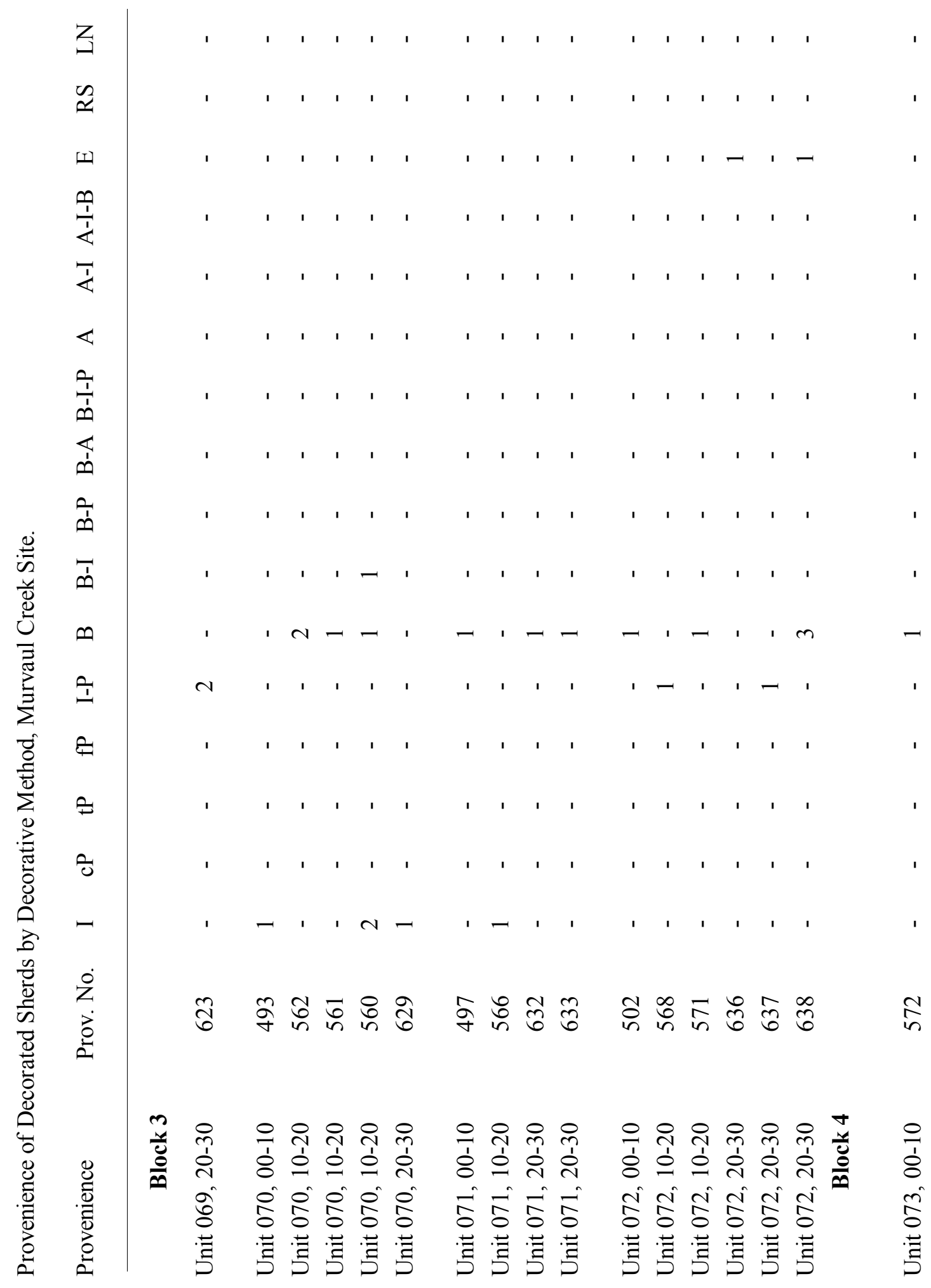




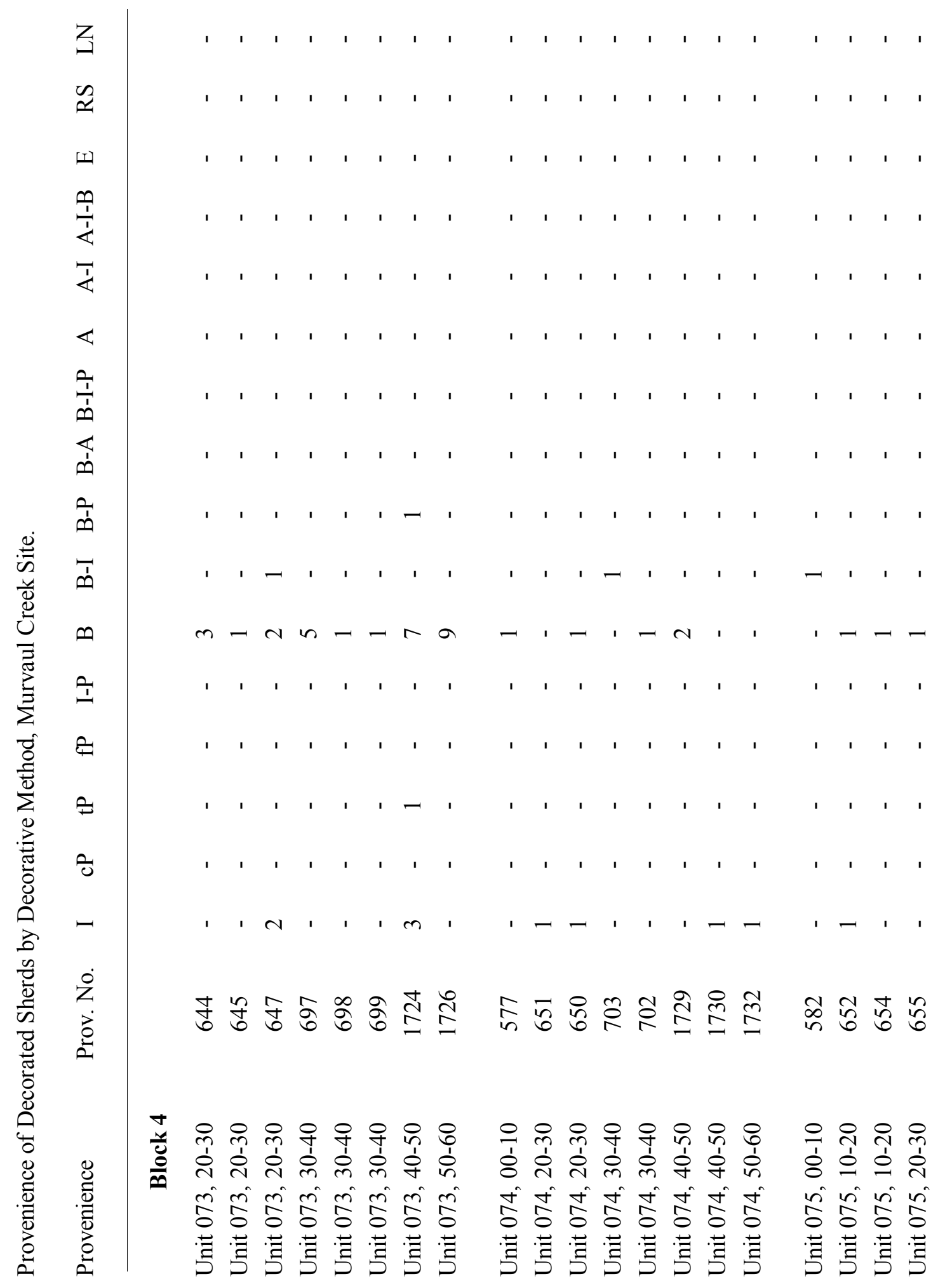




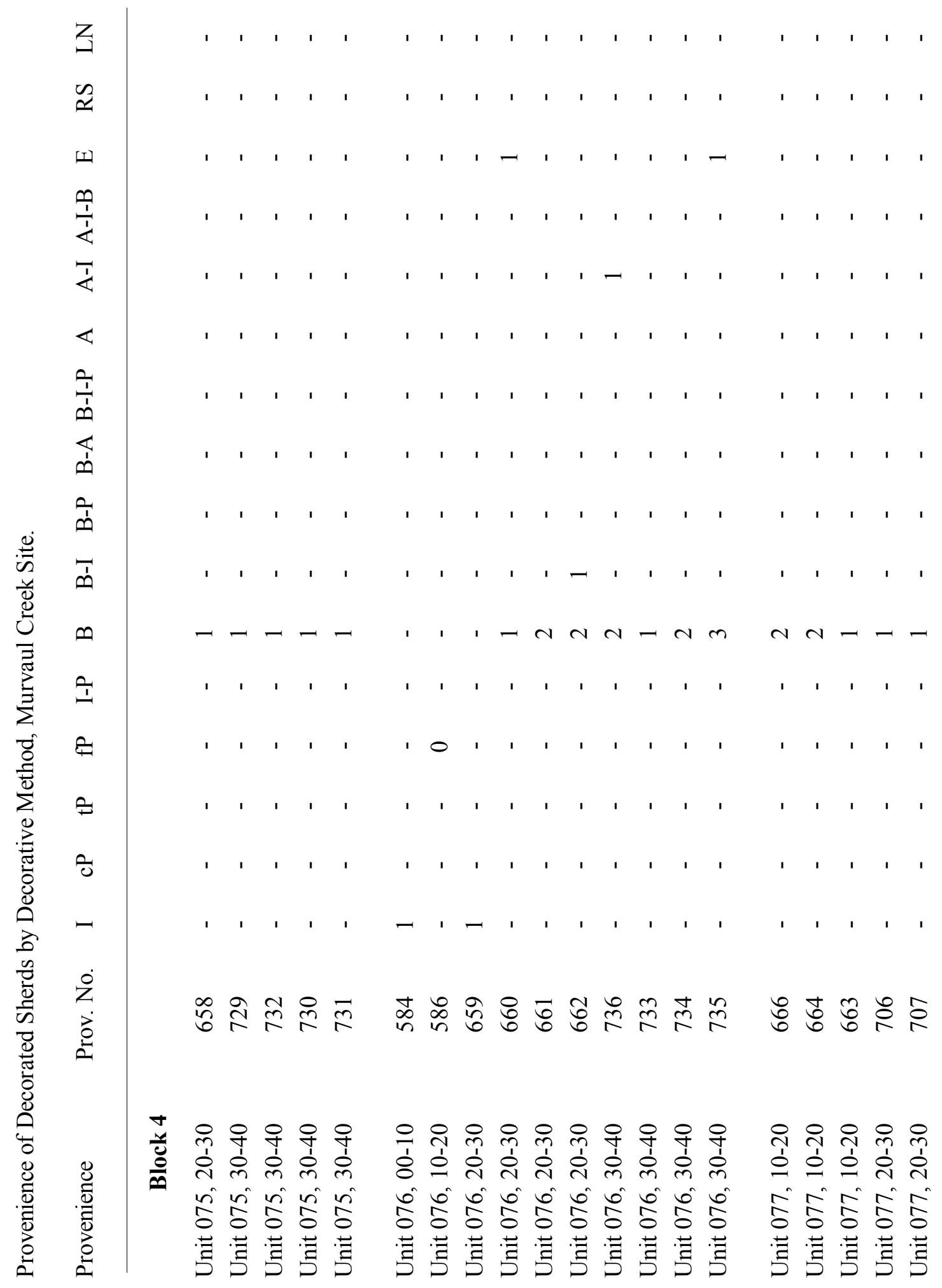




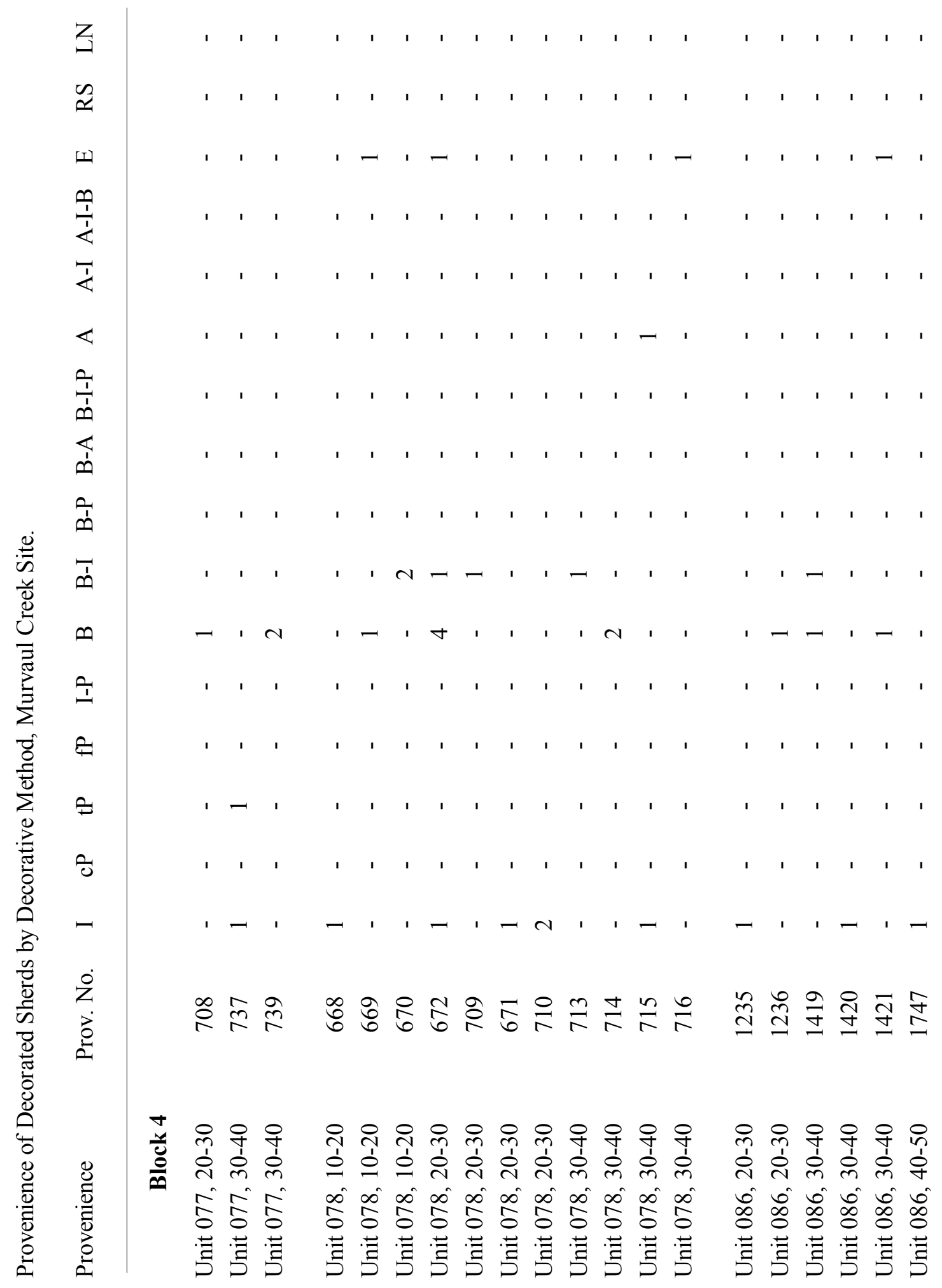




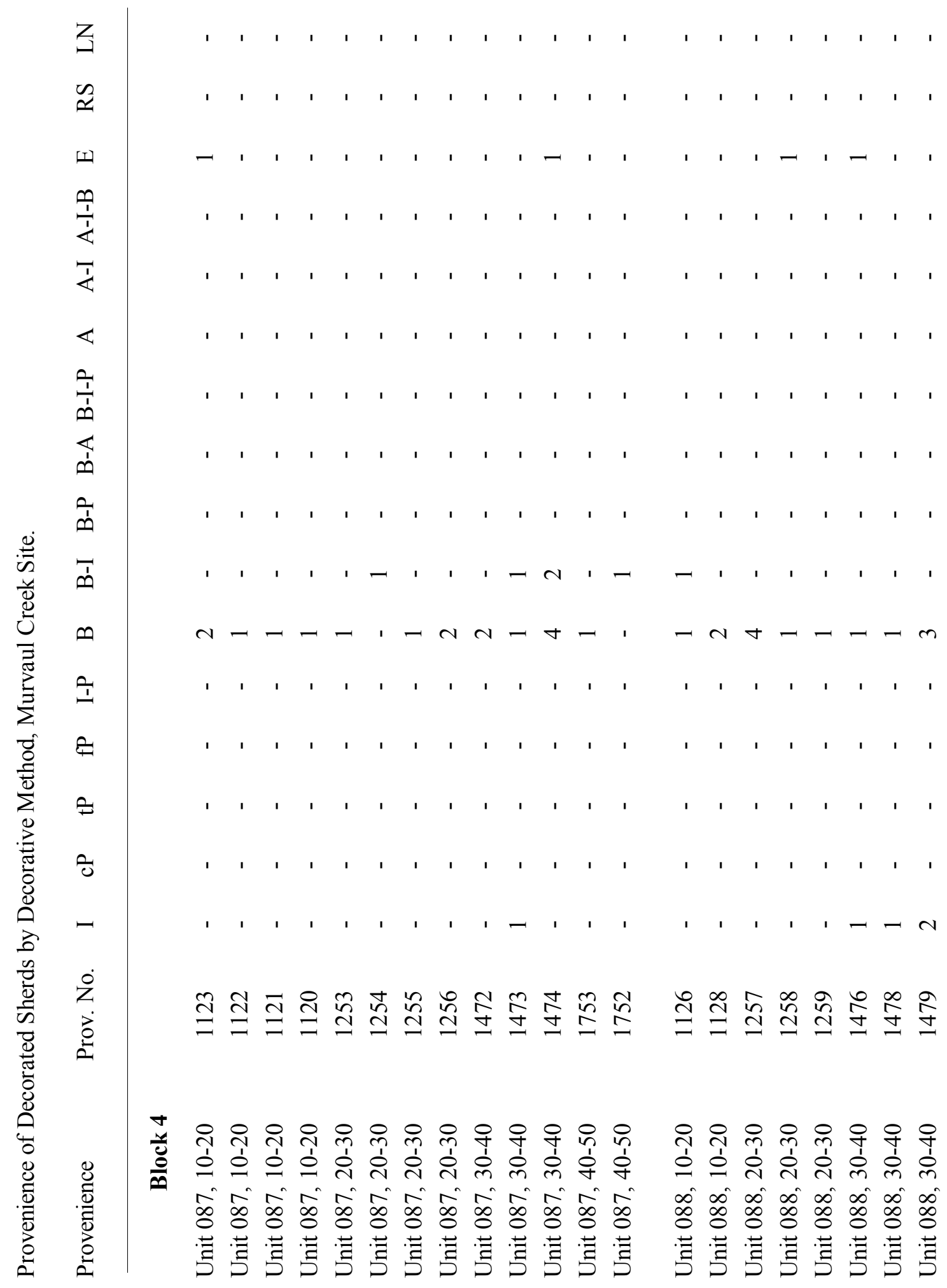




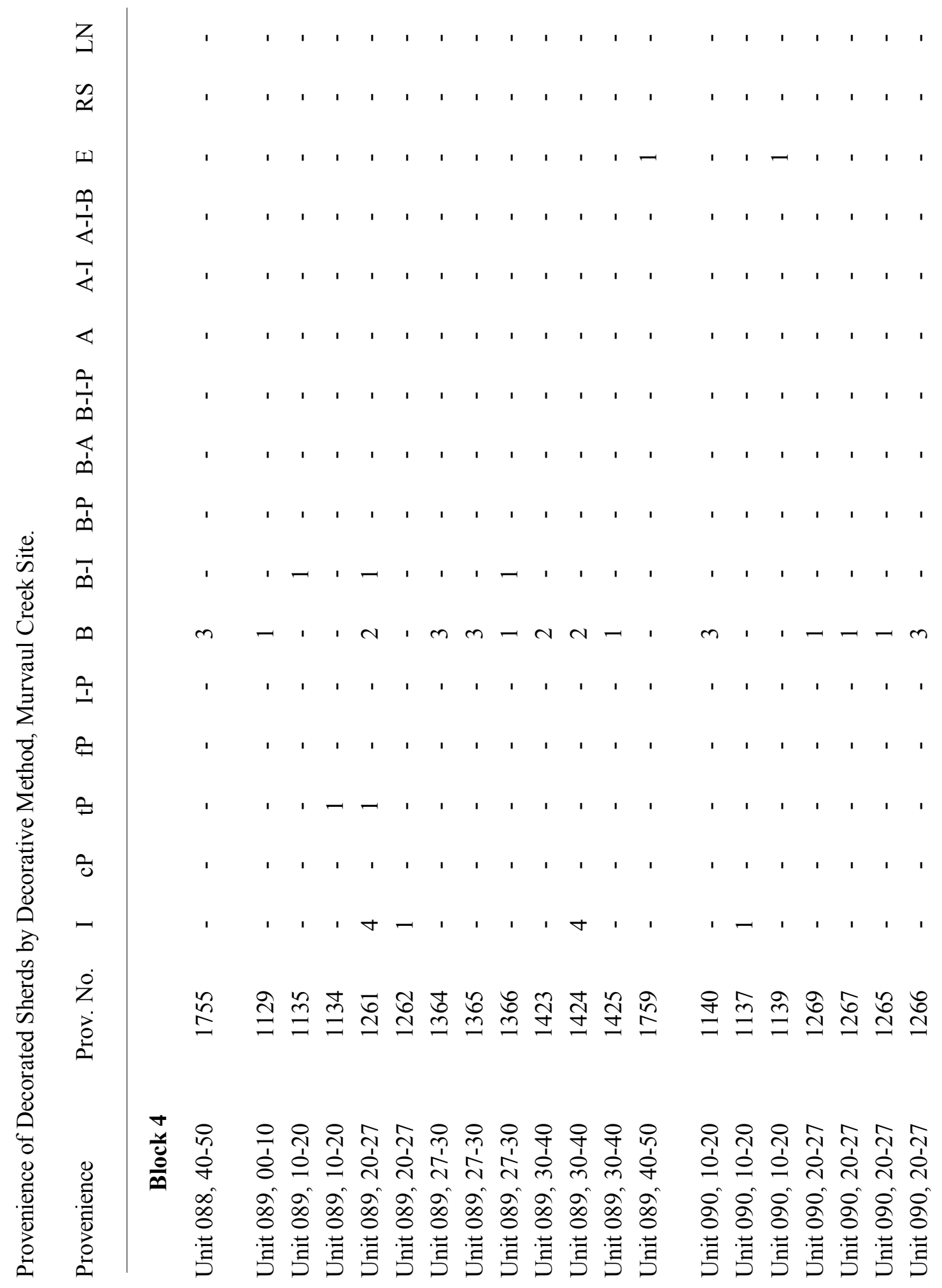




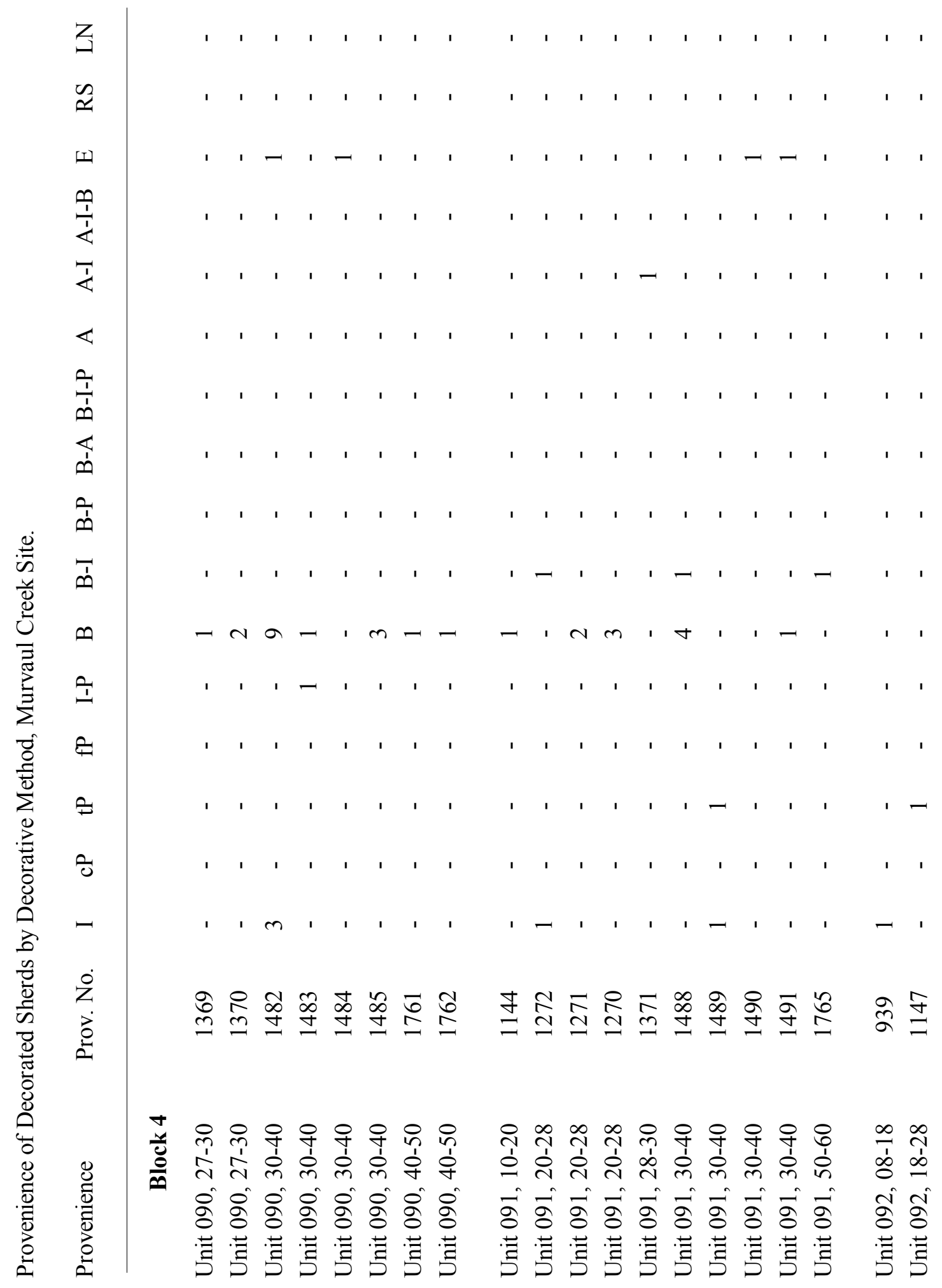




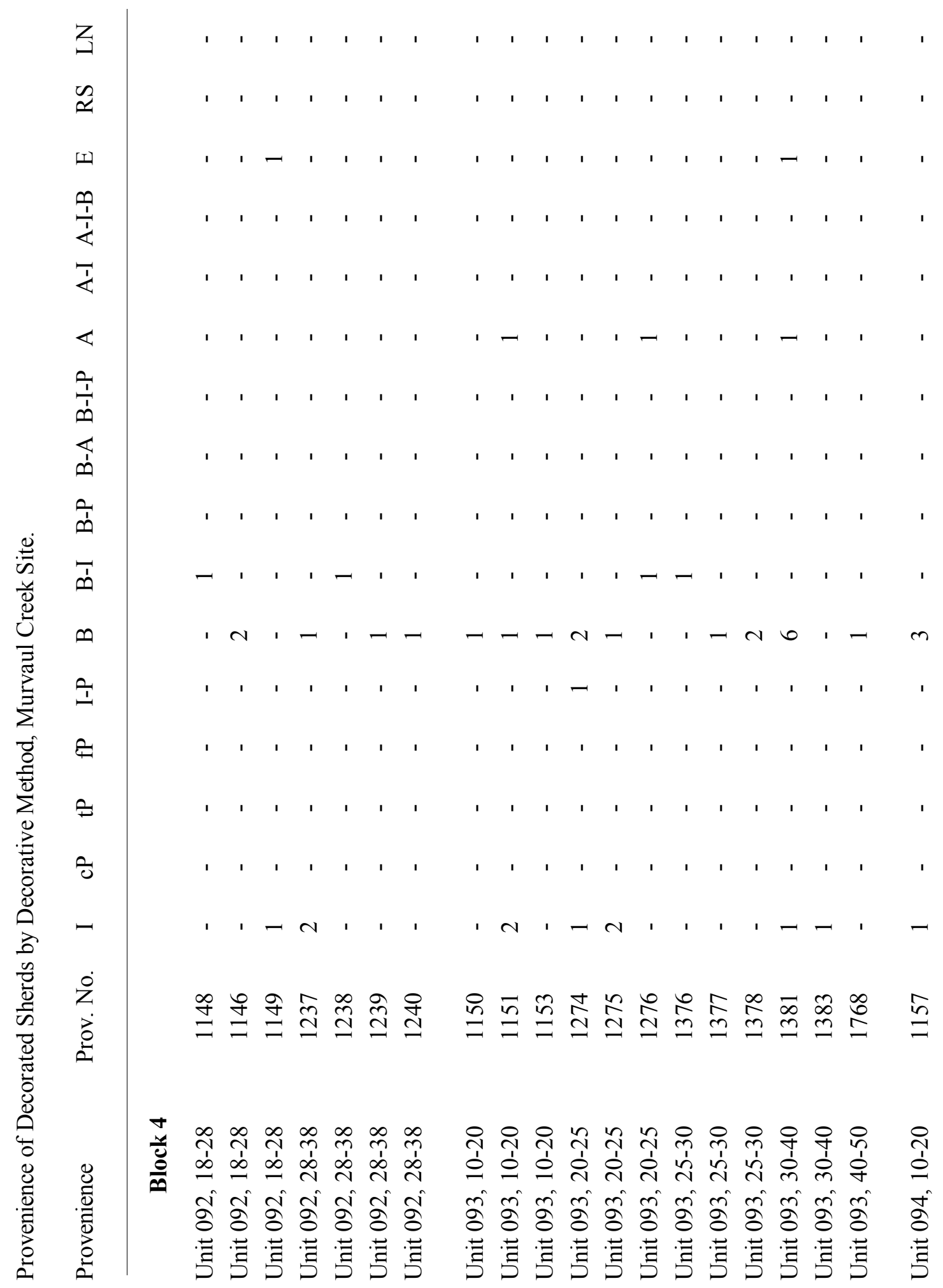




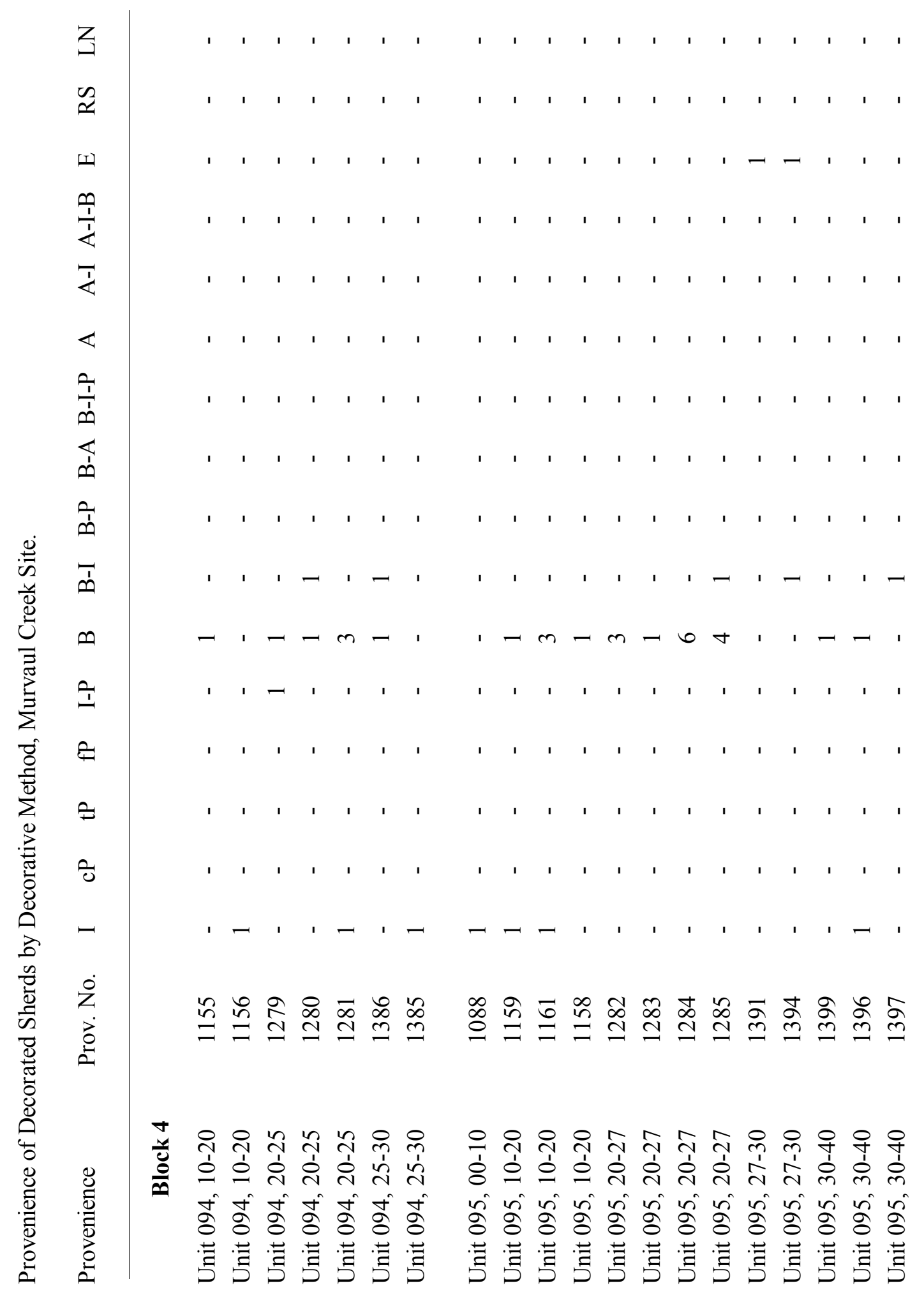




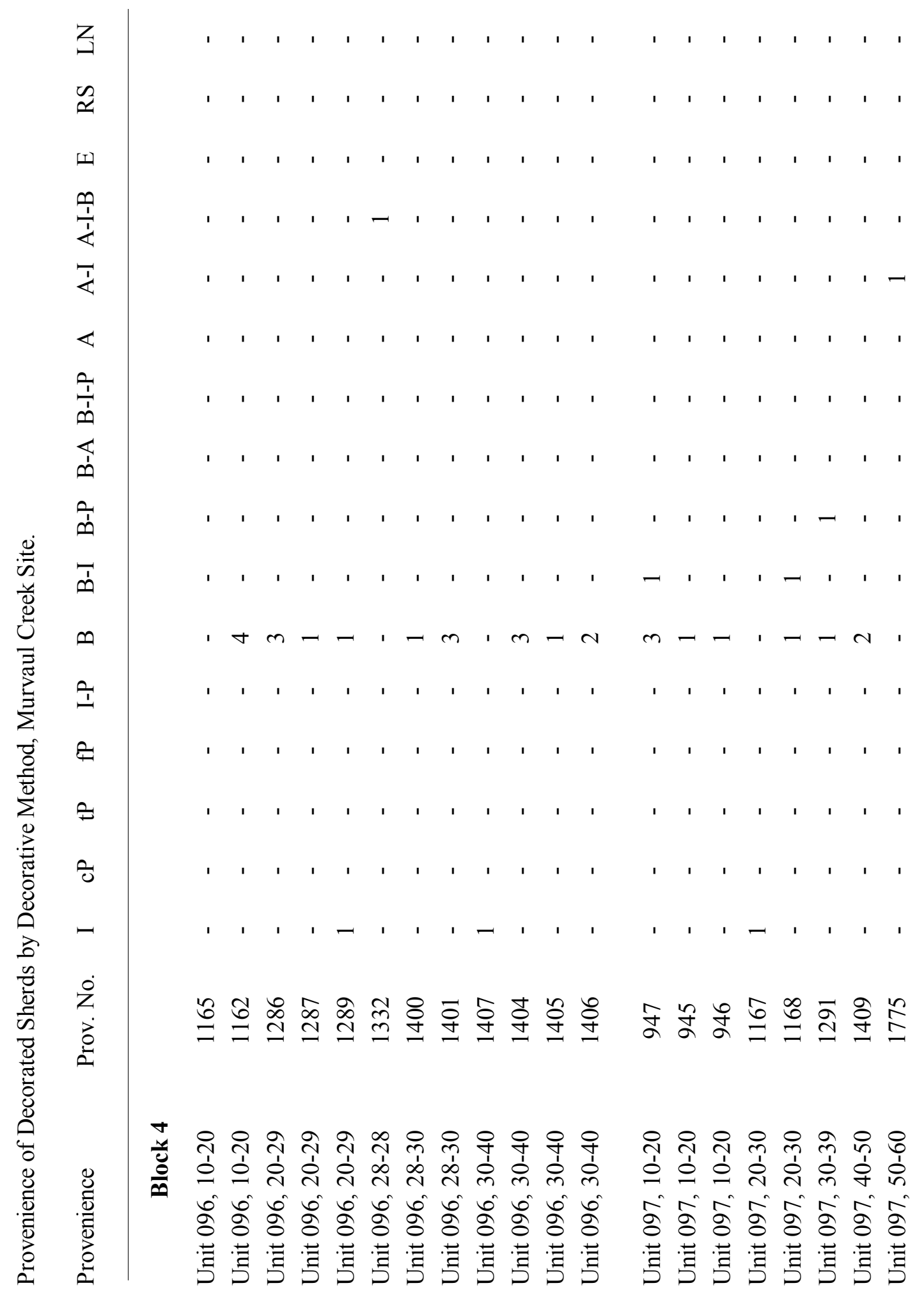




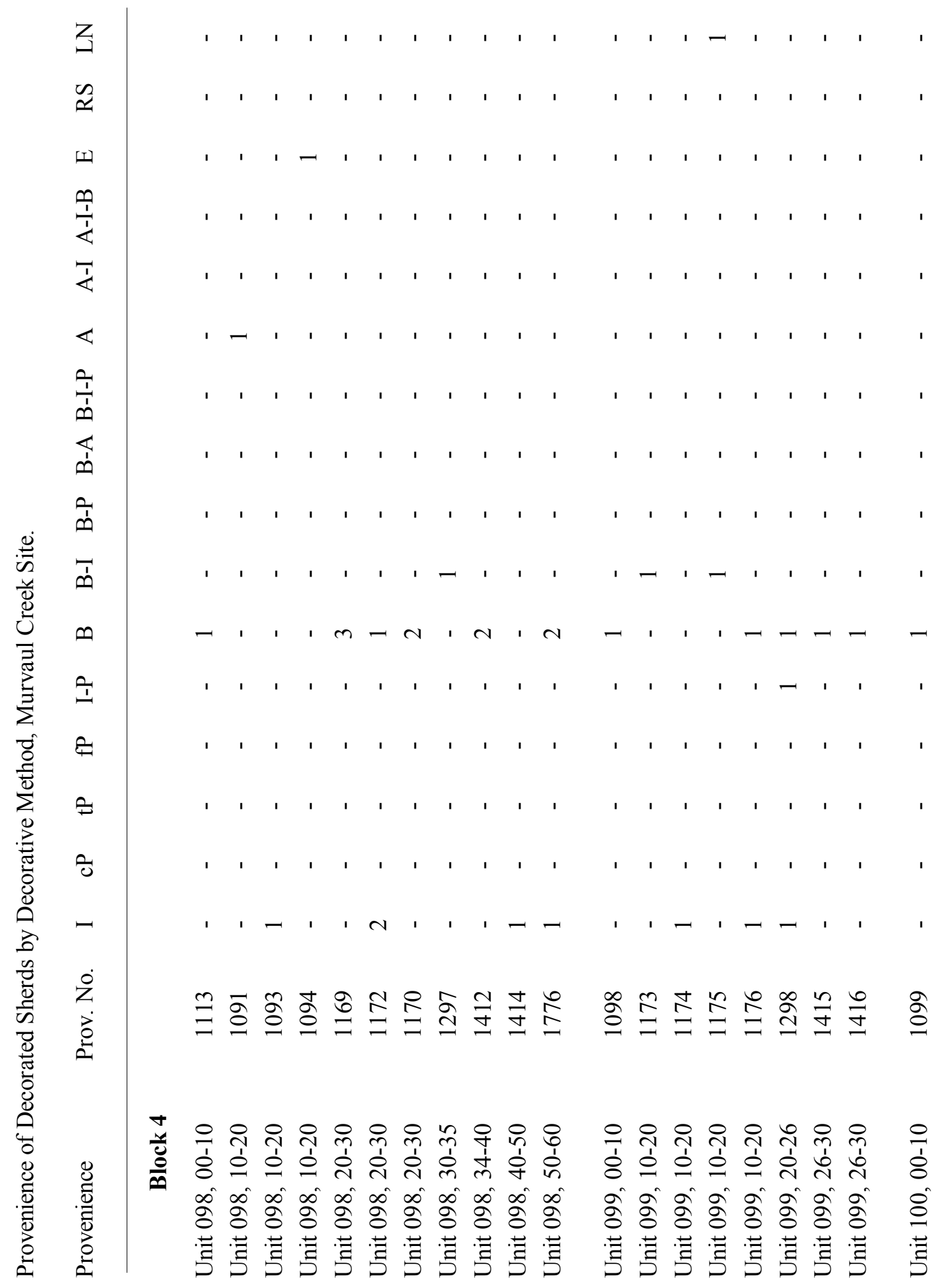









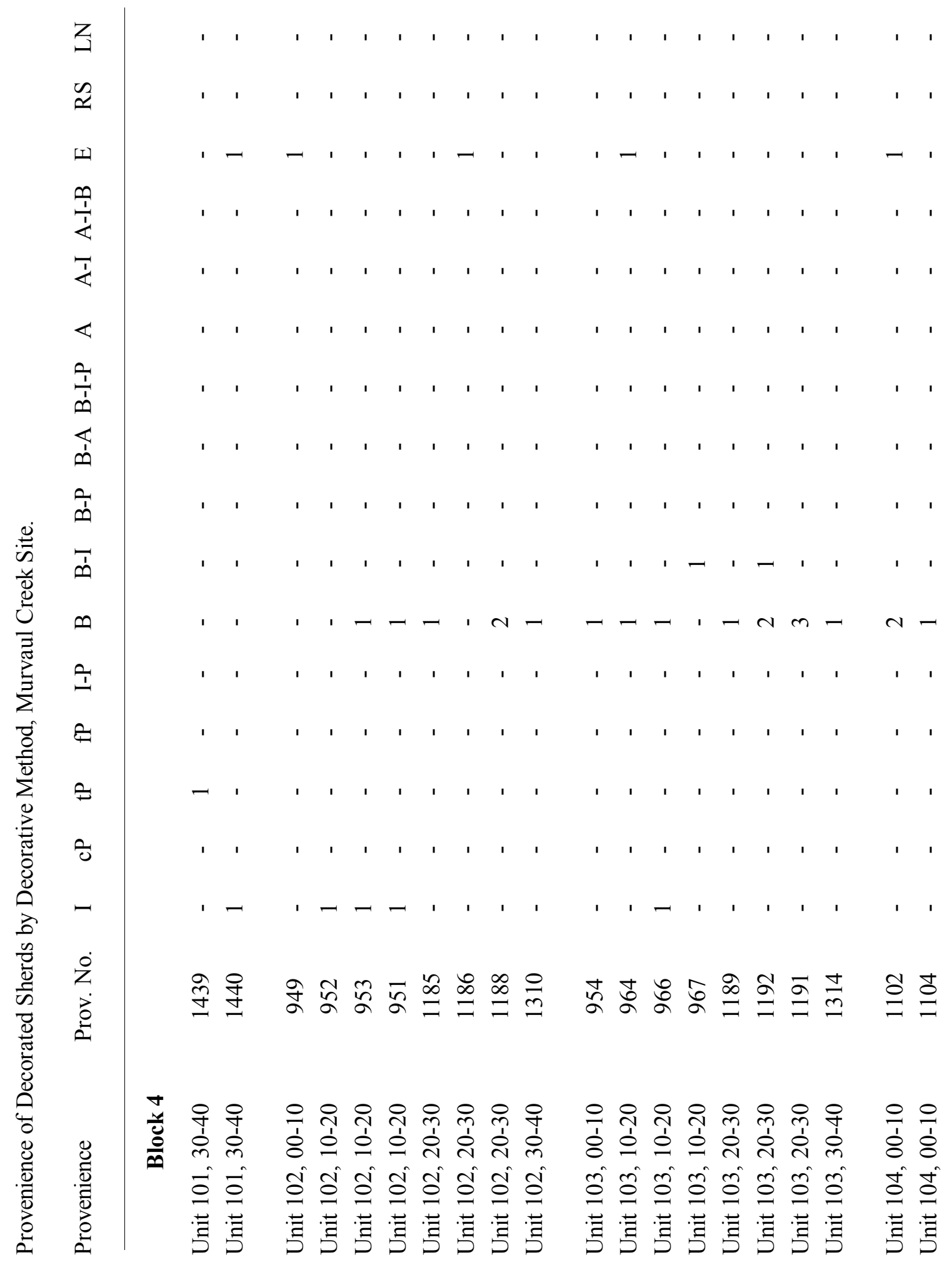




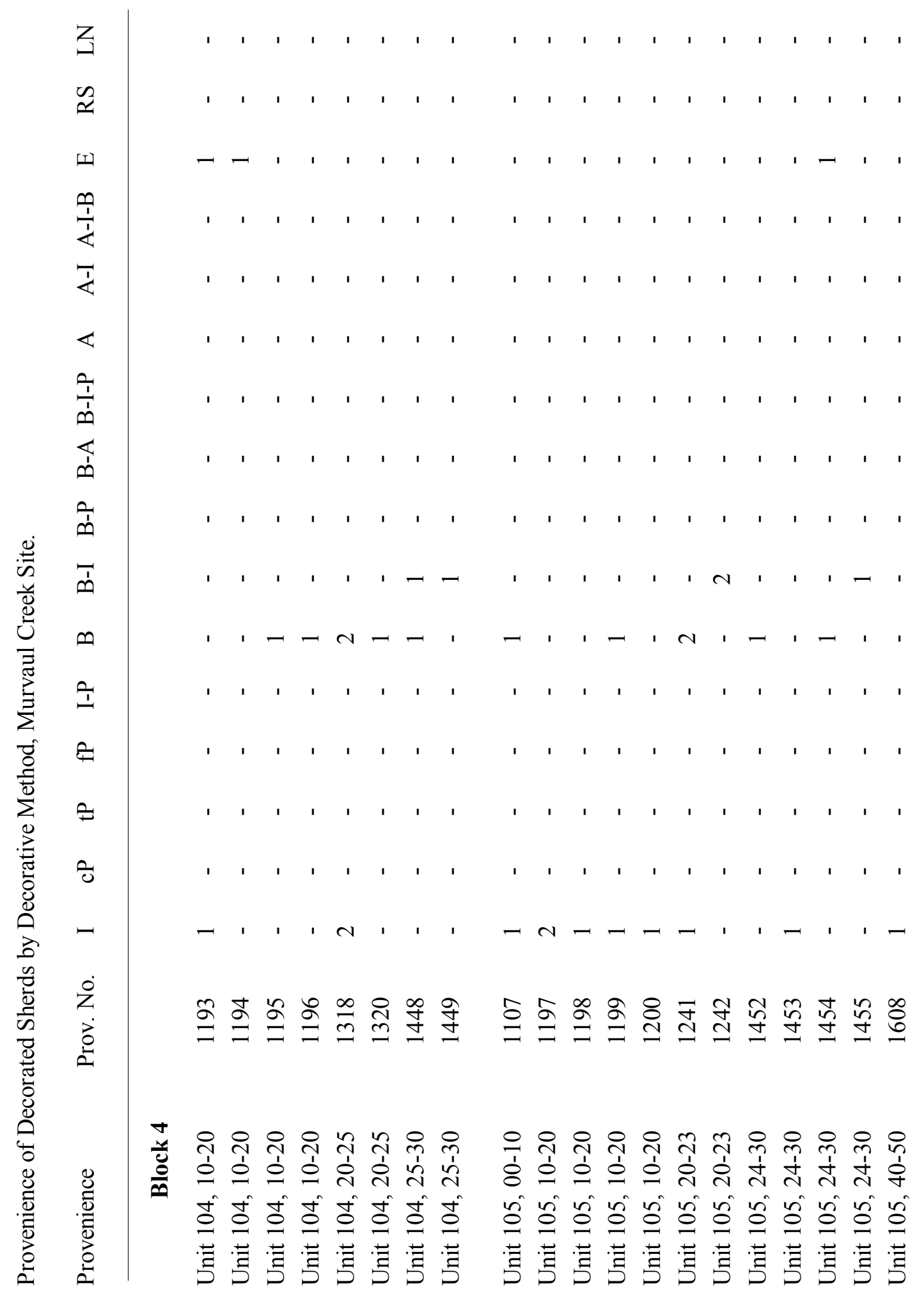




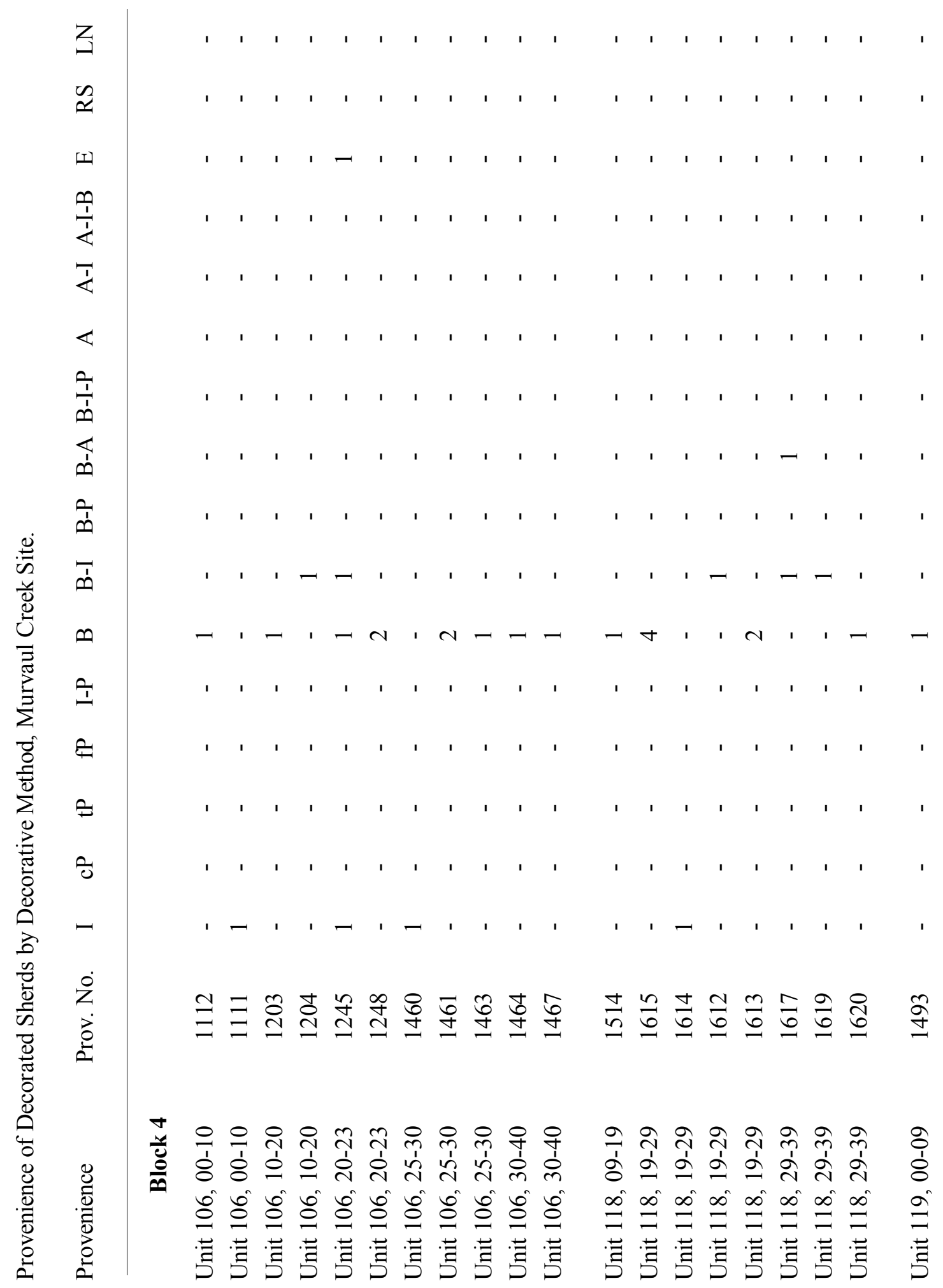




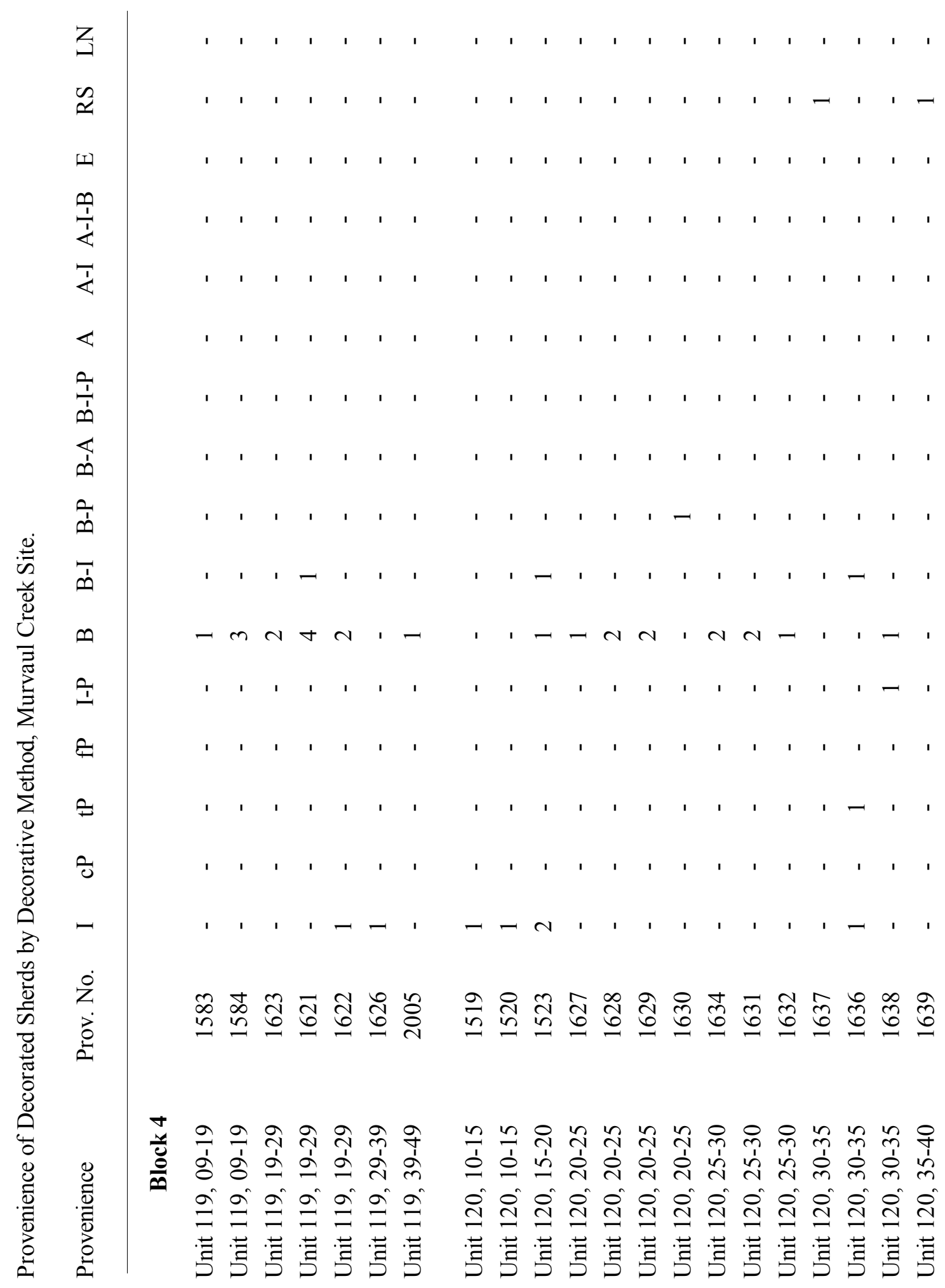




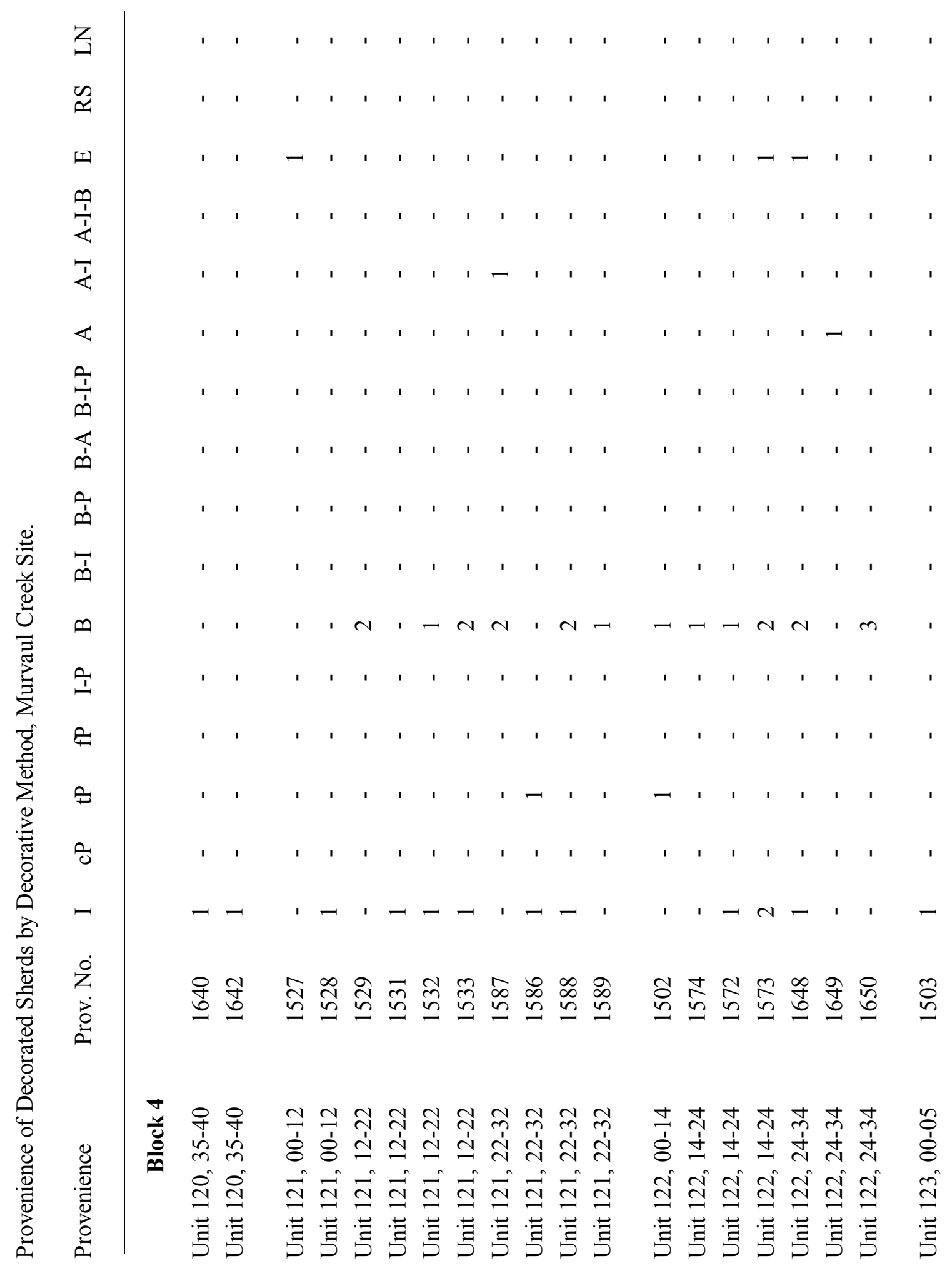




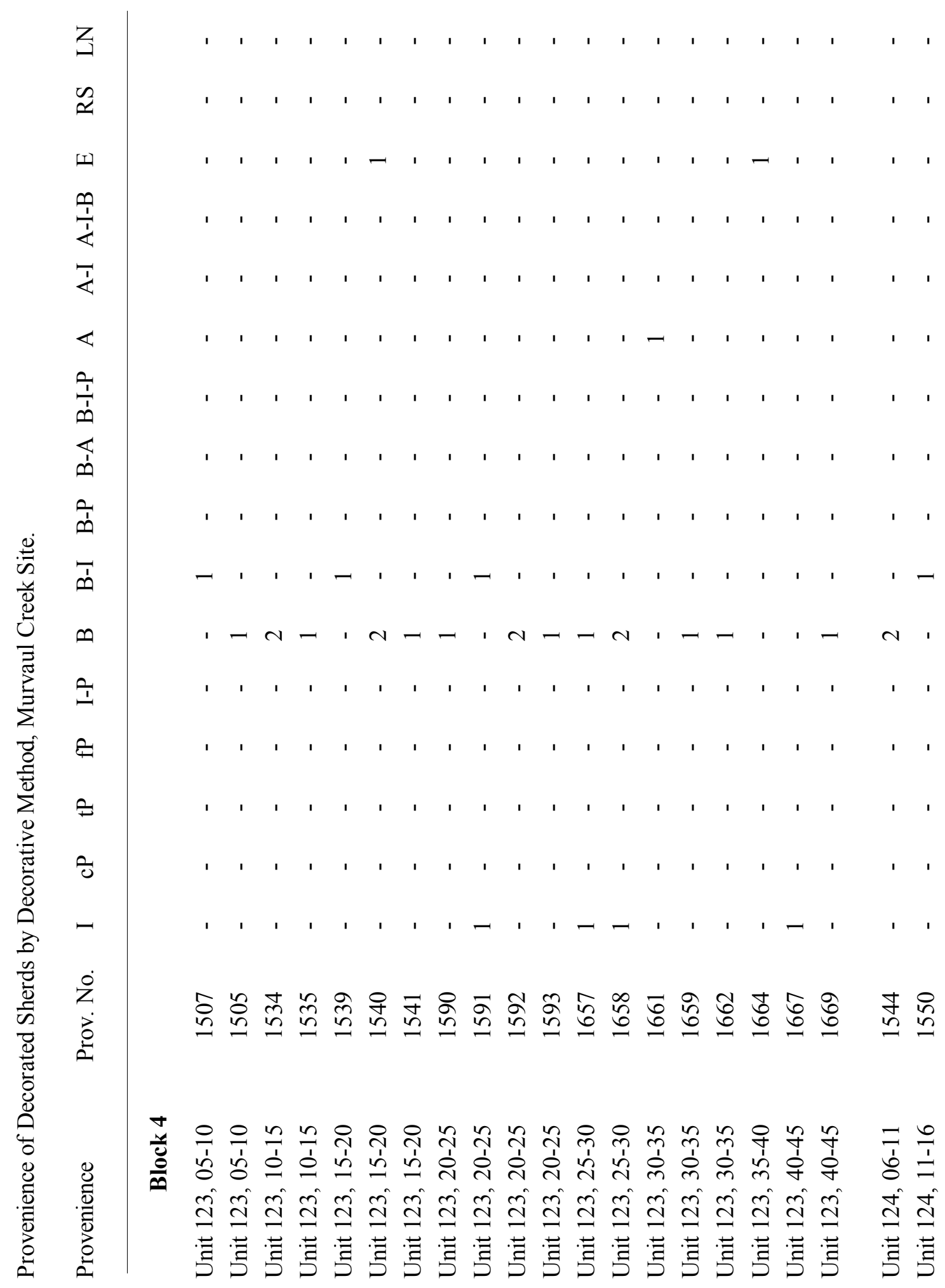




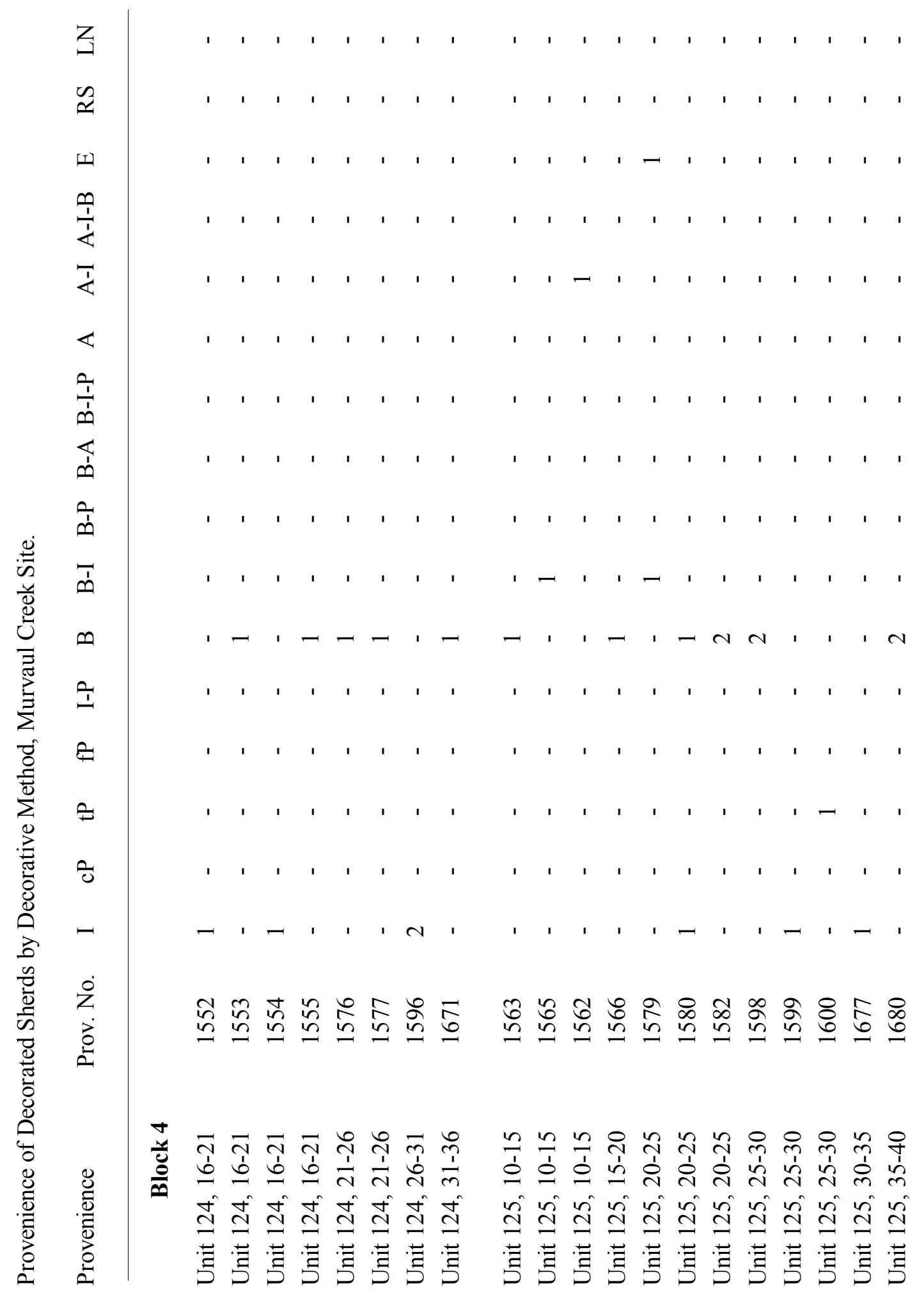




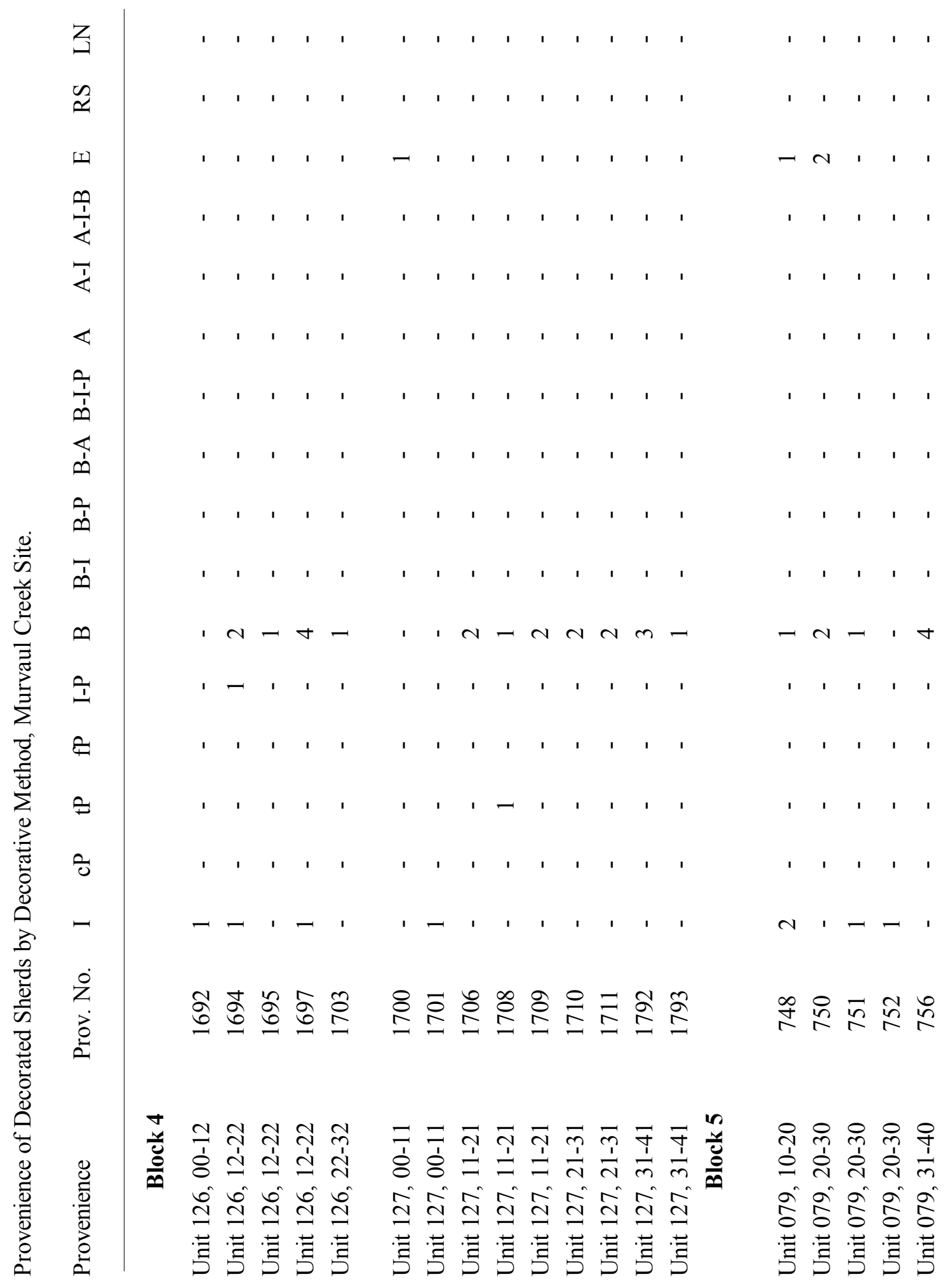




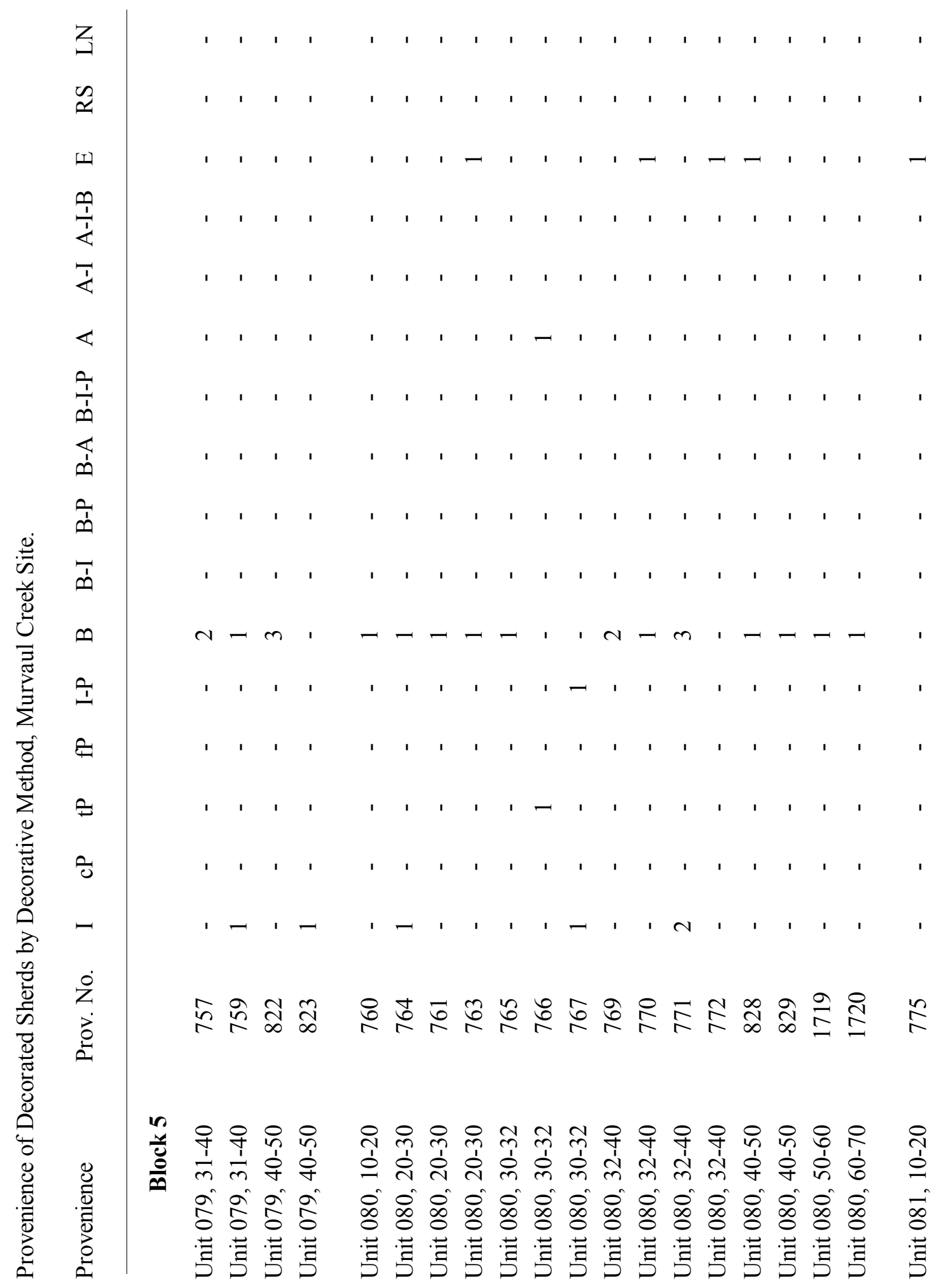




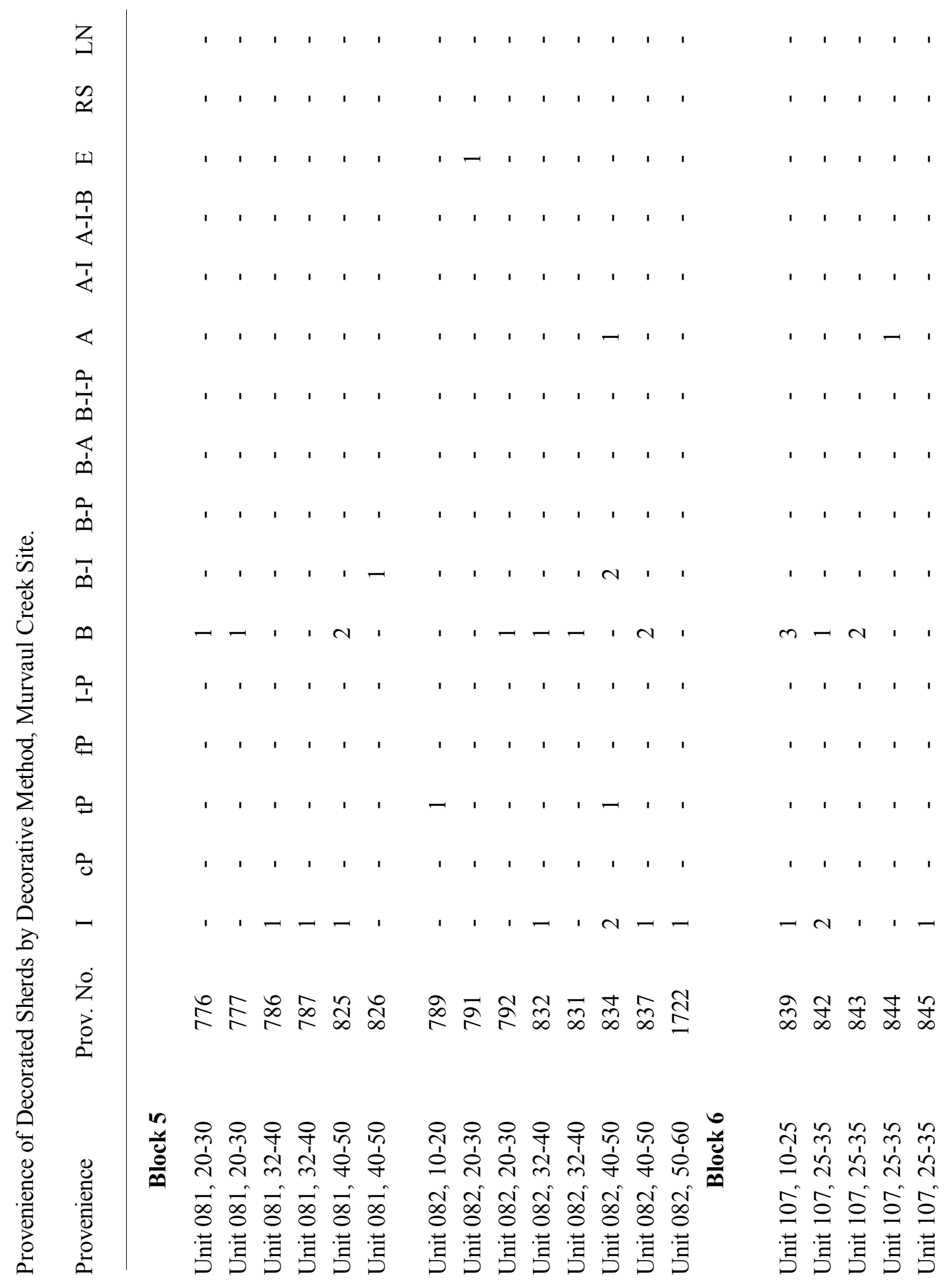




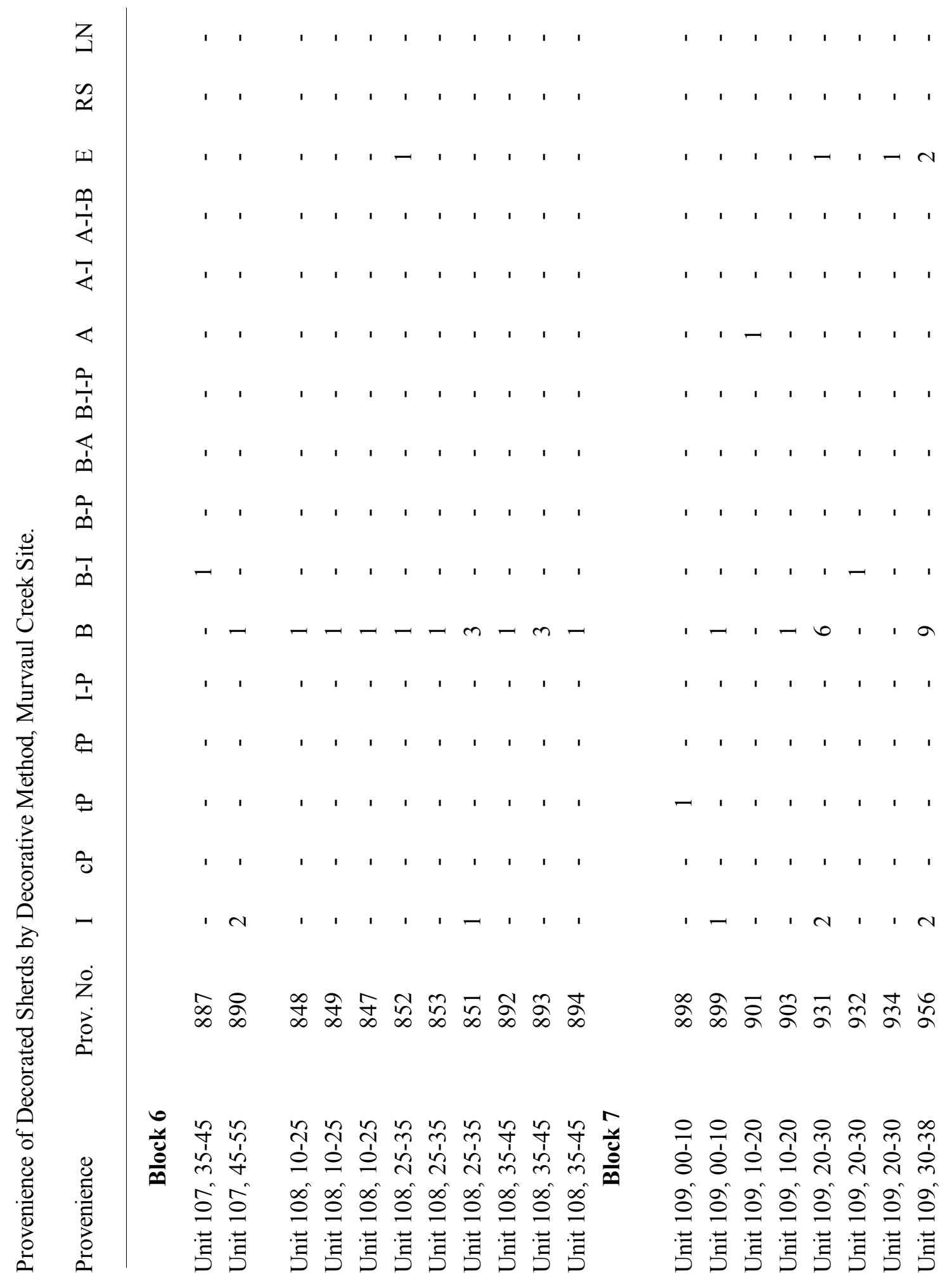









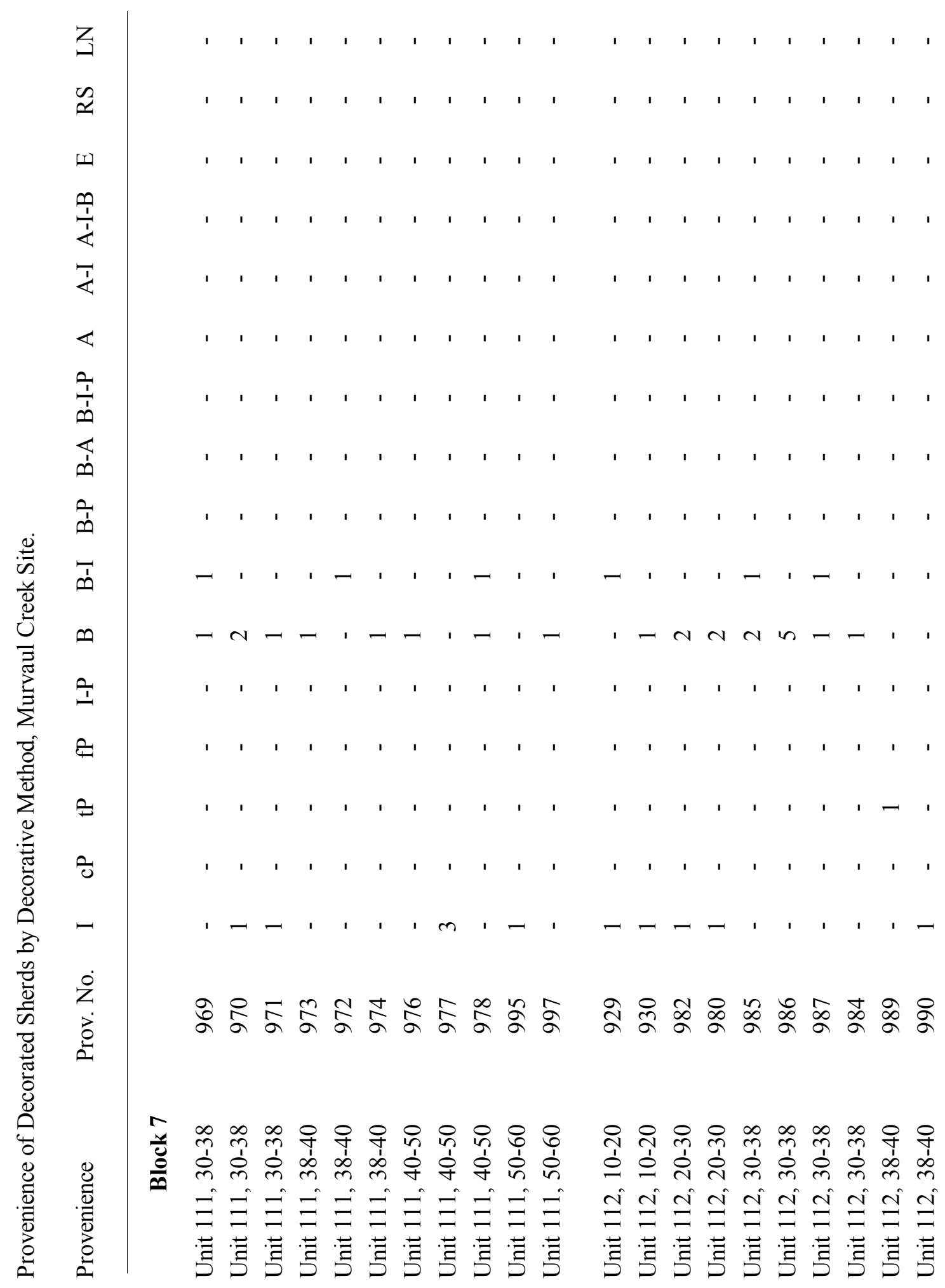




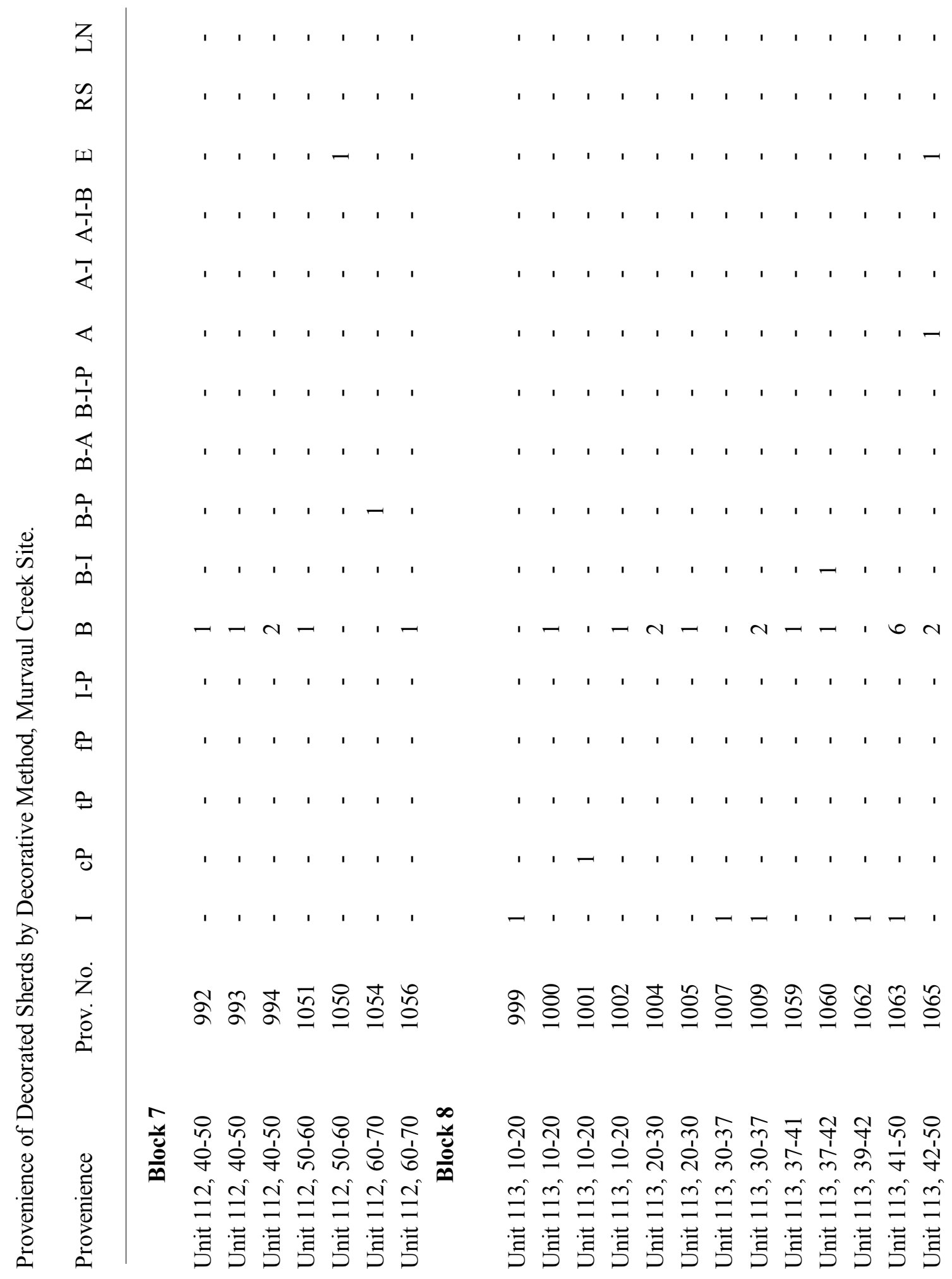




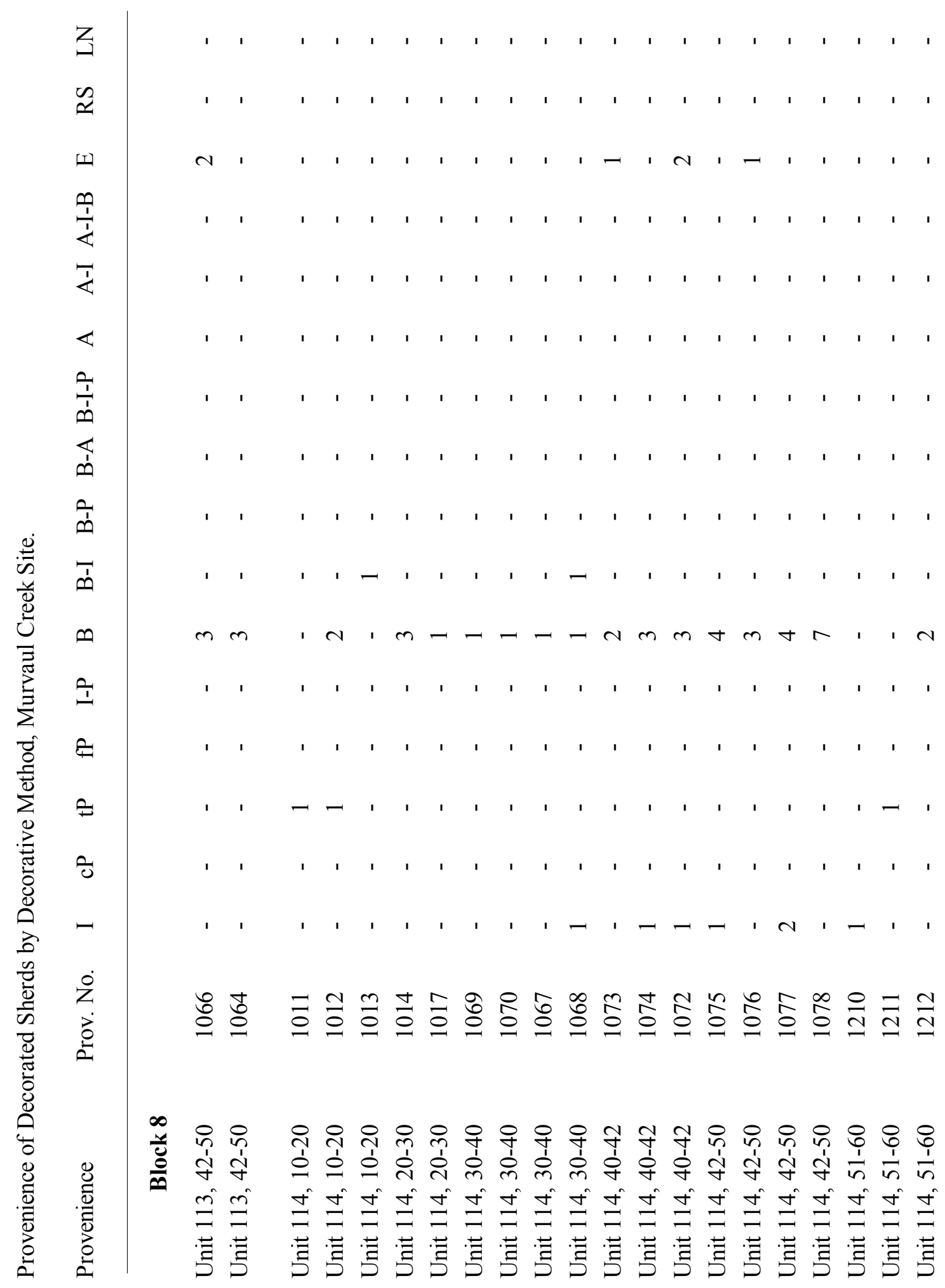




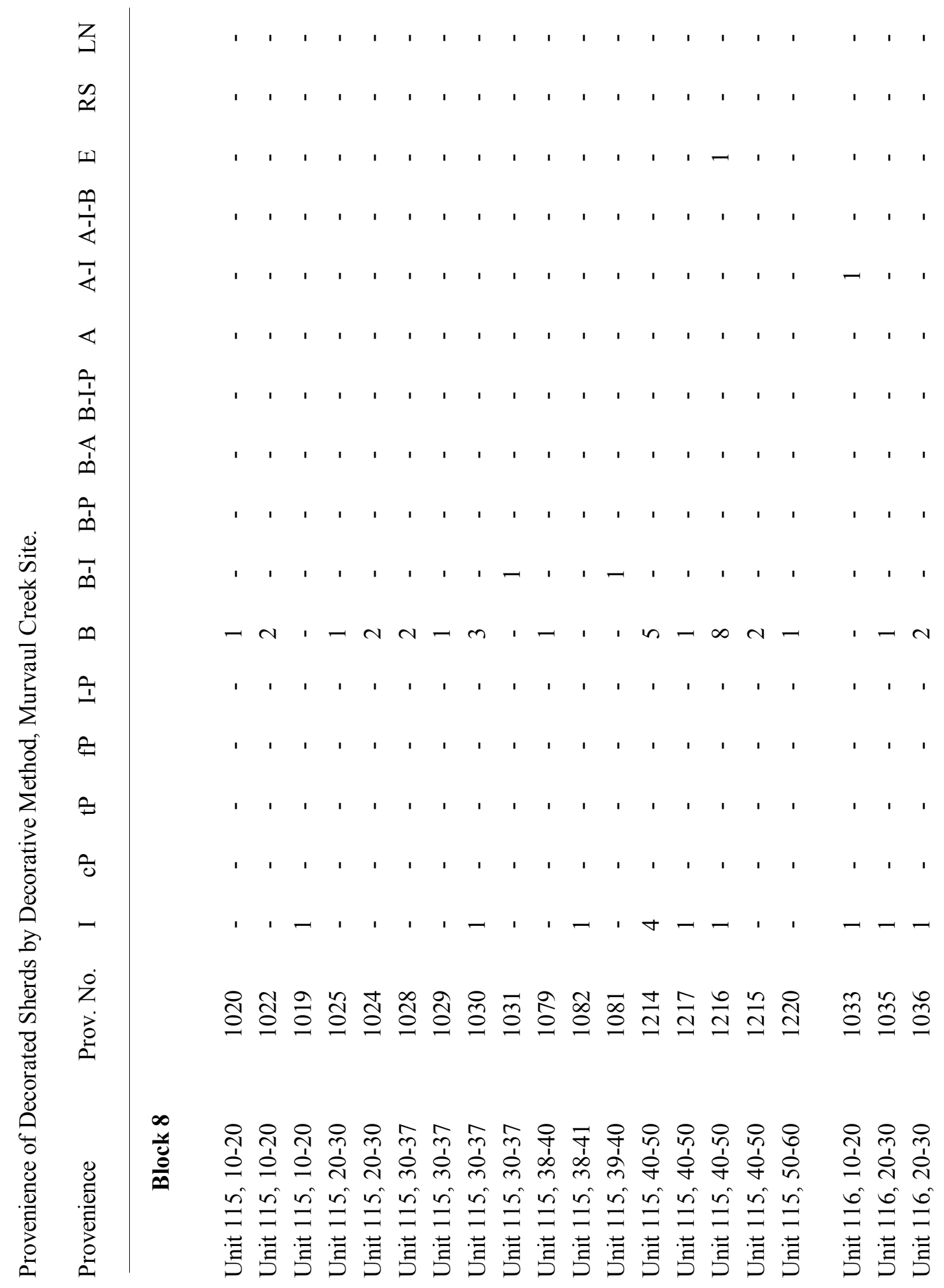




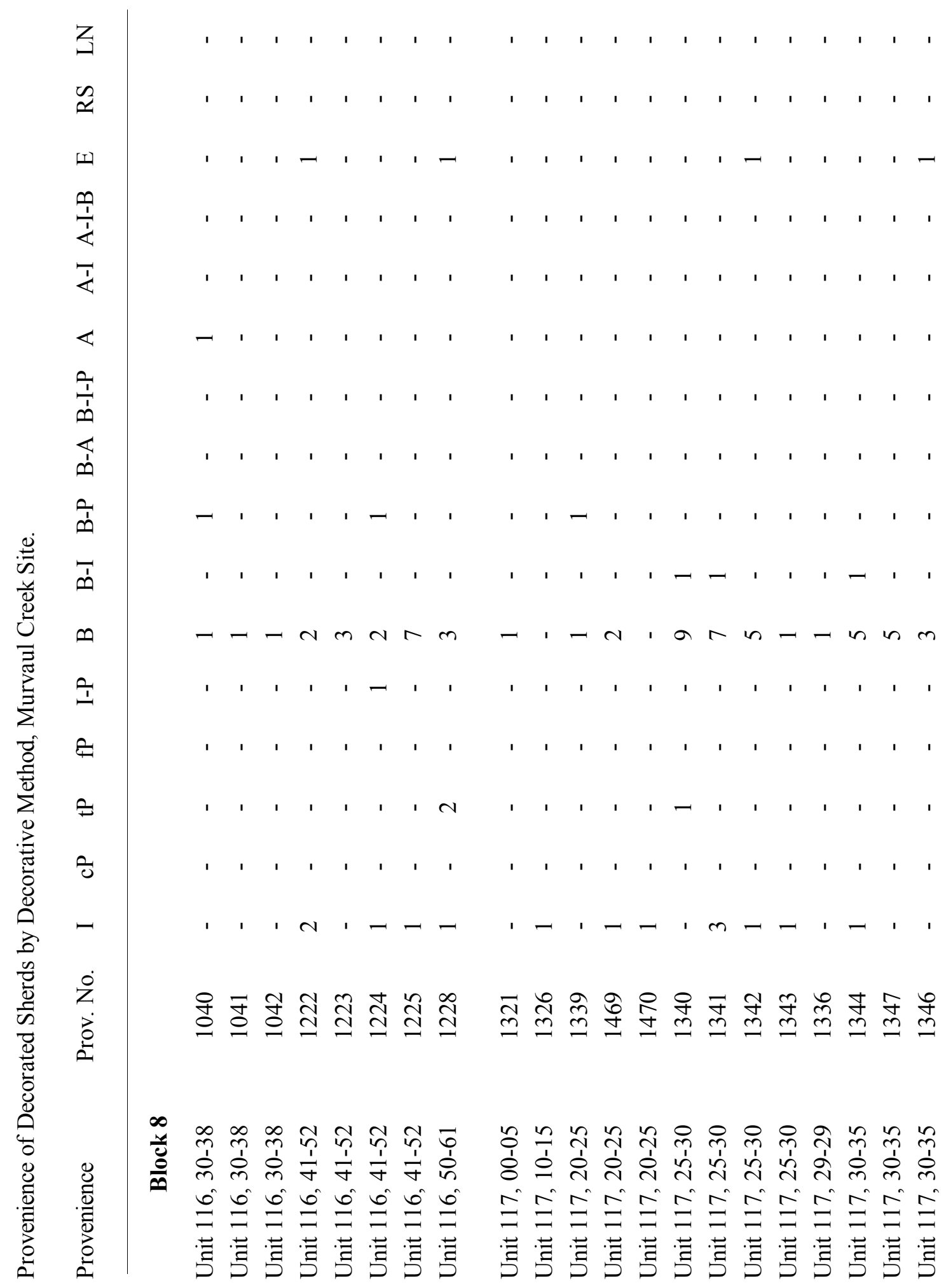




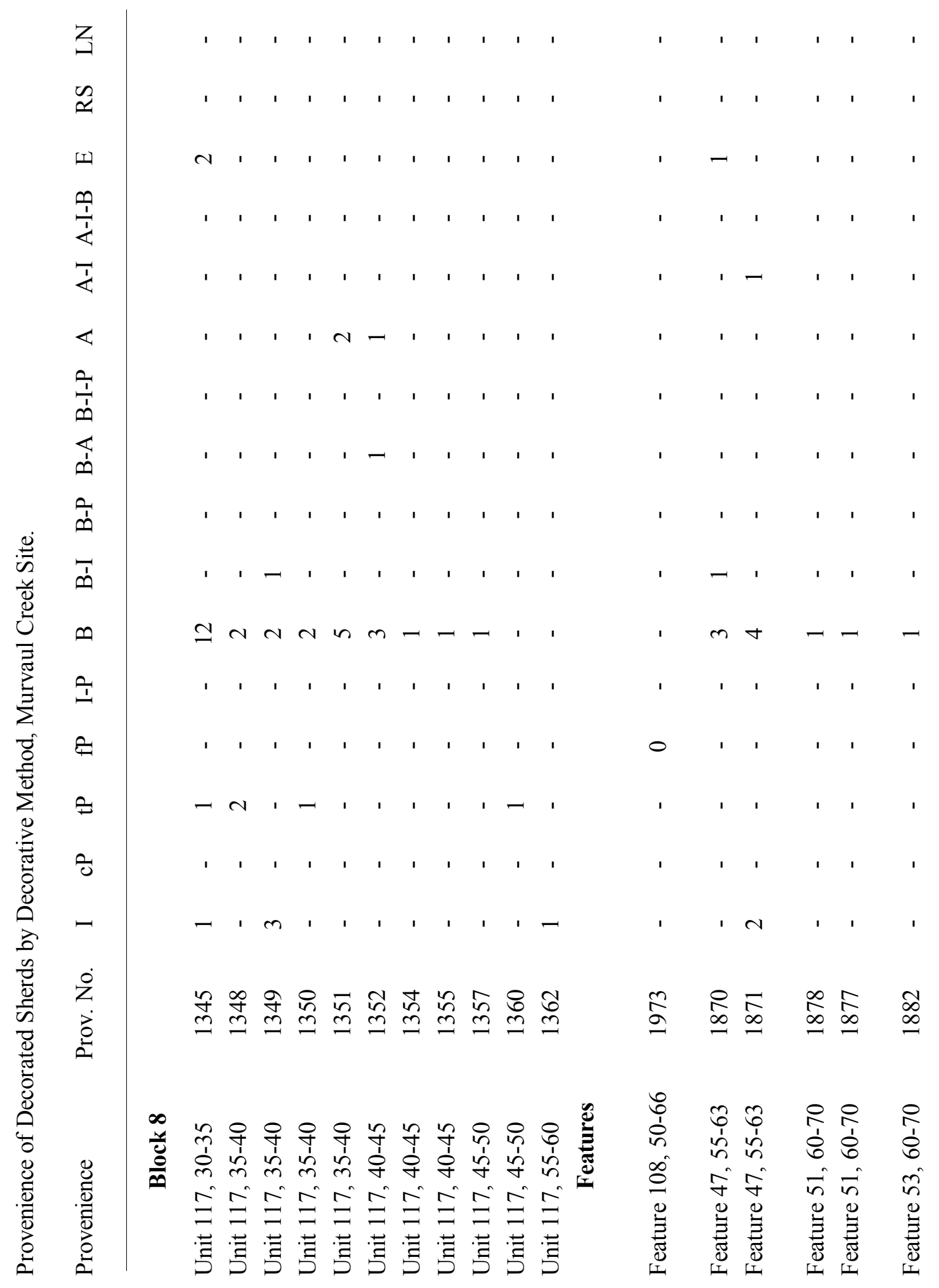









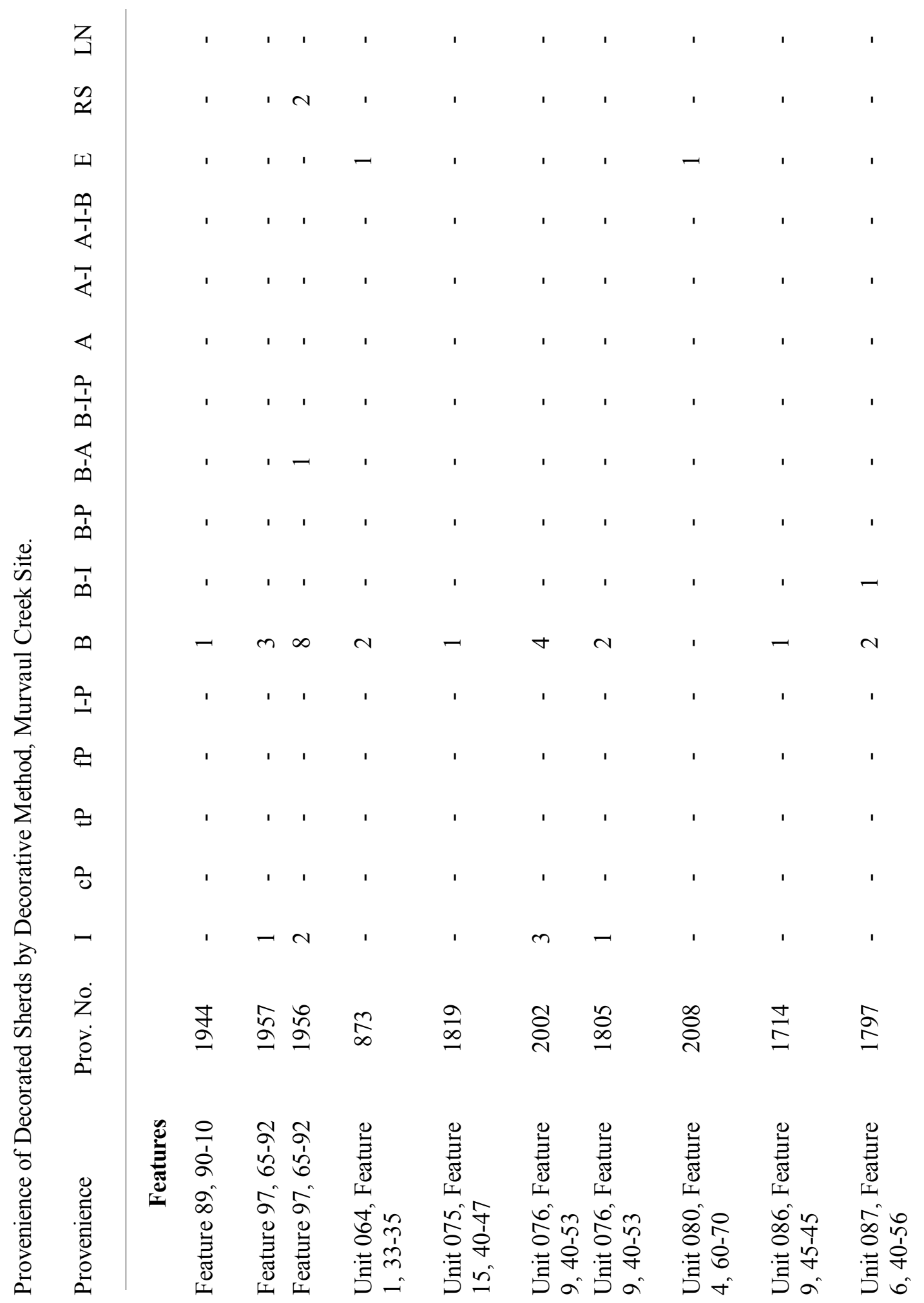




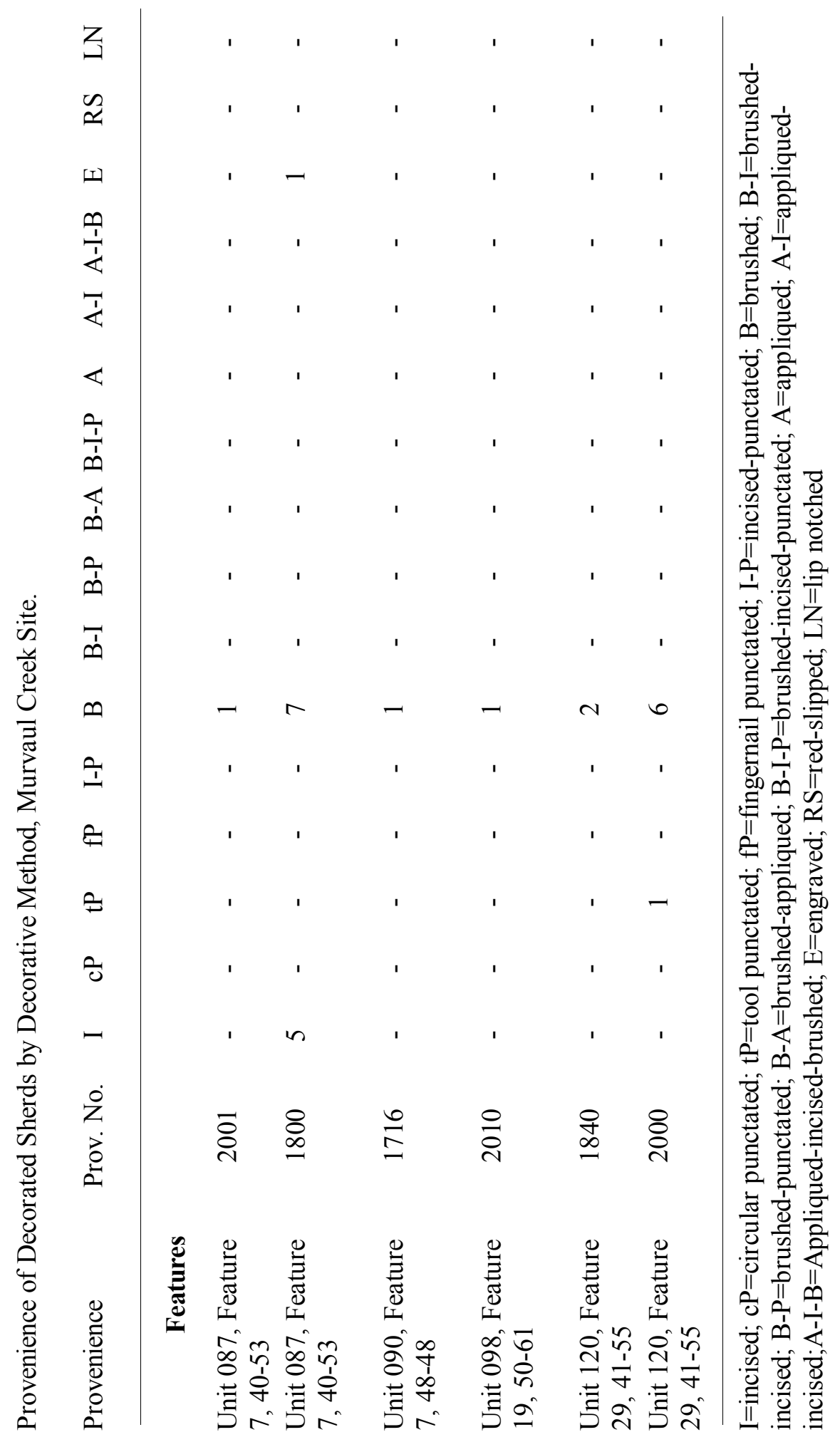





\section{A-3: DETAILED SHERD ANALYSIS}





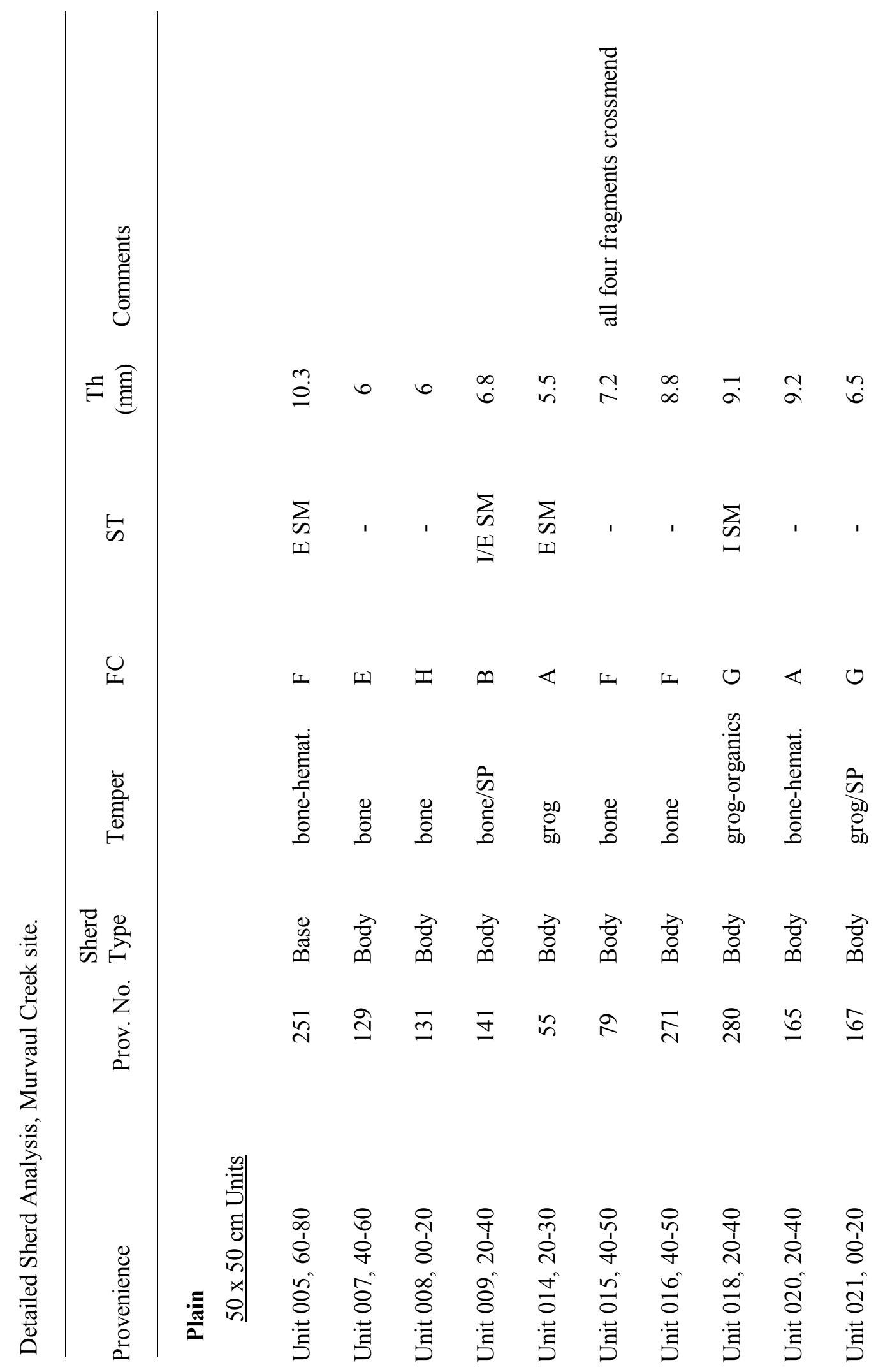









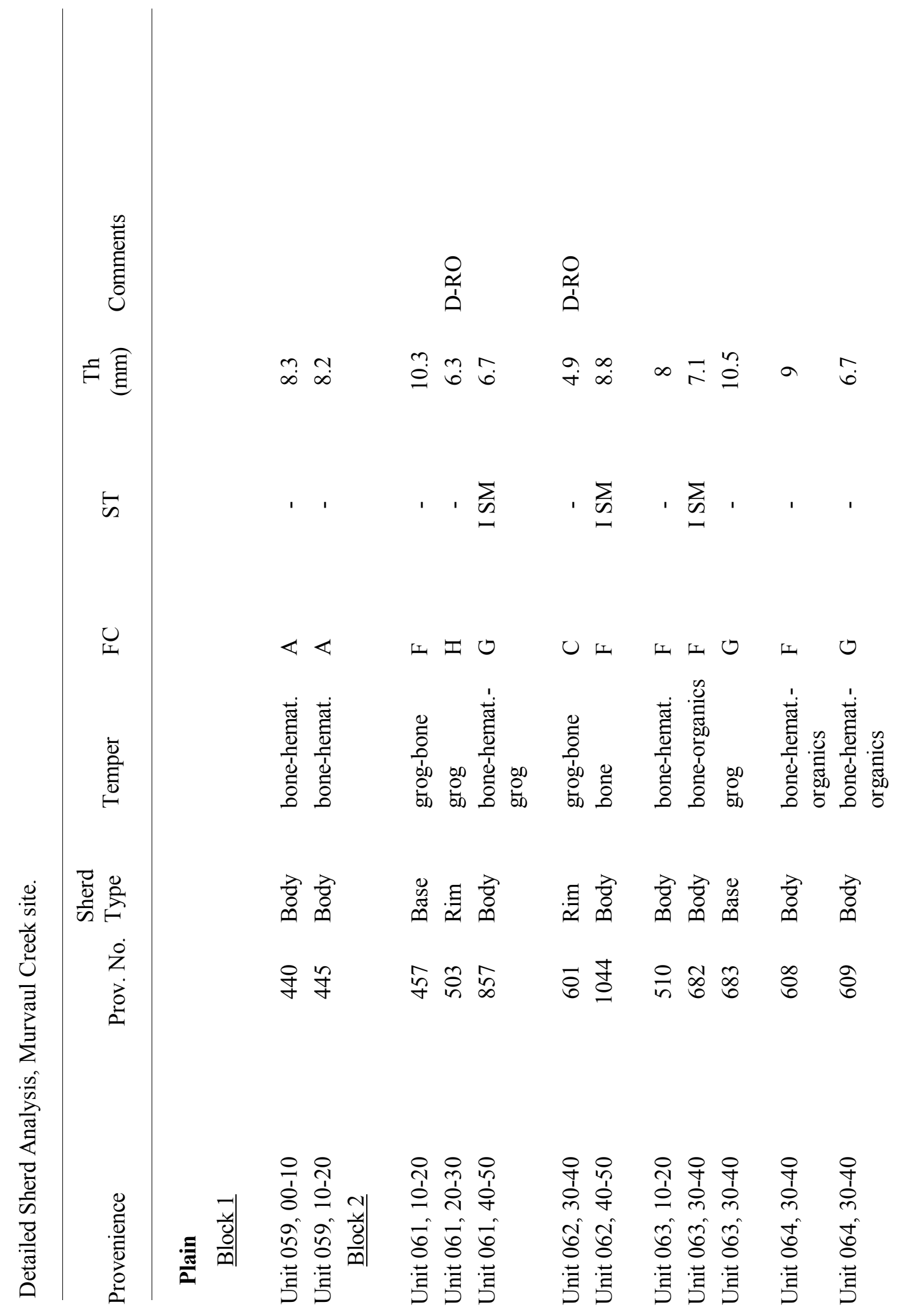




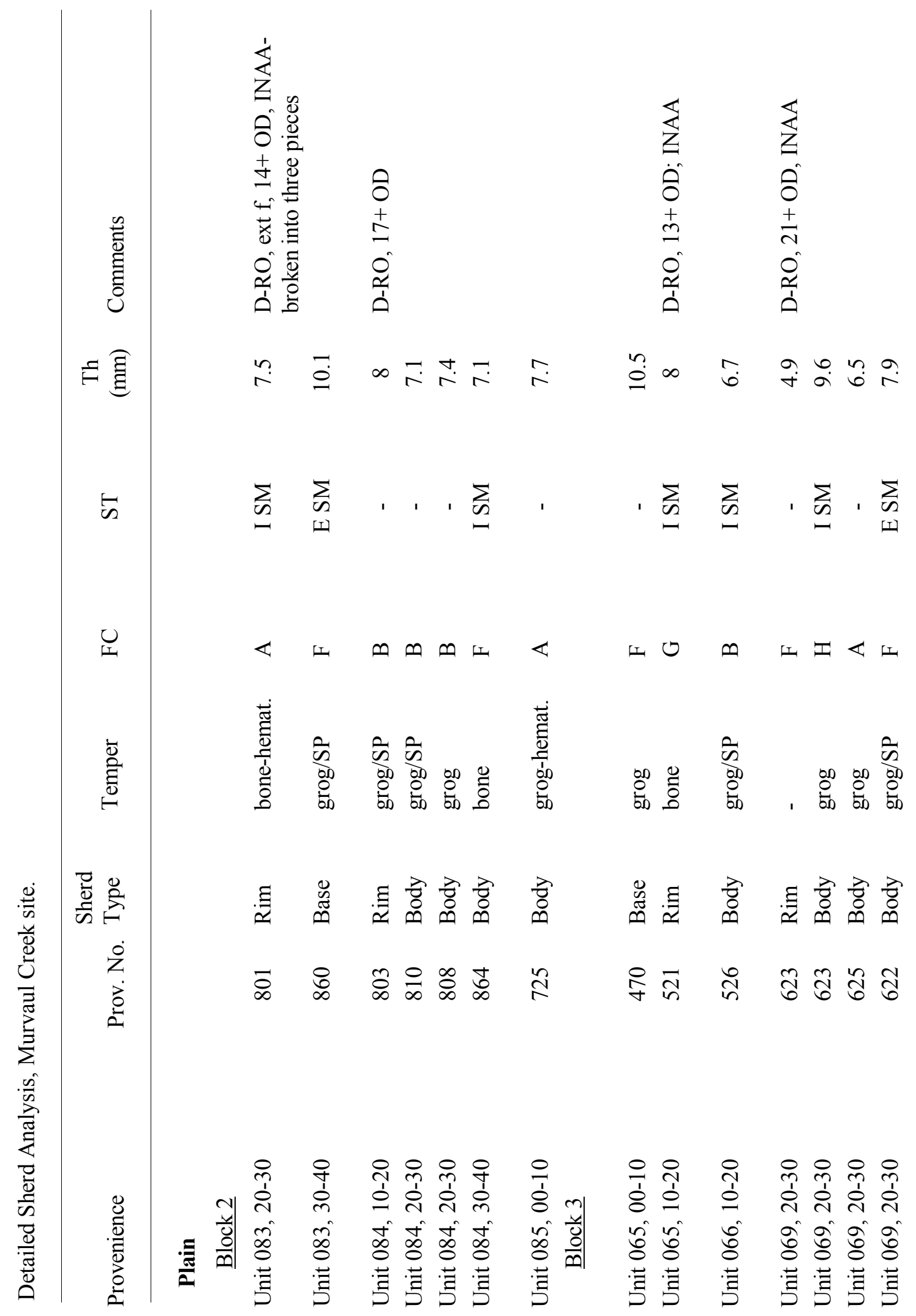




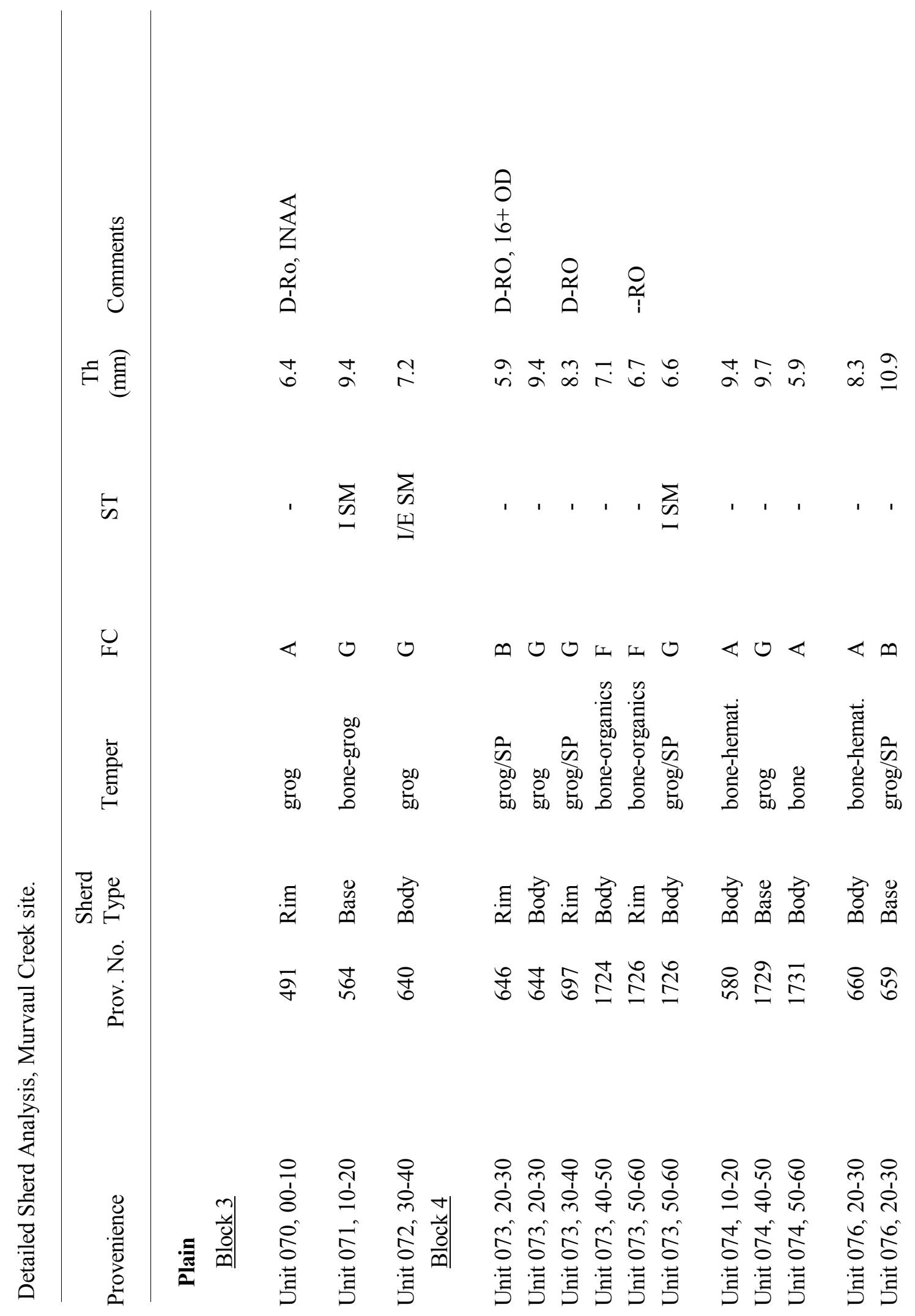




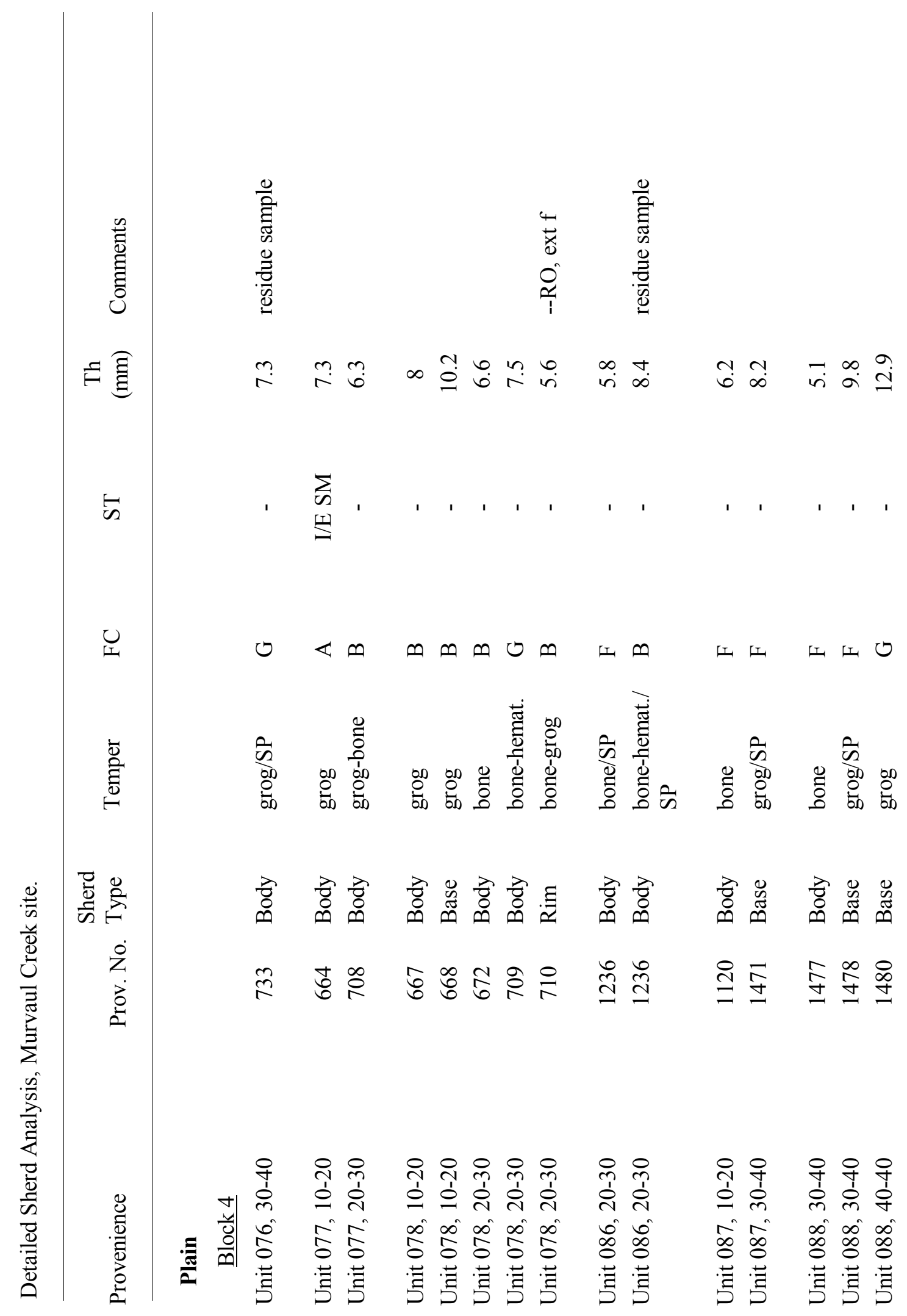




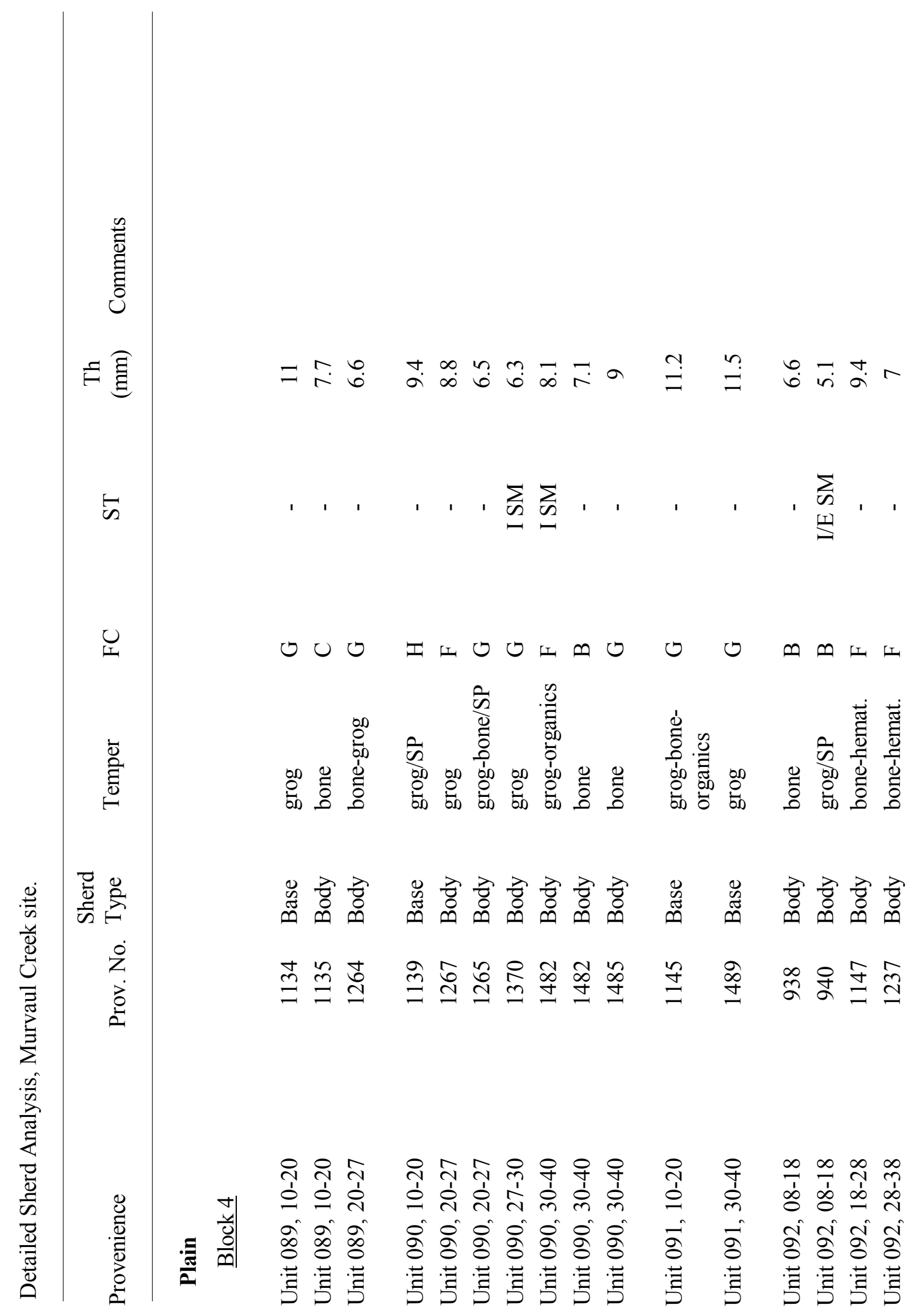




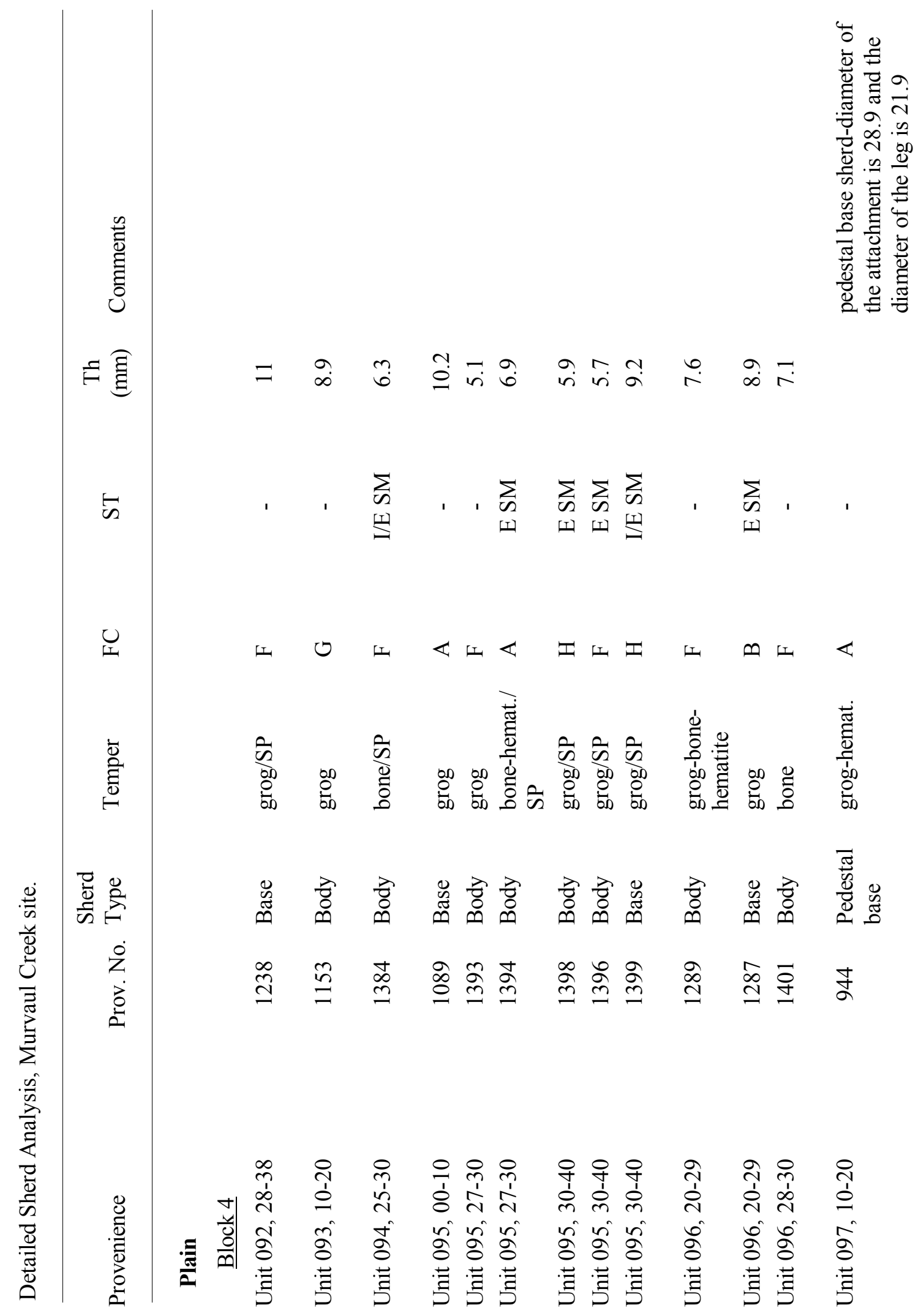




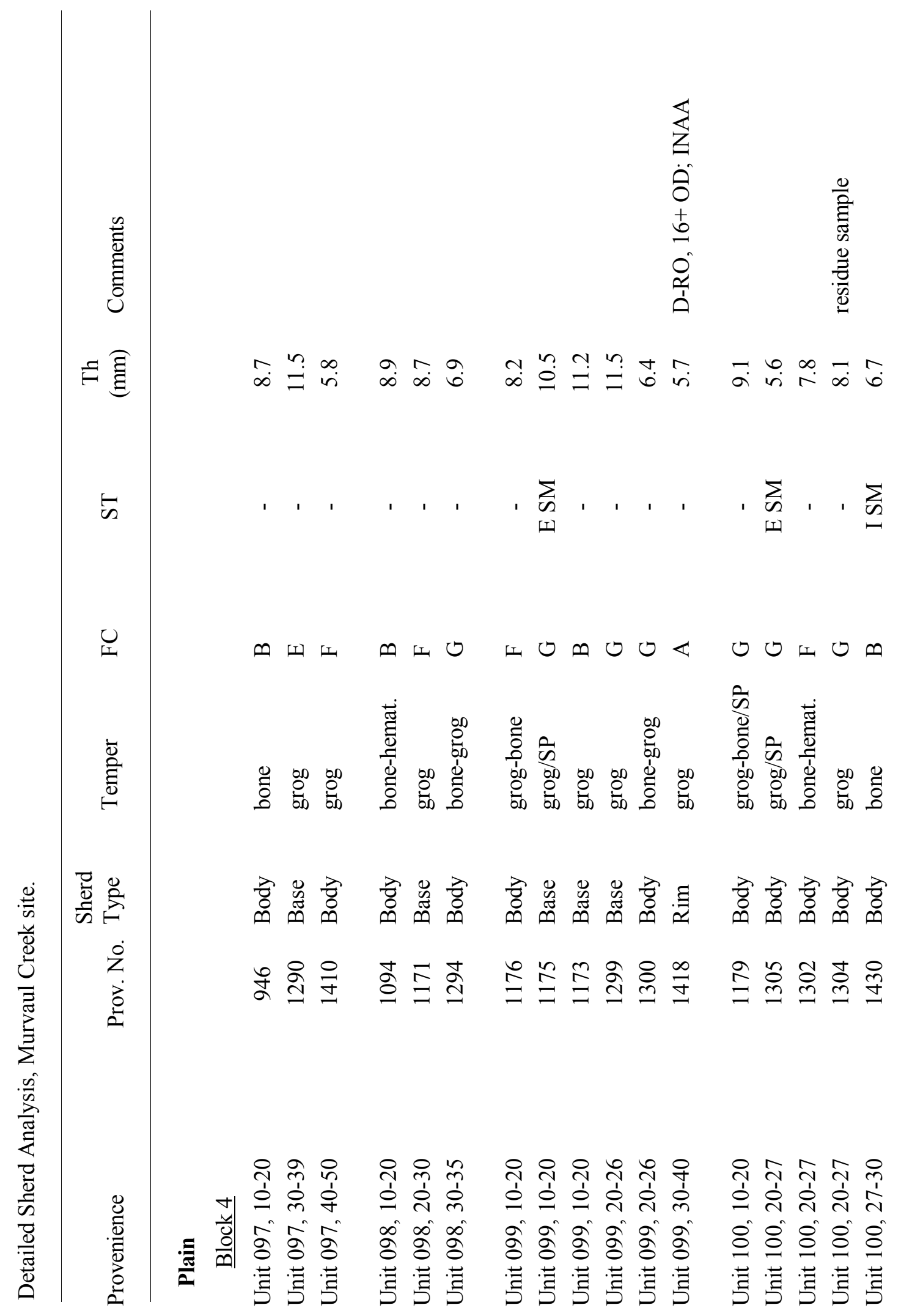




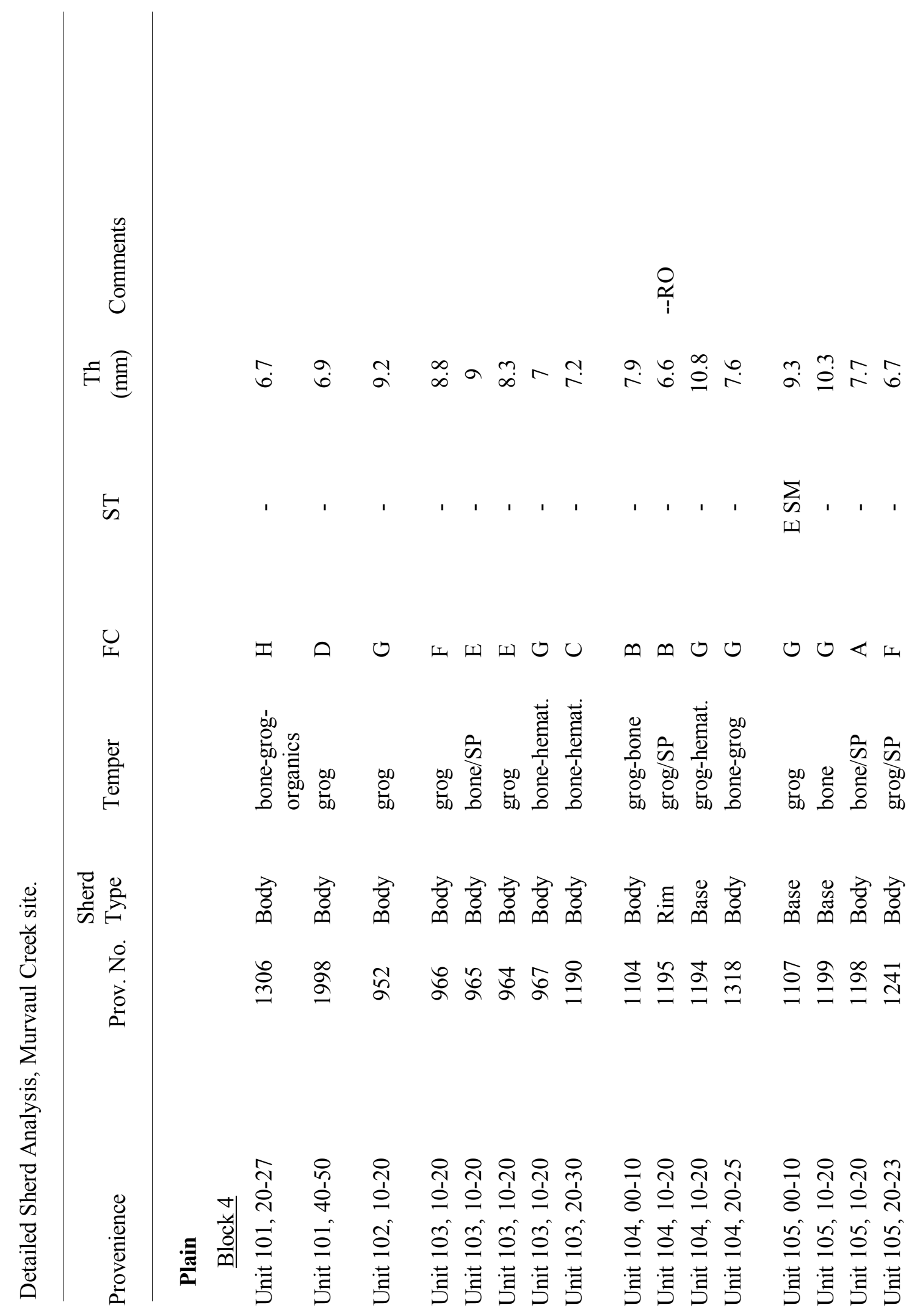









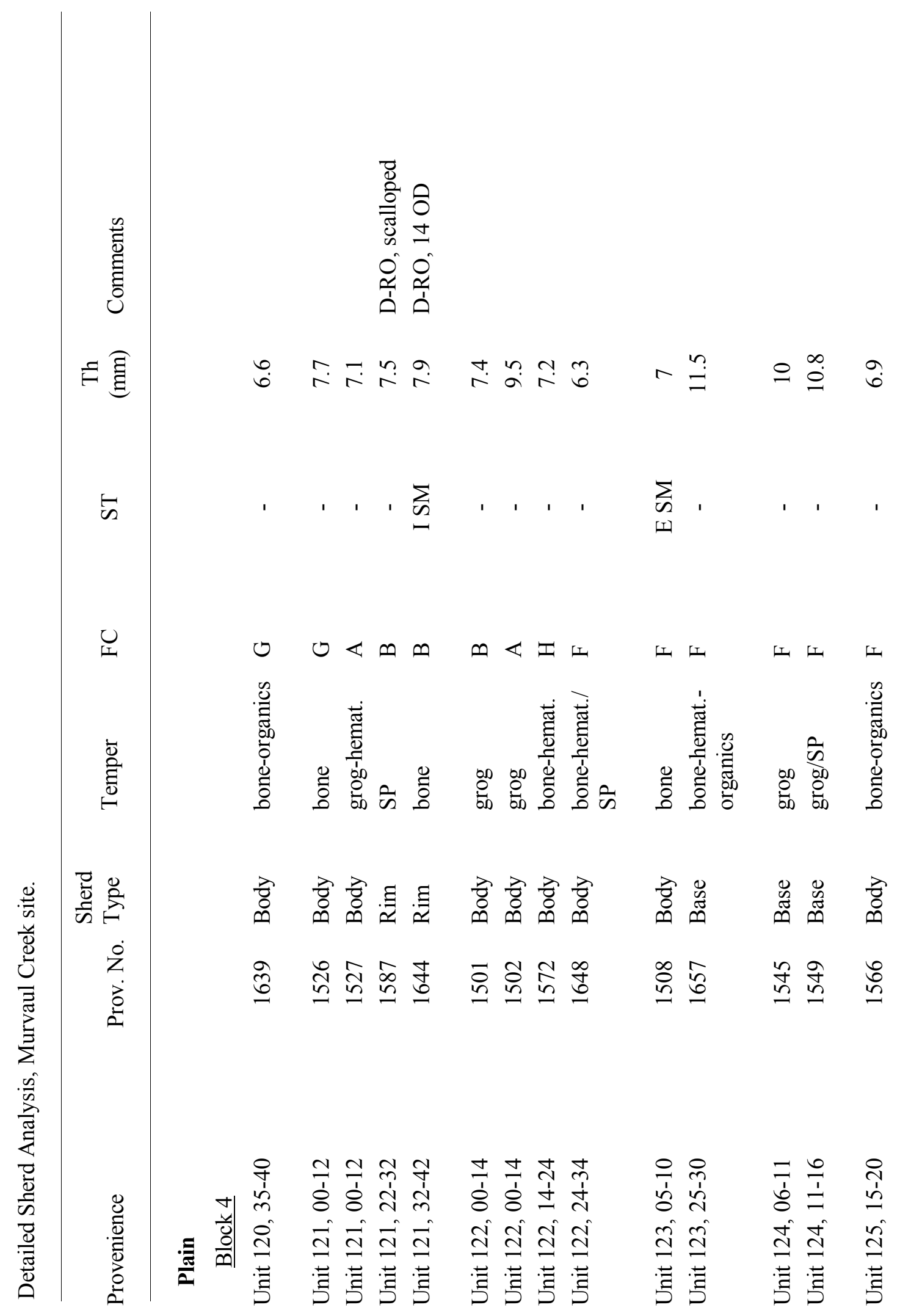




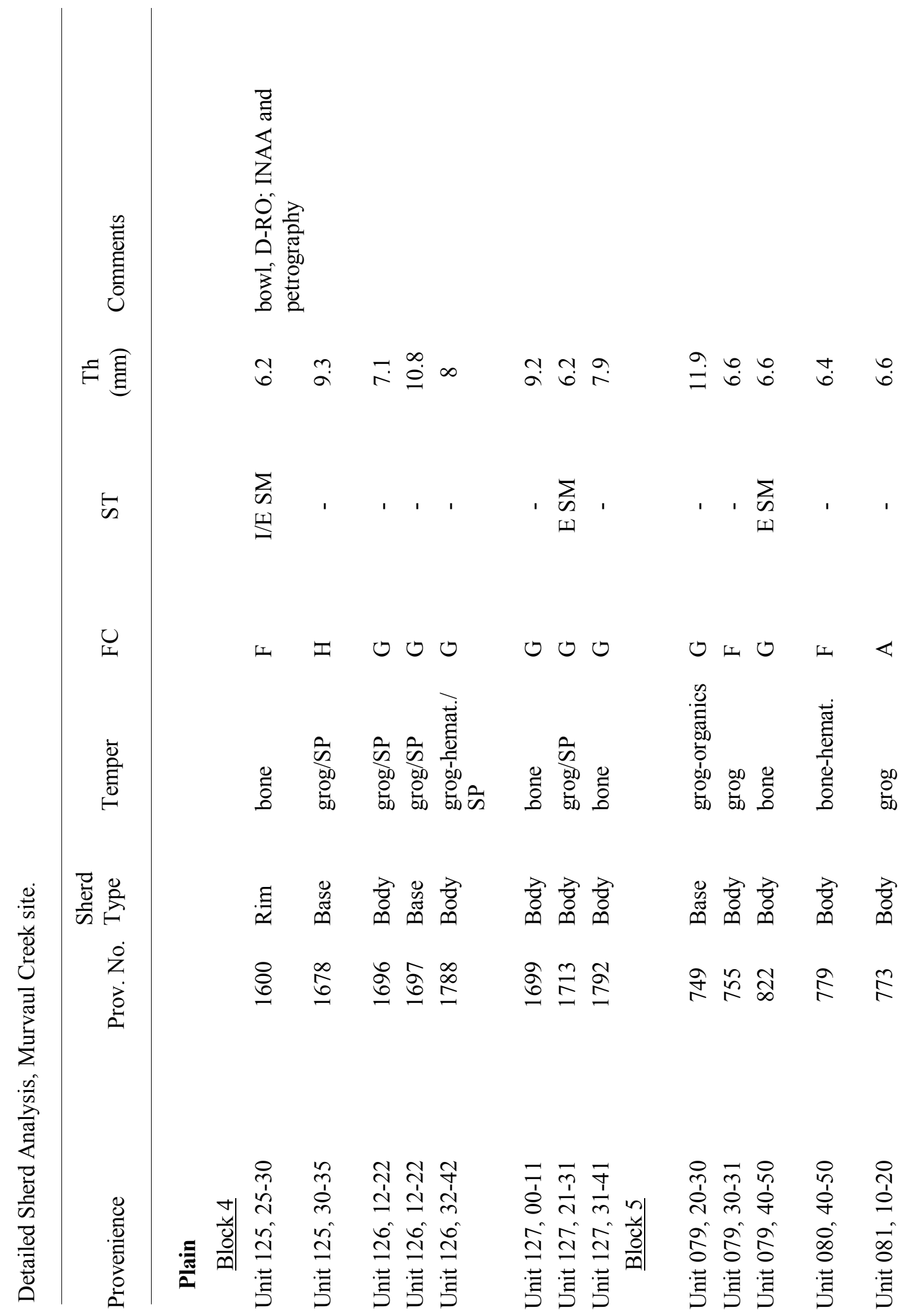




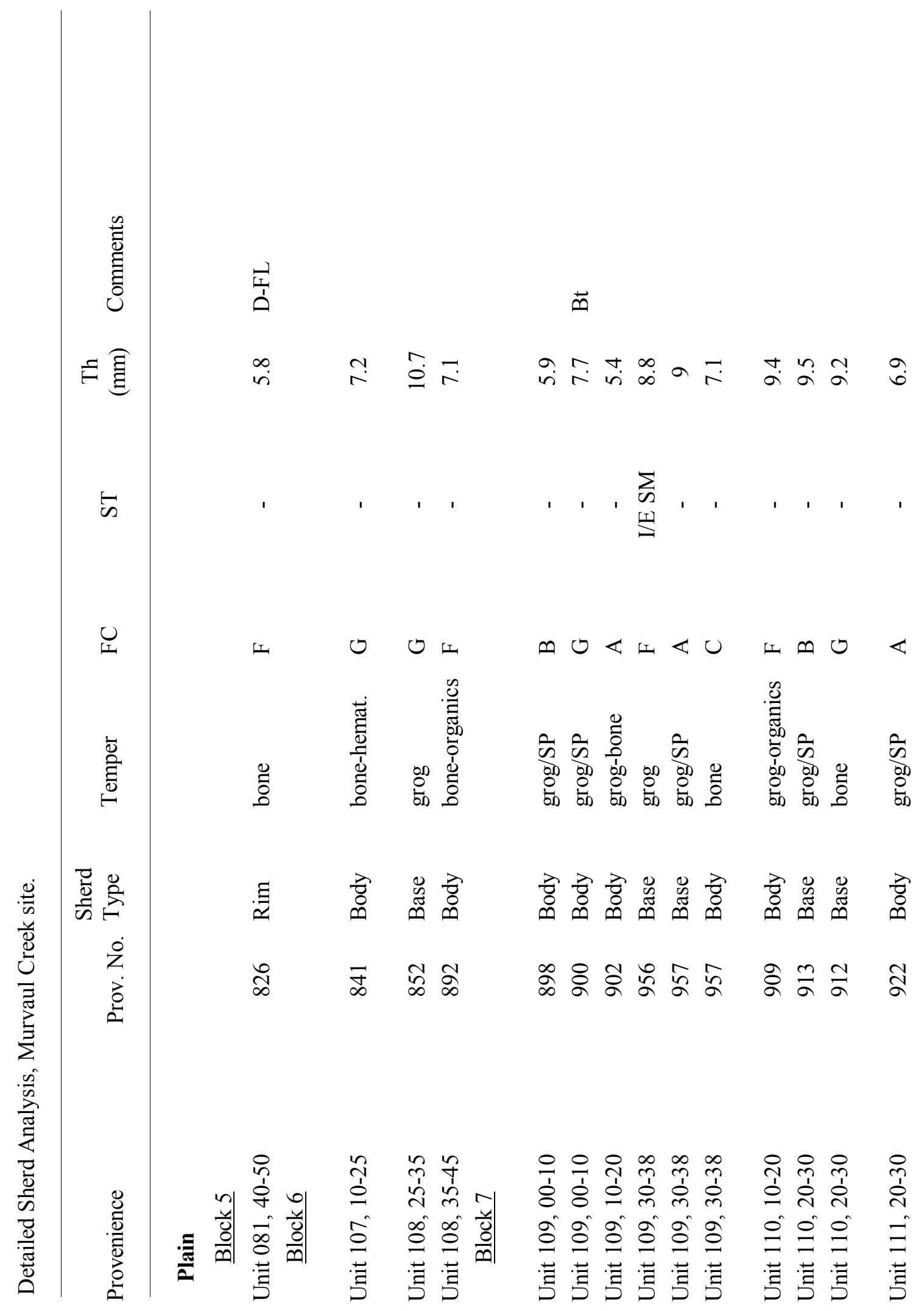




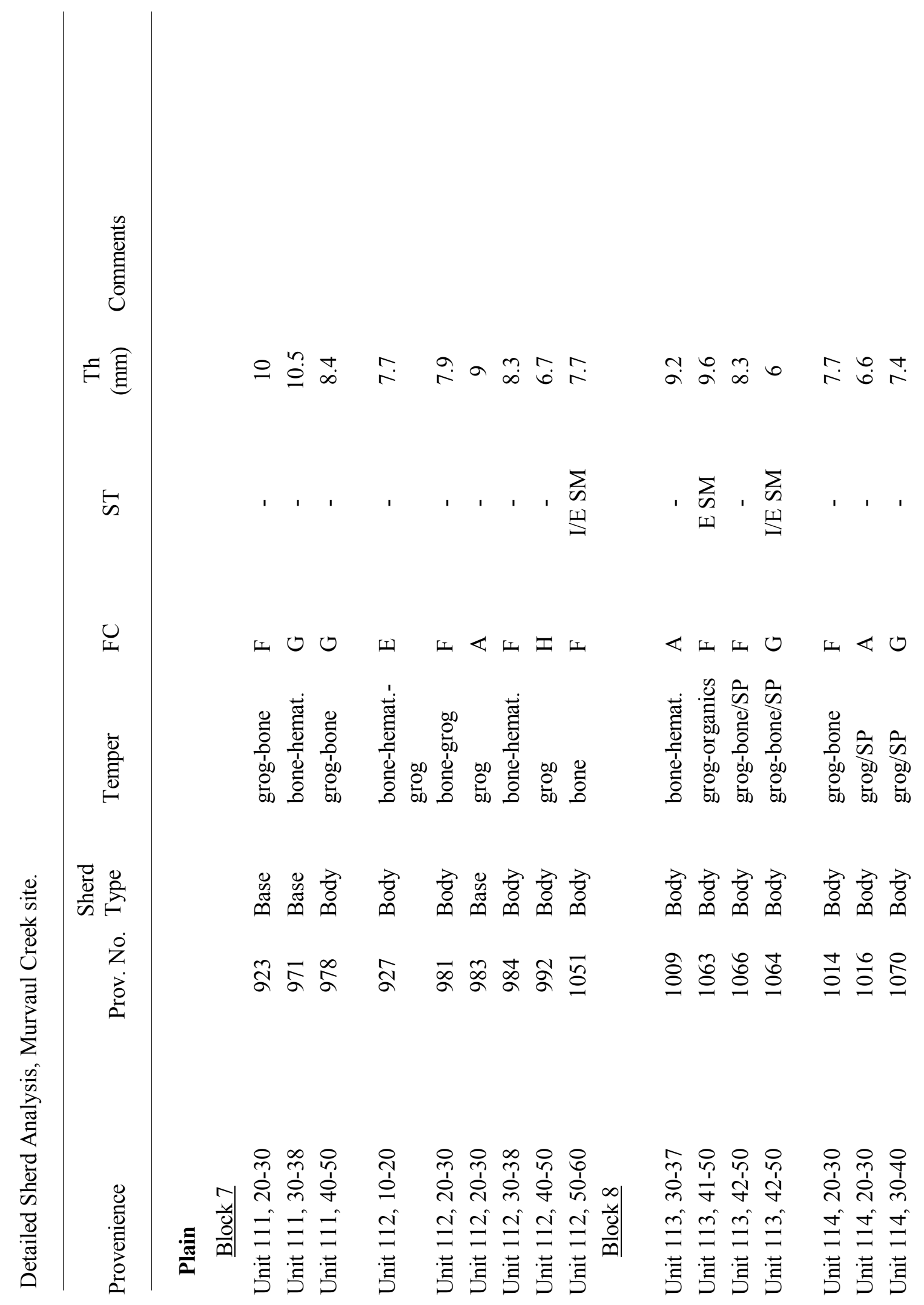




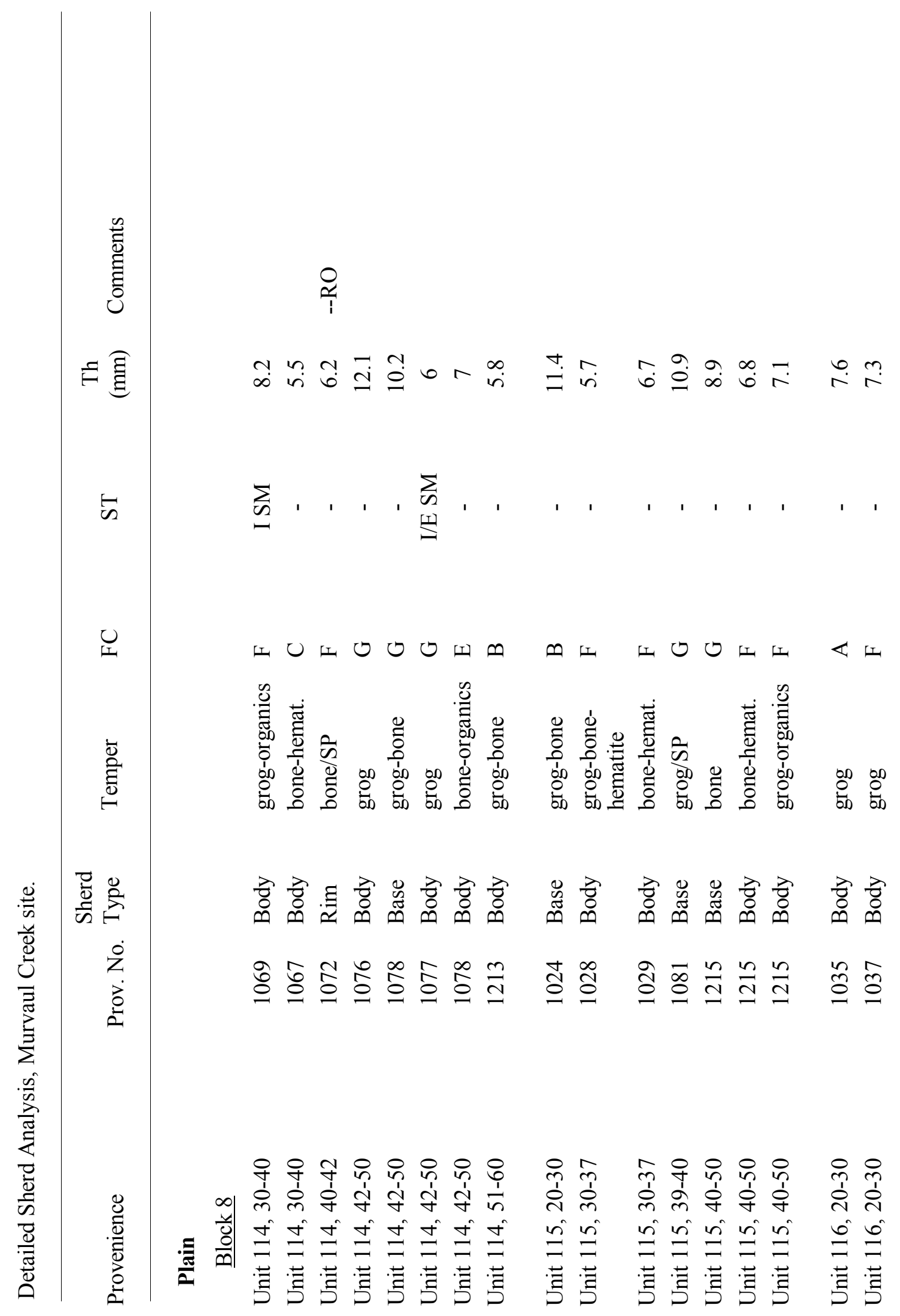




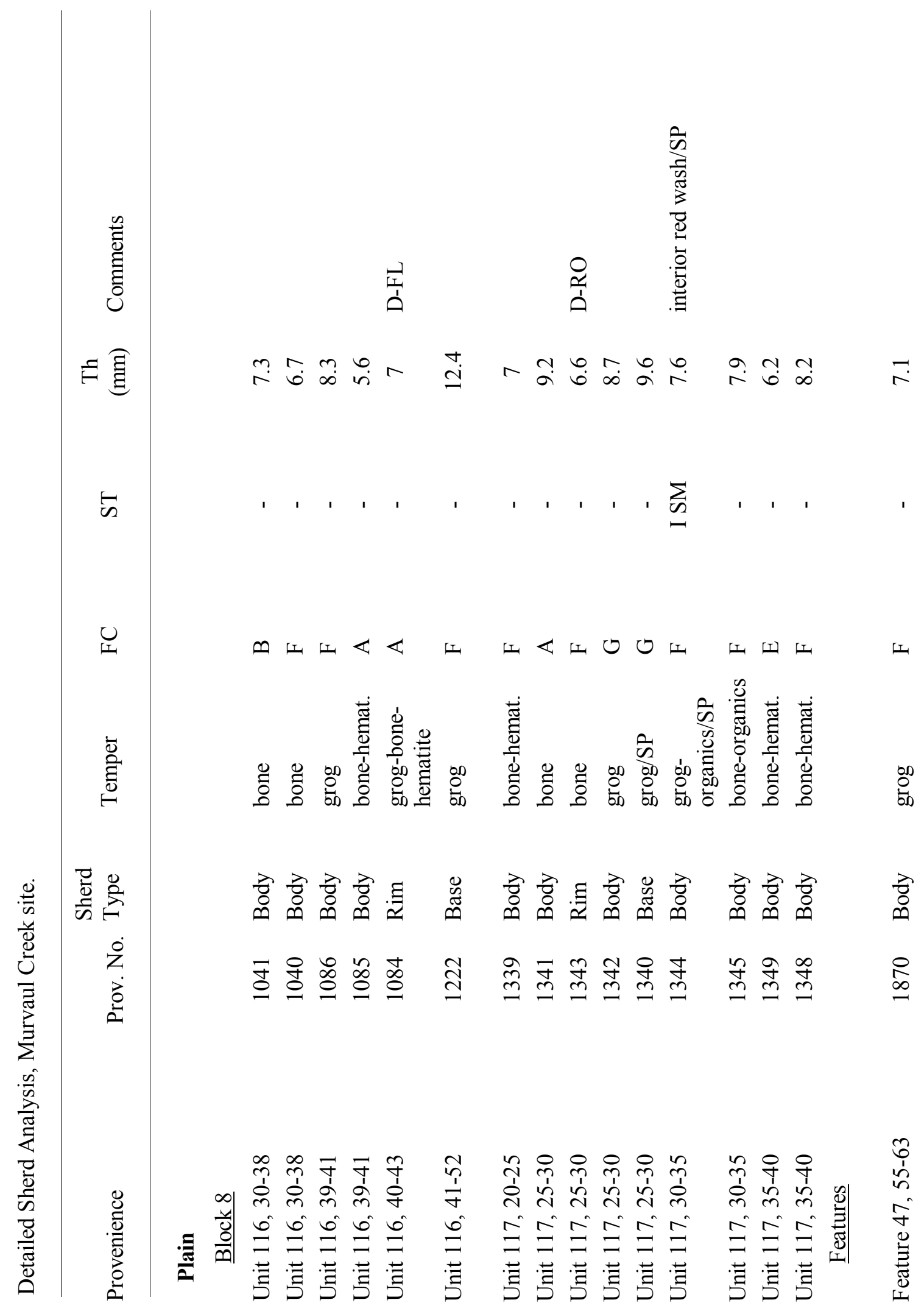




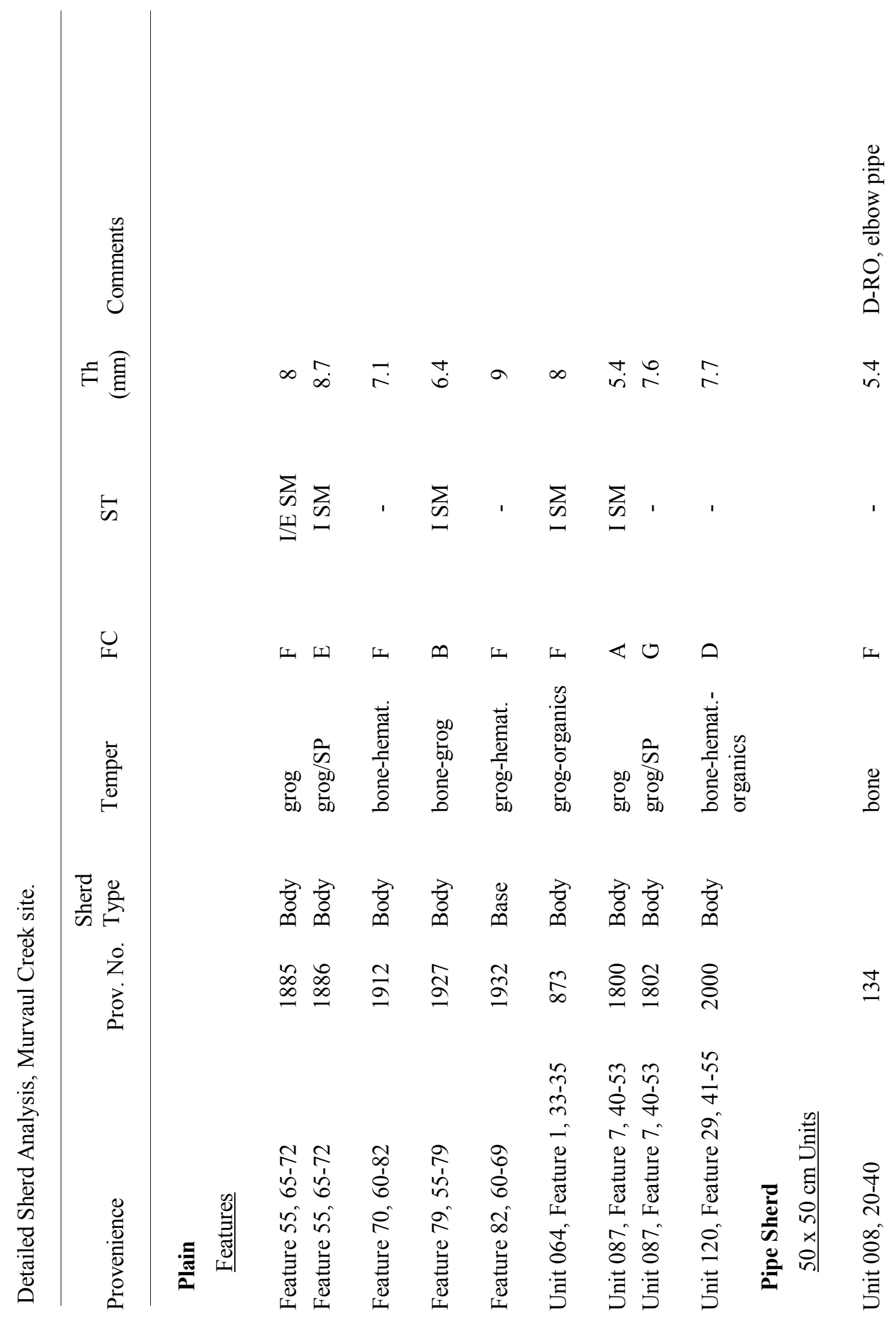




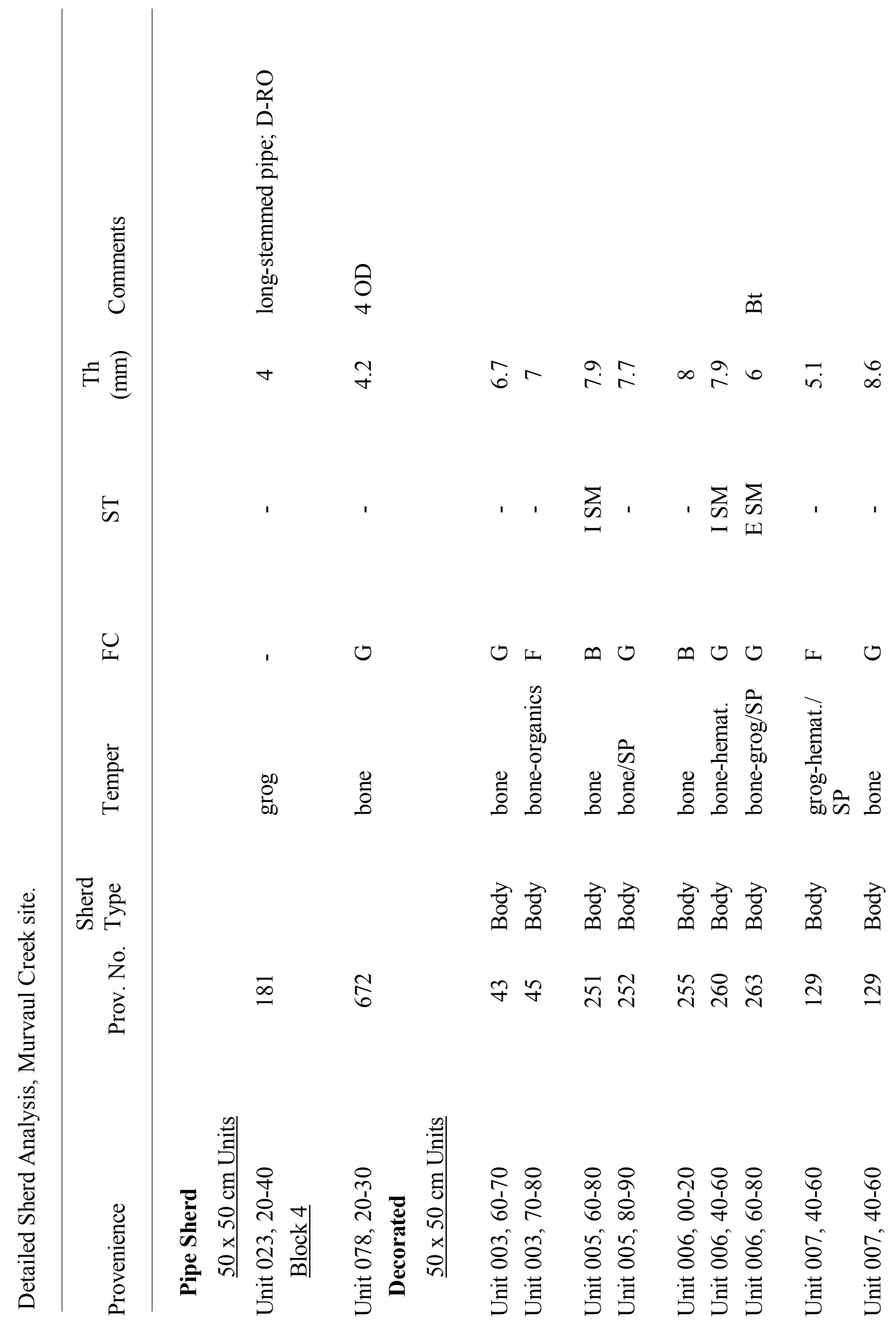




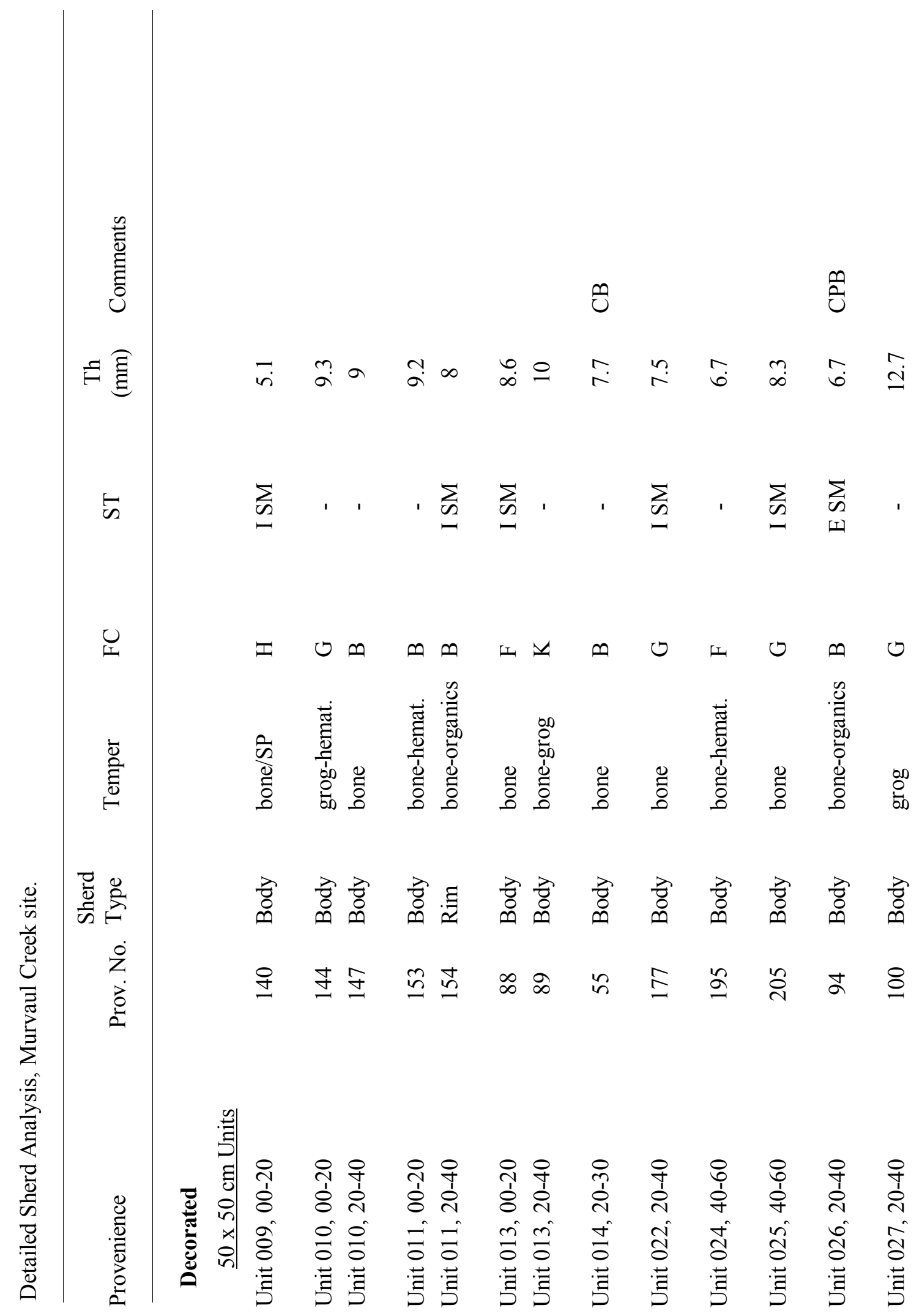




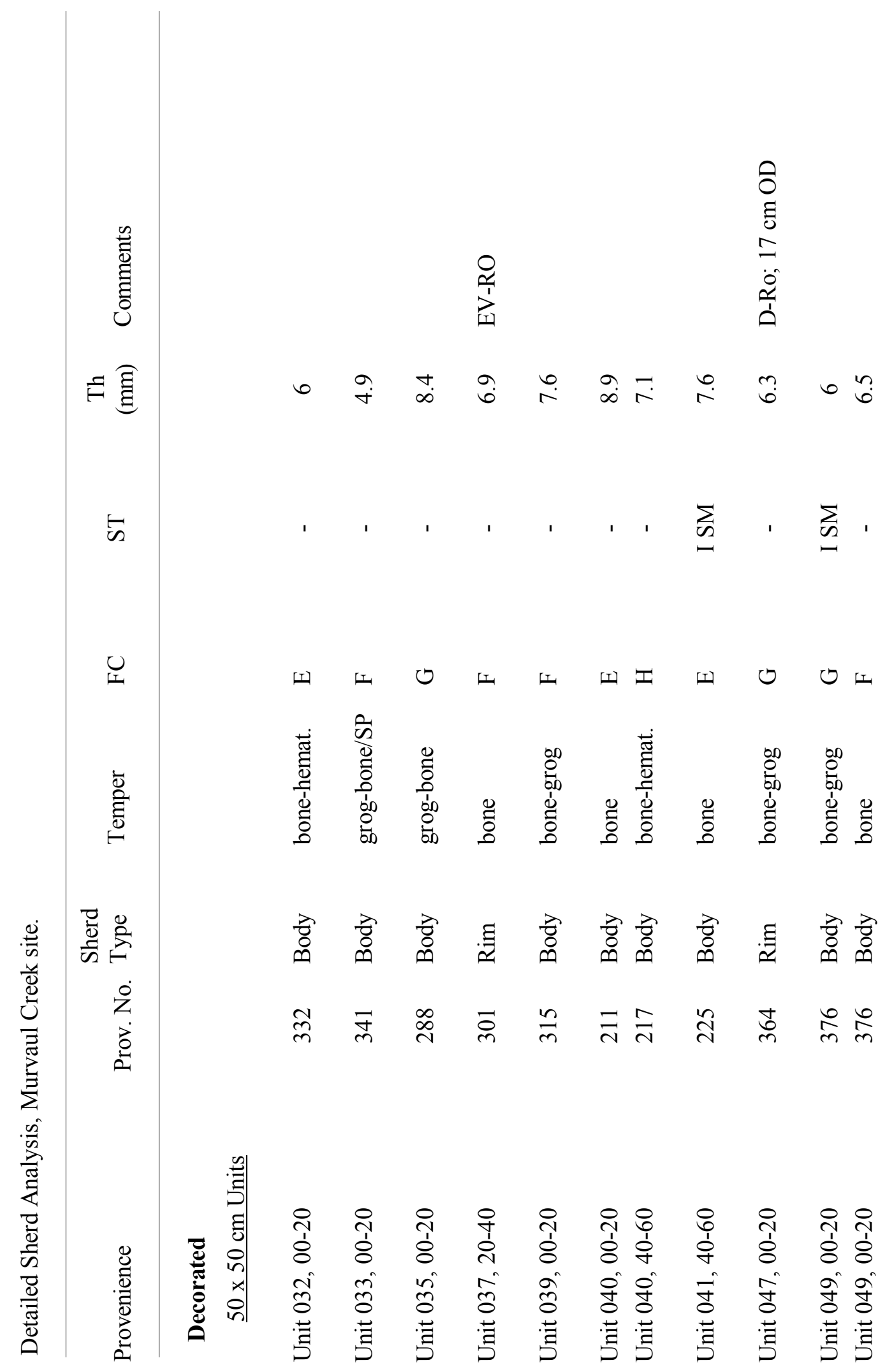




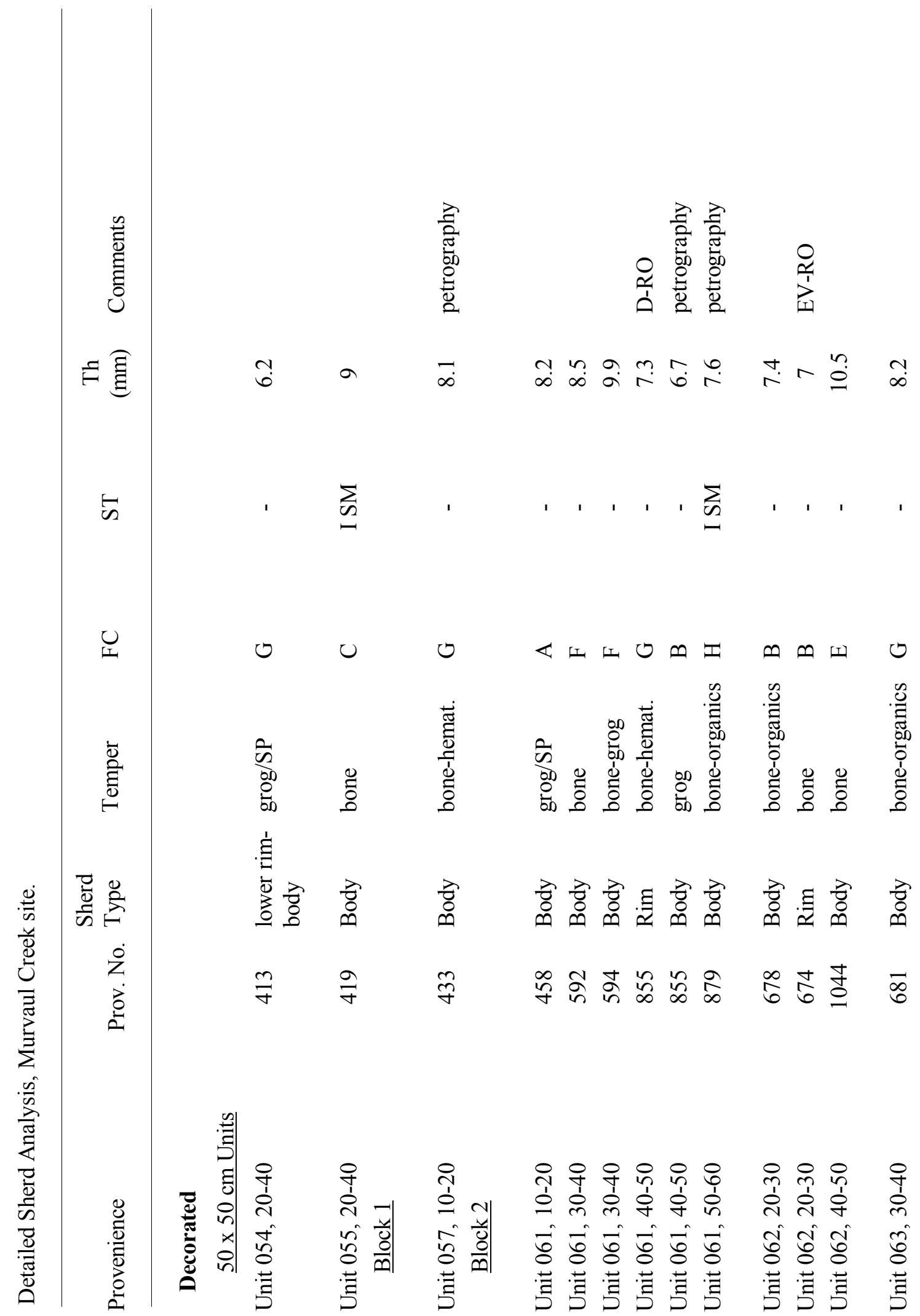




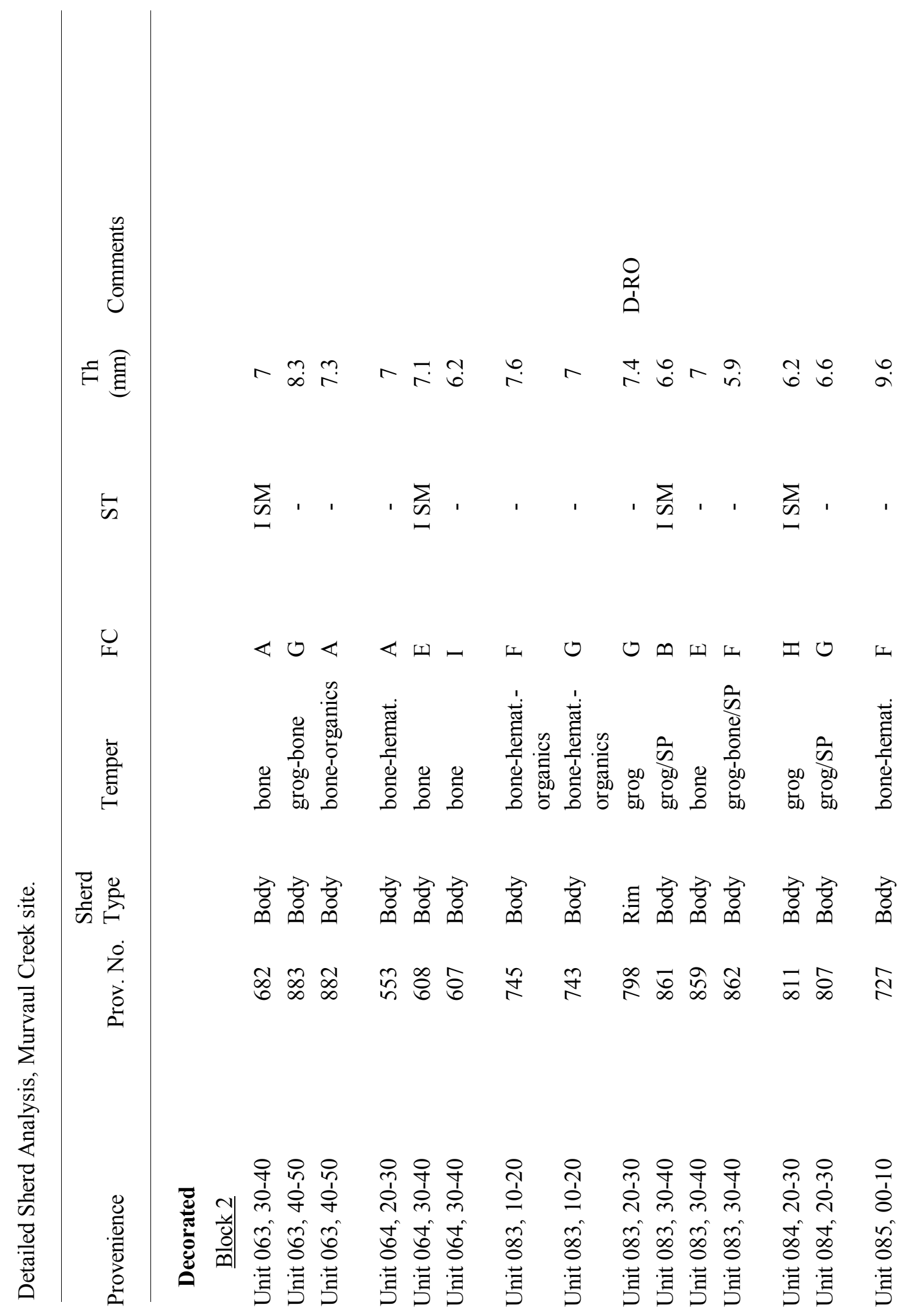




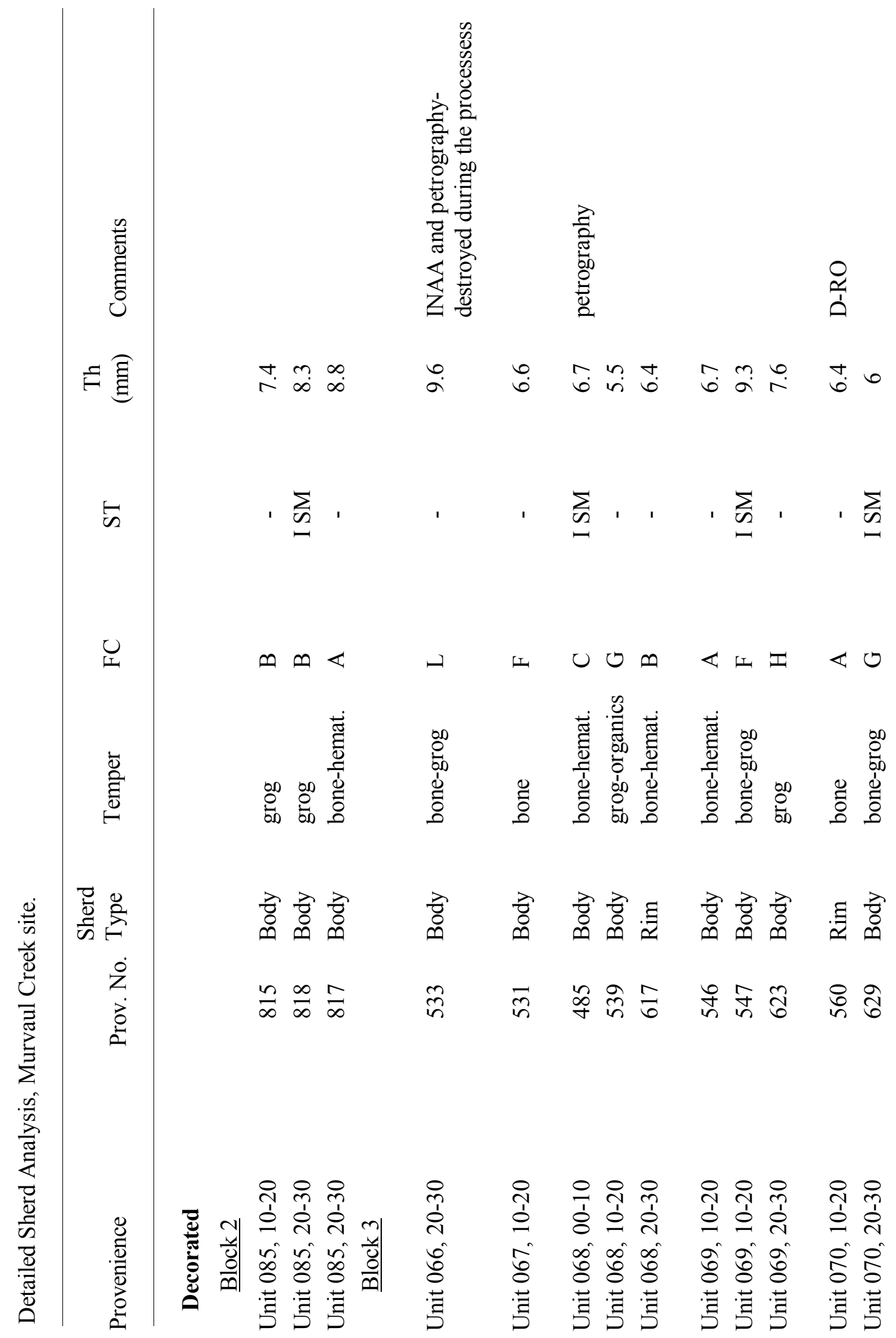




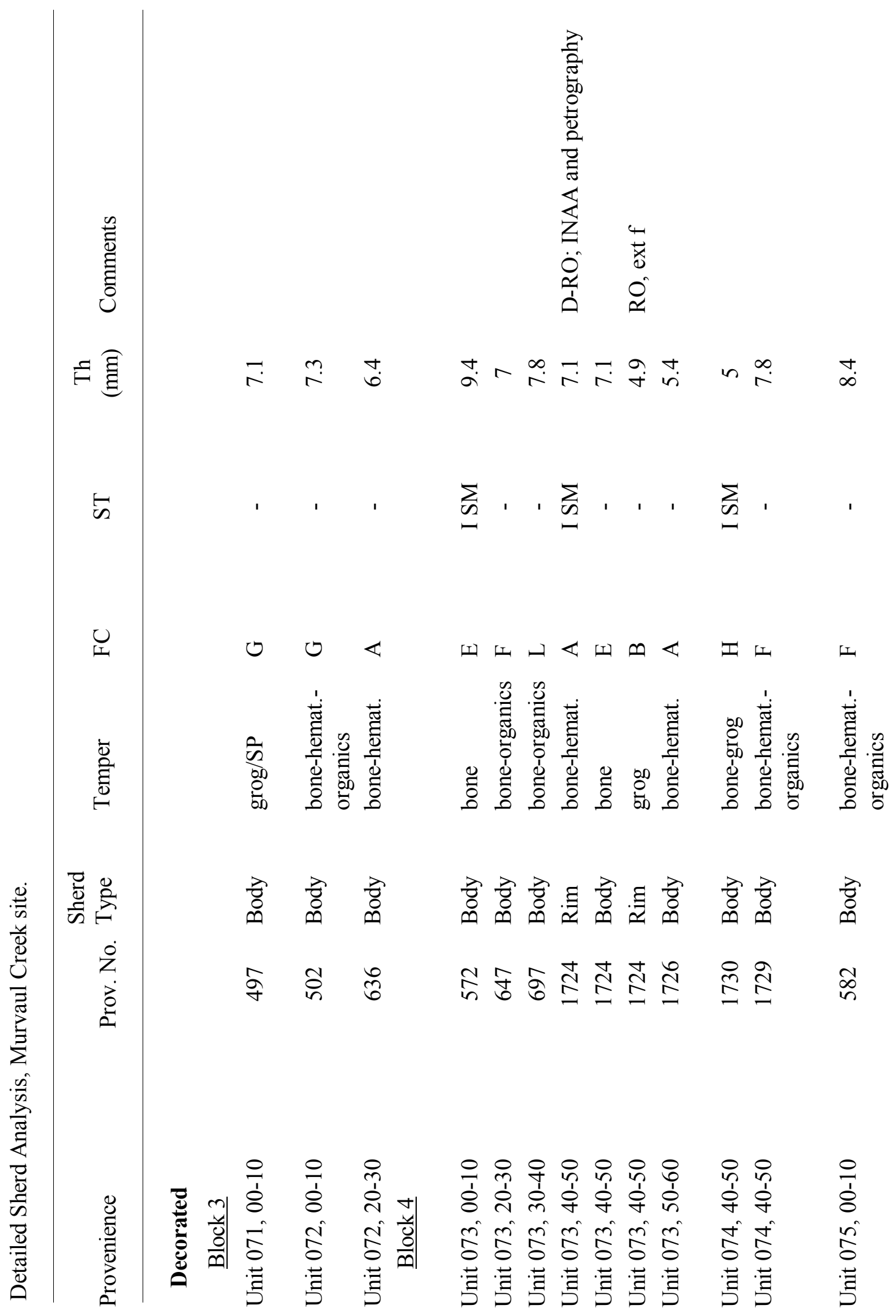




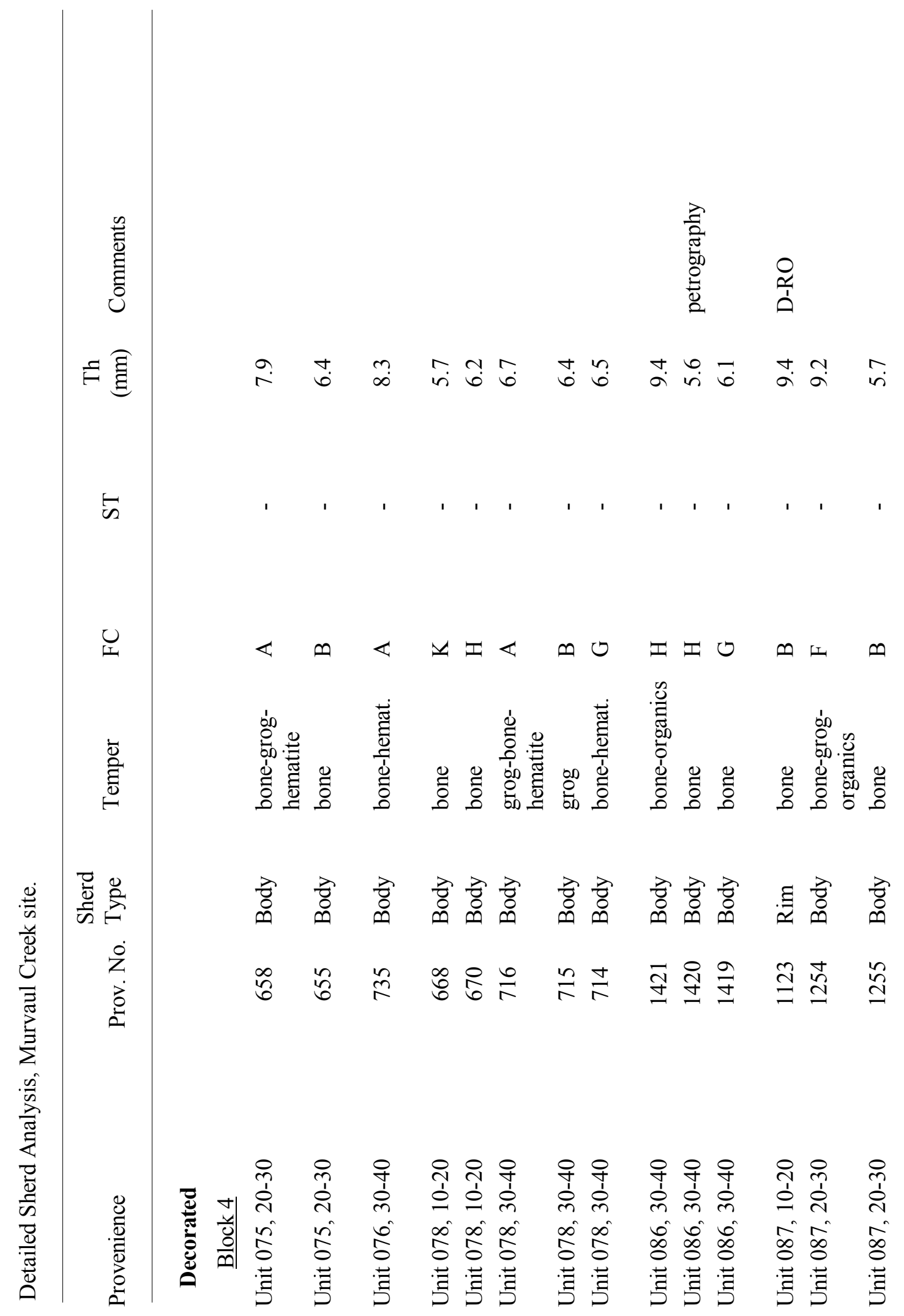




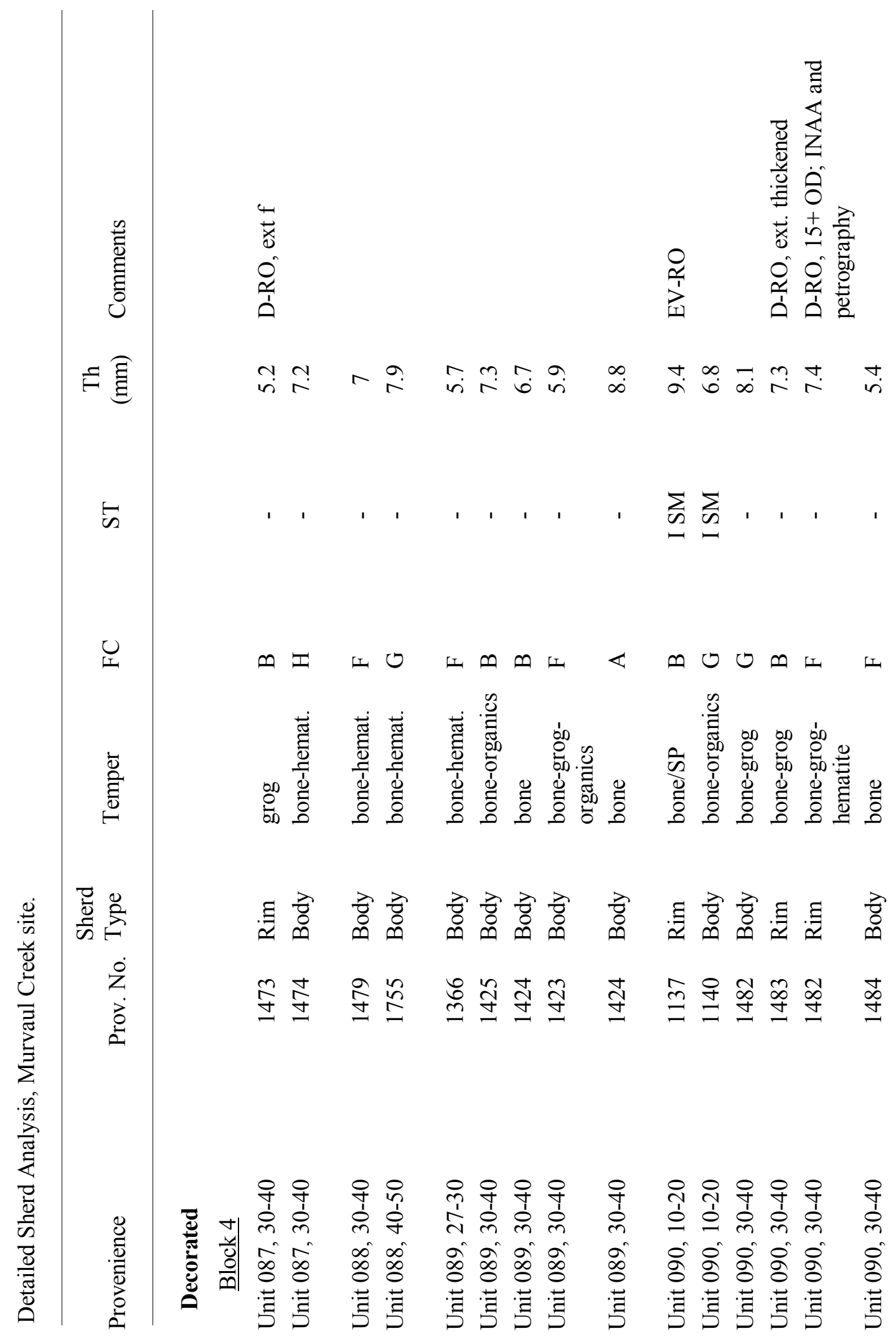




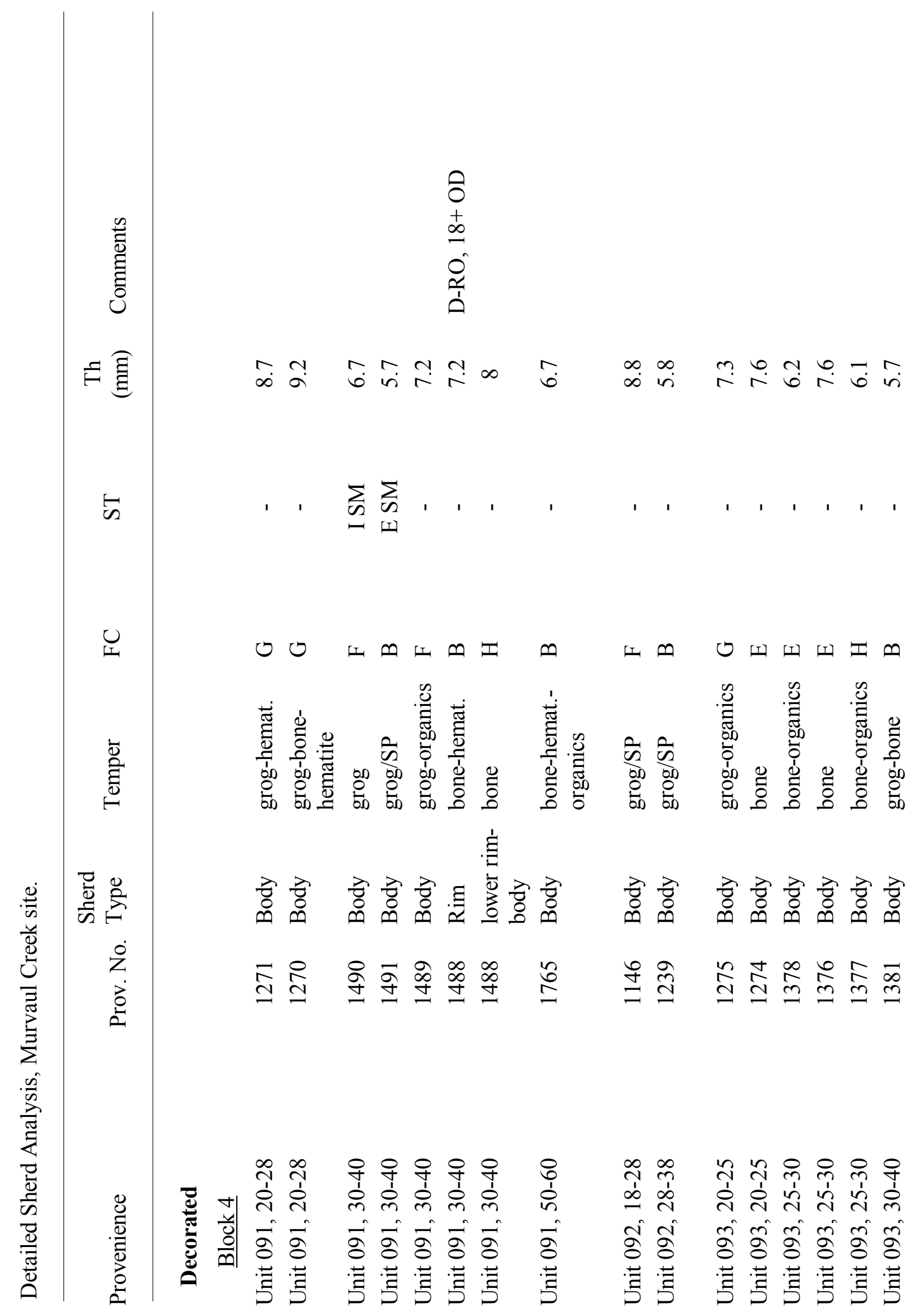




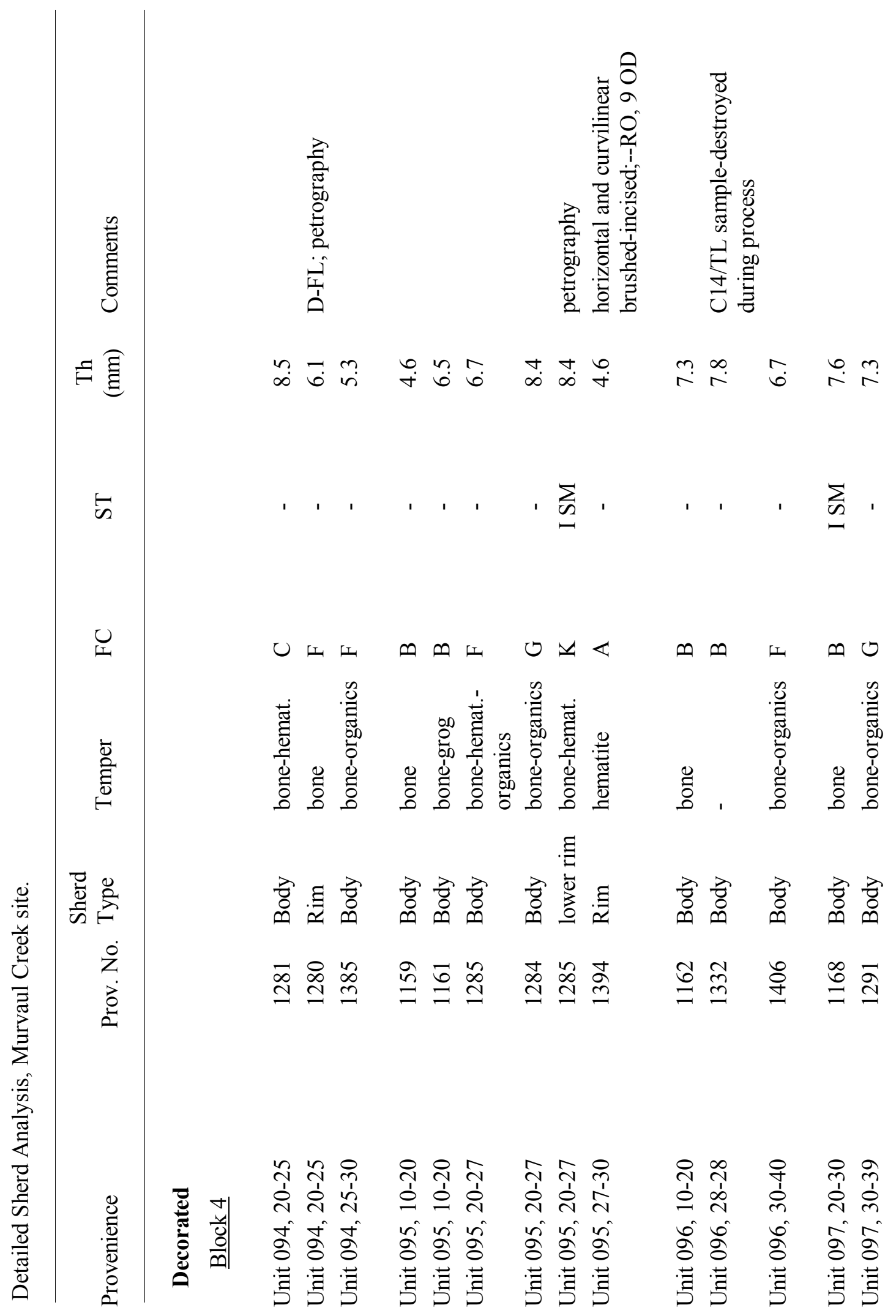




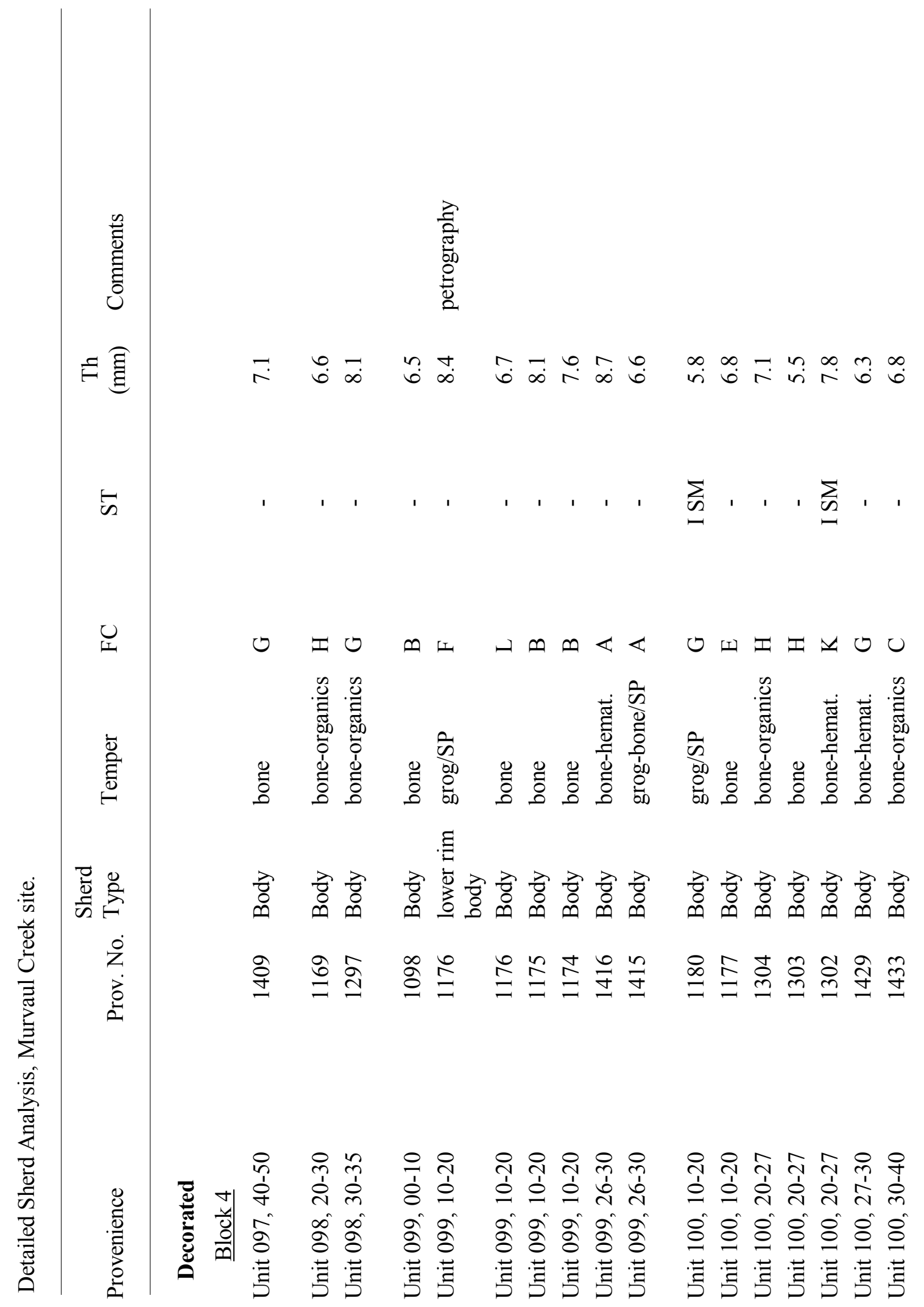




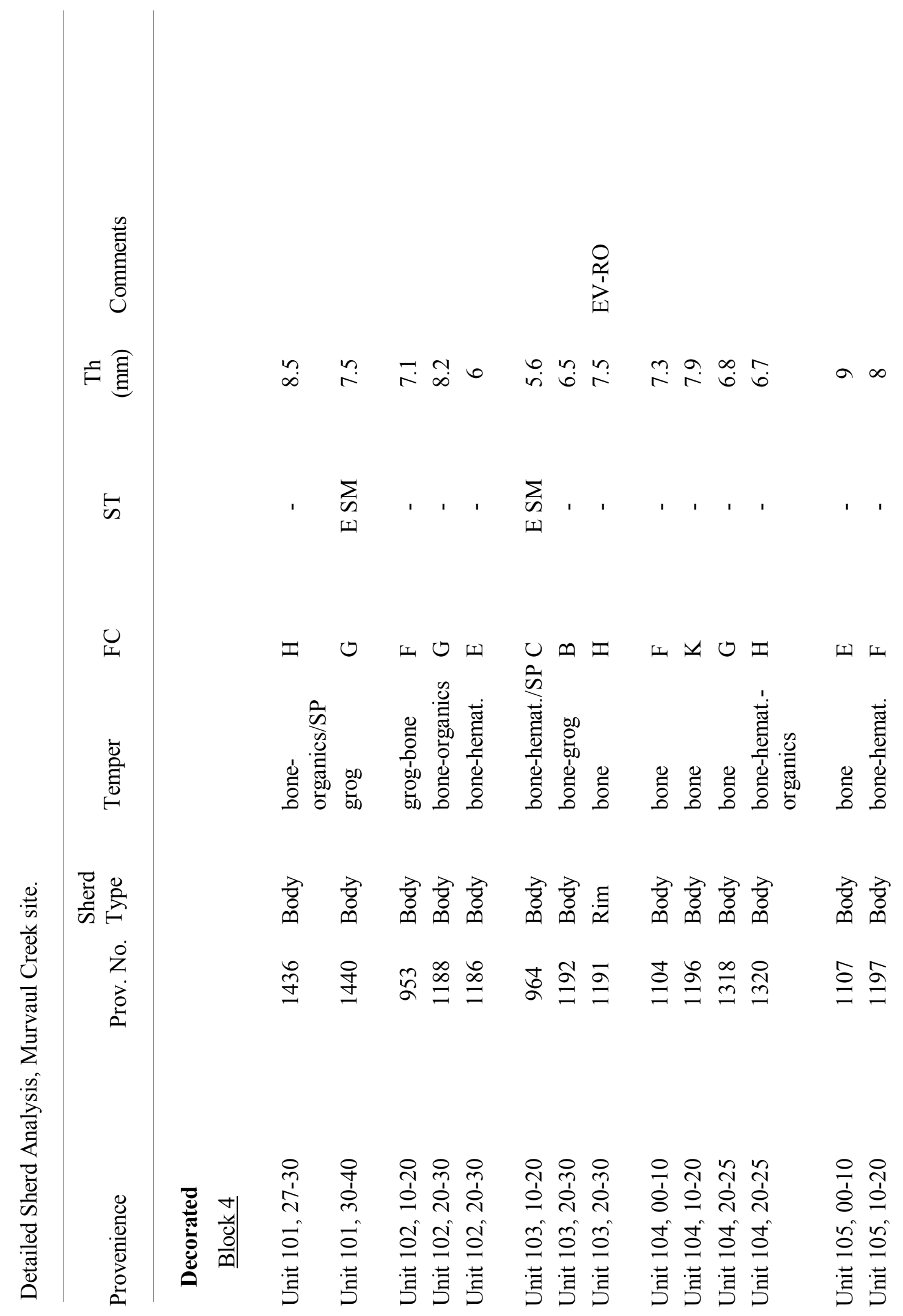









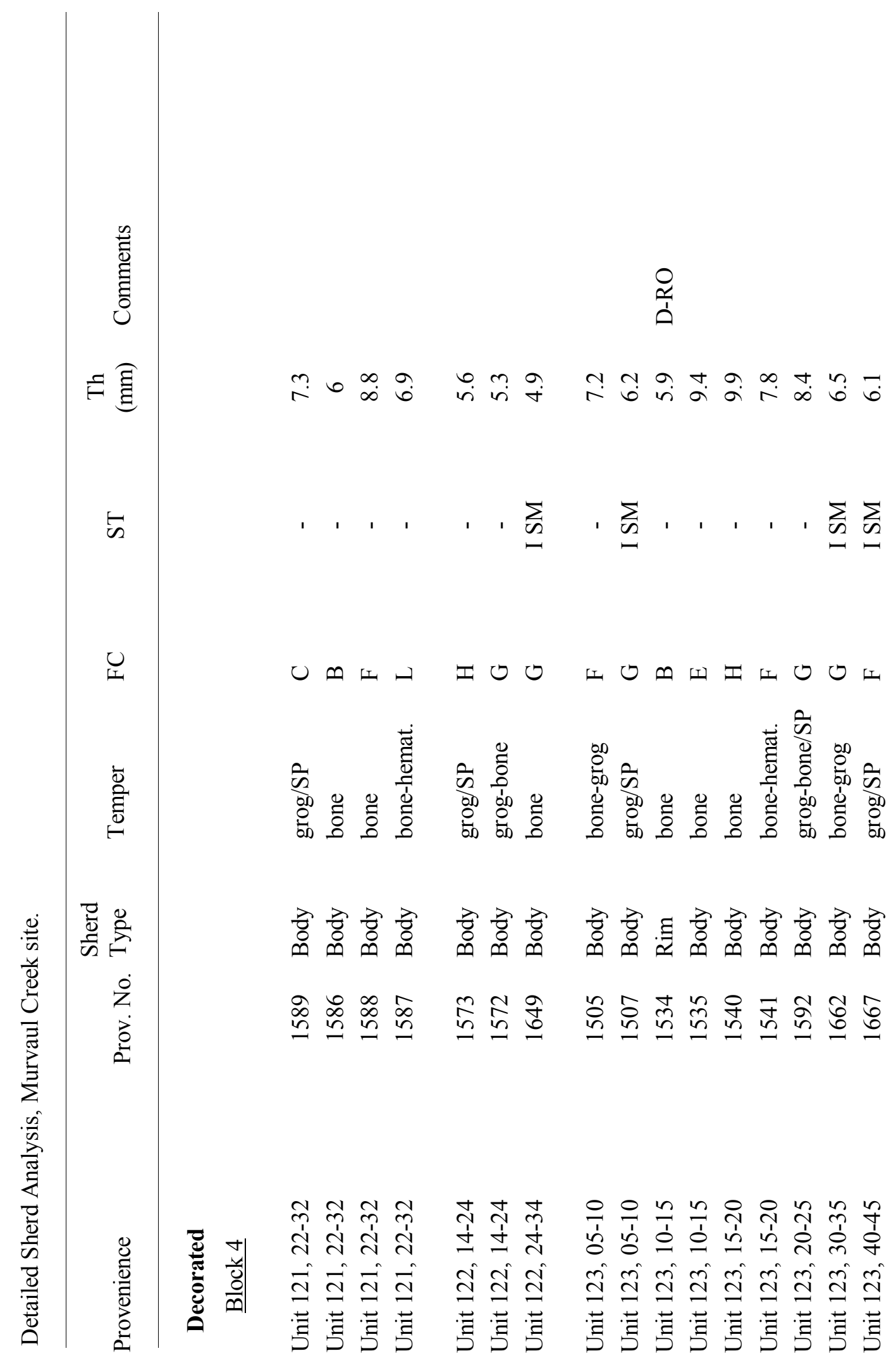




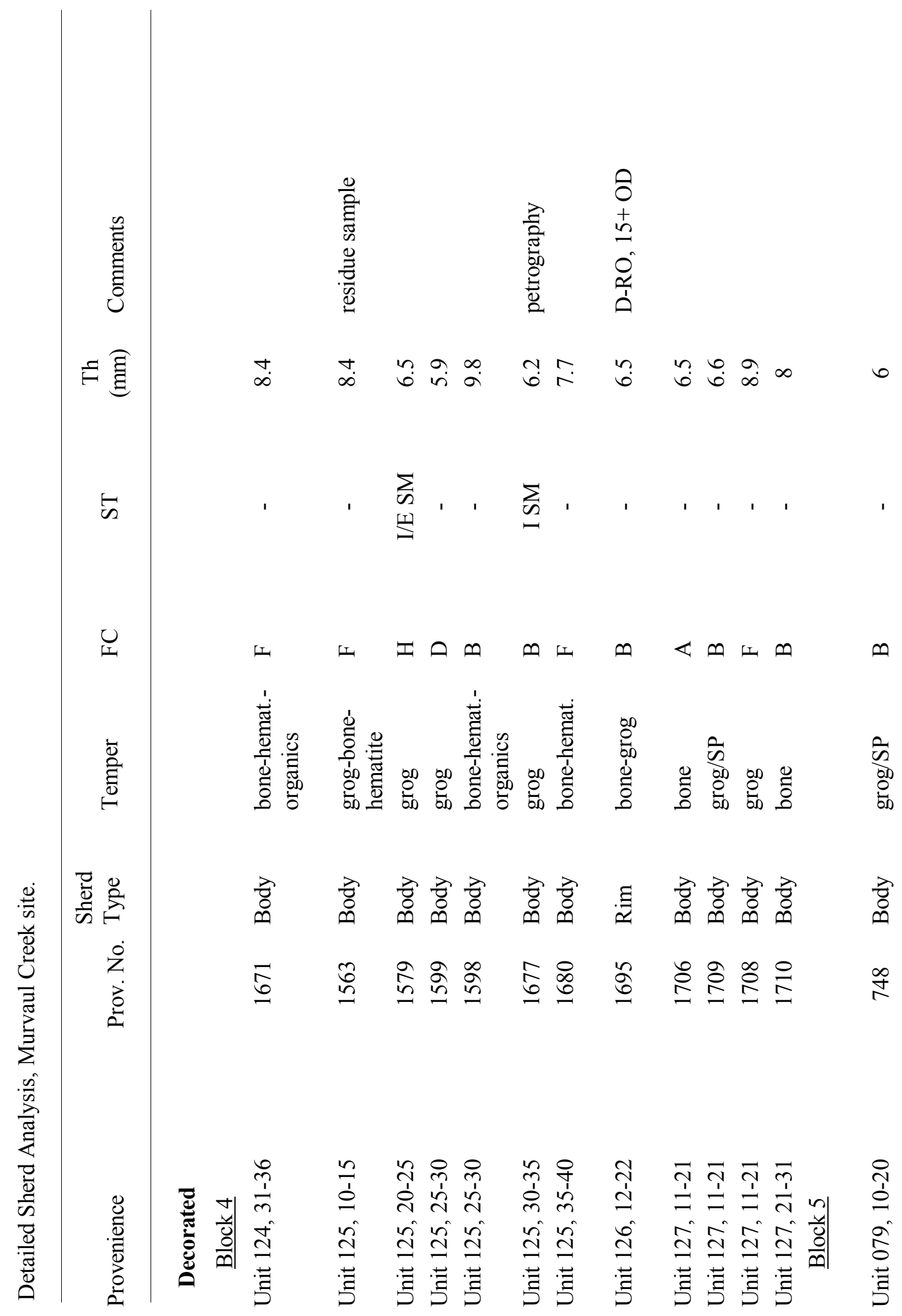




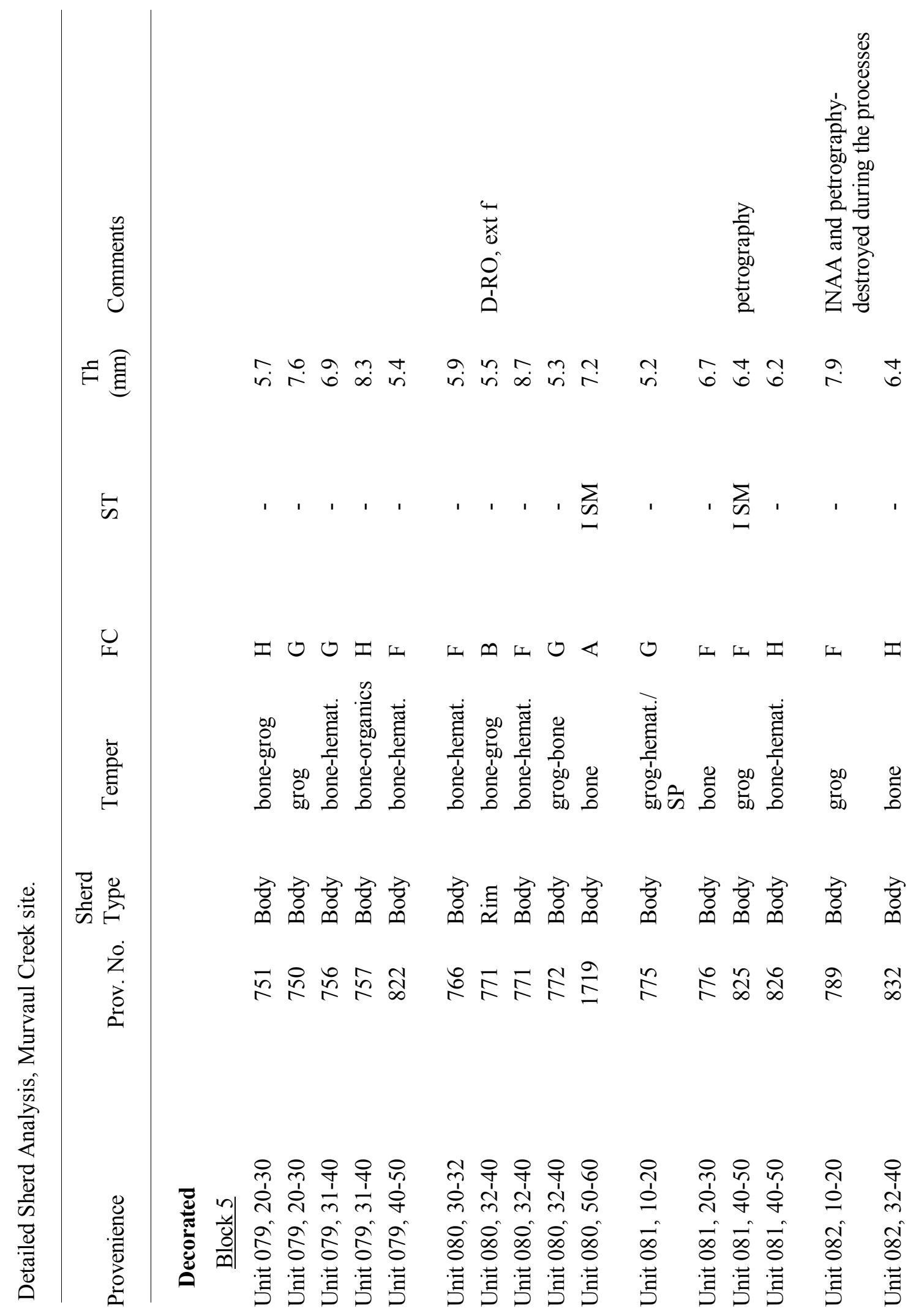




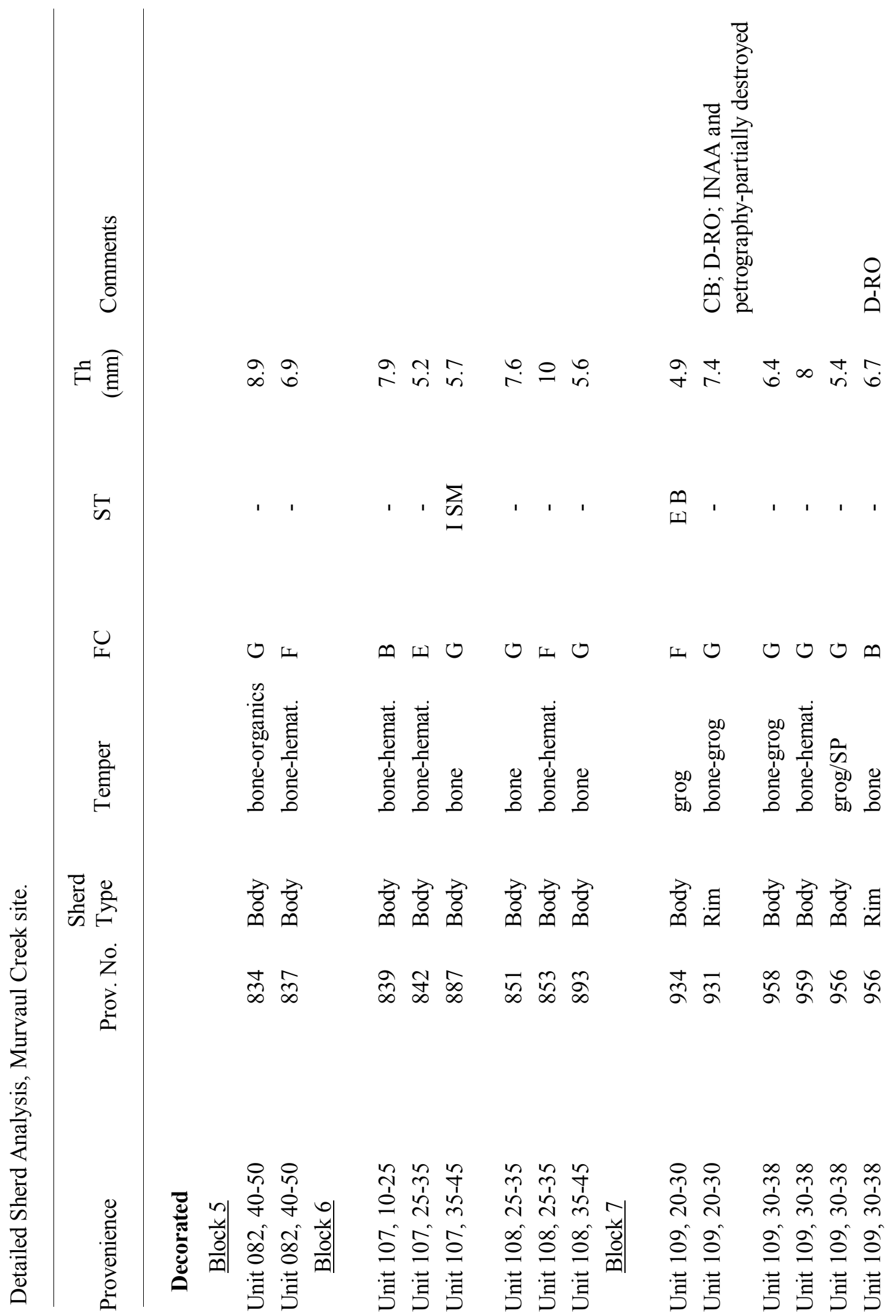









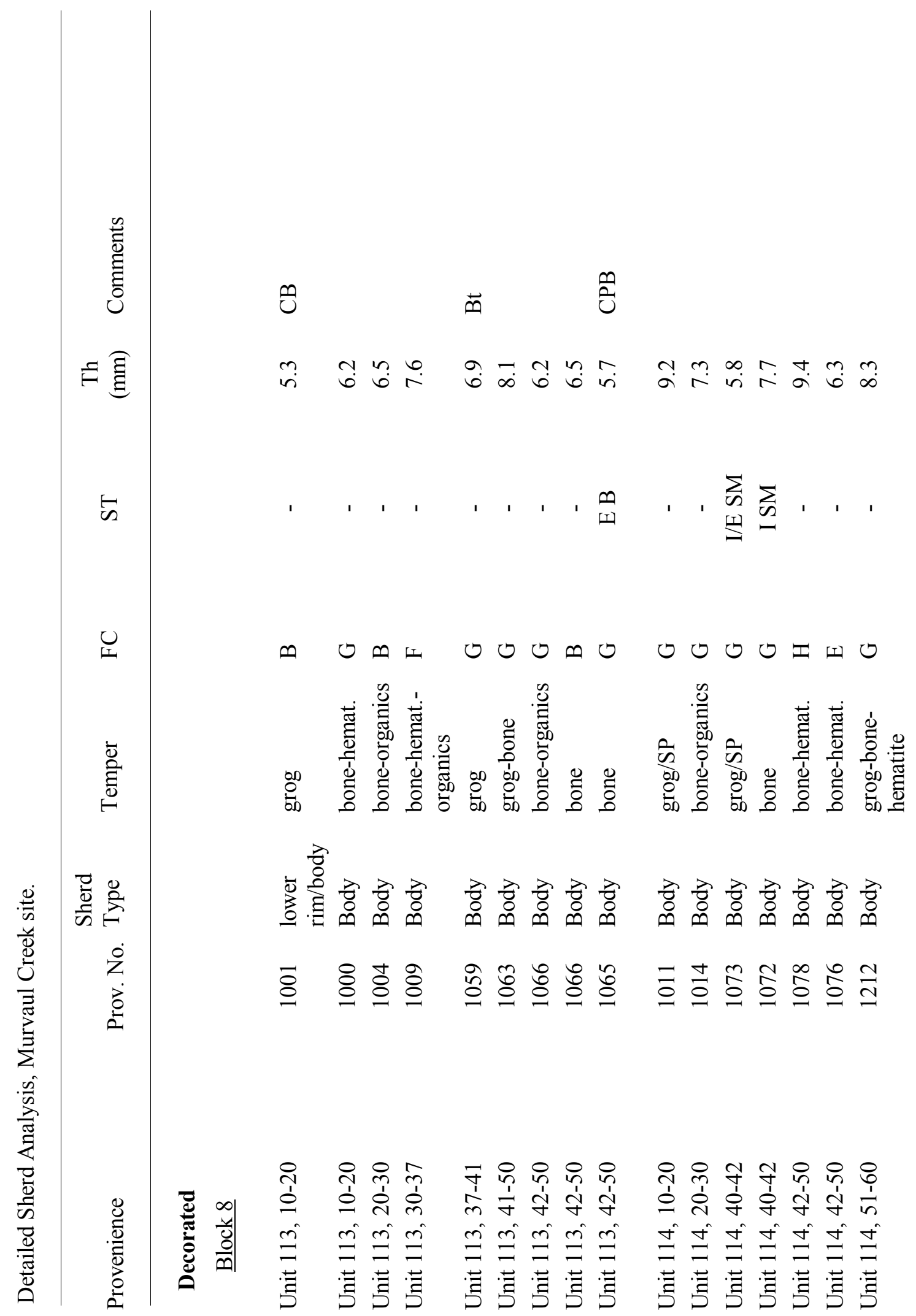




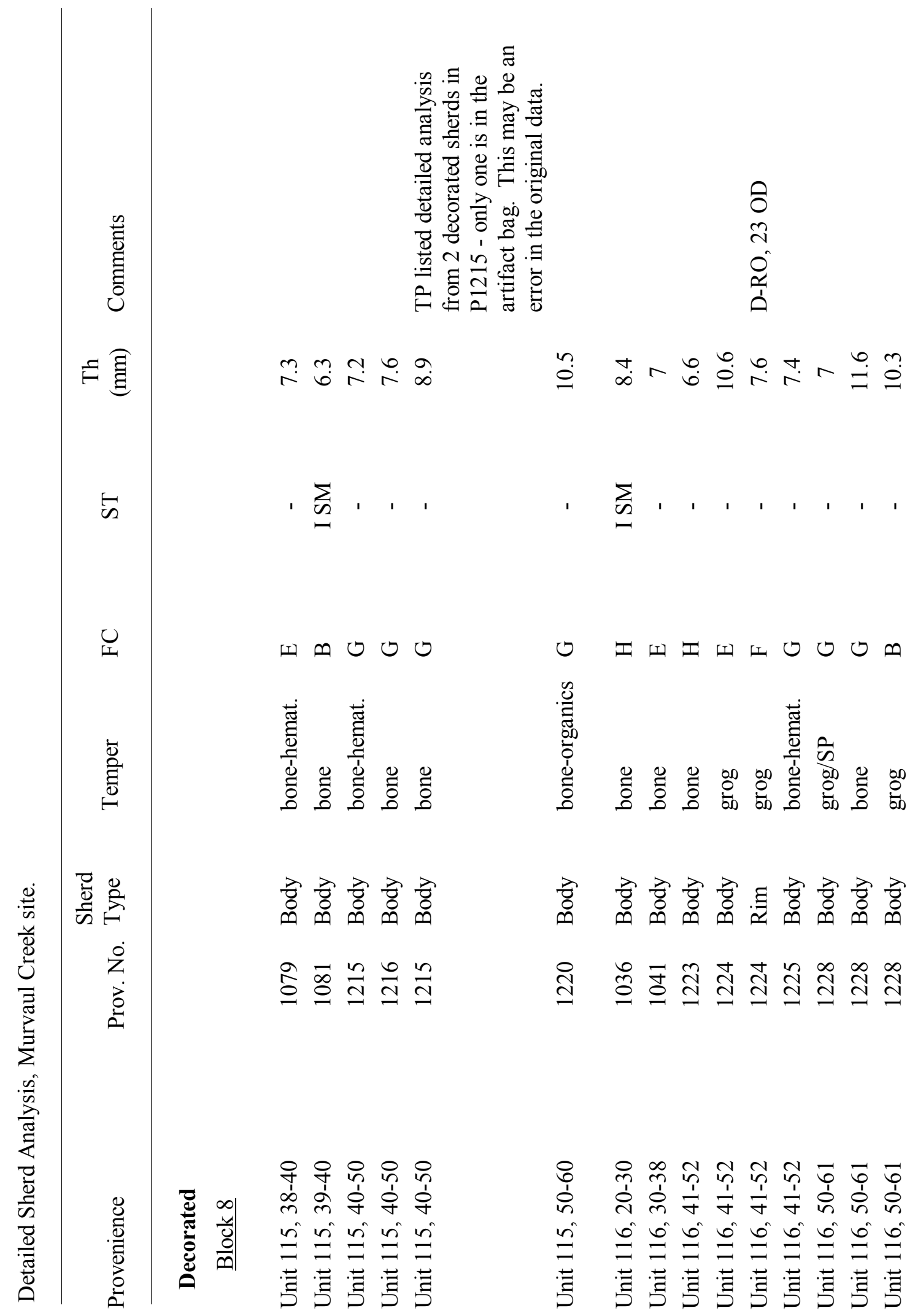




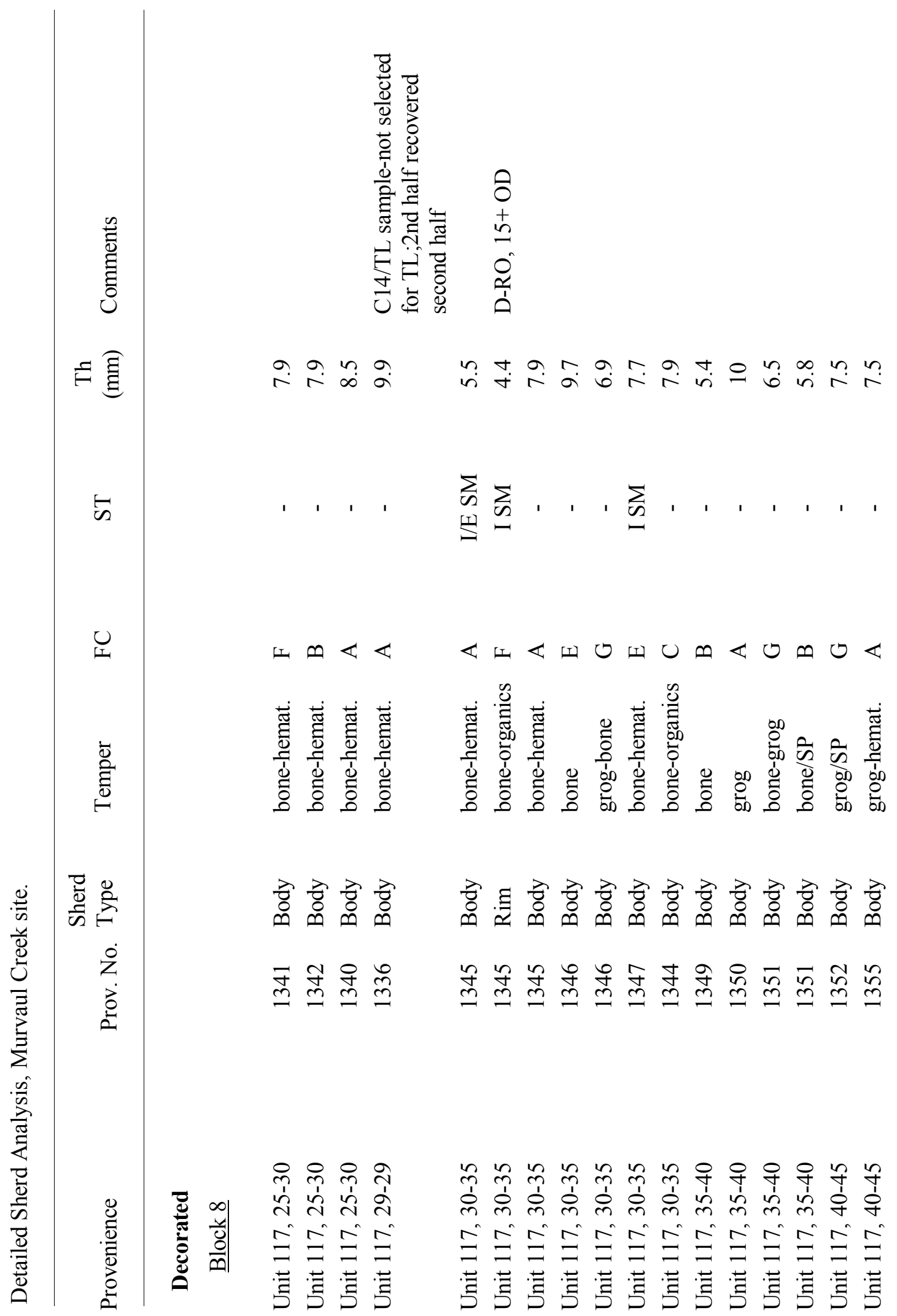




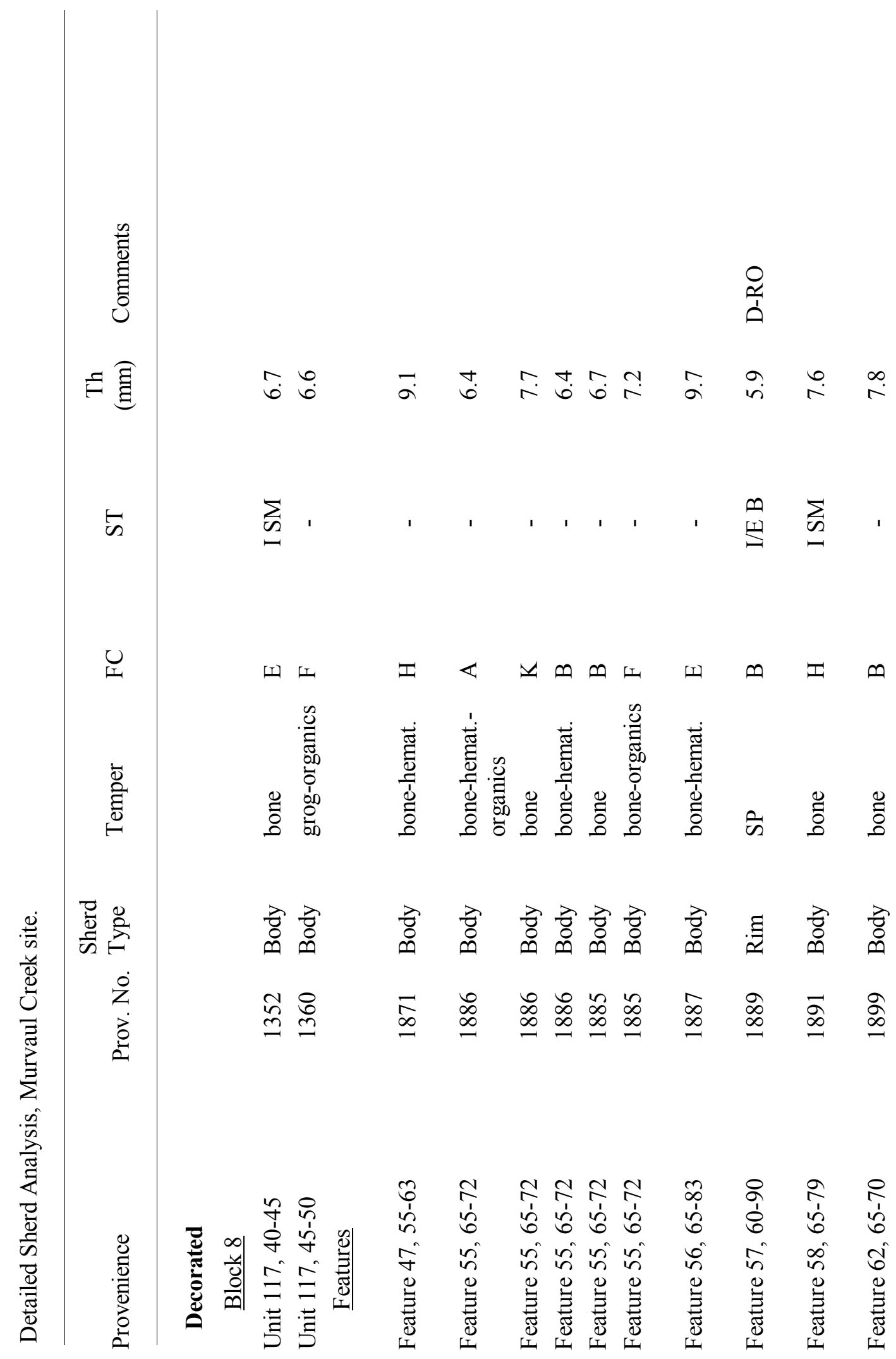




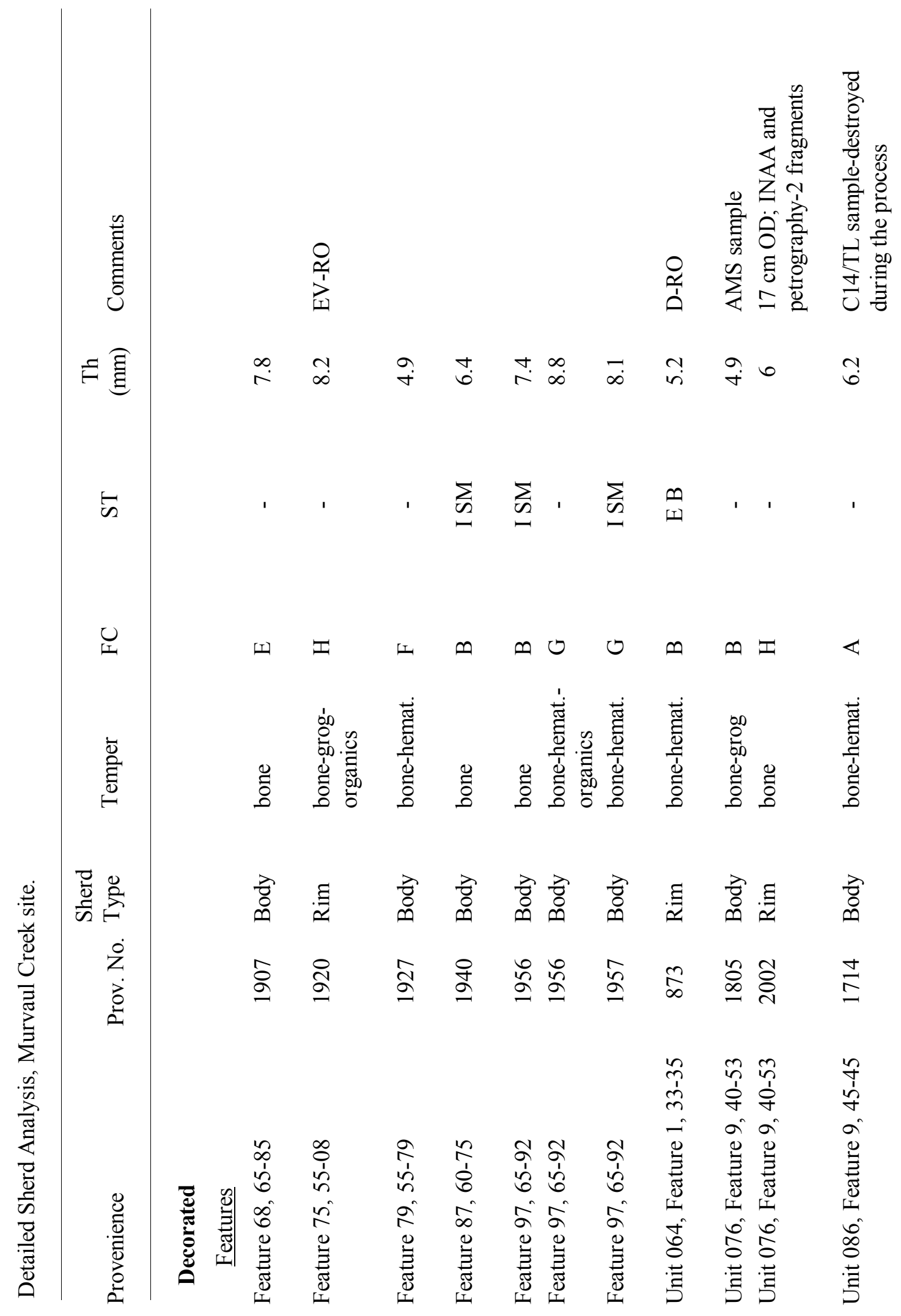




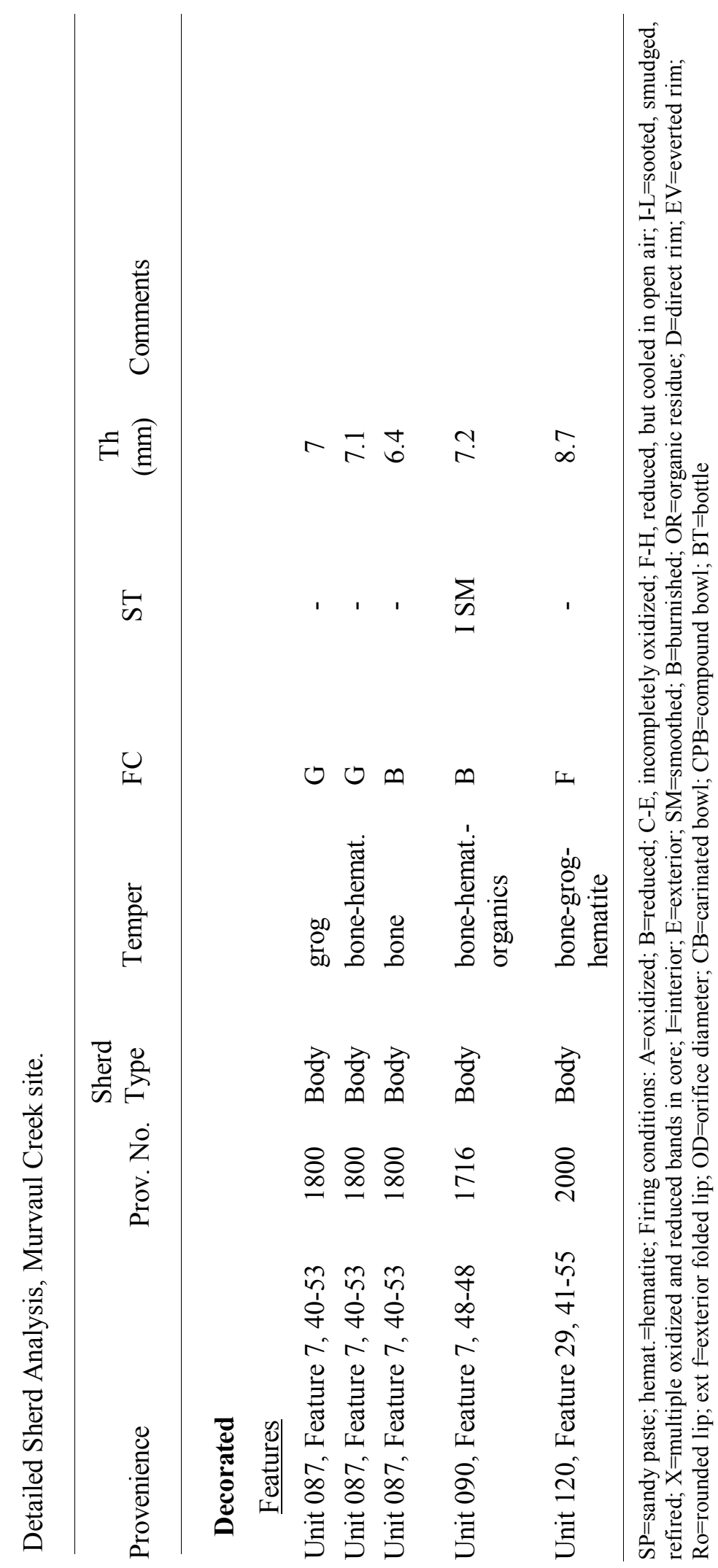



A-4: CHIPPED STONE TOOLS 

Geo-Marine, Inc.

GMI 22005.01.06.xx and 22005.00.09.xx, Data Recovery Excavations at Site 41PN175 Chipped Stone Tool Dat

\begin{tabular}{|c|c|c|c|c|c|c|c|c|c|}
\hline Unit & $\begin{array}{l}\text { Depth } \\
(\mathrm{cm})\end{array}$ & $\begin{array}{l}\text { Prov. } \\
\text { No. }\end{array}$ & $\begin{array}{l}\text { Lot } \\
\text { No. }\end{array}$ & Taxonomy & Attributes & Metric Information & Wear Patterning & Raw Material & Projectile Point Data \\
\hline \multicolumn{10}{|c|}{$50-x-50 \mathrm{~cm}$ unit } \\
\hline Unit 021 & $0-20$ & 167 & 209 & $\begin{array}{l}\text { Technology: Chipped Stone } \\
\text { Group: Tool } \\
\text { Subgroup: simple detachment-based } \\
\text { Type: } \\
\text { Subtype: Not Applicable }\end{array}$ & 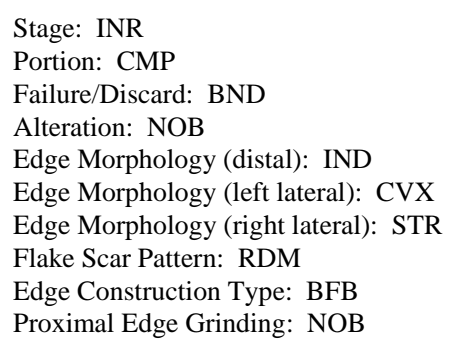 & $\begin{array}{l}\text { Quantity: } 1 \\
\text { Weight (in g): } 2.1 \\
\text { Max Length (in mm): } 25 \\
\text { Max Width (in mm): } 15 \\
\text { Max Thickness (mm): } 6 \\
\text { Edge Angle: } 25\end{array}$ & $\begin{array}{l}\text { Flake Attrition: BFB } \\
\text { Crushing: UNL } \\
\text { Smoothing: UNL } \\
\text { Polish: NPR } \\
\text { Etching/Pitting: NPR } \\
\text { Hafting Evidence: NOB }\end{array}$ & CRT & $\begin{array}{l}\text { Point Class: NAP } \\
\text { Point Ratio: 0.5991311 } \\
\text { Base/Stem Width: }\end{array}$ \\
\hline Unit 051 & $40-60$ & 394 & 298 & $\begin{array}{l}\text { Technology: Chipped Stone } \\
\text { Group: Tool } \\
\text { Subgroup: complex detachment- } \\
\text { based } \\
\text { Type: } \\
\text { Subtype: Unclassified }\end{array}$ & $\begin{array}{l}\text { Stage: FST } \\
\text { Portion: STM } \\
\text { Failure/Discard: ESH } \\
\text { Alteration: NOB } \\
\text { Edge Morphology (distal): IND } \\
\text { Edge Morhhology (eff lateral): IND } \\
\text { Edge Morphology (right lateral): IND } \\
\text { Flake Scar Pattern: IND } \\
\text { Edge Constrtuction Type: IND } \\
\text { Proximal Edge Grinding: NOB }\end{array}$ & $\begin{array}{l}\text { Quantity: } 1 \\
\text { Weight (in g): } 0.7 \\
\text { Max Length (in mm): } 8 \\
\text { Max Width (in mm): } 20 \\
\text { Max Thickness (mm): } 5 \\
\text { Edge Angle: } 30\end{array}$ & $\begin{array}{l}\text { Flake Attrition: NPR } \\
\text { Crushing: NPR } \\
\text { Smoothing: NPR } \\
\text { Polish: NPR } \\
\text { Etching/Pitting: NPR } \\
\text { Hafting Evidence: NOB }\end{array}$ & CRT & $\begin{array}{l}\text { Point Class: STM } \\
\text { Point Ratio: 2.552941 } \\
\text { Base to Blade Ratio (width): } 1 \\
\text { Base/stem ratio: } 0 \\
\text { Base Form: } 3 \\
\text { Stem Form: } 0 \\
\text { Curvature (left): } 0 \\
\text { Curvature (right): } 0 \\
\text { Shoulder Junction: } 0\end{array}$ \\
\hline \multicolumn{10}{|c|}{ Block 2} \\
\hline Unit $062 \mathrm{NW}^{1 / 1} / 4$ & $10-20$ & 507 & 341 & $\begin{array}{l}\text { Technology: Chipped Stone } \\
\text { Group: Tool } \\
\text { Subgroup: core-based } \\
\text { Type: } \\
\text { Subtype: Unclassified }\end{array}$ & 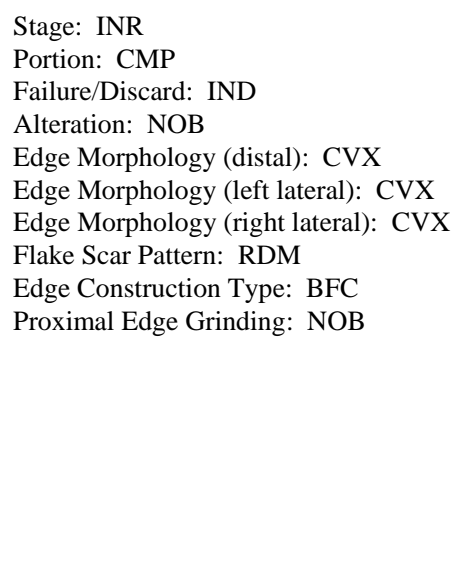 & $\begin{array}{l}\text { Quantity: } 1 \\
\text { Weight (in g): } 15.4 \\
\text { Max Length (in mm): } 44 \\
\text { Max Width (in mm): } 25 \\
\text { Max Thickness (mm): } 12 \\
\text { Edge Angle: } 60\end{array}$ & $\begin{array}{l}\text { Flake Attrition: NPR } \\
\text { Crushing: NPR } \\
\text { Smoothing: NPR } \\
\text { Polish: NPR } \\
\text { Etching/Pitting: NPR } \\
\text { Hafting Evidence: NOB }\end{array}$ & QZT & $\begin{array}{l}\text { Point Class: STM } \\
\text { Point Ratio: 0.5712986 } \\
\text { Blade Length (left): } 34.66 \\
\text { Blade Length (right): } 35.51 \\
\text { Stem Lenghth: } 11.62 \\
\text { Neck Thickness: } 9.95 \\
\text { Neck Width: } 15.35 \\
\text { Base to Blade Ratio (length): } 0.2642711 \\
\text { Base to Blade Ratio (width): } 0.580414 \\
\text { Base/stem ratio: } 2 \\
\text { Base Form: } 2 \\
\text { Stem Form: } 4 \\
\text { Curvature (left): } 2 \\
\text { Curvaturer (right): } 2 \\
\text { Shoulder (left): } 42 \\
\text { Shoulder (right): } 52 \\
\text { Shoulder Junction: } 1\end{array}$ \\
\hline Unit $062 \mathrm{SE}^{1} / 4$ & $30-40$ & 600 & 355 & $\begin{array}{l}\text { Technology: Chipped Stone } \\
\text { Group: Tool } \\
\text { Subgroup: complex detachment- } \\
\text { based } \\
\text { Type: } \\
\text { Subtype: Not Applicable }\end{array}$ & $\begin{array}{l}\text { Stage: INR } \\
\text { Portion: FRG } \\
\text { Failure/Discard: MFL } \\
\text { Alteration: NOB } \\
\text { Edge Morphology (distal): IND } \\
\text { Edge Morphology (eft lateral): IND } \\
\text { Edge Morhhology (right lateral): IND } \\
\text { Flake Scar Pattern: IND } \\
\text { Edge Construction Type: BFB } \\
\text { Proximal Edge Grinding: NOB }\end{array}$ & $\begin{array}{l}\text { Quantity: } 1 \\
\text { Weight (in g): } 5 \\
\text { Max Length (in mm): } 30 \\
\text { Max Width (in mm): } 15 \\
\text { Max Thickness (mm): } 12 \\
\text { Edge Angle: } 45\end{array}$ & $\begin{array}{l}\text { Flake Attrition: NPR } \\
\text { Crushing: NPR } \\
\text { Smoothing: NPR } \\
\text { Polish: NPR } \\
\text { Etching/Pitting: NPR } \\
\text { Hafting Evidence: NOB }\end{array}$ & CRT & $\begin{array}{l}\text { Point Class: NAP } \\
\text { Point Ratio: 0.4796962 } \\
\text { Base/Stem Width: }\end{array}$ \\
\hline
\end{tabular}


Geo-Marine, Inc.

GMI 22005.01.06.xx and 22005.00.09.xx, Data Recovery Excavations at Site 41PN175 Chipped Stone Tool Dat

\begin{tabular}{|c|c|c|c|c|c|c|c|c|c|c|}
\hline Unit & $\begin{array}{l}\text { Depth } \\
(\mathrm{cm})\end{array}$ & $\begin{array}{l}\text { Prov. } \\
\text { No. }\end{array}$ & $\begin{array}{l}\text { Lot } \\
\text { No. }\end{array}$ & Taxonomy & Attributes & Metric Information & Wear Patterning & Raw Material & Projectile Point Data & Comment \\
\hline \multicolumn{11}{|c|}{ Block 2} \\
\hline Unit $064 \mathrm{NE}^{1 / 4}$ & $30-40$ & 608 & 384 & $\begin{array}{l}\text { Technology: Chipped Stone } \\
\text { Group: Tool } \\
\text { Subgroup: complex detachment- } \\
\text { based } \\
\text { Type: } \\
\text { Subtype: Kent }\end{array}$ & $\begin{array}{l}\text { Stage: FST } \\
\text { Portion: CMP } \\
\text { FailurelDiscard: IND } \\
\text { Alteration: NOB } \\
\text { Edge Morphology (distal): STR } \\
\text { Edge Morphology (left lateral): STR } \\
\text { Edge Morphology (right latarl): STR } \\
\text { Flake Scar antern: COL } \\
\text { Edge Construction Type: BFB } \\
\text { Proximal Edge Grinding: NOB }\end{array}$ & $\begin{array}{l}\text { Quantity: } 1 \\
\text { Weight (in g): } 4.8 \\
\text { Max Length (in mm): } 38 \\
\text { Max Width (in mm): } 21 \\
\text { Max Thickness (mm): } 8 \\
\text { Edge Angle: } 35\end{array}$ & $\begin{array}{l}\text { Flake Attrition: BFB } \\
\text { Crushing: NPR } \\
\text { Smoothing: NPR } \\
\text { Polish: SHL } \\
\text { Etching/Pitting: NPR } \\
\text { Hafting Evidence: OBS }\end{array}$ & FWD & $\begin{array}{l}\text { Point Class: STM } \\
\text { Point Ratio: } 0.5444124 \\
\text { Bladede Lengnth (left): } 30.51 \\
\text { Blade Length (right): } 30.45 \\
\text { Stem Length: } 8.46 \\
\text { Neck Thickness: } 6.86 \\
\text { Neck Widthl. } 11.94 \\
\text { Base to Blade Ratio (length): } 0.221989 \\
\text { Base to Blade Ratio (width): } 0.6248804 \\
\text { Base/stem ratio: } 1 \\
\text { Base Form: } 2 \\
\text { Stem Form: } 4 \\
\text { Curvature (left): } 2 \\
\text { Curvature (right): } 3 \\
\text { Shoulder (left): } 16 \\
\text { Shoulder (right): } 28 \\
\text { Shoulder Junction: } 1\end{array}$ & Analyzed by Tomka \\
\hline Unit $085 \mathrm{SW}^{1 / 4}$ & $0-10$ & 724 & 703 & $\begin{array}{l}\text { Technology: Chipped Stone } \\
\text { Group: Tool } \\
\text { Subgroup: simple detachment-based } \\
\text { Type: } \\
\text { Subtype: Not Applicable }\end{array}$ & $\begin{array}{l}\text { Stage: INR } \\
\text { Portion: CMP } \\
\text { I FailurelDiscard: IND } \\
\text { Alteration: NOB } \\
\text { Edge Morphology (distal): STR } \\
\text { Edge Morphology (left lateral): } \\
\text { ETR } \\
\text { Ege Morphology (right lateral): CCV } \\
\text { Flade Scar Pattern: IND } \\
\text { Edge Construtucion Type: UNB } \\
\text { Proximal Edge Grinding: OBS }\end{array}$ & $\begin{array}{l}\text { Quantity: } 1 \\
\text { Weight (in g): } 0.7 \\
\text { Max Length (in mm): } 21 \\
\text { Max Width (in mm): } 12 \\
\text { Max Thickness (mm): } 3 \\
\text { Edge Angle: } 25\end{array}$ & $\begin{array}{l}\text { Flake Attrition: BFB } \\
\text { Crushing: NPR } \\
\text { Smoothing: NPR } \\
\text { Polish: SHL } \\
\text { Etching/Pitting: NPR } \\
\text { Hafting Evidence: NOB }\end{array}$ & CRT & $\begin{array}{l}\text { Point Class: NAP } \\
\text { Point Ratio: 0.5812832 } \\
\text { Base/Stem Width: }\end{array}$ & \\
\hline Unit $085 \mathrm{NW}^{1 / 4}$ & $30-40$ & 870 & 714 & $\begin{array}{l}\text { Technology: Chipped Stone } \\
\text { Group: Tool } \\
\text { Subgroup: complex detachment- } \\
\text { based } \\
\text { Type: } \\
\text { Subtype: Unclassified }\end{array}$ & $\begin{array}{l}\text { Stage: FST } \\
\text { Portion: STM } \\
\text { Failure/Discard: BND } \\
\text { Alteration: NOB } \\
\text { Edge Morphology (distal): IND } \\
\text { Edge Morphology (left lateral): IND } \\
\text { Edge Morphology (right latal): IND } \\
\text { Flake Scar antern: IND } \\
\text { Edge Construction Type: BFC } \\
\text { Proximal Edge Grinding: OBS }\end{array}$ & $\begin{array}{l}\text { Quantity: } 1 \\
\text { Weight (in g): } 0.6 \\
\text { Max Length (in mm): } 10 \\
\text { Max Width (in mm): } 19 \\
\text { Max Thickness (mm): } 5 \\
\text { Edge Angle: } 30\end{array}$ & $\begin{array}{l}\text { Flake Attrition: NPR } \\
\text { Crushing: NPR } \\
\text { Smoothing: NPR } \\
\text { Polish: NPR } \\
\text { Etching/Pitting: NPR } \\
\text { Hafting Evidence: NOB }\end{array}$ & QZT & $\begin{array}{l}\text { Point Class: STM } \\
\text { Point Ratio: } 1.875632 \\
\text { Neck Thickness: } 5.1 \\
\text { Neck Width: } 12.22 \\
\text { Base to Blade Ratio (width): } 1 \\
\text { Base/stem ratio: } 0 \\
\text { Base Form: } 2 \\
\text { Stem Form: } 3 \\
\text { Curvature (left): } 0 \\
\text { Curvature (right): } 0 \\
\text { Shoulder Junction: } 0\end{array}$ & $\begin{array}{l}\text { Archaic expanding stem, likely Ensor or other } \\
\text { subtype }\end{array}$ \\
\hline
\end{tabular}


Geo-Marine, Inc.

GMI 22005.01.06.xx and 22005.00.09.xx, Data Recovery Excavations at Site 41PN175 Chipped Stone Tool Data

\begin{tabular}{|c|c|c|c|c|c|c|c|c|c|c|}
\hline Unit & $\begin{array}{l}\text { Depth } \\
(\mathrm{cm})\end{array}$ & $\begin{array}{l}\text { Prov. } \\
\text { No. }\end{array}$ & $\begin{array}{l}\text { Lot } \\
\text { No. }\end{array}$ & Taxonomy & Attributes & Metric Information & Wear Patterning & Raw Material & Projectile Point Data & Comment \\
\hline \multicolumn{11}{|c|}{ Block 3} \\
\hline Unit $065 \mathrm{SW}^{1 / 4}$ & $0-10$ & 470 & 393 & $\begin{array}{l}\text { Technology: Chipped Stone } \\
\text { Group: Tool } \\
\text { Subgroup: complex detachment- } \\
\text { based } \\
\text { Type: } \\
\text { Subtype: Unclassified }\end{array}$ & 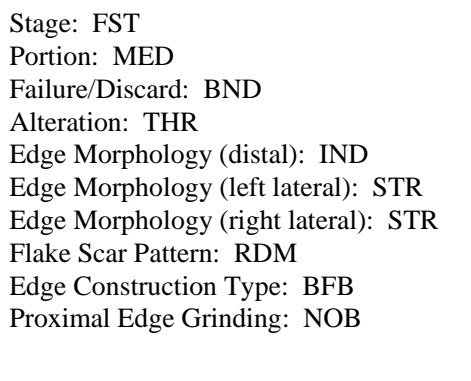 & $\begin{array}{l}\text { Quantity: } 1 \\
\text { Weight (in g): } 0.8 \\
\text { Max Length (in mm): } 16 \\
\text { Max Width (in mm): } 13 \\
\text { Max Thickness (mm): } 4 \\
\text { Edge Angle: } 20\end{array}$ & $\begin{array}{l}\text { Flake Attrition: NPR } \\
\text { Crushing: NPR } \\
\text { Smoothing: NPR } \\
\text { Polish: NPR } \\
\text { Etching/Pitting: NPR } \\
\text { Hafting Evidence: OBS }\end{array}$ & CRT & $\begin{array}{l}\text { Point Class: STM } \\
\text { Point Ratio: } 0.7953253 \\
\text { Base/Stem Width: } \\
\text { Neck Thickness: } 3.85 \\
\text { Neck Width: } 4.45 \\
\text { Base Form: } 0 . \\
\text { Stem Form: } 0 \\
\text { Curvature (left): } 3 \\
\text { Curvature (right): } 3 \\
\text { Shoulder (left): } 0 \\
\text { Shoulder Junction: } 1\end{array}$ & $\begin{array}{l}\text { since tip, right shoulder, and stem were missing } \\
\text { from impact fractures no measurements were } \\
\text { made }\end{array}$ \\
\hline Unit $066 \mathrm{NE}^{1 / 4}$ & $20-30$ & 535 & 411 & $\begin{array}{l}\text { Technology: Chipped Stone } \\
\text { Group: Tool } \\
\text { Subgroup: complex detachment- } \\
\text { based } \\
\text { Type: } \\
\text { Subtype: Not Applicable }\end{array}$ & 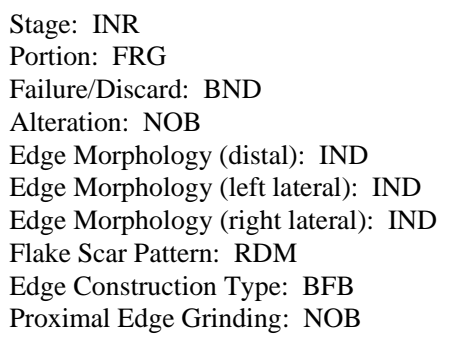 & $\begin{array}{l}\text { Quantity: } 1 \\
\text { Weight (in g): } 1.9 \\
\text { Max Length (in mm): } 19 \\
\text { Max Width (in mm): } 16 \\
\text { Max Thickness (mm): } 7 \\
\text { Edge Angle: } 35\end{array}$ & $\begin{array}{l}\text { Flake Attrition: NPR } \\
\text { Crushing: NPR } \\
\text { Smoothing: NPR } \\
\text { Polish: NPR } \\
\text { Etching/Pitting: NPR } \\
\text { Hafting Evidence: NOB }\end{array}$ & FWD & $\begin{array}{l}\text { Point Class: NAP } \\
\text { Point Ratio: 0.8196977 } \\
\text { Base/Stem Width: }\end{array}$ & \\
\hline Unit $067 \mathrm{SW}^{1 / 4}$ & $40-50$ & 687 & 428 & $\begin{array}{l}\text { Technology: Chipped Stone } \\
\text { Group: Tool } \\
\text { Subgroup: simple detachment-based } \\
\text { Type: } \\
\text { Subtype: Not Applicable }\end{array}$ & 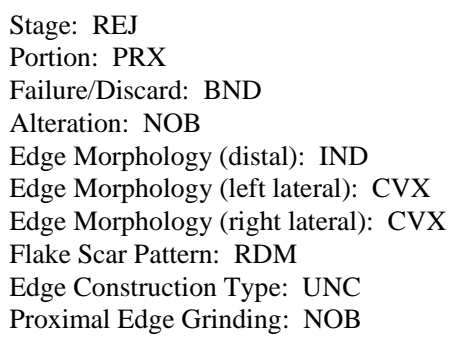 & $\begin{array}{l}\text { Quantity: } 1 \\
\text { Weight (in g): } 4.1 \\
\text { Max Length (in mm): } 24 \\
\text { Max Width (in mm): } 30 \\
\text { Max Thickness (mm): } 5 \\
\text { Edge Angle: } 25\end{array}$ & $\begin{array}{l}\text { Flake Attrition: BFC } \\
\text { Crushing: NPR } \\
\text { Smoothing: NPR } \\
\text { Polish: SHL } \\
\text { Etching/Pitting: NPR } \\
\text { Hafting Evidence: NOB }\end{array}$ & JPR & $\begin{array}{l}\text { Point Class: NAP } \\
\text { Point Ratio: } 1.240989 \\
\text { Base/Stem Width: }\end{array}$ & $\begin{array}{l}\text { small microflakes indicative of use wear are } \\
\text { present along with polish within } 1 \mathrm{~mm} \text { of the } \\
\text { edge are present on lateral and proximal edges }\end{array}$ \\
\hline Unit $069 \mathrm{SW}^{1 / 4}$ & $10-20$ & 545 & 451 & $\begin{array}{l}\text { Technology: Chipped Stone } \\
\text { Group: Tool } \\
\text { Subgroup: core-based } \\
\text { Type: } \\
\text { Subtype: Angostura }\end{array}$ & $\begin{array}{l}\text { Stage: REJ } \\
\text { Portion: BSH } \\
\text { Failure/Discard: ESH } \\
\text { Alteration: NOB } \\
\text { Edge Morphology (distal): IND } \\
\text { Edge Morphology (left lateral): STR } \\
\text { Edge Morphology (right lateral): IND } \\
\text { Flake Scar Pattern: IND } \\
\text { Edge Construction Type: IND } \\
\text { Proximal Edge Grinding: NOB }\end{array}$ & $\begin{array}{l}\text { Quantity: } 1 \\
\text { Weight (in g): } 6.4 \\
\text { Max Length (in mm): } 32 \\
\text { Max Width (in mm): } 22 \\
\text { Max Thickness (mm): } 7 \\
\text { Edge Angle: } 30\end{array}$ & $\begin{array}{l}\text { Flake Attrition: BFU } \\
\text { Crushing: NPR } \\
\text { Smoothing: NPR } \\
\text { Polish: PRX } \\
\text { Etching/Pitting: NPR } \\
\text { Hafting Evidence: OBS }\end{array}$ & CRT & $\begin{array}{l}\text { Point Class: NAP } \\
\text { Point Ratio: } 0.6915094 \\
\text { Base/Stem Width: }\end{array}$ & $\begin{array}{l}\text { Analyzed by Tomka, specimen represents a } \\
\text { projectile point that has been reworked as a } \\
\text { graver, Regional Edwards }\end{array}$ \\
\hline
\end{tabular}


Geo-Marine, Inc.

GMI 22005.01.06.xx and 22005.00.09.xx, Data Recovery Excavations at Site 41PN175 Chipped Stone Tool Dat

\begin{tabular}{|c|c|c|c|c|c|c|c|c|c|c|}
\hline Unit & $\begin{array}{c}\text { Depth } \\
(\mathrm{cm})\end{array}$ & $\begin{array}{l}\text { Prov. } \\
\text { No. }\end{array}$ & $\begin{array}{l}\text { Lot } \\
\text { No. }\end{array}$ & Taxonomy & Attributes & Metric Information & Wear Patterning & Raw Material & Projectile Point Data & Comment \\
\hline \multicolumn{11}{|l|}{ Block 3} \\
\hline Unit $070 \mathrm{SW}^{1 / 4}$ & $20-30$ & 629 & 468 & $\begin{array}{l}\text { Technology: Chipped Stone } \\
\text { Group: Tool } \\
\text { Subgroup: complex detachment- } \\
\text { based } \\
\text { Type: } \\
\text { Subtype: Not Applicable }\end{array}$ & $\begin{array}{l}\text { Stage: FST } \\
\text { Portion: MED } \\
\text { Failure/Discard: PRV } \\
\text { Alteration: NOB } \\
\text { Edge Morphology (distal): IND } \\
\text { Edge Morphologg (left lateral): CVX } \\
\text { Edge Morphology (right lateral): IND } \\
\text { Flake Scar Pattern: IND } \\
\text { Edge Construction Type: BFU } \\
\text { Proximal Edge Grinding: NOB }\end{array}$ & $\begin{array}{l}\text { Quantity: } 1 \\
\text { Weight (in g): } 4.3 \\
\text { Max Length (in mm): } 30 \\
\text { Max Width (in mm): } 21 \\
\text { Max Thickness (mm): } 6 \\
\text { Edge Angle: } 25\end{array}$ & $\begin{array}{l}\text { Flake Attrition: BFU } \\
\text { Crushing: NPR } \\
\text { Smoothing: NPR } \\
\text { Polish: SHL } \\
\text { Etching/Pitting: NPR } \\
\text { Hafting Evidence: NOB }\end{array}$ & FWD & $\begin{array}{l}\text { Point Class: NAP } \\
\text { Point Ratio: 0.70778243 } \\
\text { Base/Stem Width: }\end{array}$ & Analyzed by Tomka \\
\hline Unit 070 General & $40-50$ & 691 & 470 & $\begin{array}{l}\text { Technology: Chipped Stone } \\
\text { Group: Tool } \\
\text { Subproup: core-based } \\
\text { Type: } \\
\text { Subtype: San Patrice }\end{array}$ & 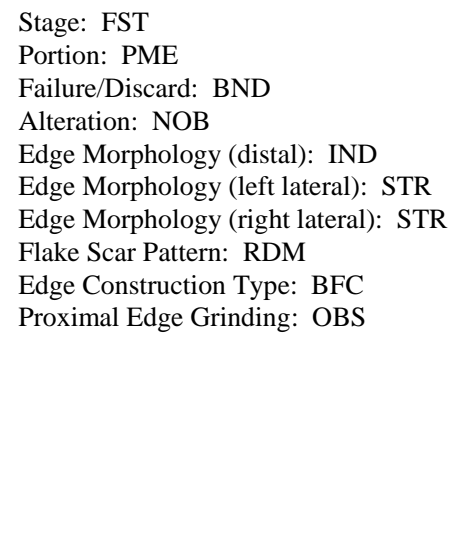 & $\begin{array}{l}\text { Quantity: } 1 \\
\text { Weight (in g): } 3.4 \\
\text { Max Length (in mm): } 34 \\
\text { Max Width (in mm): } 25 \\
\text { Max Thickness (mm): } 4 \\
\text { Edge Angle: } 25\end{array}$ & $\begin{array}{l}\text { Flake Attrition: BFB } \\
\text { Crushing: NPR } \\
\text { Smoothing: NPR } \\
\text { Polish: PRX } \\
\text { Etching/Pitting: NPR } \\
\text { Hafting Evidence: OBS }\end{array}$ & CRT & 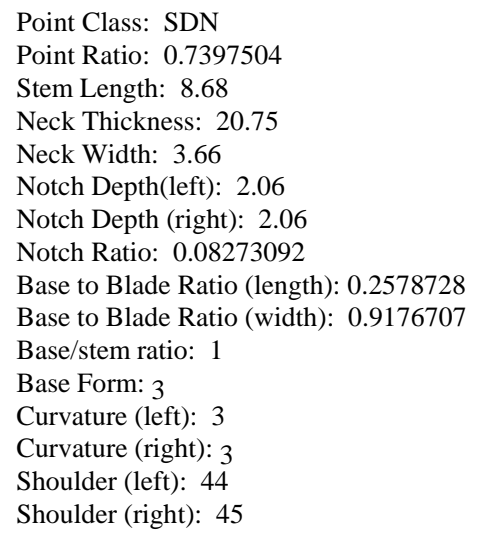 & $\begin{array}{l}\text { var. St. Johns, Analyzed by Tomka, tip missing } \\
\text { so blade lengths were not measured }\end{array}$ \\
\hline Unit $071 \mathrm{SW}^{1 / 4}$ & $30-40$ & 635 & 479 & $\begin{array}{l}\text { Technology: Chipped Stone } \\
\text { Group: Tool } \\
\text { Subgroup: simple detachment-based } \\
\text { Type: } \\
\text { Subtype: Not Applicable }\end{array}$ & $\begin{array}{l}\text { Stage: REJ } \\
\text { Portion: CMP } \\
\text { Pailure/Discard: IND } \\
\text { Alteration: THR } \\
\text { Edge Morphology (distal): STR } \\
\text { Edge Morphology (left lateral): CCV } \\
\text { Edge Morphology (right lateral): CVX } \\
\text { Flake Scar Pattern: RDM } \\
\text { Edge Construction Type: BDU } \\
\text { Proximal Edge Grinding: NOB }\end{array}$ & $\begin{array}{l}\text { Quantity: } 1 \\
\text { Weight (in g): } 3 \\
\text { Max Length (in mm): } 36 \\
\text { Max Width (in mm): } 18 \\
\text { Max Thickness (mm): } 5 \\
\text { Edge Angle: } 25\end{array}$ & $\begin{array}{l}\text { Flake Attrition: UDU } \\
\text { Crushing: NPR } \\
\text { Smoothing: NPR } \\
\text { Polish: SHD } \\
\text { Etching/Pitting: NPR } \\
\text { Hafting Evidence: NOB }\end{array}$ & CRT & $\begin{array}{l}\text { Point Class: NAP } \\
\text { Point Ratio: 0.4972543 } \\
\text { Base/Stem Width: }\end{array}$ & \\
\hline
\end{tabular}


Geo-Marine, Inc.

GMI 22005.01.06.xx and 22005.00.09.xx, Data Recovery Excavations at Site 41PN175 Chipped Stone Tool Dat

\begin{tabular}{|c|c|c|c|c|c|c|c|c|c|c|}
\hline Unit & $\begin{array}{l}\text { Depth } \\
(\mathrm{cm})\end{array}$ & $\begin{array}{l}\text { Prov. } \\
\text { No. }\end{array}$ & $\begin{array}{l}\text { Lot } \\
\text { No. }\end{array}$ & Taxonomy & Attributes & Metric Information & Wear Patterning & Raw Material & Projectile Point Data & Comment \\
\hline \multicolumn{11}{|c|}{ Block 3} \\
\hline Unit $072 \mathrm{NW}^{1 / 4}$ & $30-40$ & 641 & 493 & $\begin{array}{l}\text { Technology: Chipped Stone } \\
\text { Group: Tool } \\
\text { Subgroup: complex detachment- } \\
\text { based } \\
\text { Type: } \\
\text { Subtype: Ellis }\end{array}$ & 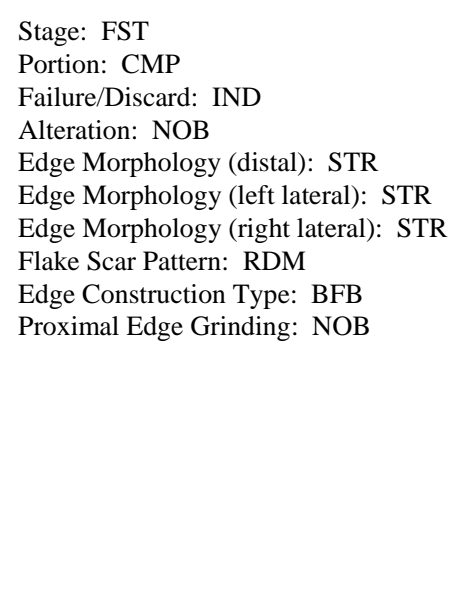 & $\begin{array}{l}\text { Quantity: } 1 \\
\text { Weight (in g): } 4.6 \\
\text { Max Length (in mm): } 33 \\
\text { Max Width (in mm): } 22 \\
\text { Max Thickness (mm): } 7 \\
\text { Edge Angle: } 35\end{array}$ & $\begin{array}{l}\text { Flake Attrition: BFB } \\
\text { Crushing: NPR } \\
\text { Smoothing: SPX } \\
\text { Polish: SHL } \\
\text { Etching/Pitting: NPR } \\
\text { Hafting Evidence: OBS }\end{array}$ & CRT & 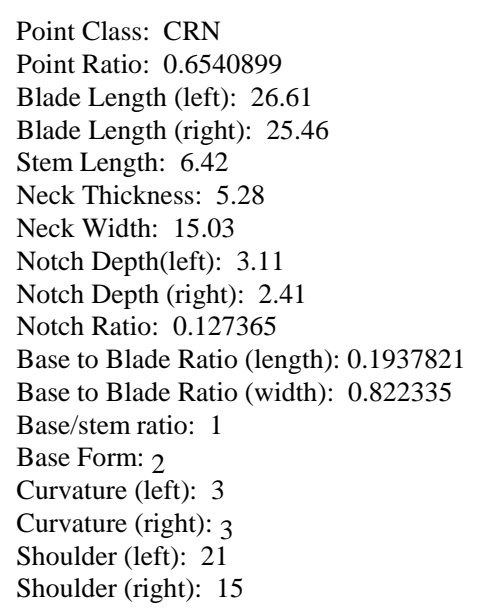 & Analyzed by Tomka \\
\hline Unit $072 \mathrm{NW}^{1 / 4}$ & $30-40$ & 641 & 493 & $\begin{array}{l}\text { Technology: Chipped Stone } \\
\text { Group: Tool } \\
\text { Subgroup: simple detachment-based } \\
\text { Type: } \\
\text { Subtype: Not Applicable }\end{array}$ & $\begin{array}{l}\text { Stage: INR } \\
\text { Portion: CMP } \\
\text { I FialurelDiscard: IND } \\
\text { Alteration: NOB } \\
\text { Edge Morphology (distal): CVX } \\
\text { Edge Morphology (left lateral): CVX } \\
\text { Edge Morphology (right latal) : CVX } \\
\text { Flake Scar Patern: RDM } \\
\text { Edge Construtuction Type: UNU } \\
\text { Proximal Edge Grinding: OBS }\end{array}$ & $\begin{array}{l}\text { Quantity: } 1 \\
\text { Weight (in g): } 7.7 \\
\text { Max Length (in mm): } 35 \\
\text { Max Width (in mm): } 22 \\
\text { Max Thickness (mm): } 10 \\
\text { Edge Angle: } 45\end{array}$ & $\begin{array}{l}\text { Flake Attrition: UFU } \\
\text { Crushing: NPR } \\
\text { Smoothing: NPR } \\
\text { Polish: DEL } \\
\text { Etching/Pitting: NPR } \\
\text { Hafting Evidence: OBS }\end{array}$ & FWD & $\begin{array}{l}\text { Point Class: NAP } \\
\text { Point Ratio: 0.6228344 } \\
\text { Base/Stem Width: }\end{array}$ & Analyzed by Tomka \\
\hline \multicolumn{11}{|c|}{ Block 4} \\
\hline Unit $073 \mathrm{SW}^{1} / 4$ & $40-50$ & 1724 & 514 & $\begin{array}{l}\text { Technology: Chipped Stone } \\
\text { Group: Tool } \\
\text { Subproup: complex detachment- } \\
\text { based } \\
\text { Type: } \\
\text { Subtype: Not Applicable }\end{array}$ & $\begin{array}{l}\text { Stage: PRF } \\
\text { Portion: PRX } \\
\text { FialurelDiscard: PRV } \\
\text { Alteration: OTH } \\
\text { Edge Morphology (distal): IND } \\
\text { Edge Morphology (left lateral): CVX } \\
\text { Edge Morphology (rithht latarl): CVX } \\
\text { Flake Scar Pattern: RDM } \\
\text { Edge Construtcion Type: BFC } \\
\text { Proximal Edge Grinding: NOB }\end{array}$ & $\begin{array}{l}\text { Quantity: } 1 \\
\text { Weight (in g): } 2.3 \\
\text { Max Length (in mm): } 30 \\
\text { Max Width (in mm): } 21 \\
\text { Max Thickness (mm): } 4 \\
\text { Edge Angle: } 25\end{array}$ & $\begin{array}{l}\text { Flake Attrition: NPR } \\
\text { Crushing: NPR } \\
\text { Smoothing: NPR } \\
\text { Polish: NPR } \\
\text { Etching/Pitting: NPR } \\
\text { Hafting Evidence: NOB }\end{array}$ & CRT & $\begin{array}{l}\text { Point Class: NAP } \\
\text { Point Ratio: 0.7000675 } \\
\text { Base/Stem Width: }\end{array}$ & Analyzed by Tomka \\
\hline
\end{tabular}


Geo-Marine, Inc.

GMI 22005.01.06.xx and 22005.00.09.xx, Data Recovery Excavations at Site 41PN175 Chipped Stone Tool Dat

\begin{tabular}{|c|c|c|c|c|c|c|c|c|c|c|}
\hline Unit & $\begin{array}{l}\text { Depth } \\
(\mathrm{cm})\end{array}$ & $\begin{array}{l}\text { Prov. } \\
\text { No. }\end{array}$ & $\begin{array}{l}\text { Lot } \\
\text { No. }\end{array}$ & Taxonomy & Attributes & Metric Information & Wear Patterning & Raw Material & Projectile Point Data & Comment \\
\hline \multicolumn{11}{|c|}{ Block 4} \\
\hline Unit $075 \mathrm{SE}^{1 / 4}$ & $30-40$ & 732 & 549 & $\begin{array}{l}\text { Technology: Chipped Stone } \\
\text { Group: Tool } \\
\text { Subgroup: simple detachment-based } \\
\text { Type: } \\
\text { Subtype: Not Applicable }\end{array}$ & $\begin{array}{l}\text { Stage: INR } \\
\text { Portion: CMP } \\
\text { Failure/Discard: IND } \\
\text { Alteration: THR } \\
\text { Edge Morphology (distal): CVX } \\
\text { Edge Morhhology (feft tateral): CVX } \\
\text { Edge Morphology (right lateral): CCV } \\
\text { Flake CCar Pattern: RDM } \\
\text { Edge Construction Type: UDB } \\
\text { Proximal Edge Grinding: NOB }\end{array}$ & $\begin{array}{l}\text { Quantity: } 1 \\
\text { Weight (in g): } 0.6 \\
\text { Max Length (in mm): } 19 \\
\text { Max Width (in mm): } 12 \\
\text { Max Thickness (mm): } 3 \\
\text { Edge Angle: } 15\end{array}$ & $\begin{array}{l}\text { Flake Attrition: UDB } \\
\text { Crushing: NPR } \\
\text { Smoothing: NPR } \\
\text { Polish: SHL } \\
\text { Etching/Pitting: NPR } \\
\text { Hafting Evidence: NOB }\end{array}$ & CRT & $\begin{array}{l}\text { Point Class: NAP } \\
\text { Point Ratio: 0.6212447 } \\
\text { Base/Stem Width: }\end{array}$ & Analyzed by Tomka \\
\hline Unit $076 \mathrm{NE}^{1 / 4}$ & $30-40$ & 735 & 559 & $\begin{array}{l}\text { Technology: Chipped Stone } \\
\text { Group: Tool } \\
\text { Subgroup: complex detachment- } \\
\text { based } \\
\text { Type: } \\
\text { Subtype: Perdiz }\end{array}$ & $\begin{array}{l}\text { Stage: FST } \\
\text { Portion: PME } \\
\text { Failure/Discard: BND } \\
\text { Alteration: NOB } \\
\text { Edge Morphology (distal): IND } \\
\text { Edge Morphology (eft tateral): STR } \\
\text { Edge Morphology (right lateral): STR } \\
\text { Flake Scar Pattern: RDM } \\
\text { Edge Construction Type: BFB } \\
\text { Proximal Edge Grinding: NOB }\end{array}$ & $\begin{array}{l}\text { Quantity: } 1 \\
\text { Weight (in g): } 0.4 \\
\text { Max Length (in mm): } 13 \\
\text { Max Width (in mm): } 12 \\
\text { Max Thickness (mm): } 3 \\
\text { Edge Angle: } 20\end{array}$ & $\begin{array}{l}\text { Flake Attrition: BDB } \\
\text { Crushing: NPR } \\
\text { Smoothing: NPR } \\
\text { Polish: SHD } \\
\text { Etching/Pitting: NPR } \\
\text { Hafting Evidence: OBS }\end{array}$ & CRT & 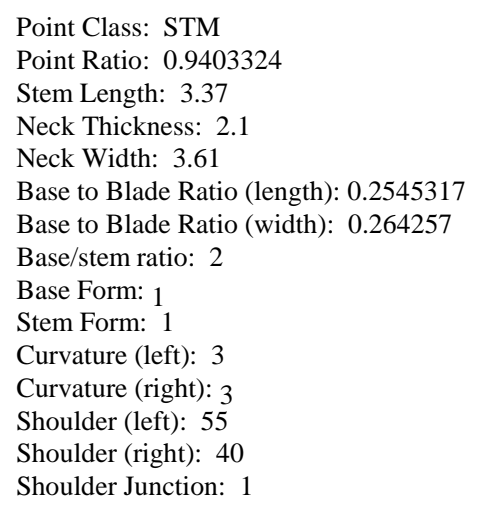 & $\begin{array}{l}\text { tip missing from impact fracture, no blade } \\
\text { measurements taken }\end{array}$ \\
\hline Unit $076 \mathrm{NW}^{1 / 4}$ & $30-40$ & 734 & 560 & $\begin{array}{l}\text { Technology: Chipped Stone } \\
\text { Group: Tool } \\
\text { Subgroup: simple detachment-based } \\
\text { Type: } \\
\text { Subtype: Not Applicable }\end{array}$ & 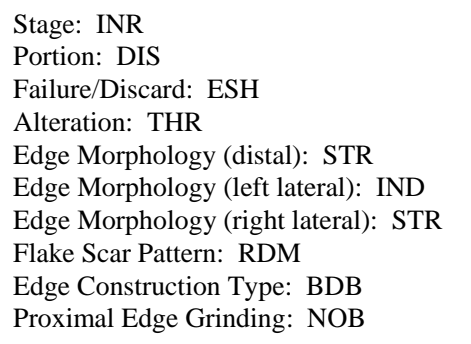 & $\begin{array}{l}\text { Quantity: } 1 \\
\text { Weight (in g): } 2.3 \\
\text { Max Length (in mm): } 23 \\
\text { Max Width (in mm): } 16 \\
\text { Max Thickness (mm): } 6 \\
\text { Edge Angle: } 35\end{array}$ & $\begin{array}{l}\text { Flake Attrition: BFB } \\
\text { Crushing: BLT } \\
\text { Smoothing: NPR } \\
\text { Polish: NPR } \\
\text { Etching/Pitting: NPR } \\
\text { Hafting Evidence: NOB }\end{array}$ & CRT & $\begin{array}{l}\text { Point Class: NAP } \\
\text { Point Ratio: 0.6949227 } \\
\text { Base/Stem Width: }\end{array}$ & \\
\hline
\end{tabular}


Geo-Marine, Inc.

GMI 22005.01.06.xx and 22005.00.09.xx, Data Recovery Excavations at Site 41PN175 Chipped Stone Tool Dat

\begin{tabular}{|c|c|c|c|c|c|c|c|c|c|c|}
\hline Unit & $\begin{array}{l}\text { Depth } \\
(\mathrm{cm})\end{array}$ & $\begin{array}{l}\text { Prov. } \\
\text { No. }\end{array}$ & $\begin{array}{l}\text { Lot } \\
\text { No. }\end{array}$ & Taxonomy & Attributes & Metric Information & Wear Patterning & Raw Material & Projectile Point Data & Comment \\
\hline \multicolumn{11}{|c|}{ Block 4} \\
\hline Unit $078 \mathrm{NE}^{1 / 4}$ & $20-30$ & 709 & 583 & $\begin{array}{l}\text { Technology: Chipped Stone } \\
\text { Group: Tool } \\
\text { Subgroup: complex detachment- } \\
\text { based } \\
\text { Type: } \\
\text { Subtype: Perdiz/Bassett }\end{array}$ & $\begin{array}{l}\text { Stage: FST } \\
\text { Portion: LEM } \\
\text { Failureliscard: BND } \\
\text { Alteration: NOB } \\
\text { Edge Morphology (distal): STR } \\
\text { Edge Morphology (left lateral): } \\
\text { Edge } \\
\text { Elge Morphology (right lateral): STR } \\
\text { Flake Scar Pattern: RDM RD } \\
\text { Edge Construction Type: BFB } \\
\text { Proximal Edge Grinding: NOB }\end{array}$ & $\begin{array}{l}\text { Quantity: } 1 \\
\text { Weight (in g): } 0.5 \\
\text { Max Length (in mm): } 18 \\
\text { Max Width (in mm): } 10 \\
\text { Max Thickness (mm): } 5 \\
\text { Edge Angle: } 30\end{array}$ & $\begin{array}{l}\text { Flake Attrition: NPR } \\
\text { Crushing: NPR } \\
\text { Smoothing: NPR } \\
\text { Polish: NPR } \\
\text { Etching/Pitting: NPR } \\
\text { Hafting Evidence: NOB }\end{array}$ & CRT & $\begin{array}{l}\text { Point Class: STM } \\
\text { Point Ratio: } 0.5196078 \\
\text { Blade Lenghth (left): } 15.95 \\
\text { Stem Length: } 4.12 \\
\text { Nemk Thickness: } 2.67 \\
\text { Neck Width: 3:52 } \\
\text { Base to Blade Ratio (length): } 0.2244009 \\
\text { Base to Blade Ratio (width): } 0.2264151 \\
\text { Bases/stem ratio: } 3 \\
\text { Base Formm: } 1 \\
\text { Stem Form: } 1 \\
\text { Curvature (left): } 2 \\
\text { Curvature (right): } 3 \\
\text { Shoulder (left): } 8 \\
\text { Shoulder Junction: } 1\end{array}$ & $\begin{array}{l}\text { Right shoulder is missing from an impact } \\
\text { fracture, corresponding measurements were not } \\
\text { made }\end{array}$ \\
\hline Unit $078 \mathrm{SW}^{1 / 4}$ & $20-30$ & 671 & 586 & $\begin{array}{l}\text { Technology: Chipped Stone } \\
\text { Group: Tool } \\
\text { Subgroup: complex detachment- } \\
\text { based } \\
\text { Type: } \\
\text { Subtype: Not Applicable }\end{array}$ & $\begin{array}{l}\text { Stage: INR } \\
\text { Portion: CMP } \\
\text { FailurelDiscard: IND } \\
\text { Alteration: NOB } \\
\text { Elge Morphology (distal): STR } \\
\text { Edge Morphology (left lateral): } \\
\text { ETR } \\
\text { Ege Morphology (right lateral): STR } \\
\text { Flake Scar Pattern: RDM } \\
\text { Edge Construtction Type: BDU } \\
\text { Proximal Edge Grinding: NOB }\end{array}$ & $\begin{array}{l}\text { Quantity: } 1 \\
\text { Weight (in g): } 1.4 \\
\text { Max Length (in mm): } 14 \\
\text { Max Width (in mm): } 15 \\
\text { Max Thickness (mm): } 6 \\
\text { Edge Angle: } 30\end{array}$ & $\begin{array}{l}\text { Flake Attrition: BFU } \\
\text { Crushing: NPR } \\
\text { Smoothing: NPR } \\
\text { Polish: NPR } \\
\text { Etching/Pitting: NPR } \\
\text { Hafting Evidence: NOB }\end{array}$ & CRT & $\begin{array}{l}\text { Point Class: NAP } \\
\text { Point Ratio: 1.066128 } \\
\text { Base/Stem Width: }\end{array}$ & \\
\hline Unit $078 \mathrm{SW}^{1 / 4}$ & $30-40$ & 713 & 590 & $\begin{array}{l}\text { Technology: Chipped Stone } \\
\text { Group: Tool } \\
\text { Subgroup: simple detachment-based } \\
\text { Type: } \\
\text { Subtype: Not Applicable }\end{array}$ & $\begin{array}{l}\text { Stage: INR } \\
\text { Portion: CMP } \\
\text { FailurelDiscard: IND } \\
\text { Alteration: NOB } \\
\text { Edge Morphology (distal): CVX } \\
\text { Edge Morphology (left lateral): } \\
\text { ETR } \\
\text { Elge Morphologg (right tateral): STR } \\
\text { Flake Scar Pattern: NAP } \\
\text { Edge Construction Nype: UFD } \\
\text { Proximal Edge Grinding: NOB }\end{array}$ & $\begin{array}{l}\text { Quantity: } 1 \\
\text { Weight (in g): } 2.1 \\
\text { Max Length (in mm): } 23 \\
\text { Max Width (in mm): } 22 \\
\text { Max Thickness (mm): } 4 \\
\text { Edge Angle: } 30\end{array}$ & $\begin{array}{l}\text { Flake Attrition: UFD } \\
\text { Crushing: NPR } \\
\text { Smoothing: NPR } \\
\text { Polish: SHD } \\
\text { Etching/Pitting: NPR } \\
\text { Hafting Evidence: NOB }\end{array}$ & CRT & $\begin{array}{l}\text { Point Class: NAP } \\
\text { Point Ratio: } 0.9773735 \\
\text { Base/Stem Width: }\end{array}$ & Analyzed by Tomka \\
\hline Unit $086 \mathrm{SW}^{1} / 4$ & $10-20$ & 1115 & 720 & $\begin{array}{l}\text { Technology: Chipped Stone } \\
\text { Group: Tool } \\
\text { Sugbroup: complex detachment- } \\
\text { based } \\
\text { Type: } \\
\text { Subtype: Not Applicable }\end{array}$ & $\begin{array}{l}\text { Stage: BLK } \\
\text { Poortion: IND } \\
\text { Failureliscard: PRV } \\
\text { Alteration: NOB } \\
\text { Edge Morphology (distall): IND } \\
\text { Edge Morphology (left lateral): STR } \\
\text { Edge Morphology (right lateral): STR } \\
\text { Flake Scar Pattern: RDM } \\
\text { Edge Construction TTpe: BFC } \\
\text { Proximal Edge Grinding: OBS }\end{array}$ & $\begin{array}{l}\text { Quantity: } 1 \\
\text { Weight (in g): } 2.7 \\
\text { Max Length (in mm): } 22 \\
\text { Max Width (in mm): } 17 \\
\text { Max Thickness (mm): } 7 \\
\text { Edge Angle: } 30\end{array}$ & $\begin{array}{l}\text { Flake Attrition: NPR } \\
\text { Crushing: NPR } \\
\text { Smoothing: PPX } \\
\text { Polish: NPR } \\
\text { Etching/Pitting: NPR } \\
\text { Hafting Evidence: NOB }\end{array}$ & CRT & $\begin{array}{l}\text { Point Class: NAP } \\
\text { Point Ratio: } 0.7916667 \\
\text { Base/Stem Width: }\end{array}$ & \\
\hline
\end{tabular}


Geo-Marine, Inc.

GMI 22005.01.06.xx and 22005.00.09.xx, Data Recovery Excavations at Site 41PN175 Chipped Stone Tool Dat

\begin{tabular}{|c|c|c|c|c|c|c|c|c|c|c|}
\hline Unit & $\begin{array}{l}\text { Depth } \\
(\mathrm{cm})\end{array}$ & $\begin{array}{l}\text { Prov. } \\
\text { No. }\end{array}$ & $\begin{array}{l}\text { Lot } \\
\text { No. }\end{array}$ & Taxonomy & Attributes & Metric Information & Wear Patterning & Raw Material & Projectile Point Data & Comment \\
\hline \multicolumn{11}{|c|}{ Block 4} \\
\hline Unit $088 \mathrm{SE}^{1 / 4}$ & $20-30$ & 1260 & 759 & $\begin{array}{l}\text { Technology: Chipped Stone } \\
\text { Group: Tool } \\
\text { Subgroup: complex detachment- } \\
\text { based } \\
\text { Type: } \\
\text { Subtype: Perdiz }\end{array}$ & $\begin{array}{l}\text { Stage: FST } \\
\text { Portion: CMP } \\
\text { Failure/Discard: BND } \\
\text { Alteration: NoB } \\
\text { Edge Morhology (distal): STR } \\
\text { Edge Morphology (left lateral): SER } \\
\text { Edge Morphology (right lateral): SER } \\
\text { Flake Scar Pattern: RDM } \\
\text { Edge Construction Type: BFB } \\
\text { Eroximal Edge Grinding: NOB }\end{array}$ & $\begin{array}{l}\text { Quantity: } 1 \\
\text { Weight (in g): } 0.5 \\
\text { Max Length (in mm): } 19 \\
\text { Max Width (in mm): } 10 \\
\text { Max Thickness (mm): } 3 \\
\text { Edge Angle: } 25\end{array}$ & $\begin{array}{l}\text { Flake Attrition: NPR } \\
\text { Crushing: NPR } \\
\text { Smoothing: NPR } \\
\text { Polish: NPR } \\
\text { Etching/Pitting: NPR } \\
\text { Hafting Evidence: OBS }\end{array}$ & CRT & 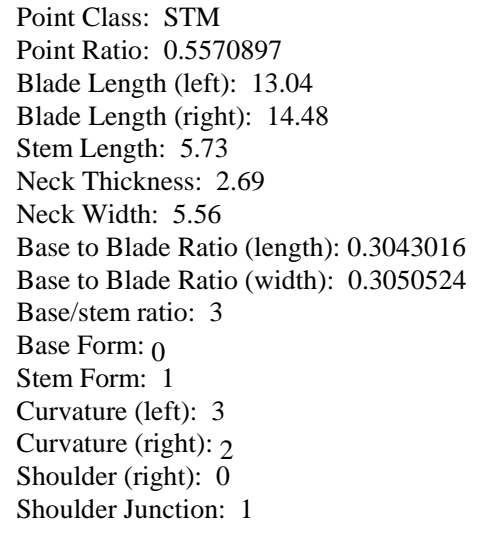 & $\begin{array}{l}\text { very small portion of base and left hhoulder } \\
\text { misssing from impact fractures, corresponding } \\
\text { measurements not taken }\end{array}$ \\
\hline Unit $090 \mathrm{SW}^{1 / 4}$ & $36-36$ & 1487 & 838 & $\begin{array}{l}\text { Technology: Chipped Stone } \\
\text { Group: Tool } \\
\text { Subgroup: complex detachment- } \\
\text { based } \\
\text { Type: } \\
\text { Subtype: Unclassified }\end{array}$ & $\begin{array}{l}\text { Stage: FST } \\
\text { Portion: CMP } \\
\text { Failure/Discard: IND } \\
\text { Alteration: NoB } \\
\text { Edge Morphology (distal): STR } \\
\text { Edge Morphology (left taterala): CVX } \\
\text { Edge Morphology (right lateral): CVX } \\
\text { Elake CCar Pattern: COL } \\
\text { Edge Construction Type: BFB } \\
\text { Edgox } \\
\text { Proximal Edge Grinding: NOB }\end{array}$ & $\begin{array}{l}\text { Quantity: } 1 \\
\text { Weight (in g): } 6.3 \\
\text { Max Length (in mm): } 39 \\
\text { Max Width (in mm): } 26 \\
\text { Max Thickness (mm): } 6 \\
\text { Edge Angle: } 30\end{array}$ & $\begin{array}{l}\text { Flake Attrition: BFU } \\
\text { Crushing: NPR } \\
\text { Smoothing: NPR } \\
\text { Polish: SHD } \\
\text { Etching/Pitting: NPR } \\
\text { Hafting Evidence: OBS }\end{array}$ & CRT & 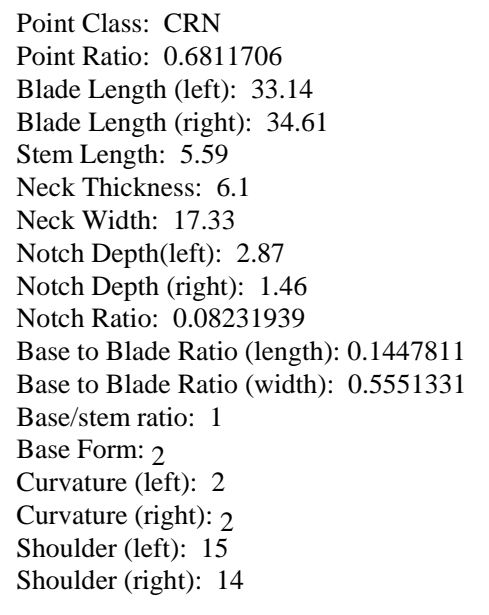 & $\begin{array}{l}\text { Analyzed by Tomka, expedient projectile point } \\
\text { resembles Kent subtype }\end{array}$ \\
\hline Unit $092 \mathrm{NW}^{1 / 4}$ & $18-28$ & 1147 & 869 & $\begin{array}{l}\text { Technology: Chipped Stone } \\
\text { Group: Tool } \\
\text { Subproup: simple detachment-based } \\
\text { Type: } \\
\text { Subtype: Not Applicable }\end{array}$ & $\begin{array}{l}\text { Stage: REJ } \\
\text { Portion: CMP } \\
\text { Failure/Discard: IND } \\
\text { Alteration: NOB } \\
\text { Edge Morphology (distal): STR } \\
\text { Edge orophology (left taterala): STR } \\
\text { Edge Morphology (right lateral): STR } \\
\text { Elake ccar Pattern. IND } \\
\text { Fdge Construction Type: UDB } \\
\text { Edyoximal Edge Grinding: NOB }\end{array}$ & $\begin{array}{l}\text { Quantity: } 1 \\
\text { Weight (in g): } 1.5 \\
\text { Max Length (in mm): } 21 \\
\text { Max Width (in mm): } 11 \\
\text { Max Thickness (mm): } 8 \\
\text { Edge Angle: } 25\end{array}$ & $\begin{array}{l}\text { Flake Attrition: UDB } \\
\text { Crushing: NPR } \\
\text { Smoothing: NPR } \\
\text { Polish: SHD } \\
\text { Etching/Pitting: NPR } \\
\text { Hafting Evidence: NOB }\end{array}$ & QZT & $\begin{array}{l}\text { Point Class: NAP } \\
\text { Point Ratio: 0.5301543 } \\
\text { Base/Stem Width: }\end{array}$ & Analyzed by Tomka \\
\hline
\end{tabular}


Geo-Marine, Inc.

GMI 22005.01.06.xx and 22005.00.09.xx, Data Recovery Excavations at Site 41PN175 Chipped Stone Tool Data

\begin{tabular}{|c|c|c|c|c|c|c|c|c|c|c|}
\hline Unit & $\begin{array}{l}\text { Depth } \\
(\mathrm{cm})\end{array}$ & $\begin{array}{l}\text { Prov. } \\
\text { No. }\end{array}$ & $\begin{array}{l}\text { Lot } \\
\text { No. }\end{array}$ & Taxonomy & Attributes & Metric Information & Wear Patterning & Raw Material & Projectile Point Data & Comment \\
\hline \multicolumn{11}{|c|}{ Block 4} \\
\hline Unit $093 \mathrm{SW}^{1 / 4}$ & $20-25$ & 1274 & 884 & $\begin{array}{l}\text { Technology: Chipped Stone } \\
\text { Group: Tool } \\
\text { Subgroup: complex detachment- } \\
\text { based } \\
\text { Type: } \\
\text { Subtype: Not Applicable }\end{array}$ & $\begin{array}{l}\text { Stage: PRF } \\
\text { Portion: DIS } \\
\text { FailureDiscard: MFL } \\
\text { Alteration: NOB } \\
\text { Edge Morphology (distal): IND } \\
\text { Edge Morphology (left lateral): CVX } \\
\text { Edge Morphology (rightl latral): STR } \\
\text { Flake Scar Pattern: RDM } \\
\text { Edge Construction Type: BFB } \\
\text { Proximal Edge Grinding: NOB }\end{array}$ & $\begin{array}{l}\text { Quantity: } 1 \\
\text { Weight (in g): } 1 \\
\text { Max Length (in mm): } 14 \\
\text { Max Width (in mm): } 18 \\
\text { Max Thickness (mm): } 5 \\
\text { Edge Angle: } 25\end{array}$ & $\begin{array}{l}\text { Flake Attrition: NPR } \\
\text { Crushing: NPR } \\
\text { Smoothing: NPR } \\
\text { Polish: NPR } \\
\text { Etching/Pitting: NPR } \\
\text { Hafting Evidence: NOB }\end{array}$ & CRT & $\begin{array}{l}\text { Point Class: NAP } \\
\text { Point Ratio: } 1.248084 \\
\text { Base/Stem Width: }\end{array}$ & \\
\hline Unit $093 \mathrm{SE}^{1 / 4}$ & $30-40$ & 1383 & 891 & $\begin{array}{l}\text { Technology: Chipped Stone } \\
\text { Group: Tool } \\
\text { Subgroup: complex detachment- } \\
\text { based } \\
\text { Type: } \\
\text { Subtype: Not Applicable }\end{array}$ & $\begin{array}{l}\text { Stage: PRF } \\
\text { Portion: DIS } \\
\text { Failure/Discard: BND } \\
\text { Alteration: THR } \\
\text { Edge Morphology (distal): CVX } \\
\text { Edge Morphology (left lateral): STR } \\
\text { Edge Morphology (right lateral): STR } \\
\text { Flake Scar Pattern: RDM } \\
\text { Edge Construction Type: BDB } \\
\text { Proximal Edge Grinding: NOB }\end{array}$ & $\begin{array}{l}\text { Quantity: } 1 \\
\text { Weight (in g): } 1.4 \\
\text { Max Length (in mm): } 17 \\
\text { Max Width (in mm): } 18 \\
\text { Max Thickness (mm): } 4 \\
\text { Edge Angle: } 25\end{array}$ & $\begin{array}{l}\text { Flake Attrition: NPR } \\
\text { Crushing: NPR } \\
\text { Smoothing: NPR } \\
\text { Polish: NPR } \\
\text { Etching/Pitting: NPR } \\
\text { Hafting Evidence: NOB }\end{array}$ & CRT & $\begin{array}{l}\text { Point Class: NAP } \\
\text { Point Ratio: 1.066589 } \\
\text { Base/Stem Width: }\end{array}$ & $\begin{array}{l}\text { distal projectile point tip, could not select Point } \\
\text { Class since missing stem/shoulder }\end{array}$ \\
\hline Unit $095 \mathrm{SE}^{1 / 4}$ & $10-20$ & 1161 & 916 & $\begin{array}{l}\text { Technology: Chipped Stone } \\
\text { Group: Tool } \\
\text { Subgroup: simple detachment-based } \\
\text { Type: } \\
\text { Subtype: Not Applicable }\end{array}$ & $\begin{array}{l}\text { Stage: INR } \\
\text { Portion: PME } \\
\text { 'Failure/Discard: ESH } \\
\text { Alteration: THR } \\
\text { Edge Morphology (distal): IND } \\
\text { Edge Morphology (left lateral): STR } \\
\text { Edge Morphology (right latral): STR } \\
\text { Flake Scar Pattern: IND } \\
\text { Edge Construction Type: UNB } \\
\text { Proximal Edge Grinding: NOB }\end{array}$ & $\begin{array}{l}\text { Quantity: } 1 \\
\text { Weight (in g): } 3.9 \\
\text { Max Length (in mm): } 36 \\
\text { Max Width (in mm): } 17 \\
\text { Max Thickness (mm): } 9 \\
\text { Edge Angle: } 35\end{array}$ & $\begin{array}{l}\text { Flake Attrition: UFB } \\
\text { Crushing: NPR } \\
\text { Smoothing: NPR } \\
\text { Polish: SHL } \\
\text { Etching/Pitting: NPR } \\
\text { Hafting Evidence: NOB }\end{array}$ & CRT & $\begin{array}{l}\text { Point Class: NAP } \\
\text { Point Ratio: 0.4729242 } \\
\text { Base/Stem Width: }\end{array}$ & Analyzed by Tomka \\
\hline Unit $095 \mathrm{NE}^{1 / 4}$ & $27-30$ & 1393 & 923 & $\begin{array}{l}\text { Technology: Chipped Stone } \\
\text { Group: Tool } \\
\text { Subgroup: complex detachment- } \\
\text { based } \\
\text { Type: } \\
\text { Subtype: Unclassified }\end{array}$ & $\begin{array}{l}\text { Stage: INR } \\
\text { Portion: DME } \\
\text { Failure/Discard: BND } \\
\text { Alteration: NOB } \\
\text { Edge Morphology (distal): IND } \\
\text { Edge Morphology (left lateral): } \\
\text { EdR } \\
\text { Ege Morphology (right latral): } \\
\text { Flake Scar PTatern: RDM } \\
\text { Edge Construtuction Type: BFB } \\
\text { Proximal Edge Grinding: NOB }\end{array}$ & $\begin{array}{l}\text { Quantity: } 1 \\
\text { Weight (in g): } 1.1 \\
\text { Max Length (in mm): } 23 \\
\text { Max Width (in mm): } 15 \\
\text { Max Thickness (mm): } 4 \\
\text { Edge Angle: } 25\end{array}$ & $\begin{array}{l}\text { Flake Attrition: NPR } \\
\text { Crushing: NPR } \\
\text { Smoothing: NPR } \\
\text { Polish: NPR } \\
\text { Etching/Pitting: NPR } \\
\text { Hafting Evidence: NOB }\end{array}$ & QZT & $\begin{array}{l}\text { Point Class: NAP } \\
\text { Point Ratio: } 0.64806687 \\
\text { Base/Stem Width: }\end{array}$ & $\begin{array}{l}\text { Distal Arrow point, since stem/shoulder were } \\
\text { missing it was not possible to select Point Class } \\
\text { (bag originally incorrectly stated P.1382) }\end{array}$ \\
\hline
\end{tabular}


Geo-Marine, Inc.

GMI 22005.01.06.xx and 22005.00.09.xx, Data Recovery Excavations at Site 41PN175 Chipped Stone Tool Data

\begin{tabular}{|c|c|c|c|c|c|c|c|c|c|c|}
\hline Unit & $\begin{array}{l}\text { Depth } \\
(\mathrm{cm})\end{array}$ & $\begin{array}{l}\text { Prov. } \\
\text { No. }\end{array}$ & $\begin{array}{l}\text { Lot } \\
\text { No. }\end{array}$ & Taxonomy & Attributes & Metric Information & Wear Patterning & Raw Material & Projectile Point Data & Comment \\
\hline \multicolumn{11}{|l|}{ Block 4} \\
\hline Unit $098 \mathrm{NE}^{1 / 4}$ & $20-30$ & 1171 & 974 & $\begin{array}{l}\text { Technology: Chipped Stone } \\
\text { Group: Tool } \\
\text { Subgroup: simple detachment-based } \\
\text { Type: } \\
\text { Subtype: Not Applicable }\end{array}$ & 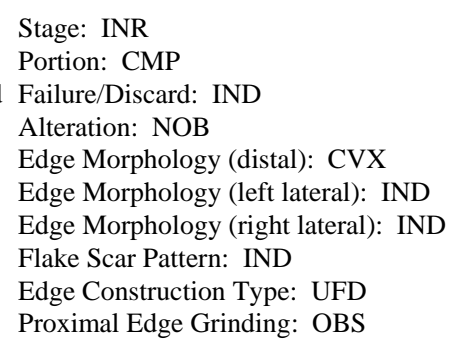 & $\begin{array}{l}\text { Quantity: } 1 \\
\text { Weight (in g): } 1.8 \\
\text { Max Length (in mm): } 17 \\
\text { Max Width (in mm): } 21 \\
\text { Max Thickness (mm): } 6 \\
\text { Edge Angle: } 30\end{array}$ & $\begin{array}{l}\text { Flake Attrition: UFB } \\
\text { Crushing: NPR } \\
\text { Smoothing: NPR } \\
\text { Polish: SHD } \\
\text { Etching/Pitting: SHD } \\
\text { Hafting Evidence: NOB }\end{array}$ & CRT & $\begin{array}{l}\text { Point Class: NAP } \\
\text { Point Ratio: } 1.252695 \\
\text { Base/Stem Width: }\end{array}$ & \\
\hline Unit $099 \mathrm{NE}^{1 / 4}$ & $10-20$ & 1175 & 991 & $\begin{array}{l}\text { Technology: Chipped Stone } \\
\text { Group: Tool } \\
\text { Subproup: simple detachment-based } \\
\text { Type: } \\
\text { Subtype: Not Applicable }\end{array}$ & $\begin{array}{l}\text { Stage: INR } \\
\text { Portion: CMP } \\
\text { Failure/Discard: IND } \\
\text { AAlteration: NOB } \\
\text { Edge Morphology (distal): IND } \\
\text { Edge Morphology (left lateral): IND } \\
\text { Edge Morphology (right laterl): CCV } \\
\text { Flake Scar antern: IND } \\
\text { Edge Construction Type: UNU } \\
\text { Proximal Edge Grinding: NOB }\end{array}$ & $\begin{array}{l}\text { Quantity: } 1 \\
\text { Weight (in g): } 0.3 \\
\text { Max Length (in mm): } 17 \\
\text { Max Width (in mm): } 9 \\
\text { Max Thickness (mm): } 3 \\
\text { Edge Angle: } 25\end{array}$ & $\begin{array}{l}\text { Flake Attrition: UFU } \\
\text { Crushing: NPR } \\
\text { Smoothing: NPR } \\
\text { Polish: SHL } \\
\text { Etching/Pitting: NPR } \\
\text { Hafting Evidence: NOB }\end{array}$ & CRT & $\begin{array}{l}\text { Point Class: NAP } \\
\text { Point Ratio: 0.5543159 } \\
\text { Base/Stem Width: }\end{array}$ & Analyzed by Tomka \\
\hline Unit $099 \mathrm{NW}^{1 / 4}$ & $10-20$ & 1174 & 992 & $\begin{array}{l}\text { Technology: Chipped Stone } \\
\text { Group: Tool } \\
\text { Subgroup: simple detachment-based } \\
\text { Type: } \\
\text { Subtype: Not Applicable }\end{array}$ & 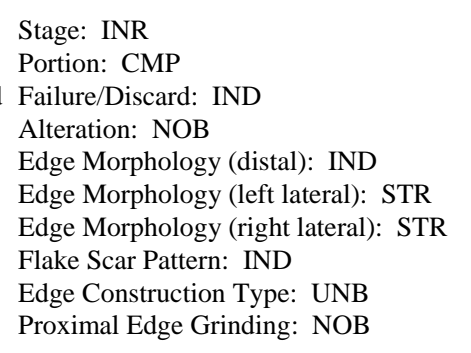 & $\begin{array}{l}\text { Quantity: } 1 \\
\text { Weight (in g): } 0.7 \\
\text { Max Length (in mm): } 20 \\
\text { Max Width (in mm): } 11 \\
\text { Max Thickness (mm): } 3 \\
\text { Edge Angle: } 25\end{array}$ & $\begin{array}{l}\text { Flake Attrition: UFU } \\
\text { Crushing: NPR } \\
\text { Smoothing: NPR } \\
\text { Polish: SHL } \\
\text { Etching/Pitting: NPR } \\
\text { Hafting Evidence: NOB }\end{array}$ & CRT & $\begin{array}{l}\text { Point Class: } \\
\text { Point Ratio: } 0.5837173 \\
\text { Base/Stem Width: }\end{array}$ & Analyzed by Tomka \\
\hline Unit 105 General & $10-20$ & 1201 & 1096 & $\begin{array}{l}\text { Technology: Chipped Stone } \\
\text { Group: Tool } \\
\text { Subgroup: simple detachment-based } \\
\text { Type: } \\
\text { Subtype: Not Applicable }\end{array}$ & $\begin{array}{l}\text { Stage: INR } \\
\text { Portion: PRX } \\
\text { l FilurelDiscard: ESH } \\
\text { Alteration: NOB } \\
\text { Edge Morphology (distall): IND } \\
\text { Edge Morphology (left lateral): STR } \\
\text { Edge Morphology (right lateral): STR } \\
\text { Flake Scar Pattern: IND } \\
\text { Edge Construction Type: UNU } \\
\text { Proximal Edge Grinding: NOB }\end{array}$ & $\begin{array}{l}\text { Quantity: } 1 \\
\text { Weight (in g): } 0.3 \\
\text { Max Length (in mm): } 11 \\
\text { Max Width (in mm): } 12 \\
\text { Max Thickness (mm): } 2 \\
\text { Edge Angle: } 15\end{array}$ & $\begin{array}{l}\text { Flake Attrition: UFU } \\
\text { Crushing: NPR } \\
\text { Smoothing: NPR } \\
\text { Polish: SHL } \\
\text { Etching/Pitting: NPR } \\
\text { Hafting Evidence: NOB }\end{array}$ & CRT & $\begin{array}{l}\text { Point Class: NAP } \\
\text { Point Ratio: } 1.101073 \\
\text { Base/Stem Width: }\end{array}$ & \\
\hline
\end{tabular}


Geo-Marine, Inc.

GMI 22005.01.06.xx and 22005.00.09.xx, Data Recovery Excavations at Site 41PN175 Chipped Stone Tool Data

\begin{tabular}{|c|c|c|c|c|c|c|c|c|c|c|}
\hline Unit & $\begin{array}{c}\text { Depth } \\
(\mathrm{cm})\end{array}$ & $\begin{array}{l}\text { Prov. } \\
\text { No. }\end{array}$ & $\begin{array}{l}\text { Lot } \\
\text { No. }\end{array}$ & Taxonomy & Attributes & Metric Information & Wear Patterning & Raw Material & Projectile Point Data & Comment \\
\hline \multicolumn{11}{|c|}{ Block 4} \\
\hline Unit $105 \mathrm{NW}^{1 / 1}$ & $10-20$ & 1198 & 1098 & $\begin{array}{l}\text { Technology: Chipped Stone } \\
\text { Group: Tool } \\
\text { Subproup: simple detachment-based } \\
\text { Type: } \\
\text { Subtype: Not Applicable }\end{array}$ & $\begin{array}{l}\text { Stage: INR } \\
\text { Portion: PME } \\
\text { I Failure/Discard: ESH } \\
\text { Alteration: NOB } \\
\text { Edge Morphology (distal): STR } \\
\text { Edge Morphology (lett lateral): CVX } \\
\text { Edge Morphology (right lateral): STR } \\
\text { Flake Scar Pattern: IND } \\
\text { Edge Construction Type: UNB } \\
\text { Proximal Edge Grinding: NOB }\end{array}$ & $\begin{array}{l}\text { Quantity: } 1 \\
\text { Weight (in g): } 1.4 \\
\text { Max Length (in mm): } 19 \\
\text { Max Width (in mm): } 17 \\
\text { Max Thickness (mm): } 5 \\
\text { Edge Angle: } 30\end{array}$ & $\begin{array}{l}\text { Flake Attrition: BFB } \\
\text { Crushing: NPR } \\
\text { Smoothing: NPR } \\
\text { Polish: SHL } \\
\text { Etching/Pitting: NPR } \\
\text { Hafting Evidence: NOB }\end{array}$ & CRT & $\begin{array}{l}\text { Point Class: NAP } \\
\text { Point Ratio: 0.9037153 } \\
\text { Base/Stem Width: }\end{array}$ & \\
\hline Unit $118 \mathrm{NE}^{1 / 4}$ & $19-29$ & 1614 & 1392 & $\begin{array}{l}\text { Technology: Chipped Stone } \\
\text { Group: Tool } \\
\text { Subgroup: complex detachment- } \\
\text { based } \\
\text { Type: } \\
\text { Subtype: Unclassified }\end{array}$ & $\begin{array}{l}\text { Stage: FST } \\
\text { Portion: MED } \\
\text { Failure/Discard: BND } \\
\text { Alteration: NOB } \\
\text { Edge Morphology (distal): IND } \\
\text { Edge Morphology (left lateral): STR } \\
\text { Edge Morphology (rightl lateral): STR } \\
\text { Flake Scar Pattern: RDM } \\
\text { Edge Construction Type: BFB } \\
\text { Proximal Edge Grinding: NOB }\end{array}$ & $\begin{array}{l}\text { Quantity: } 1 \\
\text { Weight (in g): } 0.7 \\
\text { Max Length (in mm): } 15 \\
\text { Max Width (in mm): } 15 \\
\text { Max Thickness (mm): } 4 \\
\text { Edge Angle: } 20\end{array}$ & $\begin{array}{l}\text { Flake Attrition: NPR } \\
\text { Crushing: NPR } \\
\text { Smoothing: NPR } \\
\text { Polish: NPR } \\
\text { Etching/Pitting: NPR } \\
\text { Hafting Evidence: NOB }\end{array}$ & QZT & $\begin{array}{l}\text { Point Class: STM } \\
\text { Point Ratio: 1.01178 } \\
\text { Base/Stem Width: } \\
\text { Neck Thickness: } 2.81 \\
\text { Neck Witthl: } 6.49 \\
\text { Base Form: } 0 \\
\text { Stem Form: } 0 \\
\text { Curvature (left): } 3 \\
\text { Curvature (right): } 3 \\
\text { Shoulder (left): } 5 \\
\text { Shoulder (right): } 0 \\
\text { Shoulder Junction: } 1\end{array}$ & $\begin{array}{l}\text { tip and stem are both missing from impact } \\
\text { fractures, corresponding measurements were } \\
\text { not taken }\end{array}$ \\
\hline Unit $120 \mathrm{SE}^{1 / 4}$ & $20-25$ & 1630 & 1430 & $\begin{array}{l}\text { Technology: Chipped Stone } \\
\text { Group: Tool } \\
\text { Subgroup: simple detachment-based } \\
\text { Type: } \\
\text { Subtype: Not Applicable }\end{array}$ & $\begin{array}{l}\text { Stage: INR } \\
\text { Portion: CMP } \\
\text { I Failure/Discard: IND } \\
\text { Alteration: NOB } \\
\text { Edge Morphology (distal): IND } \\
\text { Edge Morphology (lett lateral): STR } \\
\text { Edge Morphology (right lateral): CCV } \\
\text { Flake Scar Pattern: IND } \\
\text { Edge Construction Type: UNU } \\
\text { Proximal Edge Grinding: NOB }\end{array}$ & $\begin{array}{l}\text { Quantity: } 1 \\
\text { Weight (in g): } 1.7 \\
\text { Max Length (in mm): } 20 \\
\text { Max Width (in mm): } 16 \\
\text { Max Thickness (mm): } 8 \\
\text { Edge Angle: } 25\end{array}$ & $\begin{array}{l}\text { Flake Attrition: UFU } \\
\text { Crushing: NPR } \\
\text { Smoothing: NPR } \\
\text { Polish: NPR } \\
\text { Etching/Pitting: NPR } \\
\text { Hafting Evidence: NOB }\end{array}$ & FWD & $\begin{array}{l}\text { Point Class: NAP } \\
\text { Point Ratio: 0.8044776 } \\
\text { Base/Stem Width: }\end{array}$ & Analyzed by Tomka \\
\hline Unit $120 \mathrm{SW}^{1 / 4}$ & $25-30$ & 1631 & 1435 & $\begin{array}{l}\text { Technology: Chipped Stone } \\
\text { Group: Tool } \\
\text { Subgroup: complex detachment- } \\
\text { based } \\
\text { Type: } \\
\text { Subtype: Perdiz/Bassett }\end{array}$ & $\begin{array}{l}\text { Stage: FST } \\
\text { Portion: PME } \\
\text { Failure/Discard: BND } \\
\text { Alteration: NOB } \\
\text { Edge Morphology (distal): IND } \\
\text { Edge Morphology (left lateral): } \\
\text { EdRe Morphology (rightl latral): STR } \\
\text { Elake Mcar Pattern: RDM } \\
\text { Edge Construction Type: BFB } \\
\text { Proximal Edge Grinding: NOB }\end{array}$ & $\begin{array}{l}\text { Quantity: } 1 \\
\text { Weight (in g): } 0.6 \\
\text { Max Length (in mm): } 18 \\
\text { Max Width (in mm): } 15 \\
\text { Max Thickness (mm): } 4 \\
\text { Edge Angle: } 25\end{array}$ & $\begin{array}{l}\text { Flake Attrition: NPR } \\
\text { Crushing: NPR } \\
\text { Smoothing: NPR } \\
\text { Polish: NPR } \\
\text { Etching/Pitting: NPR } \\
\text { Hafting Evidence: OBS }\end{array}$ & QZT & $\begin{array}{l}\text { Point Class: STM } \\
\text { Point Ratio: } 0.8082192 \\
\text { Stem Lengnth: } 4.33 \\
\text { Neck Thickness: } 2.68 \\
\text { Neck Widtht } 3.85 \\
\text { Base to Blade Ratio (length): } 0.2372603 \\
\text { Base to Blade Ratio (width): } 0.1342373 \\
\text { Baseststem ratio: } 3 \\
\text { Base Form: } 5 \\
\text { Stem Form: } 1 \\
\text { Curvature (left): } 3 \\
\text { Curvatuare (right): } 3 \\
\text { Shoulder (left): } 20 \\
\text { Shoulder (right): } 18 \\
\text { Shoulder Junction: } 1\end{array}$ & $\begin{array}{l}\text { tip is missing, corresponding measurements } \\
\text { were not taken }\end{array}$ \\
\hline
\end{tabular}


Geo-Marine, Inc.

GMI 22005.01.06.xx and 22005.00.09.xx, Data Recovery Excavations at Site 41PN175 Chipped Stone Tool Data

\begin{tabular}{|c|c|c|c|c|c|c|c|c|c|c|}
\hline Unit & $\begin{array}{c}\text { Depth } \\
(\mathrm{cm})\end{array}$ & $\begin{array}{l}\text { Prov. } \\
\text { No. }\end{array}$ & $\begin{array}{l}\text { Lot } \\
\text { No. }\end{array}$ & Taxonomy & Attributes & Metric Information & Wear Patterning & Raw Material & Projectile Point Data & Comment \\
\hline \multicolumn{11}{|c|}{ Block 4} \\
\hline Unit $123 \mathrm{NW}^{1 / 1} 4$ & $15-20$ & 1539 & 1491 & $\begin{array}{l}\text { Technology: Chipped Stone } \\
\text { Group: Tool } \\
\text { Subproup: simple detachment-based } \\
\text { Type: } \\
\text { Subtype: Not Applicable }\end{array}$ & $\begin{array}{l}\text { Stage: INR } \\
\text { Portion: CMP } \\
\text { ' Failure/Discard: IND } \\
\text { Alteration: NOB } \\
\text { Edge Morphology (distal): IND } \\
\text { Edge Morphologg (left lateral): CCV } \\
\text { Edge Morphology (right lateral): STR } \\
\text { Flake Scar Pattern: IND } \\
\text { Edge Construction Type: UNU } \\
\text { Proximal Edge Grinding: NOB }\end{array}$ & $\begin{array}{l}\text { Quantity: } 1 \\
\text { Weight (in g): } 1 \\
\text { Max Length (in mm): } 19 \\
\text { Max Width (in mm): } 21 \\
\text { Max Thickness (mm): } 4 \\
\text { Edge Angle: } 30\end{array}$ & $\begin{array}{l}\text { Flake Attrition: BFU } \\
\text { Crushing: NPR } \\
\text { Smoothing: NPR } \\
\text { Polish: SHL } \\
\text { Etching/Pitting: NPR } \\
\text { Hafting Evidence: NOB }\end{array}$ & CRT & $\begin{array}{l}\text { Point Class: NAP } \\
\text { Point Ratio: 1.128495 } \\
\text { Base/Stem Width: }\end{array}$ & Analyzed by Tomka \\
\hline Unit $123 \mathrm{NE}^{1 / 4}$ & $30-35$ & 1661 & 1503 & $\begin{array}{l}\text { Technology: Chipped Stone } \\
\text { Group: Tool } \\
\text { Subgroup: complex detachment- } \\
\text { based } \\
\text { Type: } \\
\text { Subtype: Unclassified }\end{array}$ & $\begin{array}{l}\text { Stage: FST } \\
\text { Portion: BSH } \\
\text { Failure/Discard: BND } \\
\text { Alteration: NOB } \\
\text { Edge Morphology (distal): IND } \\
\text { Edge Morphology (left lateral): IND } \\
\text { Edge Morphology (right latal): IND } \\
\text { Flake Scar antern: RDM } \\
\text { Edge Construtction Type: IND } \\
\text { Proximal Edge Grinding: NOB }\end{array}$ & $\begin{array}{l}\text { Quantity: } 1 \\
\text { Weight (in g): } 0.6 \\
\text { Max Length (in mm): } 17 \\
\text { Max Width (in mm): } 9 \\
\text { Max Thickness (mm): } 5 \\
\text { Edge Angle: } 30\end{array}$ & $\begin{array}{l}\text { Flake Attrition: NPR } \\
\text { Crushing: NPR } \\
\text { Smoothing: NPR } \\
\text { Polish: NPR } \\
\text { Etching/Pitting: NPR } \\
\text { Hafting Evidence: NOB }\end{array}$ & CRT & $\begin{array}{l}\text { Point Class: STM } \\
\text { Point Ratio: 0.5167064 } \\
\text { BaseS/Stem Width: } \\
\text { Shoulder Junction: } 1\end{array}$ & $\begin{array}{l}\text { only the left shoulder and a small portion of the } \\
\text { base are present, corresponding measurements } \\
\text { were not taken }\end{array}$ \\
\hline Unit $125 \mathrm{NW}^{1 / 4}$ & $15-20$ & 1567 & 1551 & $\begin{array}{l}\text { Technology: Chipped Stone } \\
\text { Group: Tool } \\
\text { Subgroup: complex detachment- } \\
\text { based } \\
\text { Type: } \\
\text { Subtype: Perdiz }\end{array}$ & 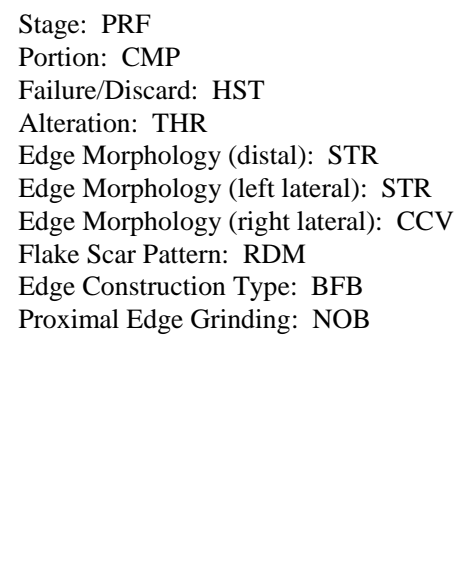 & $\begin{array}{l}\text { Quantity: } 1 \\
\text { Weight (in g): } 0.7 \\
\text { Max Length (in mm): } 20 \\
\text { Max Width (in mm): } 13 \\
\text { Max Thickness (mm): } 5 \\
\text { Edge Angle: } 30\end{array}$ & $\begin{array}{l}\text { Flake Attrition: NPR } \\
\text { Crushing: NPR } \\
\text { Smoothing: NPR } \\
\text { Polish: NPR } \\
\text { Etching/Pitting: NPR } \\
\text { Hafting Evidence: NOB }\end{array}$ & QZT & $\begin{array}{l}\text { Point Class: STM } \\
\text { Point Ratio: } 0.6527638 \\
\text { Bladede Lengnth (left): } 1.16 \\
\text { Blade Length (right): } 14.63 \\
\text { Stem Length: } 5.95 \\
\text { Neck Thickness: } 2.84 \\
\text { Neck Widthlt } 4.91 \\
\text { Base to Blade Ratio (length): } 0.298995 \\
\text { Base to Blade Ratio (width): } 0.2709777 \\
\text { Baselstem ratio: } 3 \\
\text { Base Form: } 1 \\
\text { Stem Form: } 1 \\
\text { Curvature (left): } 3 \\
\text { Curvature (right): } 4 \\
\text { Shoulder (left): } 0 \\
\text { Shoulder (right): } 0 \\
\text { Shoulder Junction: } 2\end{array}$ & \\
\hline Unit $126 \mathrm{NE}^{1 / 4}$ & $0-12$ & 1692 & 1568 & $\begin{array}{l}\text { Technology: Chipped Stone } \\
\text { Group: Tool } \\
\text { Subgroup: simple detachment-based } \\
\text { Type: } \\
\text { Subtype: Not Applicable }\end{array}$ & $\begin{array}{l}\text { Stage: INR } \\
\text { Portion: CMP } \\
\text { I Failure/Discard: IND } \\
\text { Alteration: NOB } \\
\text { Edge Morphology (distal): IND } \\
\text { Edge Morphology (left lateral): } \\
\text { ETR } \\
\text { Ege Morphology (rithht lateral): STR } \\
\text { Flake Scar Pattern: IND } \\
\text { Ege Construction Type: UNC } \\
\text { Proximal Edge Grinding: NOB }\end{array}$ & $\begin{array}{l}\text { Quantity: } 1 \\
\text { Weight (in g): } 0.8 \\
\text { Max Length (in mm): } 19 \\
\text { Max Width (in mm): } 14 \\
\text { Max Thickness (mm): } 4 \\
\text { Edge Angle: } 20\end{array}$ & $\begin{array}{l}\text { Flake Attrition: UFC } \\
\text { Crushing: NPR } \\
\text { Smoothing: NPR } \\
\text { Polish: SHL } \\
\text { Etching/Pitting: NPR } \\
\text { Hafting Evidence: NOB }\end{array}$ & CRT & $\begin{array}{l}\text { Point Class: NAP } \\
\text { Point Ratio: 0.7153135 } \\
\text { Base/Stem Width: }\end{array}$ & Analyzed by Tomka \\
\hline
\end{tabular}


Geo-Marine, Inc.

GMI 22005.01.06.xx and 22005.00.09.xx, Data Recovery Excavations at Site 41PN175 Chipped Stone Tool Data

\begin{tabular}{|c|c|c|c|c|c|c|c|c|c|c|}
\hline Unit & $\begin{array}{c}\text { Depth } \\
(\mathrm{cm})\end{array}$ & $\begin{array}{l}\text { Prov. } \\
\text { No. }\end{array}$ & $\begin{array}{l}\text { Lot } \\
\text { No. }\end{array}$ & Taxonomy & Attributes & Metric Information & Wear Patterning & Raw Material & Projectile Point Data & Comment \\
\hline \multicolumn{11}{|c|}{ Block 4} \\
\hline Unit $127 \mathrm{NW}^{1 / 4}$ & $21-31$ & 1711 & 1593 & $\begin{array}{l}\text { Technology: Chipped Stone } \\
\text { Group: Tool } \\
\text { Subgroup: complex detachment- } \\
\text { based } \\
\text { Type: } \\
\text { Subtype: Perdiz }\end{array}$ & $\begin{array}{l}\text { Stage: FST } \\
\text { Portion: PME } \\
\text { FailurelDiscard: BND } \\
\text { Alteration: NOB } \\
\text { Edge Morphology (distal): IND } \\
\text { Edge Morphologg (lett laterl): STR } \\
\text { Edge Morphology (right lateral): STR } \\
\text { Flake Scar antern: RDM } \\
\text { Edge Construction Type: BFB } \\
\text { Proximal Edge Grinding: NOB }\end{array}$ & $\begin{array}{l}\text { Quantity: } 1 \\
\text { Weight (in g): } 0.5 \\
\text { Max Length (in mm): } 15 \\
\text { Max Width (in mm): } 13 \\
\text { Max Thickness (mm): } 3 \\
\text { Edge Angle: } 25\end{array}$ & $\begin{array}{l}\text { Flake Attrition: NPR } \\
\text { Crushing: NPR } \\
\text { Smoothing: NPR } \\
\text { Polish: NPR } \\
\text { Etching/Pitting: NPR } \\
\text { Hafting Evidence: NOB }\end{array}$ & CRT & $\begin{array}{l}\text { Point Class: STM } \\
\text { Point Ratio: } 0.8327847 \\
\text { Stem Length: } 5.85 \\
\text { Neck Thickness: } 2.44 \\
\text { Neck Wicthhs } 4.16 \\
\text { Base to Blade Ratio (length): } 0.3851218 \\
\text { Base to Blade Ratio (width): } 0.1928854 \\
\text { Basestsem ratio: } 3 \\
\text { Base Form: } 1 \text {. } \\
\text { Stem Form: } 1 \\
\text { Curvature (left): } 3 \\
\text { Curvature (right): } 3 \\
\text { Shoulder (left): } 11 \\
\text { Shoulder (right): } 10 \\
\text { Shoulder Junction: } 1\end{array}$ & $\begin{array}{l}\text { tip missing from impact fracture, corresponding } \\
\text { measurments not taken }\end{array}$ \\
\hline Unit $127 \mathrm{NW}^{1 / 4}$ & $21-31$ & 1711 & 1593 & $\begin{array}{l}\text { Technology: Chipped Stone } \\
\text { Group: Tool } \\
\text { Subgroup: complex detachment- } \\
\text { based } \\
\text { Type: } \\
\text { Subtype: Perdiz/Bassett }\end{array}$ & $\begin{array}{l}\text { Stage: FST } \\
\text { Portion: CMP } \\
\text { Failure/Discard: IND } \\
\text { Alteration: NOB } \\
\text { Edge Morphology (distal): STR } \\
\text { Edge Morphologg (left lateral): STR } \\
\text { Edge Morphology (right lateral): STR } \\
\text { Flake Scar a aterer: RDM RD } \\
\text { Edge Construction Type: BFB } \\
\text { Proximal Edge Grinding: NOB }\end{array}$ & $\begin{array}{l}\text { Quantity: } 1 \\
\text { Weight (in g): } 0.6 \\
\text { Max Length (in mm): } 21 \\
\text { Max Width (in mm): } 12 \\
\text { Max Thickness (mm): } 4 \\
\text { Edge Angle: } 20\end{array}$ & $\begin{array}{l}\text { Flake Attrition: NPR } \\
\text { Crushing: NPR } \\
\text { Smoothing: NPR } \\
\text { Polish: SHD } \\
\text { Etching/Pitting: NPR } \\
\text { Hafting Evidence: OBS }\end{array}$ & FWD & $\begin{array}{l}\text { Point Class: STM } \\
\text { Point Ratio: } 0.5786705 \\
\text { Blade Lengnth (left): } 19.45 \\
\text { Blade Length (right): } 15.89 \\
\text { Stem Lenggth: } 3.59 \\
\text { Neck Thickness: } 2.48 \\
\text { Neck Widthl. } 3.85 \\
\text { Base to Blade Ratio (length): } 0.1716882 \\
\text { Base to Blade Ratio (width): } 0.1206612 \\
\text { Baselstem ratio: } 3 \\
\text { Base Form: } 5 \\
\text { Stem Form: } 1 \\
\text { Curvature (left): } 3 \\
\text { Curvature (right): } 3 \\
\text { Shoulder (left): } 6 \\
\text { Shoulder (right): } 0 \\
\text { Shoulder Junction: } 1\end{array}$ & $\begin{array}{l}\text { the right shoulder and stem are oddly formed, } \\
\text { likely the result of reworking }\end{array}$ \\
\hline Unit $127 \mathrm{SW}^{1 / 4}$ & $21-31$ & 1710 & 1595 & $\begin{array}{l}\text { Technology: Chipped Stone } \\
\text { Group: Tool } \\
\text { Subgroup: simple detachment-based } \\
\text { Type: } \\
\text { Subtype: Not Applicable }\end{array}$ & $\begin{array}{l}\text { Stage: INR } \\
\text { Portion: CMP } \\
\text { FailurelDiscard: IND } \\
\text { Alteration: NOB } \\
\text { Edge Morphology (distal): IND } \\
\text { Edge Morphology (left lateral): } \\
\text { EdRe } \\
\text { Elge Morphology (rithht lateral): IND } \\
\text { Flade Scar Pattern: IND } \\
\text { Edge Construtucion Type: UNU } \\
\text { Proximal Edge Grinding: NOB }\end{array}$ & $\begin{array}{l}\text { Quantity: } 1 \\
\text { Weight (in g): } 0.7 \\
\text { Max Length (in mm): } 24 \\
\text { Max Width (in mm): } 11 \\
\text { Max Thickness (mm): } 4 \\
\text { Edge Angle: } 20\end{array}$ & $\begin{array}{l}\text { Flake Attrition: UFU } \\
\text { Crushing: NPR } \\
\text { Smoothing: NPR } \\
\text { Polish: NPR } \\
\text { Etching/Pitting: NPR } \\
\text { Hafting Evidence: NOB }\end{array}$ & QZT & $\begin{array}{l}\text { Point Class: NAP } \\
\text { Point Ratio: 0.450211 } \\
\text { Base/Stem Width: }\end{array}$ & \\
\hline
\end{tabular}


Geo-Marine, Inc.

GMI 22005.01.06.xx and 22005.00.09.xx, Data Recovery Excavations at Site 41PN175 Chipped Stone Tool Dat

\begin{tabular}{|c|c|c|c|c|c|c|c|c|c|c|}
\hline Unit & $\begin{array}{l}\text { Depth } \\
(\mathrm{cm})\end{array}$ & $\begin{array}{l}\text { Prov. } \\
\text { No. }\end{array}$ & $\begin{array}{l}\text { Lot } \\
\text { No. }\end{array}$ & Taxonomy & Attributes & Metric Information & Wear Patterning & Raw Material & Projectile Point Data & Comment \\
\hline \multicolumn{11}{|l|}{ Block 4} \\
\hline $\begin{array}{l}\text { Unit 087, Feature } \\
7 \mathrm{SW} \mathrm{S}^{1 / 2}\end{array}$ & $40-53$ & 1802 & 1604 & $\begin{array}{l}\text { Technology: Chipped Stone } \\
\text { Group: Tool } \\
\text { Subgroup: complex detachment- } \\
\text { based } \\
\text { Type: } \\
\text { Subtype: Bonham }\end{array}$ & $\begin{array}{l}\text { Stage: FST } \\
\text { Portion: CMP } \\
\text { Failure/Discard: IND } \\
\text { Alteration: NOB } \\
\text { Edge Morphology (distal): CVX } \\
\text { Edge Morphology (left lateral): CCV } \\
\text { Edge Morphology (right latal) CCV } \\
\text { Flake Scar Pattern: RDM } \\
\text { Edge Construtucion Type: BFB } \\
\text { Proximal Edge Grinding: NOB }\end{array}$ & $\begin{array}{l}\text { Quantity: } 1 \\
\text { Weight (in g): } 0.8 \\
\text { Max Length (in mm): } 18 \\
\text { Max Width (in mm): } 13 \\
\text { Max Thickness (mm): } 5 \\
\text { Edge Angle: } 25\end{array}$ & $\begin{array}{l}\text { Flake Attrition: NPR } \\
\text { Crushing: NPR } \\
\text { Smoothing: NPR } \\
\text { Polish: SHD } \\
\text { Etching/Pitting: NPR } \\
\text { Hafting Evidence: OBS }\end{array}$ & CRT & 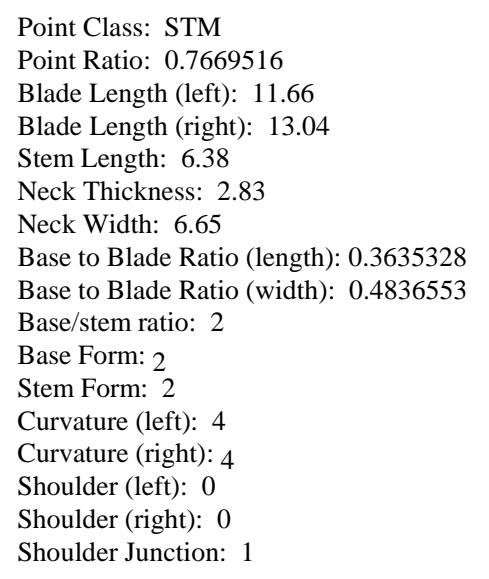 & \\
\hline \multicolumn{11}{|l|}{ Block 5} \\
\hline Unit $080 \mathrm{SW}^{1 / 4}$ & $40-50$ & 779 & 624 & $\begin{array}{l}\text { Technology: Chipped Stone } \\
\text { Group: Tool } \\
\text { Subgroup: complex detachment- } \\
\text { based } \\
\text { Type: } \\
\text { Subtype: Perdiz }\end{array}$ & $\begin{array}{l}\text { Stage: FST } \\
\text { Portion: CMP } \\
\text { Failure/Discard: IND } \\
\text { Alteration: NOB } \\
\text { Edge Morphology (distal): STR } \\
\text { Edge Morphology (left lateral): STR } \\
\text { Edge Morphology (rightl latarl): STR } \\
\text { Flake Scar Pattern: RDM } \\
\text { Edge Construtcion Type: UNB } \\
\text { Proximal Edge Grinding: NOB }\end{array}$ & $\begin{array}{l}\text { Quantity: } 1 \\
\text { Weight (in g): } 0.4 \\
\text { Max Length (in mm): } 17 \\
\text { Max Width (in mm): } 10 \\
\text { Max Thickness (mm): } 2 \\
\text { Edge Angle: } 25\end{array}$ & $\begin{array}{l}\text { Flake Attrition: BFD } \\
\text { Crushing: NPR } \\
\text { Smoothing: NPR } \\
\text { Polish: NPR } \\
\text { Etching//Pitting: NPR } \\
\text { Hafting Evidence: NOB }\end{array}$ & QZT & $\begin{array}{l}\text { Point Class: STM } \\
\text { Point Ratio: } 0.5816092 \\
\text { Blade Length (left): } 14.08 \\
\text { Blade Length (right): } 13.69 \\
\text { Stem Length: } 5.65 \\
\text { Neck Thickness: } 2.11 \\
\text { Neck Width: } 4.09 \\
\text { Base to Blade Ratio (length): } 0.3247127 \\
\text { Base to Blade Ratio (width): } 0.2835968 \\
\text { Bases/stem ratio: } 3 \\
\text { Base Formm: } 2 \\
\text { Stem Form: } 1 \\
\text { Curvature (left): } 3 \\
\text { Curvature (right): } 3 \\
\text { Shoulder (left): } 8 \\
\text { Shoulder (right): } 5 \\
\text { Shoulder Junction: } 2\end{array}$ & \\
\hline Unit $080 \mathrm{SW}^{1 / 4}$ & $40-50$ & 779 & 624 & $\begin{array}{l}\text { Technology: Chipped Stone } \\
\text { Group: Tool } \\
\text { Subgroup: complex detachment- } \\
\text { based } \\
\text { Type: } \\
\text { Subtype: Perdiz }\end{array}$ & $\begin{array}{l}\text { Stage: FST } \\
\text { Portion: PRX } \\
\text { Fialureliscard: PRV } \\
\text { Alteration: NOB } \\
\text { Edge Morphology (distal): IND } \\
\text { Edge Morphology (left lateral): IND } \\
\text { Edge Morphology (right latarl): IND } \\
\text { Flake Scar Pattern: RDM } \\
\text { Edge Construction Type: BFB } \\
\text { Proximal Edge Grinding: NOB }\end{array}$ & $\begin{array}{l}\text { Quantity: } 1 \\
\text { Weight (in g): } 0.5 \\
\text { Max Length (in mm): } 13 \\
\text { Max Width (in mm): } 17 \\
\text { Max Thickness (mm): } 3 \\
\text { Edge Angle: } 20\end{array}$ & $\begin{array}{l}\text { Flake Attrition: NPR } \\
\text { Crushing: NPR } \\
\text { Smoothing: NPR } \\
\text { Polish: NPR } \\
\text { Etching/Pitting: NPR } \\
\text { Hafting Evidence: NOB }\end{array}$ & CRT & $\begin{array}{l}\text { Point Class: STM } \\
\text { Point Ratio: } 1.273739 \\
\text { Stem Length: } 4.58 \\
\text { Neck Thickness: } 3.05 \\
\text { Neck Widtht } 5.61 \\
\text { Base to Blade Ratio (length): } 0.3397626 \\
\text { Base to Blade Ratio (width): } 0.2329645 \\
\text { Baseststem ratio: } 2 \\
\text { Base Form: } 0 \\
\text { Stem Form: } 1 \\
\text { Curvature (left): }: 0 \\
\text { Curvature (right): } 0 \\
\text { Shoulder (left): } 20 \\
\text { Shoulder (right): } 22 \\
\text { Shoulder Junction: } 1\end{array}$ & $\begin{array}{l}\text { tip missing, corresponding measurements not } \\
\text { made }\end{array}$ \\
\hline
\end{tabular}


Geo-Marine, Inc.

GMI 22005.01.06.xx and 22005.00.09.xx, Data Recovery Excavations at Site 41PN175 Chipped Stone Tool Dat

\begin{tabular}{|c|c|c|c|c|c|c|c|c|c|}
\hline Unit & $\begin{array}{l}\text { Depth } \\
(\mathrm{cm})\end{array}$ & $\begin{array}{l}\text { Prov. } \\
\text { No. }\end{array}$ & $\begin{array}{l}\text { Lot } \\
\text { No. }\end{array}$ & Taxonomy & Attributes & Metric Information & Wear Patterning & Raw Material & Projectile Point Data \\
\hline \multicolumn{10}{|c|}{ Block 5} \\
\hline Unit $081 \mathrm{SE}^{1 / 4}$ & $40-50$ & 827 & 644 & $\begin{array}{l}\text { Technology: Chipped Stone } \\
\text { Group: Tool } \\
\text { Subgroup: complex detachment- } \\
\text { based } \\
\text { Type: } \\
\text { Subtype: Not Applicable }\end{array}$ & $\begin{array}{l}\text { Stage: INR } \\
\text { Portion: PRX } \\
\text { Failure/Discard: PRV } \\
\text { Alteration: NOB } \\
\text { Edge Morphology (distal): IND } \\
\text { Edge Morphology (left lateral): } \\
\text { ERR } \\
\text { Edge Morphology (right lateral): IND } \\
\text { Flake Scar Pattern: RDM } \\
\text { Edge Construction Type: BFU } \\
\text { Proximal Edge Grinding: NOB }\end{array}$ & $\begin{array}{l}\text { Quantity: } 1 \\
\text { Weight (in g): } 2.9 \\
\text { Max Length (in mm): } 21 \\
\text { Max Width (in mm): } 15 \\
\text { Max Thickness (mm): } 9 \\
\text { Edge Angle: } 45\end{array}$ & $\begin{array}{l}\text { Flake Attrition: NPR } \\
\text { Crushing: NPR } \\
\text { Smoothing: NPR } \\
\text { Polish: NPR } \\
\text { Etching/Pitting: NPR } \\
\text { Hafting Evidence: NOB }\end{array}$ & CRT & $\begin{array}{l}\text { Point Class: NAP } \\
\text { Point Ratio: } 0.6828132 \\
\text { Base/Stem Width: }\end{array}$ \\
\hline \multicolumn{10}{|c|}{ Block 7} \\
\hline Unit $110 \mathrm{NW}^{1 / 4}$ & $20-30$ & 911 & 1185 & $\begin{array}{l}\text { Technology: Chipped Stone } \\
\text { Group: Tool } \\
\text { Subgroup: simple detachment-based } \\
\text { Type: } \\
\text { Subtype: Not Applicable }\end{array}$ & $\begin{array}{l}\text { Stage: REJ } \\
\text { Portion: CMP } \\
\text { l Failure/Discard: PLL } \\
\text { Alteration: NOB } \\
\text { Edge Morphology (distal): STR } \\
\text { Edge Morphology (left lateral): } \\
\text { EdgD } \\
\text { Edge Morphology (rithlt lateral): STR } \\
\text { Flake Scar Pattern: RDM } \\
\text { Edge Construtcion Type: BFD } \\
\text { Proximal Edge Grinding: NOB }\end{array}$ & $\begin{array}{l}\text { Quantity: } 1 \\
\text { Weight (in g): } 1.4 \\
\text { Max Length (in mm): } 22 \\
\text { Max Width (in mm): } 15 \\
\text { Max Thickness (mm): } 4 \\
\text { Edge Angle: } 20\end{array}$ & $\begin{array}{l}\text { Flake Attrition: NPR } \\
\text { Crushing: NPR } \\
\text { Smoothing: NPR } \\
\text { Polish: NPR } \\
\text { Etching/Pitting: NPR } \\
\text { Hafting Evidence: NOB }\end{array}$ & CRT & $\begin{array}{l}\text { Point Class: NAP } \\
\text { Point Ratio: 0.6806839 } \\
\text { Base/Stem Width: }\end{array}$ \\
\hline Unit $111 \mathrm{NW}^{1 / 4}$ & $0-10$ & 915 & 1193 & $\begin{array}{l}\text { Technology: Chipped Stone } \\
\text { Group: Tool } \\
\text { Subgroup: simple detachment-based } \\
\text { Type: } \\
\text { Subtype: Not Applicable }\end{array}$ & 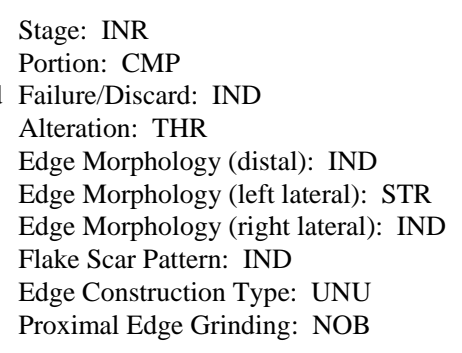 & $\begin{array}{l}\text { Quantity: } 1 \\
\text { Weight (in g): } 1.3 \\
\text { Max Length (in mm): } 24 \\
\text { Max Width (in mm): } 12 \\
\text { Max Thickness (mm): } 5 \\
\text { Edge Angle: } 30\end{array}$ & $\begin{array}{l}\text { Flake Attrition: UFU } \\
\text { Crushing: NPR } \\
\text { Smoothing: NPR } \\
\text { Polish: SHL } \\
\text { Etching/Pitting: NPR } \\
\text { Hafting Evidence: NOB }\end{array}$ & CRT & $\begin{array}{l}\text { Point Class: NAP } \\
\text { Point Ratio: } 0.4757999 \\
\text { Base/Stem Width: }\end{array}$ \\
\hline Unit $111 \mathrm{NW}^{1 / 4}$ & $10-20$ & 918 & 1196 & $\begin{array}{l}\text { Technology: Chipped Stone } \\
\text { Group: Tool } \\
\text { Subgroup: simple detachment-based } \\
\text { Type: } \\
\text { Subtype: Not Applicable }\end{array}$ & $\begin{array}{l}\text { Stage: INR } \\
\text { Portion: CMP } \\
\text { l Failure/Discard: IND } \\
\text { Alteration: THR } \\
\text { Edge Morphology (distal): STR } \\
\text { Edge Morphology (left lateral): } \\
\text { Edge Morphology } \\
\text { Elake Mcart tateral): IND } \\
\text { Flake Scartern: IND } \\
\text { Edge Construction Type: UFD } \\
\text { Proximal Edge Grinding: NOB }\end{array}$ & $\begin{array}{l}\text { Quantity: } 1 \\
\text { Weight (in g): } 0.6 \\
\text { Max Length (in mm): } 15 \\
\text { Max Width (in mm): } 14 \\
\text { Max Thickness (mm): } 3 \\
\text { Edge Angle: } 15\end{array}$ & $\begin{array}{l}\text { Flake Attrition: UFD } \\
\text { Crushing: NPR } \\
\text { Smoothing: DIS } \\
\text { Polish: SHD } \\
\text { Etching/Pitting: NPR } \\
\text { Hafting Evidence: NOB }\end{array}$ & CRT & $\begin{array}{l}\text { Point Class: NAP } \\
\text { Point Ratio: } 0.931479 \\
\text { Base/Stem Width: }\end{array}$ \\
\hline
\end{tabular}


Geo-Marine, Inc.

GMI 22005.01.06.xx and 22005.00.09.xx, Data Recovery Excavations at Site 41PN175 Chipped Stone Tool Dat

\begin{tabular}{|c|c|c|c|c|c|c|c|c|c|c|}
\hline Unit & $\begin{array}{l}\text { Depth } \\
(\mathrm{cm})\end{array}$ & $\begin{array}{l}\text { Prov. } \\
\text { No. }\end{array}$ & $\begin{array}{l}\text { Lot } \\
\text { No. }\end{array}$ & Taxonomy & Attributes & Metric Information & Wear Patterning & Raw Material & Projectile Point Data & Comment \\
\hline \multicolumn{11}{|c|}{ Block 7} \\
\hline Unit $111 \mathrm{NE}^{1} / 4$ & $50-60$ & 996 & 1215 & $\begin{array}{l}\text { Technology: Chipped Stone } \\
\text { Group: Tool } \\
\text { Subgroup: simple detachment-based } \\
\text { Type: } \\
\text { Subtype: Not Applicable }\end{array}$ & 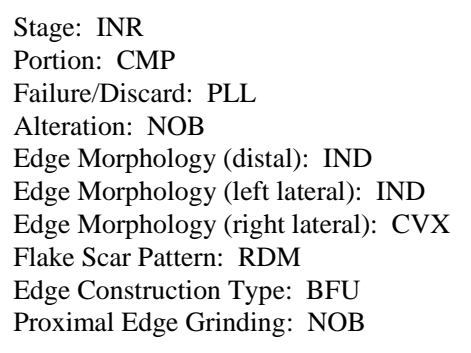 & $\begin{array}{l}\text { Quantity: } 1 \\
\text { Weight (in g): } 2 \\
\text { Max Length (in mm): } 22 \\
\text { Max Width (in mm): } 18 \\
\text { Max Thickness (mm): } 5 \\
\text { Edge Angle: } 30\end{array}$ & $\begin{array}{l}\text { Flake Attrition: BFU } \\
\text { Crushing: NPR } \\
\text { Smoothing: NPR } \\
\text { Polish: SHL } \\
\text { Etching/Pitting: NPR } \\
\text { Hafting Evidence: NOB }\end{array}$ & CRT & $\begin{array}{l}\text { Point Class: NAP } \\
\text { Point Ratio: 0.8029622 } \\
\text { Base/Stem Width: }\end{array}$ & \\
\hline Unit $111 \mathrm{SW}^{1 / 4}$ & $60-70$ & 1047 & 1220 & $\begin{array}{l}\text { Technology: Chipped Stone } \\
\text { Group: Tool } \\
\text { Subgroup: complex detachment- } \\
\text { based } \\
\text { Type: } \\
\text { Subtype: Unclassified }\end{array}$ & $\begin{array}{l}\text { Stage: FST } \\
\text { Portion: STM } \\
\text { Failure/Discard: BND } \\
\text { Alteration: NOB } \\
\text { Edge Morphology (distal): IND } \\
\text { Edge Morhhology (elt taterl): IND } \\
\text { Edge Morphology (right latel): IND } \\
\text { Flake SCar Pattern: IND } \\
\text { Edge Construction Type: BFC } \\
\text { Proximal Edge Grinding: NOB }\end{array}$ & $\begin{array}{l}\text { Quantity: } 1 \\
\text { Weight (in g): } 1.2 \\
\text { Max Length (in mm): } 12 \\
\text { Max Width (in mm): } 21 \\
\text { Max Thickness (mm): } 5 \\
\text { Edge Angle: } 25\end{array}$ & $\begin{array}{l}\text { Flake Attrition: NPR } \\
\text { Crushing: NPR } \\
\text { Smoothing: NPR } \\
\text { Polish: NPR } \\
\text { Etching/Pitting: NPR } \\
\text { Hafting Evidence: OBS }\end{array}$ & JPR & $\begin{array}{l}\text { Point Class: STM } \\
\text { Point Ratio: } 1.855903 \\
\text { Stem Length: } 11.52 \\
\text { Neck Thickness: } 5.18 \\
\text { Neck Widthe: } 14.56 \\
\text { Base to Blade Ratio (length): } 1 \\
\text { Base to Blade Ratio (width): } 1 \\
\text { Baselstem ratio: } 1 \\
\text { Base Form: } 1 \\
\text { Stem Form: } 3 \\
\text { Curvature (left): } 0 \\
\text { Curvature (right): } 0 \\
\text { Shoulder Junction: } 0\end{array}$ & $\begin{array}{l}\text { Archaic expanding stem, likely Ensor or other } \\
\text { subtype }\end{array}$ \\
\hline \multicolumn{11}{|c|}{ Block 8} \\
\hline Unit $114 \mathrm{SE}^{1 / 4}$ & $30-40$ & 1070 & 1285 & $\begin{array}{l}\text { Technology: Chipped Stone } \\
\text { Group: Tool } \\
\text { Subgroup: complex detachment- } \\
\text { based } \\
\text { Type: } \\
\text { Subtype: Not Applicable }\end{array}$ & $\begin{array}{l}\text { Stage: INR } \\
\text { Portion: CMP } \\
\text { Failure/Discard: IND } \\
\text { Alteration: NOB } \\
\text { Edge Morphology (distal): STR } \\
\text { Edge Morhhology (eft lateral): STR } \\
\text { Edge Morphology (right lateral): STR } \\
\text { Flake Scar Pattern: RDM } \\
\text { Edge Construction Type: BDB } \\
\text { Proximal Edge Grinding: NOB }\end{array}$ & $\begin{array}{l}\text { Quantity: } 1 \\
\text { Weight (in g): } 1.2 \\
\text { Max Length (in mm): } 19 \\
\text { Max Width (in mm): } 15 \\
\text { Max Thickness (mm): } 4 \\
\text { Edge Angle: } 25\end{array}$ & $\begin{array}{l}\text { Flake Attrition: BFU } \\
\text { Crushing: NPR } \\
\text { Smoothing: NPR } \\
\text { Polish: NPR } \\
\text { Etching/Pitting: NPR } \\
\text { Hafting Evidence: NOB }\end{array}$ & CRT & $\begin{array}{l}\text { Point Class: NAP } \\
\text { Point Ratio: 0.7887771 } \\
\text { Base/Stem Width: }\end{array}$ & \\
\hline Unit $115 \mathrm{SW}^{1 / 4}$ & $50-60$ & 1218 & 1323 & $\begin{array}{l}\text { Technology: Chipped Stone } \\
\text { Group: Tool } \\
\text { Subgroup: simple detachment-based } \\
\text { Type: } \\
\text { Subtype: Not Applicable }\end{array}$ & $\begin{array}{l}\text { Stage: INR } \\
\text { Portion: CMP } \\
\text { Failure/Discard: IND } \\
\text { Alteration: NOB } \\
\text { Edge Morphology (distal): STR } \\
\text { Edge Morphology (elf lateral): STR } \\
\text { Edge Morphology (right latelal): STR } \\
\text { Flake Scar Pattern: RDM } \\
\text { Edge Construction Type: UNB } \\
\text { Proximal Edge Grinding: NOB }\end{array}$ & $\begin{array}{l}\text { Quantity: } 1 \\
\text { Weight (in g): } 1.8 \\
\text { Max Length (in mm): } 22 \\
\text { Max Width (in mm): } 15 \\
\text { Max Thickness (mm): } 5 \\
\text { Edge Angle: } 30\end{array}$ & $\begin{array}{l}\text { Flake Attrition: NPR } \\
\text { Crushing: NPR } \\
\text { Smoothing: NPR } \\
\text { Polish: NPR } \\
\text { Etching/Pitting: NPR } \\
\text { Hafting Evidence: NOB }\end{array}$ & QZT & $\begin{array}{l}\text { Point Class: NAP } \\
\text { Point Ratio: 0.6784566 } \\
\text { Base/Stem Width: }\end{array}$ & \\
\hline
\end{tabular}


Geo-Marine, Inc.

GMI 22005.01.06.xx and 22005.00.09.xx, Data Recovery Excavations at Site 41PN175 Chipped Stone Tool Dat

\begin{tabular}{|c|c|c|c|c|c|c|c|c|c|c|}
\hline Unit & $\begin{array}{l}\text { Depth } \\
(\mathrm{cm})\end{array}$ & $\begin{array}{c}\text { Prov. } \\
\text { No. }\end{array}$ & $\begin{array}{l}\text { Lot } \\
\text { No. }\end{array}$ & Taxonomy & Attributes & Metric Information & Wear Patterning & Raw Material & Projectile Point Data & Comment \\
\hline \multicolumn{11}{|c|}{ Block 8} \\
\hline Unit $116 \mathrm{NW}^{1 / 4}$ & $50-61$ & 1227 & 1343 & $\begin{array}{l}\text { Technology: Chipped Stone } \\
\text { Group: Tool } \\
\text { Subgroup: complex detachment- } \\
\text { based } \\
\text { Type: } \\
\text { Subtype: Perdiz }\end{array}$ & $\begin{array}{l}\text { Stage: PRF } \\
\text { Portion: CMP } \\
\text { Failure/Discard: HST } \\
\text { Alteration: NOB } \\
\text { Edge Morphology (distal): CVX } \\
\text { Edge Morphology (left lateral): STR } \\
\text { Edge Morphology (rightl latral): STR } \\
\text { Flake Scar Pattern: RDM } \\
\text { Edge Construction Type: BFB } \\
\text { Proximal Edge Grinding: NOB }\end{array}$ & $\begin{array}{l}\text { Quantity: } 1 \\
\text { Weight (in g): } 0.6 \\
\text { Max Length (in mm): } 17 \\
\text { Max Width (in mm): } 13 \\
\text { Max Thickness (mm): } 3 \\
\text { Edge Angle: } 25\end{array}$ & $\begin{array}{l}\text { Flake Attrition: NPR } \\
\text { Crushing: NPR } \\
\text { Smoothing: NPR } \\
\text { Polish: NPR } \\
\text { Etching/Pitting: NPR } \\
\text { Hafting Evidence: NOB }\end{array}$ & FWD & $\begin{array}{l}\text { Point Class: STM } \\
\text { Point Ratio: } 0.776285 \\
\text { Bladede Lenghth (left): } 12.92 \\
\text { Blade Length (right): } 13.37 \\
\text { Stem Length: } 4.2 \text {. } \\
\text { Neck Thickness: } 2.47 \\
\text { Neck Widthl. } 5.23 \\
\text { Base to Blade Ratio (length): } 0.2453271 \\
\text { Base to Blade Ratio (width): } 0.1948834 \\
\text { Baselstem ratio: } 3 \\
\text { Base Form: } 1 \\
\text { Stem Form: } 1 \\
\text { Curvature (left): } 3 \\
\text { Curvature (right): } 3 \\
\text { Shoulder (left): } 10 \\
\text { Shoulder (right): } 10 \\
\text { Shoulder Junction: } 1\end{array}$ & likely an unfinished Perdiz point \\
\hline Unit $117 \mathrm{NW}^{1 / 4}$ & $20-25$ & 1469 & 1358 & $\begin{array}{l}\text { Technology: Chipped Stone } \\
\text { Group: Tool } \\
\text { Subgroup: complex detachment- } \\
\text { based } \\
\text { Type: } \\
\text { Subtype: Perdiz }\end{array}$ & $\begin{array}{l}\text { Stage: FST } \\
\text { Portion: FRG } \\
\text { Failure/Discard: BND } \\
\text { Alteration: NOB } \\
\text { Edge Morphology (distal): IND } \\
\text { Edge Morphology (left lateral): STR } \\
\text { Edge Morphology (right latral): IND } \\
\text { Flake Scar Pattern: RDM } \\
\text { Edge Construction Type: BFB } \\
\text { Proximal Edge Grinding: NOB }\end{array}$ & $\begin{array}{l}\text { Quantity: } 1 \\
\text { Weight (in g): } 0.2 \\
\text { Max Length (in mm): } 15 \\
\text { Max Width (in mm): } 10 \\
\text { Max Thickness (mm): } 3 \\
\text { Edge Angle: } 20\end{array}$ & $\begin{array}{l}\text { Flake Attrition: NPR } \\
\text { Crushing: NPR } \\
\text { Smoothing: NPR } \\
\text { Polish: NPR } \\
\text { Etching/Pitting: NPR } \\
\text { Hafting Evidence: NOB }\end{array}$ & CRT & $\begin{array}{l}\text { Point Class: STM } \\
\text { Point Ratio: } 0.6722296 \\
\text { Stem Length: } 5.64 \\
\text { Neck Thickness: } 2.66 \\
\text { Neck Witthl. } 5.38 \\
\text { Base to Blade Ratio (length): } 0.376502 \\
\text { Base to Blade Ratio (width): } 0.1737835 \\
\text { Base/stem ratio: } 3 \\
\text { Base Form: } 5 \\
\text { Stem Form: } 1 \\
\text { Shoulder (left): } 0 \\
\text { Shoulder Junction: } 1\end{array}$ & $\begin{array}{l}\text { impact fracture has removed the tip, one face, } \\
\text { and right lateral edge, corresponding } \\
\text { measurements were not taken }\end{array}$ \\
\hline Unit $117 \mathrm{NE}^{1 / 4}$ & $25-30$ & 1342 & 1361 & $\begin{array}{l}\text { Technology: Chipped Stone } \\
\text { Group: Tool } \\
\text { Subgroup: simple detachment-based } \\
\text { Type: } \\
\text { Subtype: Not Applicable }\end{array}$ & $\begin{array}{l}\text { Stage: INR } \\
\text { Portion: PME } \\
\text { 1 Failure/Discard: ESH } \\
\text { Alteration: NOB } \\
\text { Edge Morphology (distal): IND } \\
\text { Edge Morphology (left lateral): STR } \\
\text { Edge Morphology (right lateral): STR } \\
\text { Flake Scar Pattern: IND } \\
\text { Edge Construction Type: BFU } \\
\text { Proximal Edge Grinding: NOB }\end{array}$ & $\begin{array}{l}\text { Quantity: } 1 \\
\text { Weight (in g): } 3.5 \\
\text { Max Length (in mm): } 24 \\
\text { Max Width (in mm): } 13 \\
\text { Max Thickness (mm): } 12 \\
\text { Edge Angle: } 20\end{array}$ & $\begin{array}{l}\text { Flake Attrition: BFU } \\
\text { Crushing: UNL } \\
\text { Smoothing: NPR } \\
\text { Polish: NPR } \\
\text { Etching/Pitting: NPR } \\
\text { Hafting Evidence: NOB }\end{array}$ & CRT & $\begin{array}{l}\text { Point Class: NAP } \\
\text { Point Ratio: 0.5211499 } \\
\text { Base/Stem Width: }\end{array}$ & \\
\hline
\end{tabular}


Geo-Marine, Inc.

GMI 22005.01.06.xx and 22005.00.09.xx, Data Recovery Excavations at Site 41PN175 Chipped Stone Tool Dat

\begin{tabular}{|c|c|c|c|c|c|c|c|c|c|c|}
\hline Unit & $\begin{array}{l}\text { Depth } \\
(\mathrm{cm})\end{array}$ & $\begin{array}{l}\text { Prov. } \\
\text { No. }\end{array}$ & $\begin{array}{l}\text { Lot } \\
\text { No. }\end{array}$ & Taxonomy & Attributes & Metric Information & Wear Patterning & Raw Material & Projectile Point Data & Comment \\
\hline \multicolumn{11}{|c|}{ Feature } \\
\hline Feature $47 \mathrm{~W}^{1 / 2}$ & $55-63$ & 1871 & 1651 & $\begin{array}{l}\text { Technology: Chipped Stone } \\
\text { Group: Tool } \\
\text { Subgroup: complex detachment- } \\
\text { based } \\
\text { Type: } \\
\text { Subtype: Unclassified }\end{array}$ & $\begin{array}{l}\text { Stage: FST } \\
\text { Portion: DME } \\
\text { FailurelDiscard: BND } \\
\text { Alteration: NOB } \\
\text { Elge Morphology (distal): STR } \\
\text { Edge Morphology (left lateral): RCV } \\
\text { Edge Morphology (right lateral): RCV } \\
\text { Flake Scar Pattern: RDM RM } \\
\text { Edge Construction Type: BFB } \\
\text { Proximal Edge Grinding: NOB }\end{array}$ & $\begin{array}{l}\text { Quantity: } 1 \\
\text { Weight (in g): } 0.2 \\
\text { Max Length (in mm): } 13 \\
\text { Max Width (in mm): } 10 \\
\text { Max Thickness (mm): } 3 \\
\text { Edge Angle: } 20\end{array}$ & $\begin{array}{l}\text { Flake Attrition: NPR } \\
\text { Crushing: NPR } \\
\text { Smoothing: NPR } \\
\text { Polish: SHD } \\
\text { Etching/Pitting: NPR } \\
\text { Hafting Evidence: NOB }\end{array}$ & CRT & $\begin{array}{l}\text { Point Class: STM } \\
\text { Point Ratio: } 0.799843 \\
\text { Base/stem Width: } \\
\text { Neck Thickness: } 2.46 \\
\text { Neck Width: } 5.57 \\
\text { Base Form: } 0 . \\
\text { Stem Form: 0 } \\
\text { Curvature (left): } 5 \\
\text { Curvature (right): } 5 \\
\text { Shoulder Junction: } 0\end{array}$ & $\begin{array}{l}\text { shoulders and stem missing, no measurements } \\
\text { taken on these attributes }\end{array}$ \\
\hline Feature $47 W^{1 / 2}$ & $55-63$ & 1871 & 1651 & $\begin{array}{l}\text { Technology: Chipped Stone } \\
\text { Group: Tool } \\
\text { Subgroup: complex detachment- } \\
\text { based } \\
\text { Type: } \\
\text { Subtype: Perdiz }\end{array}$ & 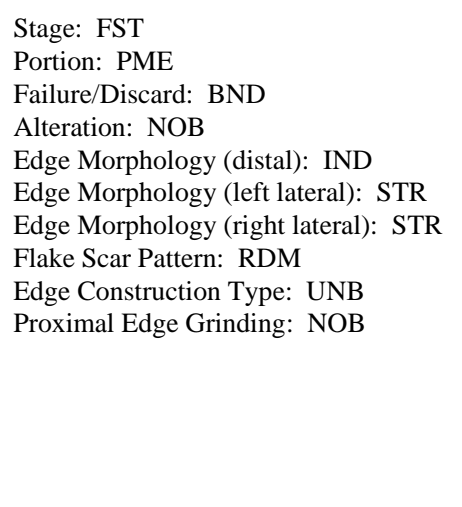 & $\begin{array}{l}\text { Quantity: } 1 \\
\text { Weight (in g): } 0.2 \\
\text { Max Length (in mm): } 10 \\
\text { Max Width (in mm): } 10 \\
\text { Max Thickness (mm): } 2 \\
\text { Edge Angle: } 15\end{array}$ & $\begin{array}{l}\text { Flake Attrition: UFU } \\
\text { Crushing: NPR } \\
\text { Smoothing: NPR } \\
\text { Polish: NPR } \\
\text { Etching/Pitting: NPR } \\
\text { Hafting Evidence: OBS }\end{array}$ & CRT & 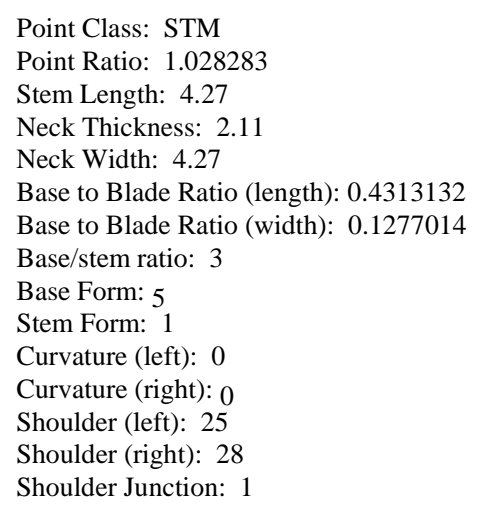 & $\begin{array}{l}\text { tip is missing from impact fracture, } \\
\text { corresponding measurements not taken }\end{array}$ \\
\hline
\end{tabular}




\section{A-5: CORES}





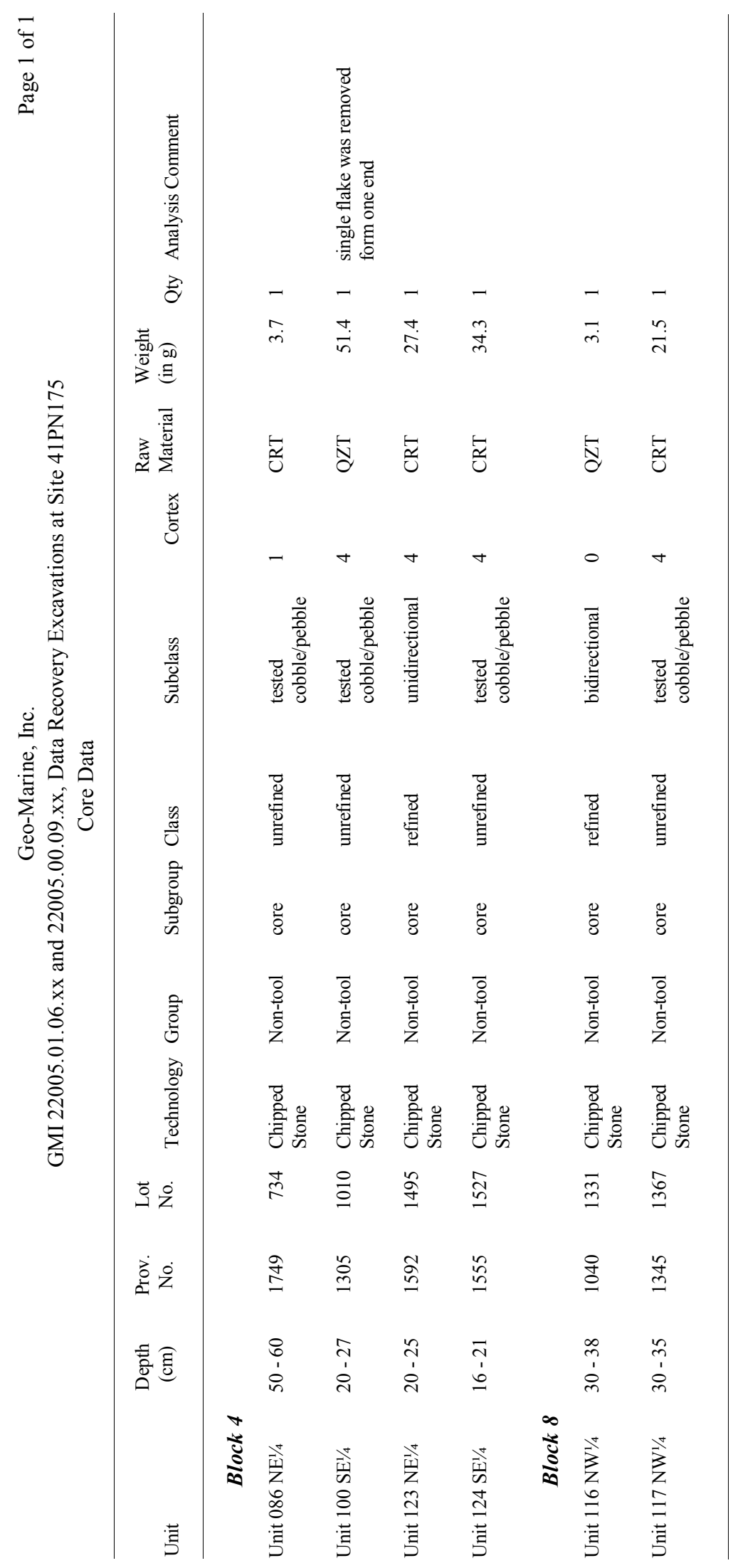



A-6: GROUNDSTONE 



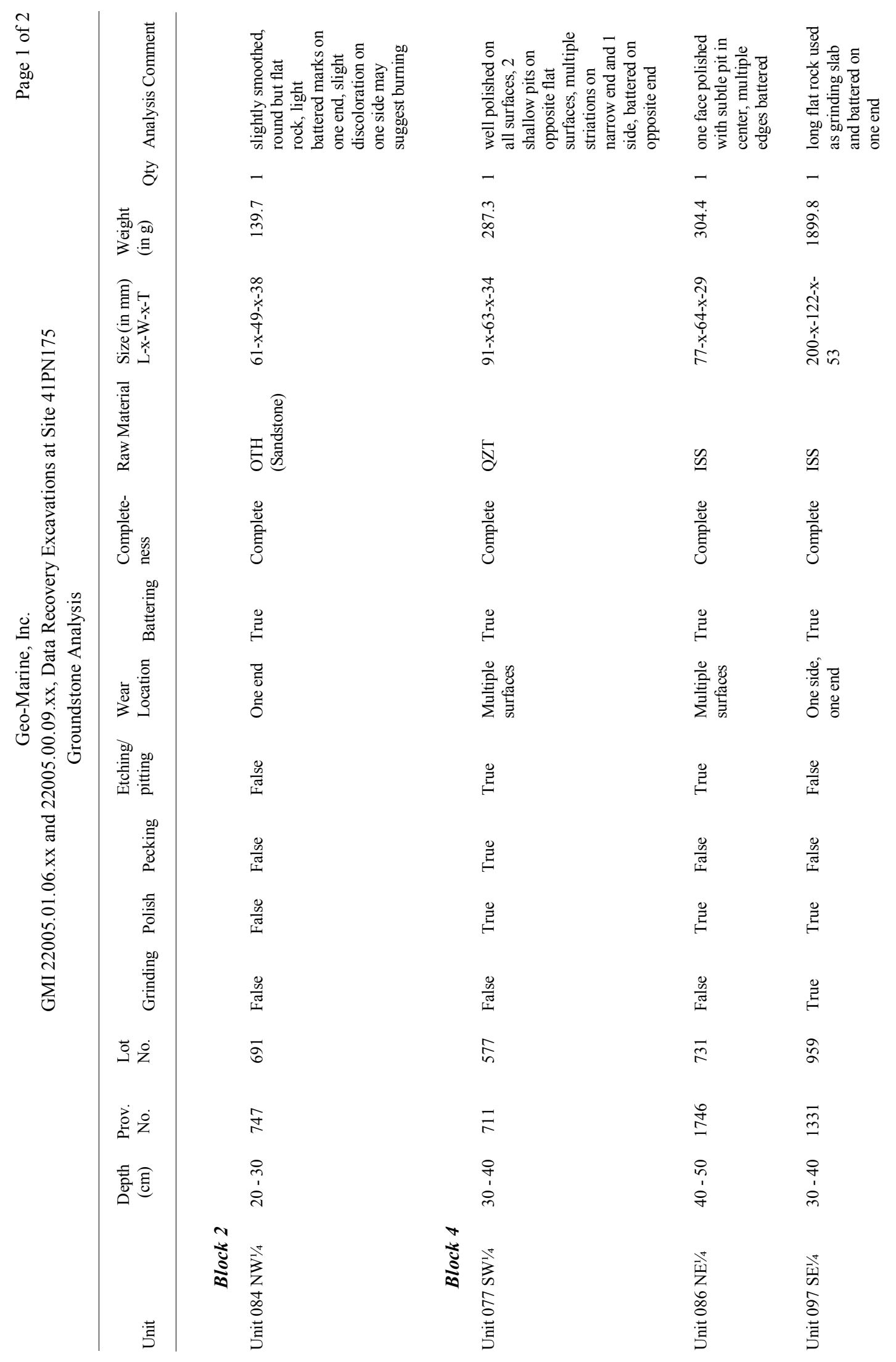




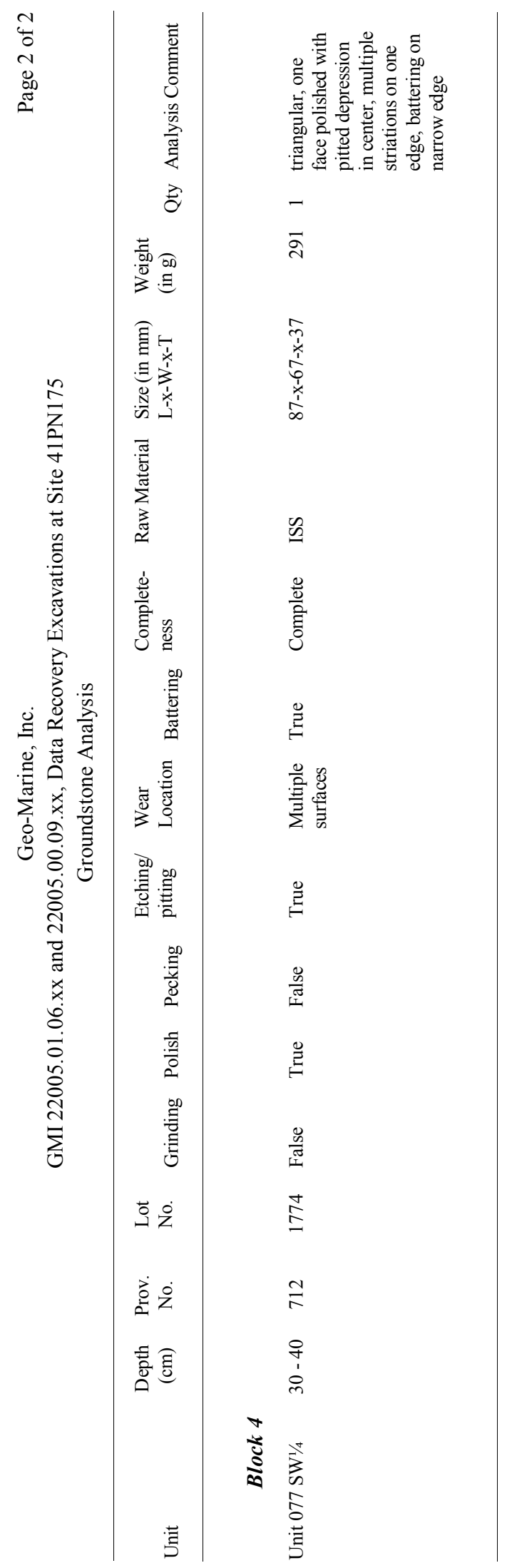


A-7: DEBITAGE 

Geo-Marine, Inc.

GMI 22005.01.06.xx and 22005.00.09.xx, Data Recovery Excavations at Site 41PN175 Debitage Analysi

\begin{tabular}{|c|c|c|c|c|c|c|c|c|c|c|c|c|c|c|c|c|c|}
\hline Unit & $\begin{array}{c}\text { Depth } \\
(\mathrm{cm})\end{array}$ & $\begin{array}{l}\text { Provenience } \\
\text { No. }\end{array}$ & $\begin{array}{l}\text { Lot } \\
\text { No. }\end{array}$ & Level of Analysis & Quantity & $\begin{array}{l}\text { Weight } \\
\text { (ing) }\end{array}$ & General Period & Region & Material & Lithology & Size Grade & $\begin{array}{c}\text { Edge } \\
\text { Modification }\end{array}$ & $\begin{array}{c}\text { Thernal } \\
\text { Alteration }\end{array}$ & Completeness & Cortex & Platform & $\begin{array}{l}\text { Minimum } \\
\text { Number of } \\
\text { Nodules }\end{array}$ \\
\hline
\end{tabular}

\begin{tabular}{lcccccc} 
Unit 001 & $10-20$ & 4 & 1773 & Unanalyzed & 1 & 5 \\
Unit 002 & $40-50$ & 30 & 145 & Unanalyzed & 1 & 0.05 \\
Unit 003 & $60-70$ & 43 & 150 & Unanalyzed & 1 & 0.6 \\
Unit 006 & $0-20$ & 256 & 160 & Unanalyzed & 1 & 0.05 \\
Unit 006 & $60-80$ & 263 & 163 & Unanalyzed & 2 & 1.5 \\
Unit 006 & $20-40$ & 259 & 161 & Unanalyzed & 1 & 0.05 \\
Unit 006 & $20-40$ & 257 & 161 & Unanalyzed & 1 & 0.6 \\
Unit 007 & $60-80$ & 130 & 167 & Unanalyzed & 1 & 3.7 \\
Unit 007 & $20-40$ & 128 & 165 & Unanalyzed & 1 & 0.2 \\
Unit 007 & $40-60$ & 129 & 166 & Unanalyzed & 1 & 2.5 \\
Unit 008 & $40-60$ & 138 & 170 & Unanalyzed & 1 & 5 \\
Unit 008 & $40-60$ & 139 & 170 & Unanalyzed & 3 & 0.05 \\
Unit 008 & $20-40$ & 136 & 169 & Unanalyzed & 4 & 0.05 \\
Unit 008 & $20-40$ & 134 & 169 & Unanalyzed & 1 & 0.4 \\
Unit 009 & $0-20$ & 140 & 171 & Unanalyzed & 1 & 5 \\
Unit 009 & $20-40$ & 141 & 172 & Unanalyzed & 3 & 0.8 \\
Unit 010 & $0-20$ & 144 & 174 & Unanalyzed & 2 & 0.6 \\
Unit 010 & $40-60$ & 152 & 176 & Unanalyzed & 2 & 0.05 \\
Unit 010 & $20-40$ & 149 & 175 & Unanalyzed & 1 & 0.05 \\
Unit 010 & $20-40$ & 147 & 175 & Unanalyzed & 1 & 0.1 \\
Unit 010 & $0-20$ & 145 & 174 & Unanalyzed & 1 & 5 \\
Unit 010 & $0-20$ & 146 & 174 & Unanalyzed & 1 & 0.05 \\
Unit 011 & $20-40$ & 154 & 178 & Unanalyzed & 1 & 0.8 \\
Unit 012 & $20-40$ & 158 & 180 & Unanalyzed & 2 & 1.2 \\
Unit 012 & $0-20$ & 157 & 179 & Unanalyzed & 1 & 0.05 \\
Unit 013 & $60-80$ & 91 & 185 & Unanalyzed & 1 & 0.2 \\
\hline & & & & & &
\end{tabular}


Geo-Marine, Inc.

GMI 22005.01.06.xx and 22005.00.09.xx, Data Recovery Excavations at Site 41PN175 Debitage Analysis

\begin{tabular}{|c|c|c|c|c|c|c|c|c|c|c|c|c|c|c|c|c|c|}
\hline Unit & $\begin{array}{c}\text { Depth } \\
(\mathrm{cm})\end{array}$ & $\begin{array}{l}\text { Provenience } \\
\text { No. }\end{array}$ & $\begin{array}{l}\text { Lot } \\
\text { No. }\end{array}$ & Level of Analysis & Quantity & $\begin{array}{l}\text { Weight } \\
\text { (ing) }\end{array}$ & General Period & Region & Material & Lithology & Size Grade & $\begin{array}{c}\text { Edge } \\
\text { Modification }\end{array}$ & $\begin{array}{l}\text { Thernal } \\
\text { Alteration }\end{array}$ & Completeness & Cortex & Platform & $\begin{array}{l}\text { Minimum } \\
\text { Number of } \\
\text { Nodules }\end{array}$ \\
\hline
\end{tabular}

$\begin{array}{lcccccc}\text { Unit } 013 & \text { 50-X-50 cm unit } & & & & & \\ \text { Unit } 014 & 30-40 & 58 & 184 & \text { Unanalyzed } & 1 & 0.8 \\ \text { Unit } 014 & 20-30 & 57 & 188 & \text { Unanalyzed } & 1 & 0.2 \\ \text { Unit } 016 & 0-20 & 269 & 199 & \text { Unanalyzzed } & 1 & 0.05 \\ \text { Unit } 019 & 0-20 & 282 & 205 & \text { Unanalyzed } & 1 & 0.4 \\ \text { Unit } 019 & 0-20 & 284 & 205 & \text { Unanalyzed } & 1 & 1.5 \\ \text { Unit } 020 & 20-40 & 165 & 208 & \text { Unanalyzed } & 1 & 0.05 \\ \text { Unit } 020 & 0-20 & 164 & 207 & \text { Unanalyzed } & 1 & 0.6 \\ \text { Unit } 021 & 20-40 & 170 & 210 & \text { Unanalyzed } & 1 & 0.4 \\ \text { Unit } 021 & 20-40 & 172 & 211 & \text { Unanalyzed } & 1 & 0.05 \\ \text { Unit } 022 & 20-40 & 177 & 214 & \text { Unanalyzed } & 8 & 2.2 \\ \text { Unit } 023 & 0-20 & 178 & 215 & \text { Unanalyzed } & 1 & 1.7 \\ \text { Unit } 023 & 0-20 & 180 & 215 & \text { Unanalyzed } & 1 & 0.05 \\ \text { Unit } 024 & 40-60 & 195 & 221 & \text { Unanalyzed } & 1 & 0.4 \\ \text { Unit } 024 & 80-90 & 197 & 223 & \text { Unanalyzed } & 1 & 5 \\ \text { Unit } 025 & 20-40 & 202 & 227 & \text { Unanalyzed } & 2 & 0.5 \\ \text { Unit } 025 & 20-40 & 204 & 227 & \text { Unanalyzed } & 2 & 0.05 \\ \text { Unit } 025 & 40-60 & 205 & 228 & \text { Unanalyzed } & 1 & 0.1 \\ \text { Unit } 025 & 0-20 & 199 & 225 & \text { Unanalyzed } & 2 & 5.3 \\ \text { Unit } 026 & 20-40 & 94 & 231 & \text { Unanalyzed } & 5 & 2.2 \\ \text { Unit } 027 & 40-60 & 103 & 235 & \text { Unanalyzed } & 1 & 1.7 \\ \text { Unit } 027 & 40-60 & 105 & 235 & \text { Unanalyzed } & 2 & 0.05 \\ \text { Unit } 032 & 0-20 & 332 & 245 & \text { Unanalyzed } & 1 & 0.1 \\ \text { Unit } 033 & 20-40 & 342 & 249 & \text { Unanalyzed } & 1 & 0.1 \\ \text { Unit } 033 & 0-20 & 341 & 248 & \text { Unanalyzed } & 4 & 6.3 \\ \text { Unit } 034 & 0-20 & 317 & 250 & \text { Unanalyzed } & 2 & 3.7\end{array}$


Geo-Marine, Inc.

\begin{tabular}{|c|c|c|c|c|c|c|c|c|c|c|c|c|c|c|c|c|c|}
\hline Unit & $\begin{array}{l}\text { Depth } \\
(\mathrm{cm})\end{array}$ & $\begin{array}{l}\text { Provenience } \\
\text { No. }\end{array}$ & $\begin{array}{l}\text { Lot } \\
\text { No. }\end{array}$ & Level of Analysis & Quantity & $\begin{array}{l}\text { Weight } \\
\text { (ing) }\end{array}$ & General Period & Region & Material & Lithology & Size Grade & $\begin{array}{c}\text { Edge } \\
\text { Modification }\end{array}$ & $\begin{array}{l}\text { Thernal } \\
\text { Alteration }\end{array}$ & Completeness & Cortex & Platform & $\begin{array}{l}\text { Minimum } \\
\text { Number of } \\
\text { Nodules }\end{array}$ \\
\hline
\end{tabular}

\begin{tabular}{|c|c|c|c|c|c|c|}
\hline \multicolumn{7}{|c|}{$50-x-50 \mathrm{~cm}$ unit } \\
\hline Unit 035 & $0-20$ & 288 & 254 & Unanalyzed & 2 & 1.1 \\
\hline Unit 035 & $20-40$ & 289 & 255 & Unanalyzed & 1 & 0.8 \\
\hline Unit 035 & $40-50$ & 290 & 256 & Unanalyzed & 1 & 5 \\
\hline Unit 036 & $0-20$ & 291 & 257 & Unanalyzed & 6 & 9.3 \\
\hline Unit 036 & $0-20$ & 293 & 257 & Unanalyzed & 1 & 0.05 \\
\hline Unit 036 & $20-40$ & 294 & 258 & Unanalyzed & 1 & 0.5 \\
\hline Unit 036 & $20-40$ & 295 & 258 & Unanalyzed & 1 & 5 \\
\hline Unit 037 & $0-20$ & 300 & 260 & Unanalyzed & 4 & 3 \\
\hline Unit 038 & $20-40$ & 306 & 263 & Unanalyzed & 3 & 1.7 \\
\hline Unit 038 & $20-40$ & 308 & 263 & Unanalyzed & 1 & 0.05 \\
\hline Unit 039 & $20-40$ & 316 & 267 & Unanalyzed & 1 & 0.3 \\
\hline Unit 040 & $0-20$ & 213 & 268 & Unanalyzed & 1 & 0.05 \\
\hline Unit 040 & $0-20$ & 211 & 268 & Unanalyzed & 1 & 0.6 \\
\hline Unit 044 & $0-20$ & 350 & 282 & Unanalyzed & 1 & 0.1 \\
\hline Unit 050 & $0-20$ & 385 & 294 & Unanalyzed & 1 & 0.8 \\
\hline Unit 050 & $20-40$ & 386 & 295 & Unanalyzed & 1 & 0.4 \\
\hline Unit 051 & $20-40$ & 391 & 297 & Unanalyzed & 2 & 0.5 \\
\hline Unit 052 & $20-40$ & 401 & 301 & Unanalyzed & 1 & 0.6 \\
\hline Unit 054 & $20-40$ & 413 & 305 & Unanalyzed & 1 & 0.3 \\
\hline \multicolumn{7}{|c|}{ Block 2} \\
\hline Unit $061 \mathrm{NE}^{1 / 4}$ & $50-60$ & 879 & 323 & Unanalyzed & 1 & 1.2 \\
\hline Unit $061 \mathrm{NW}^{1 / 4}$ & $30-40$ & 593 & 326 & Unanalyzed & 3 & 1.7 \\
\hline Unit $061 \mathrm{NW}^{1 / 4}$ & $20-30$ & 504 & 325 & Unanalyzed & 1 & 0.2 \\
\hline Unit $061 \mathrm{NW}^{1 / 4}$ & $40-50$ & 856 & 327 & Unanalyzed & 2 & 1.7 \\
\hline Unit $061 \mathrm{SE}^{1 / 4}$ & $40-50$ & 858 & 332 & Unanalyzed & 1 & 0.5 \\
\hline Unit $061 \mathrm{SW}^{1} / 4$ & $30-40$ & 592 & 336 & Unanalyzed & 2 & 0.8 \\
\hline
\end{tabular}


Geo-Marine, Inc.

GMI 22005.01.06.xx and 22005.00.09.xx, Data Recovery Excavations at Site 41PN17 Debitage Analysi

\begin{tabular}{|c|c|c|c|c|c|c|c|c|c|c|c|c|c|c|c|c|c|}
\hline Unit & $\begin{array}{c}\begin{array}{c}\text { Depth } \\
(\mathrm{cm})\end{array}\end{array}$ & $\begin{array}{l}\text { Provenience } \\
\text { No. }\end{array}$ & $\begin{array}{l}\text { Lot } \\
\text { No. }\end{array}$ & Level of Analysis & Quantity & $\begin{array}{l}\text { Weight } \\
\text { (in g) }\end{array}$ & General Period & Region & Material & Lithology & Size Grade & $\begin{array}{c}\text { Edge } \\
\text { Modification }\end{array}$ & $\begin{array}{l}\text { Thernal } \\
\text { Alteration }\end{array}$ & Completeness & Cortex & Platform & $\begin{array}{l}\text { Minimum } \\
\text { Number o } \\
\text { Nodules }\end{array}$ \\
\hline \multicolumn{18}{|l|}{ Block 2} \\
\hline Unit $061 \mathrm{SW}^{1 / 4}$ & $40-50$ & 855 & 337 & Unanalyzed & 1 & 0.3 & & & & & & & & & & & \\
\hline Unit $062 \mathrm{NE}^{1 / 4}$ & $30-40$ & 598 & 352 & Unanalyzed & 1 & 0.3 & & & & & & & & & & & \\
\hline Unit $062 \mathrm{NE}^{1 / 4}$ & $30-40$ & 599 & 353 & Unanalyzed & 2 & 2 & & & & & & & & & & & \\
\hline Unit $062 \mathrm{NE}^{1 / 4}$ & $20-30$ & 677 & 346 & Unanalyzed & 1 & 0.2 & & & & & & & & & & & \\
\hline Unit $062 \mathrm{NE}^{1 / 4}$ & $20-30$ & 676 & 345 & Unanalyzed & 2 & 2.2 & & & & & & & & & & & \\
\hline Unit $062 \mathrm{NW}^{1 / 4}$ & $20-30$ & 675 & 348 & Unanalyzed & 2 & 0.2 & & & & & & & & & & & \\
\hline Unit $062 \mathrm{SW}^{1 / 4}$ & $20-30$ & 673 & 350 & Unanalyzed & 1 & 2.5 & & & & & & & & & & & \\
\hline Unit $062 \mathrm{SW}^{1 / 4}$ & $30-40$ & 596 & 356 & Unanalyzed & 1 & 0.1 & & & & & & & & & & & \\
\hline Unit $063 \mathrm{NE}^{1 / 4}$ & $30-40$ & 682 & 369 & Analyzed & 1 & 0.04 & LPR & PWS & LOC & CRT & 4 & & I & $\mathrm{COM}$ & 0 & FLT & 1 \\
\hline Unit $063 \mathrm{SE}^{1} / 4$ & $10-20$ & 513 & 363 & Unanalyzed & 1 & 1.8 & & & & & & & & & & & \\
\hline Unit $063 \mathrm{SW}^{1 / 4}$ & $30-40$ & 680 & 372 & Analyzed & 1 & 0.3 & LPR & PWS & LOC & CRT & 4 & & I & FRG & 4 & IND & 1 \\
\hline Unit 064 General & $20-30$ & 553 & 380 & Analyzed & 1 & 0.7 & LPR & PWS & LOC & FWD & 3 & & $\mathrm{~N}$ & COM & 2 & CRT & 1 \\
\hline Unit $064 \mathrm{NE}^{1 / 4}$, Feature 1 & $33-35$ & 873 & 385 & Analyzed & 2 & 0.4 & LPR & PWS & LOC & QZT & 4 & & I & COM & 4 & CRT & 1 \\
\hline Unit $064 \mathrm{NW}^{1 / 4}$ & $30-40$ & 607 & 386 & Analyzed & 2 & 0.2 & LPR & PWS & LOC & CRT & 4 & & I & COM & 0 & FLT & 1 \\
\hline Unit $064 \mathrm{NW}^{1 / 4}$ & $10-20$ & 514 & 379 & Unanalyzed & 1 & 0.2 & & & & & & & & & & & \\
\hline Unit $064 \mathrm{SE}^{1} / 4$ & $30-40$ & 609 & 387 & Analyzed & 1 & 1 & LPR & PWS & LOC & QZT & 4 & & I & BRK & 4 & CRT & 1 \\
\hline Unit $064 \mathrm{SE}^{1 / 4}$ & $20-30$ & 552 & 383 & Analyzed & 1 & 1.1 & LPR & PWS & LOC & CRT & 3 & & $\mathrm{~N}$ & COM & 2 & CRT & 1 \\
\hline Unit $064 \mathrm{SE}^{1 / 4}$ & $20-30$ & 552 & 383 & Analyzed & 1 & 0.3 & LPR & PWS & LOC & CRT & 4 & & I & FRG & 4 & IND & 1 \\
\hline Unit $083 \mathrm{NE}^{1 / 4}$ & $30-40$ & 861 & 675 & Analyzed & 1 & 0.2 & LPR & PWS & LOC & CRT & 4 & & I & COM & 0 & FLT & 3 \\
\hline Unit $083 \mathrm{NE}^{1 / 4}$ & $30-40$ & 861 & 675 & Analyzed & 1 & 1.4 & LPR & PWS & REG & CRT & 3 & & $\mathrm{Y}$ & COM & 0 & ABR & 3 \\
\hline Unit $083 \mathrm{NE}^{1 / 4}$ & $30-40$ & 861 & 675 & Analyzed & 1 & 0.1 & LPR & PWS & LOC & FWD & 4 & & I & COM & 0 & FLT & 3 \\
\hline Unit $083 \mathrm{NW}^{1 / 4}$ & $40-50$ & 885 & 679 & Analyzed & 1 & 1 & LPR & PWS & LOC & QZT & 3 & & $\mathrm{~N}$ & FRG & 0 & IND & 1 \\
\hline Unit $083 \mathrm{SE}^{1 / 4}$ & $10-20$ & 746 & 667 & Unanalyzed & 1 & 0.3 & & & & & & & & & & & \\
\hline Unit $083 \mathrm{SE}^{1 / 4}$ & $20-30$ & 800 & 671 & Analyzed & 1 & 0.04 & LPR & PWS & LOC & CRT & 5 & & I & COM & 0 & IND & 1 \\
\hline Unit $083 \mathrm{SW}^{1 / 4}$ & $30-40$ & 859 & 678 & Analyzed & 1 & 0.3 & LPR & PWS & LOC & QZT & 4 & & I & COM & 0 & CPX & 2 \\
\hline Unit $083 \mathrm{SW}^{1} / 4$ & $30-40$ & 859 & 678 & Analyzed & 1 & 0.7 & LPR & PWS & LOC & QZT & 4 & & I & DEB & 1 & IND & 2 \\
\hline
\end{tabular}


Geo-Marine, Inc.

GMI 22005.01.06.xx and 22005.00.09.xx, Data Recovery Excavations at Site 41PN17 Debitage Analysi

\begin{tabular}{|c|c|c|c|c|c|c|c|c|c|c|c|c|c|c|c|c|c|}
\hline Unit & $\begin{array}{l}\text { Depth } \\
(\mathrm{cm})\end{array}$ & $\begin{array}{l}\text { Provenience } \\
\text { No. }\end{array}$ & $\begin{array}{l}\text { Lot } \\
\text { No. }\end{array}$ & Level of Analysis & Quantity & $\begin{array}{l}\text { Weight } \\
\text { (in g) }\end{array}$ & General Period & Region & Material & Lithology & Size Grade & $\begin{array}{c}\text { Edge } \\
\text { Modification }\end{array}$ & $\begin{array}{l}\text { Thernal } \\
\text { Alteration }\end{array}$ & Completeness & Cortex & Platform & $\begin{array}{l}\text { Minimum } \\
\text { Number of of } \\
\text { Nodules }\end{array}$ \\
\hline \multicolumn{18}{|c|}{ Block 2} \\
\hline Unit $083 \mathrm{SW}^{1 / 4}$ & $30-40$ & 859 & 678 & Analyzed & 1 & 1.6 & LPR & PWS & LOC & SLM & 3 & & $\mathrm{~N}$ & COM & 0 & $\mathrm{IND}$ & 2 \\
\hline Unit $083 \mathrm{SW}^{1 / 4}$ & $10-20$ & 743 & 668 & Unanalyzed & 3 & 8.8 & & & & & & & & & & & \\
\hline Unit $083 \mathrm{SW}^{1 / 4}$ & $20-30$ & 797 & 673 & Analyzed & 1 & 0.2 & LPR & PWS & LOC & CRT & 4 & & I & BRK & 0 & ABR & 1 \\
\hline Unit 084 General & $10-20$ & 806 & 684 & Unanalyzed & 3 & 0.8 & & & & & & & & & & & \\
\hline Unit 084 General & $20-30$ & 811 & 689 & Analyzed & 1 & 0.2 & LPR & PWS & LOC & QZT & 3 & & $\mathrm{~N}$ & COM & 4 & CRT & 2 \\
\hline Unit 084 General & $20-30$ & 811 & 689 & Analyzed & 1 & 0.04 & LPR & PWS & LOC & CRT & 4 & & I & COM & 0 & CPX & 2 \\
\hline Unit $084 \mathrm{NE}^{1 / 4}$ & $10-20$ & 804 & 685 & Unanalyzed & 5 & 1.3 & & & & & & & & & & & \\
\hline Unit $084 \mathrm{SE}^{1 / 4}$ & $20-30$ & 810 & 692 & Analyzed & 1 & 2.6 & LPR & PWS & LOC & CRT & 3 & & $\mathrm{~N}$ & COM & 4 & CRT & 1 \\
\hline Unit $084 \mathrm{SE}^{1 / 4}$ & $10-20$ & 805 & 687 & Unanalyzed & 2 & 1.8 & & & & & & & & & & & \\
\hline Unit $084 \mathrm{SW}^{1 / 4}$ & $30-40$ & 864 & 698 & Analyzed & 1 & 0.4 & LPR & PWS & REG & CRT & 4 & & I & COM & 0 & IND & 2 \\
\hline Unit $084 \mathrm{SW}^{1 / 4}$ & $30-40$ & 864 & 698 & Analyzed & 1 & 0.1 & LPR & PWS & LOC & CRT & 4 & & I & FRG & 0 & $\mathrm{IND}$ & 2 \\
\hline Unit $084 \mathrm{SW}^{1 / 4}$ & $30-40$ & 864 & 698 & Analyzed & 1 & 0.1 & LPR & PWS & LOC & CRT & 4 & & I & DEB & 0 & $\mathrm{IND}$ & 1 \\
\hline Unit $084 \mathrm{SW}^{1 / 4}$ & $10-20$ & 802 & 688 & Unanalyzed & 1 & 2.2 & & & & & & & & & & & \\
\hline Unit 085 General & $0-10$ & 728 & 699 & Unanalyzed & 1 & 0.3 & & & & & & & & & & & \\
\hline Unit $085 \mathrm{NE}^{1 / 4}$ & $30-40$ & 871 & 713 & Analyzed & 1 & 0.4 & LPR & PWS & LOC & CRT & 3 & & $\mathrm{Y}$ & COM & 0 & CPX & 1 \\
\hline Unit $085 \mathrm{NE}^{1 / 4}$ & $10-20$ & 814 & 704 & Unanalyzed & 1 & 0.2 & & & & & & & & & & & \\
\hline Unit $085 \mathrm{NE}^{1 / 4}$ & $30-40$ & 871 & 713 & Analyzed & 1 & 1.6 & LPR & PWS & LOC & CRT & 3 & & $\mathrm{~N}$ & COM & 0 & CPX & 1 \\
\hline Unit $085 \mathrm{NE}^{1 / 4}$ & $0-10$ & 726 & 700 & Unanalyzed & 2 & 1.7 & & & & & & & & & & & \\
\hline Unit $085 \mathrm{NE}^{1 / 4}$ & $30-40$ & 871 & 713 & Analyzed & 1 & 4 & LPR & PWS & LOC & CRT & 2 & ABS & $\mathrm{N}$ & COM & 0 & FAC & 1 \\
\hline Unit $085 \mathrm{NW}^{1 / 4}$ & $20-30$ & 817 & 710 & Analyzed & 1 & 0.5 & LPR & PWS & LOC & CRT & 4 & & I & BRK & 1 & FLT & 1 \\
\hline Unit $085 \mathrm{SE}^{1 / 4}$ & $0-10$ & 727 & 702 & Unanalyzed & 1 & 0.3 & & & & & & & & & & & \\
\hline Unit $085 \mathrm{SE}^{1 / 4}$ & $10-20$ & 815 & 706 & Unanalyzed & 2 & 0.8 & & & & & & & & & & & \\
\hline Unit $085 \mathrm{SW}^{1 / 4}$ & $30-40$ & 869 & 715 & Analyzed & 1 & 2.6 & LPR & PWS & LOC & CRT & 3 & & $\mathrm{~N}$ & COM & 2 & CRT & 1 \\
\hline Unit $085 \mathrm{SW}^{1 / 4}$ & $10-20$ & 812 & 707 & Unanalyzed & 2 & 2.7 & & & & & & & & & & & \\
\hline Unit $085 \mathrm{SW}^{1 / 4}$ & $30-40$ & 869 & 715 & Analyzed & 1 & 1.3 & LPR & PWS & LOC & CRT & 3 & & $\mathrm{~N}$ & COM & 0 & CPX & 1 \\
\hline Unit $085 \mathrm{SW}^{1 / 4}$ & $30-40$ & 869 & 715 & Analyzed & 1 & 2.9 & LPR & PWS & LOC & CRT & 2 & ABS & $\mathrm{N}$ & BRK & 0 & CPX & 1 \\
\hline
\end{tabular}


Geo-Marine, Inc.

GMI 22005.01.06.xx and 22005.00.09.xx, Data Recovery Excavations at Site 41PN17 Debitage Analys

\begin{tabular}{|c|c|c|c|c|c|c|c|c|c|c|c|c|c|c|c|c|c|}
\hline Unit & $\begin{array}{c}\begin{array}{c}\text { Depth } \\
(\mathrm{cm})\end{array}\end{array}$ & $\begin{array}{l}\text { Provenience } \\
\text { No. }\end{array}$ & $\begin{array}{l}\text { Lot } \\
\text { No. }\end{array}$ & Level of Analysis & Quantity & $\begin{array}{l}\text { Weight } \\
\text { (ing) }\end{array}$ & General Period & Region & Material & Lithology & Size Grade & $\begin{array}{c}\text { Edge } \\
\text { Modification }\end{array}$ & $\begin{array}{l}\text { Thernal } \\
\text { Alteration }\end{array}$ & Completeness & Cortex & Platform & $\begin{array}{l}\text { Minimun } \\
\text { Number } \\
\text { Nodules }\end{array}$ \\
\hline \multicolumn{18}{|c|}{ Block 3} \\
\hline Unit 065 General & $0-10$ & 474 & 390 & Unanalyzed & 1 & 0.8 & & & & & & & & & & & \\
\hline Unit $065 \mathrm{NE}^{1 / 4}$ & $10-20$ & 519 & 394 & Unanalyzed & 2 & 3.5 & & & & & & & & & & & \\
\hline Unit $065 \mathrm{NE}^{1 / 4}$ & $30-40$ & 612 & 400 & Analyzed & 1 & 0.4 & LPR & PWS & LOC & CRT & 4 & & I & COM & 1 & CRT & 1 \\
\hline Unit $065 \mathrm{SE}^{1 / 4}$ & $10-20$ & 521 & 396 & Unanalyzed & 1 & 0.6 & & & & & & & & & & & \\
\hline Unit $065 \mathrm{SE}^{1 / 4}$ & $20-30$ & 524 & 399 & Analyzed & 1 & 1.4 & LPR & PWS & LOC & QZT & 3 & & $\mathrm{~N}$ & COM & 1 & FLT & 1 \\
\hline Unit $065 \mathrm{SW}^{1 / 4}$ & $0-10$ & 470 & 393 & Unanalyzed & 1 & 0.6 & & & & & & & & & & & \\
\hline Unit $066 \mathrm{NE}^{1 / 4}$ & $10-20$ & 527 & 407 & Unanalyzed & 3 & 1.7 & & & & & & & & & & & \\
\hline Unit $066 \mathrm{NE}^{1 / 4}$ & $20-30$ & 535 & 411 & Analyzed & 1 & 1.1 & LPR & PWS & LOC & FWD & 4 & & I & FRG & 4 & IND & 1 \\
\hline Unit $066 \mathrm{NW}^{1 / 4}$ & $20-30$ & 534 & 412 & Analyzed & 1 & 0.7 & LPR & PWS & LOC & FWD & 3 & & $\mathrm{~N}$ & BRK & 2 & CRT & 1 \\
\hline Unit $066 \mathrm{SE}^{1 / 4}$ & $0-10$ & 478 & 406 & Unanalyzed & 1 & 0.5 & & & & & & & & & & & \\
\hline Unit $066 \mathrm{SW}^{1} / 4$ & $10-20$ & 525 & 410 & Unanalyzed & 2 & 0.5 & & & & & & & & & & & \\
\hline Unit $067 \mathrm{NE}^{1 / 4}$ & $20-30$ & 543 & 422 & Analyzed & 1 & 1.7 & LPR & PWS & LOC & QZT & 3 & & $\mathrm{~N}$ & BRK & 2 & IND & 1 \\
\hline Unit $067 \mathrm{NE}^{1 / 4}$ & $10-20$ & 531 & 418 & Unanalyzed & 3 & 1.6 & & & & & & & & & & & \\
\hline Unit $067 \mathrm{NW}^{1 / 4}$ & $0-10$ & 480 & 416 & Unanalyzed & 1 & 1.5 & & & & & & & & & & & \\
\hline Unit $067 \mathrm{NW}^{1 / 4}$ & $20-30$ & 542 & 423 & Analyzed & 1 & 4.1 & LPR & PWS & LOC & CRT & 3 & & $\mathrm{Y}$ & COM & 2 & CRT & 1 \\
\hline Unit $067 \mathrm{NW}^{1 / 4}$ & $20-30$ & 542 & 423 & Analyzed & 1 & 0.04 & LPR & PWS & LOC & CRT & 4 & & I & COM & 0 & IND & 1 \\
\hline Unit $067 \mathrm{NW}^{1 / 4}$ & $10-20$ & 530 & 419 & Unanalyzed & 1 & 0.6 & & & & & & & & & & & \\
\hline Unit $067 \mathrm{SE}^{1 / 4}$ & $20-30$ & 544 & 424 & Analyzed & 1 & 5.4 & LPR & PWS & LOC & QZT & 2 & $\mathrm{ABS}$ & $\mathrm{N}$ & COM & 4 & CRT & 1 \\
\hline Unit $067 \mathrm{SE}^{1 / 4}$ & $10-20$ & 532 & 420 & Unanalyzed & 1 & 0.2 & & & & & & & & & & & \\
\hline Unit $067 \mathrm{SE}^{1 / 4}$ & $40-50$ & 688 & 427 & Analyzed & 1 & 1.6 & LPR & PWS & LOC & CRT & 3 & & $\mathrm{~N}$ & $\mathrm{COM}$ & 0 & FAC & 1 \\
\hline Unit $068 \mathrm{NE}^{1 / 4}$ & $20-30$ & 618 & 436 & Analyzed & 1 & 2 & LPR & PWS & LOC & QZT & 2 & $\mathrm{ABS}$ & $\mathrm{N}$ & FRG & 4 & IND & 1 \\
\hline Unit $068 \mathrm{NE}^{1 / 4}$ & $10-20$ & 539 & 432 & Unanalyzed & 1 & 0.4 & & & & & & & & & & & \\
\hline Unit $068 \mathrm{NW}^{1 / 4}$ & $10-20$ & 538 & 433 & Unanalyzed & 1 & 0.8 & & & & & & & & & & & \\
\hline Unit 068 SE$^{1 / 4}$ & $20-30$ & 619 & 438 & Analyzed & 1 & 0.6 & LPR & PWS & LOC & CRT & 4 & & I & COM & 0 & FLT & 1 \\
\hline Unit 068 SE1/4 & $10-20$ & 540 & 434 & Unanalyzed & 1 & 1.5 & & & & & & & & & & & \\
\hline Unit $069 \mathrm{NE}^{1 / 4}$ & $10-20$ & 547 & 448 & Unanalyzed & 2 & 1.2 & & & & & & & & & & & \\
\hline
\end{tabular}


Geo-Marine, Inc.

GMI 22005.01.06.xx and 22005.00.09.xx, Data Recovery Excavations at Site 41PN17 Debitage Analysi

\begin{tabular}{|c|c|c|c|c|c|c|c|c|c|c|c|c|c|c|c|c|c|}
\hline Unit & $\begin{array}{c}\begin{array}{c}\text { Depth } \\
(\mathrm{cm})\end{array}\end{array}$ & $\begin{array}{l}\text { Provenience } \\
\text { No. }\end{array}$ & $\begin{array}{l}\text { Lot } \\
\text { No. }\end{array}$ & Level of Analysis & Quantity & $\begin{array}{l}\text { Weight } \\
\text { (in g) }\end{array}$ & General Period & Region & Material & Lithology & Size Grade & $\begin{array}{c}\text { Edge } \\
\text { Modification }\end{array}$ & $\begin{array}{l}\text { Thernal } \\
\text { Alteration }\end{array}$ & Completeness & Cortex & Platform & $\begin{array}{l}\text { Minimum } \\
\text { Number of } \\
\text { Nodules }\end{array}$ \\
\hline \multicolumn{18}{|c|}{ Block 3} \\
\hline Unit 069 NE'/4 & $30-40$ & 627 & 457 & Analyzed & 1 & 0.5 & LPR & PWS & REG & JPR & 3 & & $\mathrm{~N}$ & COM & 0 & FAC & 1 \\
\hline Unit $069 \mathrm{NW}^{1 / 4}$ & $10-20$ & 546 & 449 & Unanalyzed & 3 & 3.9 & & & & & & & & & & & \\
\hline Unit $069 \mathrm{SE}^{1} / 4$ & $30-40$ & 628 & 458 & Analyzed & 1 & 3.6 & LPR & PWS & LOC & CRT & 2 & ABS & $\mathrm{N}$ & DEB & 1 & IND & 1 \\
\hline Unit $069 \mathrm{SE}^{1 / 4}$ & $10-20$ & 548 & 450 & Unanalyzed & 1 & 0.8 & & & & & & & & & & & \\
\hline Unit $069 \mathrm{SW}^{1} / 4$ & $30-40$ & 626 & 459 & Analyzed & 1 & 0.5 & LPR & PWS & REG & CRT & 3 & & $\mathrm{~N}$ & FRG & 0 & IND & 1 \\
\hline Unit $069 \mathrm{SW}^{1 / 4}$ & $20-30$ & 622 & 456 & Analyzed & 1 & 0.2 & LPR & PWS & REG & CRT & 4 & & I & FRG & 0 & IND & 1 \\
\hline Unit $069 \mathrm{SW}^{1} 1 / 4$ & $10-20$ & 545 & 451 & Unanalyzed & 3 & 4 & & & & & & & & & & & \\
\hline Unit $070 \mathrm{NE}^{1 / 4}$ & $10-20$ & 562 & 464 & Unanalyzed & 1 & 0.6 & & & & & & & & & & & \\
\hline Unit $070 \mathrm{NW}^{1 / 4}$ & $10-20$ & 561 & 465 & Unanalyzed & 1 & 0.5 & & & & & & & & & & & \\
\hline Unit $070 \mathrm{SE}^{1 / 4}$ & $0-10$ & 494 & 462 & Unanalyzed & 1 & 4.4 & & & & & & & & & & & \\
\hline Unit $070 \mathrm{SW}^{1 / 4}$ & $10-20$ & 560 & 467 & Unanalyzed & 2 & 0.8 & & & & & & & & & & & \\
\hline Unit $071 \mathrm{NE}^{1 / 4}$ & $10-20$ & 565 & 472 & Unanalyzed & 4 & 2 & & & & & & & & & & & \\
\hline Unit $071 \mathrm{NW}^{1 / 4}$ & $10-20$ & 564 & 473 & Unanalyzed & 3 & 0.6 & & & & & & & & & & & \\
\hline Unit $071 \mathrm{SE}^{1 / 4}$ & $40-60$ & 693 & 480 & Analyzed & 1 & 0.5 & LPR & PWS & LOC & CRT & 3 & & $\mathrm{~N}$ & COM & 1 & CRT & 1 \\
\hline Unit $071 \mathrm{SE}^{1 / 4}$ & $20-30$ & 634 & 477 & Analyzed & 1 & 0.5 & LPR & PWS & LOC & CRT & 3 & & $\mathrm{Y}$ & COM & 0 & FLT & 1 \\
\hline Unit $071 \mathrm{SE}^{1 / 4}$ & $10-20$ & 566 & 474 & Unanalyzed & 1 & 0.1 & & & & & & & & & & & \\
\hline Unit $072 \mathrm{NE}^{1 / 4}$ & $40-60$ & 695 & 496 & Analyzed & 1 & 1.9 & LPR & PWS & REG & CRT & 2 & ABS & $\mathrm{N}$ & BRK & 0 & ABR & 1 \\
\hline Unit $072 \mathrm{SE}^{1 / 4}$ & $40-60$ & 696 & 497 & Analyzed & 1 & 0.4 & LPR & PWS & REG & CRT & 4 & & I & FRG & 0 & IND & 1 \\
\hline Unit $072 \mathrm{SE}^{1 / 4}$ & $20-30$ & 639 & 490 & Analyzed & 1 & 0.04 & LPR & PWS & LOC & CRT & 4 & & I & FRG & 0 & IND & 1 \\
\hline Unit $072 \mathrm{SW}^{1 / 4}$ & $30-40$ & 640 & 495 & Analyzed & 1 & 1.6 & LPR & PWS & LOC & QZT & 4 & & I & DEB & 0 & IND & 1 \\
\hline Unit $072 \mathrm{SW}^{1 / 4}$ & $40-60$ & 694 & 498 & Analyzed & 1 & 0.2 & LPR & PWS & LOC & CRT & 4 & & I & FRG & 0 & IND & 1 \\
\hline
\end{tabular}

$\begin{array}{lcccccc}\text { Unit } 073 \mathrm{NW}^{1 / 4} / 4 & 30-40 & 698 & 510 & \text { Unanalyzed } & 3 & 4.3 \\ \text { Unit } 073 \mathrm{NW}^{1 / 4} & 20-30 & 645 & 506 & \text { Unanalyzed } & 1 & 0.1 \\ \text { Unit } 073 \mathrm{NW}^{1 / 4} & 40-50 & 1725 & 513 & \text { Unanalyzed } & 2 & 0.6 \\ \text { Unit } 073 \mathrm{SE}^{1 / 4} & 30-40 & 700 & 511 & \text { Unanalyzed } & 1 & 5\end{array}$


Geo-Marine, Inc.

GMI 22005.01.06.xx and 22005.00.09.xx, Data Recovery Excavations at Site 41PN17 Debitage Analysi

\begin{tabular}{|c|c|c|c|c|c|c|c|c|c|c|c|c|c|c|c|c|c|}
\hline Unit & $\begin{array}{l}\text { Depth } \\
(\mathrm{cm})\end{array}$ & $\begin{array}{l}\text { Provenience } \\
\text { No. }\end{array}$ & $\begin{array}{l}\text { Lot } \\
\text { No. }\end{array}$ & Level of Analysis & Quantity & $\begin{array}{l}\text { Weight } \\
\text { (in g) }\end{array}$ & General Period & Region & Material & Lithology & Size Grade & $\begin{array}{c}\text { Edge } \\
\text { Modification }\end{array}$ & $\begin{array}{l}\text { Thernal } \\
\text { Alteration }\end{array}$ & Completeness & Cortex & Platform & $\begin{array}{l}\text { Minimun } \\
\text { Number c } \\
\text { Nodules }\end{array}$ \\
\hline \multicolumn{18}{|l|}{ Block 4} \\
\hline Unit $073 \mathrm{SW}^{1} / 4$ & $40-50$ & 1724 & 514 & Unanalyzed & 10 & 10 & & & & & & & & & & & \\
\hline Unit $073 \mathrm{SW}^{1 / 4}$ & $20-30$ & 644 & 508 & Unanalyzed & 3 & 1 & & & & & & & & & & & \\
\hline Unit $073 \mathrm{SW}^{1} / 4$ & $50-60$ & 1726 & 516 & Unanalyzed & 4 & 2.9 & & & & & & & & & & & \\
\hline Unit $073 \mathrm{SW}^{1} / 4$ & $30-40$ & 697 & 512 & Unanalyzed & 1 & 0.8 & & & & & & & & & & & \\
\hline Unit $074 \mathrm{NW}^{1 / 4}$ & $40-50$ & 1730 & 530 & Unanalyzed & 1 & 0.3 & & & & & & & & & & & \\
\hline Unit $074 \mathrm{NW}^{1 / 4}$ & $30-40$ & 702 & 527 & Unanalyzed & 2 & 0.4 & & & & & & & & & & & \\
\hline Unit $074 \mathrm{NW}^{1 / 4}$ & $50-60$ & 1732 & 533 & Unanalyzed & 2 & 1.7 & & & & & & & & & & & \\
\hline Unit $074 \mathrm{SE}^{1 / 4}$ & $20-30$ & 651 & 524 & Unanalyzed & 2 & 3.5 & & & & & & & & & & & \\
\hline Unit $074 \mathrm{SE}^{1 / 4}$ & $30-40$ & 704 & 528 & Unanalyzed & 1 & 5 & & & & & & & & & & & \\
\hline Unit $074 \mathrm{SW}^{1} / 4$ & $50-60$ & 1731 & 535 & Unanalyzed & 1 & 0.4 & & & & & & & & & & & \\
\hline Unit $074 \mathrm{SW}^{1} / 4$ & $40-50$ & 1729 & 531 & Unanalyzed & 6 & 2.5 & & & & & & & & & & & \\
\hline Unit $075 \mathrm{NE}^{1 / 4}$ & $30-40$ & 731 & 547 & Analyzed & 1 & 0.4 & LPR & PWS & LOC & CRT & 4 & & I & BRK & 4 & FLT & 1 \\
\hline Unit $075 \mathrm{NE}^{1 / 4}$ & $20-30$ & 657 & 543 & Unanalyzed & 3 & 1.1 & & & & & & & & & & & \\
\hline Unit $075 \mathrm{NW}^{1 / 4}$ & $30-40$ & 730 & 548 & Analyzed & 1 & 0.1 & LPR & PWS & LOC & QZT & 4 & & I & FRG & 0 & IND & 2 \\
\hline Unit $075 \mathrm{NW}^{1 / 4}$ & $30-40$ & 730 & 548 & Analyzed & 1 & 0.8 & LPR & PWS & LOC & CRT & 4 & & I & DEB & 4 & IND & 2 \\
\hline Unit $075 \mathrm{SE}^{1 / 4}$ & $30-40$ & 732 & 549 & Analyzed & 1 & 0.04 & LPR & PWS & LOC & CRT & 5 & & I & СOM & 0 & IND & 1 \\
\hline Unit 075 SE $1 / 4$ & $30-40$ & 732 & 549 & Analyzed & 1 & 0.04 & LPR & PWS & LOC & FWD & 5 & & I & DEB & 0 & IND & 1 \\
\hline Unit $075 \mathrm{SE}^{1} / 4$ & $30-40$ & 732 & 549 & Analyzed & 1 & 1.4 & LPR & PWS & LOC & QZT & 3 & & $\mathrm{~N}$ & FRG & 4 & IND & 1 \\
\hline Unit $075 \mathrm{SE}^{1 / 4}$ & $20-30$ & 658 & 545 & Unanalyzed & 2 & 0.1 & & & & & & & & & & & \\
\hline Unit $075 \mathrm{SW}^{1} / 4$ & $20-30$ & 655 & 546 & Unanalyzed & 2 & 0.4 & & & & & & & & & & & \\
\hline Unit 075 , Feature $10 \mathrm{~N}^{1} / 2$ & $40-50$ & 1808 & 1608 & Analyzed & 1 & 0.04 & LPR & PWS & INT & Usx & 6 & & I & DEB & 0 & IND & 1 \\
\hline Unit 075 , Feature $10 S 1 / 2$ & $40-50$ & 1810 & 1609 & Analyzed & 1 & 0.1 & LPR & PWS & LOC & CRT & 4 & & I & FRG & 1 & IND & 1 \\
\hline Unit 075, Feature 14 E⿺𠃊⿳亠丷厂 & $40-50$ & 1817 & 1613 & Analyzed & 1 & 0.04 & LPR & PWS & LOC & CRT & 5 & & I & COM & 0 & FLT & 1 \\
\hline Unit 075 , Feature $15 \mathrm{~W}^{1 / 2}$ & $40-47$ & 1819 & 1614 & Analyzed & 1 & 0.04 & LPR & PWS & LOC & QZT & 5 & & I & FRG & 1 & IND & 1 \\
\hline Unit 075 , Feature $15 \mathrm{~W}^{1 / 2}$ & $40-47$ & 1819 & 1614 & Analyzed & 1 & 0.04 & LPR & PWS & LOC & CRT & 5 & & I & СОМ & 0 & IND & 1 \\
\hline Unit 075 , Feature $15 \mathrm{~W}^{1 / 2}$ & $40-47$ & 1819 & 1614 & Analyzed & 3 & 0.04 & LPR & PWS & INT & USX & 6 & & I & DEB & 0 & IND & 2 \\
\hline
\end{tabular}


Geo-Marine, Inc.

GMI 22005.01.06.xx and 22005.00.09.xx, Data Recovery Excavations at Site 41PN175 Debitage Analysi

\begin{tabular}{|c|c|c|c|c|c|c|c|c|c|c|c|c|c|c|c|c|c|}
\hline Unit & $\begin{array}{l}\text { Depth } \\
(\mathrm{cm})\end{array}$ & $\begin{array}{l}\text { Provenience } \\
\text { No. }\end{array}$ & $\begin{array}{l}\text { Lot } \\
\text { No. }\end{array}$ & Level of Analysis & Quantity & $\begin{array}{l}\text { Weight } \\
\text { (in g) }\end{array}$ & General Period & Region & Material & Lithology & Size Grade & $\begin{array}{c}\text { Edge } \\
\text { Modification }\end{array}$ & $\begin{array}{l}\text { Thernal } \\
\text { Alteration }\end{array}$ & Completeness & Cortex & Platform & $\begin{array}{l}\text { Minimun } \\
\text { Number c } \\
\text { Nodules }\end{array}$ \\
\hline \multicolumn{18}{|l|}{ Block 4} \\
\hline Unit 075 , Feature $40 S^{1} / 2$ & $40-60$ & 1859 & 1640 & Analyzed & 1 & 0.04 & LPR & PWS & REG & CRT & 5 & & I & COM & 0 & CPX & 1 \\
\hline Unit $076 \mathrm{NE}^{1 / 4}$ & $30-40$ & 735 & 559 & Analyzed & 1 & 0.1 & LPR & PWS & LOC & CRT & 4 & & I & BRK & 0 & FLT & 1 \\
\hline Unit $076 \mathrm{NE}^{1 / 4}$ & $30-40$ & 735 & 559 & Analyzed & 1 & 0.04 & LPR & PWS & LOC & CRT & 5 & & I & COM & 0 & FLT & 1 \\
\hline Unit $076 \mathrm{NE}^{1 / 4}$ & $30-40$ & 735 & 559 & Analyzed & 1 & 0.1 & LPR & PWS & LOC & CRT & 4 & & I & COM & 0 & CPX & 1 \\
\hline Unit $076 \mathrm{NE}^{1 / 4}$ & $30-40$ & 735 & 559 & Analyzed & 1 & 0.3 & LPR & PWS & LOC & CRT & 4 & & I & BRK & 0 & CPX & 1 \\
\hline Unit $076 \mathrm{NE}^{1 / 4}$ & $50-60$ & 1741 & 565 & Analyzed & 1 & 0.8 & LPR & PWS & LOC & CRT & 4 & & I & DEB & 1 & IND & 1 \\
\hline Unit $076 \mathrm{NE}^{1 / 4}$ & $20-30$ & 661 & 555 & Unanalyzed & 2 & 0.4 & & & & & & & & & & & \\
\hline Unit $076 \mathrm{NE}^{1 / 4}$ & $30-40$ & 735 & 559 & Analyzed & 1 & 1.2 & LPR & PWS & LOC & CRT & 4 & & I & FRG & 3 & IND & 1 \\
\hline Unit $076 \mathrm{NE}^{1 / 4}$ & $30-40$ & 735 & 559 & Analyzed & 1 & 0.1 & LPR & PWS & LOC & CRT & 4 & & I & COM & 0 & FLT & 1 \\
\hline Unit $076 \mathrm{NW}^{1 / 4}$ & $20-30$ & 660 & 556 & Unanalyzed & 4 & 3 & & & & & & & & & & & \\
\hline Unit 076 NW1/4 & $30-40$ & 734 & 560 & Analyzed & 1 & 0.04 & LPR & PWS & LOC & CRT & 4 & & I & DEB & 0 & IND & 2 \\
\hline Unit 076 NW1/4 & $30-40$ & 734 & 560 & Analyzed & 1 & 0.2 & LPR & PWS & LOC & CRT & 4 & & I & сом & 3 & CRT & 2 \\
\hline Unit $076 \mathrm{NW}^{1 / 4}$ & $30-40$ & 734 & 560 & Analyzed & 1 & 0.1 & LPR & PWS & LOC & QZT & 4 & & I & COM & 0 & FLT & 2 \\
\hline Unit 076 SE$^{1 / 4}$ & $30-40$ & 736 & 561 & Analyzed & 1 & 0.1 & LPR & PWS & LOC & CRT & 4 & & I & DEB & 0 & IND & 3 \\
\hline Unit $076 \mathrm{SE}^{1 / 4}$ & $30-40$ & 736 & 561 & Analyzed & 2 & 0.2 & LPR & PWS & LOC & CRT & 4 & & I & COM & 0 & CPX & 3 \\
\hline Unit $076 \mathrm{SE}^{1 / 4}$ & $30-40$ & 736 & 561 & Analyzed & 1 & 0.04 & LPR & PWS & REG & CRT & 4 & & I & сом & 0 & FLT & 3 \\
\hline Unit $076 \mathrm{SE}^{1 / 4}$ & $20-30$ & 662 & 557 & Unanalyzed & 4 & 1.6 & & & & & & & & & & & \\
\hline Unit $076 \mathrm{SE}^{1 / 4}$ & $30-40$ & 736 & 561 & Analyzed & 1 & 0.7 & LPR & PWS & LOC & CRT & 4 & & I & COM & 4 & CRT & 3 \\
\hline Unit $076 \mathrm{SE}^{1 / 4}$ & $30-40$ & 736 & 561 & Analyzed & 1 & 0.2 & LPR & PWS & LOC & CRT & 4 & & I & СОМ & 4 & CRT & 3 \\
\hline Unit $076 \mathrm{SE}^{1 / 4}$ & $10-20$ & 586 & 554 & Unanalyzed & 1 & 1.5 & & & & & & & & & & & \\
\hline Unit $076 \mathrm{SW}^{1} / 4$ & $30-40$ & 733 & 562 & Analyzed & 2 & 0.4 & LPR & PWS & LOC & QZT & 4 & & I & FRG & 0 & IND & 1 \\
\hline Unit 076 SW'1/4 & $30-40$ & 733 & 562 & Analyzed & 1 & 0.4 & LPR & PWS & LOC & CRT & 4 & & I & FRG & 0 & $\mathrm{IND}$ & 1 \\
\hline Unit $076 \mathrm{SW}^{1 / 4}$ & $20-30$ & 659 & 558 & Unanalyzed & 4 & 1 & & & & & & & & & & & \\
\hline Unit 076 SW¹/4 & $30-40$ & 733 & 562 & Analyzed & 1 & 0.1 & LPR & PWS & LOC & CRT & 4 & & I & COM & 0 & IND & 1 \\
\hline Unit 076, Feature $13 E^{1 / 2}$ & $40-50$ & 1815 & 1612 & Analyzed & 1 & 0.04 & LPR & PWS & INT & USx & 6 & & I & DEB & 0 & IND & 1 \\
\hline Unit 076 , Feature $9 E^{1} / 2$ & $40-53$ & 1805 & 1606 & Analyzed & 19 & 0.2 & LPR & PWS & INT & USX & 6 & & I & DEB & 0 & IND & 1 \\
\hline
\end{tabular}


Geo-Marine, Inc.

GMI 22005.01.06.xx and 22005.00.09.xx, Data Recovery Excavations at Site 41PN175 Debitage Analysi

\begin{tabular}{|c|c|c|c|c|c|c|c|c|c|c|c|c|c|c|c|c|c|}
\hline Unit & $\begin{array}{l}\text { Depth } \\
(\mathrm{cm})\end{array}$ & $\begin{array}{l}\text { Provenience } \\
\text { No. }\end{array}$ & $\begin{array}{l}\text { Lot } \\
\text { No. }\end{array}$ & Level of Analysis & Quantity & $\begin{array}{l}\text { Weight } \\
\text { (in g) }\end{array}$ & General Period & Region & Material & Lithology & Size Grade & $\begin{array}{c}\text { Edge } \\
\text { Modification }\end{array}$ & $\begin{array}{l}\text { Thernal } \\
\text { Alteration }\end{array}$ & Completeness & Cortex & Platform & $\begin{array}{l}\text { Minimun } \\
\text { Number c } \\
\text { Nodules }\end{array}$ \\
\hline \multicolumn{18}{|l|}{ Block 4} \\
\hline Unit 076 , Feature $9 E^{1} / 2$ & $40-53$ & 1805 & 1606 & Analyzed & 16 & 0.5 & LPR & PWS & LOC & CRT & 5 & & I & FRG & 0 & IND & 1 \\
\hline Unit 076 , Feature 9 W'1/2 & $40-53$ & 2002 & 1766 & Analyzed & 1 & 1.2 & LPR & PWS & LOC & CRT & 4 & & I & COM & 0 & ABR & 1 \\
\hline Unit 076, Feature 9 W'1/2 & $40-53$ & 2002 & 1766 & Analyzed & 1 & 0.4 & LPR & PWS & LOC & CRT & 4 & & I & DEB & 2 & IND & 1 \\
\hline Unit 076, Feature 9 W1⁄2 & $40-53$ & 1807 & 1607 & Analyzed & 10 & 0.1 & LPR & PWS & INT & usx & 6 & & I & DEB & 0 & IND & 1 \\
\hline Unit $077 \mathrm{NE}^{1 / 4}$ & $20-30$ & 707 & 570 & Unanalyzed & 1 & 0.3 & & & & & & & & & & & \\
\hline Unit $077 \mathrm{NE}^{1 / 4}$ & $10-20$ & 665 & 566 & Unanalyzed & 1 & 0.6 & & & & & & & & & & & \\
\hline Unit $077 \mathrm{NW}^{1 / 4}$ & $20-30$ & 706 & 571 & Unanalyzed & 2 & 1.7 & & & & & & & & & & & \\
\hline Unit $077 \mathrm{NW}^{1 / 4}$ & $10-20$ & 664 & 567 & Unanalyzed & 1 & 5 & & & & & & & & & & & \\
\hline Unit $077 \mathrm{SE}^{1 / 4}$ & $10-20$ & 666 & 568 & Unanalyzed & 2 & 1 & & & & & & & & & & & \\
\hline Unit $077 \mathrm{SE}^{1 / 4}$ & $20-30$ & 708 & 572 & Unanalyzed & 1 & 0.5 & & & & & & & & & & & \\
\hline Unit $077 \mathrm{SW}^{1} / 4$ & $10-20$ & 663 & 569 & Unanalyzed & 1 & 0.6 & & & & & & & & & & & \\
\hline Unit $077 \mathrm{SW}^{1 / 4}$ & $30-40$ & 737 & 576 & Analyzed & 1 & 1.6 & LPR & PWS & LOC & CRT & 3 & & $\mathrm{~N}$ & DEB & 2 & IND & 1 \\
\hline Unit $077 \mathrm{SW}^{1 / 4}$ & $20-30$ & 705 & 573 & Unanalyzed & 3 & 3 & & & & & & & & & & & \\
\hline Unit 077, Feature $17 E^{1} / 2$ & $40-52$ & 1822 & 1616 & Analyzed & 1 & 1.5 & LPR & PWS & LOC & CRT & 4 & & I & Сом & 4 & CRT & 1 \\
\hline Unit 077, Feature $17 E^{1} / 2$ & $40-52$ & 1822 & 1616 & Analyzed & 2 & 0.04 & LPR & PWS & INT & Usx & 6 & & I & DEB & 0 & IND & 1 \\
\hline Unit 077, Feature 18 E1² & $40-48$ & 1824 & 1617 & Analyzed & 2 & 0.04 & LPR & PWS & INT & usx & 6 & & I & DEB & 0 & IND & 1 \\
\hline Unit $078 \mathrm{NE}^{1} / 4$ & $20-30$ & 709 & 583 & Unanalyzed & 3 & 0.6 & & & & & & & & & & & \\
\hline Unit $078 \mathrm{NW}^{1 / 4}$ & $30-40$ & 714 & 588 & Analyzed & 1 & 0.5 & LPR & PWS & LOC & QZT & 4 & & I & COM & 0 & CPX & 1 \\
\hline Unit $078 \mathrm{NW}^{1 / 4}$ & $30-40$ & 714 & 588 & Analyzed & 1 & 1.3 & LPR & PWS & LOC & CRT & 3 & & $\mathrm{~N}$ & FRG & 0 & IND & 1 \\
\hline Unit $078 \mathrm{NW}^{1 / 4}$ & $30-40$ & 714 & 588 & Analyzed & 1 & 0.1 & LPR & PWS & LOC & CRT & 4 & & I & СOM & 4 & CRT & 1 \\
\hline Unit $078 \mathrm{NW}^{1 / 4}$ & $30-40$ & 714 & 588 & Analyzed & 1 & 0.1 & LPR & PWS & LOC & CRT & 4 & & I & COM & 1 & CRT & 1 \\
\hline Unit $078 \mathrm{NW}^{1 / 4}$ & $40-50$ & 1744 & 591 & Analyzed & 1 & 0.1 & LPR & PWS & LOC & CRT & 4 & & I & COM & 0 & IND & 1 \\
\hline Unit $078 \mathrm{NW}^{1 / 4}$ & $20-30$ & 672 & 584 & Unanalyzed & 6 & 3 & & & & & & & & & & & \\
\hline Unit $078 \mathrm{SE}^{1 / 4}$ & $20-30$ & 710 & 585 & Unanalyzed & 2 & 0.8 & & & & & & & & & & & \\
\hline Unit $078 \mathrm{SE}^{1 / 4}$ & $30-40$ & 716 & 589 & Analyzed & 1 & 0.3 & LPR & PWS & LOC & CRT & 4 & & I & COM & 1 & CRT & 1 \\
\hline Unit $078 \mathrm{SE}^{1 / 4}$ & $30-40$ & 716 & 589 & Analyzed & 1 & 0.1 & LPR & PWS & LOC & CRT & 4 & & I & DEB & 0 & IND & 1 \\
\hline
\end{tabular}


Geo-Marine, Inc.

GMI 22005.01.06.xx and 22005.00.09.xx, Data Recovery Excavations at Site 41PN17 Debitage Analysi

\begin{tabular}{|c|c|c|c|c|c|c|c|c|c|c|c|c|c|c|c|c|c|}
\hline Unit & $\begin{array}{l}\text { Depth } \\
(\mathrm{cm})\end{array}$ & $\begin{array}{l}\text { Provenience } \\
\text { No. }\end{array}$ & $\begin{array}{l}\text { Lot } \\
\text { No. }\end{array}$ & Level of Analysis & Quantity & $\begin{array}{c}\text { Weight } \\
\text { (ing) }\end{array}$ & General Period & Region & Material & Lithology & Size Grade & $\begin{array}{c}\text { Edge } \\
\text { Modification }\end{array}$ & $\begin{array}{l}\text { Thernal } \\
\text { Alteration }\end{array}$ & Completeness & Cortex & Platform & $\begin{array}{l}\text { Minimum } \\
\text { Number of } \\
\text { Nodules }\end{array}$ \\
\hline \multicolumn{18}{|c|}{ Block 4} \\
\hline Unit 078 SW$^{1} / 4$ & $30-40$ & 713 & 590 & Analyzed & 1 & 1.9 & LPR & PWS & LOC & CRT & 3 & & $\mathrm{~N}$ & COM & 0 & FLT & 2 \\
\hline Unit 078 SW1/4 & $10-20$ & 667 & 582 & Unanalyzed & 1 & 0.2 & & & & & & & & & & & \\
\hline Unit 078 SW'1/4 & $30-40$ & 713 & 590 & Analyzed & 1 & 0.3 & LPR & PWS & LOC & QZT & 4 & & I & FRG & 1 & IND & 2 \\
\hline Unit 078 SW11/4 & $20-30$ & 671 & 586 & Unanalyzed & 1 & 0.1 & & & & & & & & & & & \\
\hline Unit $086 \mathrm{NE}^{1 / 4}$ & $30-40$ & 1421 & 725 & Analyzed & 1 & 0.1 & LPR & PWS & LOC & CRT & 4 & & I & $\mathrm{COM}$ & 0 & FLT & 1 \\
\hline Unit $086 \mathrm{NE}^{1 / 4}$ & $30-40$ & 1421 & 725 & Analyzed & 1 & 2 & LPR & PWS & LOC & QZT & 3 & & $\mathrm{Y}$ & FRG & 4 & IND & 1 \\
\hline Unit $086 \mathrm{NE}^{1 / 4}$ & $20-30$ & 1235 & 721 & Unanalyzed & 1 & 2 & & & & & & & & & & & \\
\hline Unit $086 \mathrm{NE}^{1 / 4}$ & $30-40$ & 1421 & 725 & Analyzed & 1 & 1.6 & LPR & PWS & LOC & CRT & 4 & & I & DEB & 4 & IND & 1 \\
\hline Unit $086 \mathrm{NW}^{1 / 4}$ & $20-30$ & 1234 & 722 & Unanalyzed & 3 & 0.6 & & & & & & & & & & & \\
\hline Unit $086 \mathrm{NW}^{1 / 4}$ & $30-40$ & 1420 & 726 & Analyzed & 1 & 0.3 & LPR & PWS & LOC & CRT & 4 & & I & FRG & 0 & IND & 1 \\
\hline Unit $086 \mathrm{NW}^{1 / 4}$ & $30-40$ & 1420 & 726 & Analyzed & 1 & 1.2 & LPR & PWS & LOC & CRT & 3 & & $\mathrm{~N}$ & COM & 4 & CRT & 1 \\
\hline Unit $086 \mathrm{SE}^{1 / 4}$ & $30-40$ & 1422 & 727 & Analyzed & 1 & 3 & LPR & PWS & LOC & LMS & 2 & $\mathrm{ABS}$ & $\mathrm{N}$ & $\mathrm{COM}$ & 2 & CRT & 1 \\
\hline Unit $086 \mathrm{SE}^{1 / 4}$ & $30-40$ & 1422 & 727 & Analyzed & 2 & 0.2 & LPR & PWS & LOC & CRT & 4 & & I & $\mathrm{COM}$ & 1 & CRT & 1 \\
\hline Unit $086 \mathrm{SE}^{1 / 4}$ & $30-40$ & 1422 & 727 & Analyzed & 1 & 0.04 & LPR & PWS & LOC & CRT & 5 & & I & DEB & 2 & IND & 1 \\
\hline Unit $086 \mathrm{SW}^{1} / 4$ & $30-40$ & 1419 & 728 & Analyzed & 1 & 0.3 & LPR & PWS & LOC & QZT & 4 & & I & DEB & 1 & IND & 2 \\
\hline Unit $086 \mathrm{SW}^{1} / 4$ & $30-40$ & 1419 & 728 & Analyzed & 1 & 0.1 & LPR & PWS & LOC & QZT & 4 & & I & FRG & 0 & IND & 2 \\
\hline Unit $086 \mathrm{SW}^{1 / 4}$ & $40-50$ & 1745 & 733 & Analyzed & 1 & 0.04 & LPR & PWS & LOC & CRT & 4 & & I & BRK & 1 & FLT & 1 \\
\hline Unit 086 SW¹/4 & $50-60$ & 1748 & 735 & Analyzed & 1 & 0.2 & LPR & PWS & LOC & CRT & 4 & & I & FRG & 4 & IND & 1 \\
\hline Unit $087 \mathrm{NE}^{1 / 4}$ & $30-40$ & 1473 & 744 & Analyzed & 2 & 0.2 & LPR & PWS & LOC & CRT & 4 & & I & DEB & 0 & IND & 1 \\
\hline Unit $087 \mathrm{NE}^{1 / 4}$ & $10-20$ & 1122 & 736 & Unanalyzed & 1 & 0.6 & & & & & & & & & & & \\
\hline Unit $087 \mathrm{NE}^{1 / 4}$ & $30-40$ & 1473 & 744 & Analyzed & 1 & 0.2 & LPR & PWS & LOC & CRT & 4 & & I & BRK & 4 & CRT & 1 \\
\hline Unit $087 \mathrm{NE}^{1 / 4}$ & $30-40$ & 1473 & 744 & Analyzed & 1 & 0.3 & LPR & PWS & LOC & CRT & 4 & & I & COM & 4 & CRT & 1 \\
\hline Unit $087 \mathrm{NE}^{1 / 4}$ & $20-30$ & 1255 & 740 & Unanalyzed & 1 & 5 & & & & & & & & & & & \\
\hline Unit $087 \mathrm{NW}^{1 / 4}$ & $30-40$ & 1472 & 745 & Analyzed & 1 & 0.2 & LPR & PWS & LOC & CRT & 4 & & I & $\mathrm{COM}$ & 1 & CRT & 1 \\
\hline Unit $087 \mathrm{NW}^{1 / 4}$ & $30-40$ & 1472 & 745 & Analyzed & 1 & 0.04 & LPR & PWS & LOC & CRT & 4 & & I & COM & 4 & CRT & 1 \\
\hline Unit $087 \mathrm{NW}^{1 / 4}$ & $20-30$ & 1254 & 741 & Unanalyzed & 5 & 1.2 & & & & & & & & & & & \\
\hline
\end{tabular}


Geo-Marine, Inc.

GMI 22005.01.06.xx and 22005.00.09.xx, Data Recovery Excavations at Site 41PN17 Debitage Analysis

\begin{tabular}{|c|c|c|c|c|c|c|c|c|c|c|c|c|c|c|c|c|c|}
\hline Unit & $\begin{array}{l}\text { Depth } \\
(\mathrm{cm})\end{array}$ & $\begin{array}{l}\text { Provenience } \\
\text { No. }\end{array}$ & $\begin{array}{l}\text { Lot } \\
\text { No. }\end{array}$ & Level of Analysis & Quantity & $\begin{array}{l}\text { Weight } \\
\text { (ing) }\end{array}$ & General Period & Region & Material & Lithology & Size Grade & $\begin{array}{c}\text { Edge } \\
\text { Modification }\end{array}$ & $\begin{array}{l}\text { Thernal } \\
\text { Alteration }\end{array}$ & Completeness & Cortex & Platform & $\begin{array}{l}\text { Minimum } \\
\text { Number of } \\
\text { Nodules }\end{array}$ \\
\hline \multicolumn{18}{|l|}{ Block 4} \\
\hline Unit $087 \mathrm{NW}^{1 / 4}$ & $30-40$ & 1472 & 745 & Analyzed & 1 & 0.04 & LPR & PWS & LOC & CRT & 4 & & I & BRK & 0 & $\mathrm{ABR}$ & 1 \\
\hline Unit $087 \mathrm{NW}^{1 / 4}$ & $40-50$ & 1751 & 749 & Analyzed & 1 & 0.04 & LPR & PWS & LOC & CRT & 4 & & I & FRG & 0 & IND & 1 \\
\hline Unit $087 \mathrm{NW}^{1 / 4}$ & $50-60$ & 1754 & 752 & Analyzed & 1 & 0.1 & LPR & PWS & LOC & CRT & 4 & & I & BRK & 1 & CRT & 1 \\
\hline Unit $087 \mathrm{NW}^{1 / 4}$ & $50-60$ & 1754 & 752 & Analyzed & 1 & 1.3 & LPR & PWS & LOC & CRT & 4 & & I & COM & 4 & CRT & 1 \\
\hline Unit $087 \mathrm{NW}^{1 / 4}$ & $10-20$ & 1121 & 737 & Unanalyzed & 2 & 2.7 & & & & & & & & & & & \\
\hline Unit $087 \mathrm{SE}^{1 / 4}$ & $30-40$ & 1474 & 746 & Analyzed & 1 & 0.4 & LPR & PWS & LOC & CRT & 4 & & I & DEB & 3 & IND & 1 \\
\hline Unit $087 \mathrm{SE}^{1 / 4}$ & $20-30$ & 1256 & 742 & Unanalyzed & 3 & 0.5 & & & & & & & & & & & \\
\hline Unit $087 \mathrm{SE}^{1 / 4}$ & $30-40$ & 1474 & 746 & Analyzed & 2 & 0.2 & LPR & PWS & LOC & CRT & 4 & & I & FRG & 1 & IND & 2 \\
\hline Unit $087 \mathrm{SE}^{1 / 4}$ & $30-40$ & 1474 & 746 & Analyzed & 1 & 0.3 & LPR & PWS & LOC & CRT & 4 & & I & BRK & 4 & FLT & 2 \\
\hline Unit $087 \mathrm{SE}^{1 / 4}$ & $30-40$ & 1474 & 746 & Analyzed & 2 & 0.8 & LPR & PWS & LOC & QZT & 4 & & I & DEB & 0 & IND & 2 \\
\hline Unit $087 \mathrm{SE}^{1 / 4}$ & $30-40$ & 1474 & 746 & Analyzed & 1 & 0.3 & LPR & PWS & LOC & CRT & 4 & & I & FRG & 4 & IND & 2 \\
\hline Unit $087 \mathrm{SE}^{1 / 4}$ & $40-50$ & 1753 & 750 & Analyzed & 1 & 0.1 & LPR & PWS & LOC & CRT & 4 & & I & FRG & 0 & IND & 1 \\
\hline Unit $087 \mathrm{SW}^{1 / 4}$ & $10-20$ & 1120 & 739 & Unanalyzed & 1 & 1.6 & & & & & & & & & & & \\
\hline Unit 087 , Feature $6 E^{1} / 2$ & $40-56$ & 1797 & 1601 & Analyzed & 3 & 0.1 & LPR & PWS & LOC & CRT & 5 & & I & FRG & 4 & IND & 3 \\
\hline Unit 087 , Feature $6 E^{1 / 2}$ & $40-56$ & 1797 & 1601 & Analyzed & 2 & 0.04 & LPR & PWS & LOC & CRT & 5 & & I & FRG & 1 & IND & 3 \\
\hline Unit 087 , Feature $6 E^{1} / 2$ & $40-56$ & 1797 & 1601 & Analyzed & 1 & 0.8 & LPR & PWS & LOC & QZT & 4 & & I & FRG & 2 & IND & 3 \\
\hline Unit 087 , Feature $6 E^{1} / 2$ & $40-56$ & 1797 & 1601 & Analyzed & 1 & 0.3 & LPR & PWS & REG & JPR & 4 & & I & COM & 0 & CPX & 3 \\
\hline Unit 087 , Feature $6 E^{1 / 2}$ & $40-56$ & 1797 & 1601 & Analyzed & 2 & 0.6 & LPR & PWS & LOC & CRT & 4 & & I & FRG & 1 & IND & 3 \\
\hline Unit 087, Feature $6 E^{1 / 2}$ & $40-56$ & 1797 & 1601 & Analyzed & 22 & 0.1 & LPR & PWS & LOC & usx & 6 & & I & DEB & 0 & IND & 3 \\
\hline Unit 087 , Feature $6 E^{1} / 2$ & $40-56$ & 1797 & 1601 & Analyzed & 20 & 0.4 & LPR & PWS & LOC & CRT & 5 & & I & FRG & 0 & IND & 3 \\
\hline Unit 087, Feature $6 E^{1} / 2$ & $40-56$ & 1797 & 1601 & Analyzed & 1 & 0.04 & LPR & PWS & LOC & CRT & 4 & & I & FRG & 0 & IND & 3 \\
\hline Unit 087, Feature $6 W^{1} 1 / 2$ & $40-56$ & 1799 & 1602 & Analyzed & 1 & 0.8 & LPR & PWS & LOC & CRT & 4 & & I & DEB & 3 & IND & 1 \\
\hline Unit 087 , Feature 6 W11/2 & $40-56$ & 1799 & 1602 & Analyzed & 7 & 0.1 & LPR & PWS & INT & usx & 6 & & I & DEB & 0 & IND & 1 \\
\hline Unit 087, Feature $6 W^{1} 1 / 2$ & $40-56$ & 1799 & 1602 & Analyzed & 7 & 0.2 & LPR & PWS & LOC & CRT & 5 & & I & FRG & 0 & IND & 1 \\
\hline Unit 087, Feature $7 \mathrm{NE}^{1 / 2}$ & $40-53$ & 1800 & 1603 & Analyzed & 22 & 0.2 & LPR & PWS & INT & usx & 6 & & I & DEB & 0 & IND & 1 \\
\hline Unit 087, Feature $7 \mathrm{NE}^{1} / 2$ & $40-53$ & 1800 & 1603 & Analyzed & 4 & 0.4 & LPR & PWS & LOC & CRT & 4 & & I & FRG & 0 & IND & 1 \\
\hline
\end{tabular}


Geo-Marine, Inc.

GMI 22005.01.06.xx and 22005.00.09.xx, Data Recovery Excavations at Site 41PN17 Debitage Analysi

\begin{tabular}{|c|c|c|c|c|c|c|c|c|c|c|c|c|c|c|c|c|c|}
\hline Unit & $\begin{array}{c}\text { Depth } \\
(\mathrm{cm})\end{array}$ & $\begin{array}{l}\text { Provenience } \\
\text { No. }\end{array}$ & $\begin{array}{l}\text { Lot } \\
\text { No. }\end{array}$ & Level of Analysis & Quantity & $\begin{array}{l}\text { Weight } \\
\text { (in g) }\end{array}$ & General Period & Region & Material & Lithology & Size Grade & $\begin{array}{c}\text { Edge } \\
\text { Modification }\end{array}$ & $\begin{array}{l}\text { Thernal } \\
\text { Alteration }\end{array}$ & Completeness & Cortex & Platform & $\begin{array}{l}\text { Minimu } \\
\text { Number } \\
\text { Nodul }\end{array}$ \\
\hline \multicolumn{18}{|l|}{ Block 4} \\
\hline Unit 087, Feature $7 \mathrm{NE}^{1 / 2}$ & $40-53$ & 1800 & 1603 & Analyzed & 21 & 0.5 & LPR & PWS & LOC & CRT & 5 & & I & FRG & 0 & IND & 1 \\
\hline Unit 087, Feature $7 \mathrm{NE}^{1 / 2}$ & $40-53$ & 1800 & 1603 & Analyzed & 4 & 0.8 & LPR & PWS & LOC & CRT & 4 & & I & COM & 1 & CRT & 1 \\
\hline Unit 087, Feature $7 \mathrm{NE}^{1 / 2}$ & $40-53$ & 1800 & 1603 & Analyzed & 3 & 0.2 & LPR & PWS & LOC & QZT & 4 & & I & FRG & 0 & IND & 1 \\
\hline Unit 087, Feature $7 \mathrm{SW}^{1 / 2}$ & $40-53$ & 1802 & 1604 & Analyzed & 1 & 0.1 & LPR & PWS & LOC & CRT & 4 & & I & Сом & 4 & CRT & 1 \\
\hline Unit 087, Feature $7 \mathrm{SW}^{1 / 2}$ & $40-53$ & 1802 & 1604 & Analyzed & 20 & 0.1 & LPR & PWS & INT & usx & 6 & & I & DEB & 0 & IND & 1 \\
\hline Unit 087, Feature $7 \mathrm{SW}^{1} / 2$ & $40-53$ & 1802 & 1604 & Analyzed & 16 & 0.4 & LPR & PWS & LOC & CRT & 5 & & I & FRG & 0 & IND & 1 \\
\hline Unit $088 \mathrm{NE}^{1 / 4}$ & $30-40$ & 1478 & 761 & Analyzed & 1 & 1.2 & LPR & PWS & LOC & CRT & 3 & & $\mathrm{~N}$ & DEB & 4 & IND & 1 \\
\hline Unit $088 \mathrm{NE}^{1 / 4}$ & $20-30$ & 1259 & 757 & Unanalyzed & 1 & 0.1 & & & & & & & & & & & \\
\hline Unit $088 \mathrm{NE}^{1 / 4}$ & $30-40$ & 1478 & 761 & Analyzed & 1 & 0.4 & LPR & PWS & LOC & CRT & 4 & & I & COM & 1 & FLT & 1 \\
\hline Unit $088 \mathrm{NE}^{1 / 4}$ & $40-50$ & 1756 & 797 & Analyzed & 1 & 0.9 & LPR & PWS & LOC & FWD & 3 & & $\mathrm{~N}$ & $\mathrm{COM}$ & 0 & FLT & 1 \\
\hline Unit $088 \mathrm{NW}^{1 / 4}$ & $30-40$ & 1477 & 792 & Analyzed & 1 & 0.5 & LPR & PWS & LOC & CRT & 4 & & I & COM & 2 & FLT & 1 \\
\hline Unit $088 \mathrm{NW}^{1 / 4}$ & $10-20$ & 1126 & 754 & Unanalyzed & 1 & 1.2 & & & & & & & & & & & \\
\hline Unit $088 \mathrm{NW}^{1 / 4}$ & $40-50$ & 1755 & 798 & Analyzed & 1 & 0.3 & LPR & PWS & LOC & CRT & 4 & & I & COM & 0 & $\mathrm{ABR}$ & 1 \\
\hline Unit $088 \mathrm{NW}^{1 / 4}$ & $20-30$ & 1258 & 758 & Unanalyzed & 2 & 2.7 & & & & & & & & & & & \\
\hline Unit $088 \mathrm{NW}^{1 / 4}$ & $30-40$ & 1477 & 792 & Analyzed & 1 & 0.04 & LPR & PWS & LOC & CRT & 4 & & I & COM & 0 & CPX & 1 \\
\hline Unit $088 \mathrm{NW}^{1 / 4}$ & $30-40$ & 1477 & 792 & Analyzed & 1 & 0.1 & LPR & PWS & LOC & CRT & 4 & & I & COM & 0 & FLT & 1 \\
\hline Unit $088 \mathrm{NW}^{1 / 4}$ & $30-40$ & 1477 & 792 & Analyzed & 1 & 0.8 & LPR & PWS & LOC & CRT & 4 & & I & DEB & 0 & IND & 1 \\
\hline Unit $088 \mathrm{NW}^{1 / 4}$ & $30-40$ & 1477 & 792 & Analyzed & 2 & 0.2 & LPR & PWS & LOC & CRT & 4 & & I & COM & 0 & CRT & 1 \\
\hline Unit $088 \mathrm{SE}^{1 / 4}$ & $30-40$ & 1479 & 793 & Analyzed & 2 & 0.8 & LPR & PWS & LOC & CRT & 4 & & I & COM & 0 & FLT & 1 \\
\hline Unit $088 \mathrm{SE}^{1} / 4$ & $30-40$ & 1479 & 793 & Analyzed & 2 & 0.4 & LPR & PWS & LOC & CRT & 4 & & I & COM & 1 & CRT & 1 \\
\hline Unit $088 \mathrm{SE}^{1 / 4}$ & $10-20$ & 1128 & 755 & Unanalyzed & 3 & 3.5 & & & & & & & & & & & \\
\hline Unit $088 \mathrm{SW}^{1 / 4}$ & $20-30$ & 1257 & 760 & Unanalyzed & 2 & 3.2 & & & & & & & & & & & \\
\hline Unit $088 \mathrm{SW}^{1 / 4}$ & $30-40$ & 1476 & 795 & Analyzed & 2 & 0.6 & LPR & PWS & LOC & CRT & 4 & & I & $\mathrm{COM}$ & 1 & FLT & 2 \\
\hline Unit $088 \mathrm{SW}^{1 / 4}$ & $30-40$ & 1476 & 795 & Analyzed & 1 & 0.8 & LPR & PWS & LOC & CRT & 4 & & I & FRG & 0 & IND & 2 \\
\hline Unit $088 \mathrm{SW}^{1 / 4}$ & $10-20$ & 1125 & 756 & Unanalyzed & 1 & 0.3 & & & & & & & & & & & \\
\hline Unit $088 \mathrm{SW}^{1 / 4}$ & $30-40$ & 1476 & 795 & Analyzed & 1 & 0.3 & LPR & PWS & LOC & QZT & 4 & & I & FRG & 1 & IND & 2 \\
\hline
\end{tabular}


Geo-Marine, Inc.

GMI 22005.01.06.xx and 22005.00.09.xx, Data Recovery Excavations at Site 41PN175 Debitage Analysis

\begin{tabular}{|c|c|c|c|c|c|c|c|c|c|c|c|c|c|c|c|c|c|}
\hline Unit & $\begin{array}{l}\text { Depth } \\
(\mathrm{cm})\end{array}$ & $\begin{array}{l}\text { Provenience } \\
\text { No. }\end{array}$ & $\begin{array}{l}\text { Lot } \\
\text { No. }\end{array}$ & Level of Analysis & Quantity & $\begin{array}{l}\text { Weight } \\
\text { (in g) }\end{array}$ & General Period & Region & Material & Lithology & Size Grade & $\begin{array}{c}\text { Edge } \\
\text { Modification }\end{array}$ & $\begin{array}{l}\text { Thernal } \\
\text { Alteration }\end{array}$ & Completeness & Cortex & Platform & $\begin{array}{l}\text { Minimum } \\
\text { Number of } \\
\text { Nodules }\end{array}$ \\
\hline \multicolumn{18}{|c|}{ Block 4} \\
\hline Unit 088 SW$^{1 / 4}$ & $30-40$ & 1476 & 795 & Analyzed & 1 & 2.5 & LPR & PWS & LOC & QZT & 3 & & $\mathrm{Y}$ & FRG & 1 & IND & 2 \\
\hline Unit 088 SW$^{1 / 4}$ & $50-60$ & 1758 & 799 & Analyzed & 1 & 0.04 & LPR & PWS & LOC & CRT & 5 & & I & FRG & 0 & $\mathrm{IND}$ & 1 \\
\hline Unit 088 SW11/4 & $30-40$ & 1476 & 795 & Analyzed & 1 & 0.1 & LPR & PWS & LOC & CRT & 4 & & I & COM & 0 & CPX & 2 \\
\hline Unit 089 NE $1 / 4$ & $27-30$ & 1365 & 811 & Analyzed & 1 & 0.3 & LPR & PWS & LOC & CRT & 4 & & I & COM & 4 & FLT & 1 \\
\hline Unit $089 \mathrm{NE}^{1 / 4}$ & $30-40$ & 1425 & 815 & Analyzed & 1 & 0.2 & LPR & PWS & LOC & CRT & 4 & & I & $\mathrm{COM}$ & 0 & FLT & 1 \\
\hline Unit $089 \mathrm{NE}^{1 / 4}$ & $27-30$ & 1365 & 811 & Analyzed & 1 & 0.1 & LPR & PWS & LOC & CRT & 4 & & I & COM & 0 & CPX & 1 \\
\hline Unit $089 \mathrm{NE}^{1 / 4}$ & $27-30$ & 1365 & 811 & Analyzed & 1 & 0.1 & LPR & PWS & LOC & CRT & 4 & & I & FRG & 1 & $\mathrm{IND}$ & 1 \\
\hline Unit $089 \mathrm{NE}^{1 / 4}$ & $27-30$ & 1365 & 811 & Analyzed & 1 & 1 & LPR & PWS & LOC & CRT & 4 & & I & FRG & 4 & $\mathrm{IND}$ & 1 \\
\hline Unit 089 NE¹/4 & $27-30$ & 1365 & 811 & Analyzed & 1 & 0.1 & LPR & PWS & LOC & CRT & 4 & & I & СOM & 0 & FLT & 1 \\
\hline Unit 089 NE¹/4 & $27-30$ & 1365 & 811 & Analyzed & 1 & 0.3 & LPR & PWS & LOC & QZT & 4 & & I & COM & 0 & FLT & 1 \\
\hline Unit 089 NE/4 & $20-27$ & 1263 & 807 & Unanalyzed & 2 & 0.5 & & & & & & & & & & & \\
\hline Unit $089 \mathrm{NW}^{1 / 4}$ & $30-40$ & 1424 & 816 & Analyzed & 1 & 0.5 & LPR & PWS & LOC & CRT & 3 & & $\mathrm{Y}$ & COM & 0 & ABR & 1 \\
\hline Unit $089 \mathrm{NW}^{1 / 4}$ & $20-27$ & 1262 & 808 & Unanalyzed & 2 & 0.6 & & & & & & & & & & & \\
\hline Unit $089 \mathrm{NW}^{1 / 4}$ & $27-30$ & 1364 & 812 & Analyzed & 1 & 0.1 & LPR & PWS & LOC & CRT & 4 & & I & FRG & 0 & $\mathrm{IND}$ & 2 \\
\hline Unit $089 \mathrm{NW}^{1 / 4}$ & $27-30$ & 1364 & 812 & Analyzed & 1 & 0.1 & LPR & PWS & REG & $\mathrm{NVC}$ & 4 & & I & DEB & 0 & IND & 2 \\
\hline Unit $089 \mathrm{SE}^{1 / 4}$ & $10-20$ & 1135 & 805 & Unanalyzed & 1 & 0.3 & & & & & & & & & & & \\
\hline Unit $089 \mathrm{SE}^{1} / 4$ & $0-10$ & 1131 & 801 & Unanalyzed & 1 & 0.3 & & & & & & & & & & & \\
\hline Unit $089 \mathrm{SE}^{1 / 4}$ & $27-30$ & 1366 & 813 & Analyzed & 2 & 0.6 & LPR & PWS & LOC & CRT & 4 & & I & COM & 0 & FLT & 2 \\
\hline Unit $089 \mathrm{SE}^{1 / 4}$ & $27-30$ & 1366 & 813 & Analyzed & 1 & 0.1 & LPR & PWS & LOC & QZT & 4 & & I & FRG & 0 & $\mathrm{IND}$ & 2 \\
\hline Unit 089 SW11/4 & $20-27$ & 1261 & 810 & Unanalyzed & 1 & 0.4 & & & & & & & & & & & \\
\hline Unit 089 SW11/4 & $30-40$ & 1423 & 818 & Analyzed & 1 & 0.4 & LPR & PWS & LOC & CRT & 4 & & I & FRG & 1 & $\mathrm{IND}$ & 1 \\
\hline Unit $090 \mathrm{NE}^{1 / 4}$ & $20-27$ & 1267 & 825 & Unanalyzed & 5 & 5.1 & & & & & & & & & & & \\
\hline Unit 090 NE $1 / 4$ & $27-30$ & 1369 & 829 & Analyzed & 1 & 0.1 & LPR & PWS & LOC & CRT & 4 & & I & FRG & 0 & $\mathrm{IND}$ & 2 \\
\hline Unit 090 NE $1 / 4$ & $27-30$ & 1369 & 829 & Analyzed & 1 & 0.1 & LPR & PWS & LOC & CRT & 4 & & I & BRK & 0 & FLT & 2 \\
\hline Unit $090 \mathrm{NE}^{1 / 4}$ & $30-40$ & 1484 & 833 & Analyzed & 1 & 0.04 & LPR & PWS & LOC & LMS & 4 & & I & FRG & 0 & $\mathrm{IND}$ & 1 \\
\hline Unit $090 \mathrm{NE}^{1 / 4}$ & $30-40$ & 1484 & 833 & Analyzed & 2 & 0.2 & LPR & PWS & LOC & CRT & 4 & & I & COM & 0 & IND & 1 \\
\hline
\end{tabular}


Geo-Marine, Inc.

GMI 22005.01.06.xx and 22005.00.09.xx, Data Recovery Excavations at Site 41PN17 Debitage Analysi

\begin{tabular}{|c|c|c|c|c|c|c|c|c|c|c|c|c|c|c|c|c|c|}
\hline Unit & $\begin{array}{l}\text { Depth } \\
(\mathrm{cm})\end{array}$ & $\begin{array}{l}\text { Provenience } \\
\text { No. }\end{array}$ & $\begin{array}{l}\text { Lot } \\
\text { No. }\end{array}$ & Level of Analysis & Quantity & $\begin{array}{l}\text { Weight } \\
\text { (ing) }\end{array}$ & General Period & Region & Material & Lithology & Size Grade & $\begin{array}{c}\text { Edge } \\
\text { Modification }\end{array}$ & $\begin{array}{l}\text { Thernal } \\
\text { Alteration }\end{array}$ & Completeness & Cortex & Platform & $\begin{array}{l}\text { Minimum } \\
\text { Number of } \\
\text { Nodules }\end{array}$ \\
\hline \multicolumn{18}{|c|}{ Block 4} \\
\hline Unit $090 \mathrm{NE}^{1 / 4}$ & $27-30$ & 1369 & 829 & Analyzed & 1 & 0.1 & LPR & PWS & LOC & CRT & 4 & & I & BRK & 4 & FLT & 2 \\
\hline Unit $090 \mathrm{NE}^{1 / 4}$ & $30-40$ & 1484 & 833 & Analyzed & 2 & 0.5 & LPR & PWS & LOC & CRT & 4 & & I & FRG & 1 & IND & 1 \\
\hline Unit $090 \mathrm{NE}^{1 / 4}$ & $30-40$ & 1484 & 833 & Analyzed & 3 & 1 & LPR & PWS & LOC & QZT & 4 & & I & DEB & 1 & IND & 1 \\
\hline Unit $090 \mathrm{NW}^{1 / 4}$ & $30-40$ & 1483 & 834 & Analyzed & 1 & 0.1 & LPR & PWS & LOC & QZT & 4 & & I & COM & 0 & FLT & 3 \\
\hline Unit $090 \mathrm{NW}^{1 / 4}$ & $30-40$ & 1483 & 834 & Analyzed & 1 & 6.6 & LPR & PWS & LOC & QZT & 2 & ABS & $\mathrm{N}$ & DEB & 4 & IND & 3 \\
\hline Unit $090 \mathrm{NW}^{1 / 4}$ & $30-40$ & 1483 & 834 & Analyzed & 1 & 0.1 & LPR & PWS & LOC & QZT & 4 & & I & DEB & 0 & IND & 3 \\
\hline Unit $090 \mathrm{NW}^{1 / 4}$ & $30-40$ & 1483 & 834 & Analyzed & 1 & 0.6 & LPR & PWS & LOC & CRT & 4 & & I & $\mathrm{COM}$ & 4 & CRT & 3 \\
\hline Unit $090 \mathrm{SE}^{1 / 4}$ & $27-30$ & 1370 & 831 & Analyzed & 1 & 0.04 & LPR & PWS & LOC & CRT & 4 & & I & FRG & 4 & IND & 1 \\
\hline Unit $090 \mathrm{SE}^{1 / 4}$ & $30-40$ & 1485 & 835 & Analyzed & 2 & 0.9 & LPR & PWS & LOC & CRT & 4 & & I & FRG & 0 & IND & 2 \\
\hline Unit $090 \mathrm{SE}^{1 / 4}$ & $10-20$ & 1140 & 822 & Unanalyzed & 2 & 1.5 & & & & & & & & & & & \\
\hline Unit $090 \mathrm{SE}^{1 / 4}$ & $30-40$ & 1485 & 835 & Analyzed & 1 & 0.3 & LPR & PWS & LOC & CRT & 4 & & I & BRK & 0 & CPX & 2 \\
\hline Unit $090 \mathrm{SE}^{1 / 4}$ & $30-40$ & 1485 & 835 & Analyzed & 1 & 0.1 & LPR & PWS & LOC & ISS & 4 & & I & FRG & 0 & IND & 2 \\
\hline Unit $090 \mathrm{SE}^{1 / 4}$ & $30-40$ & 1485 & 835 & Analyzed & 1 & 1.1 & LPR & PWS & LOC & CRT & 3 & & $\mathrm{~N}$ & $\mathrm{COM}$ & 1 & FLT & 2 \\
\hline Unit $090 \mathrm{SE}^{1 / 4}$ & $30-40$ & 1485 & 835 & Analyzed & 1 & 0.04 & LPR & PWS & LOC & CRT & 4 & & I & COM & 4 & CRT & 2 \\
\hline Unit $090 \mathrm{SE}^{1 / 4}$ & $27-30$ & 1370 & 831 & Analyzed & 1 & 0.4 & LPR & PWS & LOC & CRT & 4 & & I & DEB & 1 & IND & 1 \\
\hline Unit $090 \mathrm{SE}^{1 / 4}$ & $20-27$ & 1268 & 827 & Unanalyzed & 3 & 1.7 & & & & & & & & & & & \\
\hline Unit $090 \mathrm{SW}^{1 / 4}$ & $27-30$ & 1367 & 832 & Analyzed & 1 & 1.3 & LPR & PWS & LOC & CRT & 3 & & $\mathrm{~N}$ & FRG & 1 & IND & 2 \\
\hline Unit $090 \mathrm{SW}^{1 / 4}$ & $30-40$ & 1482 & 836 & Analyzed & 1 & 0.04 & LPR & PWS & REG & CRT & 5 & & I & COM & 0 & CPX & 3 \\
\hline Unit $090 \mathrm{SW}^{1 / 4}$ & $30-40$ & 1482 & 836 & Analyzed & 1 & 0.1 & LPR & PWS & LOC & CRT & 4 & & I & СОм & 1 & $\mathrm{ABR}$ & 3 \\
\hline Unit $090 \mathrm{SW}^{1 / 4}$ & $30-40$ & 1482 & 836 & Analyzed & 1 & 0.1 & LPR & PWS & LOC & CRT & 4 & & I & COM & 2 & FLT & 3 \\
\hline Unit $090 \mathrm{SW}^{1 / 4}$ & $30-40$ & 1482 & 836 & Analyzed & 1 & 0.1 & LPR & PWS & LOC & CRT & 4 & & I & BRK & 1 & CPX & 3 \\
\hline Unit $090 \mathrm{SW}^{1 / 4}$ & $10-20$ & 1137 & 823 & Unanalyzed & 4 & 1.8 & & & & & & & & & & & \\
\hline Unit $090 \mathrm{SW}^{1 / 4}$ & $30-40$ & 1482 & 836 & Analyzed & 1 & 0.1 & LPR & PWS & LOC & CRT & 4 & & I & FRG & 4 & IND & 3 \\
\hline Unit $090 \mathrm{SW}^{1 / 4}$ & $30-40$ & 1482 & 836 & Analyzed & 1 & 0.1 & LPR & PWS & LOC & CRT & 4 & & I & FRG & 1 & IND & 3 \\
\hline Unit $090 \mathrm{SW}^{1 / 4}$ & $40-50$ & 1760 & 842 & Analyzed & 1 & 0.3 & LPR & PWS & LOC & CRT & 4 & & I & DEB & 1 & IND & 1 \\
\hline Unit $090 \mathrm{SW}^{1} / 4$ & $30-40$ & 1482 & 836 & Analyzed & 2 & 0.8 & LPR & PWS & LOC & CRT & 4 & & I & FRG & 0 & IND & 3 \\
\hline
\end{tabular}


Geo-Marine, Inc.

GMI 22005.01.06.xx and 22005.00.09.xx, Data Recovery Excavations at Site 41PN175 Debitage Analysi

\begin{tabular}{|c|c|c|c|c|c|c|c|c|c|c|c|c|c|c|c|c|c|}
\hline Unit & $\begin{array}{l}\text { Depth } \\
\text { (cm) }\end{array}$ & $\begin{array}{l}\text { Provenience } \\
\text { No. }\end{array}$ & $\begin{array}{l}\text { Lot } \\
\text { No. }\end{array}$ & Level of Analysis & Quantity & $\begin{array}{l}\text { Weight } \\
\text { (ing) }\end{array}$ & General Period & Region & Material & Lithology & Size Grade & $\begin{array}{c}\text { Edge } \\
\text { Modification }\end{array}$ & $\begin{array}{l}\text { Thernal } \\
\text { Alteration }\end{array}$ & Completeness & Cortex & Platform & $\begin{array}{l}\text { Minimum } \\
\text { Number of } \\
\text { Nodules }\end{array}$ \\
\hline \multicolumn{18}{|l|}{ Block 4} \\
\hline Unit $090 \mathrm{SW}^{1} / 4$ & $30-40$ & 1482 & 836 & Analyzed & 1 & 0.3 & LPR & PWS & LOC & CRT & 4 & & I & COM & 4 & CRT & 3 \\
\hline Unit $090 \mathrm{SW}^{1} / 4$ & $30-40$ & 1482 & 836 & Analyzed & 1 & 0.4 & LPR & PWS & LOC & CRT & 4 & & I & DEB & 0 & IND & 3 \\
\hline Unit $090 \mathrm{SW}^{1} / 4$ & $30-40$ & 1482 & 836 & Analyzed & 1 & 0.9 & LPR & PWS & LOC & FWD & 3 & & $\mathrm{~N}$ & COM & 0 & FLT & 3 \\
\hline Unit $090 \mathrm{SW}^{1} / 4$ & $30-40$ & 1482 & 836 & Analyzed & 1 & 0.5 & LPR & PWS & LOC & CRT & 4 & & I & FRG & 2 & IND & 3 \\
\hline Unit $090 \mathrm{SW}^{1} / 4$ & $27-30$ & 1367 & 832 & Analyzed & 1 & 0.2 & LPR & PWS & LOC & CRT & 4 & & I & COM & 2 & CRT & 2 \\
\hline Unit $090 \mathrm{SW}^{1} / 4$ & $27-30$ & 1367 & 832 & Analyzed & 1 & 0.04 & LPR & PWS & LOC & CRT & 4 & & I & FRG & 0 & IND & 2 \\
\hline Unit $090 \mathrm{SW}^{1} / 4$ & $30-40$ & 1482 & 836 & Analyzed & 1 & 2.4 & LPR & PWS & REG & CRT & 3 & & $\mathrm{~N}$ & FRG & 0 & IND & 3 \\
\hline Unit 090, Feature $12 \mathrm{E}^{1} / 2$ & $42-51$ & 1813 & 1611 & Analyzed & 1 & 0.04 & LPR & PWS & INT & Usx & 6 & & I & DEB & 0 & IND & 1 \\
\hline Unit $091 \mathrm{NE}^{1 / 4}$ & $30-40$ & 1490 & 857 & Analyzed & 1 & 0.8 & LPR & PWS & LOC & LMS & 4 & & I & СOM & 1 & CRT & 2 \\
\hline Unit $091 \mathrm{NE}^{1} / 4$ & $30-40$ & 1490 & 857 & Analyzed & 1 & 0.5 & LPR & PWS & LOC & CRT & 4 & & I & FRG & 0 & IND & 2 \\
\hline Unit $091 \mathrm{NE}^{1 / 4}$ & $30-40$ & 1490 & 857 & Analyzed & 1 & 0.2 & LPR & PWS & LOC & CRT & 4 & & I & FRG & 0 & IND & 2 \\
\hline Unit $091 \mathrm{NE}^{1 / 4}$ & $30-40$ & 1490 & 857 & Analyzed & 1 & 0.04 & LPR & PWS & LOC & CRT & 5 & & I & COM & 0 & FLT & 2 \\
\hline Unit $091 \mathrm{NE}^{1 / 4}$ & $28-30$ & 1373 & 853 & Analyzed & 1 & 0.1 & LPR & PWS & LOC & CRT & 5 & & I & FRG & 3 & IND & 1 \\
\hline Unit $091 \mathrm{NW}^{1 / 4}$ & $20-28$ & 1271 & 850 & Unanalyzed & 4 & 1.1 & & & & & & & & & & & \\
\hline Unit $091 \mathrm{NW}^{1 / 4}$ & $30-40$ & 1489 & 858 & Analyzed & 1 & 0.1 & LPR & PWS & LOC & CRT & 4 & & I & BRK & 2 & FLT & 2 \\
\hline Unit $091 \mathrm{NW}^{1 / 4}$ & $30-40$ & 1489 & 858 & Analyzed & 1 & 0.04 & LPR & PWS & LOC & CRT & 4 & & I & COM & 0 & FLT & 2 \\
\hline Unit 091 NW'1/4 & $30-40$ & 1489 & 858 & Analyzed & 1 & 3.5 & LPR & PWS & LOC & FWD & 3 & & $\mathrm{~N}$ & COM & 4 & CRT & 2 \\
\hline Unit $091 \mathrm{NW}^{1} / 4$ & $40-50$ & 1764 & 861 & Analyzed & 1 & 0.3 & LPR & PWS & LOC & QZT & 4 & & I & COM & 0 & FLT & 1 \\
\hline Unit $091 \mathrm{NW}^{1 / 4}$ & $30-40$ & 1489 & 858 & Analyzed & 1 & 0.1 & LPR & PWS & LOC & CRT & 4 & & I & FRG & 0 & IND & 2 \\
\hline Unit $091 \mathrm{SE}^{1 / 4}$ & $28-30$ & 1374 & 855 & Analyzed & 2 & 0.1 & LPR & PWS & LOC & CRT & 4 & & I & COM & 0 & $\mathrm{CPX}$ & 1 \\
\hline Unit $091 \mathrm{SE}^{1 / 4}$ & $30-40$ & 1491 & 859 & Analyzed & 1 & 0.6 & LPR & PWS & LOC & CRT & 4 & & I & DEB & 0 & IND & 1 \\
\hline Unit $091 \mathrm{SE}^{1 / 4}$ & $30-40$ & 1491 & 859 & Analyzed & 1 & 0.9 & LPR & PWS & LOC & FWD & 4 & & I & BRK & 0 & CPX & 1 \\
\hline Unit $091 \mathrm{SE}^{1} / 4$ & $30-40$ & 1491 & 859 & Analyzed & 1 & 0.3 & LPR & PWS & LOC & CRT & 4 & & I & COM & 4 & CRT & 1 \\
\hline Unit $091 \mathrm{SE}^{1 / 4}$ & $20-28$ & 1273 & 851 & Unanalyzed & 1 & 0.6 & & & & & & & & & & & \\
\hline Unit 091 SE $1 / 4$ & $28-30$ & 1374 & 855 & Analyzed & 2 & 0.1 & LPR & PWS & LOC & CRT & 5 & & I & FRG & 0 & IND & 1 \\
\hline Unit $091 \mathrm{SW}^{1 / 4}$ & $10-20$ & 1142 & 848 & Unanalyzed & 1 & 0.4 & & & & & & & & & & & \\
\hline
\end{tabular}


Geo-Marine, Inc.

GMI 22005.01.06.xx and 22005.00.09.xx, Data Recovery Excavations at Site 41PN175 Debitage Analysi

\begin{tabular}{|c|c|c|c|c|c|c|c|c|c|c|c|c|c|c|c|c|c|}
\hline Unit & $\begin{array}{c}\text { Depth } \\
(\mathrm{cm})\end{array}$ & $\begin{array}{l}\text { Provenience } \\
\text { No. }\end{array}$ & $\begin{array}{l}\text { Lot } \\
\text { No. }\end{array}$ & Level of Analysis & Quantity & $\begin{array}{l}\text { Weight } \\
\text { (ing) }\end{array}$ & General Period & Region & Material & Lithology & Size Grade & $\begin{array}{c}\text { Edge } \\
\text { Modification }\end{array}$ & $\begin{array}{l}\text { Thernal } \\
\text { Alteration }\end{array}$ & Completeness & Cortex & Platform & $\begin{array}{l}\text { Minimun } \\
\text { Number o } \\
\text { Nodules }\end{array}$ \\
\hline \multicolumn{18}{|c|}{ Block 4} \\
\hline Unit $091 \mathrm{SW}^{1 / 4}$ & $30-40$ & 1488 & 860 & Analyzed & 1 & 1 & LPR & PWS & LOC & CRT & 4 & & I & DEB & 4 & IND & 1 \\
\hline Unit $091 \mathrm{SW}^{1 / 4}$ & $20-28$ & 1270 & 852 & Unanalyzed & 1 & 0.4 & & & & & & & & & & & \\
\hline Unit $091 \mathrm{SW}^{1 / 4}$ & $30-40$ & 1488 & 860 & Analyzed & 2 & 0.2 & LPR & PWS & LOC & CRT & 4 & & I & FRG & 0 & IND & 1 \\
\hline Unit $091 \mathrm{SW}^{1 / 4}$ & $40-50$ & 1997 & 863 & Analyzed & 1 & 0.1 & LPR & PWS & LOC & CRT & 4 & & I & COM & 0 & CPX & 1 \\
\hline Unit $092 \mathrm{NE}^{1 / 4}$ & $28-38$ & 1239 & 872 & Analyzed & 1 & 0.2 & LPR & PWS & LOC & CRT & 4 & & I & FRG & 1 & IND & 1 \\
\hline Unit $092 \mathrm{NW}^{1 / 4}$ & $28-38$ & 1238 & 873 & Analyzed & 1 & 0.4 & LPR & PWS & LOC & CRT & 4 & & I & COM & 0 & FLT & 2 \\
\hline Unit $092 \mathrm{NW}^{1 / 4}$ & $28-38$ & 1238 & 873 & Analyzed & 2 & 0.7 & LPR & PWS & LOC & CRT & 4 & & I & DEB & 2 & IND & 2 \\
\hline Unit $092 \mathrm{NW}^{1 / 4}$ & $28-38$ & 1238 & 873 & Analyzed & 1 & 0.4 & LPR & PWS & LOC & CRT & 4 & & I & COM & 4 & FLT & 2 \\
\hline Unit $092 \mathrm{NW}^{1 / 4}$ & $28-38$ & 1238 & 873 & Analyzed & 1 & 0.3 & LPR & PWS & LOC & CRT & 4 & & I & DEB & 4 & IND & 2 \\
\hline Unit $092 \mathrm{SE}^{1 / 4}$ & $18-28$ & 1149 & 870 & Unanalyzed & 2 & 3.7 & & & & & & & & & & & \\
\hline Unit $092 \mathrm{SE}^{1 / 4}$ & $28-38$ & 1240 & 874 & Analyzed & 1 & 1.2 & LPR & PWS & LOC & CRT & 3 & & $\mathrm{~N}$ & FRG & 0 & IND & 1 \\
\hline Unit $092 \mathrm{SE}^{1 / 4}$ & $28-38$ & 1240 & 874 & Analyzed & 2 & 0.7 & LPR & PWS & LOC & CRT & 4 & & I & $\mathrm{COM}$ & 1 & CRT & 1 \\
\hline Unit $092 \mathrm{SW}^{1 / 4}$ & $18-28$ & 1146 & 871 & Unanalyzed & 2 & 2.7 & & & & & & & & & & & \\
\hline Unit $092 \mathrm{SW}^{1 / 4}$ & $28-38$ & 1237 & 875 & Analyzed & 2 & 0.1 & LPR & PWS & LOC & CRT & 4 & & I & DEB & 0 & IND & 1 \\
\hline Unit $092 \mathrm{SW}^{1 / 4}$ & $28-38$ & 1237 & 875 & Analyzed & 1 & 6.4 & LPR & PWS & LOC & CRT & 2 & ABS & $\mathrm{N}$ & COM & 1 & IND & 1 \\
\hline Unit $092 \mathrm{SW}^{1 / 4}$ & $28-38$ & 1237 & 875 & Analyzed & 1 & 0.5 & LPR & PWS & LOC & CRT & 4 & & I & BRK & 4 & FLT & 1 \\
\hline Unit $092 \mathrm{SW}^{1 / 4}$ & $28-38$ & 1237 & 875 & Analyzed & 1 & 0.5 & LPR & PWS & LOC & CRT & 4 & & I & COM & 1 & CRT & 1 \\
\hline Unit $093 \mathrm{NE}^{1 / 4}$ & $10-20$ & 1152 & 877 & Unanalyzed & 1 & 0.5 & & & & & & & & & & & \\
\hline Unit $093 \mathrm{NE}^{1 / 4}$ & $20-25$ & 1276 & 881 & Unanalyzed & 4 & 1 & & & & & & & & & & & \\
\hline Unit $093 \mathrm{NE}^{1 / 4}$ & $25-30$ & 1378 & 885 & Analyzed & 1 & 0.1 & LPR & PWS & LOC & FWD & 4 & & I & FRG & 1 & IND & 1 \\
\hline Unit $093 \mathrm{NE}^{1 / 4}$ & $25-30$ & 1378 & 885 & Analyzed & 1 & 0.2 & LPR & PWS & LOC & CRT & 4 & & I & FRG & 1 & IND & 1 \\
\hline Unit $093 \mathrm{NE}^{1 / 4}$ & $25-30$ & 1378 & 885 & Analyzed & 1 & 0.2 & LPR & PWS & LOC & CRT & 4 & & I & FRG & 0 & IND & 1 \\
\hline Unit $093 \mathrm{NE}^{1 / 4}$ & $30-40$ & 1382 & 889 & Analyzed & 1 & 0.1 & LPR & PWS & LOC & FWD & 4 & & I & FRG & 0 & IND & 1 \\
\hline Unit $093 \mathrm{NE}^{1 / 4}$ & $30-40$ & 1382 & 889 & Analyzed & 1 & 5.9 & LPR & PWS & LOC & CRT & 2 & $\mathrm{ABS}$ & $\mathrm{N}$ & COM & 4 & CRT & 2 \\
\hline Unit $093 \mathrm{NE}^{1 / 4}$ & $30-40$ & 1382 & 889 & Analyzed & 1 & 0.3 & LPR & PWS & LOC & CRT & 4 & & I & DEB & 0 & IND & 1 \\
\hline Unit $093 \mathrm{NW}^{1 / 4}$ & $10-20$ & 1151 & 878 & Unanalyzed & 1 & 0.2 & & & & & & & & & & & \\
\hline
\end{tabular}


Geo-Marine, Inc.

GMI 22005.01.06.xx and 22005.00.09.xx, Data Recovery Excavations at Site 41PN175 Debitage Analysi

\begin{tabular}{|c|c|c|c|c|c|c|c|c|c|c|c|c|c|c|c|c|c|}
\hline Unit & $\begin{array}{l}\text { Depth } \\
(\mathrm{cm})\end{array}$ & $\begin{array}{l}\text { Provenience } \\
\text { No. }\end{array}$ & $\begin{array}{l}\text { Lot } \\
\text { No. }\end{array}$ & Level of Analysis & Quantity & $\begin{array}{l}\text { Weight } \\
\text { (in g) }\end{array}$ & General Period & Region & Material & Lithology & Size Grade & $\begin{array}{c}\text { Edge } \\
\text { Modification }\end{array}$ & $\begin{array}{l}\text { Thernal } \\
\text { Alteration }\end{array}$ & Completeness & Cortex & Platform & $\begin{array}{l}\text { Minimum } \\
\text { Number o } \\
\text { Nodules }\end{array}$ \\
\hline \multicolumn{18}{|c|}{ Block 4} \\
\hline Unit $093 \mathrm{NW}^{1 / 4}$ & $20-25$ & 1275 & 882 & Unanalyzed & 5 & 2.4 & & & & & & & & & & & \\
\hline Unit $093 \mathrm{NW}^{1 / 4}$ & $25-30$ & 1377 & 886 & Analyzed & 1 & 0.1 & LPR & PWS & LOC & CRT & 4 & & I & FRG & 0 & IND & 1 \\
\hline Unit $093 \mathrm{NW}^{1 / 4}$ & $25-30$ & 1377 & 886 & Analyzed & 2 & 0.1 & LPR & PWS & LOC & CRT & 4 & & I & COM & 1 & CRT & 1 \\
\hline Unit $093 \mathrm{NW}^{1 / 4}$ & $25-30$ & 1377 & 886 & Analyzed & 2 & 0.1 & LPR & PWS & LOC & CRT & 5 & & I & COM & 0 & FLT & 1 \\
\hline Unit $093 \mathrm{NW}^{1 / 4}$ & $25-30$ & 1377 & 886 & Analyzed & 1 & 0.2 & LPR & PWS & LOC & CRT & 4 & & I & COM & 4 & CRT & 1 \\
\hline Unit $093 \mathrm{NW}^{1} / 4$ & $30-40$ & 1381 & 890 & Analyzed & 1 & 0.04 & LPR & PWS & LOC & CRT & 4 & & I & COM & 4 & CRT & 1 \\
\hline Unit $093 \mathrm{NW}^{1 / 4}$ & $30-40$ & 1381 & 890 & Analyzed & 1 & 0.3 & LPR & PWS & LOC & QZT & 4 & & I & COM & 1 & CRT & 1 \\
\hline Unit $093 \mathrm{NW}^{1} / 4$ & $30-40$ & 1381 & 890 & Analyzed & 1 & 1.5 & LPR & PWS & LOC & QZT & 3 & & Y & DEB & 1 & IND & 1 \\
\hline Unit $093 \mathrm{SE}^{1 / 4}$ & $40-50$ & 1768 & 894 & Analyzed & 1 & 0.5 & LPR & PWS & LOC & CRT & 4 & & I & СOM & 3 & CRT & 1 \\
\hline Unit $093 \mathrm{SE}^{1 / 4}$ & $20-25$ & 1277 & 883 & Unanalyzed & 3 & 1.2 & & & & & & & & & & & \\
\hline Unit $093 \mathrm{SE}^{1 / 4}$ & $10-20$ & 1153 & 879 & Unanalyzed & 3 & 1.7 & & & & & & & & & & & \\
\hline Unit $093 \mathrm{SE}^{1 / 4}$ & $30-40$ & 1383 & 891 & Analyzed & 1 & 0.5 & LPR & PWS & LOC & CRT & 4 & & I & COM & 4 & CRT & 1 \\
\hline Unit $093 \mathrm{SE}^{1 / 4}$ & $25-30$ & 1379 & 887 & Analyzed & 1 & 0.04 & LPR & PWS & LOC & CRT & 4 & & I & FRG & 0 & IND & 1 \\
\hline Unit $093 \mathrm{SW}^{1} / 4$ & $25-30$ & 1376 & 888 & Analyzed & 1 & 0.3 & LPR & PWS & LOC & CRT & 4 & & I & COM & 1 & CRT & 3 \\
\hline Unit $093 \mathrm{SW}^{1 / 4}$ & $30-40$ & 1380 & 892 & Analyzed & 1 & 0.7 & LPR & PWS & LOC & CRT & 4 & & I & FRG & 4 & IND & 3 \\
\hline Unit $093 \mathrm{SW}^{1 / 4}$ & $30-40$ & 1380 & 892 & Analyzed & 2 & 0.9 & LPR & PWS & LOC & CRT & 4 & & I & COM & 4 & CRT & 3 \\
\hline Unit $093 \mathrm{SW}^{1 / 4}$ & $25-30$ & 1376 & 888 & Analyzed & 1 & 0.1 & LPR & PWS & LOC & QZT & 4 & & I & COM & 0 & FLT & 3 \\
\hline Unit 093 SW11/4 & $25-30$ & 1376 & 888 & Analyzed & 1 & 0.1 & LPR & PWS & LOC & CRT & 4 & & I & COM & 1 & FLT & 3 \\
\hline Unit 093 SW11/4 & $30-40$ & 1380 & 892 & Analyzed & 1 & 4.8 & LPR & PWS & LOC & FWD & 2 & $\mathrm{ABS}$ & $\mathrm{N}$ & СOM & 4 & CRT & 3 \\
\hline Unit $093 \mathrm{SW}^{1} / 4$ & $20-25$ & 1274 & 884 & Unanalyzed & 3 & 0.8 & & & & & & & & & & & \\
\hline Unit $093 \mathrm{SW}^{1} / 4$ & $25-30$ & 1376 & 888 & Analyzed & 1 & 0.3 & LPR & PWS & LOC & FWD & 4 & & I & DEB & 0 & IND & 3 \\
\hline Unit 094 NE $1 / 4$ & $20-25$ & 1280 & 901 & Unanalyzed & 5 & 2.5 & & & & & & & & & & & \\
\hline Unit 094 NE/4 & $10-20$ & 1156 & 897 & Unanalyzed & 2 & 2.7 & & & & & & & & & & & \\
\hline Unit $094 \mathrm{NW}^{1 / 4}$ & $25-30$ & 1385 & 906 & Analyzed & 2 & 0.1 & LPR & PWS & LOC & CRT & 4 & & I & FRG & 0 & IND & 2 \\
\hline Unit $094 \mathrm{NW}^{1 / 4}$ & $25-30$ & 1385 & 906 & Analyzed & 1 & 0.2 & LPR & PWS & LOC & QZT & 4 & & I & DEB & 0 & IND & 2 \\
\hline Unit $094 \mathrm{NW}^{1 / 4}$ & $25-30$ & 1385 & 906 & Analyzed & 1 & 0.2 & LPR & PWS & LOC & CRT & 4 & & I & COM & 0 & CPX & 2 \\
\hline
\end{tabular}


Geo-Marine, Inc.

GMI 22005.01.06.xx and 22005.00.09.xx, Data Recovery Excavations at Site 41PN175 Debitage Analysi

\begin{tabular}{|c|c|c|c|c|c|c|c|c|c|c|c|c|c|c|c|c|c|}
\hline Unit & $\begin{array}{l}\text { Depth } \\
(\mathrm{cm})\end{array}$ & $\begin{array}{l}\text { Provenience } \\
\text { No. }\end{array}$ & $\begin{array}{l}\text { Lot } \\
\text { No. }\end{array}$ & Level of Analysis & Quantity & $\begin{array}{l}\text { Weight } \\
\text { (in g) }\end{array}$ & General Period & Region & Material & Lithology & Size Grade & $\begin{array}{c}\text { Edge } \\
\text { Modification }\end{array}$ & $\begin{array}{l}\text { Thernal } \\
\text { Alteration }\end{array}$ & Completeness & Cortex & Platform & $\begin{array}{l}\text { Minimun } \\
\text { Number c } \\
\text { Nodules }\end{array}$ \\
\hline \multicolumn{18}{|c|}{ Block 4} \\
\hline Unit $094 \mathrm{NW}^{1 / 4}$ & $20-25$ & 1279 & 902 & Unanalyzed & 1 & 0.1 & & & & & & & & & & & \\
\hline Unit $094 \mathrm{NW}^{1 / 4}$ & $10-20$ & 1155 & 898 & Unanalyzed & 1 & 0.2 & & & & & & & & & & & \\
\hline Unit $094 \mathrm{SE}^{1} / 4$ & $10-20$ & 1157 & 899 & Unanalyzed & 2 & 0.4 & & & & & & & & & & & \\
\hline Unit $094 \mathrm{SE}^{1 / 4}$ & $20-25$ & 1281 & 903 & Unanalyzed & 7 & 6.8 & & & & & & & & & & & \\
\hline Unit $094 \mathrm{SE}^{1 / 4}$ & $25-30$ & 1387 & 907 & Analyzed & 1 & 0.6 & LPR & PWS & LOC & CRT & 3 & & $\mathrm{~N}$ & COM & 0 & IND & 1 \\
\hline Unit 094 SW11/4 & $25-30$ & 1384 & 908 & Analyzed & 1 & 0.4 & LPR & PWS & LOC & FWD & 4 & & I & СOM & 0 & FLT & 2 \\
\hline Unit $094 \mathrm{SW}^{1} / 4$ & $25-30$ & 1384 & 908 & Analyzed & 1 & 0.6 & LPR & PWS & LOC & CRT & 4 & & I & СOM & 0 & FLT & 2 \\
\hline Unit 094 SW11/4 & $20-25$ & 1278 & 904 & Unanalyzed & 1 & 0.5 & & & & & & & & & & & \\
\hline Unit $095 \mathrm{NE}^{1 / 4}$ & $40-50$ & 1771 & 931 & Analyzed & 1 & 0.1 & LPR & PWS & LOC & CRT & 4 & & I & FRG & 2 & IND & 1 \\
\hline Unit $095 \mathrm{NE}^{1 / 4}$ & $20-27$ & 1284 & 918 & Unanalyzed & 4 & 1.5 & & & & & & & & & & & \\
\hline Unit $095 \mathrm{NE}^{1} / 4$ & $10-20$ & 1160 & 914 & Unanalyzed & 4 & 1.8 & & & & & & & & & & & \\
\hline Unit $095 \mathrm{NE}^{1} / 4$ & $27-30$ & 1393 & 923 & Analyzed & 1 & 0.1 & LPR & PWS & LOC & QZT & 4 & & I & FRG & 0 & IND & 1 \\
\hline Unit $095 \mathrm{NW}^{1 / 4}$ & $20-27$ & 1283 & 919 & Unanalyzed & 3 & 1.1 & & & & & & & & & & & \\
\hline Unit $095 \mathrm{NW}^{1 / 4}$ & $30-40$ & 1397 & 928 & Analyzed & 1 & 0.5 & LPR & PWS & LOC & QZT & 4 & & I & FRG & 0 & IND & 1 \\
\hline Unit $095 \mathrm{NW}^{1 / 4}$ & $27-30$ & 1392 & 924 & Analyzed & 2 & 0.5 & LPR & PWS & LOC & CRT & 4 & & I & COM & 0 & IND & 1 \\
\hline Unit $095 \mathrm{NW}^{1 / 4}$ & $10-20$ & 1159 & 915 & Unanalyzed & 1 & 0.6 & & & & & & & & & & & \\
\hline Unit $095 \mathrm{SE}^{1 / 4}$ & $20-27$ & 1285 & 920 & Unanalyzed & 6 & 4.6 & & & & & & & & & & & \\
\hline Unit $095 \mathrm{SE}^{1 / 4}$ & $27-30$ & 1394 & 925 & Analyzed & 2 & 0.4 & LPR & PWS & LOC & QZT & 4 & & I & FRG & 0 & IND & 3 \\
\hline Unit $095 \mathrm{SE}^{1 / 4}$ & $30-40$ & 1399 & 929 & Analyzed & 1 & 0.8 & LPR & PWS & LOC & QZT & 3 & & $\mathrm{~N}$ & СOM & 4 & CRT & 1 \\
\hline Unit $095 \mathrm{SE}^{1 / 4}$ & $30-40$ & 1399 & 929 & Analyzed & 1 & 0.04 & LPR & PWS & LOC & QZT & 4 & & I & COM & 0 & FAC & 1 \\
\hline Unit $095 \mathrm{SE}^{1 / 4}$ & $40-50$ & 2003 & 932 & Analyzed & 1 & 0.2 & LPR & PWS & LOC & CRT & 4 & & I & FRG & 0 & IND & 1 \\
\hline Unit $095 \mathrm{SE}^{1 / 4}$ & $27-30$ & 1394 & 925 & Analyzed & 1 & 0.04 & LPR & PWS & LOC & QZT & 5 & & I & COM & 0 & CPX & 3 \\
\hline Unit $095 \mathrm{SE}^{1 / 4}$ & $27-30$ & 1394 & 925 & Analyzed & 1 & 0.1 & LPR & PWS & LOC & FWD & 4 & & I & BRK & 2 & FLT & 3 \\
\hline Unit $095 \mathrm{SE}^{1 / 4}$ & $10-20$ & 1161 & 916 & Unanalyzed & 5 & 5.5 & & & & & & & & & & & \\
\hline Unit $095 \mathrm{SE}^{1 / 4}$ & $27-30$ & 1394 & 925 & Analyzed & 1 & 0.2 & LPR & PWS & LOC & CRT & 4 & & I & DEB & 1 & IND & 3 \\
\hline Unit $095 \mathrm{SW}^{1 / 4} / 4$ & $27-30$ & 1391 & 926 & Analyzed & 1 & 0.2 & LPR & PWS & LOC & CRT & 4 & & I & DEB & 0 & IND & 1 \\
\hline
\end{tabular}


Geo-Marine, Inc.

GMI 22005.01.06.xx and 22005.00.09.xx, Data Recovery Excavations at Site 41PN175 Debitage Analysi

\begin{tabular}{|c|c|c|c|c|c|c|c|c|c|c|c|c|c|c|c|c|c|}
\hline Unit & $\begin{array}{c}\text { Depth } \\
(\mathrm{cm})\end{array}$ & $\begin{array}{l}\text { Provenience } \\
\text { No. }\end{array}$ & $\begin{array}{l}\text { Lot } \\
\text { No. }\end{array}$ & Level of Analysis & Quantity & $\begin{array}{l}\text { Weight } \\
\text { (ing) }\end{array}$ & General Period & Region & Material & Lithology & Size Grade & $\begin{array}{c}\text { Edge } \\
\text { Modification }\end{array}$ & $\begin{array}{l}\text { Thernal } \\
\text { Alteration }\end{array}$ & Completeness & Cortex & Platform & $\begin{array}{l}\text { Minimum } \\
\text { Number of } \\
\text { Nodules }\end{array}$ \\
\hline \multicolumn{18}{|c|}{ Block 4} \\
\hline Unit $095 \mathrm{SW}^{1} / 4$ & $27-30$ & 1391 & 926 & Analyzed & 1 & 0.04 & LPR & PWS & LOC & CRT & 4 & & I & COM & 4 & CRT & 1 \\
\hline Unit $095 \mathrm{SW}^{1 / 4}$ & $27-30$ & 1391 & 926 & Analyzed & 1 & 0.1 & LPR & PWS & LOC & CRT & 4 & & I & COM & 1 & FAC & 1 \\
\hline Unit $095 \mathrm{SW}^{1} / 4$ & $20-27$ & 1282 & 921 & Unanalyzed & 4 & 1.2 & & & & & & & & & & & \\
\hline Unit $095 \mathrm{SW}^{1} / 4$ & $30-40$ & 1396 & 930 & Analyzed & 1 & 0.1 & LPR & PWS & LOC & CRT & 5 & & I & FRG & 0 & IND & 1 \\
\hline Unit $096 \mathrm{NE}^{1 / 4}$ & $30-40$ & 1406 & 946 & Analyzed & 1 & 0.1 & LPR & PWS & LOC & CRT & 4 & & I & BRK & 0 & FAC & 1 \\
\hline Unit 096 NE¹/4 & $30-40$ & 1406 & 946 & Analyzed & 2 & 0.3 & LPR & PWS & LOC & CRT & 4 & & I & FRG & 2 & IND & 1 \\
\hline Unit $096 \mathrm{NE}^{1 / 4}$ & $30-40$ & 1406 & 946 & Analyzed & 2 & 0.1 & LPR & PWS & LOC & CRT & 4 & & I & FRG & 0 & IND & 1 \\
\hline Unit $096 \mathrm{NE}^{1 / 4}$ & $20-29$ & 1288 & 937 & Unanalyzed & 3 & 1.3 & & & & & & & & & & & \\
\hline Unit $096 \mathrm{NE}^{1 / 4}$ & $28-30$ & 1402 & 941 & Analyzed & 2 & 0.3 & LPR & PWS & LOC & CRT & 4 & & I & COM & 0 & FLT & 1 \\
\hline Unit $096 \mathrm{NE}^{1 / 4}$ & $28-30$ & 1402 & 941 & Analyzed & 2 & 1.1 & LPR & PWS & LOC & CRT & 4 & & I & DEB & 1 & IND & 1 \\
\hline Unit $096 \mathrm{NW}^{1 / 4}$ & $28-30$ & 1401 & 942 & Analyzed & 1 & 0.1 & LPR & PWS & LOC & QZT & 4 & & I & FRG & 0 & IND & 1 \\
\hline Unit $096 \mathrm{NW}^{1 / 4}$ & $10-20$ & 1163 & 934 & Unanalyzed & 1 & 0.8 & & & & & & & & & & & \\
\hline Unit $096 \mathrm{NW}^{1 / 4}$ & $30-40$ & 1405 & 947 & Analyzed & 2 & 0.3 & LPR & PWS & LOC & CRT & 4 & & I & COM & 3 & CRT & 2 \\
\hline Unit $096 \mathrm{NW}^{1 / 4}$ & $30-40$ & 1405 & 947 & Analyzed & 1 & 1.8 & LPR & PWS & LOC & FWD & 3 & & $\mathrm{Y}$ & COM & 2 & ABR & 2 \\
\hline Unit $096 \mathrm{NW}^{1 / 4}$ & $20-29$ & 1287 & 938 & Unanalyzed & 1 & 0.4 & & & & & & & & & & & \\
\hline Unit $096 \mathrm{SE}^{1} / 4$ & $10-20$ & 1165 & 935 & Unanalyzed & 2 & 0.3 & & & & & & & & & & & \\
\hline Unit $096 \mathrm{SE}^{1} / 4$ & $30-40$ & 1407 & 948 & Analyzed & 1 & 0.5 & LPR & PWS & LOC & CRT & 4 & & I & DEB & 0 & IND & 1 \\
\hline Unit $096 \mathrm{SE}^{1} / 4$ & $28-30$ & 1403 & 944 & Analyzed & 1 & 0.4 & LPR & PWS & LOC & CRT & 4 & & I & FRG & 0 & IND & 1 \\
\hline Unit $096 \mathrm{SE}^{1 / 4}$ & $30-40$ & 1407 & 948 & Analyzed & 1 & 0.1 & LPR & PWS & LOC & CRT & 4 & & I & COM & 0 & CPX & 1 \\
\hline Unit $096 \mathrm{SE}^{1 / 4}$ & $20-29$ & 1289 & 939 & Unanalyzed & 5 & 1.3 & & & & & & & & & & & \\
\hline Unit $096 \mathrm{SW}^{1} / 4$ & $20-29$ & 1286 & 940 & Unanalyzed & 4 & 1.7 & & & & & & & & & & & \\
\hline Unit $096 \mathrm{SW}^{1} / 4$ & $30-40$ & 1404 & 949 & Analyzed & 1 & 0.04 & LPR & PWS & LOC & CRT & 5 & & I & COM & 0 & ABR & 1 \\
\hline Unit $097 \mathrm{NE}^{1 / 4}$ & $10-20$ & 946 & 952 & Unanalyzed & 1 & 1.3 & & & & & & & & & & & \\
\hline Unit $097 \mathrm{NW}^{1 / 4}$ & $10-20$ & 945 & 953 & Unanalyzed & 1 & 0.6 & & & & & & & & & & & \\
\hline Unit $097 \mathrm{NW}^{1 / 4}$ & $50-60$ & 1774 & 967 & Unanalyzed & 1 & 1 & & & & & & & & & & & \\
\hline Unit $097 \mathrm{SW}^{1} / 4$ & $10-20$ & 944 & 955 & Unanalyzed & 1 & 4 & & & & & & & & & & & \\
\hline
\end{tabular}


Geo-Marine, Inc.

GMI 22005.01.06.xx and 22005.00.09.xx, Data Recovery Excavations at Site 41PN17 Debitage Analysi

\begin{tabular}{|c|c|c|c|c|c|c|c|c|c|c|c|c|c|c|c|c|c|}
\hline Unit & $\begin{array}{l}\text { Depth } \\
(\mathrm{cm})\end{array}$ & $\begin{array}{l}\text { Provenience } \\
\text { No. }\end{array}$ & $\begin{array}{l}\text { Lot } \\
\text { No. }\end{array}$ & Level of Analysis & Quantity & $\begin{array}{l}\text { Weight } \\
\text { (ing) }\end{array}$ & General Period & Region & Material & Lithology & Size Grade & $\begin{array}{c}\text { Edge } \\
\text { Modification }\end{array}$ & $\begin{array}{l}\text { Thernal } \\
\text { Alteration }\end{array}$ & Completeness & Cortex & Platform & $\begin{array}{l}\text { Minimur } \\
\text { Number } \\
\text { Nodule }\end{array}$ \\
\hline \multicolumn{18}{|c|}{ Block 4} \\
\hline Unit $098 \mathrm{NE}^{1 / 4}$ & $20-30$ & 1171 & 974 & Unanalyzed & 2 & 3 & & & & & & & & & & & \\
\hline Unit 098 NE1/4 & $34-40$ & 1413 & 982 & Analyzed & 1 & 0.1 & LPR & PWS & LOC & CRT & 4 & & I & BRK & 0 & FLT & 1 \\
\hline Unit $098 \mathrm{NW}^{1 / 4}$ & $34-40$ & 1412 & 983 & Analyzed & 1 & 0.04 & LPR & Pws & LOC & CRT & 5 & & I & BRK & 0 & FLT & 1 \\
\hline Unit $098 \mathrm{NW}^{1 / 4}$ & $30-35$ & 1295 & 979 & Unanalyzed & 2 & 0.6 & & & & & & & & & & & \\
\hline Unit $098 \mathrm{NW}^{1 / 4}$ & $40-50$ & 1414 & 985 & Analyzed & 1 & 0.04 & LPR & PWS & LOC & CRT & 5 & & I & $\mathrm{COM}$ & 1 & CRT & 1 \\
\hline Unit $098 \mathrm{SE}^{1 / 4}$ & $30-35$ & 1297 & 980 & Unanalyzed & 1 & 1.2 & & & & & & & & & & & \\
\hline Unit $098 \mathrm{SE}^{1 / 4}$ & $10-20$ & 1094 & 972 & Unanalyzed & 3 & 1 & & & & & & & & & & & \\
\hline Unit 098 SW$^{1} / 4$ & $20-30$ & 1169 & 977 & Unanalyzed & 2 & 0.6 & & & & & & & & & & & \\
\hline Unit 098 SW'1/4 & $10-20$ & 1091 & 973 & Unanalyzed & 3 & 0.2 & & & & & & & & & & & \\
\hline Unit $098 \mathrm{SW}^{1} / 4$ & $30-35$ & 1294 & 981 & Unanalyzed & 1 & 0.1 & & & & & & & & & & & \\
\hline Unit $099 \mathrm{NE}^{1 / 4}$ & $10-20$ & 1175 & 991 & Unanalyzed & 3 & 2.7 & & & & & & & & & & & \\
\hline Unit 099 NE1/4 & $20-26$ & 1300 & 995 & Unanalyzed & 3 & 1.7 & & & & & & & & & & & \\
\hline Unit $099 \mathrm{NW}^{1 / 4}$ & $20-26$ & 1299 & 996 & Unanalyzed & 4 & 2 & & & & & & & & & & & \\
\hline Unit $099 \mathrm{NW}^{1 / 4}$ & $10-20$ & 1174 & 992 & Unanalyzed & 1 & 0.8 & & & & & & & & & & & \\
\hline Unit $099 \mathrm{SE}^{1 / 4}$ & $20-26$ & 1301 & 997 & Unanalyzed & 4 & 1.7 & & & & & & & & & & & \\
\hline Unit $099 \mathrm{SE}^{1 / 4}$ & $10-20$ & 1176 & 993 & Unanalyzed & 1 & 0.6 & & & & & & & & & & & \\
\hline Unit $099 \mathrm{SE}^{1 / 4}$ & $26-30$ & 1417 & 1000 & Analyzed & 1 & 0.5 & LPR & PWS & LOC & QZT & 4 & & I & FRG & 2 & IND & 1 \\
\hline Unit $099 \mathrm{SW}^{1 / 4}$ & $26-30$ & 1415 & 1001 & Analyzed & 1 & 0.1 & LPR & PWS & LOC & CRT & 4 & & I & FRG & 0 & IND & 1 \\
\hline Unit $099 \mathrm{SW}^{1} / 4$ & $20-26$ & 1298 & 998 & Unanalyzed & 9 & 4 & & & & & & & & & & & \\
\hline Unit $099 \mathrm{SW}^{1} / 4$ & $10-20$ & 1173 & 994 & Unanalyzed & 1 & 1.2 & & & & & & & & & & & \\
\hline Unit $100 \mathrm{NE}^{1 / 4}$ & $27-30$ & 1429 & 1012 & Analyzed & 1 & 0.3 & LPR & PWS & LOC & CRT & 4 & & I & DEB & 0 & IND & 1 \\
\hline Unit $100 \mathrm{NE}^{1 / 4}$ & $30-40$ & 1433 & 1016 & Analyzed & 1 & 0.2 & LPR & PWS & LOC & CRT & 4 & & I & FRG & 4 & IND & 1 \\
\hline Unit $100 \mathrm{NE}^{1 / 4}$ & $27-30$ & 1429 & 1012 & Analyzed & 1 & 0.5 & LPR & PWS & LOC & CRT & 4 & & I & COM & 3 & FLT & 1 \\
\hline Unit $100 \mathrm{NE}^{1 / 4}$ & $10-20$ & 1179 & 1004 & Unanalyzed & 4 & 1.7 & & & & & & & & & & & \\
\hline Unit $100 \mathrm{NE}^{1 / 4}$ & $20-27$ & 1304 & 1008 & Unanalyzed & 8 & 4 & & & & & & & & & & & \\
\hline Unit $100 \mathrm{NW}^{1 / 4}$ & $20-27$ & 1303 & 1009 & Unanalyzed & 1 & 0.2 & & & & & & & & & & & \\
\hline
\end{tabular}


Geo-Marine, Inc.

GMI 22005.01.06.xx and 22005.00.09.xx, Data Recovery Excavations at Site 41PN17 Debitage Analysi

\begin{tabular}{|c|c|c|c|c|c|c|c|c|c|c|c|c|c|c|c|c|c|}
\hline Unit & $\begin{array}{l}\text { Depth } \\
(\mathrm{cm})\end{array}$ & $\begin{array}{l}\text { Provenience } \\
\text { No. }\end{array}$ & $\begin{array}{l}\text { Lot } \\
\text { No. }\end{array}$ & Level of Analysis & Quantity & $\begin{array}{l}\text { Weight } \\
\text { (ing) }\end{array}$ & General Period & Region & Material & Lithology & Size Grade & $\begin{array}{c}\text { Edge } \\
\text { Modification }\end{array}$ & $\begin{array}{l}\text { Thernal } \\
\text { Alteration }\end{array}$ & Completeness & Cortex & Platform & $\begin{array}{l}\text { Minimum } \\
\text { Number of } \\
\text { Nodules }\end{array}$ \\
\hline \multicolumn{18}{|c|}{ Block 4} \\
\hline Unit $100 \mathrm{NW}^{1 / 4}$ & $30-40$ & 1432 & 1017 & Analyzed & 1 & 0.5 & LPR & PWS & LOC & CRT & 4 & & I & DEB & 1 & IND & 1 \\
\hline Unit $100 \mathrm{NW}^{1 / 4}$ & $10-20$ & 1178 & 1005 & Unanalyzed & 2 & 0.6 & & & & & & & & & & & \\
\hline Unit $100 \mathrm{NW}^{1 / 4}$ & $27-30$ & 1428 & 1013 & Analyzed & 1 & 0.7 & LPR & PWS & LOC & CRT & 4 & & I & DEB & 1 & IND & 1 \\
\hline Unit $100 \mathrm{SE}^{1 / 4}$ & $10-20$ & 1180 & 1006 & Unanalyzed & 3 & 1.2 & & & & & & & & & & & \\
\hline Unit $100 \mathrm{SE}^{1 / 4}$ & $30-40$ & 1434 & 1018 & Analyzed & 1 & 1.2 & LPR & PWS & LOC & CRT & 3 & & $\mathrm{~N}$ & BRK & 0 & $\mathrm{ABR}$ & 1 \\
\hline Unit $100 \mathrm{SE}^{1 / 4}$ & $20-27$ & 1305 & 1010 & Unanalyzed & 7 & 3 & & & & & & & & & & & \\
\hline Unit $100 \mathrm{SE}^{1 / 4}$ & $27-30$ & 1430 & 1014 & Analyzed & 1 & 0.4 & LPR & PWS & LOC & CRT & 4 & & I & DEB & 1 & IND & 1 \\
\hline Unit $100 \mathrm{SW}^{1 / 4}$ & $20-27$ & 1302 & 1011 & Unanalyzed & 3 & 5 & & & & & & & & & & & \\
\hline Unit $100 \mathrm{SW}^{1 / 4}$ & $10-20$ & 1177 & 1007 & Unanalyzed & 4 & 1.7 & & & & & & & & & & & \\
\hline Unit $100 \mathrm{SW}^{1 / 4}$ & $30-40$ & 1431 & 1019 & Analyzed & 1 & 0.1 & LPR & PWS & LOC & CRT & 4 & & I & $\mathrm{COM}$ & 1 & CRT & 1 \\
\hline Unit $101 \mathrm{NE}^{1 / 4}$ & $30-40$ & 1441 & 1034 & Analyzed & 1 & 0.3 & LPR & PWS & LOC & CRT & 4 & & I & DEB & 1 & IND & 2 \\
\hline Unit $101 \mathrm{NE}^{1 / 4}$ & $10-20$ & 1183 & 1022 & Unanalyzed & 1 & 1.8 & & & & & & & & & & & \\
\hline Unit $101 \mathrm{NE}^{1 / 4}$ & $30-40$ & 1441 & 1034 & Analyzed & 1 & 0.3 & LPR & PWS & LOC & CRT & 4 & & I & DEB & 2 & IND & 2 \\
\hline Unit $101 \mathrm{NE}^{1 / 4}$ & $30-40$ & 1441 & 1034 & Analyzed & 1 & 0.1 & LPR & PWS & LOC & CRT & 4 & & I & COM & 0 & $\mathrm{ABR}$ & 2 \\
\hline Unit $101 \mathrm{NE}^{1 / 4}$ & $27-30$ & 1437 & 1030 & Analyzed & 1 & 0.3 & LPR & PWS & LOC & QZT & 4 & & I & FRG & 0 & IND & 2 \\
\hline Unit $101 \mathrm{NE}^{1 / 4}$ & $27-30$ & 1437 & 1030 & Analyzed & 1 & 0.7 & LPR & PWS & LOC & CRT & 4 & & I & FRG & 2 & IND & 2 \\
\hline Unit $101 \mathrm{NE}^{1 / 4}$ & $27-30$ & 1437 & 1030 & Analyzed & 1 & 0.1 & LPR & PWS & LOC & CRT & 4 & & I & BRK & 1 & CRT & 2 \\
\hline Unit $101 \mathrm{NE}^{1 / 4}$ & $20-27$ & 1308 & 1026 & Unanalyzed & 2 & 1.6 & & & & & & & & & & & \\
\hline Unit $101 \mathrm{NE}^{1 / 4}$ & $40-50$ & 1686 & 1038 & Analyzed & 1 & 0.2 & LPR & PWS & LOC & QZT & 4 & & I & FRG & 1 & IND & 1 \\
\hline Unit $101 \mathrm{NW}^{1 / 4}$ & $30-40$ & 1440 & 1035 & Analyzed & 2 & 0.2 & LPR & PWS & LOC & CRT & 4 & & I & COM & 4 & CRT & 1 \\
\hline Unit $101 \mathrm{NW}^{1 / 4}$ & $20-27$ & 1307 & 1027 & Unanalyzed & 4 & 1.1 & & & & & & & & & & & \\
\hline Unit $101 \mathrm{NW}^{1 / 4}$ & $30-40$ & 1440 & 1035 & Analyzed & 1 & 0.1 & LPR & PWS & LOC & CRT & 4 & & I & FRG & 0 & IND & 1 \\
\hline Unit $101 \mathrm{NW}^{1 / 4}$ & $30-40$ & 1440 & 1035 & Analyzed & 2 & 0.04 & LPR & PWS & LOC & CRT & 5 & & I & FRG & 0 & IND & 1 \\
\hline Unit $101 \mathrm{NW}^{1 / 4}$ & $30-40$ & 1440 & 1035 & Analyzed & 1 & 0.3 & LPR & PWS & LOC & CRT & 4 & & I & COM & 0 & CPX & 1 \\
\hline Unit $101 \mathrm{NW}^{1 / 4}$ & $27-30$ & 1436 & 1031 & Analyzed & 1 & 0.1 & LPR & PWS & LOC & CRT & 4 & & I & DEB & 0 & IND & 1 \\
\hline Unit $101 \mathrm{NW}^{1} / 4$ & $10-20$ & 1182 & 1023 & Unanalyzed & 5 & 1.2 & & & & & & & & & & & \\
\hline
\end{tabular}


Geo-Marine, Inc.

GMI 22005.01.06.xx and 22005.00.09.xx, Data Recovery Excavations at Site 41PN175 Debitage Analysi

\begin{tabular}{|c|c|c|c|c|c|c|c|c|c|c|c|c|c|c|c|c|c|}
\hline Unit & $\begin{array}{l}\text { Depth } \\
(\mathrm{cm})\end{array}$ & $\begin{array}{l}\text { Provenience } \\
\text { No. }\end{array}$ & $\begin{array}{l}\text { Lot } \\
\text { No. }\end{array}$ & Level of Analysis & Quantity & $\begin{array}{l}\text { Weight } \\
\text { (ing) }\end{array}$ & General Period & Region & Material & Lithology & Size Grade & $\begin{array}{c}\text { Edge } \\
\text { Modification }\end{array}$ & $\begin{array}{l}\text { Thernal } \\
\text { Alteration }\end{array}$ & Completeness & Cortex & Platform & $\begin{array}{l}\text { Minimun } \\
\text { Number o } \\
\text { Nodules }\end{array}$ \\
\hline \multicolumn{18}{|c|}{ Block 4} \\
\hline Unit $101 \mathrm{NW}^{1 / 4}$ & $27-30$ & 1436 & 1031 & Analyzed & 2 & 2 & LPR & PWS & LOC & CRT & 3 & & $\mathrm{~N}$ & COM & 1 & CRT & 1 \\
\hline Unit $101 \mathrm{NW}^{1 / 4}$ & $30-40$ & 1440 & 1035 & Analyzed & 1 & 0.04 & LPR & PWS & LOC & CRT & 5 & & I & $\mathrm{COM}$ & 4 & CRT & 1 \\
\hline Unit $101 \mathrm{SE}^{1 / 4}$ & $30-40$ & 1442 & 1036 & Analyzed & 1 & 0.04 & LPR & PWS & LOC & CRT & 5 & & I & FRG & 0 & IND & 2 \\
\hline Unit $101 \mathrm{SE}^{1 / 4}$ & $27-30$ & 1438 & 1032 & Analyzed & 1 & 0.1 & LPR & PWS & LOC & CRT & 4 & & I & COM & 1 & CPX & 2 \\
\hline Unit $101 \mathrm{SE}^{1 / 4}$ & $10-20$ & 1184 & 1024 & Unanalyzed & 3 & 0.3 & & & & & & & & & & & \\
\hline Unit $101 \mathrm{SE}^{1 / 4}$ & $27-30$ & 1438 & 1032 & Analyzed & 1 & 3.7 & LPR & PWS & LOC & QZT & 3 & & $\mathrm{Y}$ & COM & 2 & CRT & 2 \\
\hline Unit $101 \mathrm{SE}^{1 / 4}$ & $20-27$ & 1309 & 1028 & Unanalyzed & 4 & 1 & & & & & & & & & & & \\
\hline Unit $101 \mathrm{SE}^{1 / 4}$ & $27-30$ & 1438 & 1032 & Analyzed & 1 & 0.1 & LPR & PWS & LOC & QZT & 4 & & I & $\mathrm{COM}$ & 0 & ABR & 2 \\
\hline Unit $101 \mathrm{SE}^{1 / 4}$ & $30-40$ & 1442 & 1036 & Analyzed & 1 & 0.2 & LPR & PWS & LOC & QZT & 4 & & I & FRG & 0 & IND & 2 \\
\hline Unit $101 \mathrm{SE}^{1 / 4}$ & $30-40$ & 1442 & 1036 & Analyzed & 1 & 0.2 & LPR & PWS & LOC & CRT & 4 & & I & FRG & 1 & IND & 2 \\
\hline Unit $101 \mathrm{SE}^{1 / 4}$ & $30-40$ & 1442 & 1036 & Analyzed & 1 & 0.2 & LPR & PWS & LOC & CRT & 4 & & I & FRG & 3 & IND & 2 \\
\hline Unit $101 \mathrm{SW}^{1 / 4}$ & $20-27$ & 1306 & 1029 & Unanalyzed & 3 & 0.6 & & & & & & & & & & & \\
\hline Unit $101 \mathrm{SW}^{1 / 4}$ & $30-40$ & 1439 & 1037 & Analyzed & 1 & 0.2 & LPR & PWS & LOC & CRT & 4 & & I & COM & 0 & CPX & 1 \\
\hline Unit $101 \mathrm{SW}^{1 / 4}$ & $27-30$ & 1435 & 1033 & Analyzed & 1 & 0.7 & LPR & PWS & LOC & CRT & 4 & & I & DEB & 1 & IND & 1 \\
\hline Unit $101 \mathrm{SW}^{1 / 4}$ & $30-40$ & 1439 & 1037 & Analyzed & 1 & 0.04 & LPR & PWS & LOC & CRT & 5 & & I & FRG & 0 & IND & 1 \\
\hline Unit 101 SW'/4 & $27-30$ & 1435 & 1033 & Analyzed & 1 & 1.3 & LPR & PWS & LOC & CRT & 3 & & $\mathrm{~N}$ & FRG & 1 & IND & 1 \\
\hline Unit $102 \mathrm{NE}^{1 / 4}$ & $20-30$ & 1187 & 1047 & Analyzed & 1 & 0.3 & LPR & PWS & LOC & CRT & 4 & & I & FRG & 0 & IND & 2 \\
\hline Unit $102 \mathrm{NE}^{1 / 4}$ & $20-30$ & 1187 & 1047 & Analyzed & 1 & 0.2 & LPR & PWS & REG & JPR & 4 & & I & COM & 1 & FLT & 2 \\
\hline Unit $102 \mathrm{SE}^{1 / 4}$ & $0-10$ & 949 & 1042 & Unanalyzed & 2 & 0.3 & & & & & & & & & & & \\
\hline Unit $102 \mathrm{SE}^{1 / 4}$ & $20-30$ & 1188 & 1049 & Analyzed & 1 & 1.6 & LPR & PWS & REG & JPR & 4 & & I & BRK & 1 & CRT & 1 \\
\hline Unit $102 \mathrm{SE}^{1 / 4}$ & $10-20$ & 953 & 1045 & Unanalyzed & 9 & 7 & & & & & & & & & & & \\
\hline Unit $102 \mathrm{SW}^{1 / 4}$ & $10-20$ & 950 & 1046 & Unanalyzed & 1 & 0.6 & & & & & & & & & & & \\
\hline Unit $103 \mathrm{NE}^{1 / 4}$ & $30-40$ & 1315 & 1067 & Unanalyzed & 3 & 0.4 & & & & & & & & & & & \\
\hline Unit $103 \mathrm{NW}^{1 / 4}$ & $30-40$ & 1314 & 1068 & Unanalyzed & 1 & 0.8 & & & & & & & & & & & \\
\hline Unit $103 \mathrm{NW}^{1 / 4}$ & $10-20$ & 965 & 1060 & Unanalyzed & 1 & 0.2 & & & & & & & & & & & \\
\hline Unit $103 \mathrm{SE}^{1 / 4}$ & $30-40$ & 1316 & 1069 & Unanalyzed & 1 & 0.2 & & & & & & & & & & & \\
\hline
\end{tabular}


Geo-Marine, Inc.

GMI 22005.01.06.xx and 22005.00.09.xx, Data Recovery Excavations at Site 41PN17 Debitage Analysi

\begin{tabular}{|c|c|c|c|c|c|c|c|c|c|c|c|c|c|c|c|c|c|}
\hline Unit & $\begin{array}{l}\text { Depth } \\
(\mathrm{cm})\end{array}$ & $\begin{array}{l}\text { Provenience } \\
\text { No. }\end{array}$ & $\begin{array}{l}\text { Lot } \\
\text { No. }\end{array}$ & Level of Analysis & Quantity & $\begin{array}{l}\text { Weight } \\
\text { (ing) }\end{array}$ & General Period & Region & Material & Lithology & Size Grade & $\begin{array}{c}\text { Edge } \\
\text { Modification }\end{array}$ & $\begin{array}{l}\text { Thernal } \\
\text { Alteration }\end{array}$ & Completeness & Cortex & Platform & $\begin{array}{l}\text { Minimum } \\
\text { Number of } \\
\text { Nodules }\end{array}$ \\
\hline \multicolumn{18}{|c|}{ Block 4} \\
\hline Unit $104 \mathrm{NE}^{1 / 4}$ & $25-30$ & 1449 & 1086 & Analyzed & 1 & 0.2 & LPR & PWS & LOC & CRT & 4 & & I & сом & 4 & CRT & 1 \\
\hline Unit $104 \mathrm{NE}^{1 / 4}$ & $25-30$ & 1449 & 1086 & Analyzed & 1 & 0.9 & LPR & PWS & LOC & CRT & 3 & & $\mathrm{~N}$ & DEB & 1 & IND & 1 \\
\hline Unit $104 \mathrm{NW}^{1 / 4}$ & $10-20$ & 1194 & 1079 & Unanalyzed & 2 & 0.5 & & & & & & & & & & & \\
\hline Unit $104 \mathrm{NW}^{1 / 4}$ & $25-30$ & 1448 & 1087 & Analyzed & 1 & 1.1 & LPR & PWS & LOC & ISS & 4 & & I & СOM & 0 & FLT & 2 \\
\hline Unit $104 \mathrm{NW}^{1 / 4}$ & $25-30$ & 1448 & 1087 & Analyzed & 1 & 0.04 & LPR & PWS & LOC & QZT & 4 & & I & FRG & 1 & IND & 2 \\
\hline Unit $104 \mathrm{NW}^{1 / 4}$ & $20-25$ & 1318 & 1083 & Unanalyzed & 2 & 0.8 & & & & & & & & & & & \\
\hline Unit $104 \mathrm{SE}^{1 / 4}$ & $25-30$ & 1450 & 1088 & Analyzed & 1 & 0.5 & LPR & PWS & LOC & QZT & 4 & & I & сOM & 4 & CRT & 1 \\
\hline Unit $104 \mathrm{SE}^{1 / 4}$ & $10-20$ & 1196 & 1080 & Unanalyzed & 2 & 0.8 & & & & & & & & & & & \\
\hline Unit $104 \mathrm{SW}^{1 / 4}$ & $10-20$ & 1193 & 1081 & Unanalyzed & 3 & 0.5 & & & & & & & & & & & \\
\hline Unit $104 \mathrm{SW}^{1 / 4}$ & $20-25$ & 1317 & 1085 & Unanalyzed & 1 & 1 & & & & & & & & & & & \\
\hline Unit $104 \mathrm{SW}^{1 / 4}$ & $0-10$ & 1102 & 1077 & Unanalyzed & 2 & 1.7 & & & & & & & & & & & \\
\hline Unit $105 \mathrm{NE}^{1 / 4}$ & $0-10$ & 1108 & 1093 & Unanalyzed & 1 & 0.1 & & & & & & & & & & & \\
\hline Unit $105 \mathrm{NE}^{1 / 4}$ & $10-20$ & 1199 & 1097 & Unanalyzed & 1 & 0.3 & & & & & & & & & & & \\
\hline Unit $105 \mathrm{NE}^{1 / 4}$ & $30-40$ & 1458 & 1111 & Analyzed & 1 & 1.9 & LPR & PWS & LOC & CRT & 3 & & $\mathrm{~N}$ & COM & 4 & CRT & 1 \\
\hline Unit $105 \mathrm{NW}^{1 / 4}$ & $20-23$ & 1242 & 1102 & Unanalyzed & 3 & 1.1 & & & & & & & & & & & \\
\hline Unit $105 \mathrm{NW}^{1 / 4}$ & $10-20$ & 1198 & 1098 & Unanalyzed & 2 & 2 & & & & & & & & & & & \\
\hline Unit $105 \mathrm{NW}^{1 / 4}$ & $24-30$ & 1453 & 1108 & Analyzed & 1 & 0.04 & LPR & PWS & LOC & CRT & 4 & & I & FRG & 0 & IND & 1 \\
\hline Unit $105 \mathrm{NW}^{1 / 4}$ & $30-40$ & 1457 & 1112 & Analyzed & 1 & 0.04 & LPR & PWS & LOC & CRT & 4 & & I & сом & 0 & IND & 1 \\
\hline Unit $105 \mathrm{SE}^{1 / 4}$ & $30-40$ & 1459 & 1113 & Analyzed & 1 & 0.2 & LPR & PWS & LOC & CRT & 4 & & I & FRG & 0 & IND & 1 \\
\hline Unit $105 \mathrm{SE}^{1 / 4}$ & $10-20$ & 1200 & 1099 & Unanalyzed & 1 & 0.2 & & & & & & & & & & & \\
\hline Unit $105 \mathrm{SW}^{1 / 4}$ & $20-23$ & 1241 & 1105 & Unanalyzed & 2 & 2.2 & & & & & & & & & & & \\
\hline Unit $105 \mathrm{SW}^{1 / 4}$ & $0-10$ & 1106 & 1757 & Unanalyzed & 1 & 1.5 & & & & & & & & & & & \\
\hline Unit $105 \mathrm{SW}^{1 / 4}$ & $24-30$ & 1452 & 1110 & Analyzed & 1 & 0.3 & LPR & PWS & LOC & QZT & 4 & & I & COM & 1 & CRT & 1 \\
\hline Unit $105 \mathrm{SW}^{1 / 4}$ & $10-20$ & 1197 & 1100 & Unanalyzed & 3 & 0.6 & & & & & & & & & & & \\
\hline Unit $105 \mathrm{SW}^{1 / 4}$ & $24-30$ & 1452 & 1110 & Analyzed & 1 & 1.8 & LPR & PWS & LOC & QZT & 3 & & $\mathrm{~N}$ & COM & 0 & FLT & 1 \\
\hline Unit 106 General & $30-40$ & 1468 & 1133 & Analyzed & 1 & 0.04 & LPR & PWS & LOC & CRT & 4 & & I & BRK & 0 & CPX & 1 \\
\hline
\end{tabular}


Geo-Marine, Inc.

GMI 22005.01.06.xx and 22005.00.09.xx, Data Recovery Excavations at Site 41PN175 Debitage Analysi

\begin{tabular}{|c|c|c|c|c|c|c|c|c|c|c|c|c|c|c|c|c|c|}
\hline Unit & $\begin{array}{l}\text { Depth } \\
(\mathrm{cm})\end{array}$ & $\begin{array}{l}\text { Provenience } \\
\text { No. }\end{array}$ & $\begin{array}{l}\text { Lot } \\
\text { No. }\end{array}$ & Level of Analysis & Quantity & $\begin{array}{l}\text { Weight } \\
\text { (in g) }\end{array}$ & General Period & Region & Material & Lithology & Size Grade & $\begin{array}{c}\text { Edge } \\
\text { Modification }\end{array}$ & $\begin{array}{l}\text { Thernal } \\
\text { Alteration }\end{array}$ & Completeness & Cortex & Platform & $\begin{array}{l}\text { Minimum } \\
\text { Number o } \\
\text { Nodules }\end{array}$ \\
\hline \multicolumn{18}{|c|}{ Block 4} \\
\hline Unit $106 \mathrm{NE}^{1 / 4}$ & $20-23$ & 1247 & 1124 & Unanalyzed & 1 & 0.1 & & & & & & & & & & & \\
\hline Unit $106 \mathrm{NE}^{1 / 4}$ & $10-20$ & 1204 & 1120 & Unanalyzed & 1 & 0.5 & & & & & & & & & & & \\
\hline Unit $106 \mathrm{NE}^{1 / 4}$ & $25-30$ & 1462 & 1128 & Analyzed & 1 & 1.6 & LPR & PWS & LOC & QZT & 4 & & I & COM & 4 & CRT & 1 \\
\hline Unit $106 \mathrm{NW}^{1 / 4}$ & $20-23$ & 1246 & 1125 & Unanalyzed & 1 & 0.2 & & & & & & & & & & & \\
\hline Unit $106 \mathrm{NW}^{1 / 4}$ & $10-20$ & 1203 & 1121 & Unanalyzed & 1 & 0.1 & & & & & & & & & & & \\
\hline Unit $106 \mathrm{NW}^{1 / 4}$ & $25-30$ & 1461 & 1130 & Analyzed & 1 & 0.3 & LPR & PWS & LOC & CRT & 4 & & I & FRG & 0 & IND & 2 \\
\hline Unit $106 \mathrm{NW}^{1 / 4}$ & $25-30$ & 1461 & 1130 & Analyzed & 1 & 0.5 & LPR & PWS & LOC & QZT & 4 & & I & DEB & 2 & IND & 2 \\
\hline Unit $106 \mathrm{NW}^{1 / 4}$ & $25-30$ & 1461 & 1130 & Analyzed & 1 & 0.04 & LPR & PWS & LOC & CRT & 4 & & I & COM & 0 & FLT & 2 \\
\hline Unit $106 \mathrm{NW}^{1 / 4}$ & $25-30$ & 1461 & 1130 & Analyzed & 1 & 0.04 & LPR & PWS & LOC & CRT & 4 & & I & COM & 1 & CRT & 2 \\
\hline Unit $106 \mathrm{NW}^{1 / 4}$ & $25-30$ & 1461 & 1130 & Analyzed & 1 & 0.3 & LPR & PWS & LOC & CRT & 4 & & I & COM & 0 & FAC & 2 \\
\hline Unit 106 SE$^{1 / 4}$ & $25-30$ & 1463 & 1131 & Analyzed & 1 & 0.7 & LPR & PWS & LOC & QZT & 4 & & I & COM & 1 & CRT & 1 \\
\hline Unit $106 \mathrm{SE}^{1 / 4}$ & $20-23$ & 1248 & 1126 & Unanalyzed & 1 & 0.1 & & & & & & & & & & & \\
\hline Unit $106 \mathrm{SE}^{1 / 4}$ & $10-20$ & 1205 & 1122 & Unanalyzed & 3 & 1.1 & & & & & & & & & & & \\
\hline Unit $106 \mathrm{SE}^{1 / 4}$ & $0-10$ & 1112 & 1119 & Unanalyzed & 2 & 0.6 & & & & & & & & & & & \\
\hline Unit $106 \mathrm{SW}^{1 / 4}$ & $20-23$ & 1245 & 1127 & Unanalyzed & 7 & 1.1 & & & & & & & & & & & \\
\hline Unit $106 \mathrm{SW}^{1 / 4}$ & $10-20$ & 1202 & 1123 & Unanalyzed & 2 & 1.3 & & & & & & & & & & & \\
\hline Unit $106 \mathrm{SW}^{1 / 4}$ & $25-30$ & 1460 & 1132 & Analyzed & 1 & 0.1 & LPR & PWS & LOC & QZT & 4 & & I & COM & 0 & FLT & 1 \\
\hline Unit 118 General & $19-29$ & 1616 & 1391 & Analyzed & 1 & 1.2 & LPR & PWS & LOC & CRT & 4 & & I & BRK & 1 & FAC & 1 \\
\hline Unit $118 \mathrm{NE}^{1 / 4}$ & $29-39$ & 1619 & 1398 & Analyzed & 1 & 0.6 & LPR & PWS & LOC & QZT & 4 & & I & COM & 4 & FLT & 1 \\
\hline Unit $118 \mathrm{NE}^{1 / 4}$ & $19-29$ & 1614 & 1392 & Analyzed & 1 & 0.2 & LPR & PWS & LOC & QZT & 4 & & I & COM & 0 & FLT & 3 \\
\hline Unit $118 \mathrm{NE}^{1 / 4}$ & $19-29$ & 1614 & 1392 & Analyzed & 1 & 0.04 & LPR & PWS & LOC & CRT & 4 & & I & COM & 4 & CRT & 3 \\
\hline Unit $118 \mathrm{NE}^{1 / 4}$ & $19-29$ & 1614 & 1392 & Analyzed & 1 & 0.1 & LPR & PWS & LOC & CRT & 4 & & I & FRG & 0 & IND & 3 \\
\hline Unit $118 \mathrm{NE}^{1 / 4}$ & $19-29$ & 1614 & 1392 & Analyzed & 1 & 3.1 & LPR & PWS & LOC & CRT & 2 & ABS & $\mathrm{N}$ & СOM & 1 & CPX & 3 \\
\hline Unit $118 \mathrm{NE}^{1 / 4}$ & $9-19$ & 1514 & 1387 & Unanalyzed & 1 & 2.5 & & & & & & & & & & & \\
\hline Unit $118 \mathrm{NW}^{1 / 4}$ & $29-39$ & 1618 & 1399 & Analyzed & 2 & 0.4 & LPR & PWS & LOC & CRT & 4 & & I & COM & 0 & $\mathrm{ABR}$ & 1 \\
\hline Unit $118 \mathrm{NW}^{1} / 4$ & $29-39$ & 1618 & 1399 & Analyzed & 1 & 0.1 & LPR & PWS & LOC & CRT & 4 & & I & FRG & 0 & IND & 1 \\
\hline
\end{tabular}


Geo-Marine, Inc.

GMI 22005.01.06.xx and 22005.00.09.xx, Data Recovery Excavations at Site 41PN175 Debitage Analysi

\begin{tabular}{|c|c|c|c|c|c|c|c|c|c|c|c|c|c|c|c|c|c|}
\hline Unit & $\begin{array}{l}\text { Depth } \\
(\mathrm{cm})\end{array}$ & $\begin{array}{l}\text { Provenience } \\
\text { No. }\end{array}$ & $\begin{array}{l}\text { Lot } \\
\text { No. }\end{array}$ & Level of Analysis & Quantity & $\begin{array}{l}\text { Weight } \\
\text { (in g) }\end{array}$ & General Period & Region & Material & Lithology & Size Grade & $\begin{array}{c}\text { Edge } \\
\text { Modification }\end{array}$ & $\begin{array}{l}\text { Thernal } \\
\text { Alteration }\end{array}$ & Completeness & Cortex & Platform & $\begin{array}{l}\text { Minimum } \\
\text { Number of } \\
\text { Nodules }\end{array}$ \\
\hline \multicolumn{18}{|c|}{ Block 4} \\
\hline Unit $118 \mathrm{NW}^{1 / 4}$ & $29-39$ & 1618 & 1399 & Analyzed & 2 & 0.2 & LPR & PWS & LOC & CRT & 4 & & I & сом & 2 & CRT & 1 \\
\hline Unit $118 \mathrm{NW}^{1 / 4}$ & $29-39$ & 1618 & 1399 & Analyzed & 1 & 0.4 & LPR & PWS & LOC & CRT & 4 & & I & сOM & 4 & CRT & 1 \\
\hline Unit $118 \mathrm{NW}^{1 / 4}$ & $19-29$ & 1613 & 1395 & Analyzed & 1 & 0.9 & LPR & PWS & LOC & CRT & 4 & & I & сOM & 2 & FLT & 1 \\
\hline Unit $118 \mathrm{NW}^{1 / 4}$ & $19-29$ & 1613 & 1395 & Analyzed & 1 & 0.1 & LPR & PWS & LOC & CRT & 4 & & I & DEB & 0 & $\mathrm{IND}$ & 1 \\
\hline Unit $118 \mathrm{NW}^{1 / 4}$ & $19-29$ & 1613 & 1395 & Analyzed & 1 & 0.7 & LPR & PWS & LOC & CRT & 4 & & I & DEB & 3 & IND & 1 \\
\hline Unit $118 \mathrm{NW}^{1 / 4}$ & $19-29$ & 1613 & 1395 & Analyzed & 1 & 0.4 & LPR & PWS & LOC & CRT & 4 & & I & сом & 1 & CRT & 1 \\
\hline Unit $118 \mathrm{NW}^{1 / 4}$ & $19-29$ & 1613 & 1395 & Analyzed & 1 & 0.1 & LPR & PWS & LOC & CRT & 4 & & I & BRK & 1 & CRT & 1 \\
\hline Unit $118 \mathrm{NW}^{1 / 4}$ & $9-19$ & 1513 & 1388 & Unanalyzed & 2 & 0.8 & & & & & & & & & & & \\
\hline Unit $118 \mathrm{SE}^{1 / 4}$ & $29-39$ & 1620 & 1400 & Analyzed & 1 & 0.5 & LPR & PWS & LOC & CRT & 4 & & I & DEB & 3 & $\mathrm{IND}$ & 1 \\
\hline Unit 118 SW$^{1} / 4$ & $19-29$ & 1612 & 1397 & Analyzed & 3 & 1 & LPR & PWS & LOC & CRT & 4 & & I & сOM & 1 & CRT & 2 \\
\hline Unit 118 SW11/4 & $19-29$ & 1612 & 1397 & Analyzed & 1 & 1 & LPR & PWS & LOC & CRT & 4 & & I & сом & 0 & CPX & 2 \\
\hline Unit 118 SW$^{1 / 4}$ & $19-29$ & 1612 & 1397 & Analyzed & 1 & 0.1 & LPR & PWS & LOC & CRT & 4 & & I & сOM & 4 & CRT & 2 \\
\hline Unit 118 SW1/4 & $19-29$ & 1612 & 1397 & Analyzed & 1 & 1.5 & LPR & PWS & LOC & QZT & 3 & & $\mathrm{~N}$ & FRG & 3 & IND & 2 \\
\hline Unit 118 SW11/4 & $29-39$ & 1617 & 1401 & Analyzed & 2 & 4.5 & LPR & PWS & LOC & CRT & 3 & & $\mathrm{~N}$ & DEB & 3 & IND & 1 \\
\hline Unit 118 SW$^{1} / 4$ & $19-29$ & 1612 & 1397 & Analyzed & 1 & 0.2 & LPR & PWS & LOC & QZT & 4 & & I & FRG & 1 & IND & 2 \\
\hline Unit $118 \mathrm{SW}^{1 / 4}$ & $9-19$ & 1512 & 1390 & Unanalyzed & 2 & 0.5 & & & & & & & & & & & \\
\hline Unit $119 \mathrm{NE}^{1 / 4}$ & $9-19$ & 1584 & 1405 & Unanalyzed & 1 & 0.4 & & & & & & & & & & & \\
\hline Unit $119 \mathrm{NE}^{1 / 4}$ & $19-29$ & 1622 & 1408 & Unanalyzed & 2 & 0.6 & & & & & & & & & & & \\
\hline Unit $119 \mathrm{SE}^{1 / 4}$ & $0-9$ & 1494 & 1403 & Unanalyzed & 1 & 1.8 & & & & & & & & & & & \\
\hline Unit $119 \mathrm{SE}^{1 / 4}$ & $19-29$ & 1623 & 1409 & Unanalyzed & 3 & 5.3 & & & & & & & & & & & \\
\hline Unit $119 \mathrm{SE}^{1 / 4}$ & $29-39$ & 1626 & 1412 & Unanalyzed & 3 & 1.7 & & & & & & & & & & & \\
\hline Unit 119 SW11/4 & $9-19$ & 1583 & 1407 & Unanalyzed & 1 & 0.2 & & & & & & & & & & & \\
\hline Unit 119 SW1/4 & $19-29$ & 1621 & 1410 & Unanalyzed & 2 & 0.6 & & & & & & & & & & & \\
\hline Unit $119 \mathrm{SW}^{1 / 4}$ & $29-39$ & 1624 & 1413 & Unanalyzed & 4 & 1.3 & & & & & & & & & & & \\
\hline Unit $120 \mathrm{NE}^{1 / 4}$ & $30-35$ & 1637 & 1436 & Unanalyzed & 3 & 1.5 & & & & & & & & & & & \\
\hline Unit $120 \mathrm{NE}^{1 / 4}$ & $0-5$ & 1495 & 1416 & Unanalyzed & 2 & 1 & & & & & & & & & & & \\
\hline
\end{tabular}


Geo-Marine, Inc.

GMI 22005.01.06.xx and 22005.00.09.xx, Data Recovery Excavations at Site 41PN17 Debitage Analysi

\begin{tabular}{|c|c|c|c|c|c|c|c|c|c|c|c|c|c|c|c|c|c|}
\hline Unit & $\begin{array}{l}\text { Depth } \\
(\mathrm{cm})\end{array}$ & $\begin{array}{l}\text { Provenience } \\
\text { No. }\end{array}$ & $\begin{array}{l}\text { Lot } \\
\text { No. }\end{array}$ & Level of Analysis & Quantity & $\begin{array}{l}\text { Weight } \\
\text { (ing) }\end{array}$ & General Period & Region & Material & Lithology & Size Grade & $\begin{array}{c}\text { Edge } \\
\text { Modification }\end{array}$ & $\begin{array}{l}\text { Therral } \\
\text { Alteration }\end{array}$ & Completeness & Cortex & Platform & $\begin{array}{l}\text { Minimum } \\
\text { Number of } \\
\text { Nodules }\end{array}$ \\
\hline
\end{tabular}

\begin{tabular}{|c|c|c|c|c|c|c|}
\hline Block 4 & & & & & & \\
\hline Unit $120 \mathrm{NE}^{1 / 4}$ & $20-25$ & 1629 & 1428 & Unanalyzed & 1 & 0.3 \\
\hline Unit $120 \mathrm{NE}^{1 / 4}$ & $15-20$ & 1523 & 1424 & Unanalyzed & 1 & 5 \\
\hline Unit $120 \mathrm{NE}^{1 / 4}$ & $10-15$ & 1519 & 1420 & Unanalyzed & 2 & 0.8 \\
\hline Unit $120 \mathrm{NW}^{1 / 4}$ & $35-40$ & 1640 & 1441 & Unanalyzed & 3 & 0.8 \\
\hline Unit $120 \mathrm{NW}^{1 / 4}$ & $25-30$ & 1632 & 1433 & Unanalyzed & 2 & 1.6 \\
\hline Unit $120 \mathrm{NW}^{1 / 4}$ & $20-25$ & 1628 & 1429 & Unanalyzed & 2 & 1.7 \\
\hline Unit $120 \mathrm{NW}^{1 / 4}$ & $30-35$ & 1636 & 1437 & Unanalyzed & 2 & 0.5 \\
\hline Unit $120 \mathrm{NW}^{1 / 4}$ & $40-45$ & 1780 & 1444 & Unanalyzed & 1 & 0.6 \\
\hline Unit $120 \mathrm{SE}^{1} / 4$ & $30-35$ & 1638 & 1438 & Unanalyzed & 1 & 0.2 \\
\hline Unit $120 \mathrm{SE}^{1 / 4}$ & $25-30$ & 1634 & 1434 & Unanalyzed & 1 & 0.2 \\
\hline Unit $120 \mathrm{SW}^{1 / 4}$ & $25-30$ & 1631 & 1435 & Unanalyzed & 1 & 5 \\
\hline Unit $120 \mathrm{SW}^{1 / 4}$ & $20-25$ & 1627 & 1431 & Unanalyzed & 1 & 0.4 \\
\hline Unit 120, Feature $28 \mathrm{~S} 1 / 2$ & $41-53$ & 1838 & 1626 & Analyzed & 3 & 0.04 \\
\hline Unit 120, Feature $28 \mathrm{~S} 1 / 2$ & $41-53$ & 1838 & 1626 & Analyzed & 1 & 0.2 \\
\hline Unit 120, Feature $29 \mathrm{~N}^{1} / 2$ & $41-55$ & 1840 & 1627 & Analyzed & 15 & 0.6 \\
\hline Unit 120, Feature $29 \mathrm{~N}^{1 / 2}$ & $41-55$ & 2000 & 1445 & Analyzed & 1 & 0.5 \\
\hline Unit 120, Feature $29 \mathrm{~N} 1 / 2$ & $41-55$ & 1840 & 1627 & Analyzed & 10 & 0.1 \\
\hline Unit 120, Feature $29 \mathrm{~N} 1 / 2$ & $41-55$ & 2000 & 1445 & Analyzed & 1 & 1.4 \\
\hline Unit 120, Feature $29 \mathrm{~N} 1 / 2$ & $41-55$ & 1840 & 1627 & Analyzed & 1 & 0.2 \\
\hline Unit 120, Feature $29 \mathrm{~N}^{1 / 2}$ & $41-55$ & 1840 & 1627 & Analyzed & 1 & 0.2 \\
\hline Unit 120, Feature $29 \mathrm{~N}^{1} / 2$ & $41-55$ & 2000 & 1445 & Analyzed & 1 & 0.1 \\
\hline Unit 120, Feature $29 \mathrm{~N} 1 / 2$ & $41-55$ & 1840 & 1627 & Analyzed & 2 & 0.04 \\
\hline Unit 120, Feature $29 \mathrm{~N}^{1 / 2}$ & $41-55$ & 1840 & 1627 & Analyzed & 1 & 0.04 \\
\hline Unit 120, Feature $29 \mathrm{~N}^{1 / 2}$ & $41-55$ & 1840 & 1627 & Analyzed & 1 & 0.1 \\
\hline Unit 120, Feature $29 \mathrm{~N}^{1 / 2}$ & $41-55$ & 1840 & 1627 & Analyzed & 3 & 0.04 \\
\hline Unit 121 General & $0-12$ & 1570 & 1446 & Unanalyzed & 1 & 0.6 \\
\hline
\end{tabular}

$\begin{array}{ll}\text { LPR } & \text { PWS } \\ \text { LPR } & \text { PWS } \\ \text { LPR } & \text { PWS } \\ \text { LPR } & \text { PWS } \\ \text { LPR } & \text { PWS } \\ \text { LPR } & \text { PWS } \\ \text { LPR } & \text { PWS } \\ \text { LPR } & \text { PWS } \\ \text { LPR } & \text { PWS } \\ \text { LPR } & \text { PWS } \\ \text { LPR } & \text { PWS } \\ \text { LPR } & \text { PWS } \\ \text { LPR } & \text { PWS }\end{array}$

INT
LOC
LOC
LOC
INT
LOC
LOC
LOC
LOC
LOC
LOC
LOC
LOC


Geo-Marine, Inc.

GMI 22005.01.06.xx and 22005.00.09.xx, Data Recovery Excavations at Site 41PN17 Debitage Analysi

\begin{tabular}{|c|c|c|c|c|c|c|c|c|c|c|c|c|c|c|c|c|c|}
\hline Unit & $\begin{array}{l}\text { Depth } \\
(\mathrm{cm})\end{array}$ & $\begin{array}{l}\text { Provenience } \\
\text { No. }\end{array}$ & $\begin{array}{l}\text { Lot } \\
\text { No. }\end{array}$ & Level of Analysis & Quantity & $\begin{array}{l}\text { Weight } \\
\text { (in g) }\end{array}$ & General Period & Region & Material & Lithology & Size Grade & $\begin{array}{c}\text { Edge } \\
\text { Modification }\end{array}$ & $\begin{array}{l}\text { Thernal } \\
\text { Alteration }\end{array}$ & Completeness & Cortex & Platform & $\begin{array}{l}\text { Minimun } \\
\text { Number c } \\
\text { Nodules }\end{array}$ \\
\hline \multicolumn{18}{|c|}{ Block 4} \\
\hline Unit 121 General & $12-22$ & 1533 & 1451 & Unanalyzed & 1 & 0.4 & & & & & & & & & & & \\
\hline Unit $121 \mathrm{NE}^{1 / 4}$ & $12-22$ & 1531 & 1452 & Unanalyzed & 3 & 4.5 & & & & & & & & & & & \\
\hline Unit $121 \mathrm{NE}^{1 / 4}$ & $22-32$ & 1588 & 1456 & Analyzed & 1 & 0.3 & LPR & PWS & LOC & QZT & 4 & & I & COM & 4 & CRT & 1 \\
\hline Unit $121 \mathrm{NW}^{1 / 4}$ & $32-42$ & 1644 & 1461 & Analyzed & 1 & 1 & LPR & PWS & LOC & CRT & 4 & & I & COM & 4 & CRT & 1 \\
\hline Unit $121 \mathrm{NW}^{1 / 4}$ & $0-12$ & 1526 & 1448 & Unanalyzed & 2 & 0.5 & & & & & & & & & & & \\
\hline Unit $121 \mathrm{NW}^{1 / 4}$ & $22-32$ & 1587 & 1457 & Analyzed & 1 & 0.1 & LPR & PWS & LOC & QZT & 4 & & I & COM & 0 & FLT & 1 \\
\hline Unit $121 \mathrm{NW}^{1 / 4}$ & $32-42$ & 1644 & 1461 & Analyzed & 1 & 0.3 & LPR & PWS & LOC & CRT & 4 & & I & FRG & 0 & IND & 1 \\
\hline Unit $121 \mathrm{NW}^{1 / 4}$ & $12-22$ & 1530 & 1453 & Unanalyzed & 1 & 0.1 & & & & & & & & & & & \\
\hline Unit $121 \mathrm{SE}^{1 / 4}$ & $22-32$ & 1589 & 1458 & Analyzed & 1 & 0.04 & LPR & PWS & LOC & CRT & 4 & & I & COM & 0 & FLT & 1 \\
\hline Unit $121 \mathrm{SE}^{1 / 4}$ & $12-22$ & 1532 & 1454 & Unanalyzed & 2 & 1 & & & & & & & & & & & \\
\hline Unit $121 \mathrm{SW}^{1 / 4}$ & $12-22$ & 1529 & 1455 & Unanalyzed & 1 & 0.2 & & & & & & & & & & & \\
\hline Unit $121 \mathrm{SW}^{1 / 4}$ & $22-32$ & 1586 & 1459 & Analyzed & 1 & 0.04 & LPR & PWS & LOC & FWD & 4 & & I & COM & 0 & FLT & 3 \\
\hline Unit $121 \mathrm{SW}^{1 / 4}$ & $22-32$ & 1586 & 1459 & Analyzed & 1 & 0.3 & LPR & PWS & LOC & CRT & 4 & & I & FRG & 3 & IND & 3 \\
\hline Unit $121 \mathrm{SW}^{1 / 4}$ & $22-32$ & 1586 & 1459 & Analyzed & 1 & 0.1 & LPR & PWS & LOC & CRT & 4 & & I & FRG & 1 & IND & 3 \\
\hline Unit $121 \mathrm{SW}^{1 / 4}$ & $22-32$ & 1586 & 1459 & Analyzed & 1 & 0.4 & LPR & PWS & LOC & CRT & 4 & & I & FRG & 0 & IND & 3 \\
\hline Unit $121 \mathrm{SW}^{1} / 4$ & $22-32$ & 1586 & 1459 & Analyzed & 1 & 0.2 & LPR & PWS & LOC & CRT & 4 & & I & COM & 0 & CPX & 3 \\
\hline Unit $121 \mathrm{SW}^{1 / 4}$ & $22-32$ & 1586 & 1459 & Analyzed & 1 & 0.4 & LPR & PWS & LOC & CRT & 4 & & I & СOM & 1 & CRT & 3 \\
\hline Unit $121 \mathrm{SW}^{1 / 4}$ & $32-42$ & 1643 & 1463 & Analyzed & 1 & 0.04 & LPR & PWS & LOC & CRT & 5 & & I & DEB & 0 & IND & 1 \\
\hline Unit $121 \mathrm{SW}^{1 / 4}$ & $22-32$ & 1586 & 1459 & Analyzed & 1 & 0.04 & LPR & PWS & LOC & QZT & 4 & & I & COM & 0 & FLT & 3 \\
\hline Unit $121 \mathrm{SW}^{1 / 4}$ & $22-32$ & 1586 & 1459 & Analyzed & 1 & 0.3 & LPR & PWS & LOC & CRT & 4 & & I & COM & 1 & FLT & 3 \\
\hline Unit $122 \mathrm{NE}^{1 / 4}$ & $0-14$ & 1501 & 1464 & Unanalyzed & 1 & 1.2 & & & & & & & & & & & \\
\hline Unit $122 \mathrm{NE}^{1 / 4}$ & $24-34$ & 1649 & 1472 & Analyzed & 1 & 0.04 & LPR & PWS & LOC & CRT & 4 & & I & COM & 1 & CPX & 1 \\
\hline Unit $122 \mathrm{NE}^{1 / 4}$ & $24-34$ & 1649 & 1472 & Analyzed & 2 & 0.5 & LPR & PWS & LOC & CRT & 4 & & I & COM & 1 & CRT & 1 \\
\hline Unit $122 \mathrm{NE}^{1 / 4}$ & $14-24$ & 1573 & 1468 & Unanalyzed & 1 & 2.2 & & & & & & & & & & & \\
\hline Unit $122 \mathrm{NE}^{1 / 4}$ & $34-44$ & 1653 & 1476 & Analyzed & 1 & 0.3 & LPR & PWS & LOC & CRT & 4 & & I & COM & 0 & FAC & 1 \\
\hline Unit $122 \mathrm{NW}^{1} / 4$ & $24-34$ & 1648 & 1473 & Analyzed & 1 & 0.2 & LPR & PWS & LOC & CRT & 4 & & I & FRG & 0 & IND & 1 \\
\hline
\end{tabular}


Geo-Marine, Inc.

GMI 22005.01.06.xx and 22005.00.09.xx, Data Recovery Excavations at Site 41PN17 Debitage Analysi

\begin{tabular}{|c|c|c|c|c|c|c|c|c|c|c|c|c|c|c|c|c|c|}
\hline Unit & $\begin{array}{l}\text { Depth } \\
(\mathrm{cm})\end{array}$ & $\begin{array}{l}\text { Provenience } \\
\text { No. }\end{array}$ & $\begin{array}{l}\text { Lot } \\
\text { No. }\end{array}$ & Level of Analysis & Quantity & $\begin{array}{l}\text { Weight } \\
\text { (in g) }\end{array}$ & General Period & Region & Material & Lithology & Size Grade & $\begin{array}{c}\text { Edge } \\
\text { Modification }\end{array}$ & $\begin{array}{l}\text { Thernal } \\
\text { Alteration }\end{array}$ & Completeness & Cortex & Platform & $\begin{array}{l}\text { Minimun } \\
\text { Number c } \\
\text { Nodules }\end{array}$ \\
\hline \multicolumn{18}{|l|}{ Block 4} \\
\hline Unit $122 \mathrm{NW}^{1 / 4}$ & $24-34$ & 1648 & 1473 & Analyzed & 1 & 1.1 & LPR & PWS & LOC & CRT & 4 & & I & DEB & 2 & IND & 1 \\
\hline Unit $122 \mathrm{SE}^{1 / 4}$ & $0-14$ & 1502 & 1466 & Unanalyzed & 1 & 0.2 & & & & & & & & & & & \\
\hline Unit $122 \mathrm{SE}^{1 / 4}$ & $34-44$ & 1654 & 1478 & Analyzed & 1 & 1.7 & LPR & PWS & LOC & CRT & 3 & & $\mathrm{~N}$ & FRG & 4 & IND & 1 \\
\hline Unit $122 \mathrm{SE}^{1 / 4}$ & $24-34$ & 1650 & 1474 & Analyzed & 1 & 0.1 & LPR & PWS & LOC & CRT & 4 & & I & BRK & 0 & CPX & 2 \\
\hline Unit $122 \mathrm{SE}^{1 / 4}$ & $24-34$ & 1650 & 1474 & Analyzed & 1 & 0.8 & LPR & PWS & LOC & QZT & 3 & & $\mathrm{~N}$ & СOM & 1 & FLT & 2 \\
\hline Unit $122 \mathrm{SE}^{1 / 4}$ & $24-34$ & 1650 & 1474 & Analyzed & 1 & 0.1 & LPR & PWS & LOC & QZT & 4 & & I & COM & 4 & CRT & 2 \\
\hline Unit $122 \mathrm{SE}^{1 / 4}$ & $24-34$ & 1650 & 1474 & Analyzed & 1 & 0.5 & LPR & PWS & LOC & QZT & 4 & & I & Сом & 1 & CRT & 2 \\
\hline Unit $122 \mathrm{SE}^{1 / 4}$ & $14-24$ & 1574 & 1470 & Unanalyzed & 2 & 3 & & & & & & & & & & & \\
\hline Unit $122 \mathrm{SW}^{1 / 4}$ & $24-34$ & 1647 & 1475 & Analyzed & 1 & 0.1 & LPR & PWS & LOC & CRT & 4 & & I & COM & 0 & FLT & 1 \\
\hline Unit $122 \mathrm{SW}^{1} / 4$ & $14-24$ & 1571 & 1471 & Unanalyzed & 3 & 2.2 & & & & & & & & & & & \\
\hline Unit 122, Feature $31 \mathrm{~S} 1 / 2$ & $49-55$ & 1844 & 1630 & Analyzed & 1 & 0.04 & LPR & PWS & LOC & CRT & 4 & & I & FRG & 4 & IND & 1 \\
\hline Unit 122, Feature $32 \mathrm{~S}^{1} / 2$ & $49-57$ & 1845 & 1631 & Analyzed & 1 & 0.04 & LPR & PWS & LOC & CRT & 5 & & I & FRG & 1 & IND & 1 \\
\hline Unit 123 General & $20-25$ & 1594 & 1494 & Unanalyzed & 1 & 0.3 & & & & & & & & & & & \\
\hline Unit $123 \mathrm{NE}^{1 / 4}$ & $30-35$ & 1661 & 1503 & Analyzed & 1 & 0.7 & LPR & PWS & LOC & QZT & 4 & & I & COM & 0 & FLT & 1 \\
\hline Unit $123 \mathrm{NE}^{1 / 4}$ & $20-25$ & 1592 & 1495 & Unanalyzed & 3 & 1.1 & & & & & & & & & & & \\
\hline Unit $123 \mathrm{NE}^{1 / 4}$ & $25-30$ & 1657 & 1499 & Analyzed & 2 & 0.1 & LPR & PWS & LOC & CRT & 4 & & I & COM & 1 & CRT & 1 \\
\hline Unit $123 \mathrm{NE}^{1 / 4}$ & $10-15$ & 1536 & 1486 & Unanalyzed & 1 & 0.5 & & & & & & & & & & & \\
\hline Unit $123 \mathrm{NE}^{1 / 4}$ & $30-35$ & 1661 & 1503 & Analyzed & 1 & 0.1 & LPR & PWS & LOC & CRT & 4 & & I & COM & 0 & FLT & 1 \\
\hline Unit $123 \mathrm{NE}^{1 / 4}$ & $25-30$ & 1657 & 1499 & Analyzed & 2 & 1.7 & LPR & PWS & LOC & CRT & 4 & & I & COM & 2 & CPX & 1 \\
\hline Unit $123 \mathrm{NW}^{1 / 4}$ & $20-25$ & 1591 & 1496 & Unanalyzed & 1 & 0.89 & & & & & & & & & & & \\
\hline Unit $123 \mathrm{NW}^{1 / 4}$ & $35-40$ & 1664 & 1507 & Analyzed & 1 & 0.3 & LPR & PWS & LOC & CRT & 4 & & I & COM & 4 & CRT & 1 \\
\hline Unit $123 \mathrm{NW}^{1 / 4}$ & $35-40$ & 1664 & 1507 & Analyzed & 1 & 0.1 & LPR & PWS & LOC & CRT & 4 & & I & BRK & 0 & FAC & 1 \\
\hline Unit $123 \mathrm{NW}^{1 / 4}$ & $30-35$ & 1660 & 1504 & Analyzed & 2 & 0.3 & LPR & PWS & LOC & CRT & 4 & & I & DEB & 1 & IND & 1 \\
\hline Unit $123 \mathrm{NW}^{1 / 4}$ & $30-35$ & 1660 & 1504 & Analyzed & 1 & 0.3 & LPR & PWS & LOC & FWD & 4 & & I & COM & 1 & CRT & 1 \\
\hline Unit $123 \mathrm{NW}^{1 / 4}$ & $25-30$ & 1656 & 1500 & Analyzed & 1 & 0.1 & LPR & PWS & LOC & CRT & 4 & & I & BRK & 0 & FLT & 1 \\
\hline Unit $123 \mathrm{NW}^{1} / 4$ & $25-30$ & 1656 & 1500 & Analyzed & 1 & 0.2 & LPR & PWS & LOC & CRT & 4 & & I & FRG & 0 & IND & 1 \\
\hline
\end{tabular}


Geo-Marine, Inc.

GMI 22005.01.06.xx and 22005.00.09.xx, Data Recovery Excavations at Site 41PN175 Debitage Analysi

\begin{tabular}{|c|c|c|c|c|c|c|c|c|c|c|c|c|c|c|c|c|c|}
\hline Unit & $\begin{array}{l}\text { Depth } \\
(\mathrm{cm})\end{array}$ & $\begin{array}{l}\text { Provenience } \\
\text { No. }\end{array}$ & $\begin{array}{l}\text { Lot } \\
\text { No. }\end{array}$ & Level of Analysis & Quantity & $\begin{array}{l}\text { Weight } \\
\text { (in g) }\end{array}$ & General Period & Region & Material & Lithology & Size Grade & $\begin{array}{c}\text { Edge } \\
\text { Modification }\end{array}$ & $\begin{array}{l}\text { Thernal } \\
\text { Alteration }\end{array}$ & Completeness & Cortex & Platform & $\begin{array}{l}\text { Minimum } \\
\text { Number of } \\
\text { Nodules }\end{array}$ \\
\hline \multicolumn{18}{|c|}{ Block 4} \\
\hline Unit $123 \mathrm{SE}^{1 / 4}$ & $10-15$ & 1537 & 1488 & Unanalyzed & 1 & 0.1 & & & & & & & & & & & \\
\hline Unit $123 \mathrm{SE}^{1 / 4}$ & $25-30$ & 1658 & 1501 & Analyzed & 1 & 2.5 & LPR & PWS & LOC & CRT & 3 & & $\mathrm{~N}$ & DEB & 4 & IND & 1 \\
\hline Unit $123 \mathrm{SE}^{1 / 4}$ & $5-10$ & 1508 & 1484 & Unanalyzed & 2 & 1.2 & & & & & & & & & & & \\
\hline Unit $123 \mathrm{SE}^{1 / 4}$ & $30-35$ & 1662 & 1505 & Analyzed & 1 & 0.4 & LPR & PWS & LOC & QZT & 4 & & I & COM & 1 & FLT & 1 \\
\hline Unit $123 \mathrm{SE}^{1 / 4}$ & $30-35$ & 1662 & 1505 & Analyzed & 1 & 0.04 & LPR & PWS & LOC & QZT & 4 & & I & COM & 0 & FLT & 1 \\
\hline Unit $123 \mathrm{SE}^{1 / 4}$ & $20-25$ & 1593 & 1497 & Unanalyzed & 2 & 0.8 & & & & & & & & & & & \\
\hline Unit $123 \mathrm{SW}^{1 / 4}$ & $25-30$ & 1655 & 1502 & Analyzed & 1 & 0.04 & LPR & PWS & LOC & CRT & 4 & & I & COM & 0 & FLT & 1 \\
\hline Unit $123 \mathrm{SW}^{1 / 4}$ & $5-10$ & 1505 & 1485 & Unanalyzed & 1 & 0.5 & & & & & & & & & & & \\
\hline Unit $123 \mathrm{SW}^{1} 1 / 4$ & $35-40$ & 1663 & 1509 & Analyzed & 1 & 0.1 & LPR & PWS & REG & NVC & 4 & & I & COM & 0 & FLT & 1 \\
\hline Unit $123 \mathrm{SW}^{1 / 4}$ & $30-35$ & 1659 & 1506 & Analyzed & 1 & 0.2 & LPR & PWS & LOC & CRT & 4 & & I & DEB & 0 & IND & 1 \\
\hline Unit $123 \mathrm{SW}^{1 / 4}$ & $25-30$ & 1655 & 1502 & Analyzed & 1 & 0.04 & LPR & PWS & LOC & CRT & 4 & & I & COM & 1 & FLT & 1 \\
\hline Unit $123 \mathrm{SW}^{1 / 4}$ & $15-20$ & 1538 & 1493 & Unanalyzed & 1 & 0.2 & & & & & & & & & & & \\
\hline Unit $123 \mathrm{SW}^{1 / 4}$ & $20-25$ & 1590 & 1498 & Unanalyzed & 3 & 1.1 & & & & & & & & & & & \\
\hline Unit $124 \mathrm{NE}^{1 / 4}$ & $16-21$ & 1554 & 1525 & Unanalyzed & 3 & 2 & & & & & & & & & & & \\
\hline Unit $124 \mathrm{NE}^{1 / 4}$ & $6-11$ & 1546 & 1517 & Unanalyzed & 1 & 0.4 & & & & & & & & & & & \\
\hline Unit $124 \mathrm{NE}^{1 / 4}$ & $21-26$ & 1577 & 1529 & Analyzed & 1 & 0.2 & LPR & PWS & LOC & CRT & 4 & & I & BRK & 1 & CRT & \\
\hline Unit $124 \mathrm{NW}^{1 / 4}$ & $26-31$ & 1595 & 1534 & Analyzed & 1 & 0.1 & LPR & PWS & REG & CRT & 4 & & I & FRG & 0 & IND & 2 \\
\hline Unit $124 \mathrm{NW}^{1 / 4}$ & $21-26$ & 1576 & 1530 & Analyzed & 1 & 0.1 & LPR & PWS & LOC & QZT & 4 & & I & COM & 4 & CRT & 2 \\
\hline Unit $124 \mathrm{NW}^{1 / 4}$ & $21-26$ & 1576 & 1530 & Analyzed & 1 & 0.8 & LPR & PWS & LOC & FWD & 4 & & I & COM & 3 & CRT & 2 \\
\hline Unit $124 \mathrm{NW}^{1 / 4}$ & $0-6$ & 1542 & 1515 & Unanalyzed & 1 & 0.4 & & & & & & & & & & & \\
\hline Unit $124 \mathrm{NW}^{1 / 4}$ & $6-11$ & 1545 & 1518 & Unanalyzed & 2 & 1.3 & & & & & & & & & & & \\
\hline Unit $124 \mathrm{NW}^{1 / 4}$ & $11-16$ & 1549 & 1522 & Unanalyzed & 1 & 0.2 & & & & & & & & & & & \\
\hline Unit $124 \mathrm{NW}^{1 / 4}$ & $16-21$ & 1553 & 1526 & Unanalyzed & 2 & 0.6 & & & & & & & & & & & \\
\hline Unit $124 \mathrm{NW}^{1 / 4}$ & $26-31$ & 1595 & 1534 & Analyzed & 1 & 0.2 & LPR & PWS & LOC & CRT & 4 & & I & COM & 2 & CRT & 2 \\
\hline Unit $124 \mathrm{SE}^{1 / 4}$ & $26-31$ & 1597 & 1535 & Analyzed & 1 & 0.2 & LPR & PWS & LOC & CRT & 4 & & I & DEB & 0 & IND & 1 \\
\hline Unit $124 \mathrm{SE}^{1 / 4}$ & $21-26$ & 1578 & 1531 & Analyzed & 1 & 0.2 & LPR & PWS & LOC & QZT & 4 & & I & DEB & 4 & IND & 2 \\
\hline
\end{tabular}


Geo-Marine, Inc.

GMI 22005.01.06.xx and 22005.00.09.xx, Data Recovery Excavations at Site 41PN17 Debitage Analysi

\begin{tabular}{|c|c|c|c|c|c|c|c|c|c|c|c|c|c|c|c|c|c|}
\hline Unit & $\begin{array}{c}\text { Depth } \\
(\mathrm{cm})\end{array}$ & $\begin{array}{l}\text { Provenience } \\
\text { No. }\end{array}$ & $\begin{array}{l}\text { Lot } \\
\text { No. }\end{array}$ & Level of Analysis & Quantity & $\begin{array}{l}\text { Weight } \\
\text { (ing) }\end{array}$ & General Period & Region & Material & Lithology & Size Grade & $\begin{array}{c}\text { Edge } \\
\text { Modification }\end{array}$ & $\begin{array}{l}\text { Thernal } \\
\text { Alteration }\end{array}$ & Completeness & Cortex & Platform & $\begin{array}{c}\text { Minimum } \\
\text { Number of } \\
\text { Nodules }\end{array}$ \\
\hline \multicolumn{18}{|c|}{ Block 4} \\
\hline Unit $124 \mathrm{SE}^{1 / 4}$ & $21-26$ & 1578 & 1531 & Analyzed & 1 & 0.3 & LPR & PWS & LOC & CRT & 4 & & I & COM & 0 & & 2 \\
\hline Unit $124 \mathrm{SE}^{1 / 4}$ & $21-26$ & 1578 & 1531 & Analyzed & 1 & 0.5 & LPR & PWS & LOC & CRT & 4 & & I & FRG & 1 & IND & 2 \\
\hline Unit $124 \mathrm{SE}^{1 / 4}$ & $16-21$ & 1555 & 1527 & Unanalyzed & 2 & 0.4 & & & & & & & & & & & \\
\hline Unit $124 \mathrm{SE}^{1 / 4}$ & $21-26$ & 1578 & 1531 & Analyzed & 1 & 0.04 & LPR & PWS & LOC & CRT & 4 & & I & $\mathrm{COM}$ & 1 & & 2 \\
\hline Unit $124 \mathrm{SE}^{1 / 4}$ & $6-11$ & 1547 & 1519 & Unanalyzed & 1 & 0.1 & & & & & & & & & & & \\
\hline Unit $124 \mathrm{SE}^{1 / 4}$ & $21-26$ & 1578 & 1531 & Analyzed & 2 & 0.6 & LPR & PWS & LOC & CRT & 4 & & I & COM & 4 & & 2 \\
\hline Unit $124 \mathrm{SW}^{1 / 4}$ & $6-11$ & 1544 & 1520 & Unanalyzed & 1 & 0.3 & & & & & & & & & & & \\
\hline Unit $124 \mathrm{SW}^{1 / 4}$ & $16-21$ & 1552 & 1528 & Unanalyzed & 4 & 1.1 & & & & & & & & & & & \\
\hline Unit $124 \mathrm{SW}^{1 / 4}$ & $36-41$ & 1673 & 1539 & Analyzed & 1 & 0.04 & LPR & PWS & LOC & QZT & 4 & & I & $\mathrm{COM}$ & 4 & CRT & 1 \\
\hline Unit $125 \mathrm{NE}^{1 / 4}$ & $15-20$ & 1568 & 1550 & Unanalyzed & 1 & 0.4 & & & & & & & & & & & \\
\hline Unit $125 \mathrm{NE}^{1 / 4}$ & $25-30$ & 1600 & 1558 & Analyzed & 1 & 0.5 & LPR & PWS & LOC & QZT & 3 & & $\mathrm{~N}$ & COM & 0 & CPX & 1 \\
\hline Unit $125 \mathrm{NE}^{1 / 4}$ & $25-30$ & 1600 & 1558 & Analyzed & 1 & 3.5 & LPR & PWS & LOC & QZT & 3 & & $\mathrm{~N}$ & FRG & 0 & $\mathrm{IND}$ & 1 \\
\hline Unit $125 \mathrm{NE}^{1 / 4}$ & $10-15$ & 1564 & 1546 & Unanalyzed & 1 & 0.2 & & & & & & & & & & & \\
\hline Unit $125 \mathrm{NW}^{1 / 4}$ & $20-25$ & 1580 & 1555 & Analyzed & 1 & 0.2 & LPR & PWS & LOC & QZT & 4 & & I & $\mathrm{COM}$ & 0 & FLT & 1 \\
\hline Unit $125 \mathrm{NW}^{1 / 4}$ & $30-35$ & 1677 & 1563 & Analyzed & 1 & 0.4 & LPR & PWS & LOC & QZT & 4 & & I & FRG & 4 & IND & 1 \\
\hline Unit $125 \mathrm{NW}^{1 / 4}$ & $25-30$ & 1599 & 1559 & Analyzed & 1 & 0.5 & LPR & PWS & LOC & CRT & 4 & & I & COM & 0 & FLT & 1 \\
\hline Unit $125 \mathrm{NW}^{1 / 4}$ & $15-20$ & 1567 & 1551 & Unanalyzed & 3 & 0.6 & & & & & & & & & & & \\
\hline Unit $125 \mathrm{NW}^{1 / 4}$ & $25-30$ & 1599 & 1559 & Analyzed & 1 & 0.7 & LPR & PWS & LOC & CRT & 4 & & I & COM & 4 & CRT & 1 \\
\hline Unit $125 \mathrm{SE}^{1 / 4}$ & $10-15$ & 1565 & 1548 & Unanalyzed & 4 & 1.3 & & & & & & & & & & & \\
\hline Unit $125 \mathrm{SE}^{1 / 4}$ & $25-30$ & 1601 & 1560 & Analyzed & 1 & 0.04 & LPR & PWS & LOC & CRT & 4 & & I & COM & 0 & CPX & 1 \\
\hline Unit $125 \mathrm{SE}^{1 / 4}$ & $5-10$ & 1561 & 1544 & Unanalyzed & 1 & 3.5 & & & & & & & & & & & \\
\hline Unit $125 \mathrm{SE}^{1 / 4}$ & $15-20$ & 1569 & 1552 & Unanalyzed & 3 & 0.5 & & & & & & & & & & & \\
\hline Unit $125 \mathrm{SE}^{1 / 4}$ & $25-30$ & 1601 & 1560 & Analyzed & 1 & 0.5 & LPR & PWS & LOC & CRT & 4 & & I & DEB & 1 & IND & 1 \\
\hline Unit $125 \mathrm{SW}^{1 / 4}$ & $15-20$ & 1566 & 1553 & Unanalyzed & 2 & 1.3 & & & & & & & & & & & \\
\hline Unit $125 \mathrm{SW}^{1 / 4}$ & $5-10$ & 1558 & 1545 & Unanalyzed & 1 & 0.2 & & & & & & & & & & & \\
\hline Unit $125 \mathrm{SW}^{1 / 4}$ & $30-35$ & 1676 & 1565 & Analyzed & 1 & 0.04 & LPR & PWS & LOC & QZT & 4 & & I & COM & 4 & CRT & 1 \\
\hline
\end{tabular}


Geo-Marine, Inc.

GMI 22005.01.06.xx and 22005.00.09.xx, Data Recovery Excavations at Site 41PN17 Debitage Analysi

\begin{tabular}{|c|c|c|c|c|c|c|c|c|c|c|c|c|c|c|c|c|c|}
\hline Unit & $\begin{array}{l}\text { Depth } \\
(\mathrm{cm})\end{array}$ & $\begin{array}{l}\text { Provenience } \\
\text { No. }\end{array}$ & $\begin{array}{l}\text { Lot } \\
\text { No. }\end{array}$ & Level of Analysis & Quantity & $\begin{array}{l}\text { Weight } \\
\text { (ing) }\end{array}$ & General Period & Region & Material & Lithology & Size Grade & $\begin{array}{c}\text { Edge } \\
\text { Modification }\end{array}$ & $\begin{array}{l}\text { Thernal } \\
\text { Alteration }\end{array}$ & Completeness & Cortex & Platform & $\begin{array}{l}\text { Minimum } \\
\text { Number of } \\
\text { Nodules }\end{array}$ \\
\hline \multicolumn{18}{|c|}{ Block 4} \\
\hline Unit $125 \mathrm{SW}^{1 / 4}$ & $20-25$ & 1579 & 1557 & Analyzed & 1 & 0.3 & LPR & PWS & LOC & CRT & 4 & & I & COM & 0 & REJ & 1 \\
\hline Unit $126 \mathrm{NE}^{1 / 4}$ & $22-32$ & 1704 & 1576 & Analyzed & 2 & 0.3 & LPR & PWS & LOC & CRT & 4 & & I & FRG & 0 & $\mathrm{IND}$ & 1 \\
\hline Unit $126 \mathrm{NE}^{1 / 4}$ & $12-22$ & 1696 & 1572 & Unanalyzed & 2 & 0.4 & & & & & & & & & & & \\
\hline Unit $126 \mathrm{NW}^{1 / 4}$ & $22-32$ & 1703 & 1577 & Analyzed & 1 & 0.1 & LPR & PWS & LOC & CRT & 4 & & I & $\mathrm{COM}$ & 0 & FLT & 1 \\
\hline Unit $126 \mathrm{NW}^{1 / 4}$ & $22-32$ & 1703 & 1577 & Analyzed & 1 & 1.1 & LPR & PWS & LOC & CRT & 4 & & I & FRG & 3 & IND & 1 \\
\hline Unit $126 \mathrm{NW}^{1 / 4}$ & $12-22$ & 1695 & 1573 & Unanalyzed & 6 & 2.7 & & & & & & & & & & & \\
\hline Unit $126 \mathrm{NW}^{1 / 4}$ & $0-12$ & 1691 & 1569 & Unanalyzed & 3 & 2 & & & & & & & & & & & \\
\hline Unit $126 \mathrm{SE}^{1 / 4}$ & $12-22$ & 1697 & 1574 & Unanalyzed & 2 & 0.5 & & & & & & & & & & & \\
\hline Unit $126 \mathrm{SW}^{1 / 4}$ & $12-22$ & 1694 & 1575 & Unanalyzed & 7 & 3.7 & & & & & & & & & & & \\
\hline Unit $126 \mathrm{SW}^{1 / 4}$ & $32-42$ & 1787 & 1582 & Analyzed & 1 & 0.1 & LPR & PWS & LOC & CRT & 4 & & I & COM & 0 & FLT & 1 \\
\hline Unit $127 \mathrm{NE}^{1 / 4}$ & $21-31$ & 1712 & 1592 & Analyzed & 1 & 0.2 & LPR & PWS & LOC & CRT & 4 & & I & COM & 0 & CPX & 1 \\
\hline Unit $127 \mathrm{NE}^{1 / 4}$ & $21-31$ & 1712 & 1592 & Analyzed & 1 & 0.3 & LPR & PWS & LOC & CRT & 4 & & I & COM & 2 & FLT & 1 \\
\hline Unit $127 \mathrm{NE}^{1 / 4}$ & $11-21$ & 1708 & 1588 & Unanalyzed & 2 & 1.1 & & & & & & & & & & & \\
\hline Unit $127 \mathrm{NE}^{1 / 4}$ & $0-11$ & 1700 & 1584 & Unanalyzed & 3 & 2.9 & & & & & & & & & & & \\
\hline Unit $127 \mathrm{NW}^{1 / 4}$ & $11-21$ & 1707 & 1589 & Unanalyzed & 3 & 1.3 & & & & & & & & & & & \\
\hline Unit $127 \mathrm{NW}^{1 / 4}$ & $21-31$ & 1711 & 1593 & Analyzed & 1 & 0.04 & LPR & PWS & LOC & CRT & 4 & & I & BRK & 0 & FLT & 1 \\
\hline Unit $127 \mathrm{NW}^{1 / 4}$ & $21-31$ & 1711 & 1593 & Analyzed & 1 & 0.1 & LPR & PWS & LOC & CRT & 4 & & I & FRG & 2 & IND & 1 \\
\hline Unit $127 \mathrm{NW}^{1 / 4}$ & $21-31$ & 1711 & 1593 & Analyzed & 1 & 0.5 & LPR & PWS & LOC & CRT & 4 & & I & $\mathrm{COM}$ & 1 & CRT & 1 \\
\hline Unit $127 \mathrm{NW}^{1 / 4}$ & $21-31$ & 1711 & 1593 & Analyzed & 1 & 0.2 & LPR & PWS & LOC & CRT & 4 & & I & DEB & 0 & $\mathrm{IND}$ & 1 \\
\hline Unit $127 \mathrm{NW}^{1 / 4}$ & $0-11$ & 1699 & 1585 & Unanalyzed & 1 & 0.3 & & & & & & & & & & & \\
\hline Unit $127 \mathrm{SE}^{1 / 4}$ & $11-21$ & 1709 & 1590 & Unanalyzed & 1 & 3 & & & & & & & & & & & \\
\hline Unit $127 \mathrm{SE}^{1 / 4}$ & $21-31$ & 1713 & 1594 & Analyzed & 1 & 1.4 & LPR & PWS & LOC & CRT & 3 & & $\mathrm{~N}$ & COM & 4 & FLT & 1 \\
\hline Unit $127 \mathrm{SW}^{1 / 4}$ & $11-21$ & 1706 & 1591 & Unanalyzed & 3 & 1 & & & & & & & & & & & \\
\hline Unit $127 \mathrm{SW}^{1 / 4}$ & $31-41$ & 1791 & 1598 & Analyzed & 1 & 0.3 & LPR & PWS & LOC & CRT & 4 & & I & DEB & 0 & $\mathrm{IND}$ & 1 \\
\hline \multicolumn{18}{|c|}{ Block 5} \\
\hline Unit $079 \mathrm{NE}^{1 / 4}$ & $40-50$ & 823 & 604 & Analyzed & 1 & 0.6 & LPR & PWS & LOC & CRT & 4 & & I & DEB & 2 & $\mathrm{IND}$ & 1 \\
\hline
\end{tabular}


Geo-Marine, Inc.

GMI 22005.01.06.xx and 22005.00.09.xx, Data Recovery Excavations at Site 41PN175 Debitage Analysi

\begin{tabular}{|c|c|c|c|c|c|c|c|c|c|c|c|c|c|c|c|c|c|}
\hline Unit & $\begin{array}{l}\text { Depth } \\
(\mathrm{cm})\end{array}$ & $\begin{array}{l}\text { Provenience } \\
\text { No. }\end{array}$ & $\begin{array}{l}\text { Lot } \\
\text { No. }\end{array}$ & Level of Analysis & Quantity & $\begin{array}{l}\text { Weight } \\
\text { (ing) }\end{array}$ & General Period & Region & Material & Lithology & Size Grade & $\begin{array}{c}\text { Edge } \\
\text { Modification }\end{array}$ & $\begin{array}{l}\text { Thernal } \\
\text { Alteration }\end{array}$ & Completeness & Cortex & Platform & $\begin{array}{l}\text { Minimum } \\
\text { Number of } \\
\text { Nodules }\end{array}$ \\
\hline \multicolumn{18}{|c|}{ Block 5} \\
\hline Unit $079 \mathrm{NE}^{1 / 4}$ & $40-50$ & 823 & 604 & Analyzed & 1 & 0.2 & LPR & PWS & LOC & CRT & 4 & & I & DEB & 1 & IND & 1 \\
\hline Unit $079 \mathrm{NE}^{1 / 4}$ & $31-40$ & 758 & 600 & Analyzed & 1 & 0.7 & LPR & PWS & LOC & QZT & 3 & & $\mathrm{~N}$ & COM & 4 & CRT & 1 \\
\hline Unit $079 \mathrm{NE}^{1 / 4}$ & $40-50$ & 823 & 604 & Analyzed & 1 & 0.3 & LPR & PWS & LOC & CRT & 3 & & $\mathrm{~N}$ & $\mathrm{COM}$ & 4 & CRT & 1 \\
\hline Unit $079 \mathrm{NW}^{1 / 4}$ & $31-40$ & 757 & 601 & Analyzed & 1 & 0.04 & LPR & PWS & LOC & CRT & 4 & & I & COM & 1 & CRT & 2 \\
\hline Unit $079 \mathrm{NW}^{1 / 4}$ & $31-40$ & 757 & 601 & Analyzed & 1 & 1.2 & LPR & PWS & LOC & QZT & 4 & & I & COM & 4 & CRT & 2 \\
\hline Unit $079 \mathrm{NW}^{1 / 4}$ & $31-40$ & 757 & 601 & Analyzed & 1 & 0.04 & LPR & PWS & LOC & CRT & 4 & & I & COM & 4 & IND & 2 \\
\hline Unit $079 \mathrm{SE}^{1 / 4}$ & $31-40$ & 759 & 602 & Analyzed & 1 & 0.1 & LPR & PWS & LOC & QZT & 4 & & I & BRK & 0 & CPX & 1 \\
\hline Unit $079 \mathrm{SE}^{1 / 4}$ & $31-40$ & 759 & 602 & Analyzed & 1 & 0.2 & LPR & PWS & LOC & CRT & 4 & & I & BRK & 0 & CPX & 1 \\
\hline Unit $079 \mathrm{SW}^{1 / 4}$ & $31-40$ & 756 & 603 & Analyzed & 1 & 0.04 & LPR & PWS & LOC & CRT & 4 & & I & BRK & 0 & FLT & 1 \\
\hline Unit $080 \mathrm{NE}^{1 / 4}$ & $32-40$ & 771 & 617 & Analyzed & 1 & 0.8 & LPR & PWS & LOC & CRT & 4 & & I & COM & 0 & ABR & 1 \\
\hline Unit $080 \mathrm{NE}^{1 / 4}$ & $40-50$ & 828 & 621 & Analyzed & 1 & 0.2 & LPR & PWS & LOC & CRT & 4 & & I & COM & 0 & FLT & 1 \\
\hline Unit $080 \mathrm{NW}^{1 / 4}$ & $40-50$ & 780 & 622 & Analyzed & 1 & 0.2 & LPR & PWS & LOC & CRT & 4 & & I & COM & 3 & FLT & 1 \\
\hline Unit $080 \mathrm{NW}^{1 / 4}$ & $40-50$ & 780 & 622 & Analyzed & 1 & 0.5 & LPR & PWS & LOC & CRT & 4 & & I & COM & 1 & CRT & 1 \\
\hline Unit $080 \mathrm{NW}^{1 / 4}$ & $30-32$ & 766 & 614 & Unanalyzed & 1 & 0.4 & & & & & & & & & & & \\
\hline Unit $080 \mathrm{SE}^{1 / 4}$ & $20-30$ & 764 & 611 & Unanalyzed & 1 & 0.6 & & & & & & & & & & & \\
\hline Unit $080 \mathrm{SW}^{1 / 4}$ & $20-30$ & 761 & 612 & Unanalyzed & 1 & 0.1 & & & & & & & & & & & \\
\hline Unit $081 \mathrm{NE}^{1 / 4}$ & $30-32$ & 783 & 635 & Unanalyzed & 1 & 5 & & & & & & & & & & & \\
\hline Unit $081 \mathrm{NE}^{1 / 4}$ & $32-40$ & 787 & 639 & Analyzed & 1 & 0.4 & LPR & PWS & LOC & QZT & 4 & & I & BRK & 0 & CPX & 1 \\
\hline Unit $081 \mathrm{NE}^{1 / 4}$ & $20-30$ & 778 & 632 & Unanalyzed & 1 & 0.3 & & & & & & & & & & & \\
\hline Unit $081 \mathrm{NW}^{1 / 4}$ & $20-30$ & 777 & 633 & Unanalyzed & 1 & 0.1 & & & & & & & & & & & \\
\hline Unit $081 \mathrm{NW}^{1 / 4}$ & $40-50$ & 826 & 643 & Analyzed & 1 & 0.4 & LPR & PWS & REG & CRT & 3 & & Y & $\mathrm{COM}$ & 0 & FLT & 1 \\
\hline Unit $081 \mathrm{SE}^{1 / 4}$ & $30-32$ & 784 & 637 & Unanalyzed & 1 & 0.2 & & & & & & & & & & & \\
\hline Unit $081 \mathrm{SW}^{1 / 4}$ & $30-32$ & 781 & 638 & Unanalyzed & 2 & 1.3 & & & & & & & & & & & \\
\hline Unit $082 \mathrm{NE}^{1 / 4}$ & $30-32$ & 796 & 652 & Unanalyzed & 1 & 2.7 & & & & & & & & & & & \\
\hline Unit $082 \mathrm{NE}^{1 / 4}$ & $20-30$ & 793 & 648 & Unanalyzed & 1 & 0.4 & & & & & & & & & & & \\
\hline Unit $082 \mathrm{NW}^{1 / 4}$ & $40-50$ & 835 & 659 & Analyzed & 1 & 0.2 & LPR & PWS & LOC & CRT & 4 & & I & FRG & 0 & IND & 1 \\
\hline
\end{tabular}


Geo-Marine, Inc.

GMI 22005.01.06.xx and 22005.00.09.xx, Data Recovery Excavations at Site 41PN175 Debitage Analysi

\begin{tabular}{|c|c|c|c|c|c|c|c|c|c|c|c|c|c|c|c|c|c|}
\hline Unit & $\begin{array}{l}\text { Depth } \\
(\mathrm{cm})\end{array}$ & $\begin{array}{l}\text { Provenience } \\
\text { No. }\end{array}$ & $\begin{array}{l}\text { Lot } \\
\text { No. }\end{array}$ & Level of Analysis & Quantity & $\begin{array}{l}\text { Weight } \\
\text { (in g) }\end{array}$ & General Period & Region & Material & Lithology & Size Grade & $\begin{array}{c}\text { Edge } \\
\text { Modification }\end{array}$ & $\begin{array}{l}\text { Thernal } \\
\text { Alteration }\end{array}$ & Completeness & Cortex & Platform & $\begin{array}{l}\text { Minimum } \\
\text { Number of } \\
\text { Nodules }\end{array}$ \\
\hline \multicolumn{18}{|c|}{ Block 5} \\
\hline Unit $082 \mathrm{SE}^{1 / 4}$ & $40-50$ & 837 & 660 & Analyzed & 1 & 0.04 & LPR & PWS & LOC & CRT & 4 & & I & BRK & 0 & FLT & 1 \\
\hline Unit $082 \mathrm{SE}^{1 / 4}$ & $40-50$ & 837 & 660 & Analyzed & 1 & 0.1 & LPR & PWS & REG & CRT & 4 & & I & FRG & 0 & IND & 1 \\
\hline Unit $082 \mathrm{SE}^{1 / 4}$ & $32-40$ & 833 & 656 & Analyzed & 1 & 0.7 & LPR & PWS & LOC & QZT & 4 & & I & FRG & 3 & IND & 1 \\
\hline \multicolumn{18}{|c|}{ Block 6} \\
\hline Unit $107 \mathrm{NE}^{1 / 4}$ & $25-35$ & 844 & 1142 & Unanalyzed & 2 & 1.1 & & & & & & & & & & & \\
\hline Unit $107 \mathrm{SE}^{1 / 4}$ & $25-35$ & 845 & 1144 & Unanalyzed & 1 & 0.8 & & & & & & & & & & & \\
\hline Unit $107 \mathrm{SW}^{1} / 4$ & $25-35$ & 842 & 1145 & Unanalyzed & 2 & 1 & & & & & & & & & & & \\
\hline Unit 108 NE1/4 & $25-35$ & 853 & 1154 & Unanalyzed & 2 & 2.2 & & & & & & & & & & & \\
\hline Unit 108 NE1/4 & $10-25$ & 848 & 1151 & Unanalyzed & 1 & 0.2 & & & & & & & & & & & \\
\hline Unit $108 \mathrm{NE}^{1 / 4}$ & $45-55$ & 895 & 1162 & Analyzed & 1 & 0.04 & LPR & PWS & LOC & CRT & 4 & & I & COM & 0 & IND & 1 \\
\hline Unit $108 \mathrm{NE}^{1 / 4}$ & $35-45$ & 893 & 1158 & Analyzed & 1 & 1 & LPR & PWS & LOC & CRT & 4 & & I & COM & 1 & CRT & 1 \\
\hline Unit $108 \mathrm{NW}^{1 / 4}$ & $25-35$ & 852 & 1155 & Unanalyzed & 3 & 1.5 & & & & & & & & & & & \\
\hline Unit $108 \mathrm{SE}^{1 / 4}$ & $10-25$ & 849 & 1152 & Unanalyzed & 1 & 0.5 & & & & & & & & & & & \\
\hline Unit $108 \mathrm{SE}^{1 / 4}$ & $25-35$ & 854 & 1156 & Unanalyzed & 3 & 2.7 & & & & & & & & & & & \\
\hline Unit 108 SW1/4 & $10-25$ & 847 & 1153 & Unanalyzed & 1 & 0.3 & & & & & & & & & & & \\
\hline \multicolumn{18}{|c|}{ Block 7} \\
\hline Unit $109 \mathrm{NE}^{1 / 4}$ & $0-10$ & 899 & 1163 & Unanalyzed & 1 & 0.3 & & & & & & & & & & & \\
\hline Unit $109 \mathrm{NW}^{1 / 4}$ & $30-38$ & 957 & 1176 & Unanalyzed & 5 & 1.6 & & & & & & & & & & & \\
\hline Unit $109 \mathrm{NW}^{1 / 4}$ & $20-30$ & 932 & 1172 & Unanalyzed & 1 & 1 & & & & & & & & & & & \\
\hline Unit $109 \mathrm{SE}^{1 / 4}$ & $0-10$ & 900 & 1165 & Unanalyzed & 1 & 0.3 & & & & & & & & & & & \\
\hline Unit $109 \mathrm{SE}^{1 / 4}$ & $30-38$ & 959 & 1177 & Unanalyzed & 1 & 1.1 & & & & & & & & & & & \\
\hline Unit $109 \mathrm{SW}^{1 / 4}$ & $20-30$ & 931 & 1174 & Unanalyzed & 2 & 0.1 & & & & & & & & & & & \\
\hline Unit $109 \mathrm{SW}^{11 / 4}$ & $10-20$ & 901 & 1170 & Unanalyzed & 1 & 0.5 & & & & & & & & & & & \\
\hline Unit $109 \mathrm{SW}^{1} / 4$ & $30-38$ & 956 & 1178 & Unanalyzed & 3 & 0.4 & & & & & & & & & & & \\
\hline Unit $110 \mathrm{NE}^{1 / 4}$ & $10-20$ & 908 & 1180 & Unanalyzed & 3 & 1.5 & & & & & & & & & & & \\
\hline Unit $110 \mathrm{NW}^{1 / 4}$ & $20-30$ & 911 & 1185 & Unanalyzed & 1 & 1.3 & & & & & & & & & & & \\
\hline
\end{tabular}


Geo-Marine, Inc.

GMI 22005.01.06.xx and 22005.00.09.xx, Data Recovery Excavations at Site 41PN17 Debitage Analysi

\begin{tabular}{|c|c|c|c|c|c|c|c|c|c|c|c|c|c|c|c|c|c|}
\hline Unit & $\begin{array}{c}\begin{array}{c}\text { Depth } \\
(\mathrm{cm})\end{array}\end{array}$ & $\begin{array}{l}\text { Provenience } \\
\text { No. }\end{array}$ & $\begin{array}{l}\text { Lot } \\
\text { No. }\end{array}$ & Level of Analysis & Quantity & $\begin{array}{l}\text { Weight } \\
\text { (in g) }\end{array}$ & General Period & Region & Material & Lithology & Size Grade & $\begin{array}{c}\text { Edge } \\
\text { Modification }\end{array}$ & $\begin{array}{l}\text { Thernal } \\
\text { Alteration }\end{array}$ & Completeness & Cortex & Platform & $\begin{array}{l}\text { Minimun } \\
\text { Number } \\
\text { Nodules }\end{array}$ \\
\hline \multicolumn{18}{|c|}{ Block 7} \\
\hline Unit $110 \mathrm{SW}^{1 / 4}$ & $30-38$ & 960 & 1191 & Unanalyzed & 1 & 0.2 & & & & & & & & & & & \\
\hline Unit $110 \mathrm{SW}^{1 / 4}$ & $20-30$ & 910 & 1187 & Unanalyzed & 1 & 0.4 & & & & & & & & & & & \\
\hline Unit $111 \mathrm{NE}^{1 / 4}$ & $10-20$ & 919 & 1195 & Unanalyzed & 2 & 0.3 & & & & & & & & & & & \\
\hline Unit $111 \mathrm{NE}^{1 / 4}$ & $20-30$ & 923 & 1199 & Unanalyzed & 3 & 1.1 & & & & & & & & & & & \\
\hline Unit $111 \mathrm{NE}^{1} / 4$ & $0-10$ & 916 & 1192 & Unanalyzed & 1 & 0.4 & & & & & & & & & & & \\
\hline Unit $111 \mathrm{NW}^{1 / 4}$ & $20-30$ & 922 & 1200 & Unanalyzed & 2 & 0.8 & & & & & & & & & & & \\
\hline Unit $111 \mathrm{NW}^{1 / 4}$ & $30-38$ & 969 & 1204 & Unanalyzed & 2 & 0.8 & & & & & & & & & & & \\
\hline Unit $111 \mathrm{SE}^{1 / 4}$ & $40-50$ & 979 & 1213 & Analyzed & 1 & 0.1 & LPR & PWS & LOC & CRT & 4 & & I & BRK & 1 & FLT & 2 \\
\hline Unit $111 \mathrm{SE}^{1 / 4}$ & $40-50$ & 979 & 1213 & Analyzed & 1 & 0.3 & LPR & PWS & LOC & FWD & 4 & & I & FRG & 4 & IND & 2 \\
\hline Unit $111 \mathrm{SE}^{1 / 4}$ & $20-30$ & 924 & 1201 & Unanalyzed & 2 & 0.2 & & & & & & & & & & & \\
\hline Unit 111 SW1/4 & $40-50$ & 976 & 1214 & Analyzed & 1 & 0.4 & LPR & PWS & LOC & QZT & 4 & & I & COM & 4 & CRT & 1 \\
\hline Unit $111 \mathrm{SW}^{1 / 4}$ & $10-20$ & 917 & 1198 & Unanalyzed & 1 & 0.5 & & & & & & & & & & & \\
\hline Unit $111 \mathrm{SW}^{1 / 4}$ & $20-30$ & 921 & 1202 & Unanalyzed & 3 & 0.8 & & & & & & & & & & & \\
\hline Unit 111 SW1/4 & $50-60$ & 1046 & 1218 & Analyzed & 1 & 0.3 & LPR & PWS & LOC & QZT & 4 & & I & FRG & 0 & IND & 1 \\
\hline Unit 111 SW1/4 & $30-38$ & 968 & 1206 & Unanalyzed & 1 & 5 & & & & & & & & & & & \\
\hline Unit 111 SW1/4 & $38-40$ & 972 & 1210 & Analyzed & 1 & 0.04 & LPR & PWS & LOC & CRT & 5 & & I & COM & 0 & CPX & 1 \\
\hline Unit $112 \mathrm{NE}^{1 / 4}$ & $20-30$ & 982 & 1227 & Unanalyzed & 1 & 0.2 & & & & & & & & & & & \\
\hline Unit $112 \mathrm{NE}^{1 / 4}$ & $30-38$ & 986 & 1231 & Unanalyzed & 3 & 0.4 & & & & & & & & & & & \\
\hline Unit $112 \mathrm{NW}^{1 / 4}$ & $40-50$ & 992 & 1239 & Analyzed & 1 & 0.4 & LPR & PWS & LOC & QZT & 4 & & I & COM & 1 & CRT & 1 \\
\hline Unit $112 \mathrm{NW}^{1 / 4}$ & $10-20$ & 928 & 1224 & Unanalyzed & 2 & 0.6 & & & & & & & & & & & \\
\hline Unit $112 \mathrm{NW}^{1 / 4}$ & $30-38$ & 985 & 1232 & Unanalyzed & 4 & 1 & & & & & & & & & & & \\
\hline Unit $112 \mathrm{NW}^{1 / 4}$ & $60-70$ & 1055 & 1247 & Analyzed & 1 & 0.2 & LPR & PWS & LOC & CRT & 4 & & I & COM & 0 & IND & 1 \\
\hline Unit $112 \mathrm{SE}^{1 / 4}$ & $0-10$ & 926 & 1222 & Unanalyzed & 1 & 0.3 & & & & & & & & & & & \\
\hline Unit $112 \mathrm{SE}^{1 / 4}$ & $38-40$ & 990 & 1236 & Analyzed & 1 & 0.4 & LPR & PWS & LOC & QZT & 4 & & I & COM & 0 & FLT & 1 \\
\hline Unit $112 \mathrm{SE}^{1 / 4}$ & $50-60$ & 1053 & 1244 & Analyzed & 1 & 0.04 & LPR & PWS & LOC & CRT & 4 & & I & COM & 1 & FLT & 1 \\
\hline Unit $112 \mathrm{SW}^{1 / 4}$ & $20-30$ & 980 & 1230 & Unanalyzed & 1 & 1.8 & & & & & & & & & & & \\
\hline
\end{tabular}


Geo-Marine, Inc.

GMI 22005.01.06.xx and 22005.00.09.xx, Data Recovery Excavations at Site 41PN17 Debitage Analysi

\begin{tabular}{|c|c|c|c|c|c|c|c|c|c|c|c|c|c|c|c|c|c|}
\hline Unit & $\begin{array}{l}\text { Depth } \\
(\mathrm{cm})\end{array}$ & $\begin{array}{l}\text { Provenience } \\
\text { No. }\end{array}$ & $\begin{array}{l}\text { Lot } \\
\text { No. }\end{array}$ & Level of Analysis & Quantity & $\begin{array}{l}\text { Weight } \\
\text { (in g) }\end{array}$ & General Period & Region & Material & Lithology & Size Grade & $\begin{array}{c}\text { Edge } \\
\text { Modification }\end{array}$ & $\begin{array}{l}\text { Thernal } \\
\text { Alteration }\end{array}$ & Completeness & Cortex & Platform & $\begin{array}{l}\text { Minimum } \\
\text { Number of } \\
\text { Nodules }\end{array}$ \\
\hline \multicolumn{18}{|c|}{ Block 7} \\
\hline Unit $112 \mathrm{SW}^{1 / 4}$ & $40-50$ & 991 & 1241 & Analyzed & 1 & 0.7 & LPR & PWS & LOC & CRT & 4 & & I & COM & 1 & FLT & 1 \\
\hline \multicolumn{18}{|c|}{ Block 8} \\
\hline Unit $113 \mathrm{NE}^{1 / 4}$ & $42-50$ & 1065 & 1266 & Analyzed & 1 & 0.2 & LPR & PWS & LOC & QZT & 4 & & I & FRG & 0 & IND & 2 \\
\hline Unit $113 \mathrm{NE}^{1 / 4}$ & $42-50$ & 1065 & 1266 & Analyzed & 1 & 1.1 & LPR & PWS & LOC & CRT & 3 & & $\mathrm{Y}$ & COM & 1 & CRT & 2 \\
\hline Unit $113 \mathrm{NE}^{1 / 4}$ & $42-50$ & 1065 & 1266 & Analyzed & 1 & 0.3 & LPR & PWS & LOC & CRT & 4 & & I & COM & 1 & CRT & 2 \\
\hline Unit $113 \mathrm{NE}^{1 / 4}$ & $37-42$ & 1061 & 1262 & Unanalyzed & 2 & 1.5 & & & & & & & & & & & \\
\hline Unit $113 \mathrm{NE}^{1 / 4}$ & $30-37$ & 1008 & 1258 & Unanalyzed & 3 & 1 & & & & & & & & & & & \\
\hline Unit $113 \mathrm{NE}^{1 / 4}$ & $50-60$ & 1208 & 1270 & Analyzed & 1 & 0.4 & LPR & PWS & LOC & FWD & 4 & & I & COM & 1 & CRT & 1 \\
\hline Unit $113 \mathrm{NE}^{1 / 4}$ & $50-60$ & 1208 & 1270 & Analyzed & 1 & 0.5 & LPR & PWS & LOC & CRT & 3 & & $\mathrm{Y}$ & COM & 0 & CPX & 1 \\
\hline Unit $113 \mathrm{NW}^{1 / 4}$ & $30-37$ & 1007 & 1259 & Unanalyzed & 1 & 0.2 & & & & & & & & & & & \\
\hline Unit $113 \mathrm{SE}^{1 / 4}$ & $42-50$ & 1066 & 1268 & Analyzed & 1 & 0.1 & LPR & PWS & LOC & CRT & 4 & & I & COM & 2 & CRT & 2 \\
\hline Unit $113 \mathrm{SE}^{1 / 4}$ & $42-50$ & 1066 & 1268 & Analyzed & 1 & 0.04 & LPR & PWS & REG & CRT & 4 & & I & FRG & 0 & IND & 2 \\
\hline Unit $113 \mathrm{SE}^{1 / 4}$ & $42-50$ & 1066 & 1268 & Analyzed & 1 & 1.3 & LPR & PWS & LOC & CRT & 3 & & $\mathrm{Y}$ & DEB & 2 & IND & 2 \\
\hline Unit $113 \mathrm{SE}^{1 / 4}$ & $39-42$ & 1062 & 1264 & Unanalyzed & 2 & 0.4 & & & & & & & & & & & \\
\hline Unit $113 \mathrm{SE}^{1 / 4}$ & $42-50$ & 1066 & 1268 & Analyzed & 1 & 0.1 & LPR & PWS & REG & CRT & 4 & & I & COM & 0 & CPX & 2 \\
\hline Unit $113 \mathrm{SE}^{1 / 4}$ & $30-37$ & 1009 & 1260 & Unanalyzed & 1 & 0.8 & & & & & & & & & & & \\
\hline Unit $113 \mathrm{SW}^{1 / 4}$ & $30-37$ & 1006 & 1261 & Unanalyzed & 2 & 1.2 & & & & & & & & & & & \\
\hline Unit $113 \mathrm{SW}^{1 / 4}$ & $10-20$ & 999 & 1253 & Unanalyzed & 1 & 0.2 & & & & & & & & & & & \\
\hline Unit $113 \mathrm{SW}^{1 / 4}$ & $41-50$ & 1063 & 1269 & Analyzed & 1 & 0.1 & LPR & PWS & LOC & QZT & 4 & & I & $\mathrm{COM}$ & 3 & FLT & 2 \\
\hline Unit $113 \mathrm{SW}^{1 / 4}$ & $41-50$ & 1063 & 1269 & Analyzed & 1 & 2.6 & LPR & PWS & LOC & QZT & 3 & & $\mathrm{Y}$ & COM & 0 & CPX & 2 \\
\hline Unit $113 \mathrm{SW}^{1 / 4}$ & $41-50$ & 1063 & 1269 & Analyzed & 1 & 0.3 & LPR & PWS & LOC & CRT & 4 & & I & COM & 0 & FLT & 2 \\
\hline Unit $113 \mathrm{SW}^{1 / 4}$ & $41-50$ & 1063 & 1269 & Analyzed & 1 & 0.2 & LPR & PWS & LOC & CRT & 4 & & I & COM & 0 & CPX & 2 \\
\hline Unit $114 \mathrm{NE}^{1 / 4}$ & $42-50$ & 1077 & 1291 & Analyzed & 1 & 1.1 & LPR & PWS & LOC & CRT & 4 & & I & DEB & 4 & IND & 1 \\
\hline Unit $114 \mathrm{NE}^{1 / 4}$ & $42-50$ & 1077 & 1291 & Analyzed & 2 & 0.1 & LPR & PWS & LOC & CRT & 4 & & I & BRK & 0 & FLT & 1 \\
\hline Unit $114 \mathrm{NE}^{1 / 4}$ & $42-50$ & 1077 & 1291 & Analyzed & 1 & 0.4 & LPR & PWS & LOC & CRT & 4 & & I & FRG & 1 & IND & 1 \\
\hline
\end{tabular}


Geo-Marine, Inc.

GMI 22005.01.06.xx and 22005.00.09.xx, Data Recovery Excavations at Site 41PN17 Debitage Analysi

\begin{tabular}{|c|c|c|c|c|c|c|c|c|c|c|c|c|c|c|c|c|c|}
\hline Unit & $\begin{array}{c}\text { Depth } \\
(\mathrm{cm})\end{array}$ & $\begin{array}{l}\text { Provenience } \\
\text { No. }\end{array}$ & $\begin{array}{l}\text { Lot } \\
\text { No. }\end{array}$ & Level of Analysis & Quantity & $\begin{array}{l}\text { Weight } \\
\text { (ing) }\end{array}$ & General Period & Region & Material & Lithology & Size Grade & $\begin{array}{c}\text { Edge } \\
\text { Modification }\end{array}$ & $\begin{array}{l}\text { Thernal } \\
\text { Alteration }\end{array}$ & Completeness & Cortex & Platform & $\begin{array}{l}\text { Minimum } \\
\text { Number of } \\
\text { Nodules }\end{array}$ \\
\hline \multicolumn{18}{|c|}{ Block 8} \\
\hline Unit $114 \mathrm{NE}^{1 / 4}$ & $42-50$ & 1077 & 1291 & Analyzed & 1 & 0.2 & LPR & PWS & LOC & CRT & 4 & & I & BRK & 1 & CRT & 1 \\
\hline Unit $114 \mathrm{NW}^{1 / 4}$ & $30-40$ & 1068 & 1284 & Unanalyzed & 2 & 1.6 & & & & & & & & & & & \\
\hline Unit $114 \mathrm{NW}^{1 / 4}$ & $40-42$ & 1072 & 1288 & Unanalyzed & 7 & 3.2 & & & & & & & & & & & \\
\hline Unit $114 \mathrm{NW}^{1 / 4}$ & $42-50$ & 1076 & 1292 & Analyzed & 2 & 0.9 & LPR & PWS & LOC & QZT & 4 & & I & DEB & 0 & IND & 1 \\
\hline Unit $114 \mathrm{SE}^{1 / 4}$ & $42-50$ & 1078 & 1293 & Analyzed & 1 & 1.3 & LPR & PWS & LOC & CRT & 4 & & I & DEB & 0 & IND & 1 \\
\hline Unit $114 \mathrm{SE}^{1 / 4}$ & $40-42$ & 1074 & 1289 & Unanalyzed & 1 & 0.3 & & & & & & & & & & & \\
\hline Unit $114 \mathrm{SE}^{1 / 4}$ & $30-40$ & 1070 & 1285 & Unanalyzed & 4 & 1.7 & & & & & & & & & & & \\
\hline Unit $114 \mathrm{SW}^{1 / 4}$ & $42-50$ & 1075 & 1294 & Analyzed & 1 & 0.6 & LPR & PWS & LOC & QZT & 4 & & I & BRK & 0 & FLT & 1 \\
\hline Unit $114 \mathrm{SW}^{1 / 4}$ & $40-42$ & 1071 & 1290 & Unanalyzed & 6 & 2 & & & & & & & & & & & \\
\hline Unit $114 \mathrm{SW}^{1 / 4}$ & $20-30$ & 1014 & 1282 & Unanalyzed & 3 & 3.2 & & & & & & & & & & & \\
\hline Unit $114 \mathrm{SW}^{1 / 4}$ & $30-40$ & 1067 & 1286 & Unanalyzed & 1 & 0.4 & & & & & & & & & & & \\
\hline Unit 115 General & $20-30$ & 1027 & 1303 & Unanalyzed & 2 & 1.6 & & & & & & & & & & & \\
\hline Unit $115 \mathrm{NE}^{1 / 4}$ & $20-30$ & 1025 & 1304 & Unanalyzed & 1 & 0.4 & & & & & & & & & & & \\
\hline Unit $115 \mathrm{NE}^{1 / 4}$ & $50-60$ & 1220 & 1320 & Analyzed & 1 & 0.5 & LPR & PWS & LOC & QZT & 4 & & I & FRG & 1 & IND & 1 \\
\hline Unit $115 \mathrm{NE}^{1 / 4}$ & $30-37$ & 1030 & 1308 & Unanalyzed & 2 & 3.7 & & & & & & & & & & & \\
\hline Unit $115 \mathrm{NE}^{1 / 4}$ & $39-40$ & 1081 & 1312 & Unanalyzed & 2 & 0.6 & & & & & & & & & & & \\
\hline Unit $115 \mathrm{NW}^{1 / 4}$ & $30-37$ & 1029 & 1309 & Unanalyzed & 3 & 2.2 & & & & & & & & & & & \\
\hline Unit $115 \mathrm{NW}^{1 / 4}$ & $40-50$ & 1215 & 1317 & Analyzed & 1 & 0.1 & LPR & PWS & LOC & CRT & 4 & & I & FRG & 0 & IND & 1 \\
\hline Unit $115 \mathrm{NW}^{1 / 4}$ & $10-20$ & 1020 & 1300 & Unanalyzed & 1 & 0.1 & & & & & & & & & & & \\
\hline Unit $115 \mathrm{NW}^{1 / 4}$ & $40-50$ & 1215 & 1317 & Analyzed & 1 & 0.7 & LPR & PWS & LOC & CRT & 4 & & I & BRK & 3 & CRT & 1 \\
\hline Unit $115 \mathrm{NW}^{1 / 4}$ & $40-50$ & 1215 & 1317 & Analyzed & 1 & 1.1 & LPR & PWS & LOC & CRT & 3 & & $\mathrm{~N}$ & DEB & 0 & IND & 1 \\
\hline Unit $115 \mathrm{SE}^{1 / 4}$ & $40-50$ & 1217 & 1318 & Analyzed & 1 & 0.5 & LPR & PWS & LOC & CRT & 3 & & $\mathrm{~N}$ & COM & 3 & FLT & 1 \\
\hline Unit $115 \mathrm{SE}^{1 / 4}$ & $40-50$ & 1217 & 1318 & Analyzed & 1 & 0.04 & LPR & PWS & LOC & CRT & 4 & & I & FRG & 0 & IND & 1 \\
\hline Unit $115 \mathrm{SE}^{1 / 4}$ & $40-50$ & 1217 & 1318 & Analyzed & 1 & 0.6 & LPR & PWS & LOC & CRT & 3 & & $\mathrm{~N}$ & COM & 0 & FLT & 1 \\
\hline Unit $115 \mathrm{SE}^{1 / 4}$ & $30-37$ & 1031 & 1310 & Unanalyzed & 1 & 2.5 & & & & & & & & & & & \\
\hline Unit $115 \mathrm{SW}^{1 / 4}$ & $40-50$ & 1214 & 1319 & Analyzed & 2 & 1.3 & LPR & PWS & LOC & QZT & 4 & & I & DEB & 3 & IND & 2 \\
\hline
\end{tabular}


Geo-Marine, Inc.

GMI 22005.01.06.xx and 22005.00.09.xx, Data Recovery Excavations at Site 41PN17 Debitage Analysi

\begin{tabular}{|c|c|c|c|c|c|c|c|c|c|c|c|c|c|c|c|c|c|}
\hline Unit & $\begin{array}{l}\text { Depth } \\
(\mathrm{cm})\end{array}$ & $\begin{array}{l}\text { Provenience } \\
\text { No. }\end{array}$ & $\begin{array}{l}\text { Lot } \\
\text { No. }\end{array}$ & Level of Analysis & Quantity & $\begin{array}{l}\text { Weight } \\
\text { (ing) }\end{array}$ & General Period & Region & Material & Lithology & Size Grade & $\begin{array}{c}\text { Edge } \\
\text { Modification }\end{array}$ & $\begin{array}{l}\text { Thernal } \\
\text { Alteration }\end{array}$ & Completeness & Cortex & Platform & $\begin{array}{l}\text { Minimun } \\
\text { Number o } \\
\text { Nodules }\end{array}$ \\
\hline \multicolumn{18}{|c|}{ Block 8} \\
\hline Unit $115 \mathrm{SW}^{1 / 4}$ & $38-40$ & 1079 & 1315 & Unanalyzed & 1 & 0.2 & & & & & & & & & & & \\
\hline Unit $115 \mathrm{SW}^{1 / 4}$ & $40-50$ & 1214 & 1319 & Analyzed & 1 & 2.7 & LPR & PWS & LOC & CRT & 2 & $\mathrm{ABS}$ & $\mathrm{Y}$ & COM & 4 & CRT & 2 \\
\hline Unit $115 \mathrm{SW}^{1 / 4}$ & $20-30$ & 1023 & 1307 & Unanalyzed & 1 & 3.2 & & & & & & & & & & & \\
\hline Unit $115 \mathrm{SW}^{1 / 4}$ & $30-37$ & 1028 & 1311 & Unanalyzed & 3 & 0.6 & & & & & & & & & & & \\
\hline Unit 116 General & $20-30$ & 1038 & 1325 & Unanalyzed & 1 & 0.5 & & & & & & & & & & & \\
\hline Unit $116 \mathrm{NE}^{1 / 4}$ & $50-61$ & 1228 & 1342 & Analyzed & 1 & 0.2 & LPR & PWS & LOC & QZT & 4 & & I & FRG & 0 & IND & 1 \\
\hline Unit $116 \mathrm{NE}^{1 / 4}$ & $39-41$ & 1085 & 1334 & Unanalyzed & 1 & 0.2 & & & & & & & & & & & \\
\hline Unit $116 \mathrm{NE}^{1 / 4}$ & $41-52$ & 1224 & 1338 & Analyzed & 1 & 0.2 & LPR & PWS & LOC & CRT & 4 & & I & COM & 0 & CPX & 1 \\
\hline Unit $116 \mathrm{NW}^{1 / 4} / 4$ & $41-52$ & 1223 & 1339 & Analyzed & 1 & 0.7 & LPR & PWS & LOC & CRT & 3 & & $\mathrm{~N}$ & BRK & 1 & CRT & 1 \\
\hline Unit $116 \mathrm{NW}^{1 / 4} / 4$ & $40-43$ & 1084 & 1335 & Unanalyzed & 1 & 0.6 & & & & & & & & & & & \\
\hline Unit $116 \mathrm{NW}^{1 / 4}$ & $20-30$ & 1035 & 1327 & Unanalyzed & 5 & 3.5 & & & & & & & & & & & \\
\hline Unit $116 \mathrm{SE}^{1 / 4}$ & $41-52$ & 1225 & 1340 & Analyzed & 1 & 0.1 & LPR & PWS & LOC & CRT & 4 & & I & FRG & 0 & IND & 2 \\
\hline Unit $116 \mathrm{SE}^{1 / 4}$ & $30-38$ & 1042 & 1332 & Unanalyzed & 1 & 0.4 & & & & & & & & & & & \\
\hline Unit $116 \mathrm{SE}^{1 / 4}$ & $41-52$ & 1225 & 1340 & Analyzed & 1 & 0.04 & LPR & PWS & LOC & CRT & 4 & & I & BRK & 1 & CRT & 2 \\
\hline Unit $116 \mathrm{SE}^{1 / 4}$ & $41-52$ & 1225 & 1340 & Analyzed & 1 & 0.9 & LPR & PWS & LOC & CRT & 3 & & $\mathrm{~N}$ & COM & 4 & FLT & 2 \\
\hline Unit $116 \mathrm{SE}^{1 / 4}$ & $41-52$ & 1225 & 1340 & Analyzed & 1 & 0.4 & LPR & PWS & LOC & CRT & 4 & & I & DEB & 2 & IND & 2 \\
\hline Unit $116 \mathrm{SE}^{1 / 4}$ & $41-52$ & 1225 & 1340 & Analyzed & 1 & 0.5 & LPR & PWS & LOC & QZT & 4 & & I & DEB & 1 & IND & 2 \\
\hline Unit $116 \mathrm{SW}^{1 / 4}$ & $41-52$ & 1222 & 1341 & Analyzed & 1 & 0.1 & LPR & PWS & LOC & CRT & 4 & & I & FRG & 4 & IND & 1 \\
\hline Unit $116 \mathrm{SW}^{1 / 4}$ & $20-30$ & 1034 & 1329 & Unanalyzed & 1 & 1 & & & & & & & & & & & \\
\hline Unit $116 \mathrm{SW}^{1 / 4}$ & $41-52$ & 1222 & 1341 & Analyzed & 3 & 0.6 & LPR & PWS & LOC & CRT & 4 & & I & FRG & 0 & IND & 1 \\
\hline Unit $116 \mathrm{SW}^{1 / 4}$ & $41-52$ & 1222 & 1341 & Analyzed & 1 & 1.2 & LPR & PWS & LOC & CRT & 3 & & $\mathrm{~N}$ & $\mathrm{COM}$ & 0 & FLT & 1 \\
\hline Unit $116 \mathrm{SW}^{1 / 4}$ & $40-43$ & 1083 & 1337 & Unanalyzed & 3 & 3 & & & & & & & & & & & \\
\hline Unit 117 General & $40-45$ & 1356 & 1374 & Analyzed & 1 & 0.7 & LPR & PWS & LOC & CRT & 4 & & I & COM & 4 & CRT & 1 \\
\hline Unit $117 \mathrm{NE}^{1 / 4}$ & $30-35$ & 1346 & 1366 & Analyzed & 3 & 2.4 & LPR & PWS & LOC & CRT & 4 & & I & FRG & 4 & IND & 1 \\
\hline Unit $117 \mathrm{NE}^{1 / 4}$ & $30-35$ & 1346 & 1366 & Analyzed & 1 & 0.5 & LPR & PWS & LOC & CRT & 4 & & I & COM & 1 & CRT & 1 \\
\hline Unit $117 \mathrm{NE}^{1 / 4}$ & $25-30$ & 1342 & 1361 & Unanalyzed & 7 & 6.3 & & & & & & & & & & & \\
\hline
\end{tabular}


Geo-Marine, Inc.

GMI 22005.01.06.xx and 22005.00.09.xx, Data Recovery Excavations at Site 41PN17 Debitage Analysi

\begin{tabular}{|c|c|c|c|c|c|c|c|c|c|c|c|c|c|c|c|c|c|}
\hline Unit & $\begin{array}{l}\text { Depth } \\
(\mathrm{cm})\end{array}$ & $\begin{array}{l}\text { Provenience } \\
\text { No. }\end{array}$ & $\begin{array}{l}\text { Lot } \\
\text { No. }\end{array}$ & Level of Analysis & Quantity & $\begin{array}{l}\text { Weight } \\
\text { (in g) }\end{array}$ & General Period & Region & Material & Lithology & Size Grade & $\begin{array}{c}\text { Edge } \\
\text { Modification }\end{array}$ & $\begin{array}{l}\text { Thernal } \\
\text { Alteration }\end{array}$ & Completeness & Cortex & Platform & $\begin{array}{l}\text { Minimum } \\
\text { Number o } \\
\text { Nodules }\end{array}$ \\
\hline \multicolumn{18}{|c|}{ Block 8} \\
\hline Unit $117 \mathrm{NE}^{1 / 4}$ & $20-25$ & 1470 & 1357 & Unanalyzed & 1 & 0.6 & & & & & & & & & & & \\
\hline Unit $117 \mathrm{NE}^{1 / 4}$ & $15-20$ & 1329 & 1353 & Unanalyzed & 1 & 0.2 & & & & & & & & & & & \\
\hline Unit $117 \mathrm{NE}^{1 / 4}$ & $40-45$ & 1354 & 1375 & Analyzed & 1 & 0.6 & LPR & PWS & LOC & QZT & 4 & & I & COM & 3 & CRT & 1 \\
\hline Unit $117 \mathrm{NW}^{1 / 4}$ & $30-35$ & 1345 & 1367 & Analyzed & 1 & 0.8 & LPR & PWS & LOC & CRT & 4 & & I & DEB & 2 & IND & 2 \\
\hline Unit $117 \mathrm{NW}^{1 / 4}$ & $35-40$ & 1349 & 1371 & Analyzed & 1 & 0.5 & LPR & PWS & LOC & CRT & 4 & & I & BRK & 1 & CRT & 1 \\
\hline Unit $117 \mathrm{NW}^{1 / 4}$ & $30-35$ & 1345 & 1367 & Analyzed & 1 & 0.1 & LPR & PWS & LOC & CRT & 4 & & I & FRG & 4 & IND & 2 \\
\hline Unit $117 \mathrm{NW}^{1 / 4}$ & $30-35$ & 1345 & 1367 & Analyzed & 1 & 0.2 & LPR & PWS & LOC & CRT & 4 & & I & СOM & 1 & CRT & 2 \\
\hline Unit $117 \mathrm{NW}^{1 / 4}$ & $25-30$ & 1341 & 1362 & Unanalyzed & 1 & 0.6 & & & & & & & & & & & \\
\hline Unit $117 \mathrm{NW}^{1 / 4}$ & $30-35$ & 1345 & 1367 & Analyzed & 1 & 0.5 & LPR & PWS & LOC & CRT & 3 & & $\mathrm{~N}$ & COM & 0 & FAC & 2 \\
\hline Unit $117 \mathrm{NW}^{1 / 4}$ & $30-35$ & 1345 & 1367 & Analyzed & 1 & 0.2 & LPR & PWS & LOC & CRT & 4 & & I & FRG & 0 & IND & 2 \\
\hline Unit $117 \mathrm{NW}^{1 / 4}$ & $20-25$ & 1469 & 1358 & Unanalyzed & 2 & 0.5 & & & & & & & & & & & \\
\hline Unit $117 \mathrm{NW}^{1 / 4}$ & $30-35$ & 1345 & 1367 & Analyzed & 1 & 1 & LPR & PWS & LOC & CRT & 4 & & I & DEB & 2 & IND & 2 \\
\hline Unit $117 \mathrm{NW}^{1 / 4}$ & $35-40$ & 1349 & 1371 & Analyzed & 2 & 2.6 & LPR & PWS & LOC & CRT & 3 & & $\mathrm{~N}$ & COM & 4 & CRT & 1 \\
\hline Unit $117 \mathrm{NW}^{1 / 4}$ & $35-40$ & 1349 & 1371 & Analyzed & 2 & 0.9 & LPR & PWS & LOC & CRT & 4 & & I & FRG & 0 & IND & 1 \\
\hline Unit $117 \mathrm{NW}^{1 / 4}$ & $10-15$ & 1324 & 1350 & Unanalyzed & 1 & 0.8 & & & & & & & & & & & \\
\hline Unit $117 \mathrm{NW}^{1 / 4}$ & $40-45$ & 1353 & 1376 & Analyzed & 1 & 0.6 & LPR & PWS & LOC & FWD & 3 & & $\mathrm{~N}$ & COM & 0 & FLT & 3 \\
\hline Unit $117 \mathrm{NW}^{1 / 4}$ & $40-45$ & 1353 & 1376 & Analyzed & 1 & 0.7 & LPR & PWS & REG & CRT & 3 & & $\mathrm{~N}$ & FRG & 0 & IND & 3 \\
\hline Unit $117 \mathrm{NW}^{1 / 4}$ & $40-45$ & 1353 & 1376 & Analyzed & 1 & 0.9 & LPR & PWS & LOC & CRT & 3 & & $\mathrm{Y}$ & DEB & 2 & IND & 3 \\
\hline Unit $117 \mathrm{NW}^{1 / 4}$ & $40-45$ & 1353 & 1376 & Analyzed & 1 & 0.3 & LPR & PWS & LOC & CRT & 4 & & I & DEB & 0 & IND & 3 \\
\hline Unit $117 \mathrm{NW}^{1 / 4}$ & $30-35$ & 1345 & 1367 & Analyzed & 1 & 0.1 & LPR & PWS & LOC & CRT & 4 & & I & COM & 4 & CRT & 2 \\
\hline Unit $117 \mathrm{SE}^{1 / 4}$ & $30-35$ & 1347 & 1368 & Analyzed & 3 & 1.2 & LPR & PWS & LOC & CRT & 4 & & I & COM & 1 & FLT & 2 \\
\hline Unit $117 \mathrm{SE}^{1} / 4$ & $15-20$ & 1330 & 1355 & Unanalyzed & 2 & 1.1 & & & & & & & & & & & \\
\hline Unit $117 \mathrm{SE}^{1 / 4}$ & $25-30$ & 1343 & 1364 & Unanalyzed & 11 & 8 & & & & & & & & & & & \\
\hline Unit $117 \mathrm{SE}^{1 / 4}$ & $30-35$ & 1347 & 1368 & Analyzed & 1 & 0.1 & LPR & PWS & LOC & CRT & 4 & & I & FRG & 0 & IND & 2 \\
\hline Unit $117 \mathrm{SE}^{1 / 4}$ & $30-35$ & 1347 & 1368 & Analyzed & 2 & 0.7 & LPR & PWS & LOC & CRT & 4 & & I & DEB & 1 & IND & 2 \\
\hline Unit $117 \mathrm{SE}^{1 / 4}$ & $30-35$ & 1347 & 1368 & Analyzed & 1 & 0.3 & LPR & PWS & LOC & CRT & 4 & & I & COM & 4 & CRT & 2 \\
\hline
\end{tabular}


Geo-Marine, Inc.

GMI 22005.01.06.xx and 22005.00.09.xx, Data Recovery Excavations at Site 41PN17 Debitage Analysi

\begin{tabular}{|c|c|c|c|c|c|c|c|c|c|c|c|c|c|c|c|c|c|}
\hline Unit & $\begin{array}{l}\text { Depth } \\
(\mathrm{cm})\end{array}$ & $\begin{array}{l}\text { Provenience } \\
\text { No. }\end{array}$ & $\begin{array}{l}\text { Lot } \\
\text { No. }\end{array}$ & Level of Analysis & Quantity & $\begin{array}{l}\text { Weight } \\
\text { (ing) }\end{array}$ & General Period & Region & Material & Lithology & Size Grade & $\begin{array}{c}\text { Edge } \\
\text { Modification }\end{array}$ & $\begin{array}{l}\text { Thernal } \\
\text { Alteration }\end{array}$ & Completeness & Cortex & Platform & $\begin{array}{l}\text { Minimum } \\
\text { Number o } \\
\text { Nodules }\end{array}$ \\
\hline \multicolumn{18}{|c|}{ Block 8} \\
\hline Unit $117 \mathrm{SE}^{1 / 4}$ & $30-35$ & 1347 & 1368 & Analyzed & 1 & 0.1 & LPR & PWS & LOC & CRT & 4 & & I & FRG & 1 & IND & 2 \\
\hline Unit $117 \mathrm{SE}^{1 / 4}$ & $30-35$ & 1347 & 1368 & Analyzed & 1 & 0.1 & LPR & PWS & LOC & CRT & 4 & & I & FRG & 0 & IND & 2 \\
\hline Unit $117 \mathrm{SE}^{1} / 4$ & $35-40$ & 1351 & 1372 & Analyzed & 1 & 0.5 & LPR & PWS & LOC & CRT & 4 & & I & FRG & 4 & IND & 1 \\
\hline Unit $117 \mathrm{SE}^{1 / 4}$ & $35-40$ & 1351 & 1372 & Analyzed & 1 & 4.4 & LPR & PWS & LOC & CRT & 3 & & $\mathrm{~N}$ & COM & 3 & CRT & 1 \\
\hline Unit $117 \mathrm{SE}^{1} / 4$ & $30-35$ & 1347 & 1368 & Analyzed & 1 & 1.5 & LPR & PWS & LOC & CRT & 4 & & I & FRG & 3 & IND & 2 \\
\hline Unit $117 \mathrm{SW}^{1 / 4}$ & $15-20$ & 1327 & 1356 & Unanalyzed & 1 & 0.6 & & & & & & & & & & & \\
\hline Unit $117 \mathrm{SW}^{1 / 4}$ & $25-30$ & 1340 & 1365 & Unanalyzed & 1 & 0.4 & & & & & & & & & & & \\
\hline Unit $117 \mathrm{SW}^{1 / 4}$ & $35-40$ & 1348 & 1373 & Analyzed & 1 & 0.1 & LPR & PWS & LOC & CRT & 4 & & I & FRG & 0 & IND & 1 \\
\hline Unit $117 \mathrm{SW}^{1 / 4}$ & $10-15$ & 1323 & 1352 & Unanalyzed & 1 & 0.2 & & & & & & & & & & & \\
\hline Unit $117 \mathrm{SW}^{1 / 4}$ & $30-35$ & 1344 & 1369 & Analyzed & 1 & 0.1 & LPR & PWS & LOC & CRT & 4 & & I & COM & 1 & IND & 1 \\
\hline Unit $117 \mathrm{SW}^{1 / 4}$ & $30-35$ & 1344 & 1369 & Analyzed & 1 & 0.2 & LPR & PWS & LOC & CRT & 4 & & I & COM & 0 & CPX & 1 \\
\hline Unit $117 \mathrm{SW}^{1 / 4}$ & $30-35$ & 1344 & 1369 & Analyzed & 2 & 0.6 & LPR & PWS & LOC & CRT & 4 & & I & DEB & 3 & IND & 1 \\
\hline \multicolumn{18}{|c|}{ Feature } \\
\hline Feature $100 \mathrm{E}^{1 / 2}$ & $50-57$ & 1962 & 1741 & Analyzed & 1 & 1.8 & LPR & PWS & LOC & CRT & 3 & & $\mathrm{~N}$ & COM & 4 & CRT & 1 \\
\hline Feature $103 \mathrm{~N} \mathrm{~N}^{1 / 2}$ & $50-54$ & 1966 & 1745 & Analyzed & 1 & 0.04 & LPR & PWS & INT & USX & 6 & & I & DEB & 0 & IND & 1 \\
\hline Feature $105 \mathrm{NW}^{1 / 2}$ & $50-60$ & 1969 & 1747 & Analyzed & 2 & 0.04 & LPR & PWS & INT & USX & 6 & & I & DEB & 0 & IND & 1 \\
\hline Feature $105 \mathrm{SE}^{1 / 2}$ & $50-60$ & 1968 & 1748 & Analyzed & 1 & 0.04 & LPR & PWS & INT & USX & 6 & & I & DEB & 0 & IND & 1 \\
\hline Feature $108 \mathrm{E}^{1 / 2}$ & $50-66$ & 1973 & 1752 & Analyzed & 1 & 0.04 & LPR & PWS & INT & USX & 6 & & I & DEB & 0 & IND & 1 \\
\hline Feature $108 \mathrm{~W}^{1 / 2}$ & $50-66$ & 1974 & 1753 & Analyzed & 1 & 0.04 & LPR & PWS & LOC & QZT & 5 & & I & COM & 0 & IND & 1 \\
\hline Feature $45 \mathrm{~N}^{1 / 2}$ & $55-65$ & 1867 & 1647 & Analyzed & 1 & 0.04 & LPR & PWS & INT & USX & 6 & & I & DEB & 0 & IND & 1 \\
\hline Feature $46 \mathrm{~W}^{1 / 2}$ & $55-59$ & 1868 & 1649 & Analyzed & 1 & 0.04 & LPR & PWS & INT & USX & 6 & & I & DEB & 0 & IND & 1 \\
\hline Feature $47 \mathrm{E}^{1} / 2$ & $55-63$ & 1870 & 1650 & Analyzed & 3 & 0.04 & LPR & PWS & LOC & CRT & 5 & & I & COM & 0 & CPX & 2 \\
\hline Feature $47 \mathrm{E}^{1 / 2}$ & $55-63$ & 1870 & 1650 & Analyzed & 1 & 0.3 & LPR & PWS & LOC & FWD & 4 & & I & COM & 1 & CRT & 2 \\
\hline Feature $47 \mathrm{E}^{1 / 2}$ & $55-63$ & 1870 & 1650 & Analyzed & 1 & 1.5 & LPR & PWS & LOC & CRT & 3 & & $\mathrm{~N}$ & COM & 1 & CRT & 2 \\
\hline Feature $47 \mathrm{E}^{1} / 2$ & $55-63$ & 1870 & 1650 & Analyzed & 2 & 0.04 & LPR & PWS & LOC & CRT & 5 & & I & FRG & 4 & IND & 2 \\
\hline Feature $47 E^{1} / 2$ & $55-63$ & 1870 & 1650 & Analyzed & 11 & 0.5 & LPR & PWS & LOC & CRT & 5 & & I & FRG & 0 & IND & 2 \\
\hline
\end{tabular}


Geo-Marine, Inc.

GMI 22005.01.06.xx and 22005.00.09.xx, Data Recovery Excavations at Site 41PN175 Debitage Analysi

\begin{tabular}{|c|c|c|c|c|c|c|c|c|c|c|c|c|c|c|c|c|c|}
\hline Unit & $\begin{array}{c}\text { Depth } \\
(\mathrm{cm})\end{array}$ & $\begin{array}{l}\text { Provenience } \\
\text { No. }\end{array}$ & $\begin{array}{l}\text { Lot } \\
\text { No. }\end{array}$ & Level of Analysis & Quantity & $\begin{array}{l}\text { Weight } \\
\text { (in g) }\end{array}$ & General Period & Region & Material & Lithology & Size Grade & $\begin{array}{c}\text { Edge } \\
\text { Modification }\end{array}$ & $\begin{array}{l}\text { Thernal } \\
\text { Alteration }\end{array}$ & Completeness & Cortex & Platform & $\begin{array}{l}\text { Minimum } \\
\text { Number of } \\
\text { Nodules }\end{array}$ \\
\hline \multicolumn{18}{|c|}{ Feature } \\
\hline Feature $47 \mathrm{E}^{1 / 2}$ & $55-63$ & 1870 & 1650 & Analyzed & 11 & 0.1 & LPR & PWS & INT & USX & 6 & & I & DEB & 0 & IND & 2 \\
\hline Feature $47 \mathrm{~W} 1 / 2$ & $55-63$ & 1871 & 1651 & Analyzed & 7 & 0.04 & LPR & PWS & INT & USX & 6 & & I & DEB & 0 & IND & 1 \\
\hline Feature $47 \mathrm{~W} 1 / 2$ & $55-63$ & 1871 & 1651 & Analyzed & 1 & 0.2 & LPR & PWS & LOC & CRT & 4 & & I & DEB & 0 & IND & 1 \\
\hline Feature $47 \mathrm{~W} 1 / 2$ & $55-63$ & 1871 & 1651 & Analyzed & 1 & 0.04 & LPR & PWS & LOC & CRT & 5 & & I & FRG & 0 & IND & 1 \\
\hline Feature $47 \mathrm{~W} 1 / 2$ & $55-63$ & 1871 & 1651 & Analyzed & 1 & 0.04 & LPR & PWS & LOC & CRT & 4 & & I & FRG & 4 & IND & 1 \\
\hline Feature $47 \mathrm{~W} 1 / 2$ & $55-63$ & 1871 & 1651 & Analyzed & 1 & 1.8 & LPR & PWS & LOC & QZT & 3 & & $\mathrm{Y}$ & DEB & 0 & IND & 1 \\
\hline Feature $47 \mathrm{~W} 1 / 2$ & $55-63$ & 1871 & 1651 & Analyzed & 1 & 0.4 & LPR & PWS & LOC & FWD & 4 & & I & COM & 0 & FLT & 1 \\
\hline Feature $47 \mathrm{~W} 1 / 2$ & $55-63$ & 1871 & 1651 & Analyzed & 3 & 0.8 & LPR & PWS & LOC & CRT & 4 & & I & FRG & 1 & IND & 1 \\
\hline Feature $47 \mathrm{~W} 1 / 2$ & $55-63$ & 1871 & 1651 & Analyzed & 2 & 0.04 & LPR & PWS & LOC & CRT & 5 & & I & FRG & 4 & IND & 1 \\
\hline Feature $47 \mathrm{~W}^{1 / 2}$ & $55-63$ & 1871 & 1651 & Analyzed & 1 & 0.04 & LPR & PWS & LOC & CRT & 5 & & I & FRG & 1 & IND & 1 \\
\hline Feature $51 \mathrm{~N}^{1 / 2}$ & $60-70$ & 1878 & 1658 & Analyzed & 2 & 0.04 & LPR & PWS & INT & USX & 6 & & I & DEB & 0 & IND & 1 \\
\hline Feature $51 \mathrm{~N}^{1 / 2}$ & $60-70$ & 1878 & 1658 & Analyzed & 1 & 0.04 & LPR & PWS & LOC & CRT & 5 & & I & $\mathrm{COM}$ & 0 & CPX & 1 \\
\hline Feature $51 \mathrm{~S}^{1 / 2}$ & $60-70$ & 1877 & 1657 & Analyzed & 2 & 0.04 & LPR & PWS & INT & USX & 6 & & I & DEB & 0 & IND & 1 \\
\hline Feature $53 \mathrm{E}^{1 / 2}$ & $60-70$ & 1881 & 1662 & Analyzed & 1 & 2.7 & LPR & PWS & LOC & CRT & 3 & & $\mathrm{~N}$ & COM & 4 & FLT & 1 \\
\hline Feature $53 \mathrm{E}^{1 / 2}$ & $60-70$ & 1881 & 1662 & Analyzed & 4 & 0.04 & LPR & PWS & INT & USX & 6 & & I & DEB & 0 & IND & 1 \\
\hline Feature $53 \mathrm{~W} \mathbf{W}^{1 / 2}$ & $60-70$ & 1882 & 1661 & Analyzed & 1 & 1 & LPR & PWS & LOC & FWD & 4 & & I & DEB & 2 & IND & 2 \\
\hline Feature $53 \mathrm{~W} 1 / 2$ & $60-70$ & 1882 & 1661 & Analyzed & 1 & 0.04 & LPR & PWS & INT & USX & 6 & & I & DEB & 0 & IND & 2 \\
\hline Feature $53 \mathrm{~W}{ }^{1 / 2}$ & $60-70$ & 1882 & 1661 & Analyzed & 1 & 0.1 & LPR & PWS & LOC & CRT & 4 & & I & $\mathrm{COM}$ & 1 & CPX & 2 \\
\hline Feature $53 \mathrm{~W}^{1 / 2}$ & $60-70$ & 1882 & 1661 & Analyzed & 3 & 0.04 & LPR & PWS & LOC & CRT & 4 & & I & FRG & 0 & IND & 2 \\
\hline Feature $54 \mathrm{E}^{1 / 2}$ & $60-65$ & 1884 & 1664 & Analyzed & 1 & 0.04 & LPR & PWS & LOC & QZT & 5 & & I & COM & 1 & IND & 1 \\
\hline Feature $55 \mathrm{~N} 1 / 2$ & $65-72$ & 1886 & 1666 & Analyzed & 1 & 0.1 & LPR & PWS & LOC & CRT & 4 & & I & BRK & 0 & CPX & 1 \\
\hline Feature $55 \mathrm{~N}^{1 / 2}$ & $65-72$ & 1886 & 1666 & Analyzed & 1 & 0.6 & LPR & PWS & LOC & CRT & 4 & & I & COM & 4 & CRT & 1 \\
\hline Feature $55 \mathrm{~N}^{1 / 2}$ & $65-72$ & 1886 & 1666 & Analyzed & 1 & 1.3 & LPR & PWS & LOC & CRT & 3 & & $\mathrm{Y}$ & COM & 0 & FLT & 1 \\
\hline Feature $55 \mathrm{~N} 1 / 2$ & $65-72$ & 1886 & 1666 & Analyzed & 1 & 0.2 & LPR & PWS & LOC & CRT & 4 & & I & FRG & 0 & IND & 1 \\
\hline Feature $55 \mathrm{~S} / 2$ & $65-72$ & 1885 & 1665 & Analyzed & 1 & 0.04 & LPR & PWS & LOC & CRT & 4 & & I & FRG & 4 & IND & 1 \\
\hline Feature $55 \mathrm{~S} 1 / 2$ & $65-72$ & 1885 & 1665 & Analyzed & 1 & 0.5 & LPR & PWS & LOC & CRT & 4 & & I & COM & 2 & CRT & 1 \\
\hline
\end{tabular}


Geo-Marine, Inc.

GMI 22005.01.06.xx and 22005.00.09.xx, Data Recovery Excavations at Site 41PN175 Debitage Analysi

\begin{tabular}{|c|c|c|c|c|c|c|c|c|c|c|c|c|c|c|c|c|c|}
\hline Unit & $\begin{array}{c}\text { Depth } \\
(\mathrm{cm})\end{array}$ & $\begin{array}{l}\text { Provenience } \\
\text { No. }\end{array}$ & $\begin{array}{l}\text { Lot } \\
\text { No. }\end{array}$ & Level of Analysis & Quantity & $\begin{array}{l}\text { Weight } \\
\text { (in g) }\end{array}$ & General Period & Region & Material & Lithology & Size Grade & $\begin{array}{c}\text { Edge } \\
\text { Modification }\end{array}$ & $\begin{array}{l}\text { Thernal } \\
\text { Alteration }\end{array}$ & Completeness & Cortex & Platform & $\begin{array}{l}\text { Minimum } \\
\text { Number of } \\
\text { Nodules }\end{array}$ \\
\hline \multicolumn{18}{|c|}{ Feature } \\
\hline Feature $55 \mathrm{~S} 1 / 2$ & $65-72$ & 1885 & 1665 & Analyzed & 7 & 0.3 & LPR & PWS & LOC & CRT & 5 & & I & FRG & 0 & IND & 1 \\
\hline Feature $55 \mathrm{~S} 1 / 2$ & $65-72$ & 1885 & 1665 & Analyzed & 1 & 0.1 & LPR & PWS & REG & CRT & 4 & & I & $\mathrm{COM}$ & 0 & FLT & 1 \\
\hline Feature $55 \mathrm{~S} 1 / 2$ & $65-72$ & 1885 & 1665 & Analyzed & 3 & 0.8 & LPR & PWS & LOC & CRT & 4 & & I & FRG & 0 & IND & 1 \\
\hline Feature $55 \mathrm{~S} 1 / 2$ & $65-72$ & 1885 & 1665 & Analyzed & 4 & 0.04 & LPR & PWS & INT & USX & 6 & & I & DEB & 0 & IND & 1 \\
\hline Feature $56 \mathrm{E}^{1} / 2$ & $65-83$ & 1887 & 1668 & Analyzed & 8 & 0.04 & LPR & PWS & INT & usx & 6 & & I & DEB & 0 & IND & 2 \\
\hline Feature $56 \mathrm{E}^{1} / 2$ & $65-83$ & 1887 & 1668 & Analyzed & 2 & 0.04 & LPR & PWS & LOC & CRT & 5 & & I & FRG & 0 & IND & 2 \\
\hline Feature $56 \mathrm{E}^{1} / 2$ & $65-83$ & 1887 & 1668 & Analyzed & 1 & 0.04 & LPR & PWS & LOC & QZT & 5 & & I & DEB & 0 & IND & 2 \\
\hline Feature $56 \mathrm{~W}^{1 / 2}$ & $65-83$ & 1888 & 1667 & Analyzed & 2 & 0.04 & LPR & PWS & LOC & CRT & 5 & & I & FRG & 1 & IND & 1 \\
\hline Feature $56 \mathrm{~W}^{1 / 2}$ & $65-83$ & 1888 & 1667 & Analyzed & 4 & 0.04 & LPR & PWS & LOC & CRT & 5 & & I & FRG & 0 & IND & 1 \\
\hline Feature $56 \mathrm{~W}^{1 / 2}$ & $65-83$ & 1888 & 1667 & Analyzed & 2 & 0.1 & LPR & PWS & LOC & CRT & 4 & & I & FRG & 0 & IND & 1 \\
\hline Feature $56 \mathrm{~W} 1 / 2$ & $65-83$ & 1888 & 1667 & Analyzed & 12 & 0.1 & LPR & PWS & INT & USX & 6 & & I & DEB & 0 & IND & 1 \\
\hline Feature $57 \mathrm{E}^{1} / 2$ & $60-90$ & 1889 & 1669 & Analyzed & 1 & 0.04 & LPR & PWS & LOC & CRT & 5 & & I & $\mathrm{COM}$ & 4 & CRT & 1 \\
\hline Feature $57 \mathrm{E}^{1} / 2$ & $60-90$ & 1889 & 1669 & Analyzed & 2 & 0.04 & LPR & PWS & LOC & CRT & 5 & & I & FRG & 0 & IND & 1 \\
\hline Feature $57 \mathrm{E}^{1} / 2$ & $60-90$ & 1889 & 1669 & Analyzed & 7 & 0.04 & LPR & PWS & INT & USX & 6 & & I & DEB & 0 & IND & 1 \\
\hline Feature $57 \mathrm{~W}^{1 / 2}$ & $60-90$ & 1890 & 1670 & Analyzed & 1 & 3 & LPR & PWS & LOC & QZT & 3 & & $\mathrm{Y}$ & DEB & 2 & IND & 3 \\
\hline Feature $57 \mathrm{~W}^{1 / 2}$ & $60-90$ & 1890 & 1670 & Analyzed & 1 & 0.1 & LPR & PWS & LOC & CRT & 4 & & I & COM & 0 & CPX & 3 \\
\hline Feature $57 \mathrm{~W}^{1 / 2}$ & $60-90$ & 1890 & 1670 & Analyzed & 1 & 0.04 & LPR & PWS & LOC & CRT & 5 & & I & FRG & 1 & IND & 3 \\
\hline Feature $57 \mathrm{~W}^{1 / 2}$ & $60-90$ & 1890 & 1670 & Analyzed & 2 & 0.04 & LPR & PWS & LOC & CRT & 5 & & I & FRG & 0 & IND & 3 \\
\hline Feature $57 \mathrm{~W}^{1 / 2}$ & $60-90$ & 1890 & 1670 & Analyzed & 1 & 0.04 & LPR & PWS & REG & CRT & 5 & & I & $\mathrm{COM}$ & 0 & CPX & 3 \\
\hline Feature $57 \mathrm{~W}^{1 / 2}$ & $60-90$ & 1890 & 1670 & Analyzed & 6 & 0.04 & LPR & PWS & INT & USX & 6 & & I & DEB & 0 & IND & 3 \\
\hline Feature $58 \mathrm{~N}^{1 / 2}$ & $65-79$ & 1891 & 1671 & Analyzed & 5 & 0.3 & LPR & PWS & LOC & CRT & 5 & & I & DEB & 0 & IND & 1 \\
\hline Feature $58 \mathrm{~N}^{1 / 2}$ & $65-79$ & 1891 & 1671 & Analyzed & 14 & 0.1 & LPR & PWS & INT & USX & 6 & & I & DEB & 0 & IND & 1 \\
\hline Feature 58 S1⁄2 & $65-79$ & 1892 & 1672 & Analyzed & 1 & 1.3 & LPR & PWS & LOC & QZT & 3 & & $\mathrm{~N}$ & COM & 0 & FLT & 2 \\
\hline Feature $58 \mathrm{~S} 1 / 2$ & $65-79$ & 1892 & 1672 & Analyzed & 7 & 0.04 & LPR & PWS & INT & USX & 6 & & I & DEB & 0 & IND & 2 \\
\hline Feature $58 \mathrm{~S}^{1 / 2}$ & $65-79$ & 1892 & 1672 & Analyzed & 1 & 0.04 & LPR & PWS & LOC & CRT & 5 & & I & COM & 0 & FLT & 2 \\
\hline Feature $59 \mathrm{NE}^{1 / 2}$ & $65-74$ & 1894 & 1674 & Analyzed & 1 & 0.04 & LPR & PWS & INT & USX & 6 & & I & DEB & 0 & IND & 1 \\
\hline
\end{tabular}


Geo-Marine, Inc.

GMI 22005.01.06.xx and 22005.00.09.xx, Data Recovery Excavations at Site 41PN175 Debitage Analysi

\begin{tabular}{|c|c|c|c|c|c|c|c|c|c|c|c|c|c|c|c|c|c|}
\hline Unit & $\begin{array}{c}\text { Depth } \\
(\mathrm{cm})\end{array}$ & $\begin{array}{l}\text { Provenience } \\
\text { No. }\end{array}$ & $\begin{array}{l}\text { Lot } \\
\text { No. }\end{array}$ & Level of Analysis & Quantity & $\begin{array}{l}\text { Weight } \\
\text { (in g) }\end{array}$ & General Period & Region & Material & Lithology & Size Grade & $\begin{array}{c}\text { Edge } \\
\text { Modification }\end{array}$ & $\begin{array}{l}\text { Thernal } \\
\text { Alteration }\end{array}$ & Completeness & Cortex & Platform & $\begin{array}{l}\text { Minimum } \\
\text { Number of } \\
\text { Nodules }\end{array}$ \\
\hline \multicolumn{18}{|c|}{ Feature } \\
\hline Feature $59 \mathrm{SW}^{1} / 2$ & $65-74$ & 1893 & 1673 & Analyzed & 1 & 0.04 & LPR & PWS & LOC & CRT & 5 & & I & FRG & 0 & IND & 1 \\
\hline Feature $60 \mathrm{~N} 1 / 2$ & $65-70$ & 1896 & 1676 & Analyzed & 2 & 0.04 & LPR & PWS & INT & USX & 6 & & I & DEB & 0 & IND & 1 \\
\hline Feature $62 \mathrm{E}^{1 / 2}$ & $65-70$ & 1899 & 1679 & Analyzed & 1 & 0.04 & LPR & PWS & INT & USX & 6 & & I & DEB & 0 & IND & 1 \\
\hline Feature $62 \mathrm{~W}^{1 / 2}$ & $65-70$ & 1900 & 1680 & Analyzed & 1 & 0.04 & LPR & PWS & LOC & CRT & 5 & & I & COM & 0 & IND & 1 \\
\hline Feature $68 \mathrm{E}^{1 / 2}$ & $65-85$ & 1907 & 1688 & Analyzed & 1 & 0.04 & LPR & PWS & INT & USX & 6 & & I & DEB & 0 & IND & 1 \\
\hline Feature $70 \mathrm{~N} 1 / 2$ & $60-82$ & 1912 & 1692 & Analyzed & 1 & 0.3 & LPR & PWS & LOC & QZT & 4 & & I & DEB & 0 & IND & 1 \\
\hline Feature $72 \mathrm{NW}^{1 / 2}$ & $60-74$ & 1915 & 1695 & Analyzed & 1 & 0.04 & LPR & PWS & INT & usx & 6 & & I & DEB & 0 & IND & 1 \\
\hline Feature $72 \mathrm{NW}^{1 / 2}$ & $60-74$ & 1915 & 1695 & Analyzed & 1 & 0.04 & LPR & PWS & LOC & CRT & 5 & & I & $\mathrm{COM}$ & 0 & IND & 1 \\
\hline Feature $72 \mathrm{SE}^{1 / 2}$ & $60-74$ & 1914 & 1694 & Analyzed & 4 & 0.04 & LPR & PWS & INT & USX & 6 & & I & DEB & 0 & IND & 1 \\
\hline Feature $72 \mathrm{SE}^{1 / 2}$ & $60-74$ & 1914 & 1694 & Analyzed & 1 & 6.4 & LPR & PWS & LOC & CRT & 2 & ABS & $\mathrm{Y}$ & COM & 2 & FAC & 1 \\
\hline Feature $73 \mathrm{NW}^{1 / 2}$ & $60-74$ & 1917 & 1697 & Analyzed & 3 & 0.04 & LPR & PWS & INT & USX & 6 & & I & DEB & 0 & IND & 1 \\
\hline Feature $73 \mathrm{NW}^{1 / 2}$ & $60-74$ & 1917 & 1697 & Analyzed & 2 & 0.04 & LPR & PWS & LOC & CRT & 5 & & I & FRG & 0 & IND & 1 \\
\hline Feature $73 \mathrm{NW}^{1 / 2}$ & $60-74$ & 1917 & 1697 & Analyzed & 1 & 0.04 & LPR & PWS & LOC & CRT & 5 & & I & FRG & 3 & IND & 1 \\
\hline Feature $73 \mathrm{SE}^{1 / 2}$ & $60-74$ & 1916 & 1696 & Analyzed & 1 & 0.04 & LPR & PWS & LOC & CRT & 5 & & I & FRG & 0 & IND & 1 \\
\hline Feature $73 \mathrm{SE}^{1 / 2}$ & $60-74$ & 1916 & 1696 & Analyzed & 2 & 0.04 & LPR & PWS & INT & USX & 6 & & I & DEB & 0 & IND & 1 \\
\hline Feature $75 \mathrm{~W}^{1 / 2}$ & $\begin{array}{l}55- \\
108\end{array}$ & 1920 & 1699 & Analyzed & 7 & 0.1 & LPR & PWS & INT & usx & 6 & & I & DEB & 0 & IND & 1 \\
\hline Feature $75 \mathrm{~W}^{1 / 2}$ & $\begin{array}{l}55- \\
108\end{array}$ & 1920 & 1699 & Analyzed & 1 & 0.04 & LPR & PWS & LOC & CRT & 5 & & I & $\mathrm{COM}$ & 1 & CRT & 1 \\
\hline Feature $75 \mathrm{~W}^{1 / 2}$ & $\begin{array}{l}55- \\
108\end{array}$ & 1920 & 1699 & Analyzed & 1 & 0.1 & LPR & PWS & LOC & CRT & 4 & & I & COM & 2 & CRT & 1 \\
\hline Feature $75 \mathrm{~W}^{1 / 2}$ & $\begin{array}{l}55- \\
108\end{array}$ & 1920 & 1699 & Analyzed & 5 & 0.1 & LPR & PWS & LOC & CRT & 5 & & I & FRG & 0 & IND & 1 \\
\hline Feature $76 \mathrm{~N}^{1 / 2}$ & $55-77$ & 1922 & 1701 & Analyzed & 2 & 0.1 & LPR & PWS & LOC & CRT & 5 & & I & DEB & 0 & IND & 1 \\
\hline Feature $76 \mathrm{~N} 1 / 2$ & $55-77$ & 1922 & 1701 & Analyzed & 2 & 0.04 & LPR & PWS & INT & usx & 6 & & I & DEB & 0 & IND & 1 \\
\hline Feature $76 \mathrm{~S}^{1 / 2}$ & $55-77$ & 1921 & 1700 & Analyzed & 1 & 0.1 & LPR & PWS & LOC & CRT & 4 & & I & COM & 0 & CPX & 1 \\
\hline Feature $76 \mathrm{~S}^{1 / 2}$ & $55-77$ & 1921 & 1700 & Analyzed & 1 & 2.2 & LPR & PWS & LOC & CRT & 3 & & $\mathrm{Y}$ & DEB & 1 & IND & 1 \\
\hline Feature $78 \mathrm{~S} 1 / 2$ & $55-75$ & 1924 & 1703 & Analyzed & 1 & 0.04 & LPR & PWS & LOC & CRT & 5 & & I & FRG & 0 & IND & 1 \\
\hline
\end{tabular}


Geo-Marine, Inc.

GMI 22005.01.06.xx and 22005.00.09.xx, Data Recovery Excavations at Site 41PN175 Debitage Analysi

\begin{tabular}{|c|c|c|c|c|c|c|c|c|c|c|c|c|c|c|c|c|c|}
\hline Unit & $\begin{array}{c}\text { Depth } \\
(\mathrm{cm})\end{array}$ & $\begin{array}{l}\text { Provenience } \\
\text { No. }\end{array}$ & $\begin{array}{l}\text { Lot } \\
\text { No. }\end{array}$ & Level of Analysis & Quantity & $\begin{array}{l}\text { Weight } \\
\text { (ing) }\end{array}$ & General Period & Region & Material & Lithology & Size Grade & $\begin{array}{c}\text { Edge } \\
\text { Modification }\end{array}$ & $\begin{array}{l}\text { Thernal } \\
\text { Alteration }\end{array}$ & Completeness & Cortex & Platform & $\begin{array}{c}\text { Minimum } \\
\text { Number of } \\
\text { Nodules }\end{array}$ \\
\hline \multicolumn{18}{|c|}{ Feature } \\
\hline Feature $79 \mathrm{~S}^{1 / 2}$ & $55-79$ & 1927 & 1706 & Analyzed & 1 & 0.1 & LPR & PWS & LOC & QZT & 4 & & I & FRG & 0 & IND & 1 \\
\hline Feature $79 \mathrm{~S}^{1 / 2}$ & $55-79$ & 1927 & 1706 & Analyzed & 15 & 0.1 & LPR & PWS & INT & USX & 6 & & I & DEB & 0 & IND & 15 \\
\hline Feature $79 \mathrm{~S}^{1 / 2}$ & $55-79$ & 1927 & 1706 & Analyzed & 1 & 0.04 & LPR & PWS & LOC & QZT & 5 & & I & FRG & 0 & IND & 1 \\
\hline Feature $82 \mathrm{~S}^{1 / 2}$ & $60-69$ & 1931 & 1711 & Analyzed & 2 & 0.04 & LPR & PWS & INT & USX & 6 & & I & DEB & 0 & IND & 1 \\
\hline Feature $85 \mathrm{~S}^{1 / 2}$ & $60-73$ & 1936 & 1715 & Analyzed & 1 & 0.04 & LPR & PWS & LOC & CRT & 5 & & I & FRG & 0 & IND & 1 \\
\hline Feature $86 \mathrm{NW}^{1 / 2}$ & $60-67$ & 1938 & 1718 & Analyzed & 1 & 1.3 & LPR & PWS & LOC & CRT & 4 & & I & $\mathrm{COM}$ & 1 & CRT & 3 \\
\hline Feature $86 \mathrm{NW}^{1 / 2}$ & $60-67$ & 1938 & 1718 & Analyzed & 2 & 0.2 & LPR & PWS & LOC & CRT & 4 & & I & $\mathrm{COM}$ & 0 & FLT & 3 \\
\hline Feature $86 \mathrm{NW}^{1 / 2}$ & $60-67$ & 1938 & 1718 & Analyzed & 1 & 0.4 & LPR & PWS & REG & CRT & 4 & & I & FRG & 0 & IND & 3 \\
\hline Feature $86 \mathrm{SE}^{1 / 2}$ & $60-67$ & 1939 & 1717 & Analyzed & 1 & 0.5 & LPR & PWS & LOC & QZT & 4 & & I & DEB & 0 & IND & 1 \\
\hline Feature $87 E^{1 / 2}$ & $60-75$ & 1940 & 1720 & Analyzed & 1 & 0.04 & LPR & PWS & INT & USX & 6 & & I & DEB & 0 & IND & 1 \\
\hline Feature $87 \mathrm{E}^{1 / 2}$ & $60-75$ & 1940 & 1720 & Analyzed & 1 & 0.04 & LPR & PWS & LOC & CRT & 5 & & I & COM & 0 & FLT & 1 \\
\hline Feature $87 \mathrm{~W}^{1 / 2}$ & $60-75$ & 1941 & 1719 & Analyzed & 4 & 0.04 & LPR & PWS & INT & USX & 6 & & I & DEB & 0 & IND & 1 \\
\hline Feature $88 \mathrm{~S}^{1 / 2}$ & $\begin{array}{l}90- \\
124\end{array}$ & 1942 & 1721 & Analyzed & 1 & 0.2 & LPR & PWS & LOC & CRT & 4 & & I & COM & 0 & CPX & 1 \\
\hline Feature $88 \mathrm{~S} 5 / 2$ & $\begin{array}{l}90- \\
124\end{array}$ & 1942 & 1721 & Analyzed & 1 & 0.04 & LPR & PWS & INT & USX & 6 & & I & DEB & 0 & IND & 1 \\
\hline Feature $93 \mathrm{~S} \mathrm{~S}^{1} / 2$ & $50-54$ & 1950 & 1729 & Analyzed & 1 & 0.04 & LPR & PWS & LOC & QZT & 4 & & I & $\mathrm{COM}$ & 4 & CRT & 2 \\
\hline Feature $93 \mathrm{~S}^{1 / 2}$ & $50-54$ & 1950 & 1729 & Analyzed & 1 & 0.04 & LPR & PWS & INT & USX & 6 & & I & DEB & 0 & IND & 2 \\
\hline Feature $93 \mathrm{~S}^{1 / 2}$ & $50-54$ & 1950 & 1729 & Analyzed & 1 & 1.9 & LPR & PWS & LOC & CHL & 4 & & I & FRG & 4 & IND & 2 \\
\hline Feature $97 \mathrm{E}^{1 / 2}$ & $65-92$ & 1956 & 1735 & Analyzed & 1 & 0.1 & LPR & PWS & LOC & CRT & 4 & & I & FRG & 0 & IND & 1 \\
\hline Feature $97 \mathrm{E}^{1 / 2}$ & $65-92$ & 1956 & 1735 & Analyzed & 2 & 0.04 & LPR & PWS & LOC & CRT & 5 & & I & FRG & 0 & IND & 1 \\
\hline Feature $97 \mathrm{E}^{1 / 2}$ & $65-92$ & 1956 & 1735 & Analyzed & 22 & 0.2 & LPR & PWS & INT & USX & 6 & & I & DEB & 0 & IND & 1 \\
\hline Feature $97 \mathrm{~W}^{1 / 2}$ & $65-92$ & 1957 & 1736 & Analyzed & 6 & 0.1 & LPR & PWS & LOC & CRT & 5 & & I & FRG & 0 & IND & 1 \\
\hline Feature $97 \mathrm{~W}^{1 / 2}$ & $65-92$ & 1957 & 1736 & Analyzed & 3 & 0.1 & LPR & PWS & LOC & CRT & 5 & & I & FRG & 1 & IND & 1 \\
\hline Feature $97 \mathrm{~W}^{1 / 2}$ & $65-92$ & 1957 & 1736 & Analyzed & 9 & 0.04 & LPR & PWS & INT & USX & 6 & & I & DEB & 0 & IND & 1 \\
\hline Feature $99 \mathrm{E}^{1 / 2}$ & $50-54$ & 1959 & 1738 & Analyzed & 2 & 0.04 & LPR & PWS & INT & USX & 6 & & I & DEB & 0 & IND & 1 \\
\hline
\end{tabular}


A-8: FIRE-CRACKED ROCK 

Geo-Marine, Inc.

GMI 22005.01.06.xx and 22005.00.09.xx, Data Recovery Excavations at Site 41PN175

FCR Analysis Data

\begin{tabular}{|c|c|c|c|c|c|c|c|c|}
\hline Unit Type & $\begin{array}{c}\text { Depth } \\
(\mathrm{cm})\end{array}$ & $\begin{array}{c}\text { Prov. } \\
\text { No. }\end{array}$ & $\begin{array}{l}\text { Lot } \\
\text { No. }\end{array}$ & $\begin{array}{c}\text { Artifact } \\
\text { No. }\end{array}$ & Class & Raw Material & Qty & $\begin{array}{l}\text { Weight } \\
\text { (in g) }\end{array}$ \\
\hline
\end{tabular}

$50 \times 50 \mathrm{~cm}$ Units

$\begin{array}{lccccclcc}\text { Unit } 007 & 20-40 & 128 & 165 & 9 & \text { FCR } & \text { Chert } & 1 & 2 \\ \text { Unit } 014 & 20-30 & 55 & 188 & 21 & \text { FCR } & \text { Ironized Sandstone } & 1 & 2.5 \\ \text { Unit } 026 & 20-40 & 94 & 231 & 21 & \text { FCR } & \text { Quartzite } & 1 & 7\end{array}$

Block 2

\begin{tabular}{|c|c|c|c|c|c|c|c|}
\hline Unit $083 \mathrm{SE}^{1 / 4}$ & $30-40$ & 862 & 677 & 3 & FCR & Quartzite & 1 \\
\hline Unit $084 \mathrm{SE}^{1 / 4}$ & $30-40$ & 867 & 697 & 18 & FCR & Quartzite & 1 \\
\hline Unit $084 \mathrm{SE}^{1 / 4}$ & $30-40$ & 867 & 697 & 19 & FCR & Ironized Sandstone & 1 \\
\hline Unit $083 \mathrm{NW}^{1} / 4$ & $10-20$ & 744 & 1769 & 1 & FCR & Quartzite & 1 \\
\hline
\end{tabular}

Block 3

\begin{tabular}{|c|c|c|c|c|c|c|c|c|}
\hline Unit $065 \mathrm{NW}^{1} / 4$ & $0-10$ & 471 & 392 & 1 & FCR & Quartzite & 1 & 5.2 \\
\hline Unit $065 \mathrm{NE}^{1 / 4}$ & $10-20$ & 519 & 394 & 2 & FCR & Silt-stone & 1 & 4 \\
\hline Unit $065 \mathrm{NE}^{1} / 4$ & $10-20$ & 519 & 394 & 3 & FCR & Ironized Sandstone & 1 & 35.6 \\
\hline Unit $070 \mathrm{NE}^{1} / 4$ & $0-10$ & 493 & 460 & 3 & FCR & Chert & 1 & 3.2 \\
\hline Unit $071 \mathrm{SE}^{1 / 4}$ & $10-20$ & 566 & 474 & 4 & FCR & Chert & 1 & 4 \\
\hline Unit $071 \mathrm{SW}^{1} / 4$ & $20-30$ & 631 & 478 & 1 & FCR & Fossilized Wood & 1 & 2.6 \\
\hline Unit $071 \mathrm{SW}^{1} / 4$ & $20-30$ & 631 & 478 & 2 & FCR & Fossilized Wood & 1 & 1.5 \\
\hline \multicolumn{9}{|c|}{ Block 4} \\
\hline Unit $073 \mathrm{NE}^{1} / 4$ & $20-30$ & 646 & 505 & 4 & FCR & Quartzite & 1 & 1.9 \\
\hline Unit $073 \mathrm{SW}^{1} / 4$ & $50-60$ & 1726 & 516 & 15 & FCR & Quartzite & 1 & 4.3 \\
\hline Unit $074 \mathrm{NW}^{1} / 4$ & $30-40$ & 702 & 527 & 6 & FCR & Quartzite & 1 & 3.3 \\
\hline Unit $075 \mathrm{SE}^{1 / 4}$ & $20-30$ & 658 & 545 & 6 & FCR & Fossilized Wood & 1 & 22 \\
\hline Unit $078 \mathrm{NE}^{1} / 4$ & $30-40$ & 715 & 587 & 9 & FCR & Quartzite & 1 & 7 \\
\hline Unit $086 \mathrm{NE}^{1} / 4$ & $40-50$ & 1746 & 731 & 2 & FCR & Fossilized Wood & 1 & 122.6 \\
\hline Unit $087 \mathrm{NW}^{1} / 4$ & $20-30$ & 1254 & 741 & 8 & FCR & Quartzite & 1 & 23.2 \\
\hline Unit $087 \mathrm{SE}^{1 / 4}$ & $20-30$ & 1256 & 742 & 6 & FCR & Silt-stone & 1 & 2.2 \\
\hline Unit $087 \mathrm{NW}^{1} / 4$ & $30-40$ & 1472 & 745 & 13 & FCR & Ironized Sandstone & 1 & 8 \\
\hline Unit $087 \mathrm{NW}^{1} / 4$ & $30-40$ & 1472 & 745 & 14 & FCR & Ironized Sandstone & 1 & 2 \\
\hline Unit $088 \mathrm{SE}^{1 / 4}$ & $30-40$ & 1479 & 793 & 18 & FCR & Quartzite & 1 & 11.4 \\
\hline
\end{tabular}


Geo-Marine, Inc.

GMI 22005.01.06.xx and 22005.00.09.xx, Data Recovery Excavations at Site 41PN175

FCR Analysis Data

\begin{tabular}{|c|c|c|c|c|c|c|c|c|}
\hline Unit Type & $\begin{array}{l}\text { Depth } \\
(\mathrm{cm})\end{array}$ & $\begin{array}{l}\text { Prov. } \\
\text { No. }\end{array}$ & $\begin{array}{l}\text { Lot } \\
\text { No. }\end{array}$ & $\begin{array}{c}\text { Artifact } \\
\text { No. }\end{array}$ & Class & Raw Material & Qty & $\begin{array}{l}\text { Weight } \\
\text { (in g) }\end{array}$ \\
\hline \multicolumn{9}{|c|}{ Block 4} \\
\hline Unit $093 \mathrm{NW}^{1} 1 / 4$ & $30-40$ & 1381 & 890 & 24 & FCR & Ironized Sandstone & 1 & 4.2 \\
\hline Unit $096 \mathrm{SW}^{1} / 4$ & $10-20$ & 1162 & 936 & 10 & FCR & Quartzite & 1 & 2.5 \\
\hline Unit 099 NE $1 / 4$ & $0-10$ & 1097 & 987 & 1 & FCR & Ironized Sandstone & 1 & 5 \\
\hline Unit $099 \mathrm{NE}^{1 / 4}$ & $20-26$ & 1300 & 995 & 4 & FCR & Ironized Sandstone & 1 & 2.7 \\
\hline Unit $100 \mathrm{NE}^{1 / 4}$ & $10-20$ & 1179 & 1004 & 14 & FCR & Ironized Sandstone & 1 & 1.4 \\
\hline Unit $100 \mathrm{NE}^{1 / 4}$ & $27-30$ & 1429 & 1012 & 9 & FCR & Quartzite & 1 & 1.7 \\
\hline Unit $102 \mathrm{NW}^{1} \frac{1}{4}$ & $10-20$ & 951 & 1044 & 5 & FCR & Chert & 1 & 2.9 \\
\hline Unit $104 \mathrm{SE}^{1 / 4} 4$ & $10-20$ & 1196 & 1080 & 10 & FCR & Ironized Sandstone & 1 & 3.1 \\
\hline Unit $105 \mathrm{SE} 1 / 4$ & $10-20$ & 1200 & 1099 & 11 & FCR & Ironized Sandstone & 1 & 1.8 \\
\hline Unit $106 \mathrm{NW}^{1} / 4$ & $10-20$ & 1203 & 1121 & 7 & FCR & Chert & 1 & 1.1 \\
\hline Unit $118 \mathrm{SW}^{1 / 4}$ & $9-19$ & 1512 & 1390 & 5 & FCR & Chert & 1 & 4.9 \\
\hline Unit $119 \mathrm{SE} 1 / 4$ & $9-19$ & 1585 & 1406 & 5 & FCR & Chert & 1 & 10.3 \\
\hline Unit $122 \mathrm{NW}^{1 / 4}$ & $14-24$ & 1572 & 1469 & 11 & FCR & Chert & 1 & 2.1 \\
\hline Unit $122 \mathrm{SW}^{1 / 4}$ & $24-34$ & 1647 & 1475 & 10 & $\mathrm{FCR}$ & Chert & 1 & 6.9 \\
\hline Unit $125 \mathrm{SE}^{1 / 4}$ & $15-20$ & 1569 & 1552 & 6 & $\mathrm{FCR}$ & Ironized Sandstone & 1 & 1.5 \\
\hline Unit $126 \mathrm{SE} 1 / 4$ & $12-22$ & 1697 & 1574 & 17 & FCR & Chert & 1 & 1 \\
\hline \multicolumn{9}{|c|}{ Block 5} \\
\hline Unit $080 \mathrm{NW}^{1} / 4$ & $32-40$ & 770 & 618 & 7 & FCR & Silt-stone & 1 & 3.4 \\
\hline Unit $082 \mathrm{SW}^{1 / 4}$ & $32-40$ & 830 & 657 & 1 & FCR & Chert & 1 & 3.9 \\
\hline \multicolumn{9}{|c|}{ Block 6} \\
\hline Unit $108 \mathrm{NW}^{1 / 4}$ & $25-35$ & 852 & 1155 & 8 & FCR & Chert & 1 & 4.3 \\
\hline \multicolumn{9}{|c|}{ Block 7} \\
\hline Unit $109 \mathrm{SW}^{1 / 4}$ & $30-38$ & 956 & 1178 & 35 & FCR & Chert & 1 & 4.2 \\
\hline \multicolumn{9}{|c|}{ Block 8} \\
\hline Unit $114 \mathrm{SE} 1 / 4$ & $10-20$ & 1013 & 1277 & 6 & FCR & Ironized Sandstone & 1 & 3 \\
\hline Unit $114 \mathrm{NE}^{1 / 4}$ & $20-30$ & 1016 & 1279 & 10 & FCR & Ironized Sandstone & 1 & 1.6 \\
\hline Unit $114 \mathrm{SW}^{1 / 4}$ & $20-30$ & 1014 & 1282 & 11 & FCR & Quartzite & 1 & 5.4 \\
\hline Unit $116 \mathrm{SE}^{1 / 4}$ & $50-61$ & 1229 & 1344 & 4 & FCR & Chert & 1 & 0.1 \\
\hline
\end{tabular}


Geo-Marine, Inc.

GMI 22005.01.06.xx and 22005.00.09.xx, Data Recovery Excavations at Site 41PN175

FCR Analysis Data

\begin{tabular}{|c|c|c|c|c|c|c|c|c|}
\hline Unit Type & $\begin{array}{l}\text { Depth } \\
(\mathrm{cm})\end{array}$ & $\begin{array}{l}\text { Prov. } \\
\text { No. }\end{array}$ & $\begin{array}{l}\text { Lot } \\
\text { No. }\end{array}$ & $\begin{array}{l}\text { Artifact } \\
\text { No. }\end{array}$ & Class & Raw Material & Qty & $\begin{array}{l}\text { Weight } \\
\text { (in g) }\end{array}$ \\
\hline \multicolumn{9}{|l|}{ Block 8} \\
\hline Unit $117 \mathrm{SE}^{1 / 4}$ & $25-30$ & 1343 & 1364 & 17 & FCR & Ironized Sandstone & 1 & 5.4 \\
\hline Unit $117 \mathrm{NE}^{1 / 4}$ & $30-35$ & 1346 & 1366 & 16 & FCR & Chert & 1 & 1.3 \\
\hline Unit $117 \mathrm{NE}^{1 / 4}$ & $30-35$ & 1346 & 1366 & 17 & FCR & Ironized Sandstone & 1 & 13.3 \\
\hline Unit $117 \mathrm{NW}^{1 / 4}$ & $30-35$ & 1345 & 1367 & 33 & FCR & Chert & 1 & 2 \\
\hline Unit $117 \mathrm{NW}^{1 / 4}$ & $30-35$ & 1345 & 1367 & 35 & FCR & Ironized Sandstone & 1 & 119.4 \\
\hline Unit $117 \mathrm{SE}^{1 / 4}$ & $30-35$ & 1347 & 1368 & 27 & FCR & Chert & 1 & 4.5 \\
\hline \multicolumn{9}{|l|}{ Features } \\
\hline $\begin{array}{l}\text { Unit } 087 \text {, Feature } 7 \\
\mathrm{NE}^{1 / 2}\end{array}$ & $40-53$ & 1800 & 1603 & 33 & FCR & Chert & 1 & 0.4 \\
\hline $\begin{array}{l}\text { Unit } 087 \text {, Feature } 7 \\
\mathrm{NE}^{1 / 2}\end{array}$ & $40-53$ & 1800 & 1603 & 34 & FCR & Ironized Sandstone & 1 & 11.5 \\
\hline $\begin{array}{l}\text { Unit } 087 \text {, Feature } 7 \\
\mathrm{NE}^{1 / 2}\end{array}$ & $40-53$ & 1800 & 1603 & 35 & FCR & $\begin{array}{l}\text { Unidentified Metamorphic- } \\
\text { ironstone }\end{array}$ & 1 & 9.4 \\
\hline $\begin{array}{l}\text { Unit } 087 \text {, Feature } 7 \\
\mathrm{NE}^{1 / 2}\end{array}$ & $40-53$ & 1800 & 1603 & 36 & FCR & $\begin{array}{l}\text { Unidentified Metamorphic- } \\
\text { ironstone }\end{array}$ & 1 & 1.2 \\
\hline $\begin{array}{l}\text { Unit } 120 \text {, Feature } 29 \\
\mathrm{~N}^{1 / 2}\end{array}$ & $41-55$ & 1840 & 1627 & 21 & FCR & Silt-stone & 1 & 4.8 \\
\hline $\begin{array}{l}\text { Unit } 076 \text {, Feature } 9 \\
\mathrm{~W}^{1 / 2}\end{array}$ & $40-53$ & 2002 & 1766 & 12 & FCR & Quartzite & 1 & 74.7 \\
\hline $\begin{array}{l}\text { Unit } 120 \mathrm{NW}^{1} 1 / 4, \\
\text { Feature } 29 \mathrm{~N}^{1} 1 / 2\end{array}$ & $48-48$ & 2006 & 1767 & 1 & FCR & Chert & 1 & 162.6 \\
\hline
\end{tabular}



A-9: ECOFACTS 



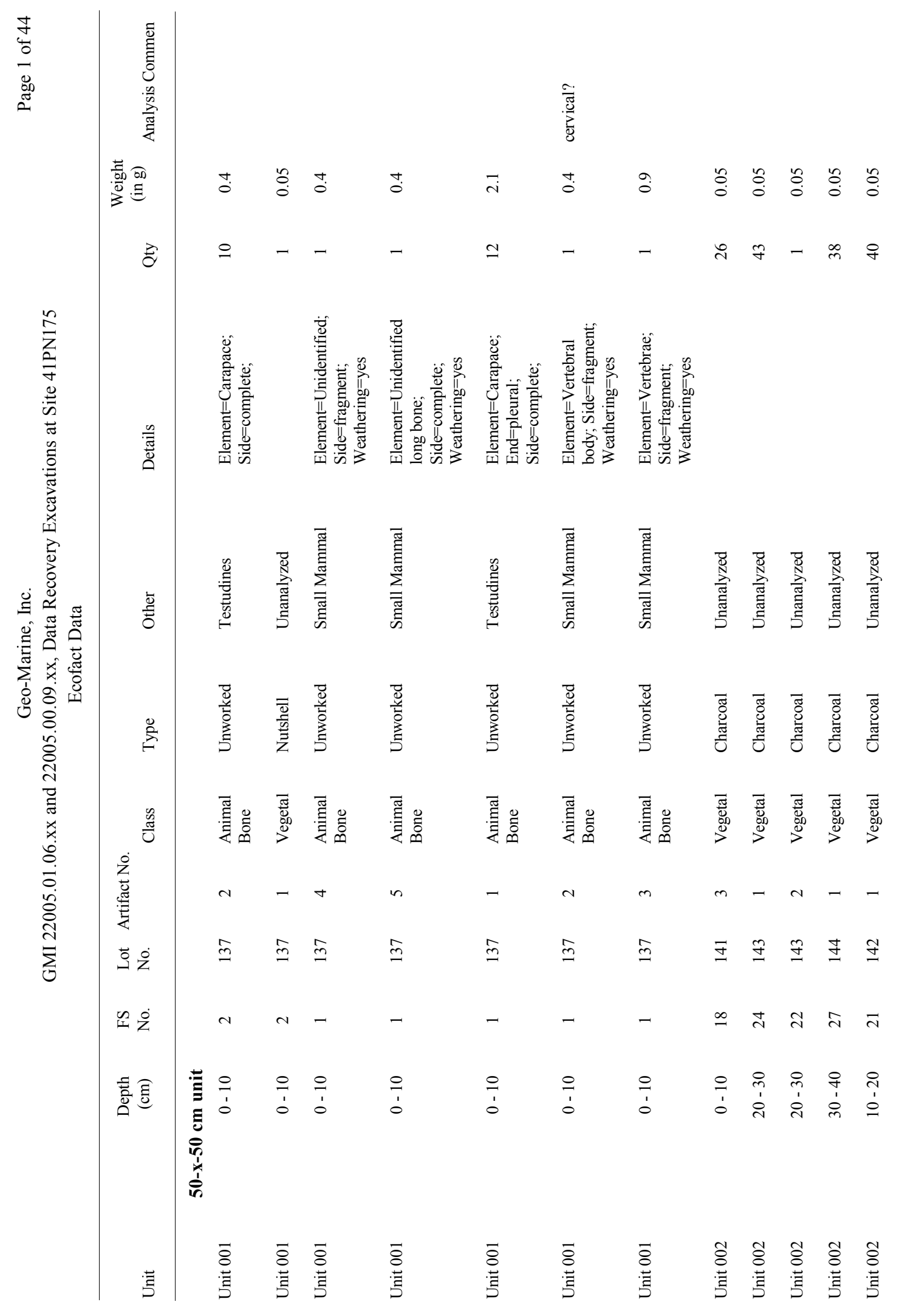









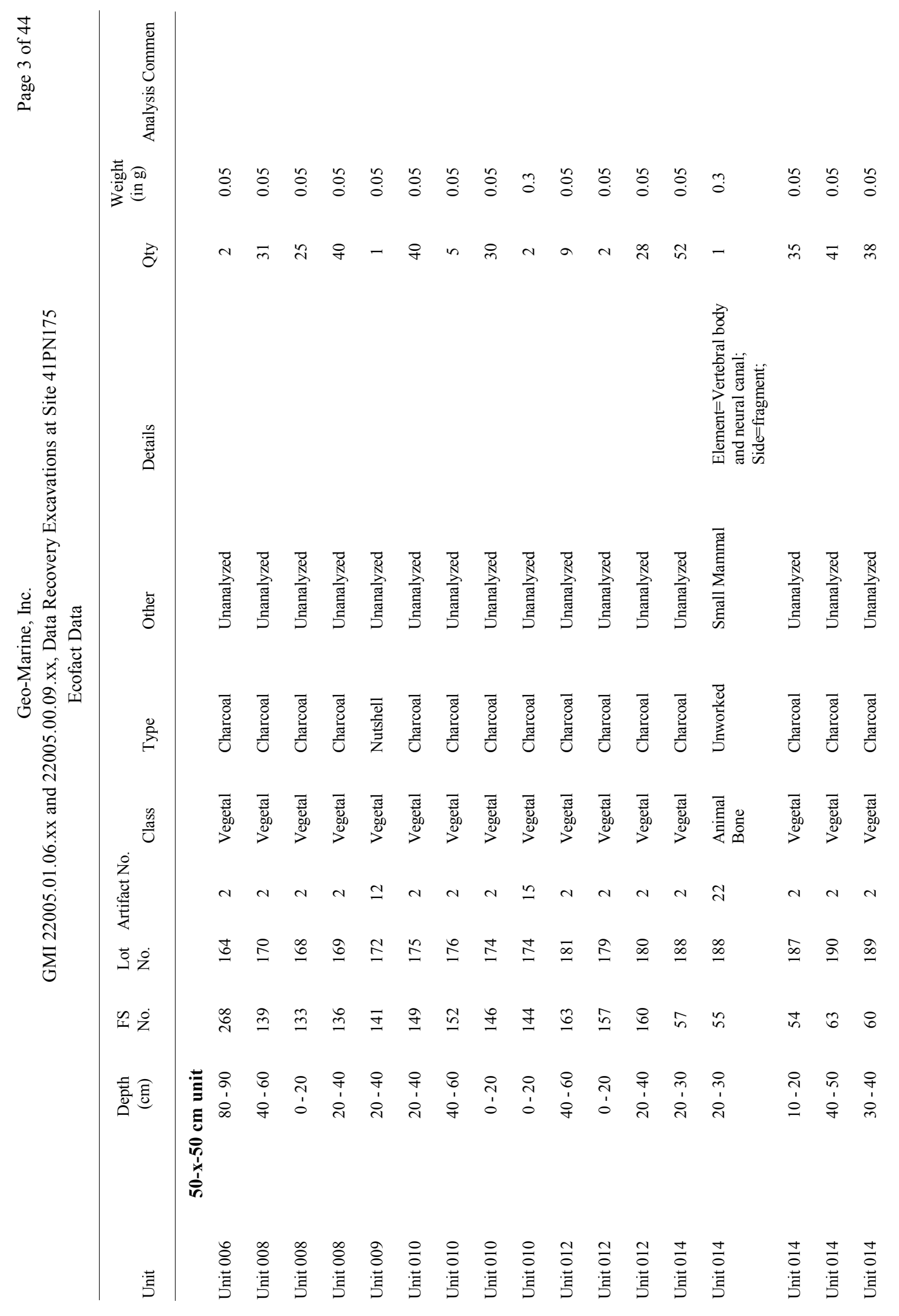




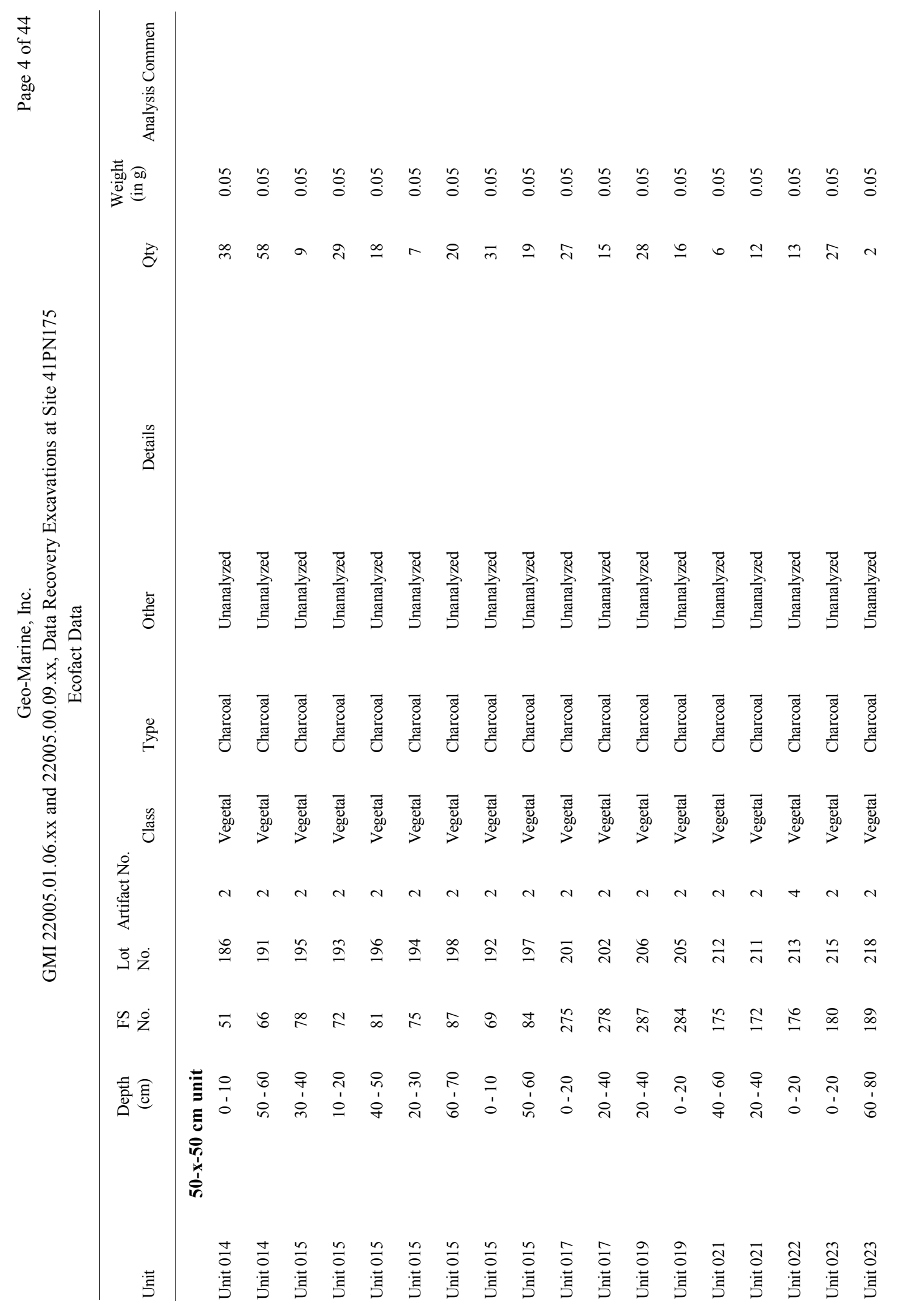




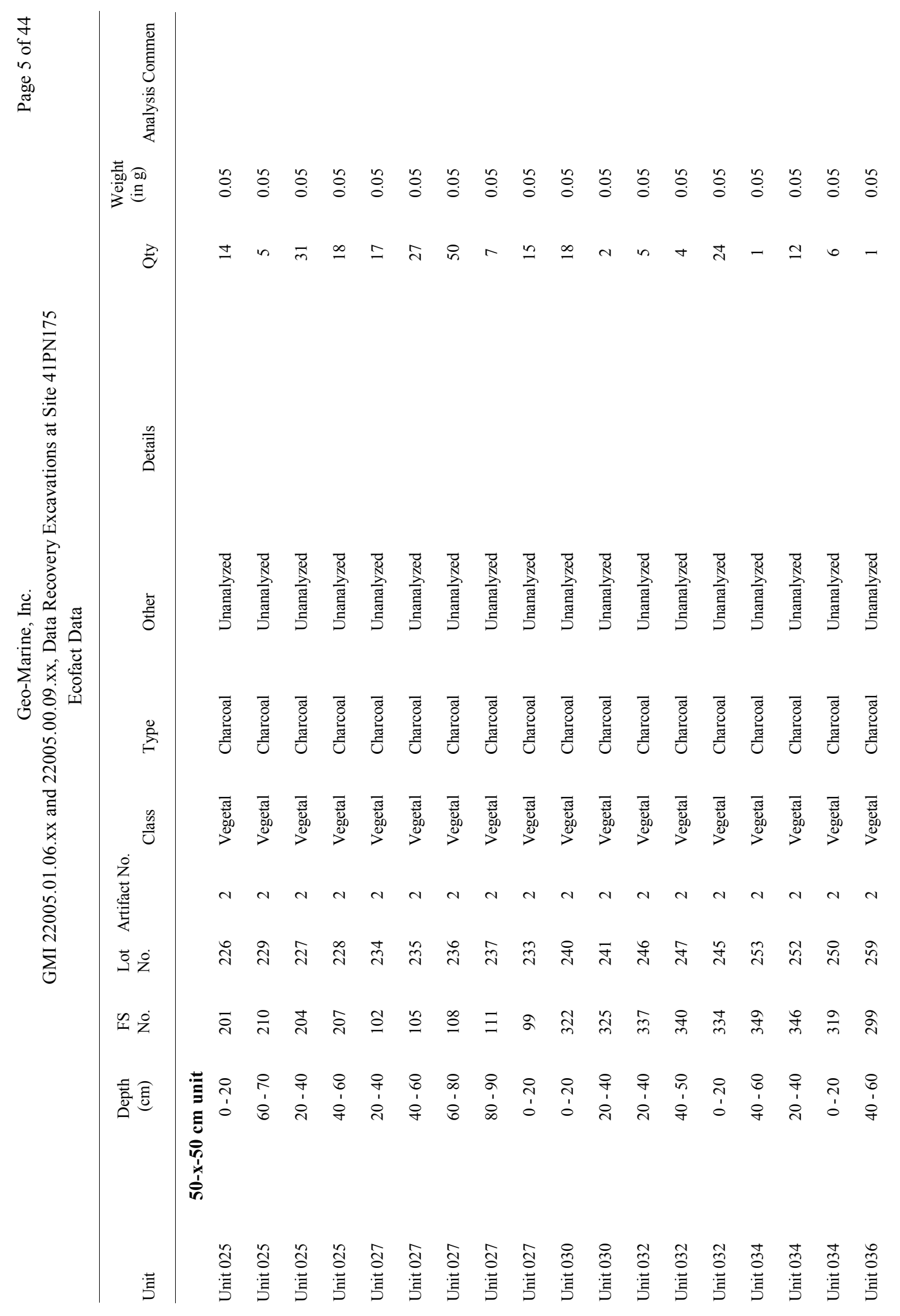









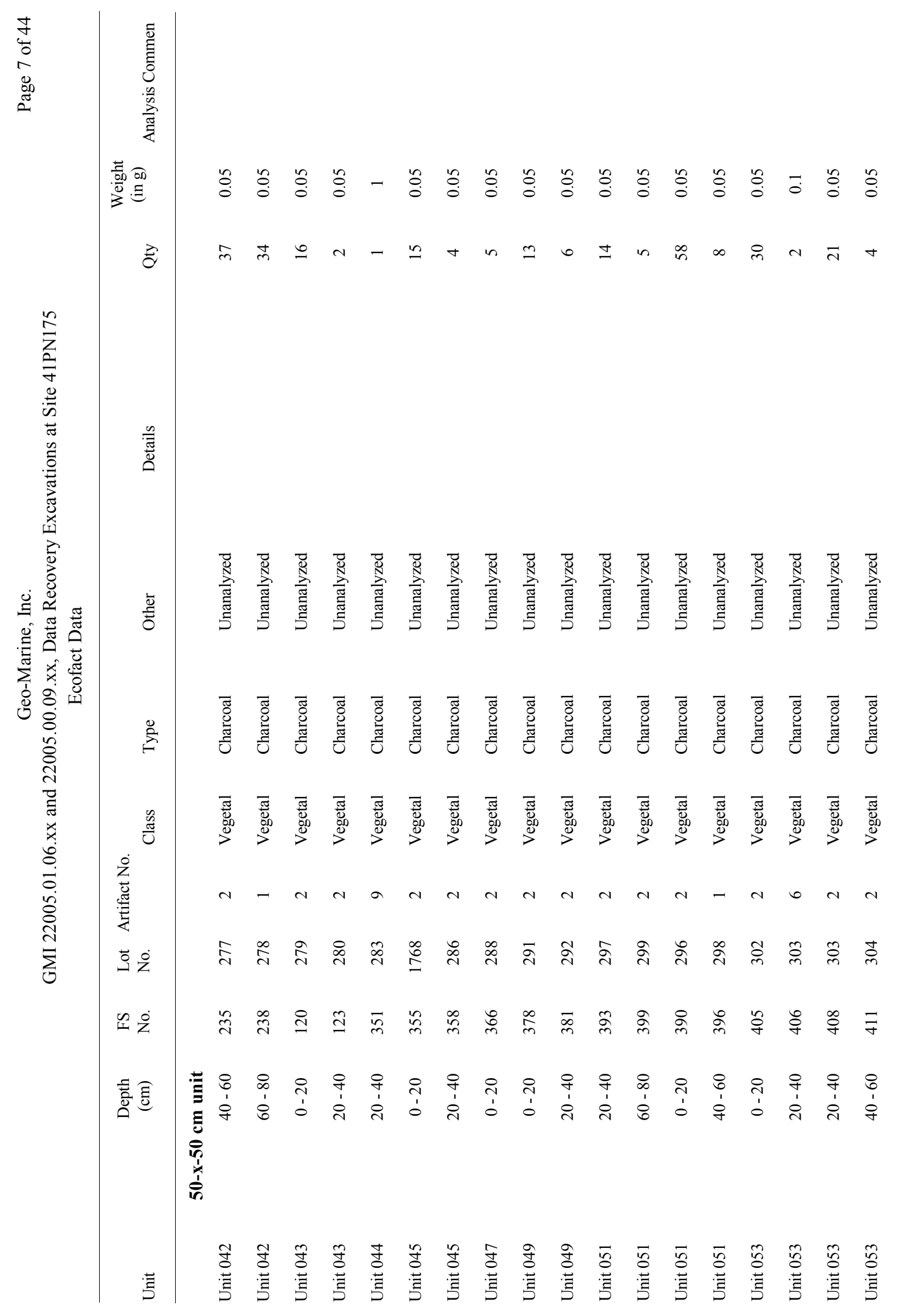




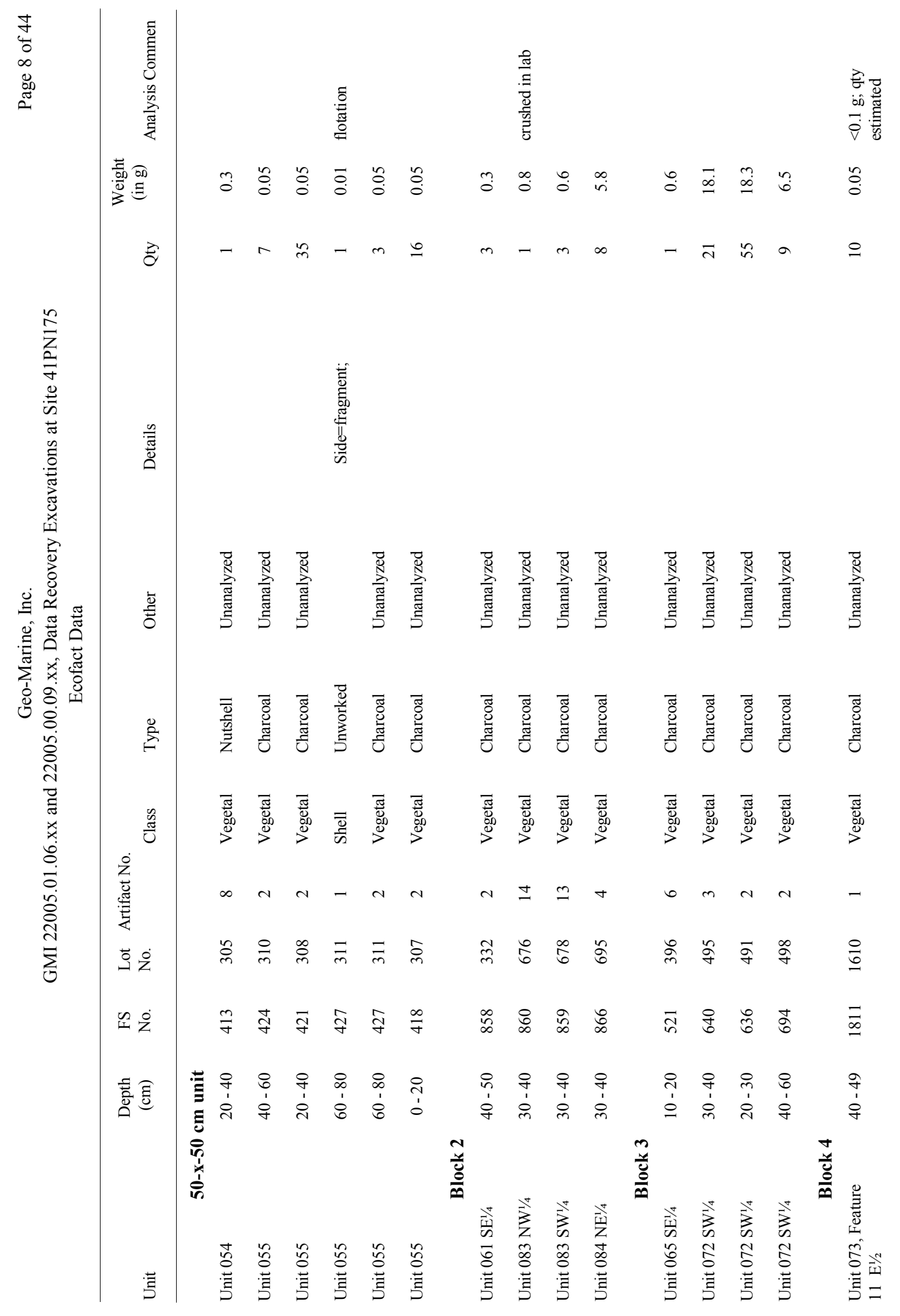




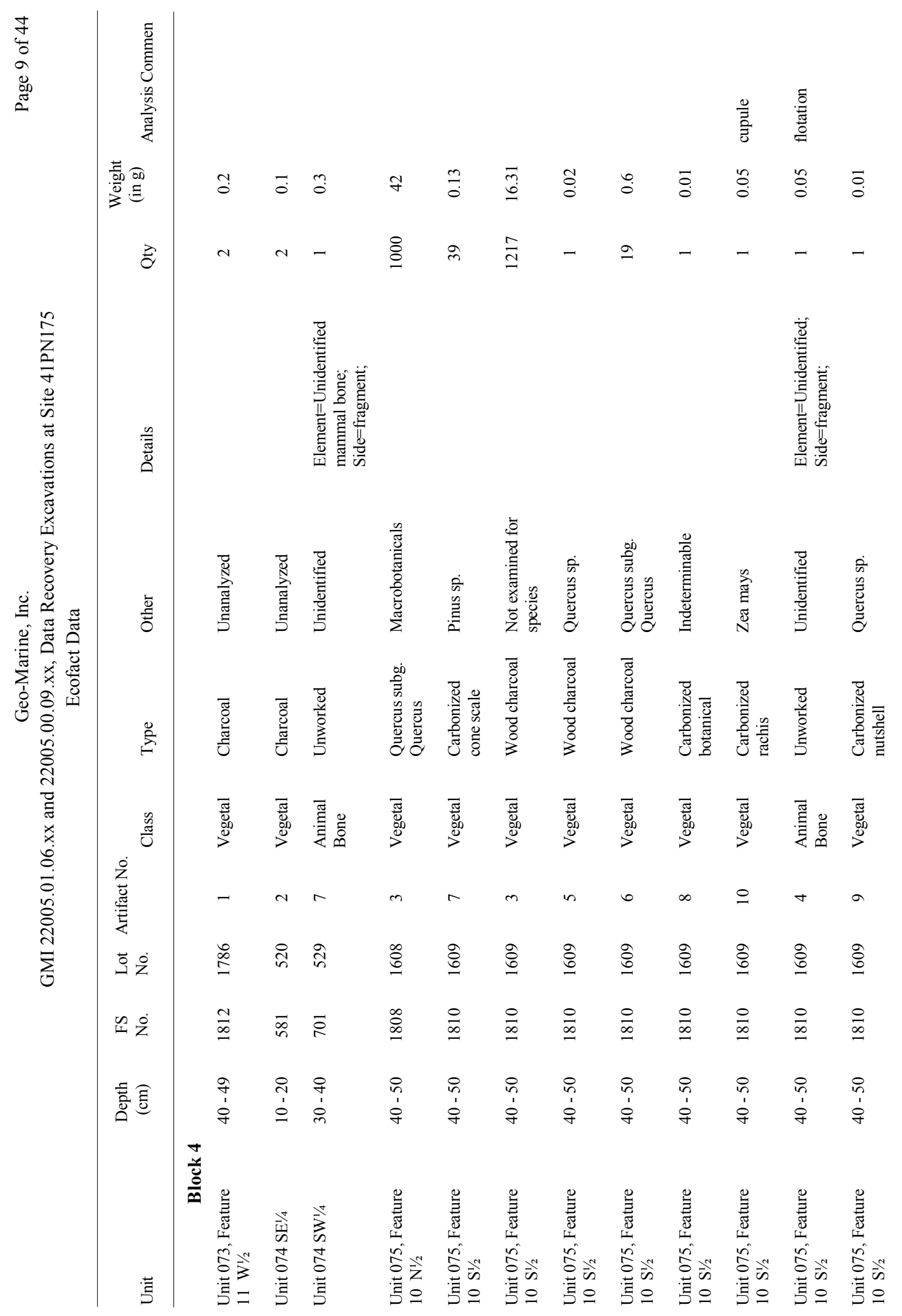









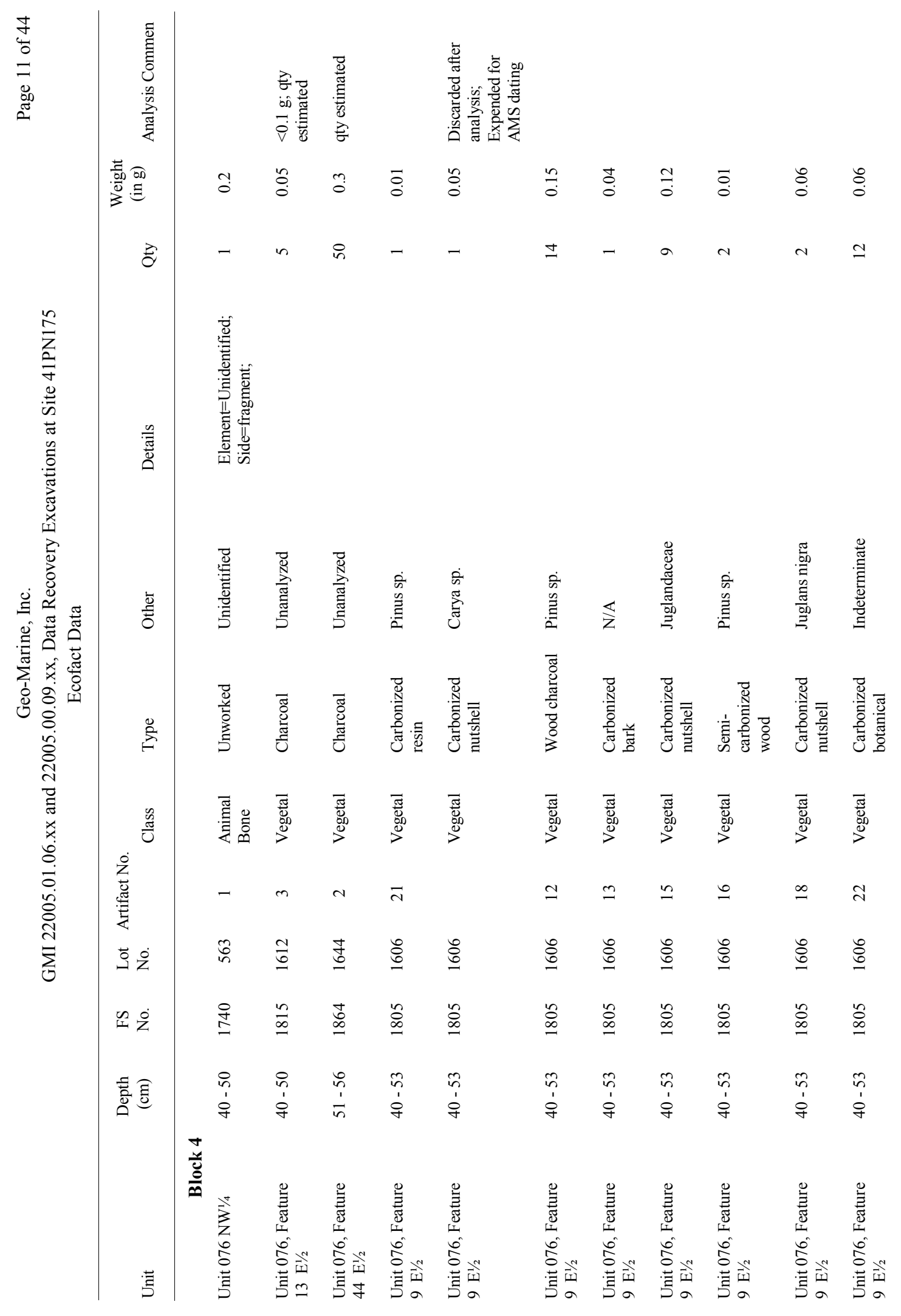




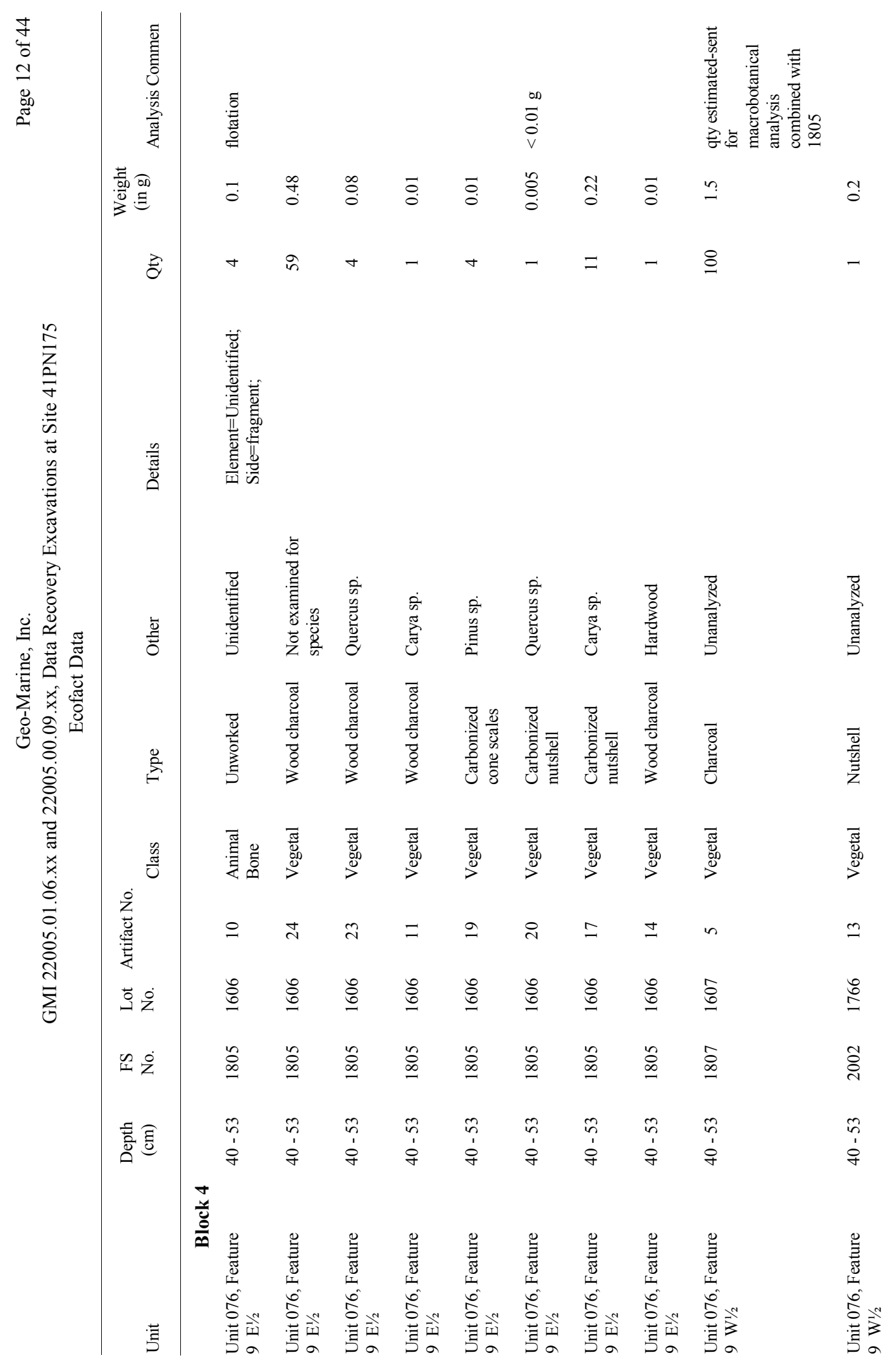




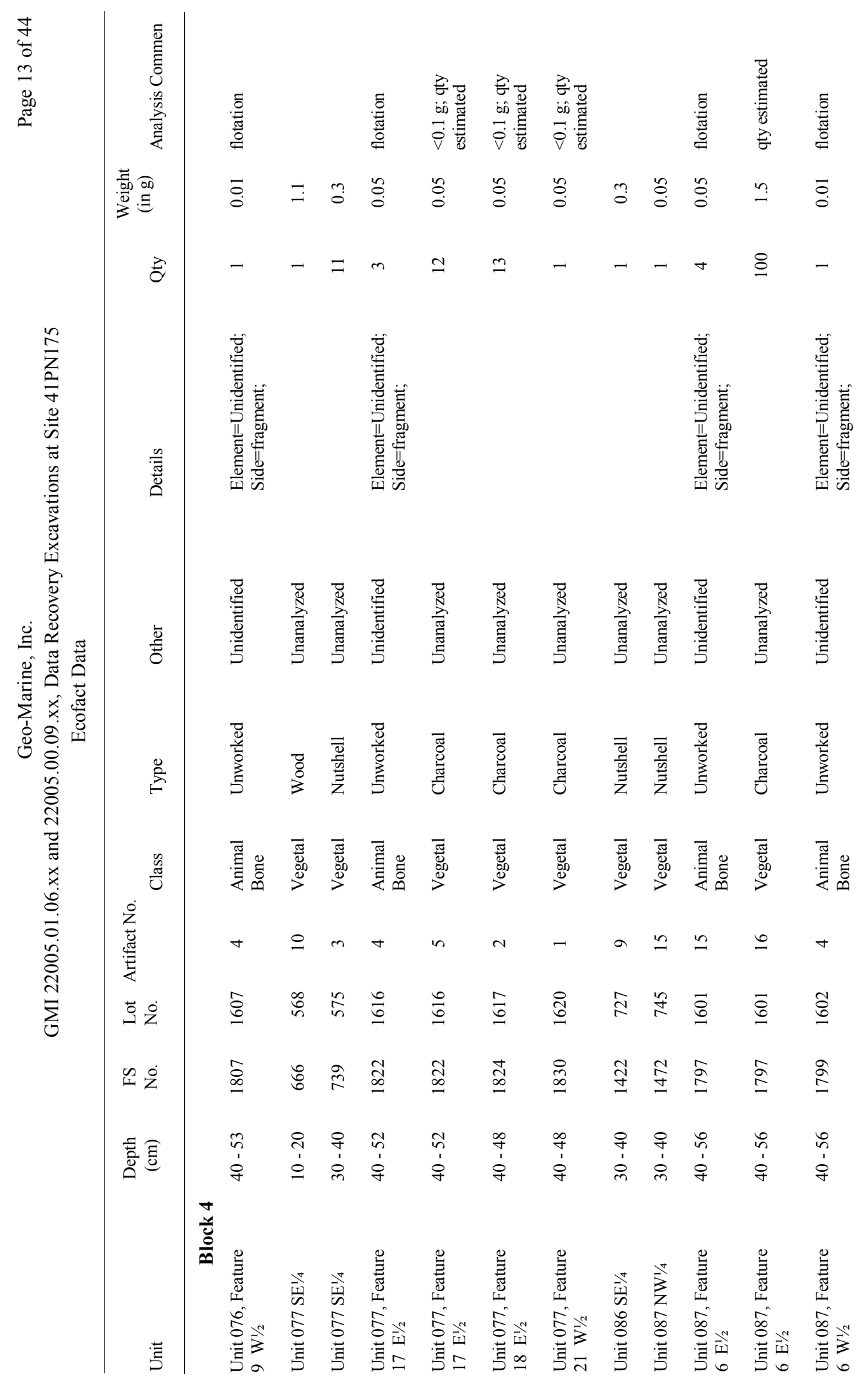




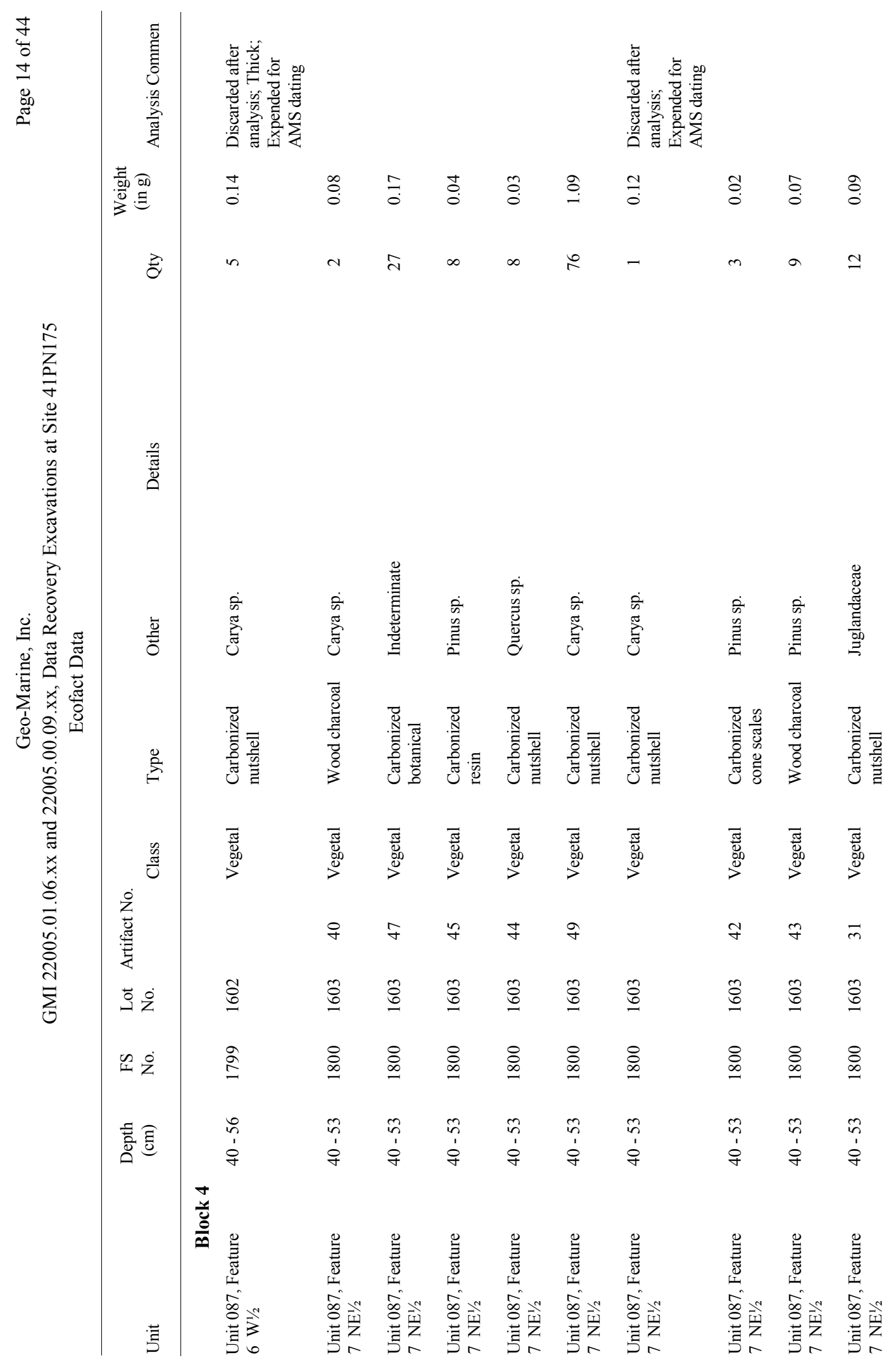




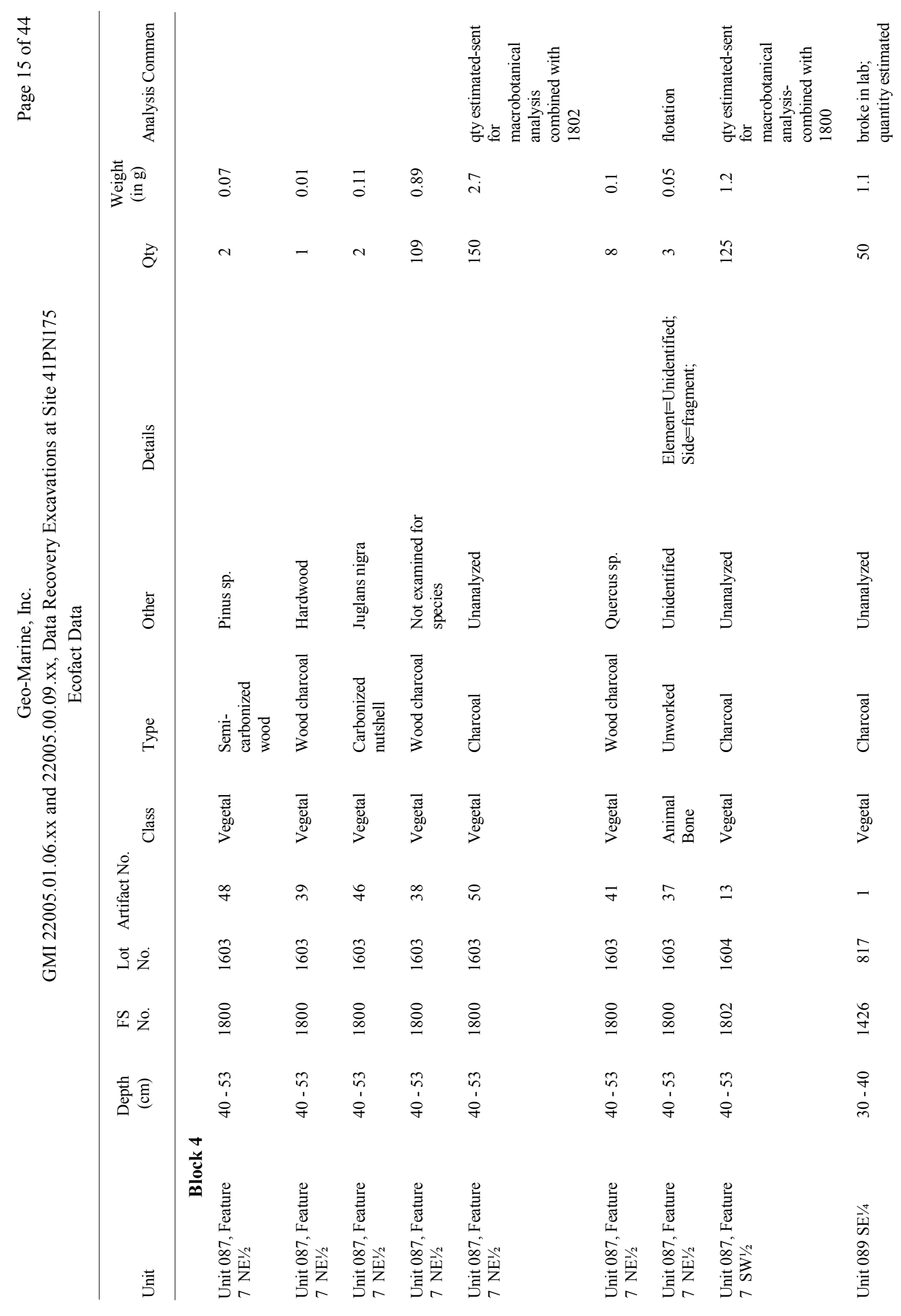




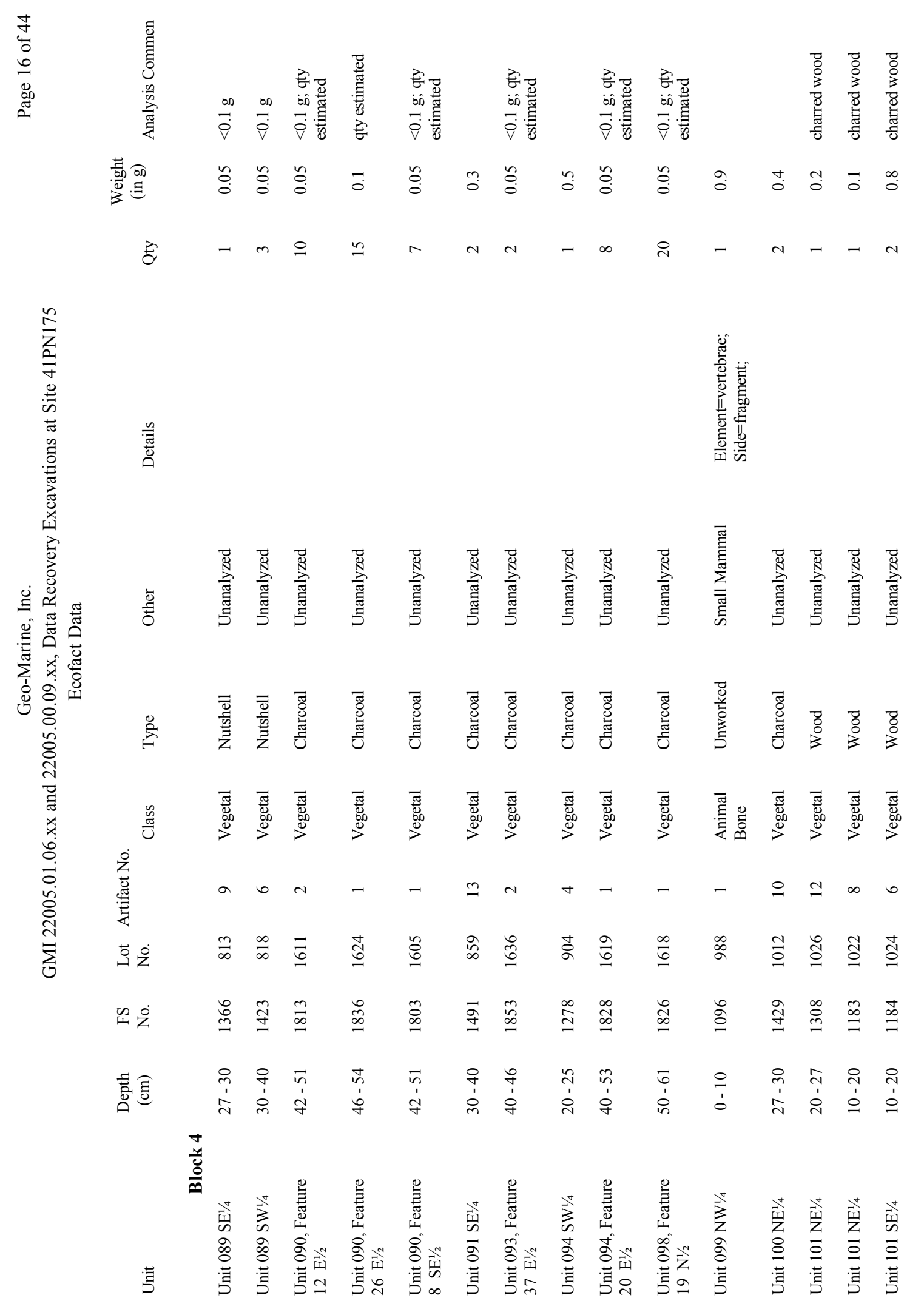




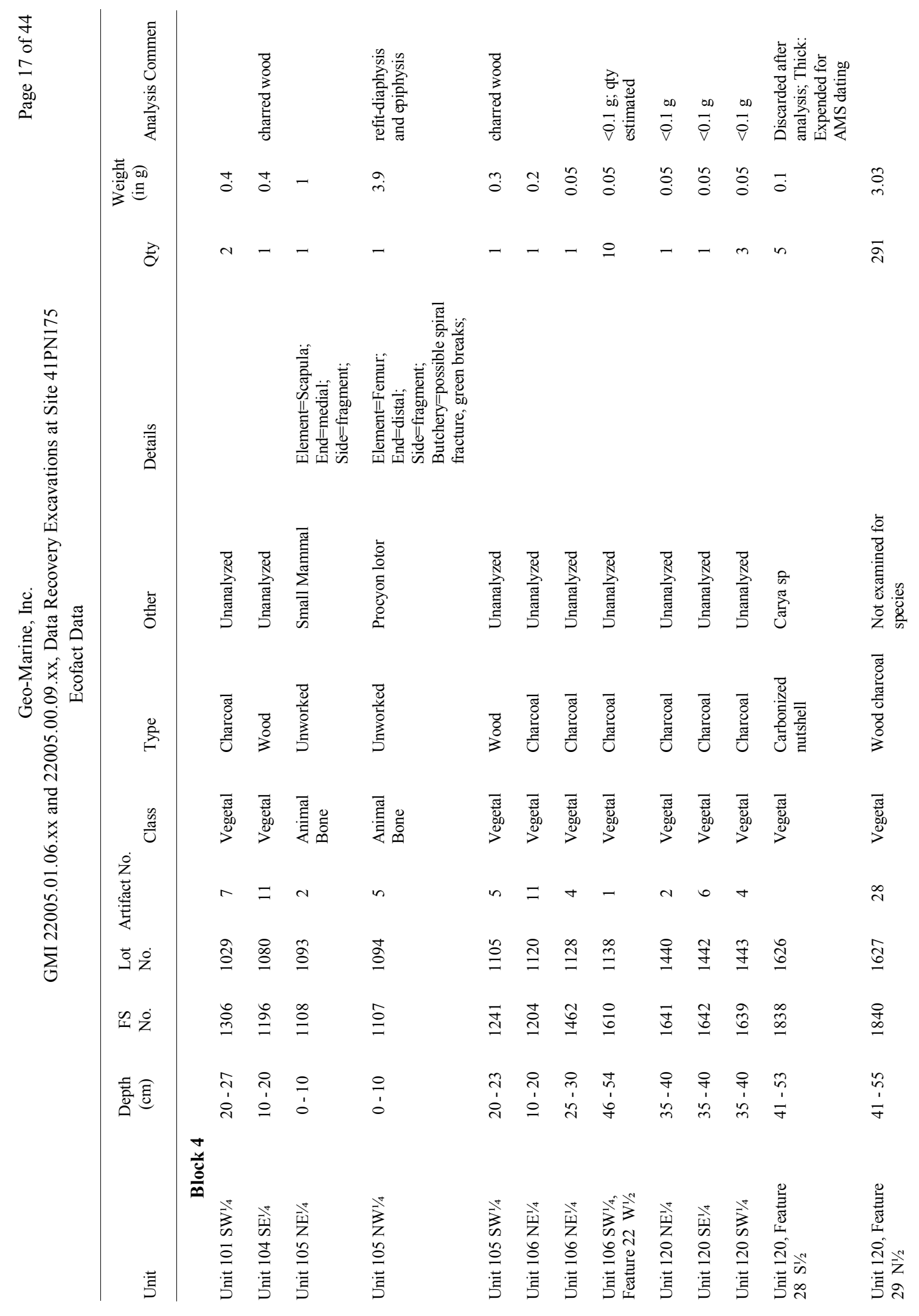




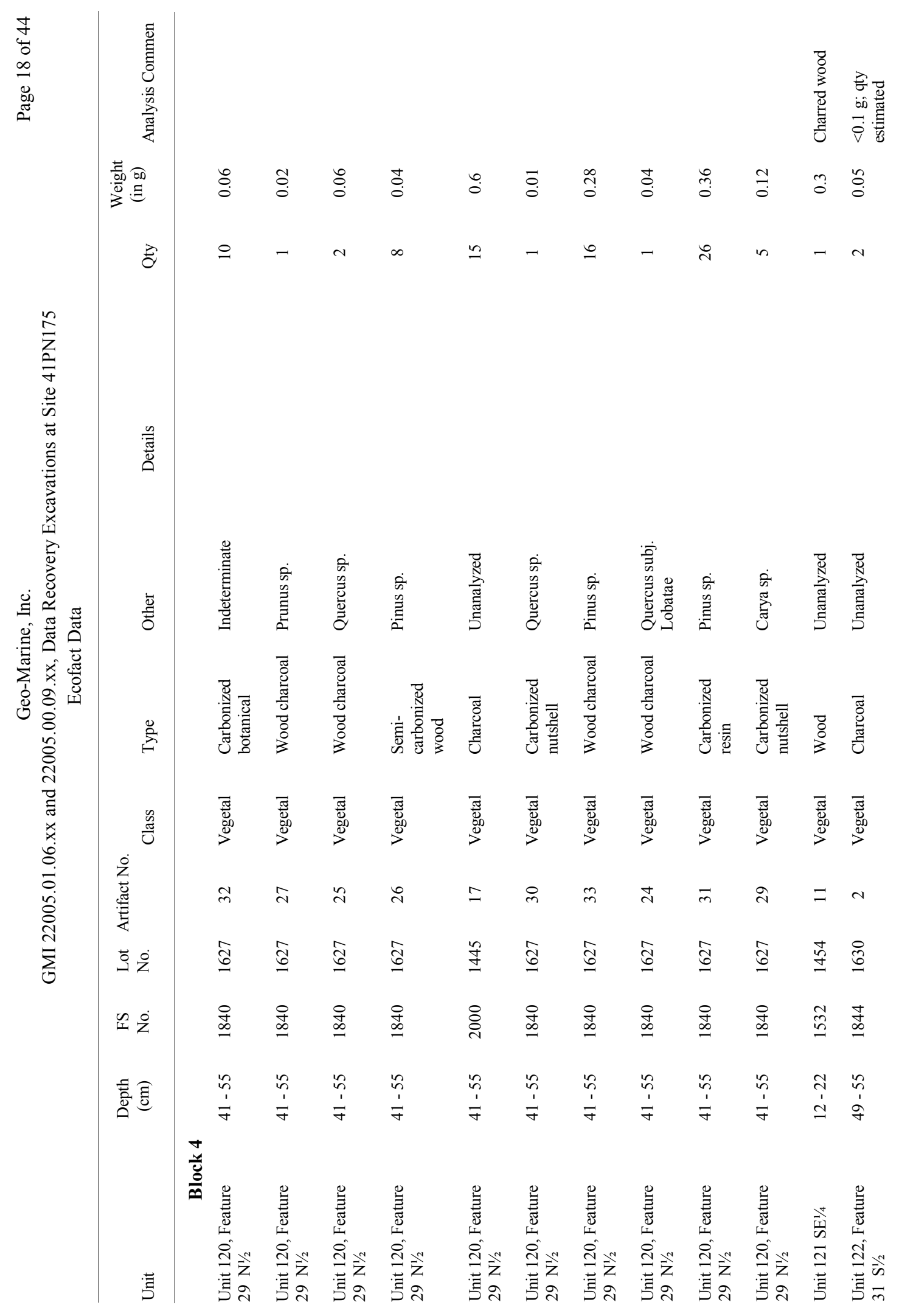




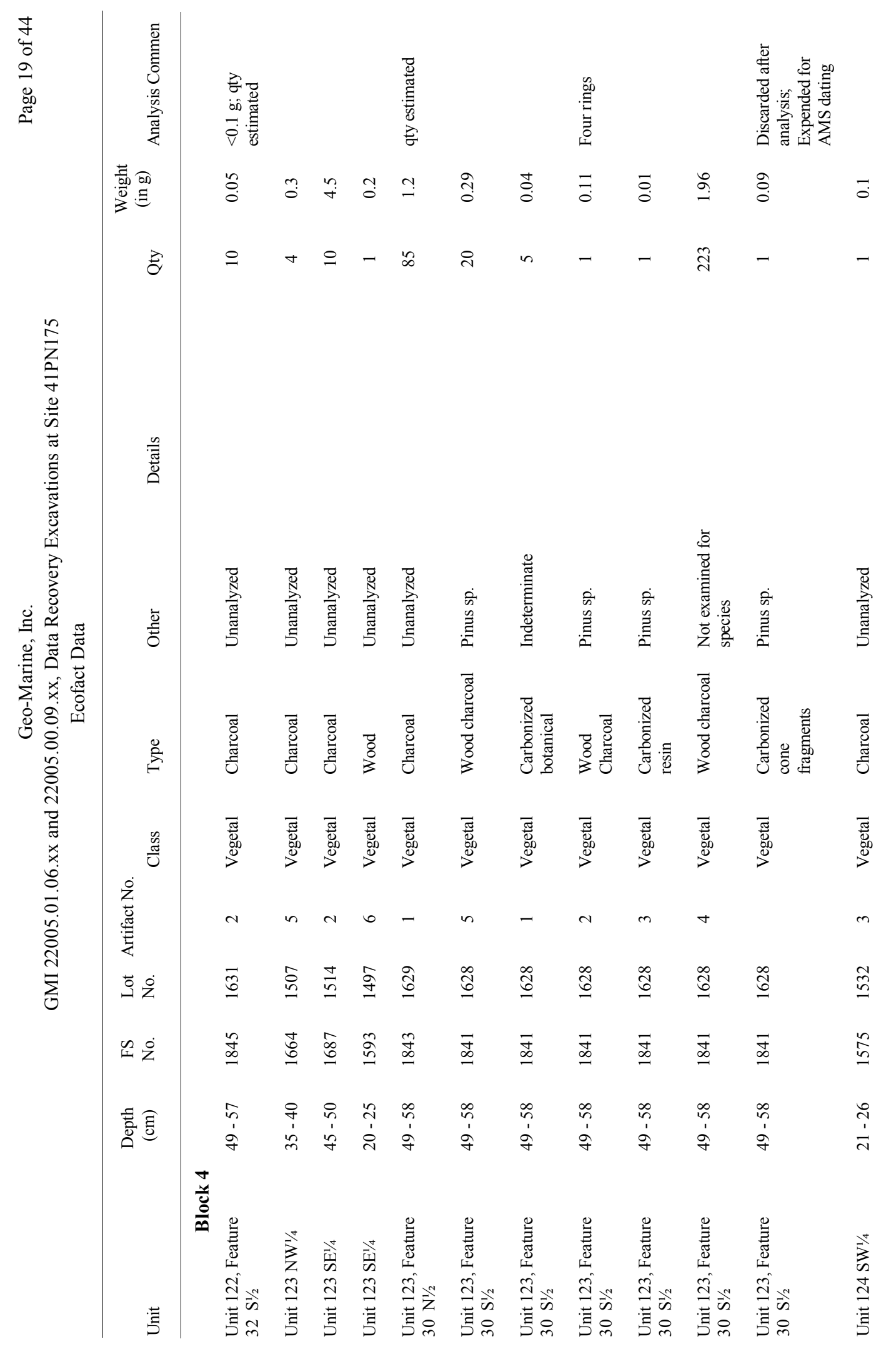




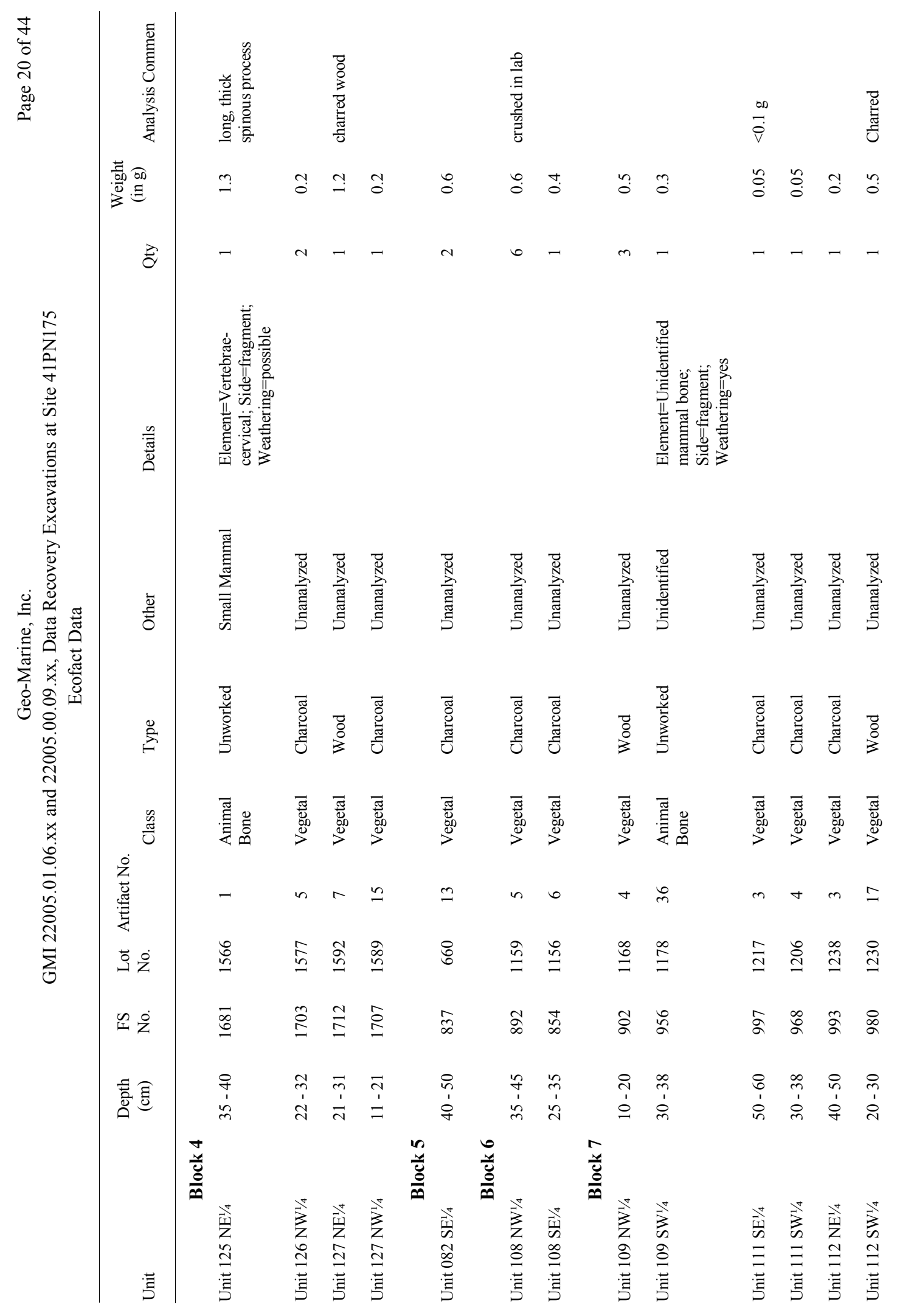




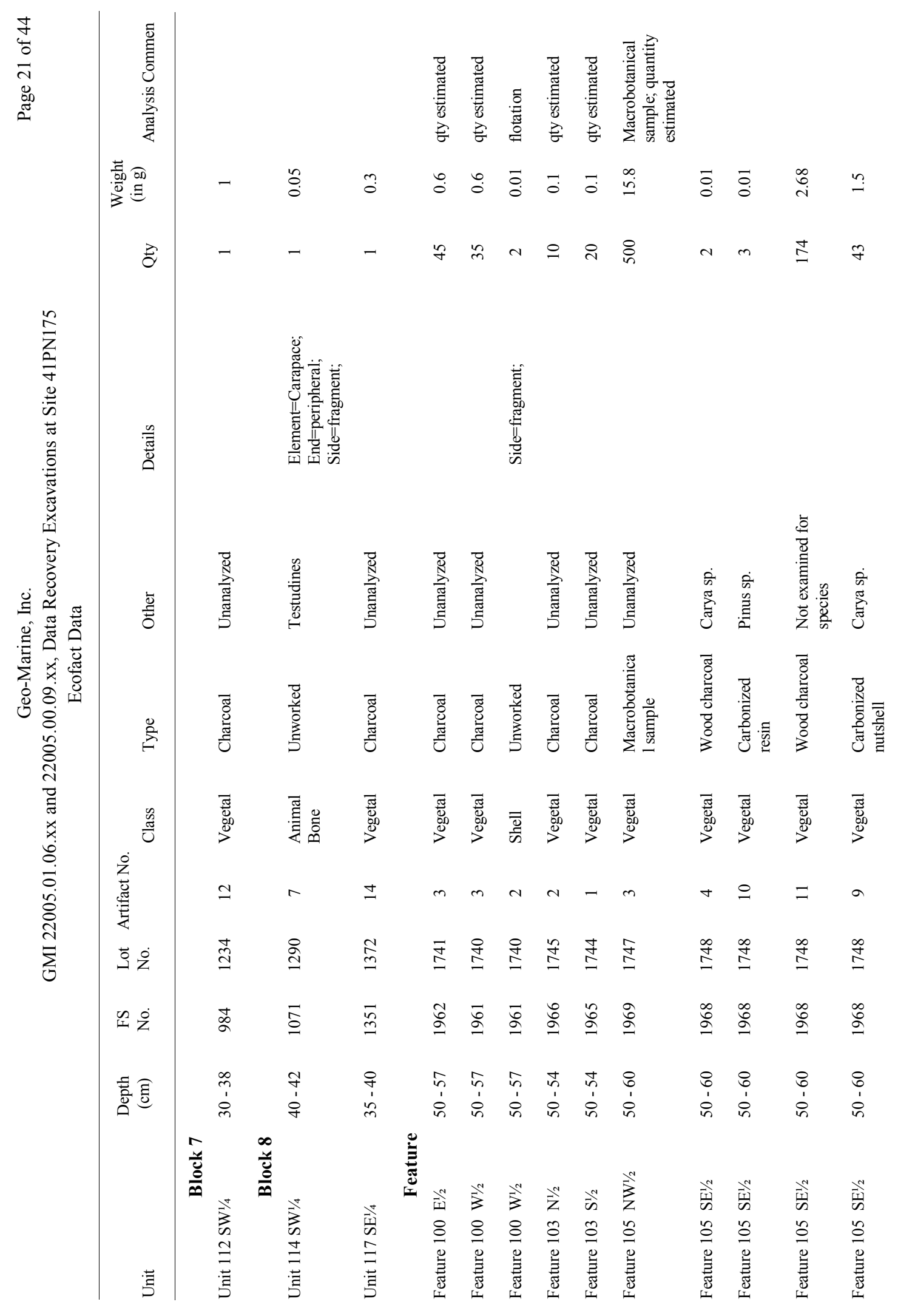




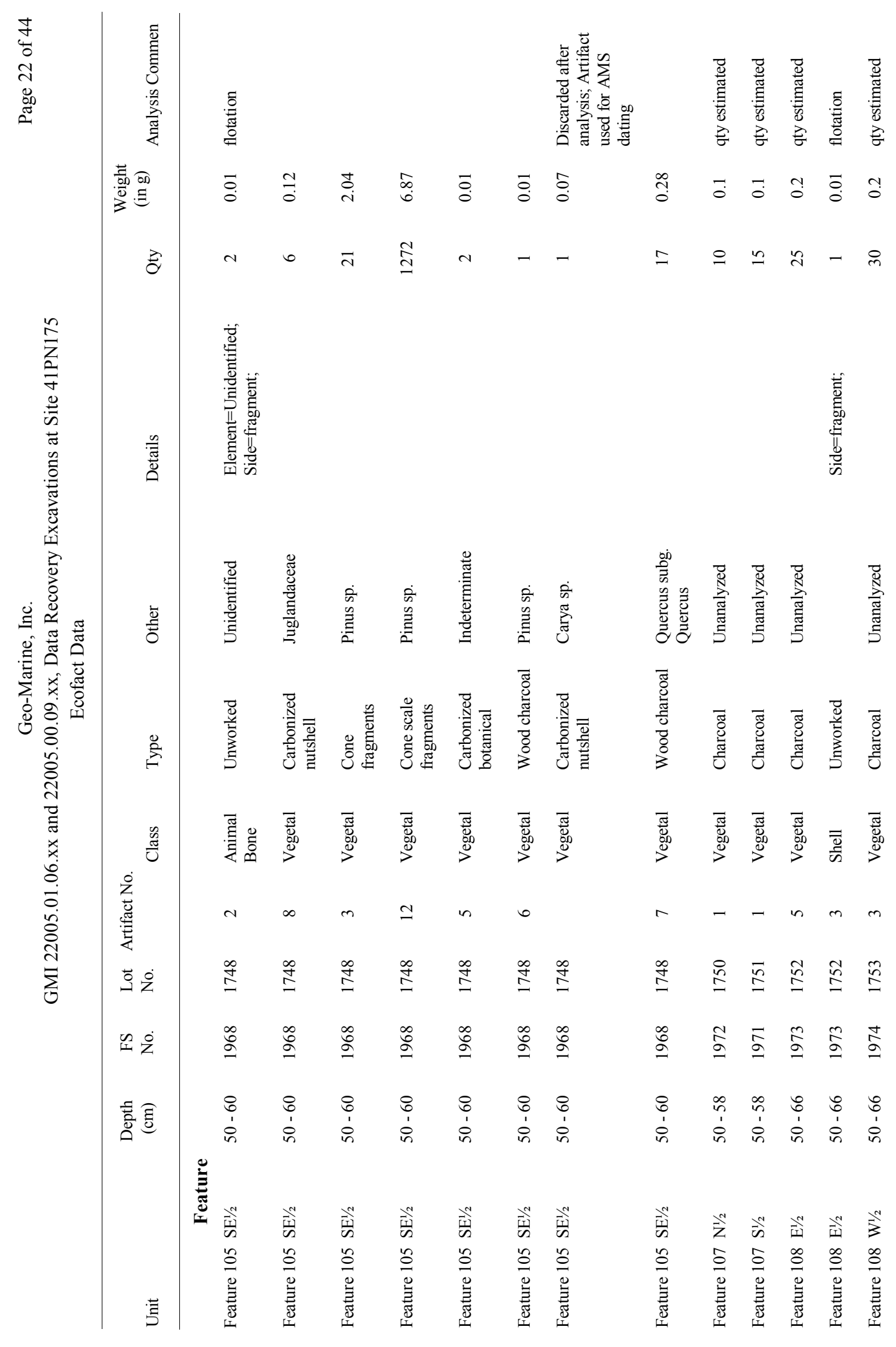




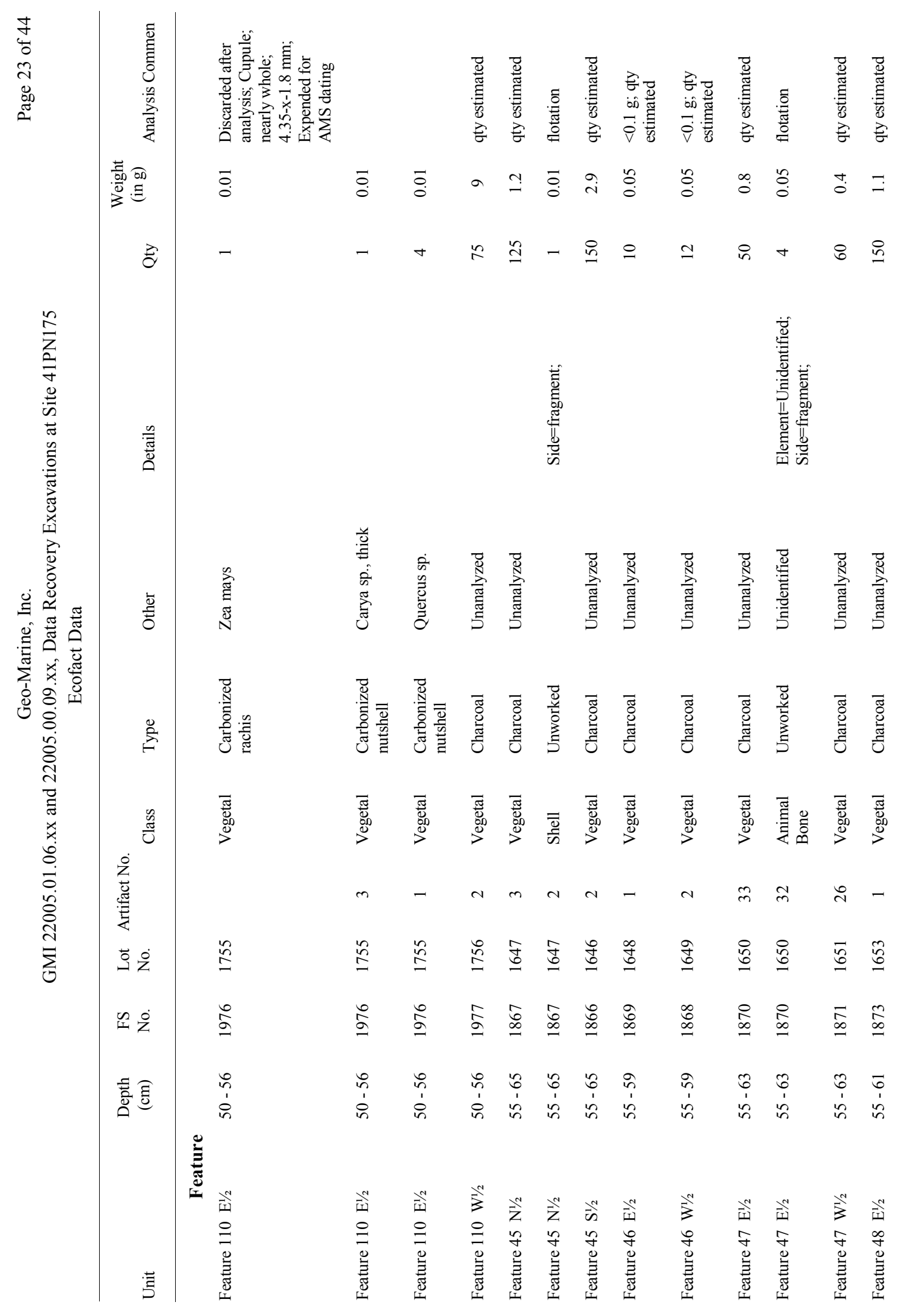




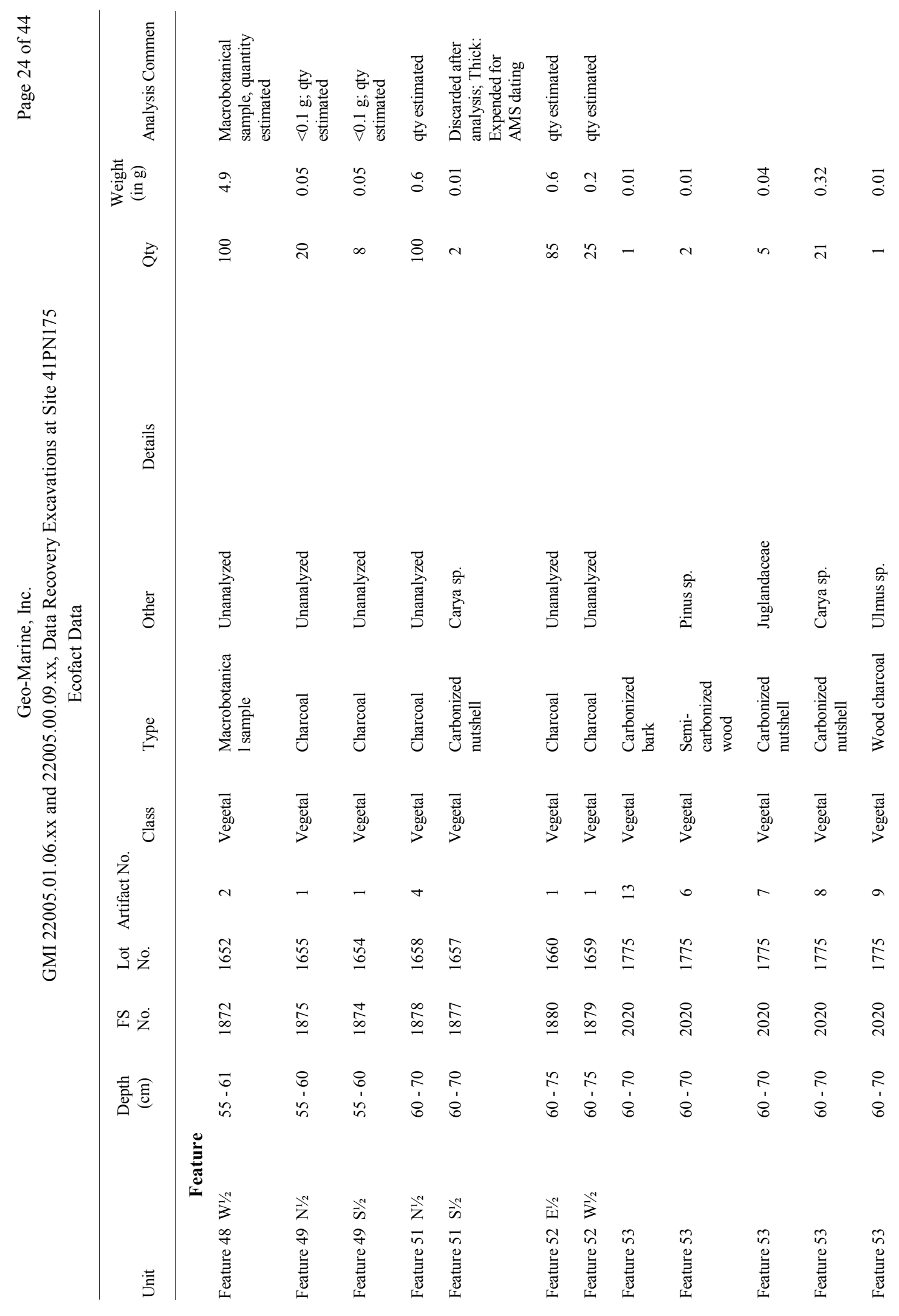




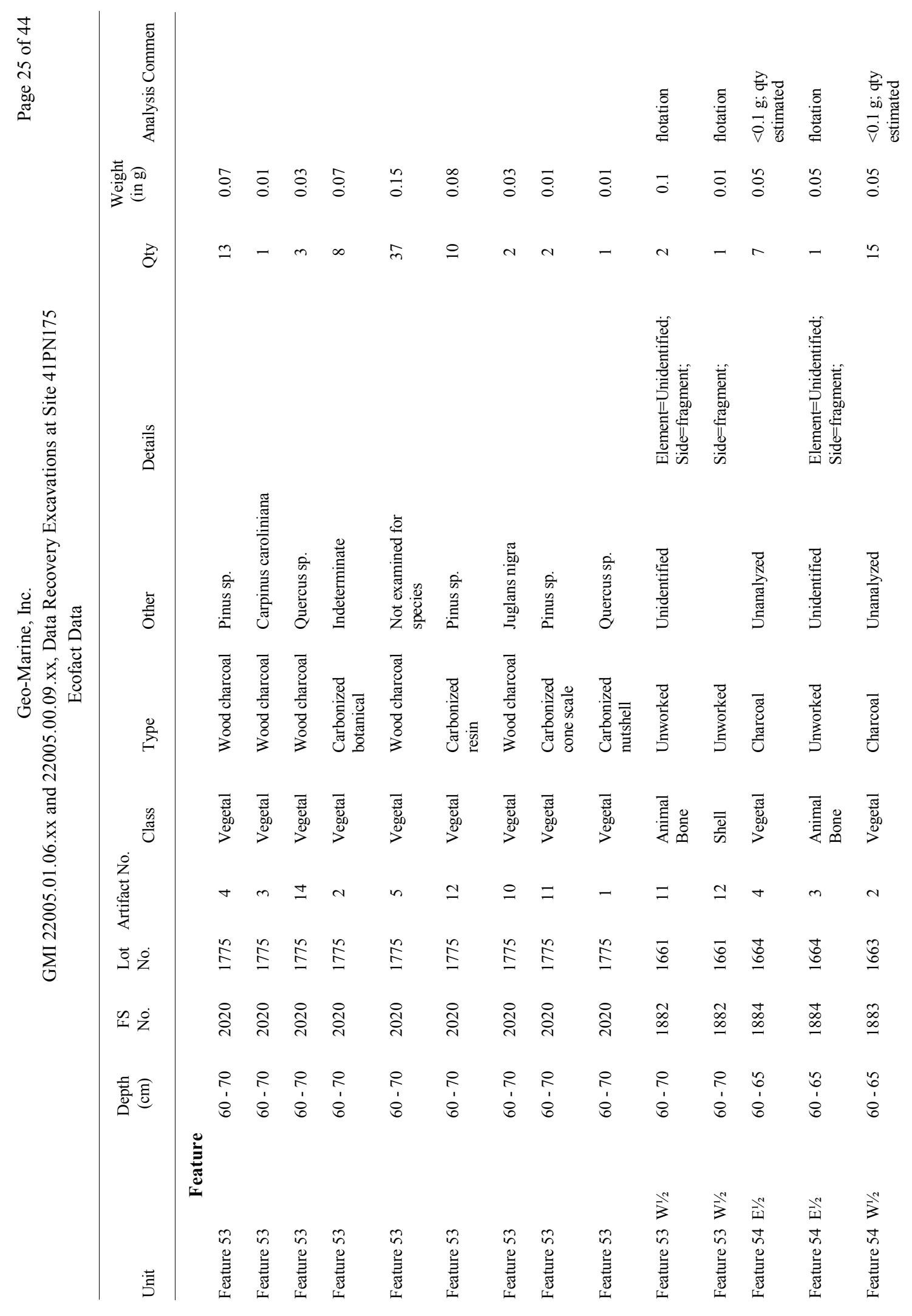




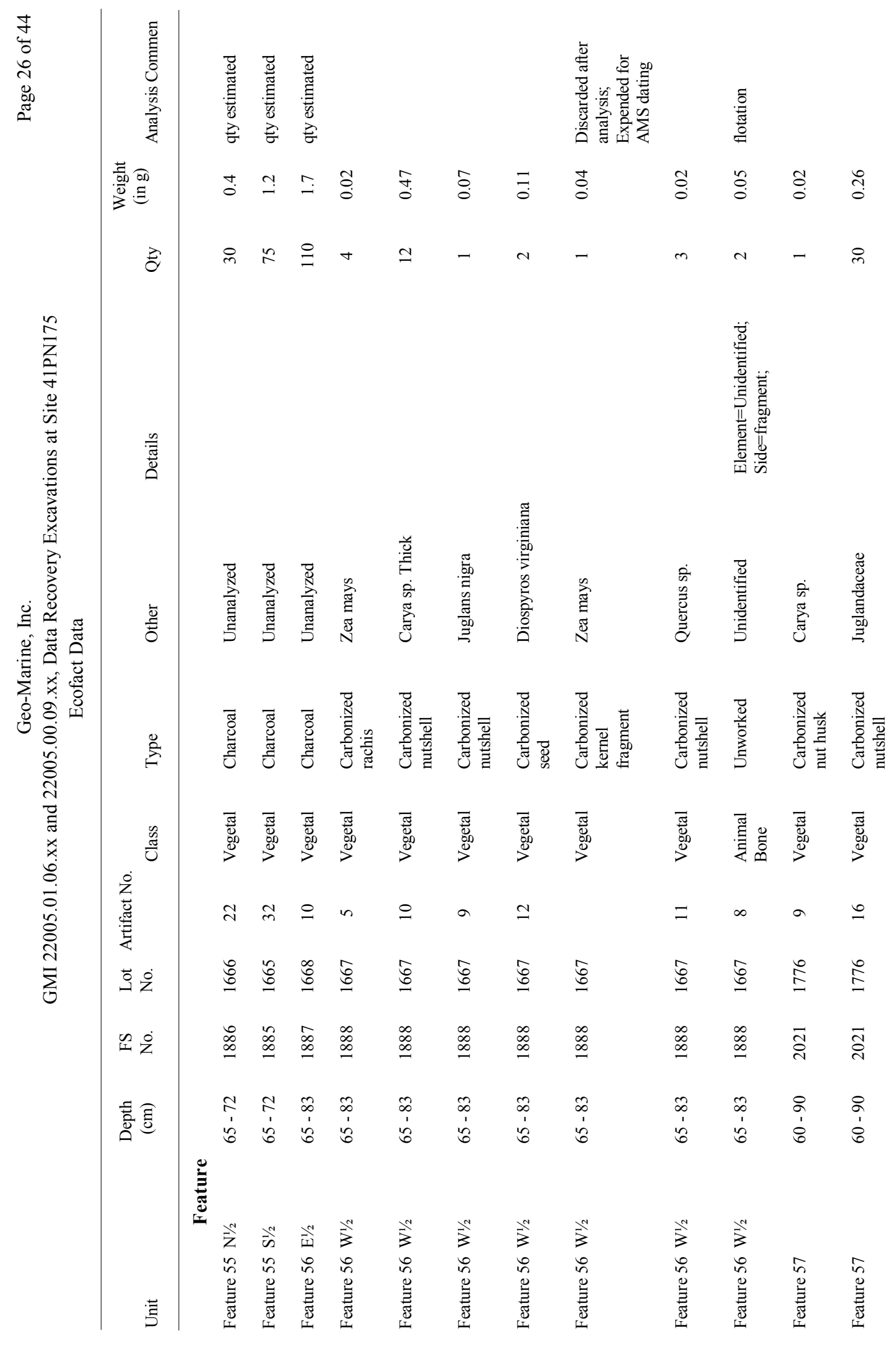




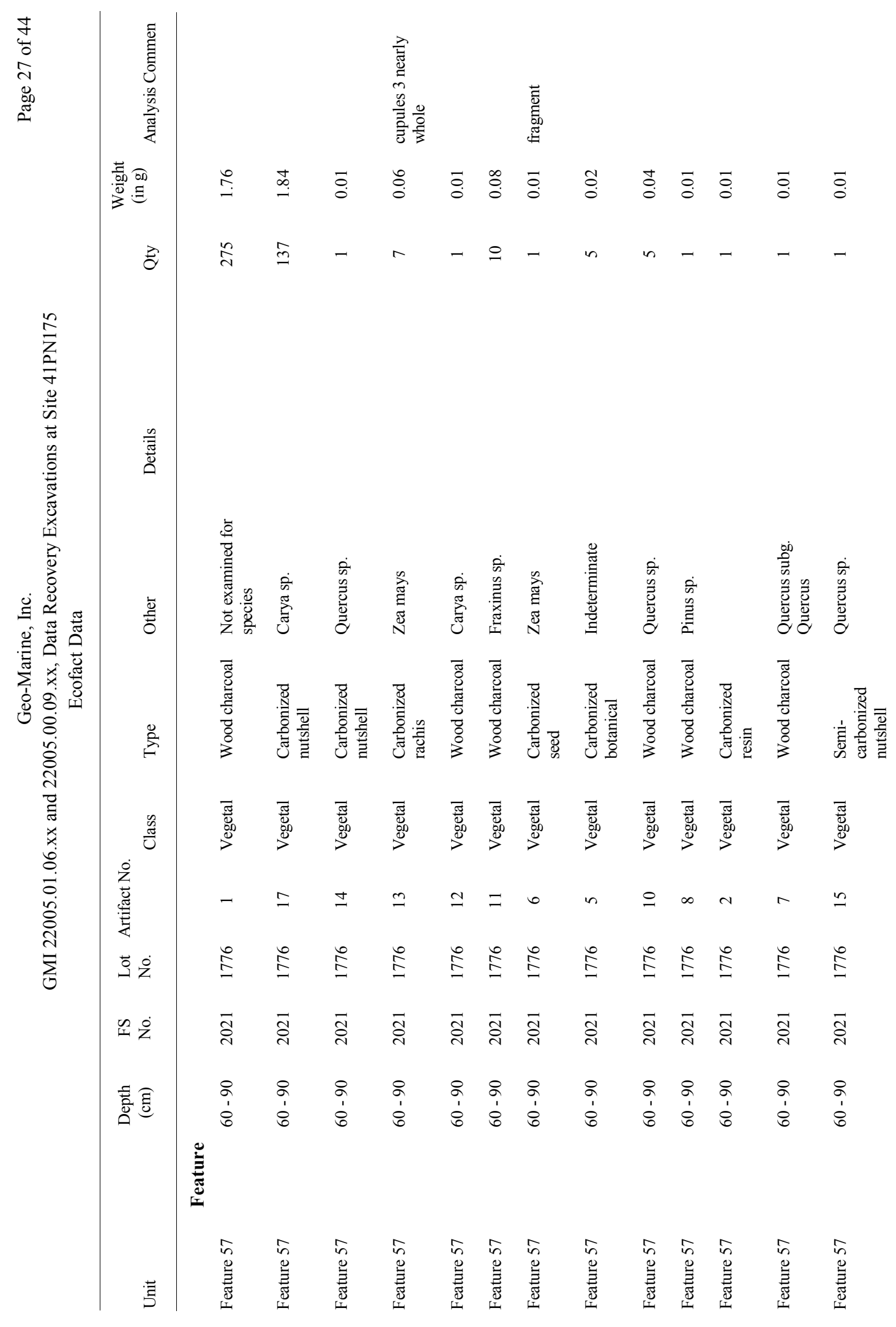




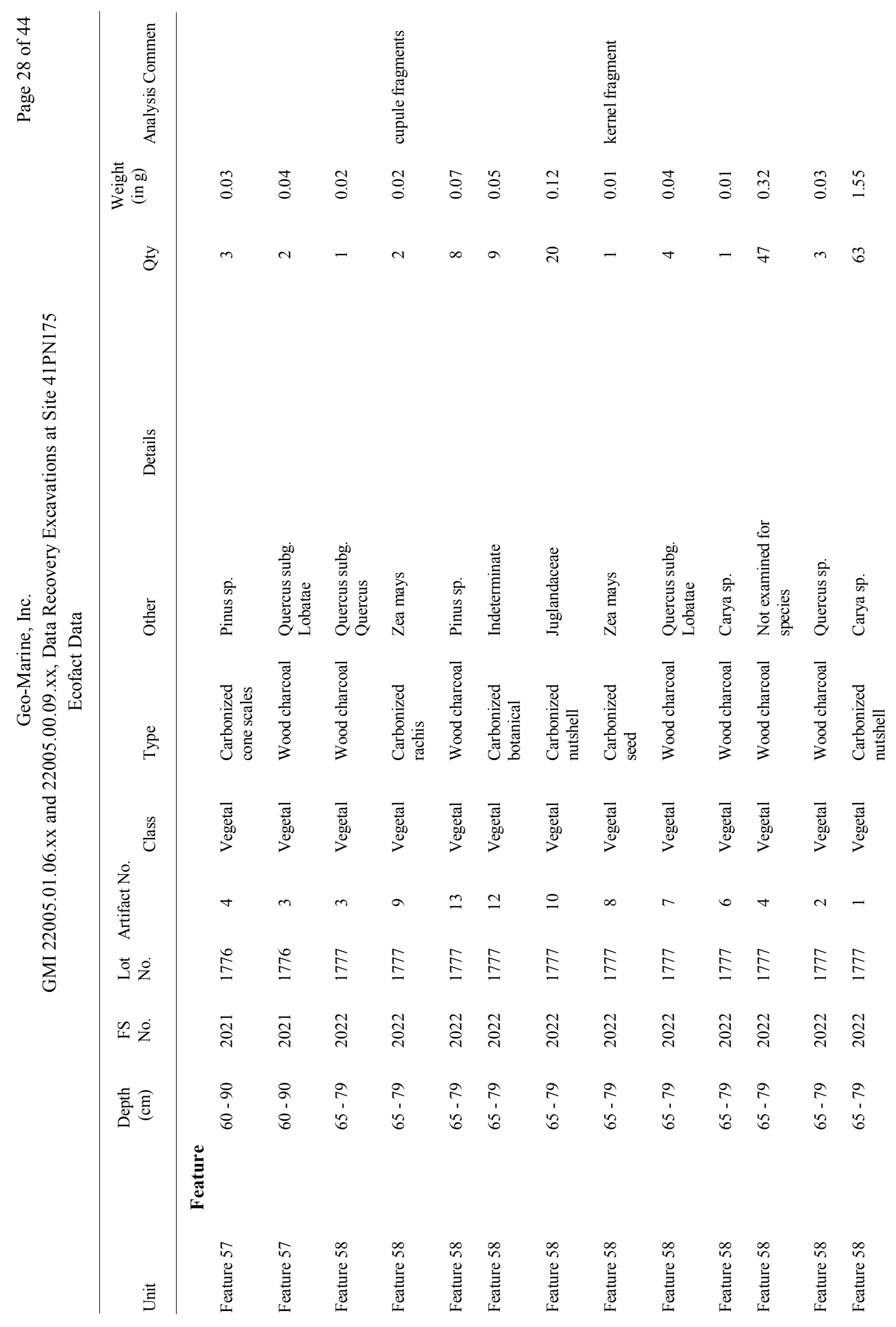




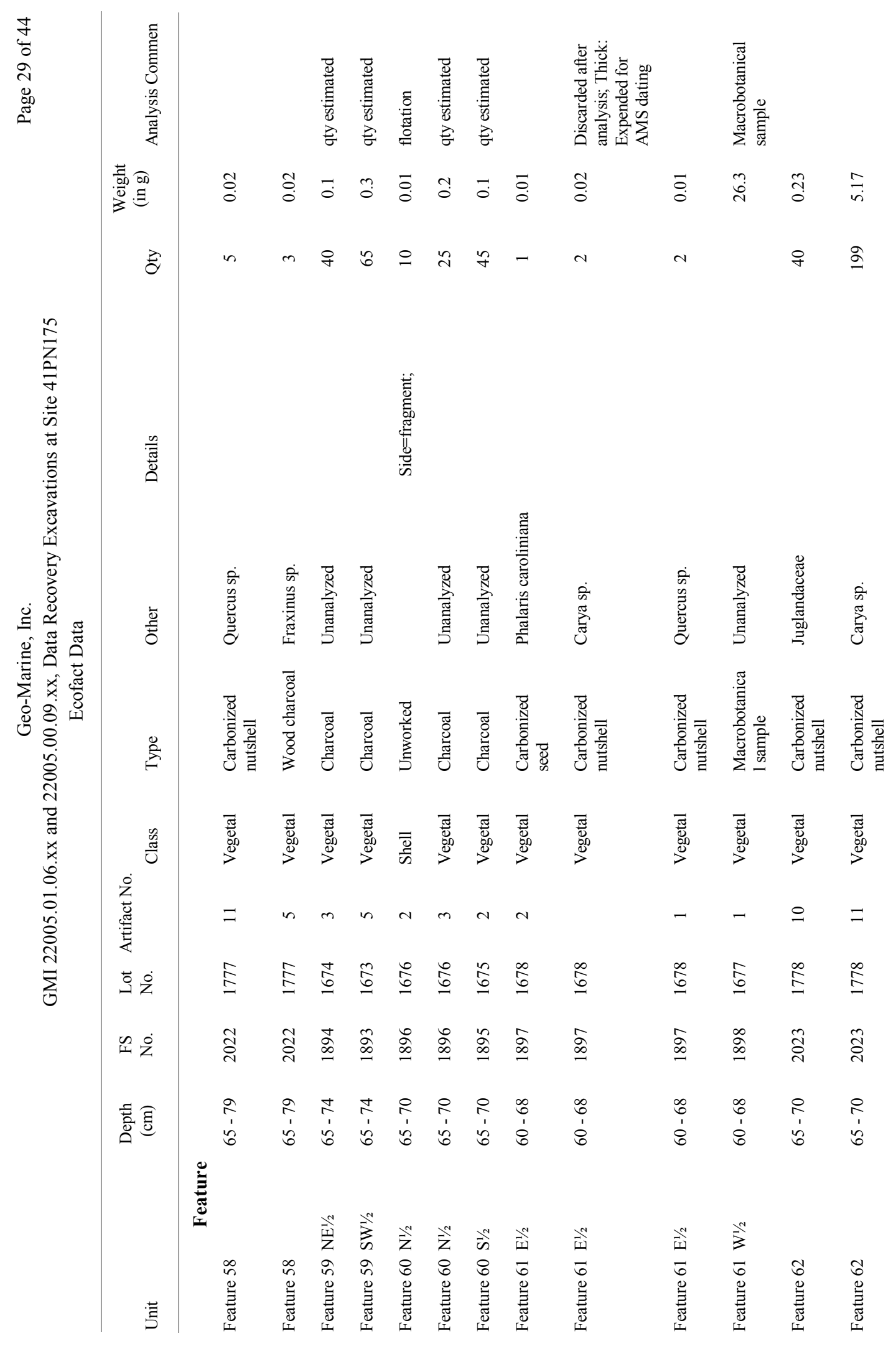




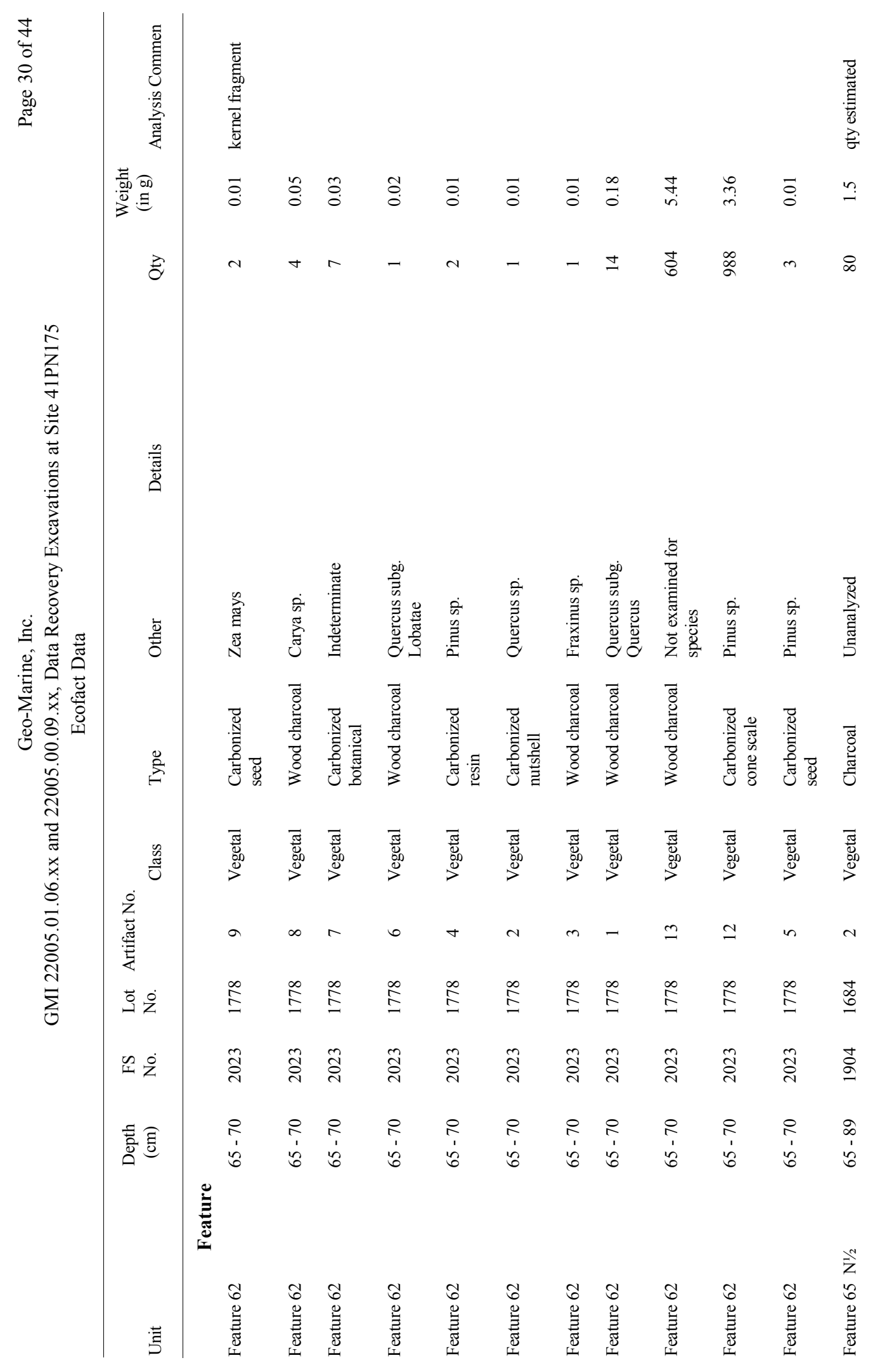




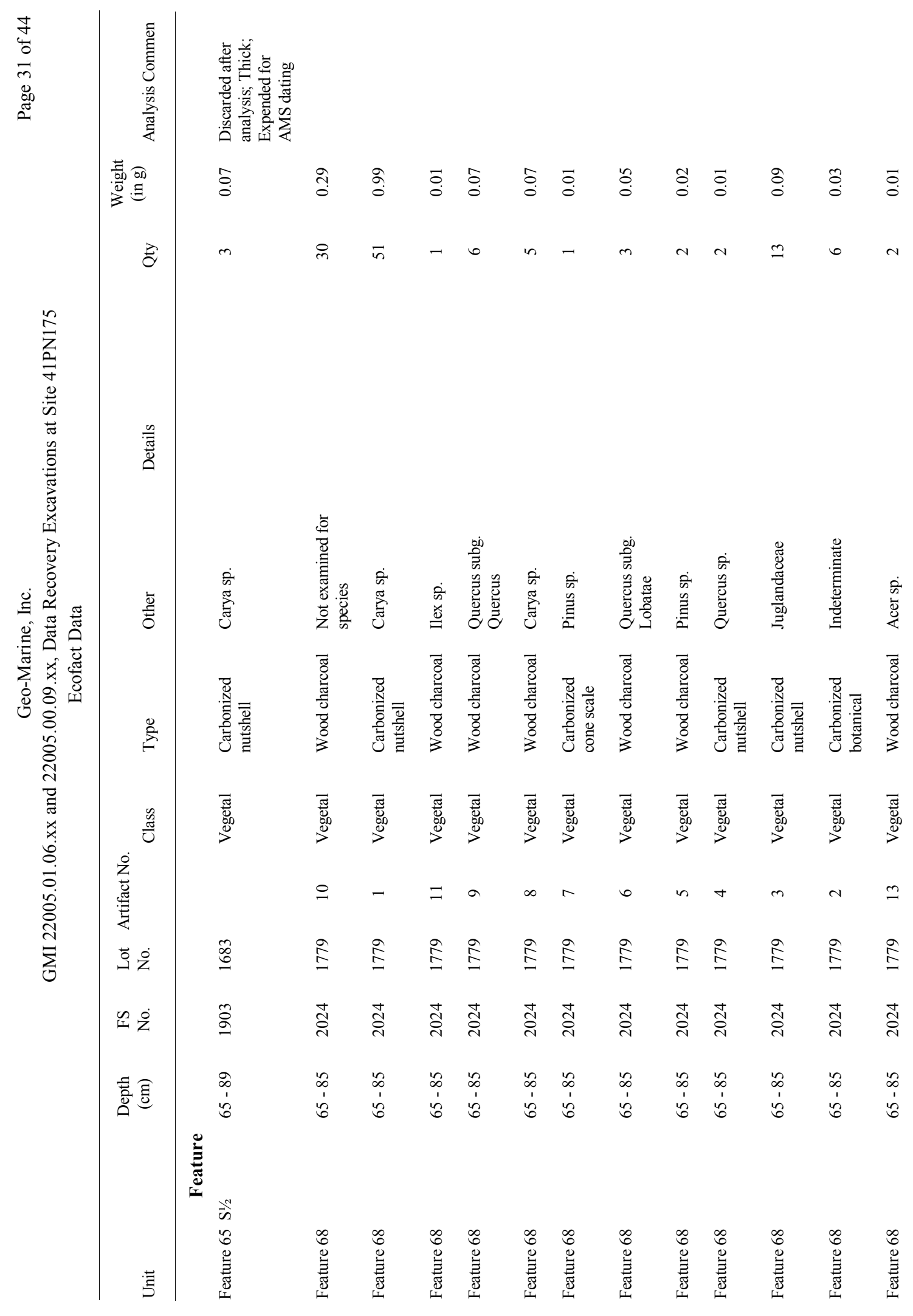




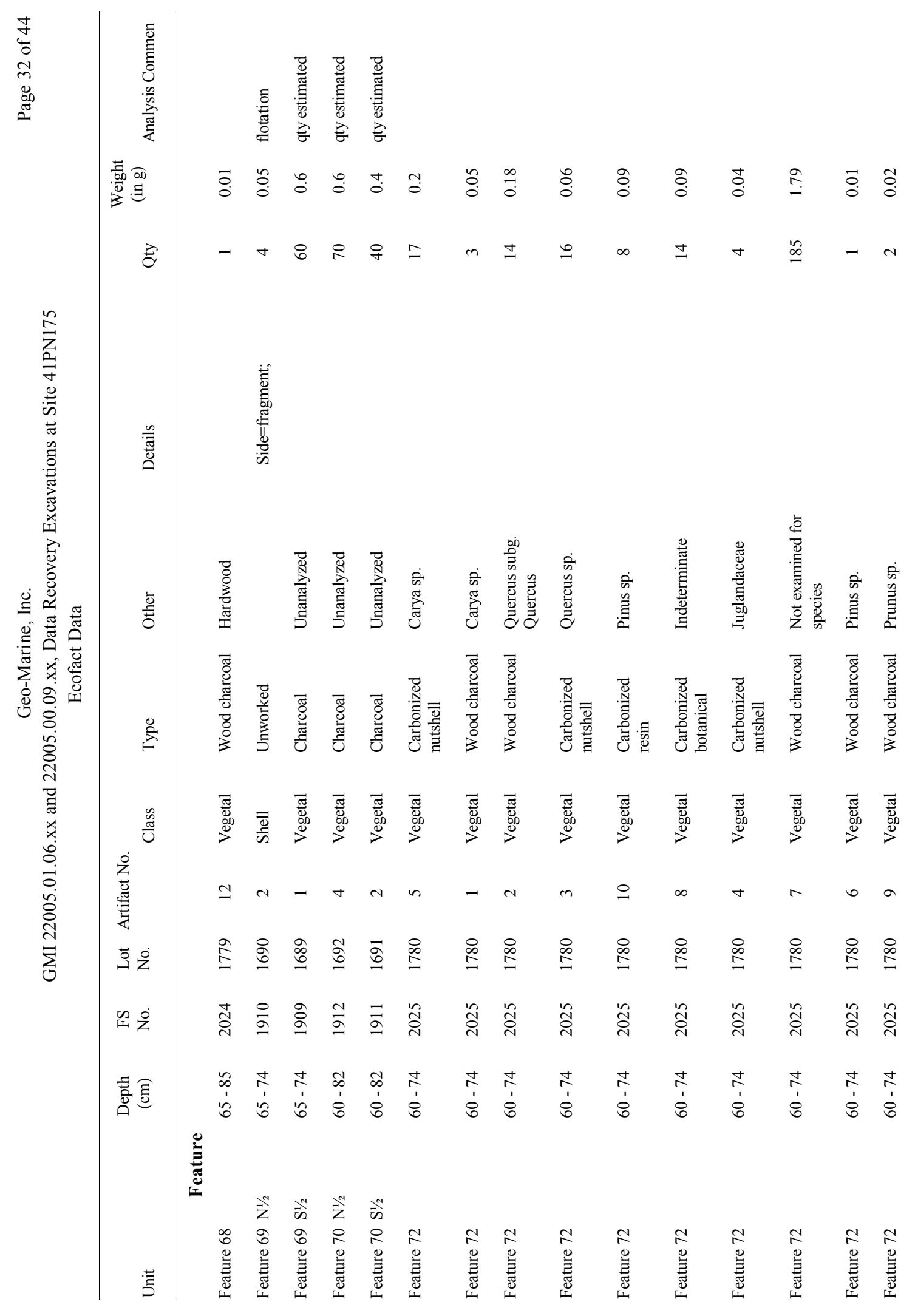









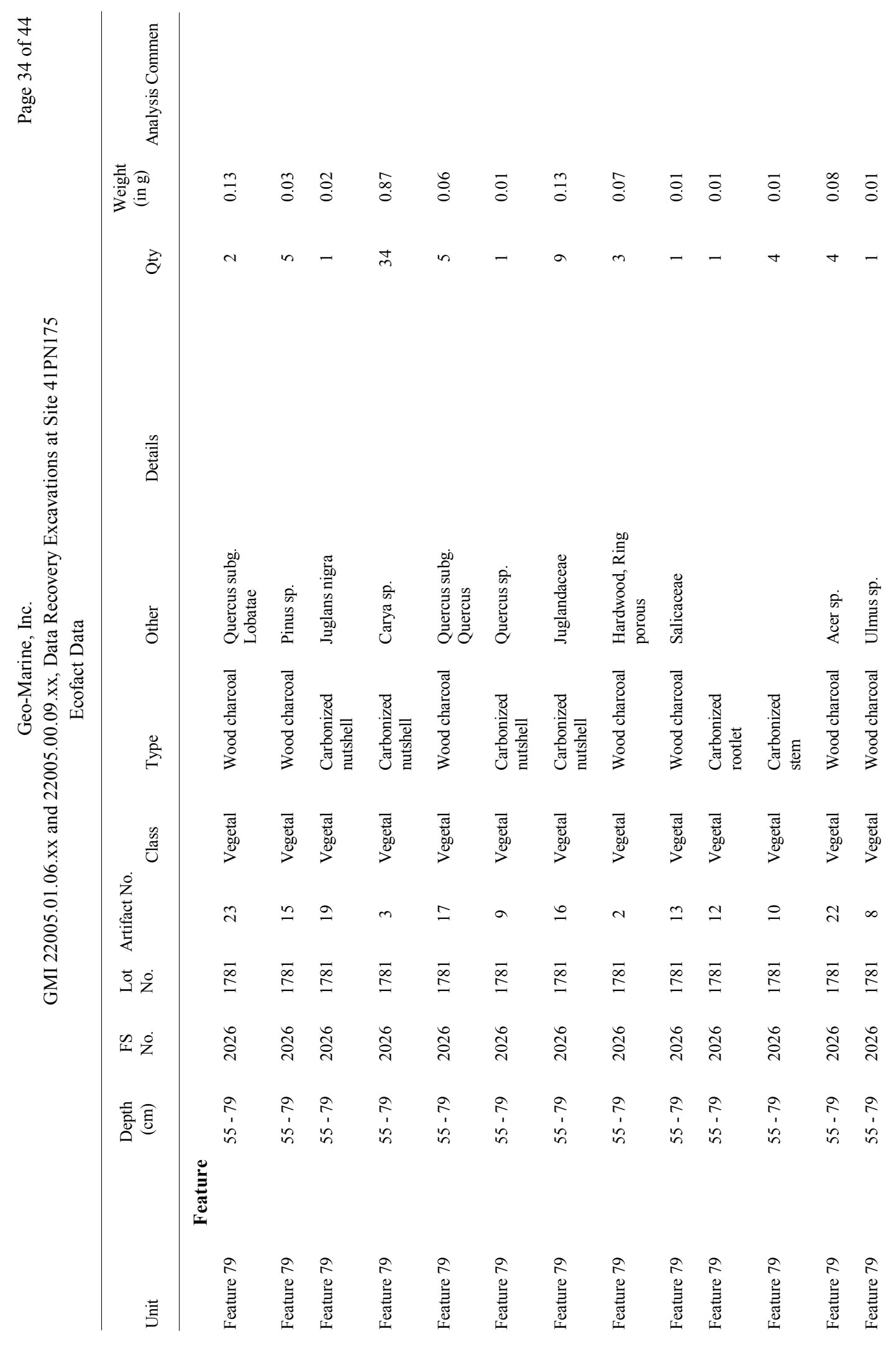




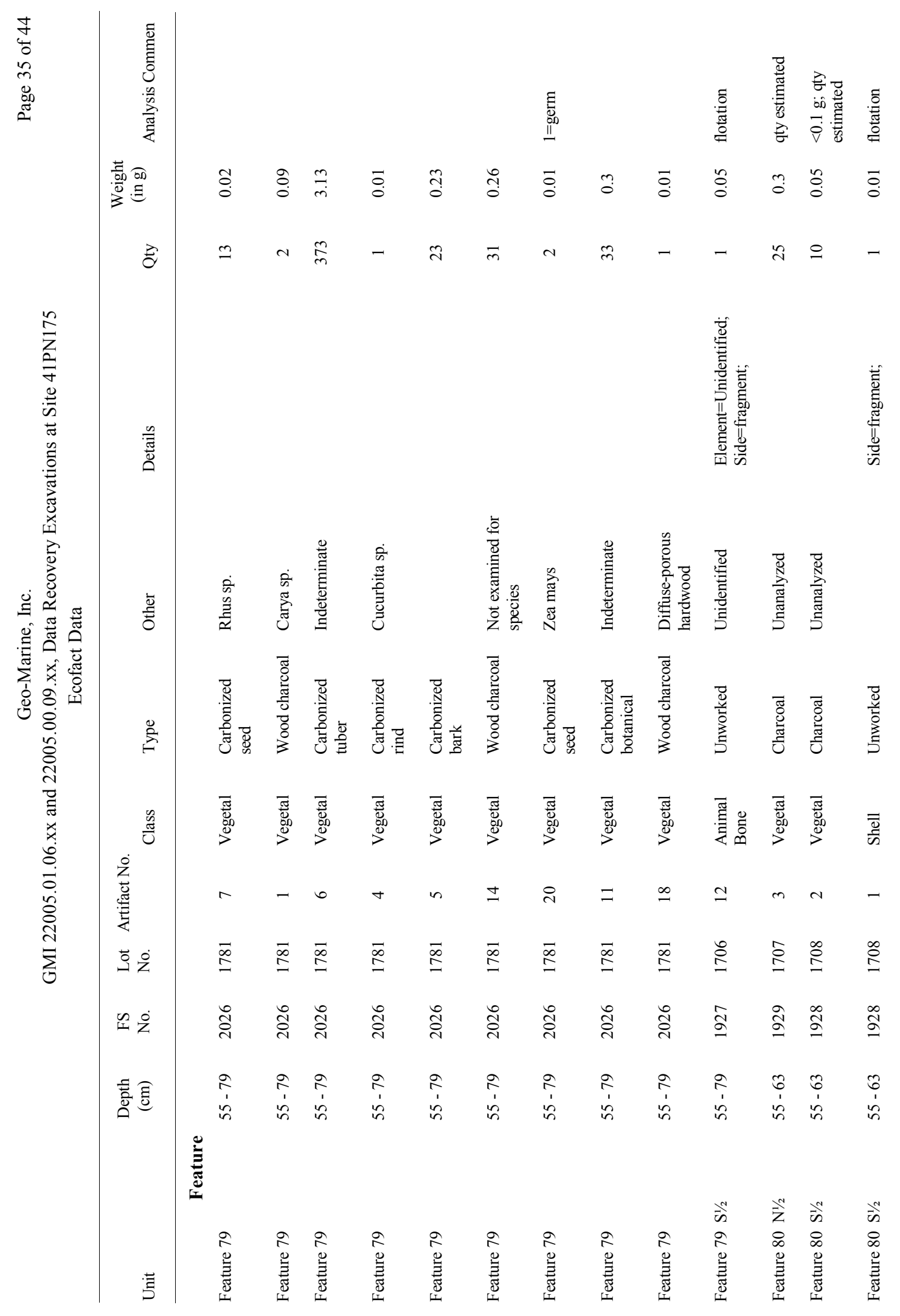




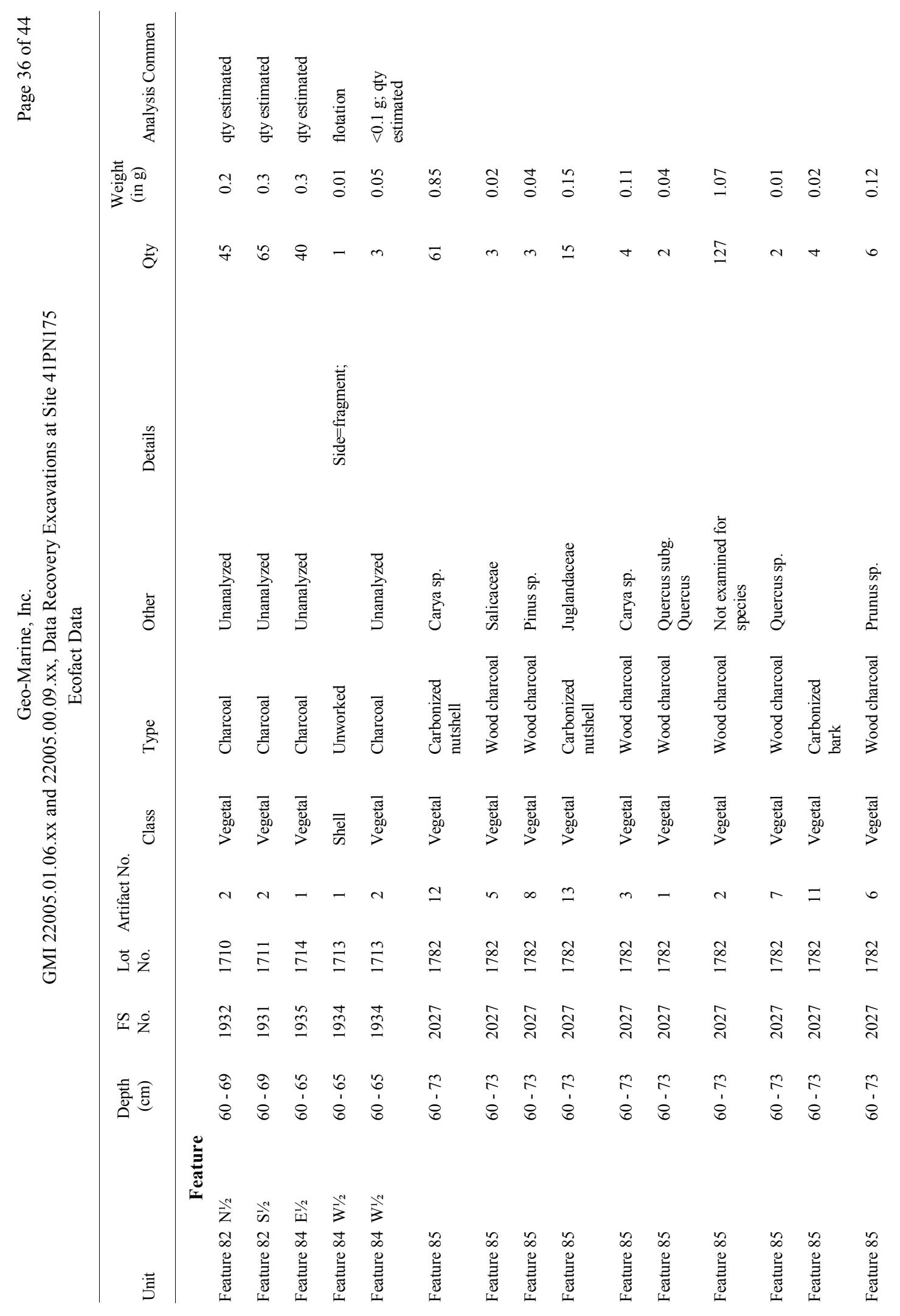




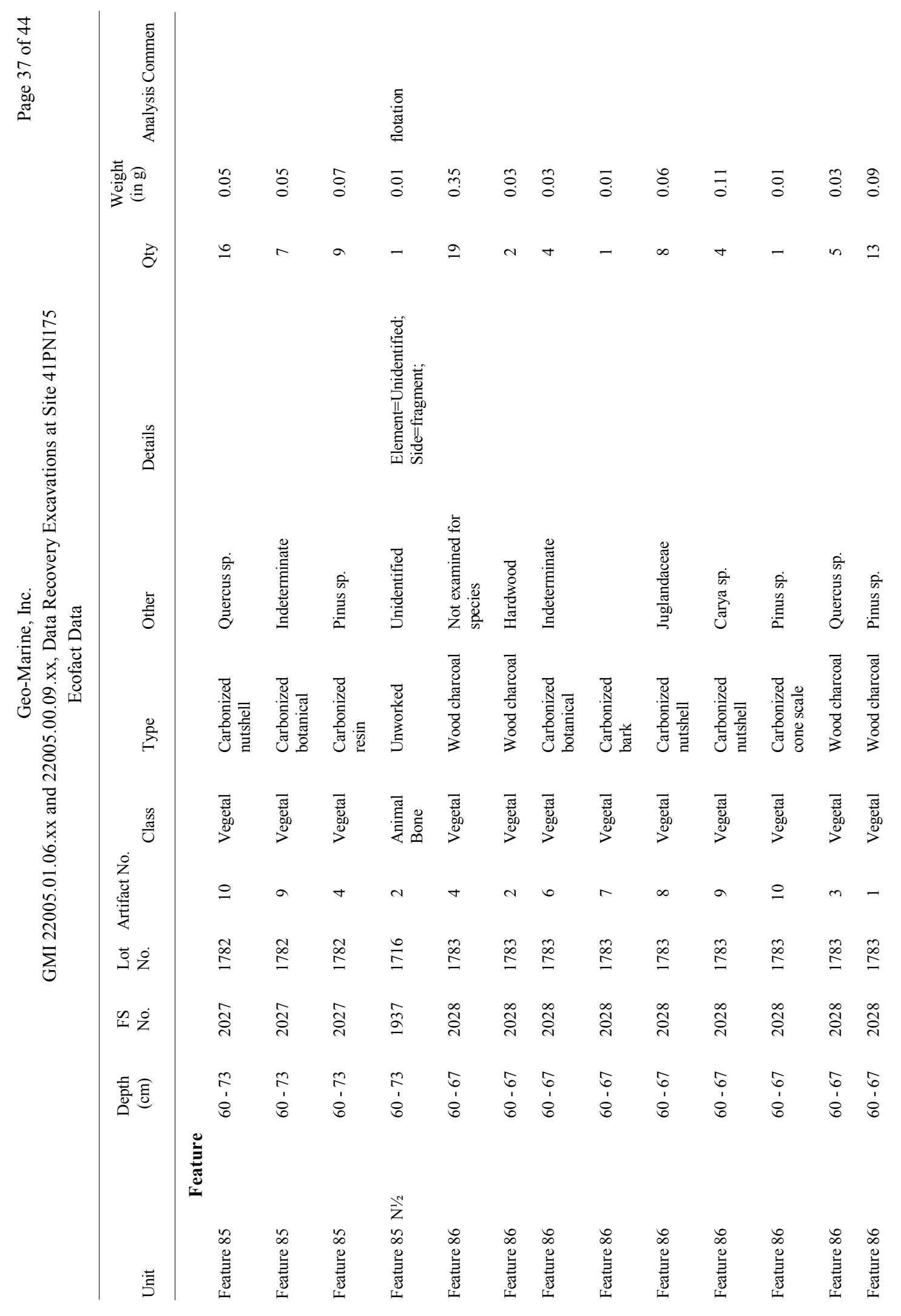




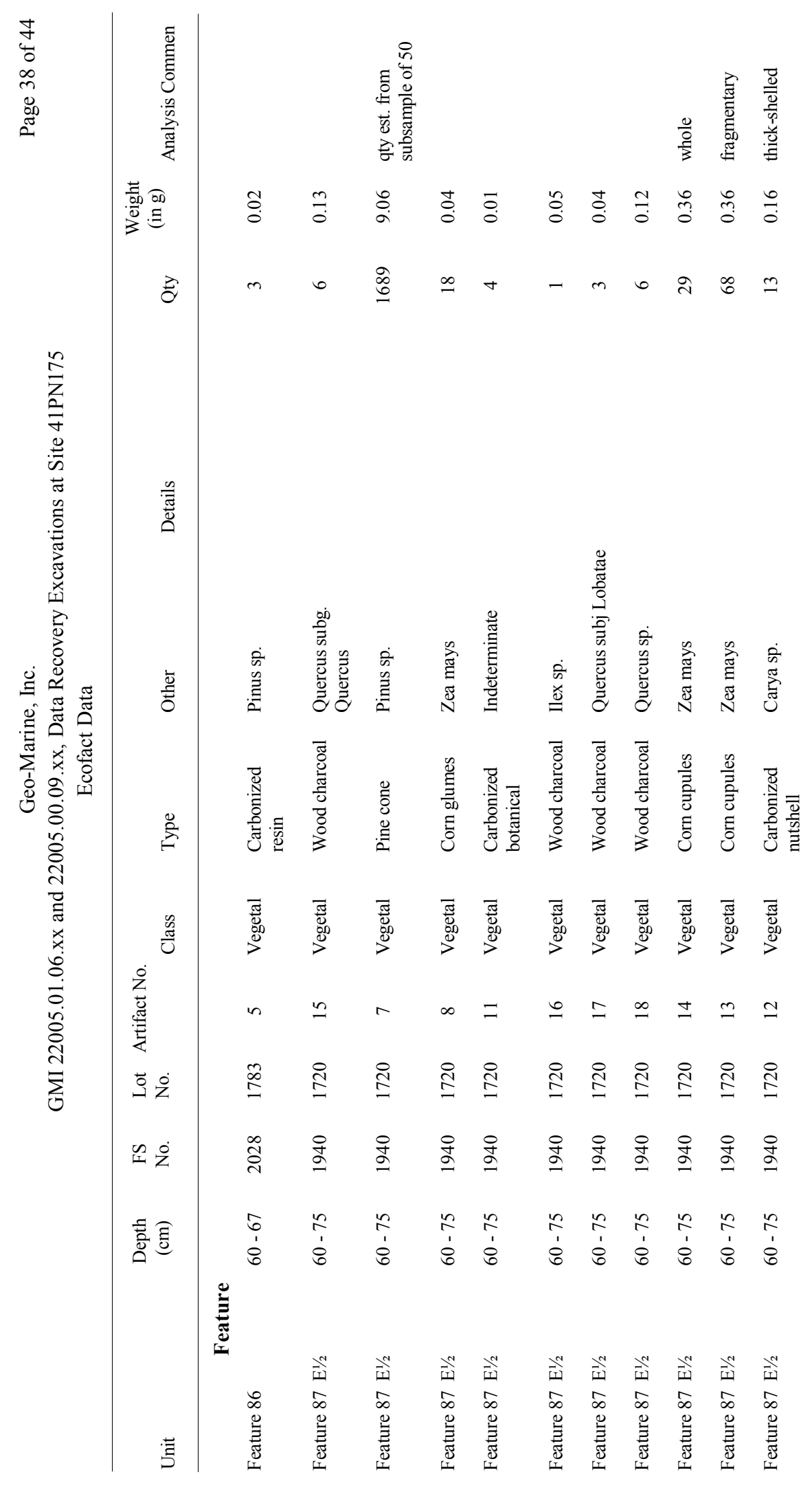




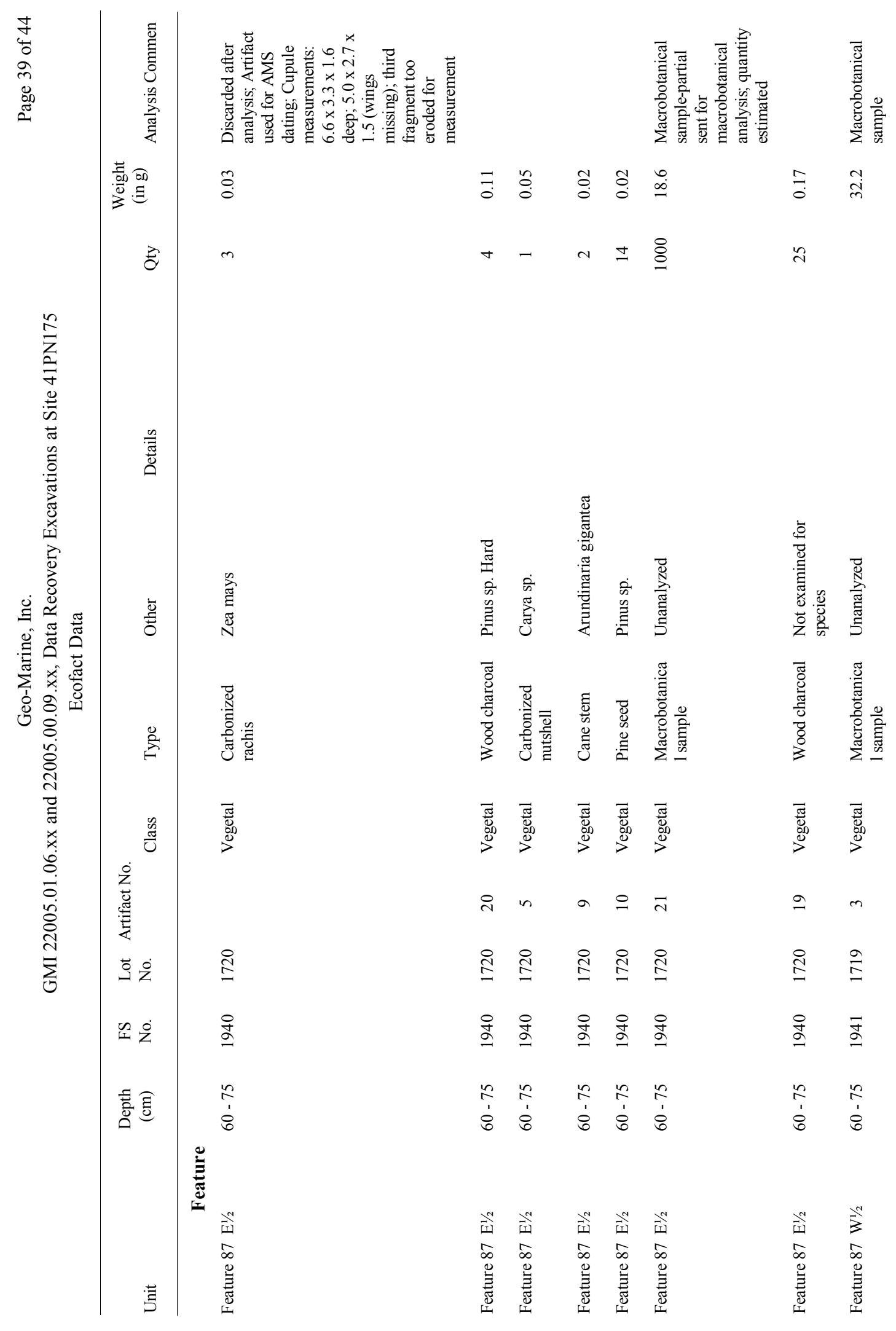




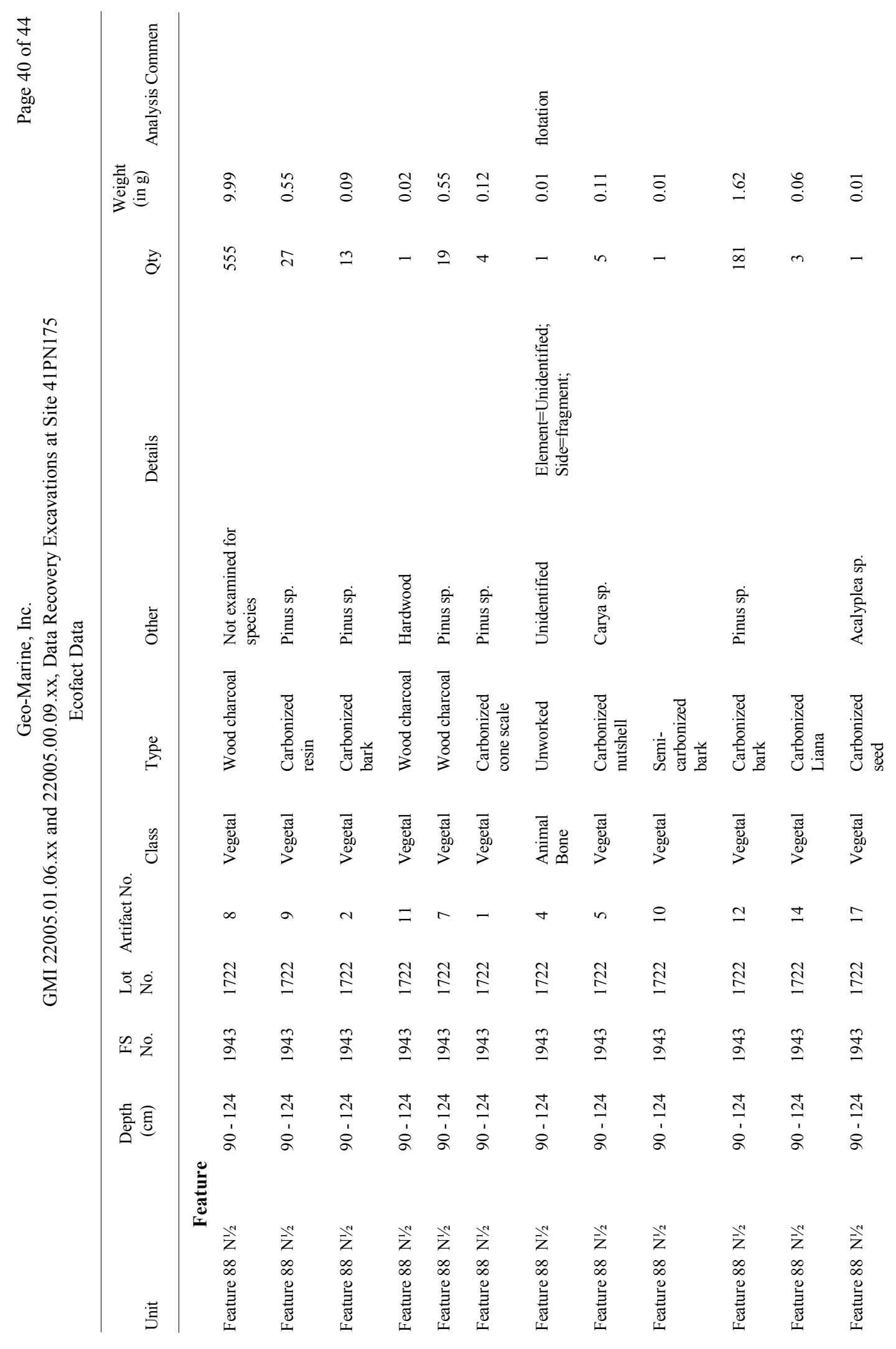









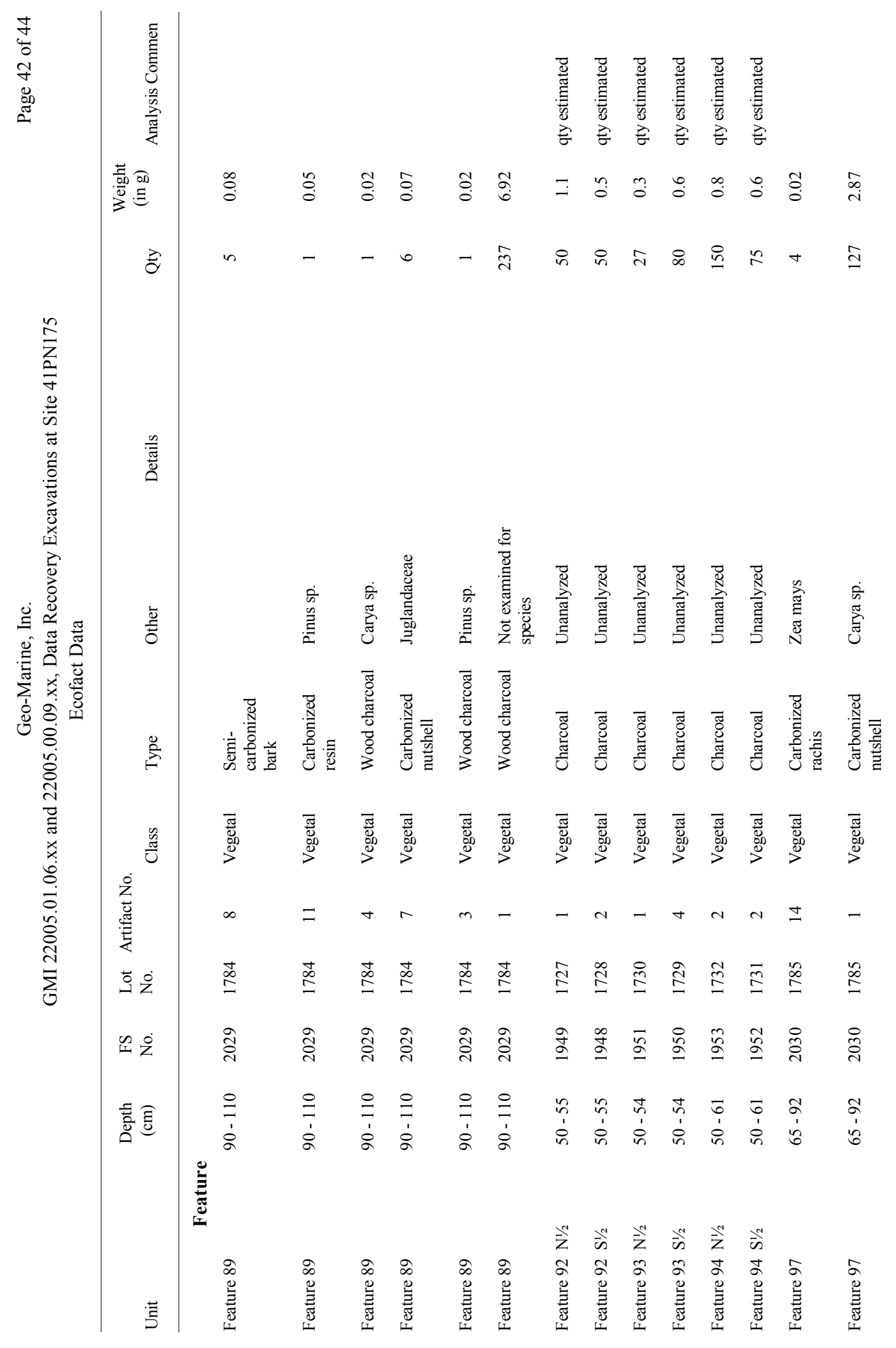




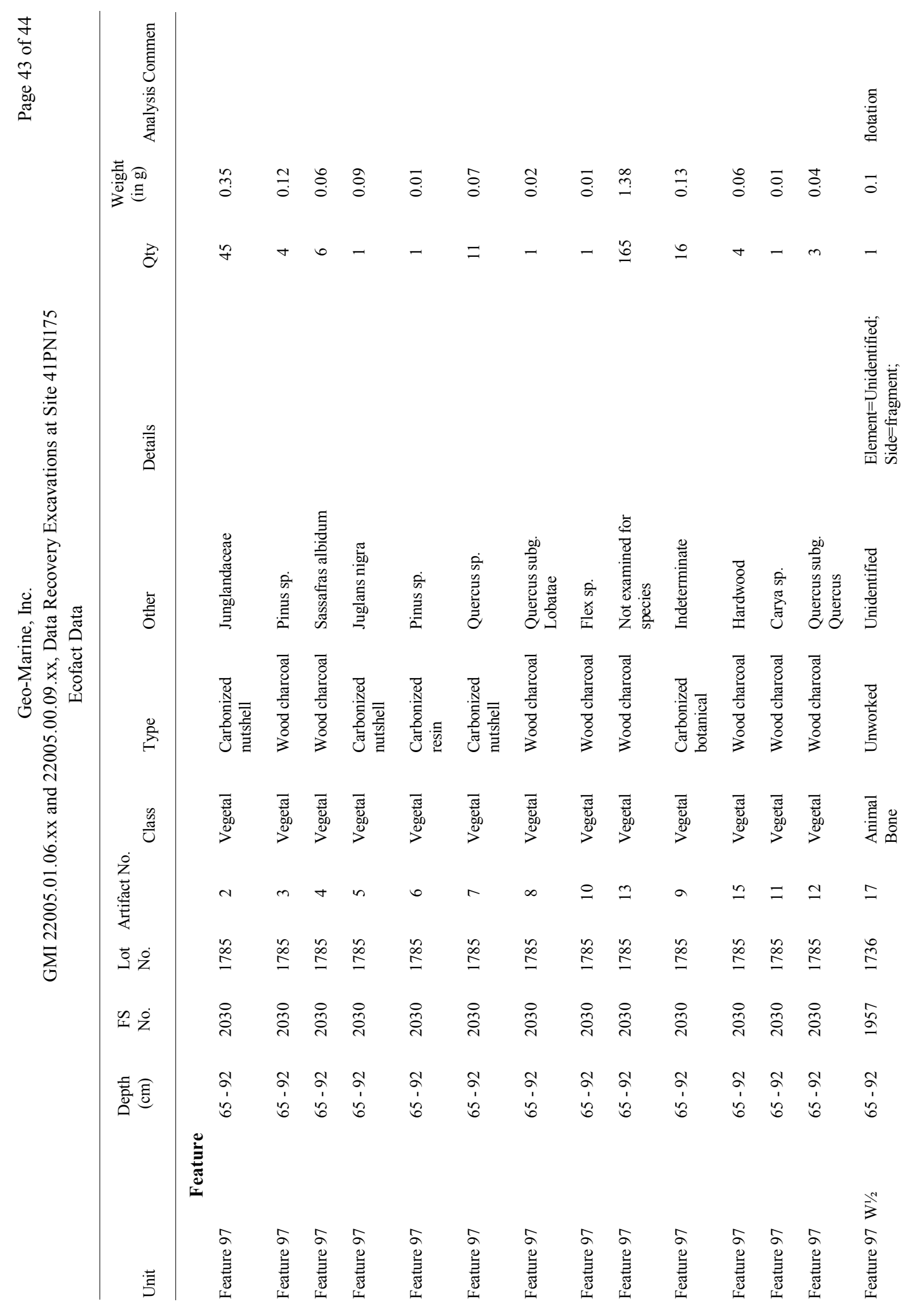




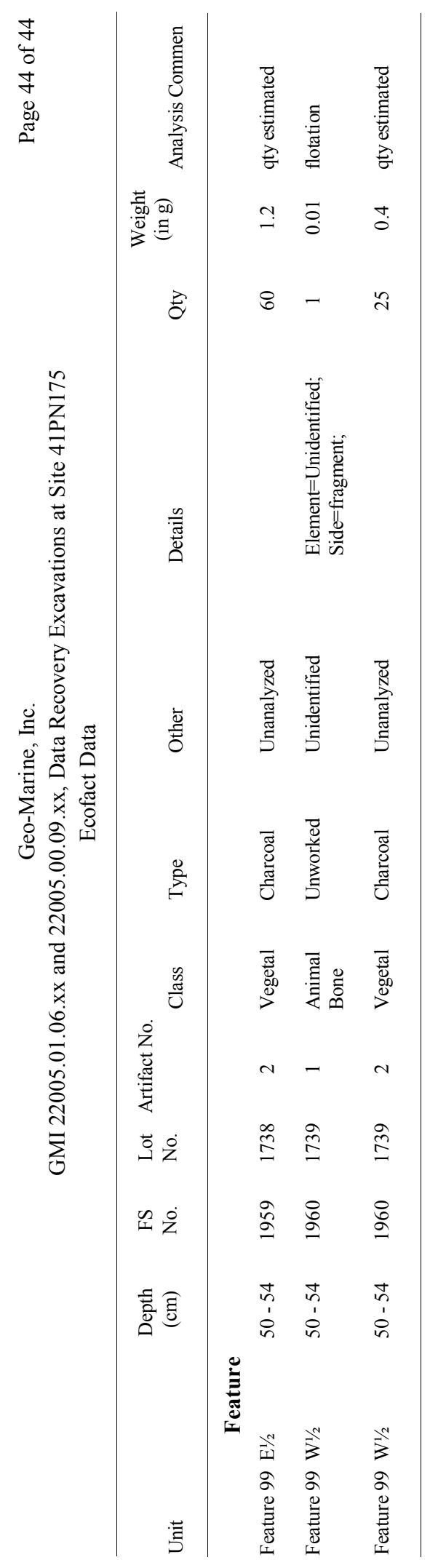




\section{A-10: HISTORIC ARTIFACTS}





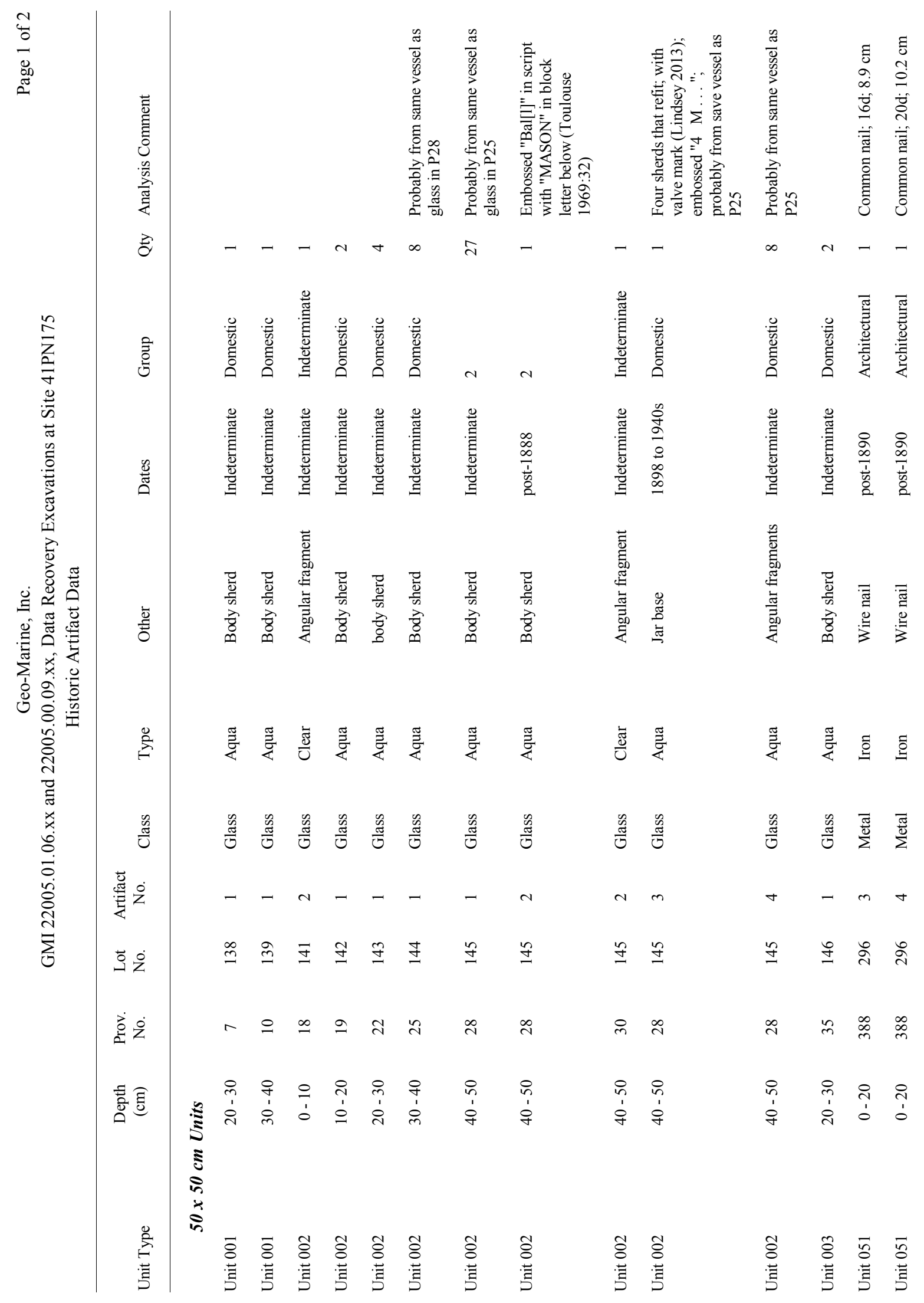




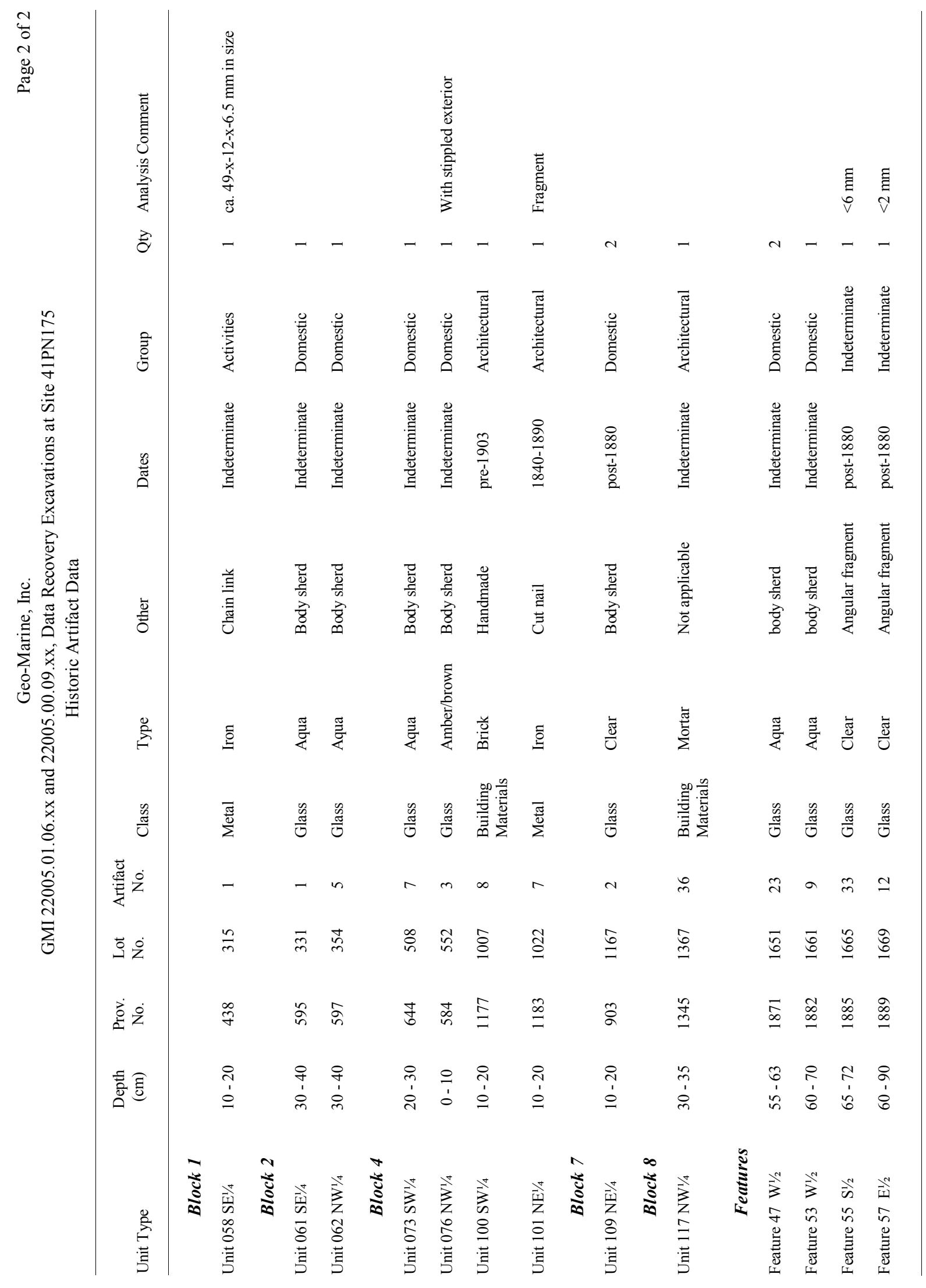




\section{APPENDIX B: RADIOCARBON, OSL, AND THERMOLUMINESCENCE}



B-1: RADIOCARBON 





Consistent Accuracy ... ... Delivered On-time

March 15, 2013

\section{Dr. James Abbott}

Texas Department of Transportation

Cultural Resource Management

Environmental Affairs Division

125 East 11th Street

Austin, TX 78701

USA

RE: Radiocarbon Dating Results For Samples 41PN175-1232, 41PN175-1332, 41PN175-1336, 41PN175-1714, 41PN175-1799, 41PN175-1800, 41PN175-1805, 41PN175-1810, 41PN175-1838, 41PN175-1841, 41PN175-1877, 41PN175-1888, 41PN175-1897, 41PN175-1903, 41PN175-1920, 41PN175-1940, 41PN175-1943, 41PN175-1968, 41PN175-1976, 41PN175-1982-2, 41PN175-1982-5, 41PN175-1982-7, 41PN175-1982-12, 41PN175-1982-14, 41PN175-1984-5, 41PN175-1984-8, 41PN175-1984-12

\section{Dear Dr. Abbott:}

Enclosed are the radiocarbon dating results for 27 samples recently sent to us. They each provided plenty of carbon for accurate measurements and all the analyses proceeded normally. As usual, the method of analysis is listed on the report with the results and calibration data is provided where applicable.

The web directory containing the table of results and PDF download also contains pictures including, most importantly the portion actually analyzed. These can be saved by opening them and right clicking. Also a cvs spreadsheet download option is available and a quality assurance report is posted for each set of results. This report contains expected vs measured values for 3-5 working standards analyzed simultaneously with your samples.

All results reported are accredited to ISO-17025 standards and all analyses were performed entirely here in our laboratories. Since Beta is not a teaching laboratory, only graduates trained in accordance with the strict protocols of the ISO-17025 program participated in the analyses. When interpreting the results, please consider any communications you may have had with us regarding the samples.

If you have specific questions about the analyses, please contact us. Your inquiries are always welcome.

Our invoice will be emailed separately. Please, forward it to the appropriate officer or send VISA charge authorization. Thank you. As always, if you have any questions or would like to discuss the results, don't hesitate to contact me.

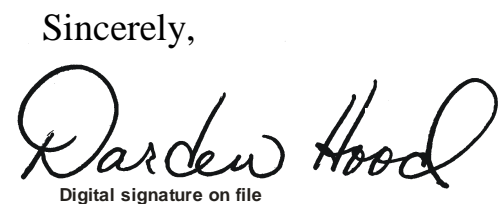




\section{REPORT OF RADIOCARBON DATING ANALYSES}

Dr. James Abbott

Report Date: 3/15/2013

Texas Department of Transportation

Material Received: 3/6/2013

Sample Data

Measured
Radiocarbon Age

$610+/-30 \mathrm{BP}$

Beta - 344076

SAMPLE : 41PN175-1232

ANALYSIS : AMS-Standard delivery

MATERIAL/PRETREATMENT : (bulk sherd organics): acid washes

2 SIGMA CALIBRATION :
Cal AD 1300 to 1370 (Cal BP 650 to 580) AND Cal AD 1380 to 1410 (Cal BP 570 to 540)
Conventional

Radiocarbon Age $\left(^{*}\right)$
Beta - 344077

SAMPLE : 41PN175-1332

ANALYSIS : AMS-Standard delivery

MATERIAL/PRETREATMENT : (bulk sherd organics): acid washes

2 SIGMA CALIBRATION : $\quad$ Cal AD 1430 to 1480 (Cal BP 520 to 470)
$13 \mathrm{C} / 12 \mathrm{C}$

Ratio

$-26.3 \mathrm{o} / \mathrm{oo}$

$590+/-30 \mathrm{BP}$
Beta - 344078

SAMPLE : 41PN175-1336

ANALYSIS : AMS-Standard delivery

MATERIAL/PRETREATMENT : (bulk sherd organics): acid washes

2 SIGMA CALIBRATION :
Cal AD 1260 to 1290 (Cal BP 690 to 660)
$720+/-30 \mathrm{BP}$

$-24.6 \mathrm{o} / \mathrm{oo}$

$730+/-30$ BP
Beta - 344079

SAMPLE : 41PN175-1714

ANALYSIS : AMS-Standard delivery

MATERIAL/PRETREATMENT : (bulk sherd organics): acid washes 2 SIGMA CALIBRATION :

Cal AD 970 to 1030 (Cal BP 980 to 920 )
$-27.2 \mathrm{o} / \mathrm{oo}$

$430+/-30$ BP
Dates are reported as RCYBP (radiocarbon years before present, "present" = AD 1950). By international convention, the modern reference standard was $95 \%$ the $14 \mathrm{C}$ activity of the National Institute of Standards and Technology (NIST) Oxalic Acid (SRM 4990C) and calculated using the Libby $14 \mathrm{C}$ half-life (5568 years). Quoted errors represent 1 relative standard deviation statistics (68\% probability) counting errors based on the combined measurements of the sample, background, and modern reference standards. Measured $13 \mathrm{C} / 12 \mathrm{C}$ ratios (delta $13 \mathrm{C}$ ) were calculated relative to the PDB-1 standard.
The Conventional Radiocarbon Age represents the Measured Radiocarbon Age corrected for isotopic fractionation, calculated using the delta $13 \mathrm{C}$. On rare occasion where the Conventional Radiocarbon Age was calculated using an assumed delta $13 \mathrm{C}$, the ratio and the Conventional Radiocarbon Age will be followed by "*". The Conventional Radiocarbon Age is not calendar calibrated. When available, the Calendar Calibrated result is calculated from the Conventional Radiocarbon Age and is listed as the "Two Sigma Calibrated Result" for each sample. 


\section{REPORT OF RADIOCARBON DATING ANALYSES}

$$
\text { Sample Data }
$$

\author{
Measured \\ Radiocarbon Age
}

$13 \mathrm{C} / 12 \mathrm{C}$

Ratio
Conventional

Radiocarbon Age $\left(^{*}\right)$

\section{Beta - 344080}

$360+/-30$ BP

$-25.5 \mathrm{o} / \mathrm{oo}$

$350+/-30 \mathrm{BP}$

SAMPLE : 41PN175-1799

ANALYSIS : AMS-Standard delivery

MATERIAL/PRETREATMENT : (charred material): acid/alkali/acid

2 SIGMA CALIBRATION :

Cal AD 1450 to 1640 (Cal BP 500 to 310)

Beta - 344081

$350+/-30$ BP

$-26.4 \mathrm{o} / \mathrm{oo}$

$330+/-30 \mathrm{BP}$

SAMPLE : 41PN175-1800

ANALYSIS : AMS-Standard delivery

MATERIAL/PRETREATMENT : (charred material): acid/alkali/acid

2 SIGMA CALIBRATION : $\quad$ Cal AD 1460 to 1650 (Cal BP 490 to 300)

Beta -344082

$350+/-30 \mathrm{BP}$

$-23.6 \mathrm{o} / \mathrm{oo}$

$370+/-30 \mathrm{BP}$

SAMPLE : 41PN175-1805

ANALYSIS : AMS-Standard delivery

MATERIAL/PRETREATMENT : (charred material): acid/alkali/acid

2 SIGMA CALIBRATION : $\quad$ Cal AD 1450 to 1530 (Cal BP 500 to 420) AND Cal AD 1540 to 1550 (Cal BP 410 to 400)

Cal AD 1550 to 1630 (Cal BP 400 to 320)

Beta - 344083

$430+/-30 \mathrm{BP}$

$-26.3 \mathrm{o} / \mathrm{oo}$

$410+/-30 \mathrm{BP}$

SAMPLE : 41PN175-1810

ANALYSIS : AMS-Standard delivery

MATERIAL/PRETREATMENT : (charred material): acid/alkali/acid

2 SIGMA CALIBRATION : $\quad$ Cal AD 1440 to 1500 (Cal BP 510 to 450) AND Cal AD 1500 to 1510 (Cal BP 450 to 440)

Cal AD 1600 to 1620 (Cal BP 350 to 330)

Dates are reported as RCYBP (radiocarbon years before present, "present" = AD 1950). By international convention, the modern reference standard was $95 \%$ the $14 \mathrm{C}$ activity of the National Institute of Standards and Technology (NIST) Oxalic Acid (SRM 4990C) and calculated using the Libby $14 \mathrm{C}$ half-life (5568 years). Quoted errors represent 1 relative standard deviation statistics (68\% probability) counting errors based on the combined measurements of the sample, background, and modern reference standards. Measured $13 \mathrm{C} / 12 \mathrm{C}$ ratios (delta $13 \mathrm{C}$ ) were calculated relative to the PDB-1 standard.
The Conventional Radiocarbon Age represents the Measured Radiocarbon Age corrected for isotopic fractionation, calculated using the delta $13 \mathrm{C}$. On rare occasion where the Conventional Radiocarbon Age was calculated using an assumed delta 13C, the ratio and the Conventional Radiocarbon Age will be followed by "*”. The Conventional Radiocarbon Age is not calendar calibrated. When available, the Calendar Calibrated result is calculated from the Conventional Radiocarbon Age and is listed as the "Two Sigma Calibrated Result" for each sample. 


\section{REPORT OF RADIOCARBON DATING ANALYSES}

\section{Sample Data}

\author{
Measured \\ Radiocarbon Age
}

$13 \mathrm{C} / 12 \mathrm{C}$

Ratio
Conventional

Radiocarbon Age $\left(^{*}\right)$

\section{Beta - 344084}

$340+/-30$ BP

$-23.5 \mathrm{o} / \mathrm{oo}$

$360+/-30$ BP

SAMPLE : 41PN175-1838

ANALYSIS : AMS-Standard delivery

MATERIAL/PRETREATMENT : (charred material): acid/alkali/acid

2 SIGMA CALIBRATION :

Cal AD 1450 to 1640 (Cal BP 500 to 310)

Beta -344085

$1330+/-30 \mathrm{BP}$

$-29.0 \mathrm{o} / \mathrm{oo}$

$1260+/-30 \mathrm{BP}$

SAMPLE : 41PN175-1841

ANALYSIS : AMS-Standard delivery

MATERIAL/PRETREATMENT : (charred material): acid/alkali/acid

2 SIGMA CALIBRATION : $\quad$ Cal AD 670 to 780 (Cal BP 1280 to 1170) AND Cal AD 790 to 810 (Cal BP 1160 to 1140)

Cal AD 850 to 850 (Cal BP 1100 to 1100)

Beta - 344086

$440+/-30$ BP

$-26.3 \mathrm{o} / \mathrm{oo}$

$420+/-30 \mathrm{BP}$

SAMPLE : 41PN175-1877

ANALYSIS : AMS-Standard delivery

MATERIAL/PRETREATMENT : (charred material): acid/alkali/acid

2 SIGMA CALIBRATION : $\quad$ Cal AD 1430 to 1490 (Cal BP 520 to 460) AND Cal AD 1600 to 1610 (Cal BP 350 to 340)

Beta - 344087

$30+/-30$ BP

-7.8 o/oo

$310+/-30 \mathrm{BP}$

SAMPLE : 41PN175-1888

ANALYSIS : AMS-Standard delivery

MATERIAL/PRETREATMENT : (charred material): acid/alkali/acid

2 SIGMA CALIBRATION : $\quad$ Cal AD 1480 to 1650 (Cal BP 470 to 300)

Dates are reported as RCYBP (radiocarbon years before present, "present" = AD 1950). By international convention, the modern reference standard was $95 \%$ the $14 \mathrm{C}$ activity of the National Institute of Standards and Technology (NIST) Oxalic Acid (SRM 4990C) and calculated using the Libby $14 \mathrm{C}$ half-life (5568 years). Quoted errors represent 1 relative standard deviation statistics (68\% probability) counting errors based on the combined measurements of the sample, background, and modern reference standards. Measured $13 \mathrm{C} / 12 \mathrm{C}$ ratios (delta $13 \mathrm{C}$ ) were calculated relative to the PDB-1 standard.
The Conventional Radiocarbon Age represents the Measured Radiocarbon Age corrected for isotopic fractionation, calculated using the delta $13 \mathrm{C}$. On rare occasion where the Conventional Radiocarbon Age was calculated using an assumed delta 13C, the ratio and the Conventional Radiocarbon Age will be followed by "*". The Conventional Radiocarbon Age is not calendar calibrated. When available, the Calendar Calibrated result is calculated from the Conventional Radiocarbon Age and is listed as the "Two Sigma Calibrated Result" for each sample. 


\section{REPORT OF RADIOCARBON DATING ANALYSES}

$$
\text { Sample Data }
$$

\author{
Measured \\ Radiocarbon Age
}

$13 \mathrm{C} / 12 \mathrm{C}$

Ratio
Conventional

Radiocarbon Age $\left(^{*}\right)$

\section{Beta -344088}

$400+/-30$ BP

$-27.1 \mathrm{o} / \mathrm{oo}$

$370+/-30 \mathrm{BP}$

SAMPLE : 41PN175-1897

ANALYSIS : AMS-Standard delivery

MATERIAL/PRETREATMENT : (charred material): acid/alkali/acid

2 SIGMA CALIBRATION :

Cal AD 1450 to 1530 (Cal BP 500 to 420) AND Cal AD 1540 to 1550 (Cal BP 410 to 400)

Cal AD 1550 to 1630 (Cal BP 400 to 320)

Beta - 344089

$580+/-30 \mathrm{BP}$

-25.9 o/oo

$570+/-30 \mathrm{BP}$

SAMPLE : 41PN175-1903

ANALYSIS : AMS-Standard delivery

MATERIAL/PRETREATMENT : (charred material): acid/alkali/acid

2 SIGMA CALIBRATION : $\quad$ Cal AD 1300 to 1360 (Cal BP 640 to 590) AND Cal AD 1380 to 1420 (Cal BP 570 to 530)

Beta -344090

$310+/-30$ BP

$-23.4 \mathrm{o} / \mathrm{oo}$

$340+/-30 \mathrm{BP}$

SAMPLE : 41PN175-1920

ANALYSIS : AMS-Standard delivery

MATERIAL/PRETREATMENT : (charred material): acid/alkali/acid

2 SIGMA CALIBRATION : $\quad$ Cal AD 1450 to 1640 (Cal BP 500 to 310)

Beta - 344091

$100+/-30 \mathrm{BP}$

$-10.0 \mathrm{o} / \mathrm{oo}$

$350+/-30 \mathrm{BP}$

SAMPLE : 41PN175-1940

ANALYSIS : AMS-Standard delivery

MATERIAL/PRETREATMENT : (charred material): acid/alkali/acid

2 SIGMA CALIBRATION : $\quad$ Cal AD 1450 to 1640 (Cal BP 500 to 310)

Dates are reported as RCYBP (radiocarbon years before present, "present" = AD 1950). By international convention, the modern reference standard was $95 \%$ the $14 \mathrm{C}$ activity of the National Institute of Standards and Technology (NIST) Oxalic Acid (SRM 4990C) and calculated using the Libby $14 \mathrm{C}$ half-life (5568 years). Quoted errors represent 1 relative standard deviation statistics (68\% probability) counting errors based on the combined measurements of the sample, background, and modern reference standards. Measured $13 \mathrm{C} / 12 \mathrm{C}$ ratios (delta $13 \mathrm{C}$ ) were calculated relative to the PDB-1 standard.
The Conventional Radiocarbon Age represents the Measured Radiocarbon Age corrected for isotopic fractionation, calculated using the delta 13C. On rare occasion where the Conventional Radiocarbon Age was calculated using an assumed delta $13 \mathrm{C}$, the ratio and the Conventional Radiocarbon Age will be followed by "*". The Conventional Radiocarbon Age is not calendar calibrated. When available, the Calendar Calibrated result is calculated from the Conventional Radiocarbon Age and is listed as the "Two Sigma Calibrated Result" for each sample. 


\section{REPORT OF RADIOCARBON DATING ANALYSES}

Dr. James Abbott

Report Date: 3/15/2013

\section{Sample Data}

\author{
Measured \\ Radiocarbon Age
}

$13 \mathrm{C} / 12 \mathrm{C}$

Ratio
Conventional

Radiocarbon Age $\left(^{*}\right)$

Beta -344092

$1220+/-30$ BP

$-24.2 \mathrm{o} / \mathrm{oo}$

$1230+/-30 \mathrm{BP}$

SAMPLE : 41PN175-1943

ANALYSIS : AMS-Standard delivery

MATERIAL/PRETREATMENT : (charred material): acid/alkali/acid

2 SIGMA CALIBRATION : $\quad$ Cal AD 690 to 880 (Cal BP 1260 to 1060)

Beta -344093

$360+/-30$ BP

$-24.7 \mathrm{o} / \mathrm{oo}$

$360+/-30 \mathrm{BP}$

SAMPLE : 41PN175-1968

ANALYSIS : AMS-Standard delivery

MATERIAL/PRETREATMENT : (charred material): acid/alkali/acid

2 SIGMA CALIBRATION : $\quad$ Cal AD 1450 to 1640 (Cal BP 500 to 310)

Beta - 344094

$400+/-30$ BP

-9.4 o/oo

$660+/-30 \mathrm{BP}$

SAMPLE : 41PN175-1976

ANALYSIS : AMS-Standard delivery

MATERIAL/PRETREATMENT : (charred material): acid/alkali/acid

2 SIGMA CALIBRATION : $\quad$ Cal AD 1280 to 1320 (Cal BP 670 to 630) AND Cal AD 1350 to 1390 (Cal BP 600 to 560)

Beta -344095

$290+/-30$ BP

-26.6 o/oo

$260+/-30 \mathrm{BP}$

SAMPLE : 41PN175-1982-2

ANALYSIS : AMS-Standard delivery

MATERIAL/PRETREATMENT : (charred material): acid/alkali/acid

2 SIGMA CALIBRATION : $\quad$ Cal AD 1520 to 1560 (Cal BP 420 to 390) AND Cal AD 1630 to 1670 (Cal BP 320 to 280)

Cal AD 1780 to 1800 (Cal BP 170 to 150) AND Cal AD 1950 to 1950 (Cal BP 0 to 0 )

Dates are reported as RCYBP (radiocarbon years before present, "present" = AD 1950). By international convention, the modern reference standard was $95 \%$ the $14 \mathrm{C}$ activity of the National Institute of Standards and Technology (NIST) Oxalic Acid (SRM 4990C) and calculated using the Libby $14 \mathrm{C}$ half-life (5568 years). Quoted errors represent 1 relative standard deviation statistics (68\% probability) counting errors based on the combined measurements of the sample, background, and modern reference standards. Measured $13 \mathrm{C} / 12 \mathrm{C}$ ratios (delta 13C) were calculated relative to the PDB-1 standard.
The Conventional Radiocarbon Age represents the Measured Radiocarbon Age corrected for isotopic fractionation, calculated using the delta $13 \mathrm{C}$. On rare occasion where the Conventional Radiocarbon Age was calculated using an assumed delta 13C, the ratio and the Conventional Radiocarbon Age will be followed by "*”. The Conventional Radiocarbon Age is not calendar calibrated. When available, the Calendar Calibrated result is calculated from the Conventional Radiocarbon Age and is listed as the "Two Sigma Calibrated Result" for each sample. 


\section{REPORT OF RADIOCARBON DATING ANALYSES}

Dr. James Abbott

Report Date: 3/15/2013

\section{Sample Data}

\author{
Measured \\ Radiocarbon Age
}

$13 \mathrm{C} / 12 \mathrm{C}$

Ratio
Conventional

Radiocarbon Age $\left(^{*}\right)$

\section{Beta - 344096}

$340+/-30$ BP

$-26.8 \mathrm{o} / \mathrm{oo}$

$310+/-30 \mathrm{BP}$

SAMPLE : 41PN175-1982-5

ANALYSIS : AMS-Standard delivery

MATERIAL/PRETREATMENT : (charred material): acid/alkali/acid

2 SIGMA CALIBRATION : $\quad$ Cal AD 1480 to 1650 (Cal BP 470 to 300)

Beta - 344097

$150+/-30 \mathrm{BP}$

$-25.4 \mathrm{o} / \mathrm{oo}$

$140+/-30 \mathrm{BP}$

SAMPLE : 41PN175-1982-7

ANALYSIS : AMS-Standard delivery

MATERIAL/PRETREATMENT : (charred material): acid/alkali/acid

2 SIGMA CALIBRATION : $\quad$ Cal AD 1670 to 1780 (Cal BP 280 to 170) AND Cal AD 1800 to 1890 (Cal BP 150 to 60)

Cal AD 1900 to 1950 (Cal BP 50 to 0) AND Cal AD 1950 to post 1950 (Cal BP 0 to post 1950)

Beta -344098

$1650+/-30 \mathrm{BP}$

$-25.8 \mathrm{o} / \mathrm{oo}$

$1640+/-30 \mathrm{BP}$

SAMPLE : 41PN175-1982-12

ANALYSIS : AMS-Standard delivery

MATERIAL/PRETREATMENT : (charred material): acid/alkali/acid

2 SIGMA CALIBRATION : $\quad$ Cal AD 350 to 370 (Cal BP 1600 to 1580) AND Cal AD 380 to 440 (Cal BP 1570 to 1510)

Cal AD 490 to 510 (Cal BP 1460 to 1440) AND Cal AD 520 to 530 (Cal BP 1430 to 1420)

Beta -344099

$1790+/-30$ BP

$-23.3 \mathrm{o} / \mathrm{oo}$

$1820+/-30$ BP

SAMPLE : 41PN175-1982-14

ANALYSIS : AMS-Standard delivery

MATERIAL/PRETREATMENT : (charred material): acid/alkali/acid

2 SIGMA CALIBRATION : $\quad$ Cal AD 130 to 250 (Cal BP 1820 to 1700)

Dates are reported as RCYBP (radiocarbon years before present, "present" = AD 1950). By international convention, the modern reference standard was $95 \%$ the $14 \mathrm{C}$ activity of the National Institute of Standards and Technology (NIST) Oxalic Acid (SRM 4990C) and calculated using the Libby $14 \mathrm{C}$ half-life (5568 years). Quoted errors represent 1 relative standard deviation statistics (68\% probability) counting errors based on the combined measurements of the sample, background, and modern reference standards. Measured $13 \mathrm{C} / 12 \mathrm{C}$ ratios (delta $13 \mathrm{C}$ ) were calculated relative to the PDB-1 standard.
The Conventional Radiocarbon Age represents the Measured Radiocarbon Age corrected for isotopic fractionation, calculated using the delta $13 \mathrm{C}$. On rare occasion where the Conventional Radiocarbon Age was calculated using an assumed delta 13C, the ratio and the Conventional Radiocarbon Age will be followed by "*". The Conventional Radiocarbon Age is not calendar calibrated. When available, the Calendar Calibrated result is calculated from the Conventional Radiocarbon Age and is listed as the "Two Sigma Calibrated Result" for each sample. 


\section{REPORT OF RADIOCARBON DATING ANALYSES}

\section{Sample Data}

\author{
Measured \\ Radiocarbon Age
}

$13 \mathrm{C} / 12 \mathrm{C}$

Ratio
Conventional

Radiocarbon Age $\left(^{*}\right)$

Beta -344100

$560+/-30$ BP

$-25.6 \mathrm{o} / \mathrm{oo}$

$550+/-30 \mathrm{BP}$

SAMPLE : 41PN175-1984-5

ANALYSIS : AMS-Standard delivery

MATERIAL/PRETREATMENT : (charred material): acid/alkali/acid

2 SIGMA CALIBRATION : $\quad$ Cal AD 1320 to 1350 (Cal BP 630 to 600) AND Cal AD 1390 to 1430 (Cal BP 560 to 520)

Beta - 344101

$360+/-30 \mathrm{BP}$

$-25.4 \mathrm{o} / \mathrm{oo}$

$350+/-30 \mathrm{BP}$

SAMPLE : 41PN175-1984-8

ANALYSIS : AMS-Standard delivery

MATERIAL/PRETREATMENT : (charred material): acid/alkali/acid

2 SIGMA CALIBRATION : $\quad$ Cal AD 1450 to 1640 (Cal BP 500 to 310)

Beta -344102

$580+/-30$ BP

$-24.9 \mathrm{o} / \mathrm{oo}$

$580+/-30 \mathrm{BP}$

SAMPLE : 41PN175-1984-12

ANALYSIS : AMS-Standard delivery

MATERIAL/PRETREATMENT : (charred material): acid/alkali/acid

2 SIGMA CALIBRATION : $\quad$ Cal AD 1300 to 1370 (Cal BP 650 to 580) AND Cal AD 1380 to 1420 (Cal BP 570 to 530)

Dates are reported as RCYBP (radiocarbon years before present, "present" = AD 1950). By international convention, the modern reference standard was $95 \%$ the $14 \mathrm{C}$ activity of the National Institute of Standards and Technology (NIST) Oxalic Acid (SRM 4990C) and calculated using the Libby $14 \mathrm{C}$ half-life (5568 years). Quoted errors represent 1 relative standard deviation statistics (68\% probability) counting errors based on the combined measurements of the sample, background, and modern reference standards. Measured $13 \mathrm{C} / 12 \mathrm{C}$ ratios (delta 13C) were calculated relative to the PDB-1 standard.
The Conventional Radiocarbon Age represents the Measured Radiocarbon Age corrected for isotopic fractionation, calculated using the delta 13C. On rare occasion where the Conventional Radiocarbon Age was calculated using an assumed delta 13C, the ratio and the Conventional Radiocarbon Age will be followed by "*”. The Conventional Radiocarbon Age is not calendar calibrated. When available, the Calendar Calibrated result is calculated from the Conventional Radiocarbon Age and is listed as the "Two Sigma Calibrated Result" for each sample. 


\section{CALIBRATION OF RADIOCARBON AGE TO CALENDAR YEARS}

\section{(V ariables: C13/C12=-26.3:lab. mult $=1$ )}

\section{Laboratory number: Beta-344076}

\section{Conventional radiocarbon age: $\quad \mathbf{5 9 0 \pm 3 0} \mathrm{BP}$}

2 Sigma calib rated results: Cal AD 1300 to 1370 (Cal BP 650 to 580) and (95\% probability) Cal AD 1380 to 1410 (Cal BP 570 to 540)

Intercept data

Intercepts of radiocarbon age with calibration curve:

Cal AD 1320 (Cal BP 630) and

Cal AD 1340 (Cal BP 610) and

Cal AD 1390 (Cal BP 560)

1 Sigma calibrated results: Cal AD 1310 to 1360 (Cal BP 640 to 590) and

(68\% probability) Cal AD 1390 to 1400 (Cal BP 560 to 540)



References:

Database used INTCALO9

References to INTCALO9 database

Heaton,et.al.,2009, Radiocarbon 51(4): 1151-1164, Reimer,et.al, 2009, Radiocarbon 51(4):1111-1150, Stuiver, et.al,1993, Radiocarbon 35(1):13 7-1 89, Oesch ger,et.al., 1975,Tellus 27:168-192

Mathematics used for calibration scenario A Simplified Approach to Calibrating C14 Dates Talma, A. S., Vogel, J. C., 1993, Radiocarbon 35(2):317-322 


\section{CALIBRATION OF RADIOCARBON AGE TO CALENDAR YEARS}

\section{(V ariables: C13/C12=-27.2:lab. mult $=1$ )}

\section{Laboratory number: Beta-344077}

\section{Conventional radiocarbon age: $\quad 430 \pm 30$ BP}

2 Sigma calibrated result: Cal AD 1430 to 1480 (Cal BP 520 to 470) (95\% probability)

Intercept data

Intercept of radiocarbon age

with calibration curve: Cal AD 1450 (Cal BP 500)

1 Sigma calibrated result: Cal AD 1440 to 1450 (Cal BP 510 to 500)

(68\% probability)

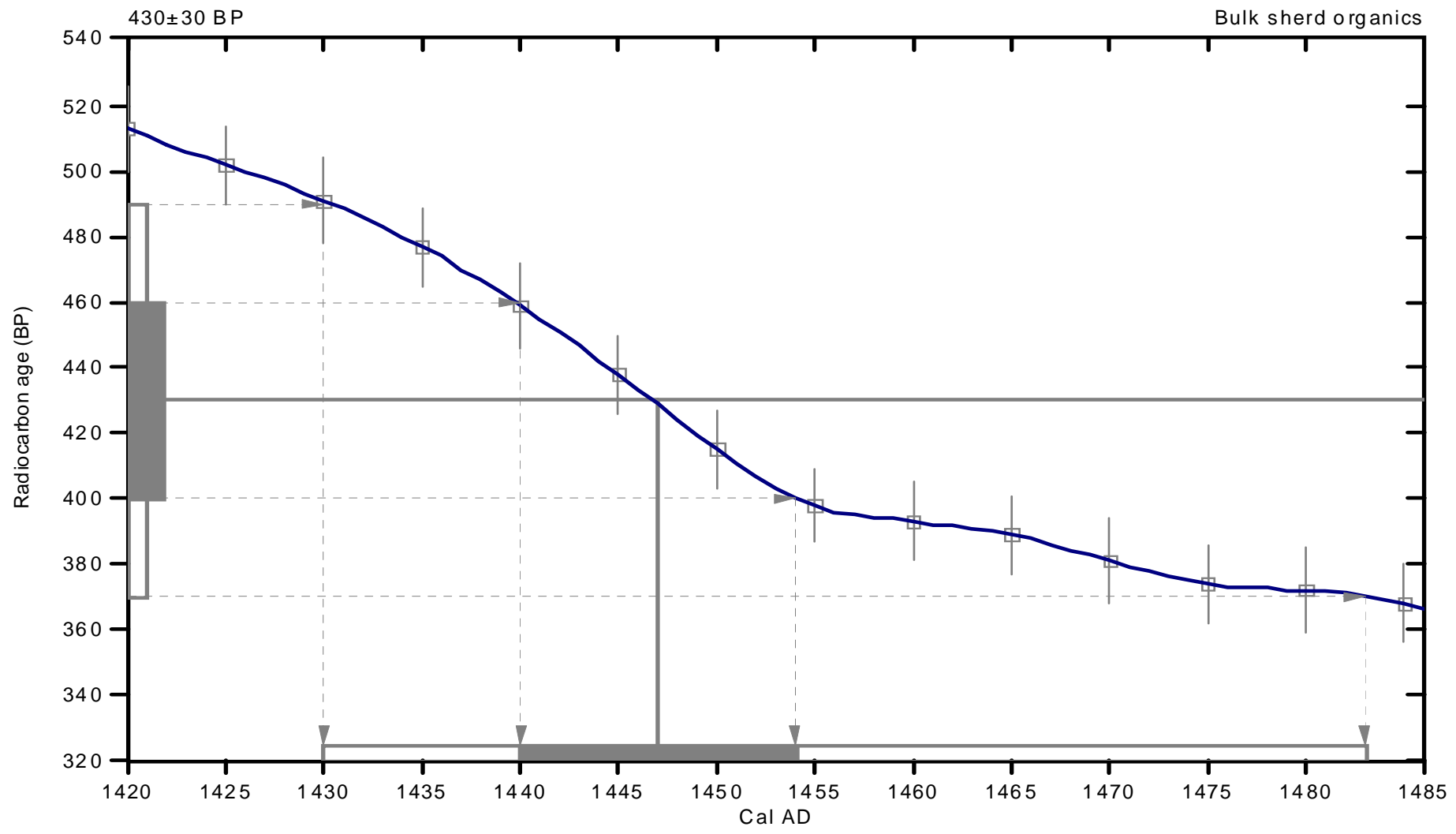

References:

Database used INTCALO 9

References to INTCALO9 database

Heaton, et.al.,2009, Radiocarbon 51(4):1151-1164, Reimer,et.al, 2009, Radiocarbon 51(4):1111-1150, Stuiver, et.al,1993, Radiocarbon 35(1):13 7-189, Oeschger,et.al., 1975, Tellus 27:168-192

Mathematics used for calibration scenario A Simplified Approach to Calibrating C14 Dates Talma, A. S., Vogel, J. C., 1993, Radiocarbon 35(2):317-322 


\section{CALIBRATION OF RADIOCARBON AGE TO CALENDAR YEARS}

\section{(V ariables: C13/C12=-24.6:lab. mult $=1$ )}

\section{Laboratory number: Beta-344078}

\section{Conventional radiocarbon age: $730 \pm 30 \mathrm{BP}$}

\section{Sigma calibrated result: Cal AD 1260 to 1290 (Cal BP 690 to 660) (95\% probability)}

Intercept data

Intercept of radiocarbon age

with calibration curve:

Cal AD 1280 (Cal BP 670)

1 Sigma calibrated result: Cal AD 1270 to 1280 (Cal BP 680 to 670)

(68\% probability)

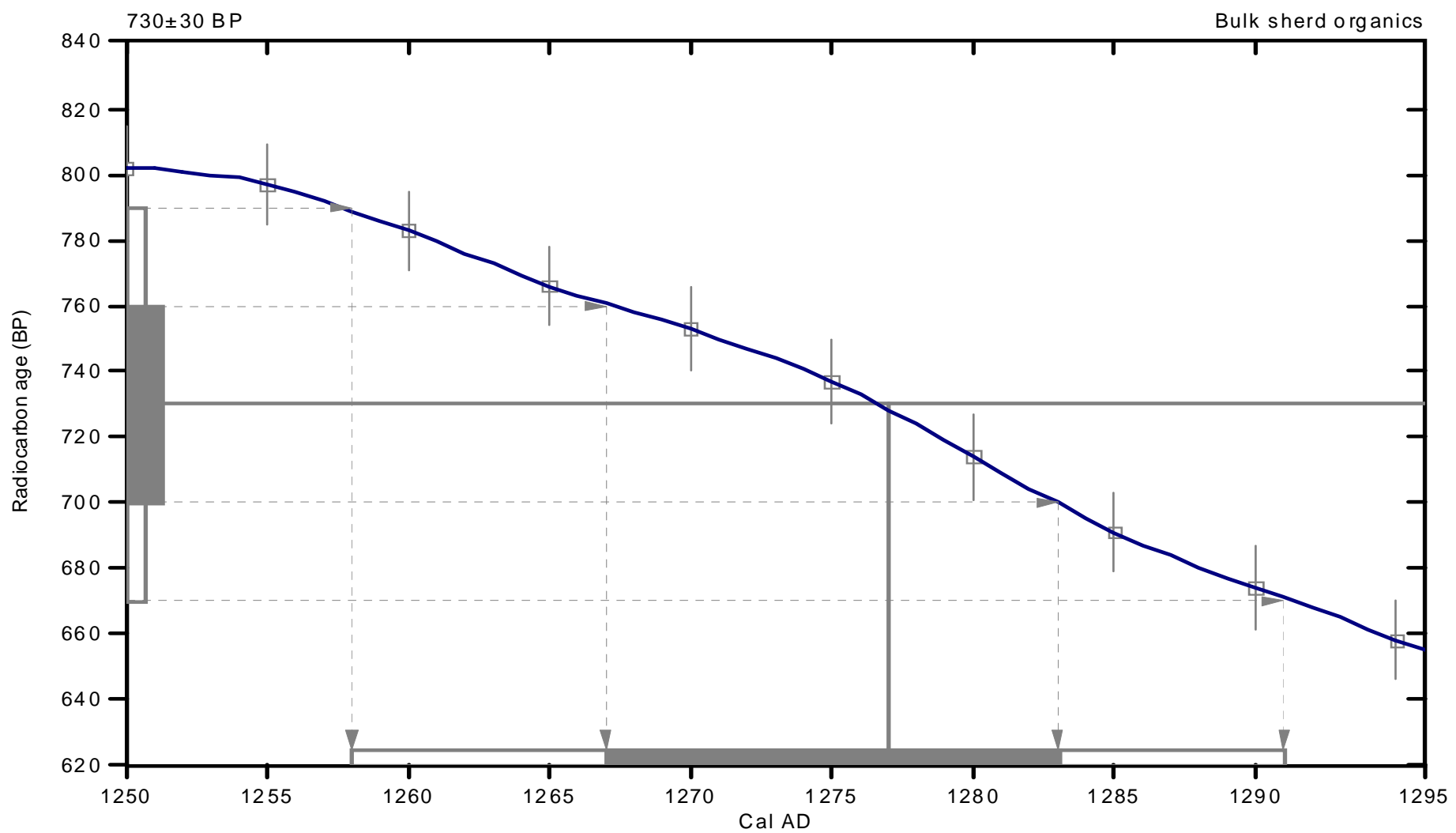

References:

Database used INTCALO9

References to INTCALO9 database

Heaton,et.al.,2009, Radiocarbon 51(4): 1151-1164, Reimer,et.al, 2009, Radiocarbon 51(4):1111-1150, Stuiver, et.al,1993, Radiocarbon 35(1):13 7-189, Oesch ger,et.al., 1975,Tellus 27:168-192

Mathematics used for calibration scenario A Simplified Approach to Calibrating C14 Dates Talma, A. S., Vogel, J. C., 1993, Radiocarbon 35(2):317-322 


\section{CALIBRATION OF RADIOCARBON AGE TO CALENDAR YEARS}

(Variables: C13/C 12=-26.7:lab. mult $=1$ )

Laboratory number: Beta-344079

Conventional radiocarbon age: $1030 \pm 30 \mathrm{BP}$

2 Sigma calibrated result: Cal A D 970 to 1030 (Cal BP 980 to 920 ) (95\% probability)

Intercept data

Intercept of radiocarbon age

with calibration curve: Cal AD 1020 (Cal BP 930)

1 Sigma calibrated result: Cal AD 990 to 1020 (Cal B P 960 to 930)

(68\% probability)

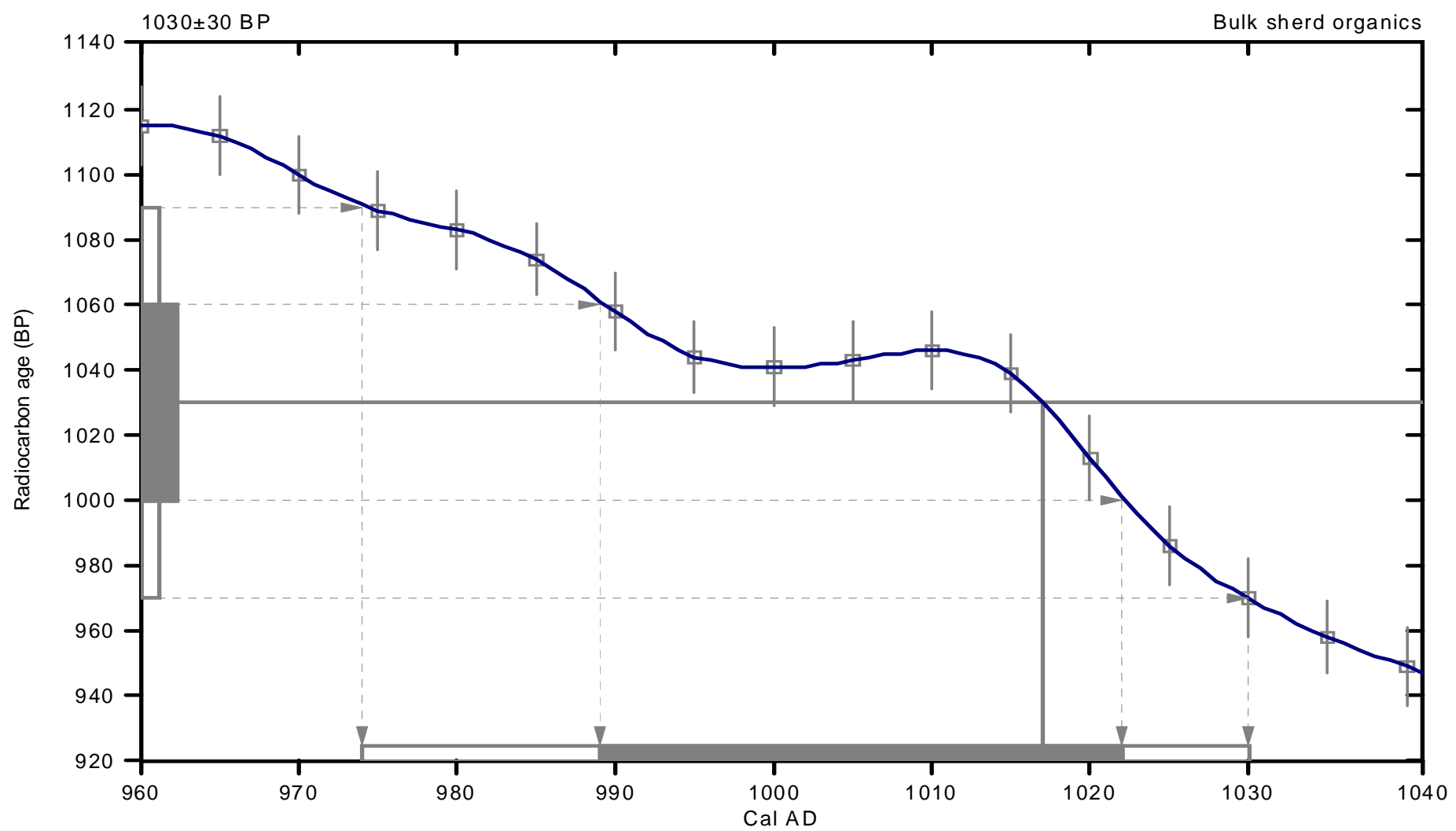

References:

Database used INTCALO9

References to INTCALO9 database

Heaton,et.al.,2009, Radiocarbon 51(4):1151-1164, Reimer,et.al, 2009, Radiocarbon 51(4):1111-1150,

Stuiver,et.al,1993, Radiocarbon 35(1):137-189, Oeschger,et.al.,1975,Tellus 27:168-192

Mathematics used for calibration scenario

A Simplified Approach to Calibrating C14 Dates

Talma, A. S., Vogel, J. C., 1993, Radiocarbon 35(2):317-322

\section{Beta Analytic Radiocarbon Dating Laboratory}

4985 S.W.74th Court, Miami, Florida 33155 -Tel: (305)667-5167•Fax: (305)663-0964・E-Mail: beta@ radiocarbon.com 


\section{CALIBRATION OF RADIOCARBON AGE TO CALENDAR YEARS}

\section{(V ariables: C13/C12=-25.5:lab. mult $=1$ )}

\section{Laboratory number: Beta-344080}

\section{Conventional radiocarbon age: $\quad 350 \pm 30$ BP}

2 Sigma calibrated result: Cal AD 1450 to 1640 (Cal BP 500 to 310 ) (95\% probability)

Intercept data

Intercepts of radiocarbon age with calibration curve:

Cal AD 1500 (Cal BP 450) and

Cal AD 1500 (Cal BP 450) and

Cal AD 1510 (Cal BP 440) and

Cal AD 1600 (Cal BP 350) and

Cal AD 1620 (Cal BP 330)

1 Sigma calibrated results: Cal AD 1470 to 1520 (Cal BP 480 to 420) and

(68\% probability) Cal AD 1560 to 1630 (Cal BP 390 to 320)

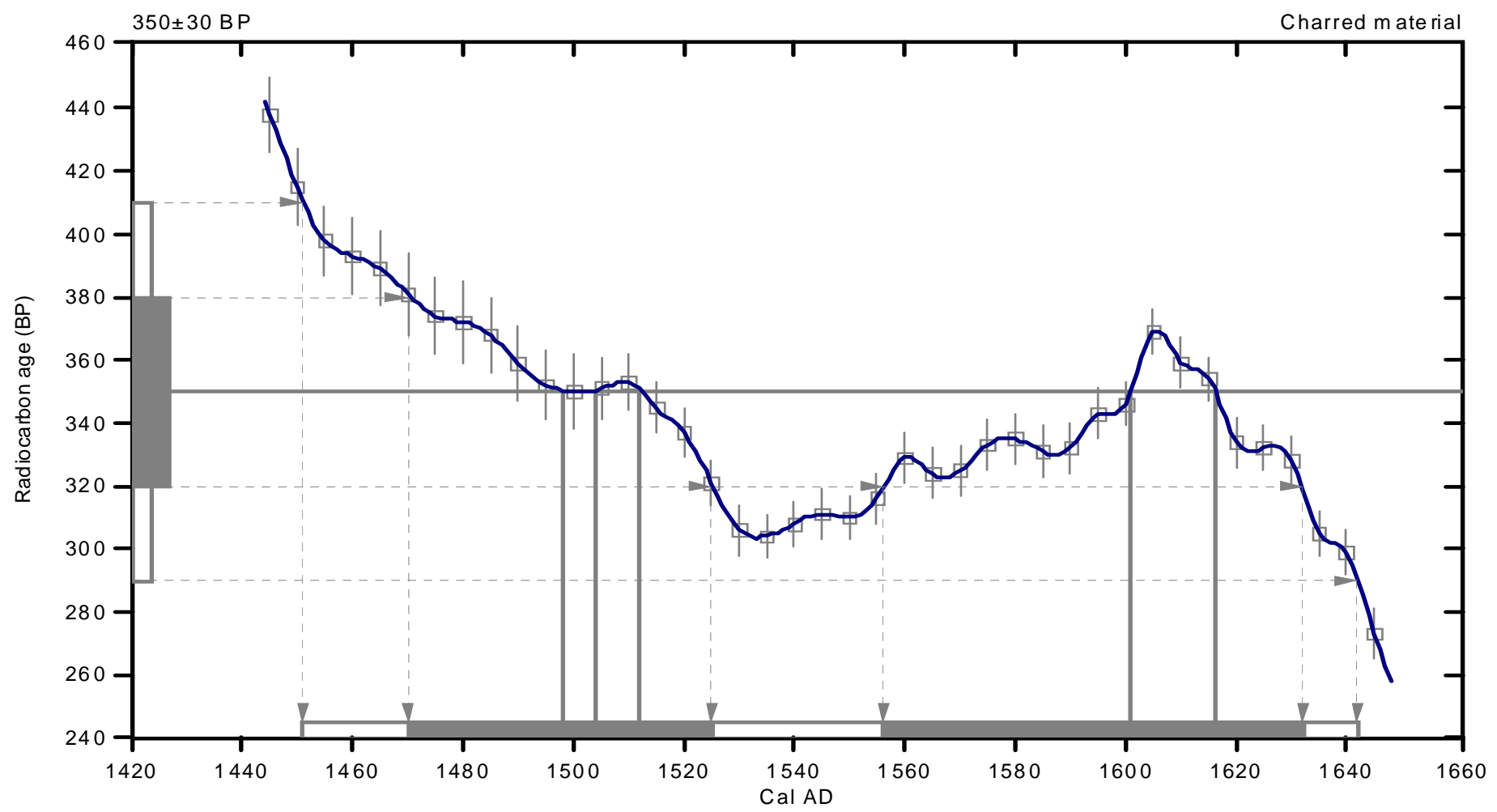

References:

Database used INTCALO 9

References to INTCALO9 database

Heaton, et.al.,2009, Radiocarbon 51(4):1151-1164, Reimer,et.al, 2009, Radiocarbon 51(4):1111-1150, Stuiver, et.al,1993, Radiocarbon 35(1):13 7-189, Oeschger,et.al., 1975, Tellus 27:168-192

Mathematics used for calibration scenario A Simplified Approach to Calibrating C14 Dates Talma, A. S., Vogel, J. C., 1993, Radiocarbon 35(2):317-322 


\section{CALIBRATION OF RADIOCARBON AGE TO CALENDAR YEARS}

\section{(V ariables: C13/C12=-26.4:lab. mult $=1$ )}

\section{Laboratory number: Beta-344081}

\section{Conventional radiocarbon age: $\quad 330 \pm 30$ BP}

2 Sigma calibrated result: Cal AD 1460 to 1650 (Cal BP 490 to 300 ) (95\% probability)

In tercept data

Intercepts of radiocarbon age with calibration curve:

Cal AD 1520 (Cal BP 430) and

Cal AD 1570 (Cal BP 380) and

Cal AD 1590 (Cal BP 360) and

Cal AD 1590 (Cal BP 360) and

Cal AD 1630 (Cal BP 320)

1 Sigma calibrated results: Cal AD 1490 to 1600 (Cal BP 460 to 350) and (68\% probability) Cal AD 1610 to 1640 (Cal BP 340 to 310)

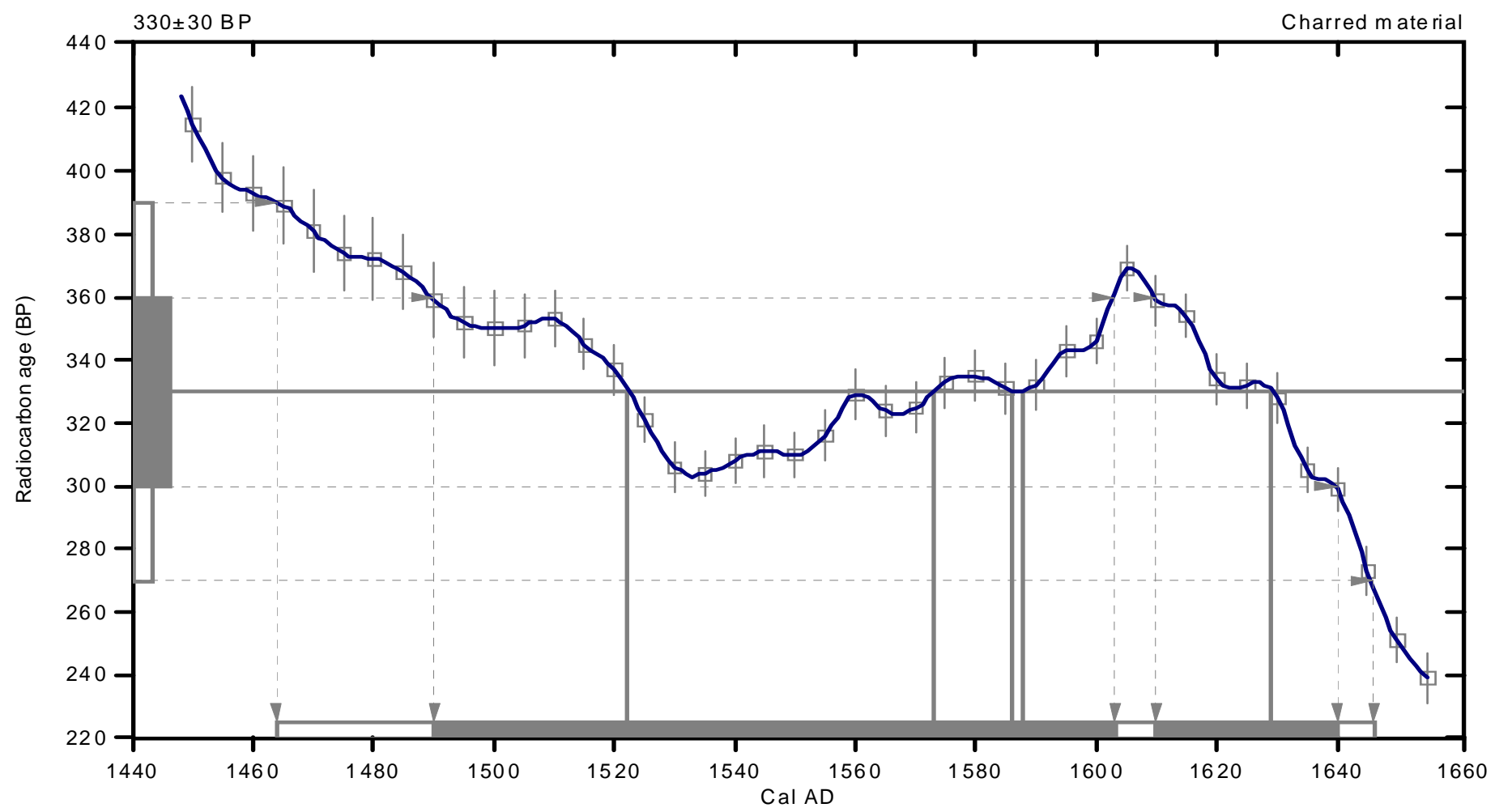

References:

Database used INTCALO 9

References to INTCALO9 database

Heaton, et.al.,2009, Radiocarbon 51(4):1151-1164, Reimer,et.al, 2009, Radiocarbon 51(4):1111-1150, Stuiver, et.al,1993, Radiocarbon 35(1):13 7-189, Oeschger,et.al., 1975, Tellus 27:168-192

Mathematics used for calibration scenario A Simplified Approach to Calibrating C14 Dates Talma, A. S., Vogel, J. C., 1993, Radiocarbon 35(2):317-322

\section{Beta Analytic Radiocarbon Dating Laboratory}




\section{CALIBRATION OF RADIOCARBON AGE TO CALENDAR YEARS}

\section{(V ariables: C13/C12=-23.6:lab. mult $=1$ )}

\section{Laboratory number: Beta-344082}

\section{Conventional radiocarbon age: $\quad 370 \pm 30 \mathrm{BP}$}

2 Sigma calib rated results: Cal AD 1450 to 1530 (Cal BP 500 to 420 ) and (95\% probability) Cal AD 1540 to 1550 (Cal BP 410 to 400) and

Cal AD 1550 to 1630 (Cal BP 400 to 320)

Intercept data

Intercept of radiocarbon age with calibration curve: Cal AD 1480 (Cal BP 470)

1 Sigma calibrated results: Cal AD 1450 to 1520 (Cal BP 500 to 430) and

(68\% probability) Cal AD 1590 to 1620 (Cal BP 360 to 330)

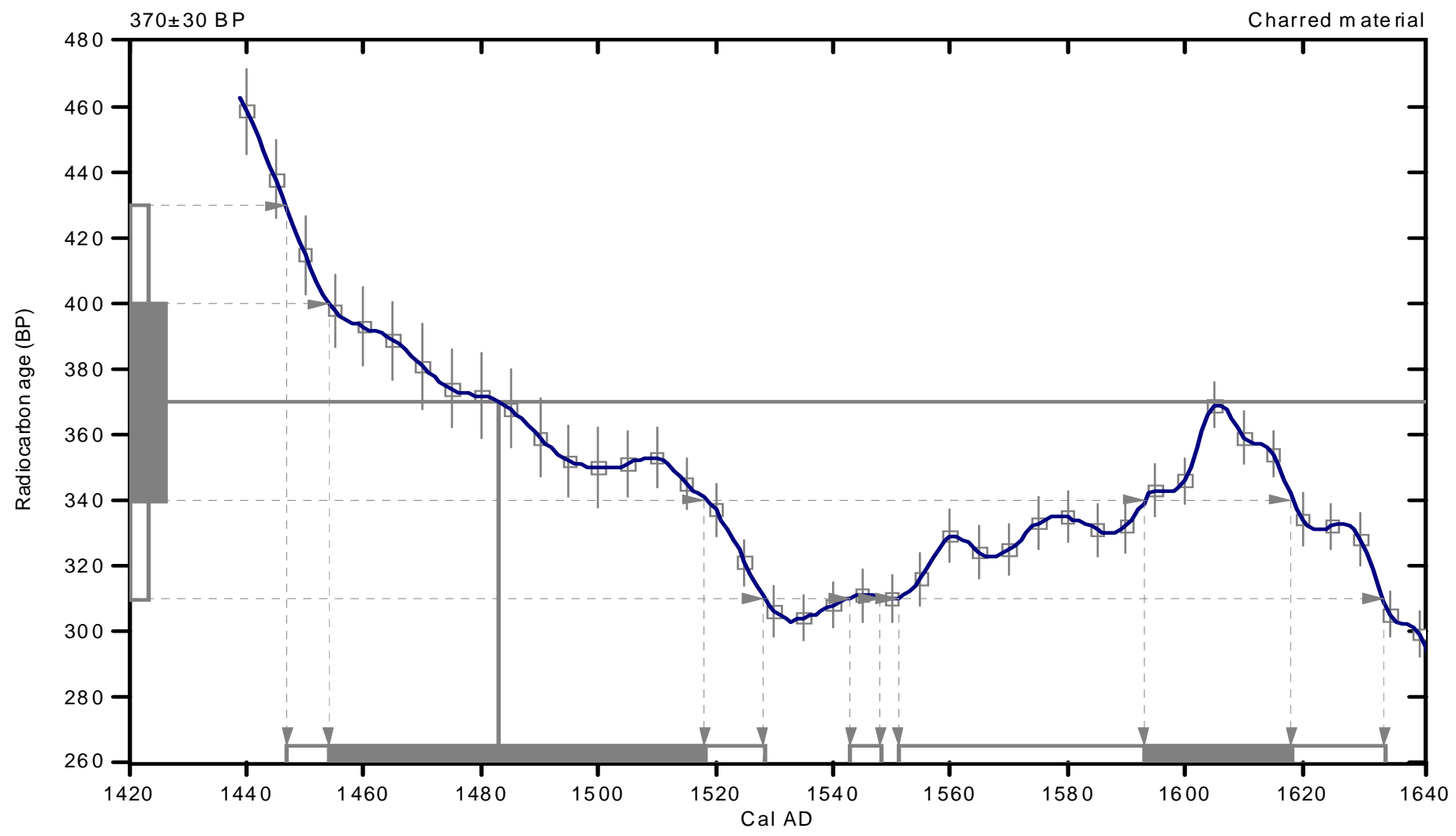

References:

Database used INTCALO9

References to INTCALO9 database

Heaton,et.al.,2009, Radiocarbon 51(4): 1151-1164, Reimer,et.al, 2009, Radiocarbon 51(4):1111-1150, Stuiver, et.al,1993, Radiocarbon 35(1):137-189, Oeschger,et.al., 1975,Tellus 27:168-192

Mathematics used for calibration scenario A Simplified Approach to Calibrating C14 Dates Talma, A. S., Vogel, J. C., 1993, Radiocarbon 35(2):317-322 


\section{CALIBRATION OF RADIOCARBON AGE TO CALENDAR YEARS}

\section{(V ariables: C13/C12=-26.3:lab. mult $=1$ )}

\section{Laboratory number: Beta -344083}

\section{Conventional radiocarbon age: $410 \pm 30$ BP}

2 Sigma calib rated results: Cal AD 1440 to 1500 (Cal BP 510 to 450) and (95\% probability) Cal AD 1500 to 1510 (Cal BP 450 to 440) and

Cal AD 1600 to 1620 (Cal BP 350 to 330)

Intercept data

Intercept of radiocarbon age

with calibration curve: Cal AD 1450 (Cal BP 500)

1 Sigma calibrated result: Cal AD 1440 to 1470 (Cal BP 510 to 480)

(68\% probability)

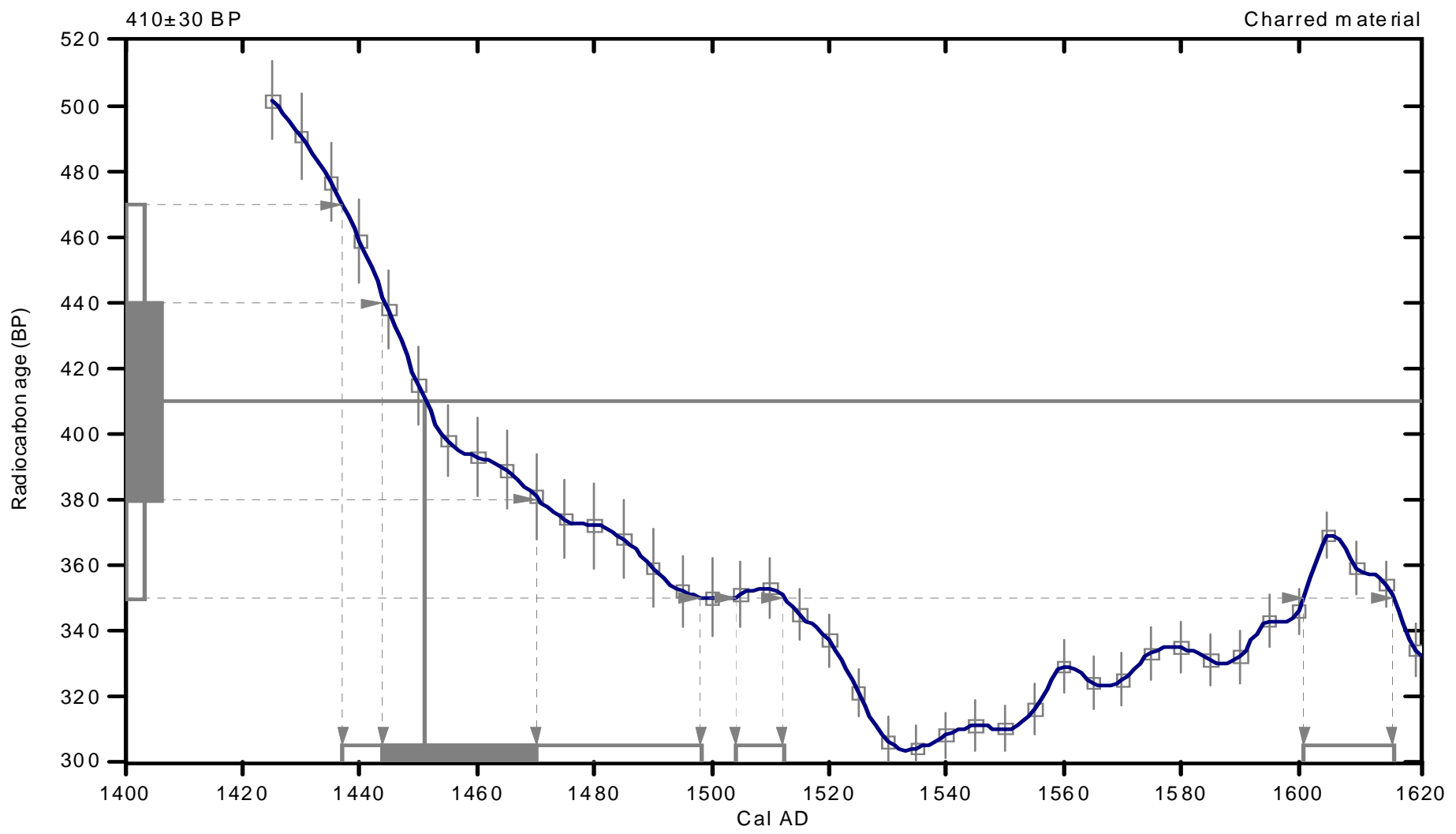

References:

Database used INTCALO9

References to INTCALO9 database

Heaton,et.al.,2009, Radiocarbon 51(4): 1151-1164, Reimer,et.al, 2009, Radiocarbon 51(4):1111-1150, Stuiver, et.al,1993, Radiocarbon 35(1):13 7-189, Oesch ger,et.al., 1975,Tellus 27:168-192

Mathematics used for calibration scenario A Simplified Approach to Calibrating C14 Dates Talma, A. S., Vogel, J. C., 1993, Radiocarbon 35(2):317-322

\section{Beta Analytic Radiocarbon Dating Laboratory}




\section{CALIBRATION OF RADIOCARBON AGE TO CALENDAR YEARS}

\section{(V ariables: C13/C12=-23.5:lab. mult $=1$ )}

\section{Laboratory number: Beta-344084}

\section{Conventional radiocarbon age: $\quad 360 \pm 30 \mathrm{BP}$}

2 Sigma calibrated result: Cal AD 1450 to 1640 (Cal BP 500 to 310 ) (95\% probability)

Intercept data

Intercepts of radiocarbon age with calibration curve:

Cal AD 1490 (Cal BP 460) and

Cal AD 1600 (Cal BP 350) and

Cal AD 1610 (Cal BP 340)

1 Sigma calibrated results: Cal AD 1460 to 1520 (Cal BP 490 to 430) and

(68\% probability) Cal AD 1570 to 1590 (Cal BP 380 to 360) and Cal AD 1590 to 1630 (Cal BP 360 to 320)

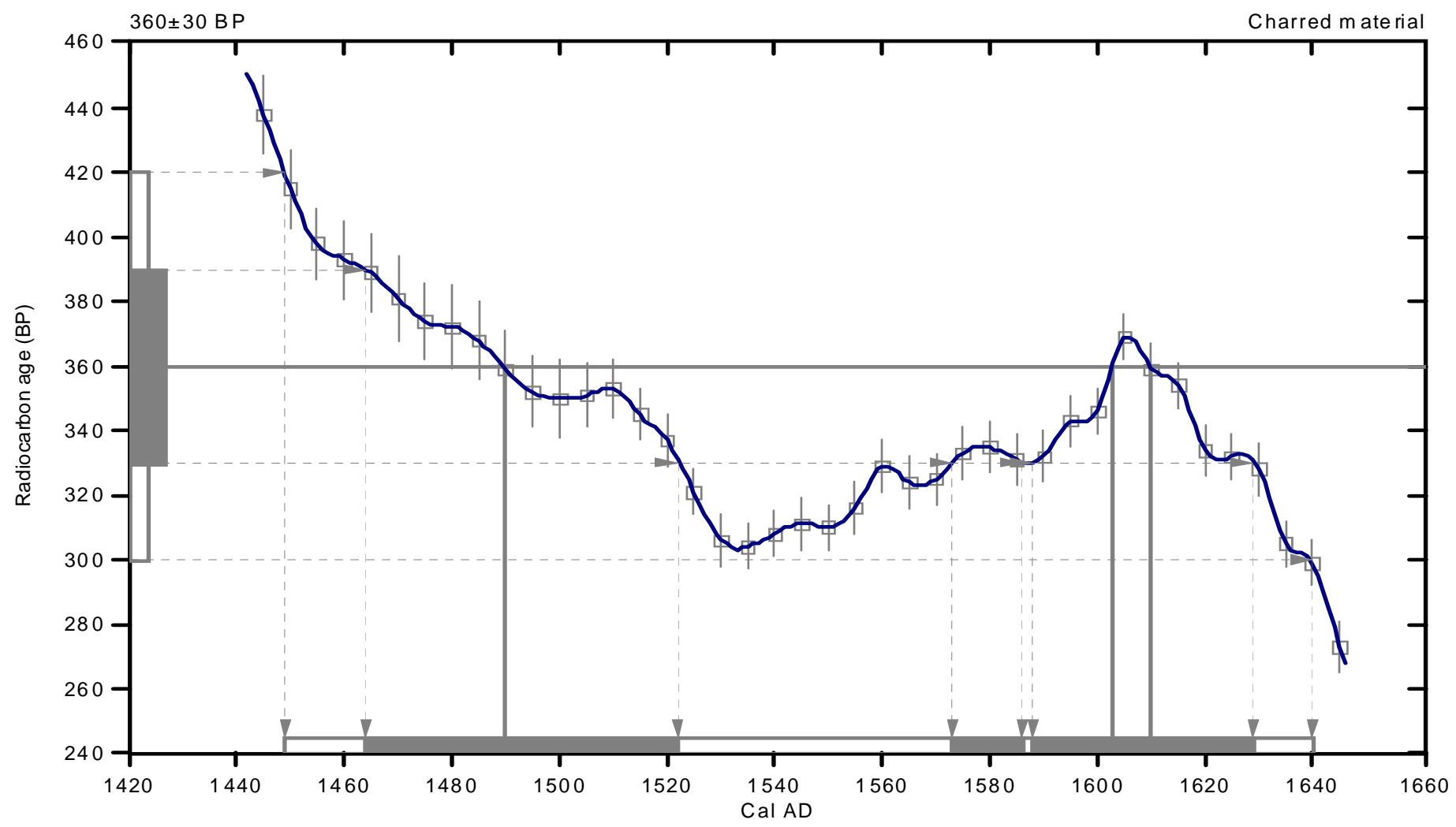

References:

Database used INTCALO 9

References to INTCALO9 database

Heaton,et.al.,2009, Radiocarbon 51(4):1151-1164, Reimer,et.al, 2009, Radiocarbon 51(4):1111-1150, Stuiver, et.al,1993, Radiocarbon 35(1):13 7-189, Oeschger,et.al., 1975, Tellus 27:168-192

Mathematics used for calibration scenario A Simplified Approach to Calibrating C14 Dates Talma, A. S., Vogel, J. C., 1993, Radiocarbon 35(2):317-322 


\section{CALIBRATION OF RADIOCARBON AGE TO CALENDAR YEARS}

(V ariables: C13/C12=-29:1ab. mult=1)

Laboratory number: Beta -344085

Conventional radiocarbon age: $1260 \pm 30$ B P

2 Sigma calibrated results: Cal AD 670 to 780 (Cal BP 1280 to 1170) and (95\% probability) Cal AD 790 to 810 (Cal BP 1160 to 1140) and

Cal AD 850 to 850 (Cal BP 1100 to 1100)

In tercept data

Intercepts of radiocarbon age

with calibration curve: Cal AD 720 (Cal BP 1230) and

Cal AD 740 (Cal BP 1210) and

Cal AD 770 (Cal BP 1180)

1 Sigma calibrated result: Cal AD 690 to 780 (Cal BP 1260 to 1170)

(68\% probability)

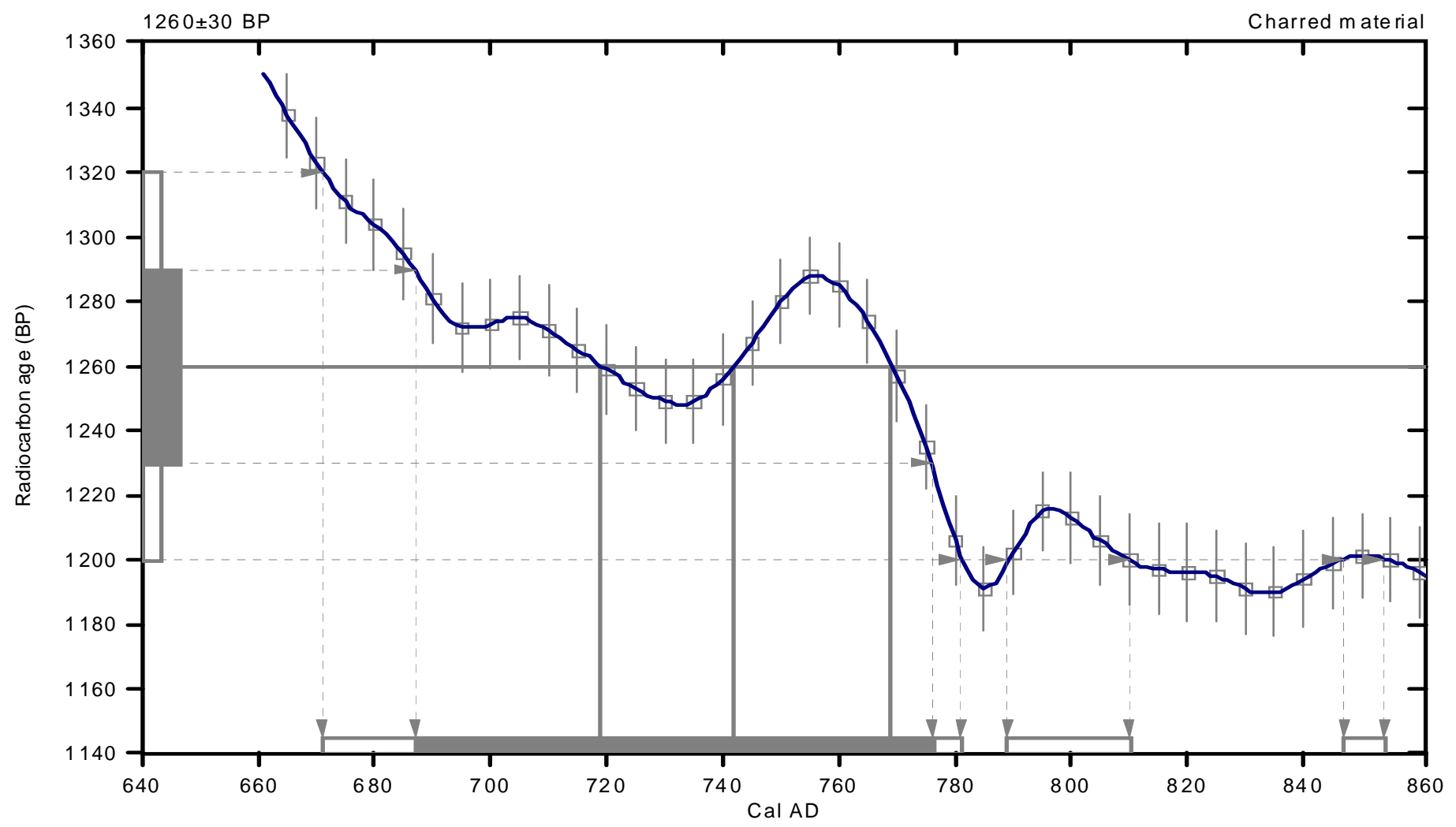

References:

Database used INTCALO9

References to INTCALO9 database

Heaton,et.al.,2009, Radiocarbon 51(4): 1151-1164, Reimer,et.al, 2009, Radiocarbon 51(4):1111-1150,

Stuiver, et.al,1993, Radiocarbon 35(1):13 7-189, Oeschger,et.al., 1975,Tellus 27:168-192

Mathematics used for calibration scenario A Simplified Approach to Calibrating C14 Dates

Talma, A. S., Vogel, J. C., 1993, Radiocarbon 35(2):317-322 


\section{CALIBRATION OF RADIOCARBON AGE TO CALENDAR YEARS}

\section{(V ariables: C13/C12=-26.3:lab. mult $=1$ )}

\section{Laboratory number: Beta-344086}

\section{Conventional radiocarbon age: $420 \pm 30$ BP}

2 Sigma calibrated results: Cal AD 1430 to 1490 (Cal BP 520 to 460) and (95\% probability) Cal AD 1600 to 1610 (Cal BP 350 to 340)

Intercept data

Intercept of radiocarbon age

with calibration curve:

Cal AD 1450 (Cal BP 500)

1 Sigma calibrated result: Cal AD 1440 to 1460 (Cal BP 510 to 490)

(68\% probability)

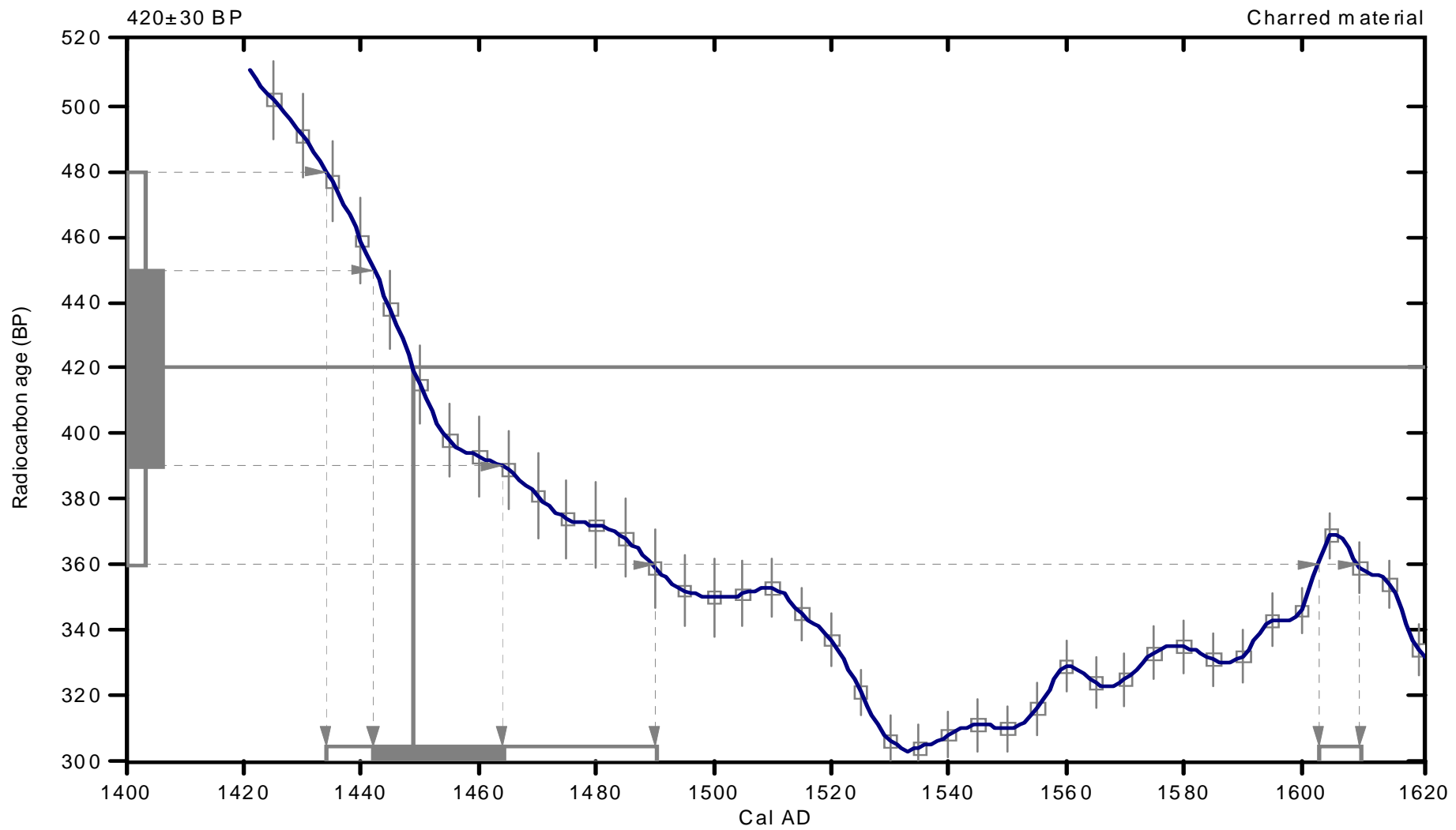

References:

Database used INTCALO9

References to INTCALO9 database

Heaton,et.al.,2009, Radiocarbon 51(4): 1151-1164, Reimer,et.al, 2009, Radiocarbon 51(4):1111-1150, Stuiver, et.al,1993, Radiocarbon 35(1):137-189, Oeschger,et.al., 1975,Tellus 27:168-192

Mathematics used for calibration scenario A Simplified Approach to Calibrating C14 Dates Talma, A. S., Vogel, J. C., 1993, Radiocarbon 35(2):317-322 


\section{CALIBRATION OF RADIOCARBON AGE TO CALENDAR YEARS}

(Variables: C 13/C12=-7.8:1ab. mult $=1$ )

Laboratory number: Beta-344087

Conventional radiocarbon age: $310 \pm 30$ BP

2 Sigma calibrated result: Cal AD 1480 to 1650 (Cal BP 470 to 300 ) (95\% probability)

Intercept data

Intercepts of radiocarbon age

with calibration curve: Cal AD 1530 (Cal BP 420) and

Cal AD 1540 (Cal BP 410) and

Cal AD 1550 (Cal BP 400) and

Cal AD 1550 (Cal BP 400) and

Cal AD 1630 (Cal BP 320)

1 Sigma calibrated results: Cal AD 1520 to 1590 (Cal BP 430 to 360) and

(68\% probability) Cal AD 1620 to 1640 (Cal BP 330 to 310)

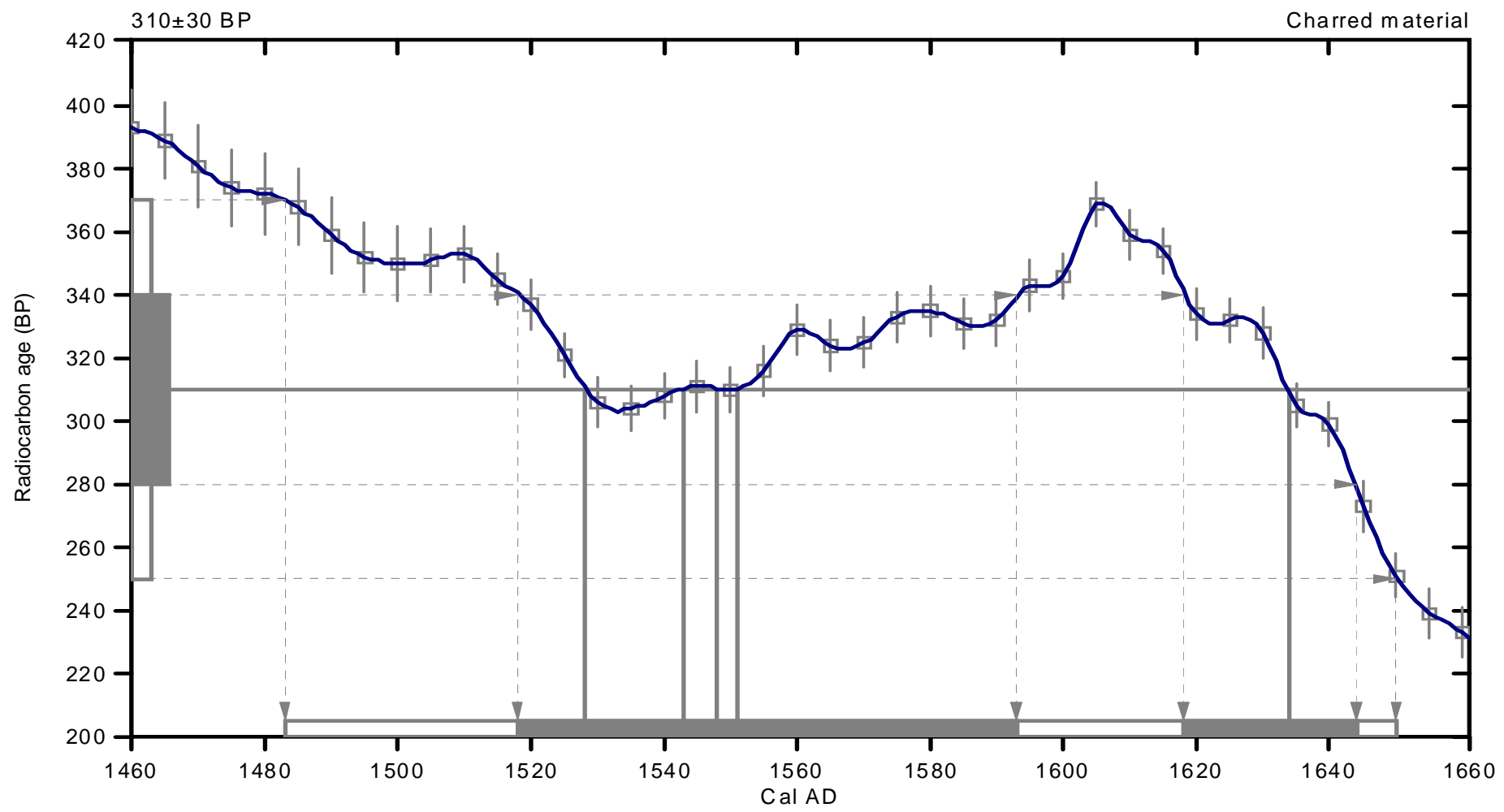

References:

Database used INTCALO9

References to INTCALO9 database

Heaton,et.al.,2009, Radiocarbon 51(4):1151-1164, Reimer,et.al, 2009, Radiocarbon 51(4):1111-1150, Stuiver,et.al,1993, Radiocarbon 35(1):137-189, Oesch ger,et.al.,1975, Tellus 27:168-192

Mathematics used for calibration scenario A Simplified Approach to Calibrating C14 Dates

Talma, A. S., Vogel, J. C., 1993, Radiocarbon 35(2):317-322

\section{Beta Analytic Radiocarbon Dating Laboratory}

4985 S.W.74th Court, Miami, Florida 33155 -Tel: (305)667-5167•Fax: (305)663-0964・E-Mail: beta@ radiocarbon.com 


\section{CALIBRATION OF RADIOCARBON AGE TO CALENDAR YEARS}

\section{(V ariables: C13/C12=-27.1:lab. mult $=1$ )}

\section{Laboratory number: Beta-344088}

\section{Conventional radiocarbon age: $\quad 370 \pm 30 \mathrm{BP}$}

2 Sigma calib rated results: Cal AD 1450 to 1530 (Cal BP 500 to 420 ) and (95\% probability) Cal AD 1540 to 1550 (Cal BP 410 to 400) and

Cal AD 1550 to 1630 (Cal BP 400 to 320)

Intercept data

Intercept of radiocarbon age with calibration curve: Cal AD 1480 (Cal BP 470)

1 Sigma calibrated results: Cal AD 1450 to 1520 (Cal BP 500 to 430) and

(68\% probability) Cal AD 1590 to 1620 (Cal BP 360 to 330)

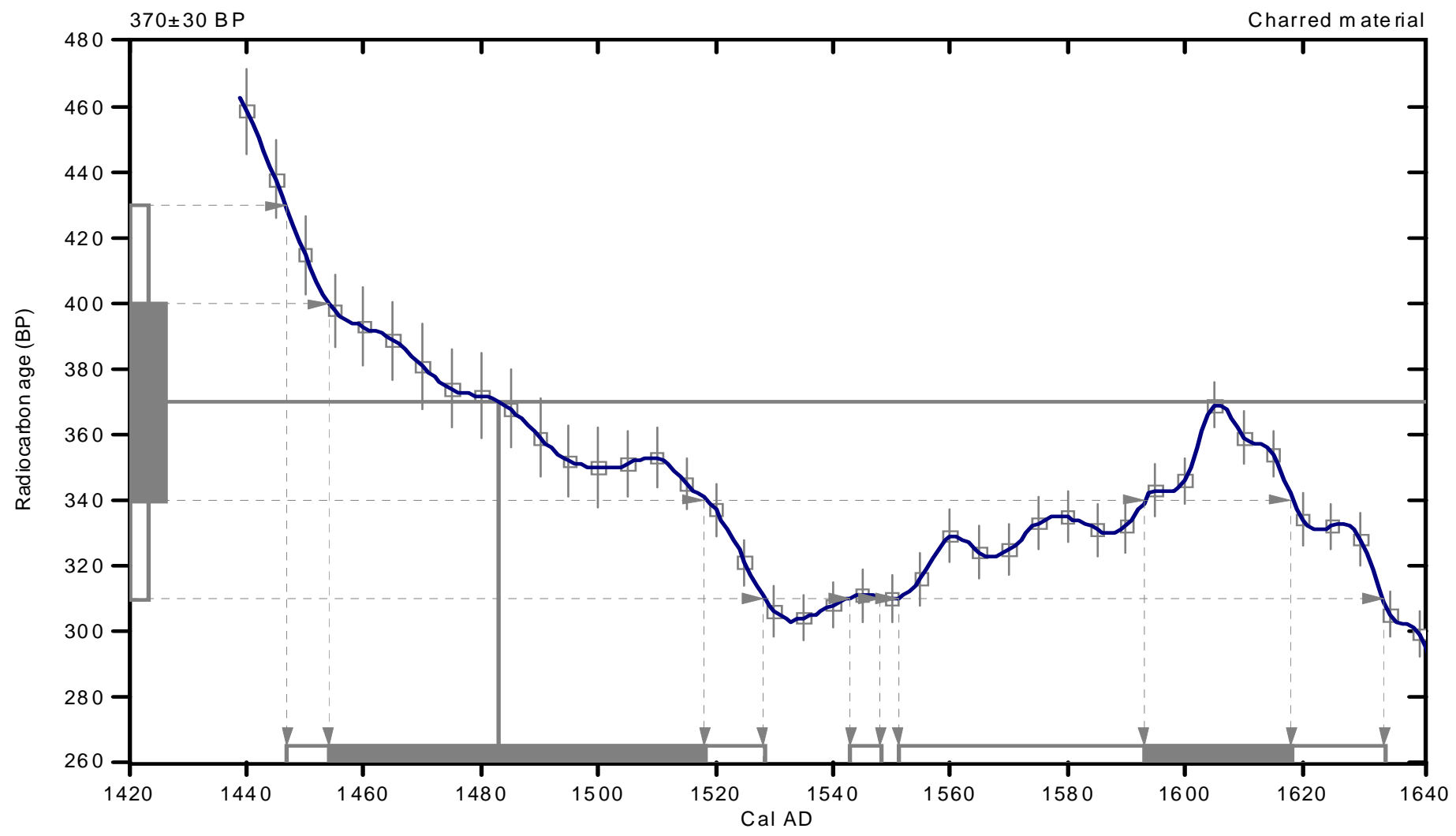

References:

Database used INTCALO9

References to INTCALO9 database

Heaton,et.al.,2009, Radiocarbon 51(4): 1151-1164, Reimer,et.al, 2009, Radiocarbon 51(4):1111-1150, Stuiver, et.al,1993, Radiocarbon 35(1):137-189, Oeschger,et.al., 1975,Tellus 27:168-192

Mathematics used for calibration scenario A Simplified Approach to Calibrating C14 Dates Talma, A. S., Vogel, J. C., 1993, Radiocarbon 35(2):317-322 


\section{CALIBRATION OF RADIOCARBON AGE TO CALENDAR YEARS}

\section{(V ariables: C13/C12=-25.9:lab. mult $=1$ )}

\section{Laboratory number: Beta-344089}

\section{Conventional radiocarbon age: $\quad \mathbf{5 7 0} \pm 30 \mathrm{BP}$}

2 Sigma calibrated results: Cal AD 1300 to 1360 (Cal BP 640 to 590) and (95\% probability) Cal AD 1380 to 1420 (Cal BP 570 to 530)

Intercept data

Intercept of radiocarbon age

with calibration curve:

Cal AD 1400 (Cal BP 550)

1 Sigma calibrated results: Cal AD 1320 to 1350 (Cal BP 630 to 600) and

(68\% probability) Cal AD 1390 to 1410 (Cal BP 560 to 540)

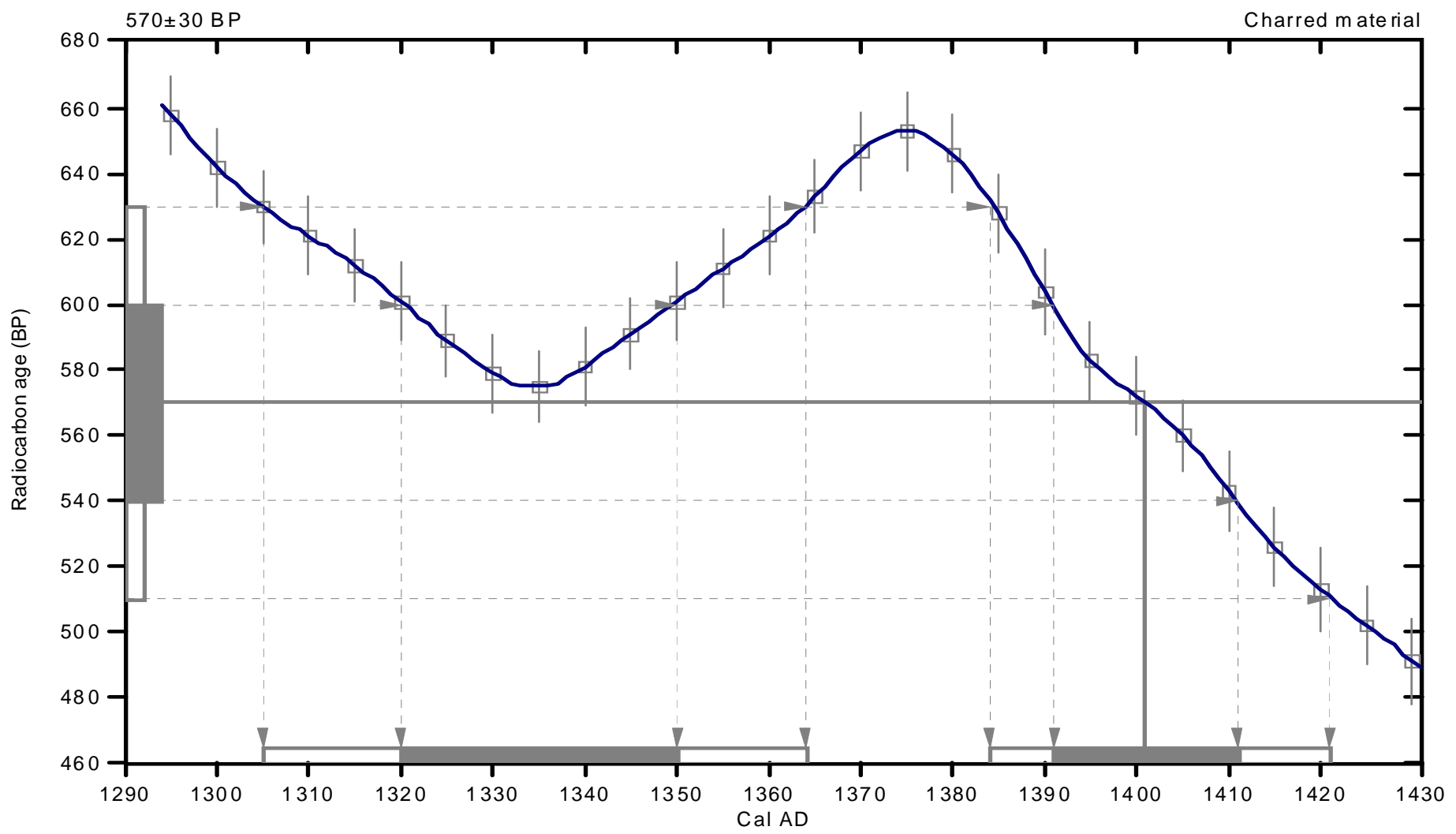

References:

Database used INTCALO9

References to INTCALO9 database

Heaton,et.al.,2009, Radiocarbon 51(4): 1151-1164, Reimer,et.al, 2009, Radiocarbon 51(4):1111-1150, Stuiver, et.al,1993, Radiocarbon 35(1):13 7-1 89, Oesch ger,et.al., 1975,Tellus 27:168-192

Mathematics used for calibration scenario A Simplified Approach to Calibrating C14 Dates Talma, A. S., Vogel, J. C., 1993, Radiocarbon 35(2):317-322 


\section{CALIBRATION OF RADIOCARBON AGE TO CALENDAR YEARS}

\section{(V ariables: C13/C12=-23.4:lab. mult $=1$ )}

\section{Laboratory number: Beta-344090}

\section{Conventional radiocarbon age: $\quad 340 \pm 30$ BP}

\section{Sigma calibrated result: Cal AD 1450 to 1640 (Cal BP 500 to 310 ) (95\% probability)}

In tercept data

Intercepts of radiocarbon age with calibration curve:

Cal AD $1520($ Cal BP 430) and

Cal AD 1590 (Cal BP 360) and

Cal AD 1620 (Cal BP 330)

1 Sigma calibrated results: Cal AD 1480 to 1530 (Cal BP 470 to 420) and

(68\% probability) Cal AD 1540 to 1550 (Cal BP 410 to 400) and Cal AD 1550 to 1630 (Cal BP 400 to 320)

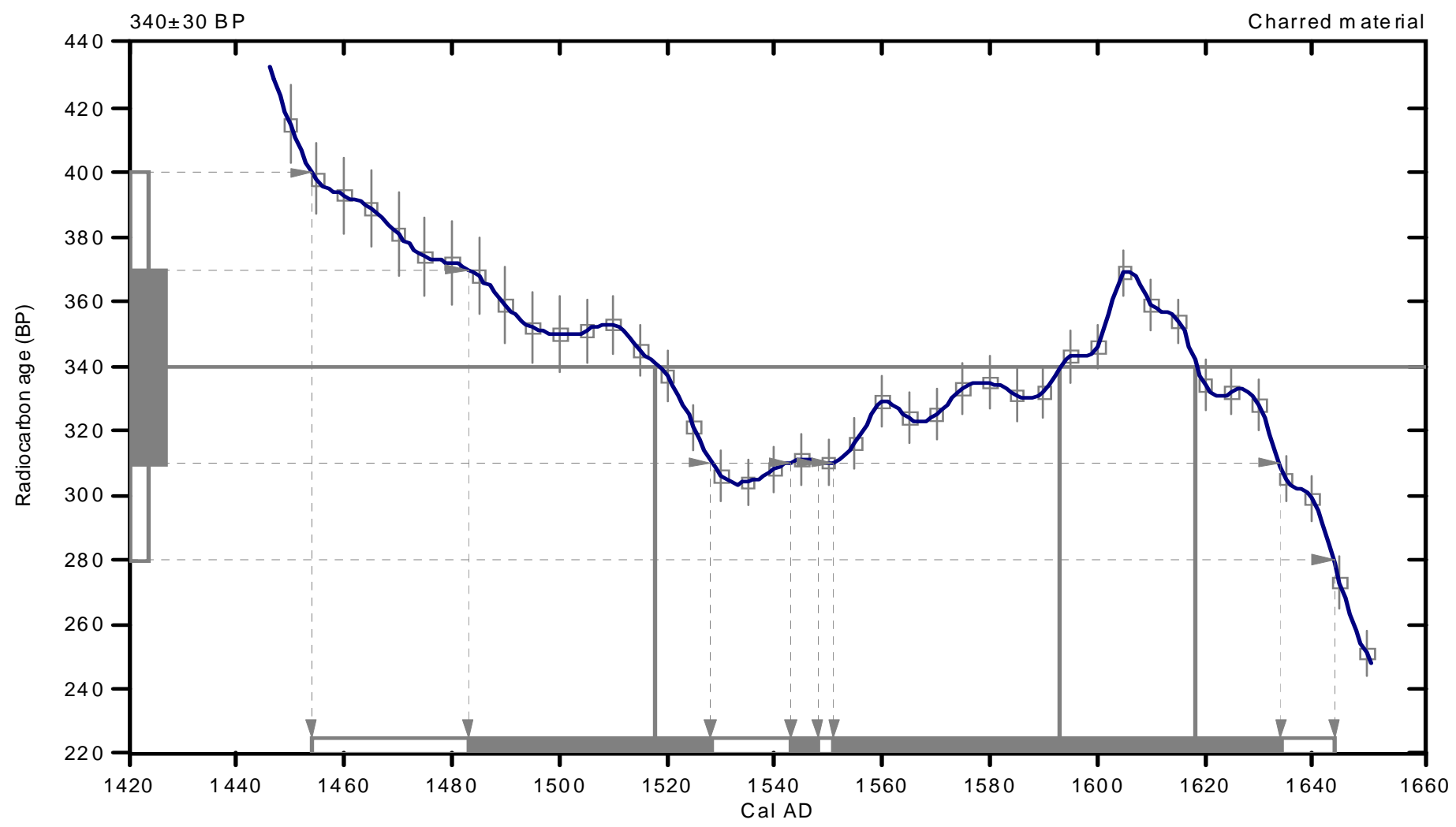

References:

Database used INTCALO 9

References to INTCALO9 da tabase

Heaton,et.al.,2009, Radiocarbon 51(4):1151-1164, Reimer,et.al, 2009, Radiocarbon 51(4):1111-1150, Stuiver, et.al,1993, Radiocarbon 35(1):13 7-189, Oeschger,et.al., 1975, Tellus 27:168-192

Mathematics used for calibration scenario A Simplified Approach to Calibrating C14 Dates Talma, A. S., Vogel, J. C., 1993, Radiocarbon 35(2):317-322 


\section{CALIBRATION OF RADIOCARBON AGE TO CALENDAR YEARS}

(V ariables: C13/C12=-10:1ab. mult $=1$ )

Laboratory number: Beta-344091

Conventional radiocarbon age: $\quad 350 \pm 30 \mathrm{BP}$

2 Sigma calib rated result: Cal AD 1450 to 1640 (Cal BP 500 to 310 ) (95\% probability)

In tercept data

Intercepts of radiocarbon age

with calibration curve: Cal AD 1500 (Cal BP 450) and

Cal AD 1500 (Cal BP 450) and

Cal AD 1510 (Cal BP 440) and

Cal AD 1600 (Cal BP 350) and

Cal AD 1620 (Cal BP 330)

1 Sigma calibrated results: Cal AD 1470 to 1520 (Cal BP 480 to 420) and

(68\% probability) Cal AD 1560 to 1630 (Cal BP 390 to 320)

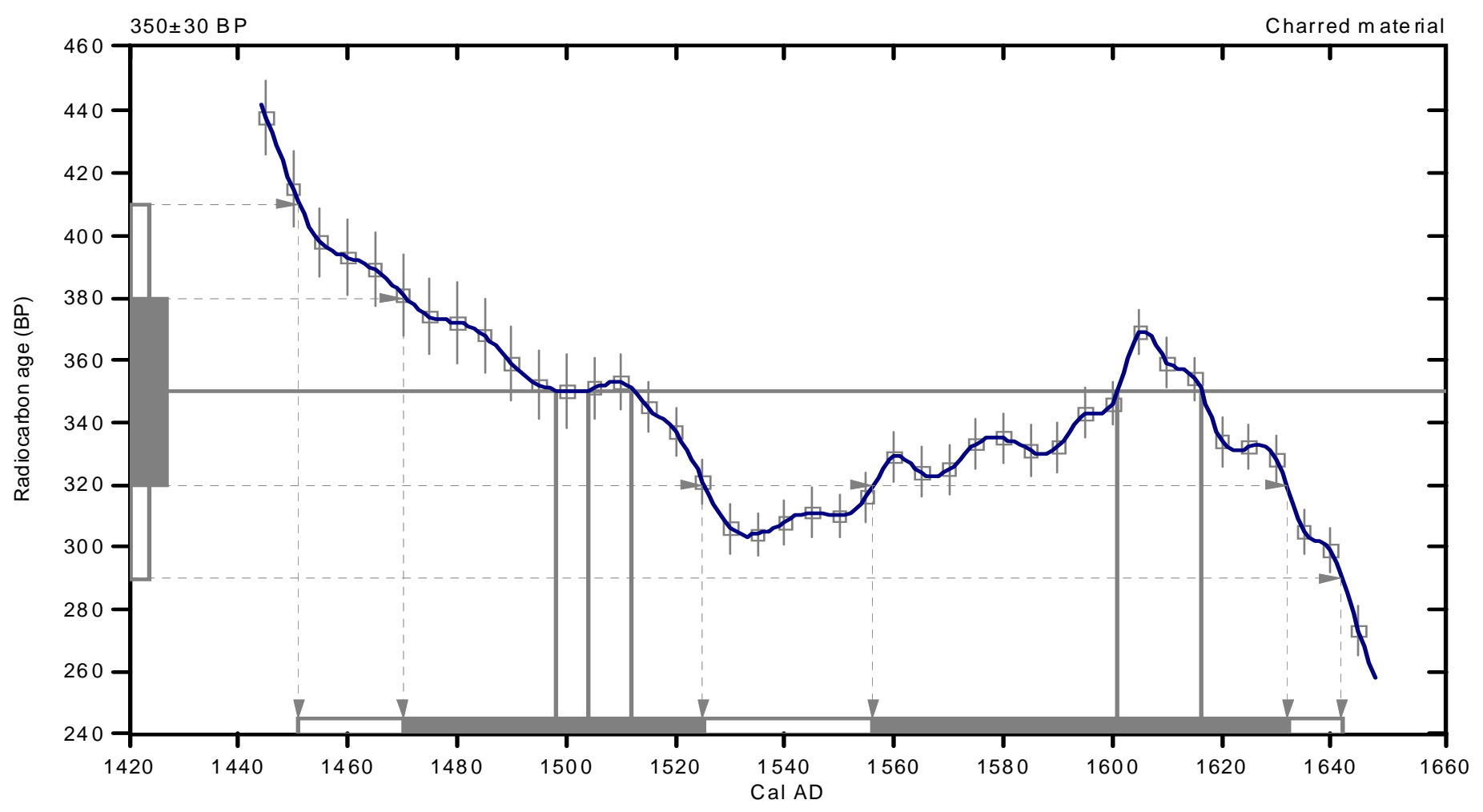

References:

Database used INTCALO9

References to INTCALO9 database

Heaton,et.al.,2009, Radiocarbon 51(4): 1151-1164, Reimer,et.al, 2009, Radiocarbon 51(4):1111-1150,

Stuiver,et.al,1993, Radiocarbon 35(1):13 7-189, Oesch ger,et.al., 1975,Tellus 27:168-192

Mathematics used for calibration scenario

A Simplified Approach to Calibrating C14 Dates

Talma, A. S., Vogel, J. C., 1993, Radiocarbon 35(2):317-322 


\section{CALIBRATION OF RADIOCARBON AGE TO CALENDAR YEARS}

\section{(V ariables: C13/C12=-24.2:lab. mult $=1$ )}

\section{Laboratory number: Beta-344092}

\section{Conventional radiocarbon age: $1230 \pm 30$ B P}

\section{Sigma calibrated result: Cal AD 690 to 880 (Cal BP 1260 to 1060) (95\% probability)}

Intercept data

Intercept of radiocarbon age

with calibration curve:

Cal AD 780 (Cal BP 1170)

1 Sigma calibrated results: Cal AD 720 to 740 (Cal BP 1230 to 1210) and

(68\% probability) Cal AD 770 to 780 (Cal BP 1180 to 1170 ) and

Cal AD 790 to 810 (Cal BP 1160 to 1140 ) and

Cal AD 850 to 850 (Cal BP 1100 to 1100 )

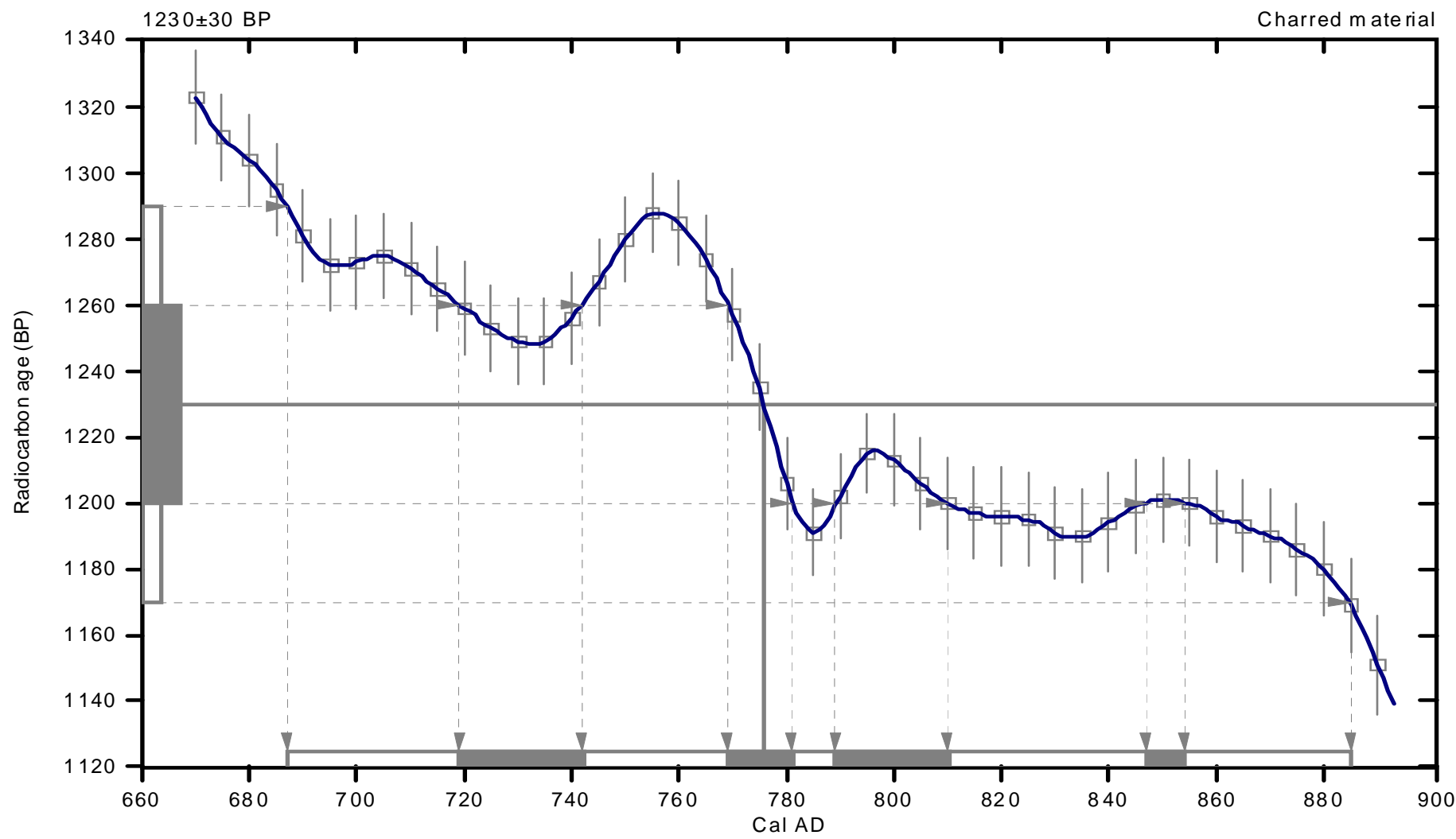

References:

Database used INTCALO9

References to INTCALO9 database

Heaton,et.al.,2009, Radiocarbon 51(4): 1151-1164, Reimer,et.al, 2009, Radiocarbon 51(4):1111-1150, Stuiver, et.al,1993, Radiocarbon 35(1):13 7-189, Oesch ger,et.al., 1975,Tellus 27:168-192

Mathematics used for calibration scenario A Simplified Approach to Calibrating C14 Dates Talma, A. S., Vogel, J. C., 1993, Radiocarbon 35(2):317-322 


\section{CALIBRATION OF RADIOCARBON AGE TO CALENDAR YEARS}

\section{(V ariables: C13/C12=-24.7:lab. mult $=1$ )}

\section{Laboratory number: Beta-344093}

\section{Conventional radiocarbon age: $\quad 360 \pm 30$ BP}

\section{Sigma calibrated result: Cal AD 1450 to 1640 (Cal BP 500 to 310) (95\% probability)}

Intercept data

Intercepts of radiocarbon age with calibration curve:

Cal AD 1490 (Cal BP 460) and

Cal AD 1600 (Cal BP 350) and

Cal AD 1610 (Cal BP 340)

1 Sigma calibrated results: Cal AD 1460 to 1520 (Cal BP 490 to 430) and

(68\% probability) Cal AD 1570 to 1590 (Cal BP 380 to 360) and Cal AD 1590 to 1630 (Cal BP 360 to 320)

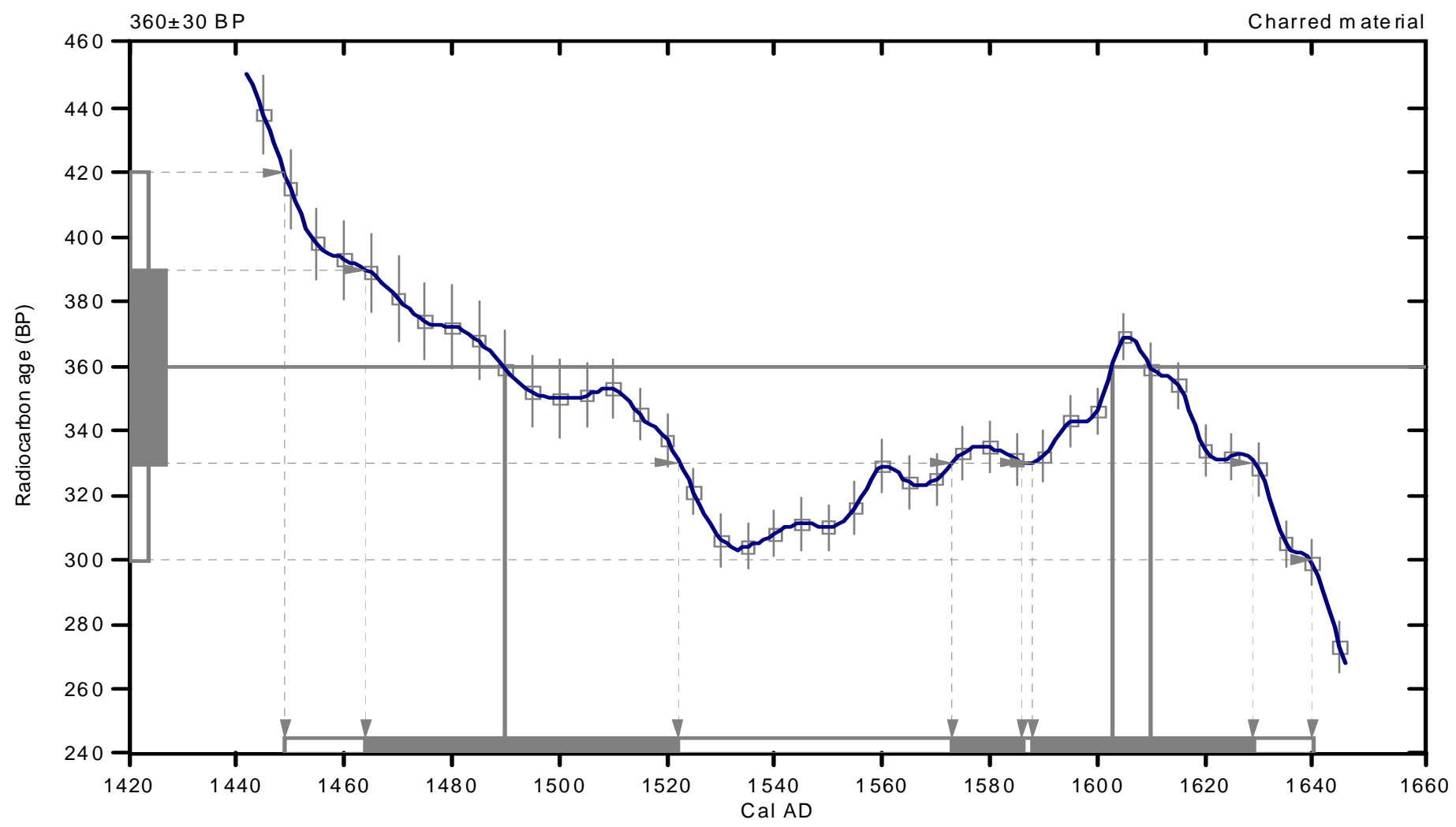

References:

Database used INTCALO 9

References to INTCALO9 database

Heaton,et.al.,2009, Radiocarbon 51(4):1151-1164, Reimer,et.al, 2009, Radiocarbon 51(4):1111-1150, Stuiver, et.al,1993, Radiocarbon 35(1):13 7-189, Oeschger,et.al., 1975, Tellus 27:168-192

Mathematics used for calibration scenario A Simplified Approach to Calibrating C14 Dates Talma, A. S., Vogel, J. C., 1993, Radiocarbon 35(2):317-322 


\title{
CALIBRATION OF RADIOCARBON AGE TO CALENDAR YEARS
}

\author{
(V ariables: $\mathrm{C} 13 / \mathrm{C} 12=-9 \cdot 4: 1 \mathrm{ab} . \mathrm{mult}=1)$
}

\section{Laboratory number: Beta-344094}

\section{Conventional radiocarbon age: $660 \pm 30 \mathrm{BP}$}

\section{Sigma calib rated results: Cal AD 1280 to 1320 (Cal BP 670 to 630) and (95\% probability) Cal AD 1350 to 1390 (Cal BP 600 to 560)}

Intercept data

Intercept of radiocarbon age with calibration curve:

Cal AD 1290 (Cal BP 660)

1 Sigma calibrated results: Cal AD 1280 to 1300 (Cal BP 660 to 640) and

(68\% probability) Cal AD 1360 to 1380 (Cal BP 590 to 570)

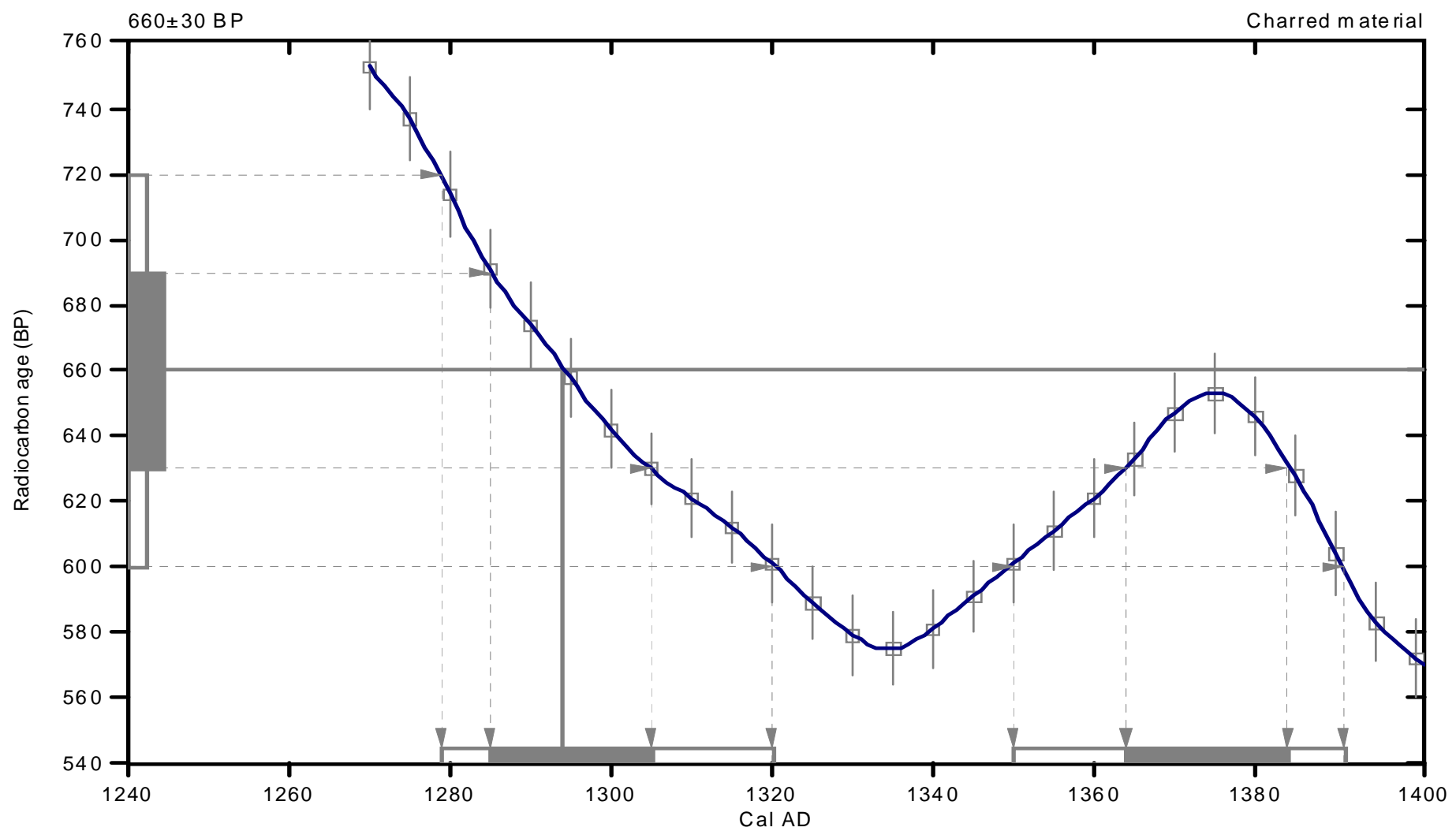

References:

Database used INTCALO9

References to INTCALO9 database

Heaton,et.al.,2009, Radiocarbon 51(4): 1151-1164, Reimer,et.al, 2009, Radiocarbon 51(4):1111-1150, Stuiver, et.al,1993, Radiocarbon 35(1):13 7-189, Oesch ger,et.al., 1975,Tellus 27:168-192

Mathematics used for calibration scenario A Simplified Approach to Calibrating C14 Dates Talma, A. S., Vogel, J. C., 1993, Radiocarbon 35(2):317-322 


\section{CALIBRATION OF RADIOCARBON AGE TO CALENDAR YEARS}

\section{(V ariables: C13/C12=-26.6:lab. mult $=1$ )}

\section{Laboratory number: Beta-344095}

\section{Conventional radiocarbon age: $260 \pm 30$ BP}

2 Sigma calibrated results: Cal AD 1520 to 1560 (Cal BP 420 to 390 ) and (95\% probability) Cal AD 1630 to 1670 (Cal BP 320 to 280) and

Cal AD 1780 to 1800 (Cal BP 170 to 150) and

Cal AD 1950 to 1950 (Cal BP 0 to 0 )

In tercept data

Intercept of radiocarbon age

with calibration curve: Cal AD 1650 (Cal BP 300)

1 Sigma calibrated result: Cal AD 1640 to 1660 (Cal BP 310 to 290)

(68\% probability)

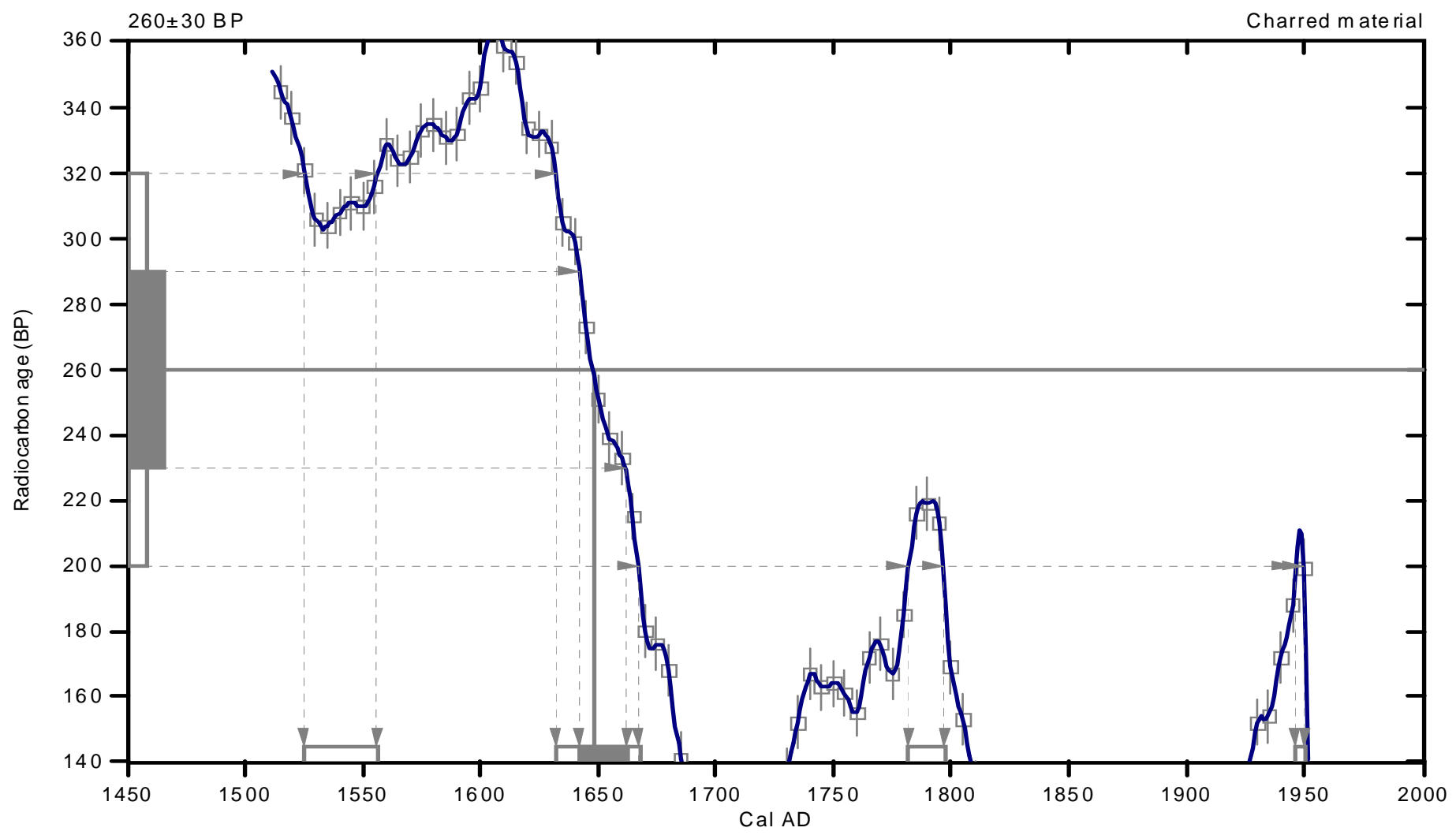

References:

Database used INTCALO 9

References to INTCALO9 database

Heaton,et.al.,2009, Radiocarbon 51(4): 1151-1164, Reimer,et.al, 2009, Radiocarbon 51(4):1111-1150, Stuiver, et.al,1993, Radiocarbon 35(1):13 7-189, Oesch ger,et.al., 1975,Tellus 27:168-192

Mathematics used for calibration scenario A Simplified Approach to Calibrating C14 Dates Talma, A. S., Vogel, J. C., 1993, Radiocarbon 35(2):317-322 


\section{CALIBRATION OF RADIOCARBON AGE TO CALENDAR YEARS}

\section{(Variables: C13/C 12=-26.8:lab. mult $=1$ )}

\section{Laboratory number: Beta-344096}

\section{Conventional radiocarbon age: $\quad 310 \pm 30$ B P}

\section{Sigma calibrated result: Cal A D 1480 to 1650 (C al BP 470 to 300 )}

(95\% probability)

Intercept data

Intercepts of radiocarbon age with calibration curve:

Cal AD 1530 (Cal BP 420) and

Cal AD 1540 (Cal BP 410) and

Cal AD 1550 (Cal BP 400) and

Cal AD 1550 (Cal BP 400) and

Cal AD 1630 (Cal BP 320)

1 Sigma calibrated results: Cal AD 1520 to 1590 (Cal BP 430 to 360) and

(68\% probability) Cal AD 1620 to 1640 (Cal B P 330 to 310 )

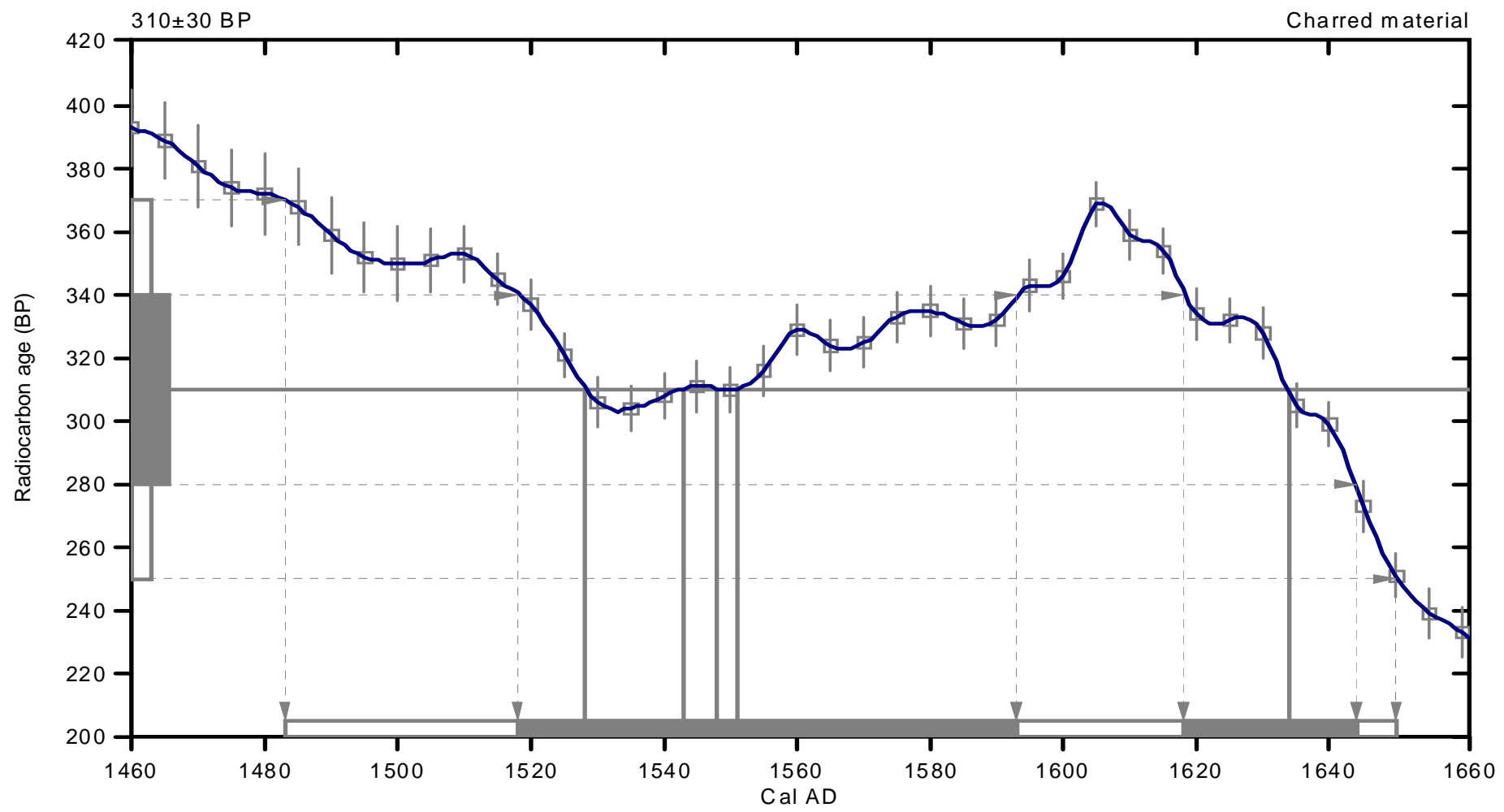

References:

Database used INTCALO9

References to INTCALOS database

Heaton,et.al.,2009, Radiocarbon 51(4):1151-1164, Reimer,et.al, 2009, Radiocarbon 51(4):1111-1150, Stuiver,et.al,1993, Radiocarbon 35(1):137-189, Oesch ger,et.al.,1975, Tellus 27:168-192

Mathematics used for calibration scenario A Simplified Approach to Calibrating C14 Dates Talma, A. S., Vogel, J. C., 1993, Radiocarbon 35(2):317-322

\section{Beta Analytic Radiocarbon Dating Laboratory}

4985 S.W.74th Court, Miami, Florida 33155 •Tel:(305)667-5167•Fax: (305)663-0964・E-Mail: beta@ radiocarbon.com 


\title{
CALIBRATION OF RADIOCARBON AGE TO CALENDAR YEARS
}

\author{
(V ariables: $\mathrm{C} 13 / \mathrm{C} 12=-25.4:$ lab. mult $=1)$
}

\section{Laboratory number: Beta-344097}

\section{Conventional radiocarbon age: $140 \pm 30 \mathrm{BP}$}

\section{Sigma calib rated results: Cal AD 1670 to 1780 (Cal BP 280 to 170) and (95\% probability) Cal AD 1800 to 1890 (Cal BP 150 to 60) and \\ Cal AD 1900 to 1950 (Cal BP 50 to 0) and \\ Cal AD 1950 to post 1950 (C al BP 0 to post 1950)}

Intercept data

Intercepts of radiocarbon age with calibration curve:

1 Sigma calibrated results: (68\% probability)
Cal AD 1680 (Cal BP 260) and

Cal AD 1730 (Cal BP 220) and

Cal AD 1810 (Cal BP 140) and

Cal AD 1930 (Cal BP 20) and

Cal AD Post 1950

Cal AD 1680 to 1700 (Cal BP 270 to 250) and Cal AD 1720 to 1760 (Cal BP 230 to 190) and Cal AD 1770 to 1780 (Cal BP 180 to 170 ) and Cal AD 1800 to 1820 (Cal BP 150 to 140 ) and Cal AD 1830 to 1880 (Cal BP 120 to 70 ) and Cal AD 1920 to 1940 (Cal BP 30 to 10) and Cal AD Post 1950

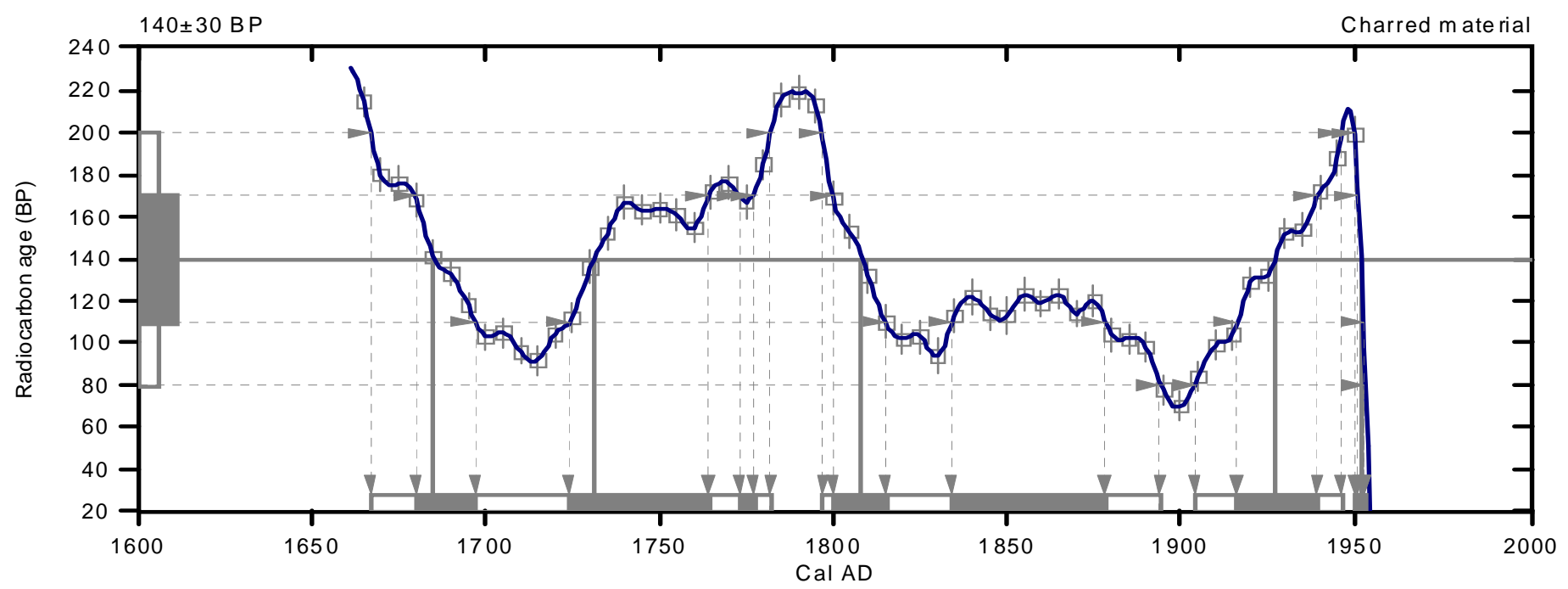

References:

Database used INTCALO 9

References to INTCALO9 database Heaton,et.al.,2009, Radiocarbon 51(4):1151-1164, Reimer,et.al, 2009, Radiocarbon 51(4):1111-1150, Stuiver, et.al,1993, Radiocarbon 35(1):13 7-189, Oesch ger,et.al., 1975,Tellus 27:168-192

Mathematics used for calibration scenario A Simplified Approach to Calibrating C14 Dates Talma, A. S., Vogel, J. C., 1993, Radiocarbon 35(2):317-322 


\section{CALIBRATION OF RADIOCARBON AGE TO CALENDAR YEARS}

\section{(V ariables: C13/C12=-25.8:lab. mult $=1$ )}

Laboratory number: Beta-344098

Conventional radiocarbon age: $1640 \pm 30$ B P

2 Sigma calib rated results: Cal AD 350 to 370 (Cal BP 1600 to 1580) and (95\% probability) Cal AD 380 to 440 (Cal BP 1570 to 1510) and

Cal AD 490 to 510 (Cal BP 1460 to 1440) and

Cal AD 520 to 530 (Cal BP 1430 to 1420)

Intercept data

Intercept of radiocarbon age

with calibration curve: Cal AD 410 (Cal BP 1540)

1 Sigma calibrated result: Cal AD 390 to 430 (Cal BP 1560 to 1520)

(68\% probability)

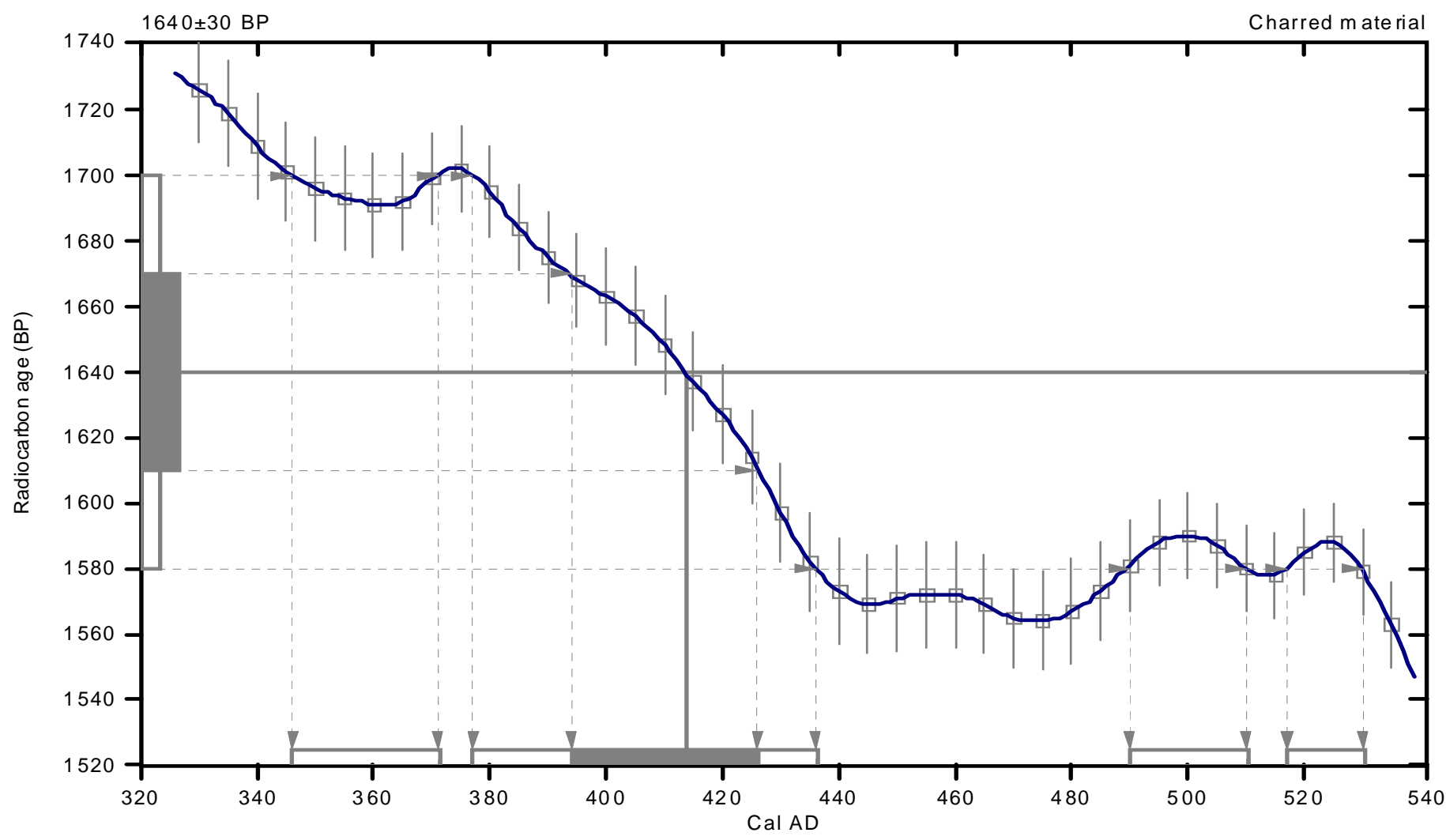

References:

Database used INTCALO 9

References to INTCALO9 database

Heaton,et.al.,2009, Radiocarbon 51(4): 1151-1164, Reimer,et.al, 2009, Radiocarbon 51(4):1111-1150, Stuiver, et.al,1993, Radiocarbon 35(1):13 7-189, Oesch ger,et.al., 1975,Tellus 27:168-192

Mathematics used for calibration scenario A Simplified Approach to Calibrating C14 Dates Talma, A. S., Vogel, J. C., 1993, Radiocarbon 35(2):317-322 


\section{CALIBRATION OF RADIOCARBON AGE TO CALENDAR YEARS}

\section{(V ariables: C13/C12=-23.3:lab. mult $=1$ )}

\section{Laboratory number: Beta-344099}

\section{Conventional radiocarbon age: $1820 \pm 30$ B P}

2 Sigma calibrated result: Cal AD 130 to 250 (Cal BP 1820 to 1700) (95\% probability)

Intercept data

Intercept of radiocarbon age

with calibration curve: Cal AD 220 (Cal BP 1730)

1 Sigma calibrated result: Cal AD 130 to 240 (Cal BP 1820 to 1710 )

(68\% probability)

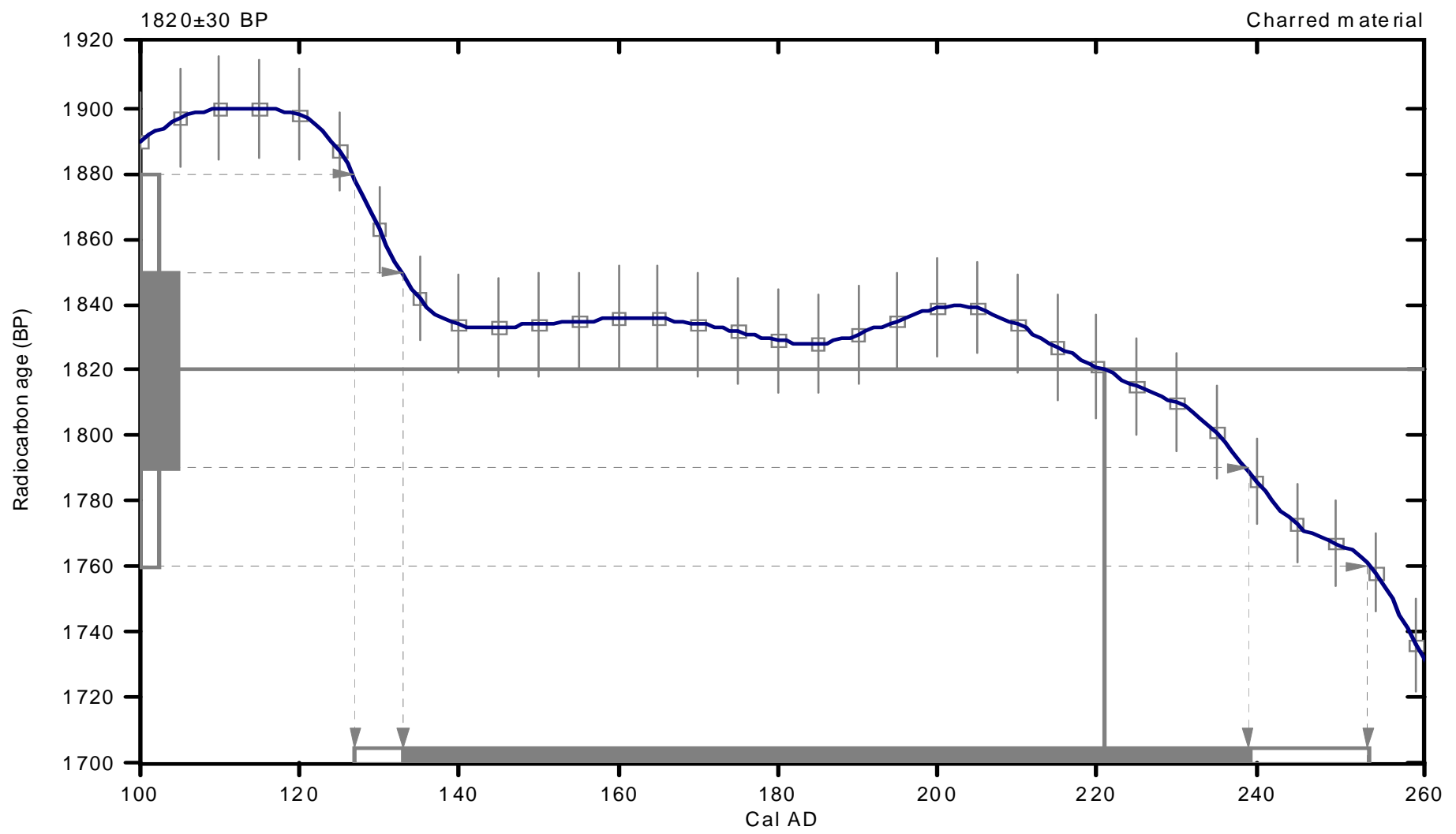

References:

Database used INTCALO9

References to INTCALO9 database

Heaton,et.al.,2009, Radiocarbon 51(4): 1151-1164, Reimer,et.al, 2009, Radiocarbon 51(4):1111-1150, Stuiver, et.al,1993, Radiocarbon 35(1):13 7-189, Oesch ger,et.al., 1975,Tellus 27:168-192

Mathematics used for calibration scenario A Simplified Approach to Calibrating C14 Dates Talma, A. S., Vogel, J. C., 1993, Radiocarbon 35(2):317-322 


\section{CALIBRATION OF RADIOCARBON AGE TO CALENDAR YEARS}

\section{(V ariables: C13/C12=-25.6:lab. mult $=1$ )}

\section{Laboratory number: Beta-344100}

\section{Conventional radiocarbon age: $\quad 550 \pm 30$ BP}

2 Sigma calibrated results: Cal AD 1320 to 1350 (Cal BP 630 to 600) and (95\% probability) Cal AD 1390 to 1430 (Cal BP 560 to 520)

Intercept data

Intercept of radiocarbon age with calibration curve:

1 Sigma calibrated results: Cal AD 1330 to 1340 (Cal BP 620 to 610) and

(68\% probability) Cal AD 1400 to 1420 (Cal BP 550 to 530)

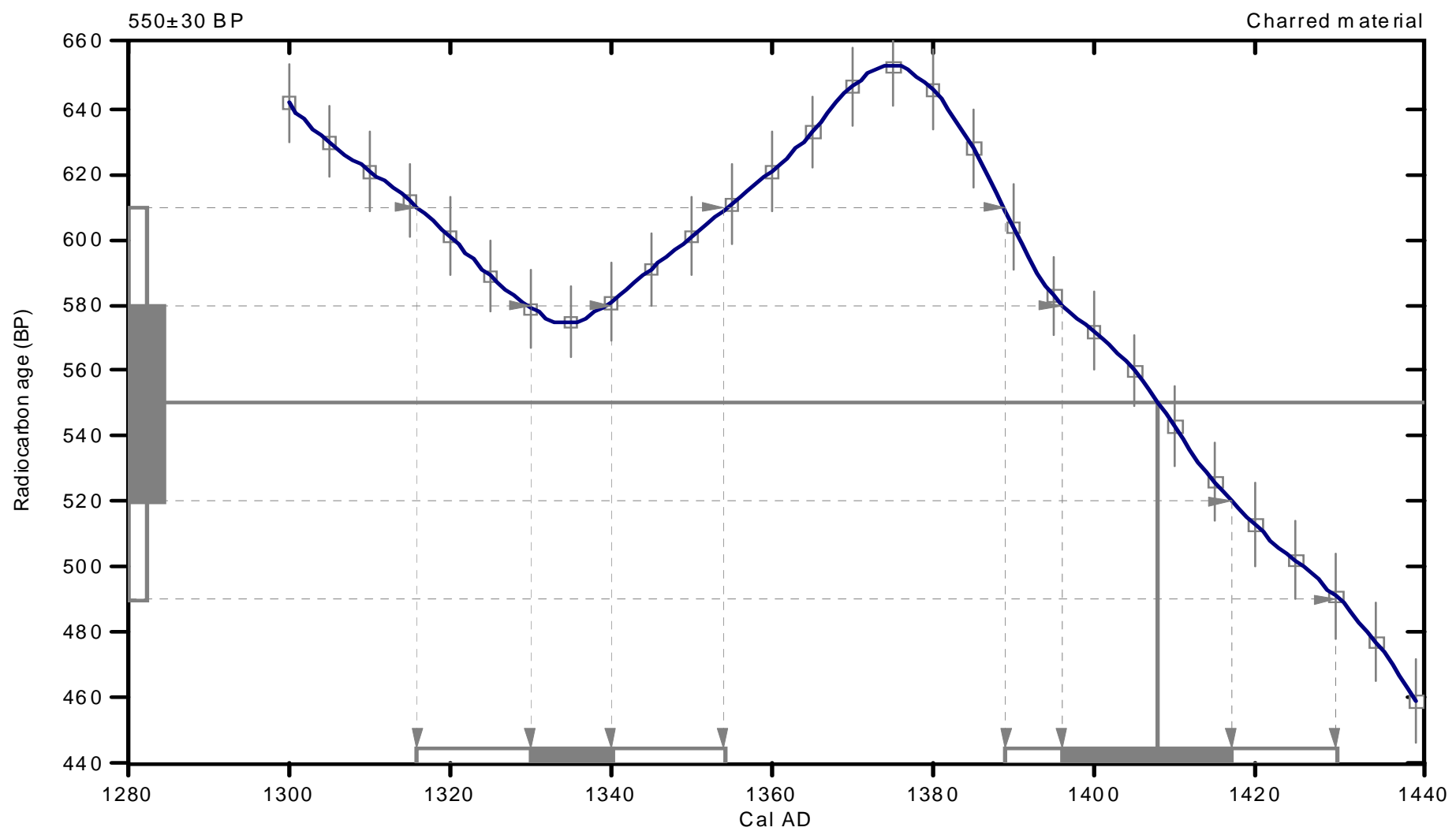

References:

Database used INTCALO9

References to INTCALO9 database

Heaton,et.al.,2009, Radiocarbon 51(4): 1151-1164, Reimer,et.al, 2009, Radiocarbon 51(4):1111-1150, Stuiver, et.al,1993, Radiocarbon 35(1):13 7-189, Oesch ger,et.al., 1975,Tellus 27:168-192

Mathematics used for calibration scenario A Simplified Approach to Calibrating C14 Dates Talma, A. S., Vogel, J. C., 1993, Radiocarbon 35(2):317-322 


\section{CALIBRATION OF RADIOCARBON AGE TO CALENDAR YEARS}

\section{(V ariables: C13/C12=-25.4:lab. mult $=1$ )}

\section{Laboratory number: Beta-344101}

\section{Conventional radiocarbon age: $\quad 350 \pm 30$ BP}

2 Sigma calibrated result: Cal AD 1450 to 1640 (Cal BP 500 to 310 ) (95\% probability)

Intercept data

Intercepts of radiocarbon age with calibration curve:

Cal AD 1500 (Cal BP 450) and

Cal AD 1500 (Cal BP 450) and

Cal AD 1510 (Cal BP 440) and

Cal AD 1600 (Cal BP 350) and

Cal AD 1620 (Cal BP 330)

1 Sigma calibrated results: Cal AD 1470 to 1520 (Cal BP 480 to 420) and

(68\% probability) Cal AD 1560 to 1630 (Cal BP 390 to 320)

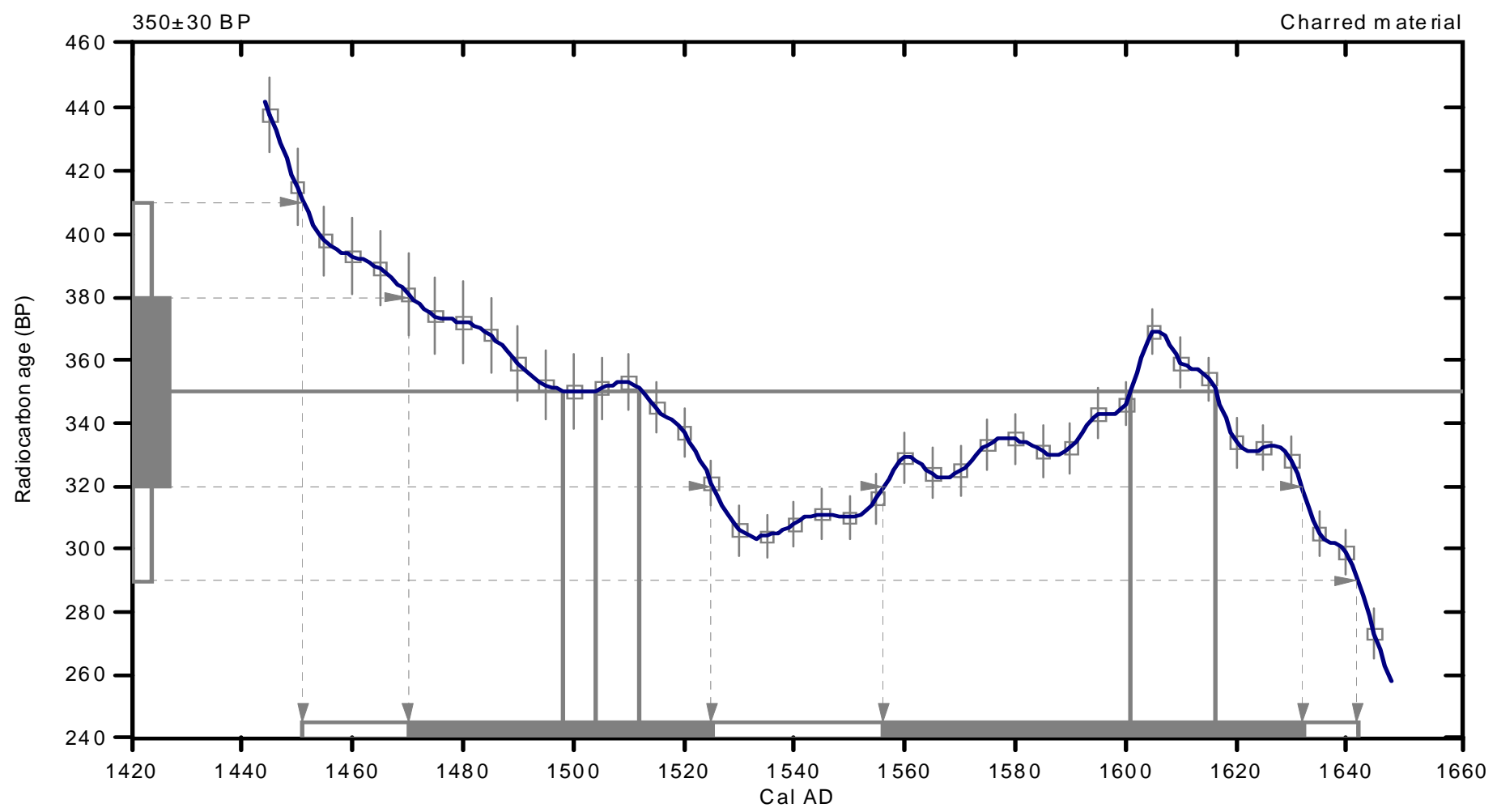

References:

Database used INTCALO 9

References to INTCALO9 database

Heaton,et.al.,2009, Radiocarbon 51(4):1151-1164, Reimer,et.al, 2009, Radiocarbon 51(4):1111-1150, Stuiver, et.al,1993, Radiocarbon 35(1):13 7-189, Oeschger,et.al., 1975, Tellus 27:168-192

Mathematics used for calibration scenario A Simplified Approach to Calibrating C14 Dates Talma, A. S., Vogel, J. C., 1993, Radiocarbon 35(2):317-322 


\section{CALIBRATION OF RADIOCARBON AGE TO CALENDAR YEARS}

\section{(V ariables: C13/C12=-24.9:lab. mult $=1$ )}

\section{Laboratory number: Beta-344102}

\section{Conventional radiocarbon age: $\quad 580 \pm 30$ BP}

\section{Sigma calibrated results: Cal AD 1300 to 1370 (Cal BP 650 to 580) and (95\% probability) Cal AD 1380 to 1420 (Cal BP 570 to 530)}

Intercept data

Intercepts of radiocarbon age with calibration curve:

Cal AD 1330 (Cal BP 620) and

Cal AD 1340 (Cal BP 610) and

Cal AD 1400 (Cal BP 550)

1 Sigma calibrated results: Cal AD 1320 to 1350 (Cal BP 630 to 600) and

(68\% probability) Cal AD 1390 to 1410 (Cal BP 560 to 540)

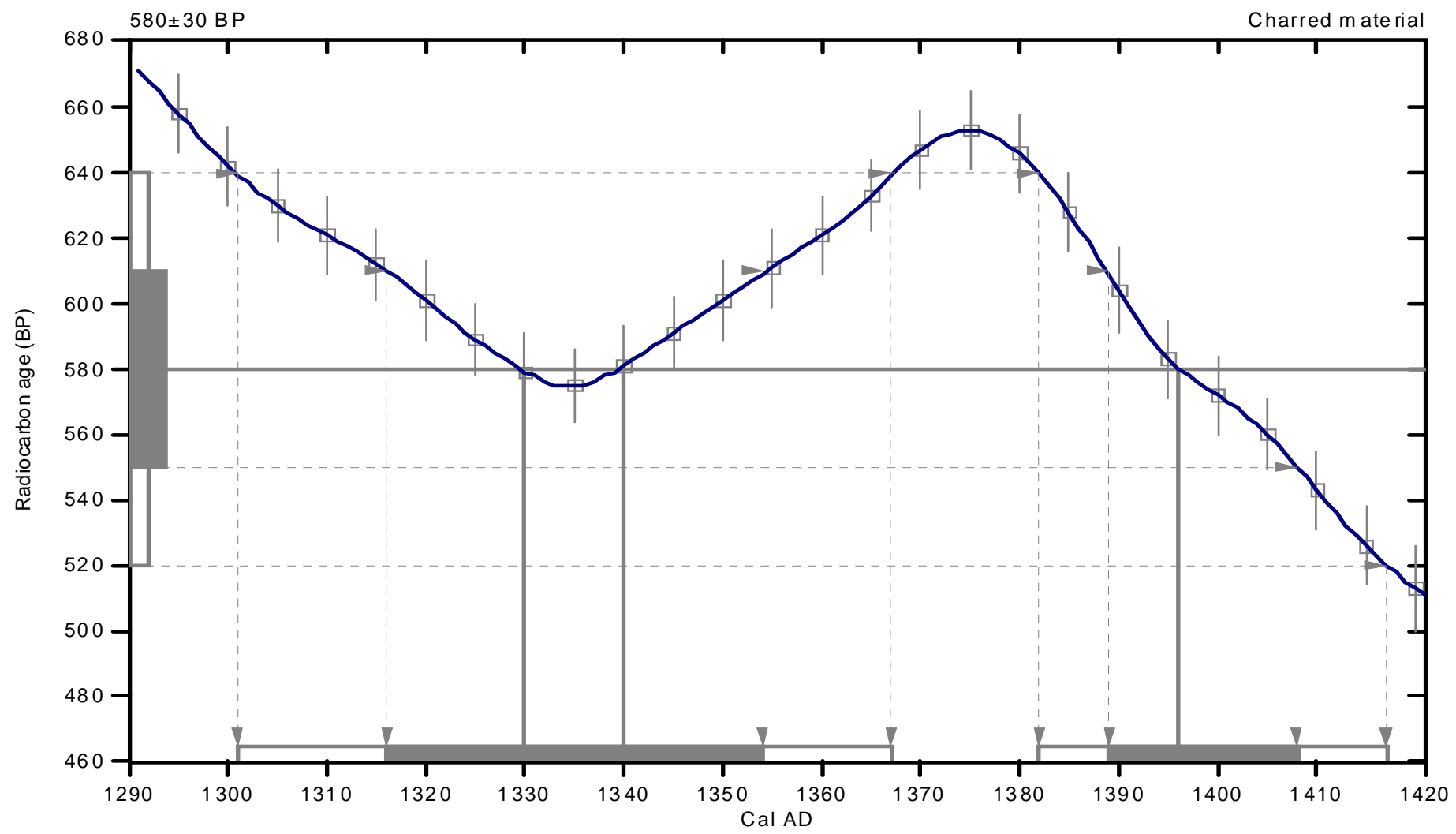

References:

Database used INTCALO9

References to INTCALO9 database

Heaton,et.al.,2009, Radiocarbon 51(4): 1151-1164, Reimer,et.al, 2009, Radiocarbon 51(4):1111-1150, Stuiver, et.al,1993, Radiocarbon 35(1):13 7-1 89, Oesch ger,et.al., 1975,Tellus 27:168-192

Mathematics used for calibration scenario A Simplified Approach to Calibrating C14 Dates Talma, A. S., Vogel, J. C., 1993, Radiocarbon 35(2):317-322 

June 19, 2013

\section{Dr. James Abbott}

Texas Department of Transportation

Cultural Resource Management

Environmental Affairs Division

125 East 11th Street

Austin, TX 78701

USA

RE: Radiocarbon Dating Results For Samples 41PN175-597, 41PN175-623, 41PN175-1224, 41PN1751579, 41PN175-1587, 41PN175-1805

Dear Dr. Abbott:

Enclosed are the radiocarbon dating results for six samples recently sent to us. They each provided plenty of carbon for accurate measurements and all the analyses proceeded normally. As usual, the method of analysis is listed on the report with the results and calibration data is provided where applicable.

The web directory containing the table of results and PDF download also contains pictures including, most importantly the portion actually analyzed. These can be saved by opening them and right clicking. Also a cvs spreadsheet download option is available and a quality assurance report is posted for each set of results. This report contains expected vs measured values for 3-5 working standards analyzed simultaneously with your samples.

All results reported are accredited to ISO-17025 standards and all analyses were performed entirely here in our laboratories. Since Beta is not a teaching laboratory, only graduates trained in accordance with the strict protocols of the ISO-17025 program participated in the analyses. When interpreting the results, please consider any communications you may have had with us regarding the samples. If you have specific questions about the analyses, please contact us. Your inquiries are always welcome.

Our invoice will be emailed separately. Please, forward it to the appropriate officer or send VISA charge authorization. Thank you. As always, if you have any questions or would like to discuss the results, don't hesitate to contact me.

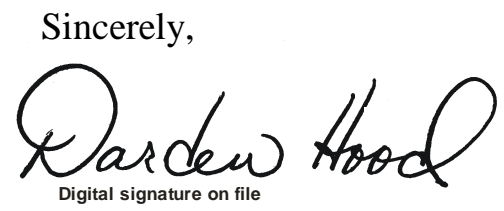




\section{REPORT OF RADIOCARBON DATING ANALYSES}

Dr. James Abbott

Report Date: 6/19/2013

Texas Department of Transportation

Material Received: 6/13/2013

Sample Data

Measured
Radiocarbon Age

$850+/-30 \mathrm{BP}$

Beta - 351054

SAMPLE : 41PN175-597

ANALYSIS : AMS-Standard delivery

MATERIAL/PRETREATMENT : (bulk sherd organics): acid washes

2 SIGMA CALIBRATION : $\quad$ Cal AD 1220 to 1280 (Cal BP 740 to 670)
$13 \mathrm{C} / 12 \mathrm{C}$
Ratio

$-29.4 \mathrm{o} / \mathrm{oo}$

$780+/-30 \mathrm{BP}$
Beta - 351055

SAMPLE : 41PN175-623

ANALYSIS : AMS-Standard delivery

MATERIAL/PRETREATMENT : (bulk sherd organics): acid washes

2 SIGMA CALIBRATION : $\quad$ Cal AD 1270 to 1310 (Cal BP 680 to 640) AND Cal AD 1360 to 1390 (Cal BP 590 to 560$)$
Conventional

Radiocarbon Age $\left(^{*}\right)$
Beta - 351056

SAMPLE : 41PN175-1224

ANALYSIS : AMS-Standard delivery

MATERIAL/PRETREATMENT : (bulk sherd organics): acid washes

2 SIGMA CALIBRATION : $\quad$ Cal AD 1280 to 1320 (Cal BP 670 to 630) AND Cal AD 1350 to 1390 (Cal BP 600 to 560 )
$740+/-30 \mathrm{BP}$
$-29.5 \mathrm{o} / \mathrm{oo}$
$670+/-30 \mathrm{BP}$

Beta - 351057

$490+/-30$ BP

-28.9 o/oo

$430+/-30 \mathrm{BP}$

SAMPLE : 41PN175-1579

ANALYSIS : AMS-Standard delivery

MATERIAL/PRETREATMENT : (bulk sherd organics): acid washes

2 SIGMA CALIBRATION :

Cal AD 1430 to 1480 (Cal BP 520 to 470)

Dates are reported as RCYBP (radiocarbon years before present, "present" = AD 1950). By international convention, the modern reference standard was $95 \%$ the $14 \mathrm{C}$ activity of the National Institute of Standards and Technology (NIST) Oxalic Acid (SRM 4990C) and calculated using the Libby $14 \mathrm{C}$ half-life (5568 years). Quoted errors represent 1 relative standard deviation statistics (68\% probability) counting errors based on the combined measurements of the sample, background, and modern reference standards. Measured $13 \mathrm{C} / 12 \mathrm{C}$ ratios (delta 13C) were calculated relative to the PDB-1 standard.
The Conventional Radiocarbon Age represents the Measured Radiocarbon Age corrected for isotopic fractionation, calculated using the delta $13 \mathrm{C}$. On rare occasion where the Conventional Radiocarbon Age was calculated using an assumed delta 13C, the ratio and the Conventional Radiocarbon Age will be followed by "*". The Conventional Radiocarbon Age is not calendar calibrated. When available, the Calendar Calibrated result is calculated from the Conventional Radiocarbon Age and is listed as the "Two Sigma Calibrated Result" for each sample. 


\section{REPORT OF RADIOCARBON DATING ANALYSES}

Dr. James Abbott

Report Date: 6/19/2013

\section{Sample Data}

\author{
Measured \\ Radiocarbon Age
}

\author{
$13 \mathrm{C} / 12 \mathrm{C}$ \\ Ratio
}

Conventional

Radiocarbon Age $\left.{ }^{*}\right)$

Beta - 351058

$750+/-30 \mathrm{BP}$

$-29.1 \mathrm{o} / \mathrm{oo}$

$680+/-30 \mathrm{BP}$

SAMPLE : 41PN175-1587

ANALYSIS : AMS-Standard delivery

MATERIAL/PRETREATMENT : (bulk sherd organics): acid washes

2 SIGMA CALIBRATION : $\quad$ Cal AD 1270 to 1310 (Cal BP 680 to 640) AND Cal AD 1360 to 1390 (Cal BP 590 to 560)

Beta - 351059

$440+/-30 \mathrm{BP}$

-29.5 o/oo

$370+/-30 \mathrm{BP}$

SAMPLE : 41PN175-1805

ANALYSIS : AMS-Standard delivery

MATERIAL/PRETREATMENT : (bulk sherd organics): acid washes

2 SIGMA CALIBRATION : $\quad$ Cal AD 1450 to 1530 (Cal BP 500 to 420) AND Cal AD 1540 to 1550 (Cal BP 410 to 400)

Cal AD 1550 to 1630 (Cal BP 400 to 320)

Dates are reported as RCYBP (radiocarbon years before present, "present" = AD 1950). By international convention, the modern reference standard was $95 \%$ the $14 \mathrm{C}$ activity of the National Institute of Standards and Technology (NIST) Oxalic Acid (SRM 4990C) and calculated using the Libby $14 \mathrm{C}$ half-life (5568 years). Quoted errors represent 1 relative standard deviation statistics (68\% probability) counting errors based on the combined measurements of the sample, background, and modern reference standards. Measured $13 \mathrm{C} / 12 \mathrm{C}$ ratios (delta 13C) were calculated relative to the PDB-1 standard.
The Conventional Radiocarbon Age represents the Measured Radiocarbon Age corrected for isotopic fractionation, calculated using the delta 13C. On rare occasion where the Conventional Radiocarbon Age was calculated using an assumed delta $13 \mathrm{C}$, the ratio and the Conventional Radiocarbon Age will be followed by "*". The Conventional Radiocarbon Age is not calendar calibrated. When available, the Calendar Calibrated result is calculated from the Conventional Radiocarbon Age and is listed as the "Two Sigma Calibrated Result" for each sample. 


\section{CALIBRATION OF RADIOCARBON AGE TO CALENDAR YEARS}

\section{(V ariables: C13/C12=-29.4:lab. mult $=1$ )}

\section{Laboratory number: Beta-351054}

\section{Conventional radiocarbon age: $\quad 780 \pm 30$ BP}

2 Sigma calibrated result: Cal AD 1220 to 1280 (Cal BP 740 to 670) (95\% probability)

Intercept data

Intercept of radiocarbon age

with calibration curve: Cal AD 1260 (Cal BP 690)

1 Sigma calibrated result: Cal AD 1220 to 1270 (Cal BP 730 to 680)

(68\% probability)

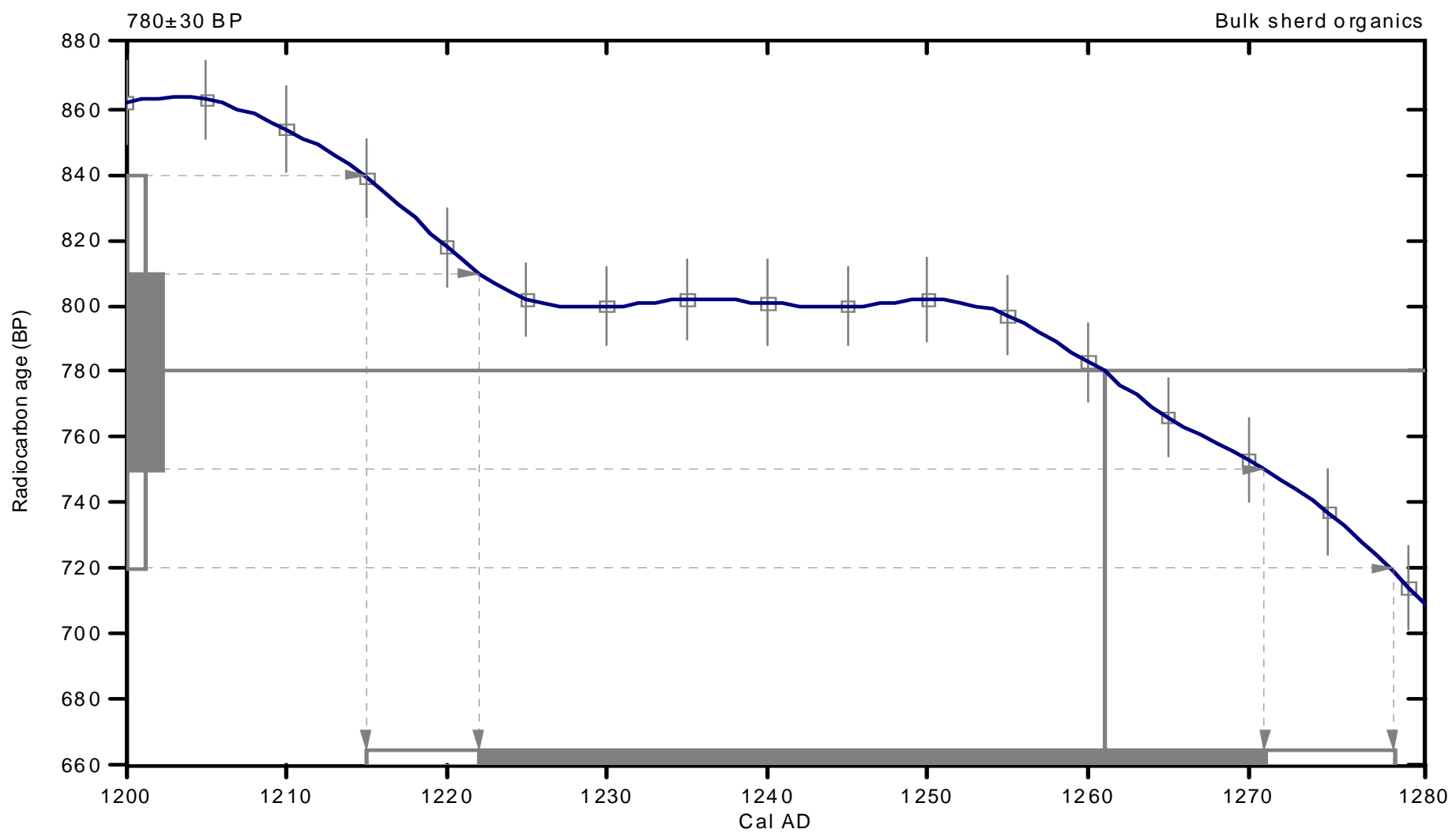

References:

Database used INTCALO9

References to INTCALO9 database

Heaton,et.al.,2009, Radiocarbon 51(4): 1151-1164, Reimer,et.al, 2009, Radiocarbon 51(4):1111-1150, Stuiver, et.al,1993, Radiocarbon 35(1):13 7-189, Oesch ger,et.al., 1975,Tellus 27:168-192

Mathematics used for calibration scenario A Simplified Approach to Calibrating C14 Dates Talma, A. S., Vogel, J. C., 1993, Radiocarbon 35(2):317-322 


\section{CALIBRATION OF RADIOCARBON AGE TO CALENDAR YEARS}

\section{(V ariables: C13/C12=-29.5:lab. mult $=1$ )}

\section{Laboratory number: Beta-351055}

\section{Conventional radiocarbon age: $\quad 680 \pm 30$ BP}

2 Sigma calibrated results: Cal AD 1270 to 1310 (Cal BP 680 to 640) and (95\% probability) Cal AD 1360 to 1390 (Cal BP 590 to 560)

Intercept data

Intercept of radiocarbon age with calibration curve:

Cal AD 1290 (Cal BP 660)

1 Sigma calibrated results: Cal AD 1280 to 1300 (Cal BP 670 to 650) and

(68\% probability) Cal AD 1370 to 1380 (Cal BP 580 to 570)

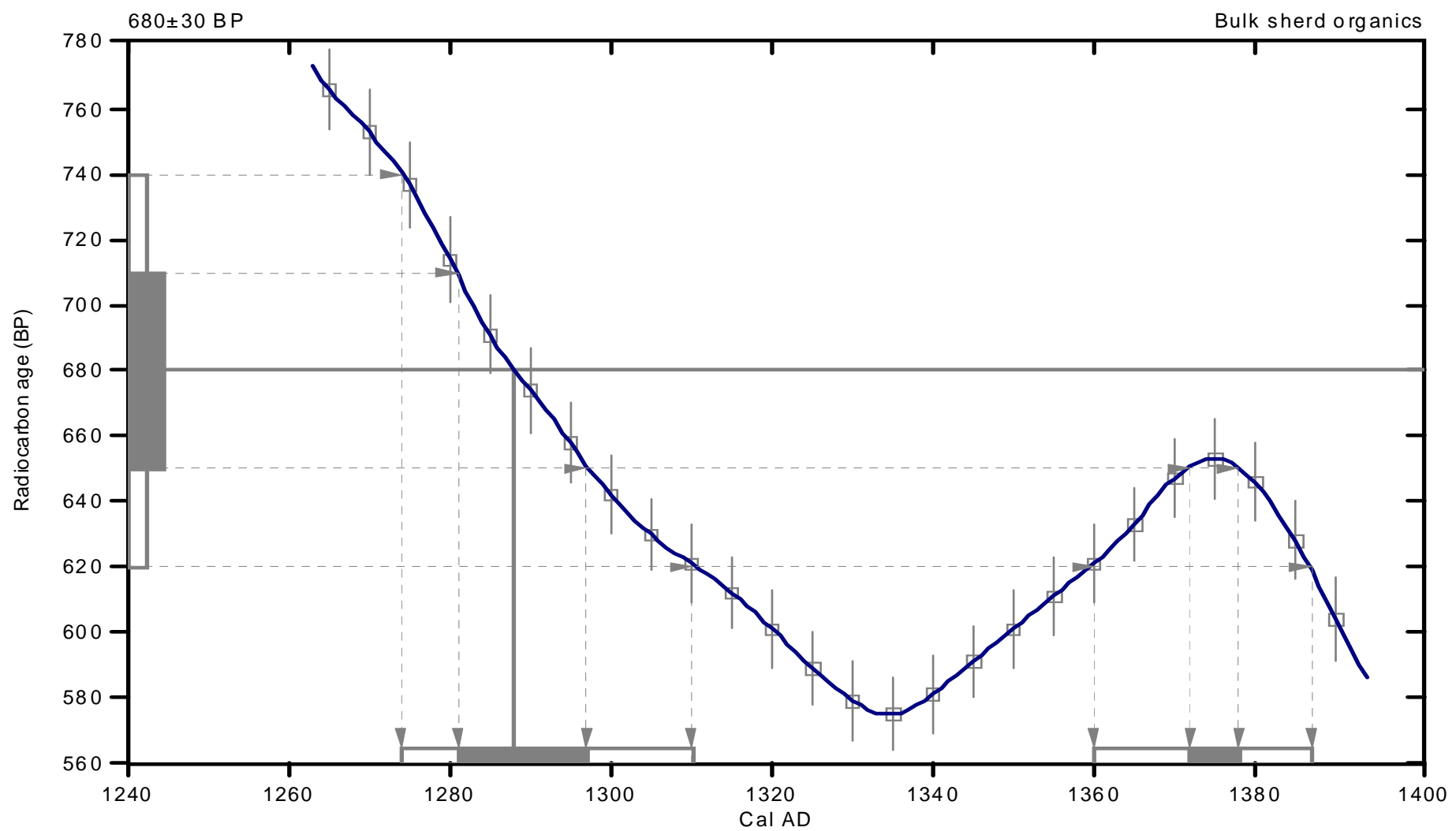

References:

Database used INTCALO9

References to INTCALO9 database

Heaton,et.al.,2009, Radiocarbon 51(4): 1151-1164, Reimer,et.al, 2009, Radiocarbon 51(4):1111-1150, Stuiver, et.al,1993, Radiocarbon 35(1):13 7-189, Oesch ger,et.al., 1975,Tellus 27:168-192

Mathematics used for calibration scenario A Simplified Approach to Calibrating C14 Dates Talma, A. S., Vogel, J. C., 1993, Radiocarbon 35(2):317-322 


\section{CALIBRATION OF RADIOCARBON AGE TO CALENDAR YEARS}

\section{(V ariables: C13/C12=-29.5:lab. mult $=1$ )}

\section{Laboratory number: Beta-351056}

\section{Conventional radiocarbon age: $\quad 670 \pm 30 \mathrm{BP}$}

2 Sigma calib rated results: Cal AD 1280 to 1320 (Cal BP 670 to 630) and (95\% probability) Cal AD 1350 to 1390 (Cal BP 600 to 560)

Intercept data

Intercept of radiocarbon age with calibration curve:

Cal AD 1290 (Cal BP 660)

1 Sigma calibrated results: Cal AD 1280 to 1300 (Cal BP 670 to 650) and

(68\% probability) Cal AD 1370 to 1380 (Cal BP 580 to 570)

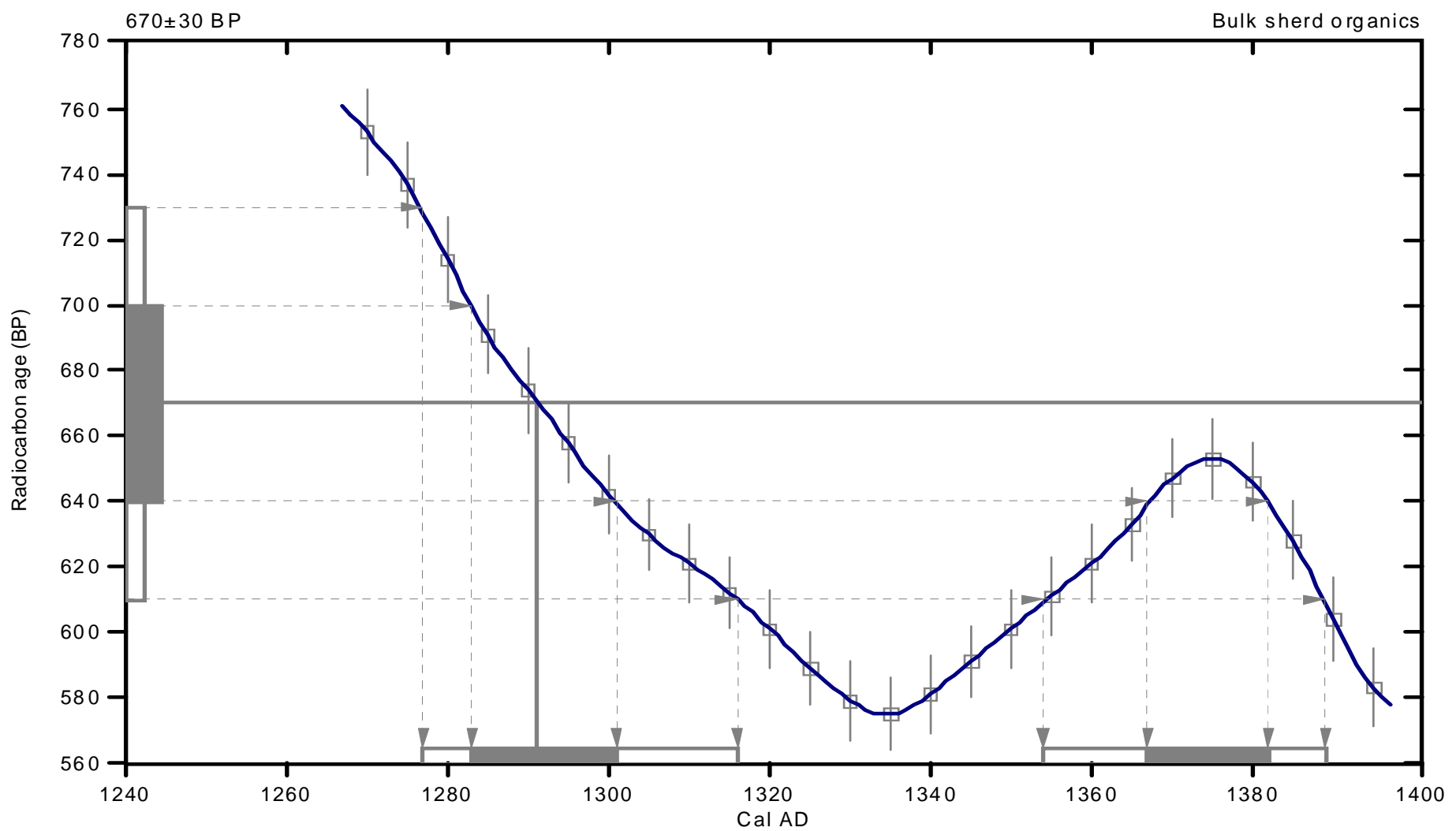

References:

Database used INTCALO9

References to INTCALO9 database

Heaton,et.al.,2009, Radiocarbon 51(4): 1151-1164, Reimer,et.al, 2009, Radiocarbon 51(4):1111-1150, Stuiver, et.al,1993, Radiocarbon 35(1):137-189, Oeschger,et.al., 1975,Tellus 27:168-192

Mathematics used for calibration scenario A Simplified Approach to Calibrating C14 Dates Talma, A. S., Vogel, J. C., 1993, Radiocarbon 35(2):317-322 


\section{CALIBRATION OF RADIOCARBON AGE TO CALENDAR YEARS}

\section{(V ariables: C13/C12=-28.9:lab. mult $=1$ )}

\section{Laboratory number: Beta-351057}

\section{Conventional radiocarbon age: $430 \pm 30$ BP}

2 Sigma calib rated result: Cal AD 1430 to 1480 (Cal BP 520 to 470) (95\% probability)

Intercept data

Intercept of radiocarbon age

with calibration curve: Cal AD 1450 (Cal BP 500)

1 Sigma calibrated result: Cal AD 1440 to 1450 (Cal BP 510 to 500)

(68\% probability)

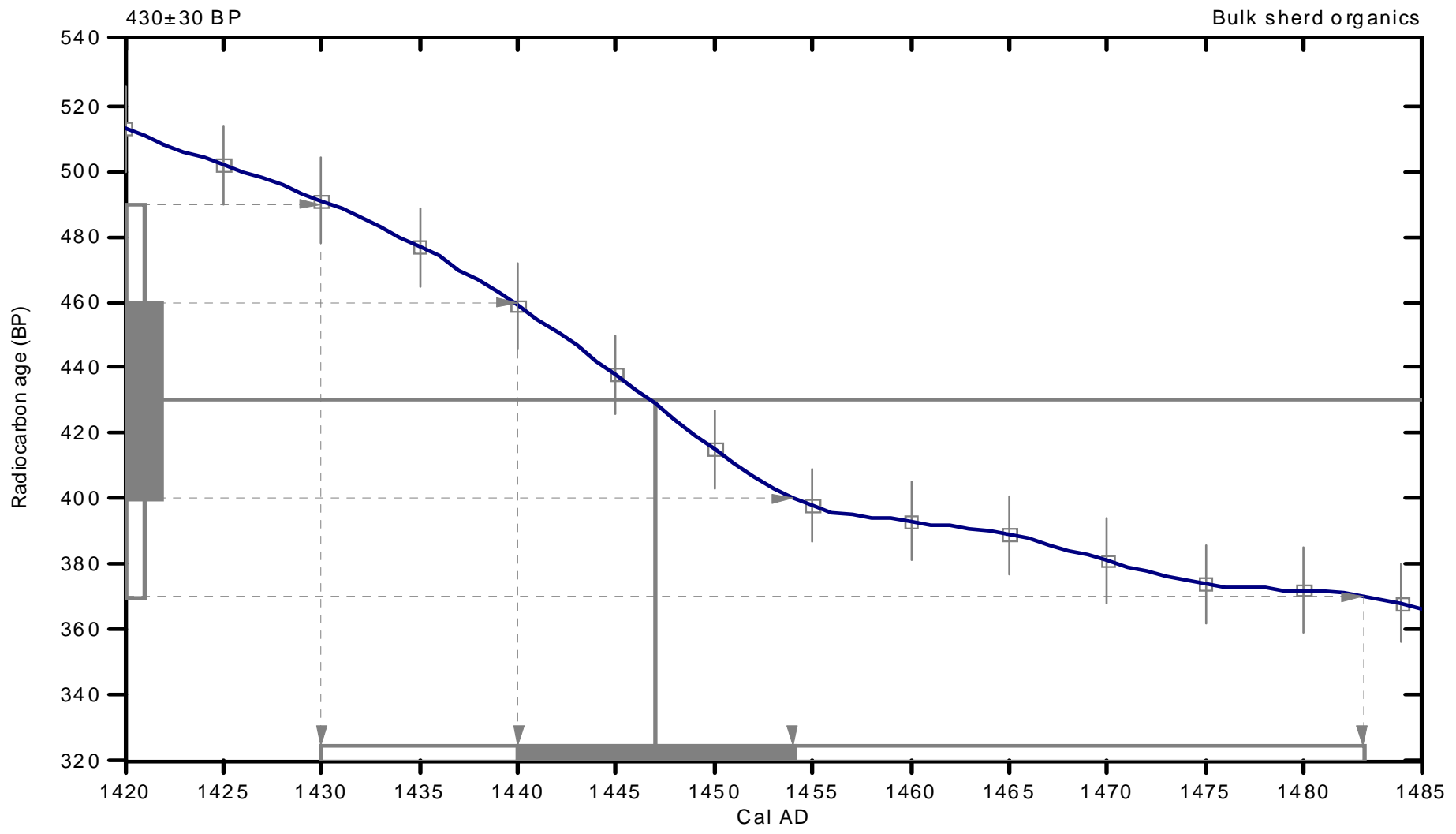

References:

Database used INTCALO 9

References to INTCALO9 database

Heaton, et.al.,2009, Radiocarbon 51(4):1151-1164, Reimer,et.al, 2009, Radiocarbon 51(4):1111-1150, Stuiver, et.al,1993, Radiocarbon 35(1):13 7-189, Oeschger,et.al., 1975, Tellus 27:168-192

Mathematics used for calibration scenario A Simplified Approach to Calibrating C14 Dates Talma, A. S., Vogel, J. C., 1993, Radiocarbon 35(2):317-322

\section{Beta Analytic Radiocarbon Dating Laboratory}




\section{CALIBRATION OF RADIOCARBON AGE TO CALENDAR YEARS}

(Variables: C13/C12=-29.1:1ab. mult=1)

\section{Laboratory number: B eta-351058}

\section{Conventional radiocarbon age: $\quad 680 \pm 30 \mathrm{BP}$}

2 Sigma calibrated results: Cal AD 1270 to 1310 (Cal B P 680 to 640) and (95\% probability) Cal AD 1360 to 1390 (Cal B P 590 to 560)

Intercept data

Intercept of radiocarbon age

with calibration curve: Cal AD 1290 (Cal BP 660)

1 Sigma calibrated results: Cal AD 1280 to 1300 (Cal BP 670 to 650) and

(68\% probability) Cal AD 1370 to 1380 (Cal BP 580 to 570)

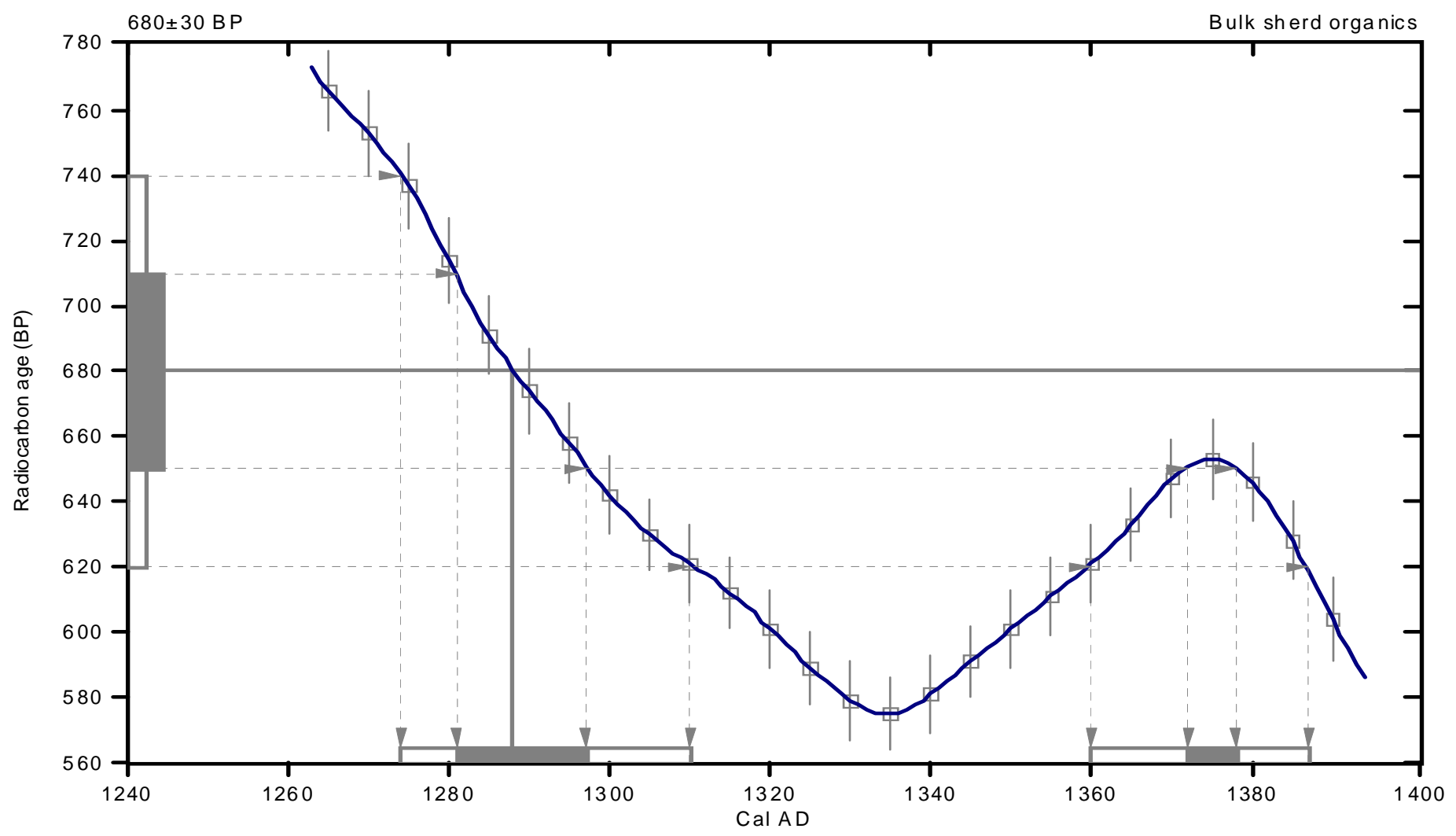

References:

Database used INTCALO9

References to INTCALO9 database

Heaton,et.al.,2009, Radiocarbon 51(4):1151-1164, Reimer,et.al, 2009, Radiocarbon 51(4):1111-1150, Stuiver,et.al,1993, Radiocarbon 35(1):1-244, Oeschger,et.al.,1975,Tellus 2 7:168-192

Mathematics used for calibration scenario A Simplified Approach to Calibrating C14 Dates Talma, A. S., Vogel, J. C., 1993, Radiocarbon 35(2):317-322 


\section{CALIBRATION OF RADIOCARBON AGE TO CALENDAR YEARS}

\section{(V ariables: C13/C12=-29.5:lab. mult $=1$ )}

Laboratory number: Beta-351059

\section{Conventional radiocarbon age: $\quad 370 \pm 30 \mathrm{BP}$}

2 Sigma calib rated results: Cal AD 1450 to 1530 (Cal BP 500 to 420 ) and (95\% probability) Cal AD 1540 to 1550 (Cal BP 410 to 400 ) and

Cal AD 1550 to 1630 (Cal BP 400 to 320)

Intercept data

Intercept of radiocarbon age with calibration curve: Cal AD 1480 (Cal BP 470)

1 Sigma calibrated results: Cal AD 1450 to 1520 (Cal BP 500 to 430) and (68\% probability) Cal AD 1590 to 1620 (Cal BP 360 to 330)



References:

Database used INTCALO9

References to INTCALO9 database

Heaton,et.al.,2009, Radiocarbon 51(4): 1151-1164, Reimer,et.al, 2009, Radiocarbon 51(4):1111-1150, Stuiver, et.al,1993, Radiocarbon 35(1):13 7-189, Oesch ger,et.al., 1975,Tellus 27:168-192

Mathematics used for calibration scenario A Simplified Approach to Calibrating C14 Dates Talma, A. S., Vogel, J. C., 1993, Radiocarbon 35(2):317-322

\section{Beta Analytic Radiocarbon Dating Laboratory}



B-2: OSL 



\title{
Quartz Optical Dating Report
}

\author{
$18^{\text {th }}$ July, 2012
}

41 FN 175, East Texas

Abstract: Optical luminescence dating (OSL) at the single grain level was applied to coarse quartz grains extracted from three samples taken from the 41 FN 175 site, East Texas. All samples responded acceptably to OSL measurement but analysis of sample replicates indicated all samples had appreciable palaeodose scatter. This is taken to indicate either partial bleaching prior to burial or post-depositional disturbance. Whilst efforts have been made to mitigate the effects of this and ages have been calculated, results for these samples should be treated with some caution. The best estimates of ages range from $0.23 \pm 0.02 \mathrm{ka}$ (Shfd12032) to $0.94 \pm 0.08 \mathrm{ka}$ (Shfd12033).

1. Introduction: Three samples from the $41 \mathrm{FN} 175$ site, East Texas were submitted for OSL dating by Dr Charles Frederick. All luminescence work was carried out at the Sheffield Centre for International Drylands Research (SCIDR) luminescence laboratory. The samples are assumed not to have been exposed to sunlight during sampling or transportation to the laboratory. Upon arrival, each sample was allocated a Sheffield laboratory number (Table 1). This report provides a brief summary of the procedures employed and results obtained for samples.

Table 1. Sample descriptive data.

\begin{tabular}{cccccc}
\hline Lab No. & Field Reference & $\begin{array}{c}\text { Latitude } \\
(\mathrm{N})\end{array}$ & $\begin{array}{c}\text { Longitude } \\
\left({ }^{\circ} \mathrm{W}\right)\end{array}$ & $\begin{array}{c}\text { Altitude } \\
(\mathrm{m})\end{array}$ & $\begin{array}{c}\text { Sampling Depth } \\
(\mathrm{cm} \text { below surface) }\end{array}$ \\
Shfd12031 & Sample 1 & 32.04 & 94.37 & 72 & 30 \\
Shfd12032 & Sample 2 & 32.04 & 94.37 & 72 & 23 \\
Shfd12033 & Sample 3 & 32.04 & 94.37 & 72 & 45
\end{tabular}

In order to derive an optically stimulated luminescence (OSL) age both the palaeodose (De - the amount of absorbed dose since the sample was buried) and the dose rate (the estimated radiation flux for the sedimentary bodies) have to be determined. Aitken (1998) gives a detailed explanation of both these parameters. To calculate an age, the palaeodose (expressed in Grays) is divided by the annual dose rate (Grays/yr). An inherent assumption in these age calculations is that the sediment was fully reset or 'bleached' by exposure to sunlight during the last transport event or whilst in situ prior to burial and that no post-depositional sediment disturbance has occurred. As part of this investigation, efforts have been taken to establish if these sediments have been bleached prior to burial or disturbed by, for example, bioturbation. As the OSL signal measured at the small single aliquot level of measurement is an average of $\sim 2000$ grains the true distribution of De values may be masked. This is of particular significance in heterogeneously dosed samples (e.g. poorly reset/bleached) in which grains with a high De signal will dominate the signal at the expense of grains containing a true burial De. The De of grains recently exhumed and bleached due to bioturbation (referred to as zero-dosed grains) are also masked at the single aliquot level. Thus $\mathrm{Dr}$ Charles Frederick requested samples underwent OSL measurement at the single grain level of analysis.

2. Dose Rate Analysis: Naturally occurring potassium (K), thorium (Th), rubidium (Rb) and uranium $(\mathrm{U})$ are the main contributors of dose to sedimentary quartz. The concentrations of these elements were determined by inductively 
coupled plasma mass spectrometry (ICP) at SGS laboratories Ontario Canada (Table 2). Elemental concentrations were converted to annual dose rates using data from Adamiec and Aitken (1998), Marsh et al. (2002), and Aitken (1998). This took into account attenuation factors relating to sediment grain sizes used, density and palaeomoisture. It has been assumed that the samples formed part of a thick homogeneous unit with no gamma contribution (other than from cosmogenic sources) being received by the samples from other unsampled sedimentary units. Attenuation of dose by moisture used the present-day moisture values as measured in the laboratory with a $3 \%$ error to incorporate fluctuations through time (Table 2). The contribution to dose rates from cosmic sources was calculated using the expression published in Prescott and Hutton (1994; Table 2). Cosmic dose is calculated as a linear decay curve at depths below $50 \mathrm{~cm}$. Above this depth, errors in calculation may lead to an under-estimation of the cosmic dose contribution. As samples were collected from within the top $50 \mathrm{~cm}$ of sediment a small error in the calculated cosmic dose rate can be expected.

The dose rates calculated are based on analyses of the sediment sampled at the present day. This assumption is only valid if no movement and/or reprecipitation of the four key elements has taken place since sediment burial and the adjacent sediments to those sampled had similar dose rates. Further analysis would have to be undertaken to establish whether the latter is true and if radioactive disequilibrium is present in the dose rate. It also assumes that the sediments submitted for analyses were representative in terms of radioactivity of sediments within a $50 \mathrm{~cm}$ sphere of each OSL sample as all this sediment would be contributing a gamma dose to the OSL samples.

Table 2. Summary of results - Dosimetry related data.

\begin{tabular}{lccccccc}
\hline Lab Code & $\begin{array}{c}\mathbf{U} \\
\text { (PPM) }\end{array}$ & $\begin{array}{c}\text { Th } \\
(\mathrm{PPM})\end{array}$ & $\begin{array}{c}\mathbf{R b} \\
(\mathrm{PPM})\end{array}$ & $\begin{array}{c}\mathbf{K} \\
(\%)\end{array}$ & $\begin{array}{c}\mathbf{D}_{\text {cosmic }}{ }^{+} \\
(\mathrm{Gy} / \mathrm{ka})\end{array}$ & $\begin{array}{c}\text { Moisture } \\
(\%)\end{array}$ & $\begin{array}{c}\text { Dose rate }^{\dagger} \\
(\mathrm{Gy} / \mathrm{ka})\end{array}$ \\
\hline Shfd12031 & 2.00 & 4.4 & 2.78 & 0.6 & $0.193 \pm 0.01$ & 4.7 & $1.536 \pm 0.059$ \\
Shfd12032 & 2.04 & 4.6 & 2.69 & 0.8 & $0.195 \pm 0.01$ & 4.2 & $1.756 \pm 0.070$ \\
Shfd12033 & 2.49 & 5.8 & 3.82 & 0.8 & $0.190 \pm 0.009$ & 10.3 & $1.811 \pm 0.071$ \\
\hline
\end{tabular}

+ Cosmic dose is calculated as a linear decay curve at depths below $50 \mathrm{~cm}$. Above this depth, errors in calculation may lead to an under-estimation of the cosmic dose contribution.

${ }^{\dagger}$ Total Dose is attenuated for grain size, density and moisture and assuming a saturation value prior to 13 ka before present.

3. Palaeodose Determination: The samples were prepared under subdued red lighting following the procedure to extract and clean quartz outlined in Bateman and Catt (1996). Prepared aliquots of the samples were taken from within a size range of $125-180 \mu \mathrm{m}$ reflecting the dominant size within each sample. All OSL measurements were carried out using a Risø TL DA-15 single grain laser luminescence reader with radiation doses administered using a calibrated ${ }^{90}$ strontium beta source. Grains were mounted in $300 \mu \mathrm{m}$ pits with 100 pits per $9.6 \mathrm{~mm}$ stainless steel aliquot. A focussed $532 \mathrm{~nm} \mathrm{Nd:YVO}$ laser provided the stimulation and luminescence detection was through a Hoya U-340 filter placed in front of the photomultiplier tube. All grains were analysed using the single aliquot regenerative (SAR) approach (Murray and Wintle 2000, 2003), in which an interpolative growth curve is constructed using data derived from repeated measurements of a single aliquot which has been given various laboratory irradiations (Figure $1 \mathrm{a}$ and $1 \mathrm{~b}$ ). The most appropriate preheat temperature for the site was derived experimentally using single aliquots and a dose recovery test with a range of preheat temperatures (after Murray and Wintle, 2003). As Figure 2 shows the 
$160{ }^{\circ} \mathrm{C}$ for $10 \mathrm{~s}$ preheat recovers the $11.6 \mathrm{~Gy}$ dose within a few percent. The purity of the quartz extract was checked using infrared stimulated luminescence.

(a)

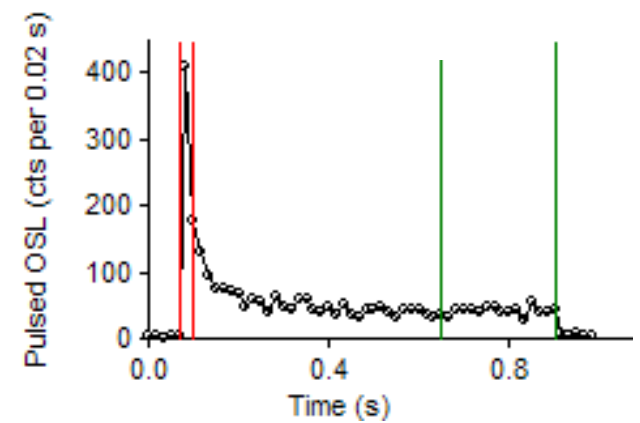

(b)

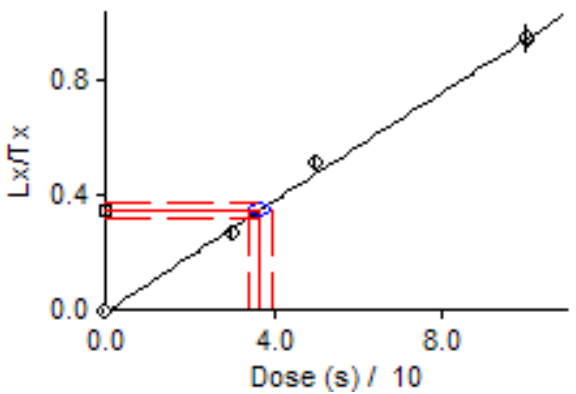

Figure 1: Examples of single grain OSL data (a) single grain OSL decay of naturally acquired signal for sample Shfd12033 (b) Single grain SAR growth curve for sample Shfd12033. Note red lines in (a) and (c) indicate block of data used as OSL signal and green lines indicate block of data used as OSL background. Red lines in (b) and (d) indicate where naturally acquired OSL signal intercepts with SAR growth curve (and associated uncertainties) from which the naturally acquired dose can be calculated.

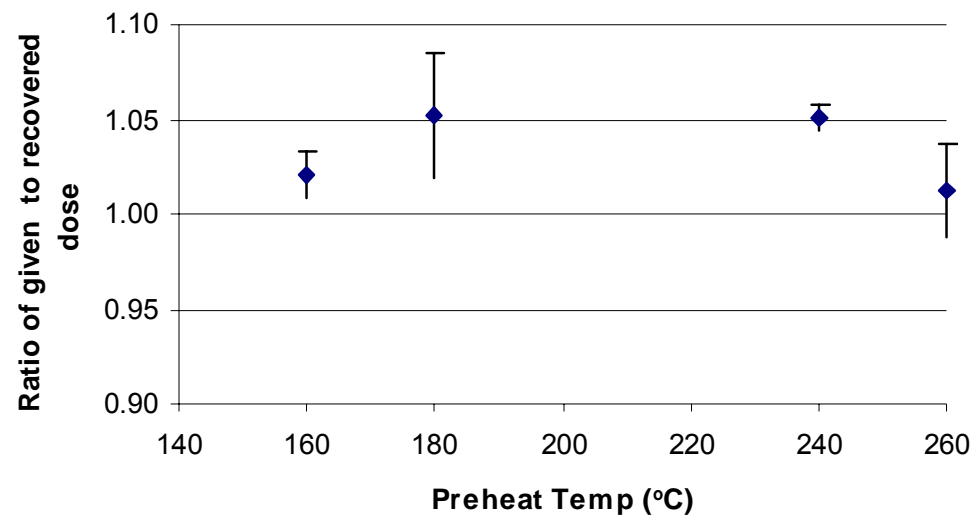

Figure 2 Results of different preheat temperatures in recovering a 11.6 Gy beta radiation dose from sample Shfd12049.

With all single grain OSL analyses many grains exhibit insufficient OSL signal to be utilised and/or are too poorly behaved for the De to be accurately measured. In this study, De values from individual grains were only accepted they exhibited an OSL signal measurable above background, good growth with dose and the error on the test dose used within the SAR protocol was less than $20 \%$. It was found that the samples exhibited a good proportion of grains with detectable signal and which were well behaved and sufficiently sensitive to laboratory dose that they yielded reliable palaeodoses. In order to get sufficient data, 800 grains were measured for samples Shfd12031 and Shfd12033 and 1000 grains were measured for sample Shfd12032.

4. Sedimentary bleaching behaviour: The effects of incomplete bleaching of the sediment during the last period of transport or exposure in situ can be profound. Typically, poorly bleached sediments retain a significant level of residual signal from previous phases of sedimentary cycling, leading to inherent inaccuracies in the calculation of a palaeodose value. By plotting the replicate data for each sample as a probability density function some assessment of whether older or younger material has been included in the sample measurements can be made (Figure 3). In principle a well bleached unpost-depositionally disturbed sample should have replicate palaeodose (De) data which is normally distributed and highly reproducible (See Bateman et al. 2003, Fig 3; Bateman et al 2007a). Where post-depositional disturbance or incomplete bleaching prior to sample burial has occurred skewing of this distribution may occur and/or 
replicate reproducibility may be lower (Bateman et al 2007a; Bateman et al. 2007b). In the case of poorly bleached material skewing should be evident with a high De tail (e.g. Olley et al. 2004).
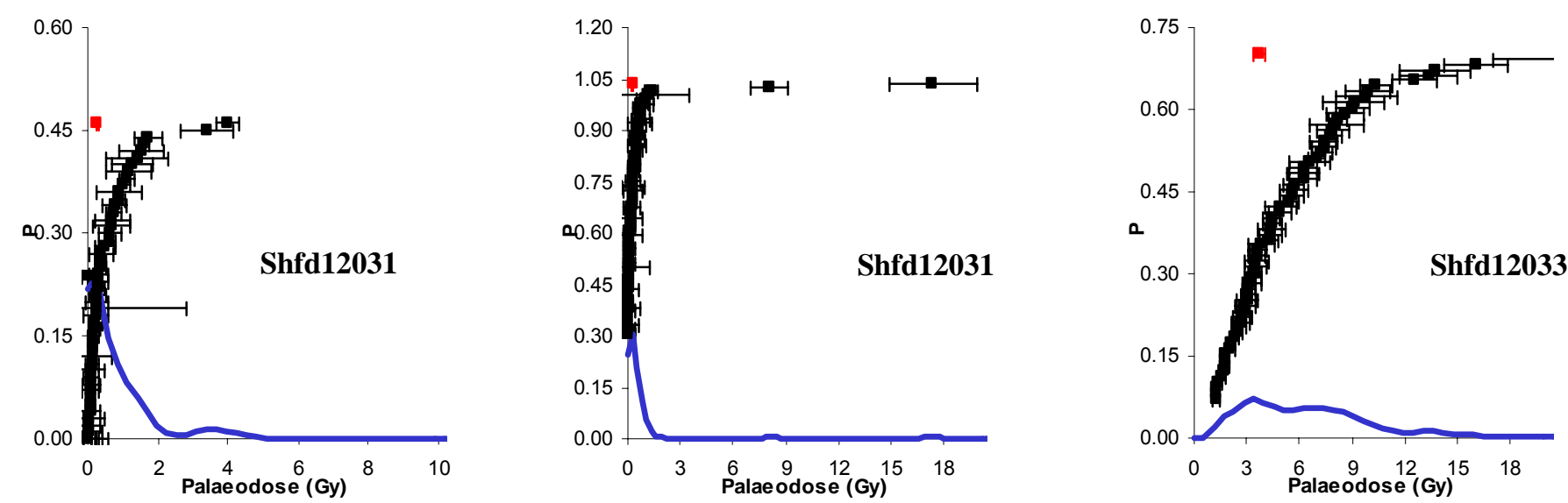

Figure 3: Examples of combined probability density functions for the single grain OSL measurements showing degree of inter-aliquot scatter. Also plotted are individual grain De (black) and the unweighted mean De (red).

As Figure 3 and Table 3 (see also appendix) shows, the De distributions measured for all the samples have some grains with high uncertainties, overall have high OD values, have zero De values and have multiple De modes. This is taken to indicate that the deposits have either undergone some post-depositional disturbance since deposition and/or include unbleached grains. Samples Shfd12031 and Shfd12032 intercept with zero De showing a component of grains within uncertainties of zero whilst Shfd12033 is skewed and more likely to have an unreset component (or grains moved up profile by bioturbation). In order to try and better understand the De distrubutions, the De values for all samples were statistically analysed using the finite mixture model (FMM: Roberts at al 2000). This model attempts to extract the different multiple components contained within the De distributions. Results from this (excluding any component representing less than $10 \%$ of data as per Bateman et al 2010) are shown in Table 3.

Table 3. Results of Single grain level of analysis with Finite Mixture modelling used on De data to extract multiple components for each sample. Dominant $\mathrm{De}$ and age derived from it highlighted in bold.

\begin{tabular}{lccccccc}
\hline Lab Code & Field Ref. & $\begin{array}{c}\text { Depth } \\
(\mathbf{c m})\end{array}$ & $\begin{array}{c}\text { FMM } \\
\text { component }\end{array}$ & $\begin{array}{c}\text { De } \\
(\mathrm{Gy})\end{array}$ & $\begin{array}{c}\text { Proportion } \\
\text { of grains } \\
(\%)\end{array}$ & $\begin{array}{c}\text { Dose rate } \\
(\mathrm{Gy} / \mathrm{ka})\end{array}$ & $\begin{array}{c}\text { Age } \\
(\mathrm{ka})\end{array}$ \\
\hline Shfd12031 & Sample 1 & 30 & 1 & $\mathbf{0 . 4 3} \pm \mathbf{0 . 0 8}$ & 59 & $1.536 \pm 0.059$ & $\mathbf{0 . 2 8} \pm \mathbf{0 . 0 5}$ \\
& & & 2 & $1.18 \pm 0.13$ & 35 & $1.536 \pm 0.059$ & $0.77 \pm 0.09$ \\
Shfd12032 & Sample 2 & 23 & 1 & $\mathbf{0 . 4 0 \pm 0 . 0 4}$ & 84 & $1.756 \pm 0.070$ & $\mathbf{0 . 2 3} \pm \mathbf{0 . 0 2}$ \\
& & & 2 & $0.94 \pm 0.20$ & 12 & $1.756 \pm 0.070$ & $0.54 \pm 0.12$ \\
Shfd12033 & Sample 3 & 45 & 1 & $1.71 \pm 0.12$ & 19 & $1.592 \pm 0.082$ & $0.94 \pm 0.08$ \\
& & & 2 & $3.51 \pm 0.23$ & 36 & $1.592 \pm 0.082$ & $\mathbf{1 . 9 4} \pm \mathbf{0 . 1 5}$ \\
& & & 3 & $7.36 \pm 0.39$ & 37 & $1.592 \pm 0.082$ & $4.06 \pm 0.27$
\end{tabular}

a only component representing more than $10 \%$ of De data are reported.

${ }^{\dagger}$ Total Dose is attenuated for grain size, density and moisture as well as assuming sample saturation prior to 13 ka before present.

${ }^{\ddagger}$ De extracted using the central age model as good reproducibility 
For partially bleached samples it has been argued that the first De mode should closest relate to the true burial age. This is suggested to be the case for sample Shfd12033. For disturbed sediments the dominant (that incorporating the results of the most number of aliquots) should closest relate to the true burial age (Bateman et al. 2007a,b). This is suggested to be the case for samples Shfd12031 and Shfd12032.

5. Age Calculation and Conclusions: Ages are quoted in ka from the present day (2012) and are presented with one sigma confidence intervals which incorporate systematic uncertainties with the dosimetry data, uncertainties with the palaeomoisture content and errors associated with the De determination. Table 3 shows the final OSL age estimates for each component recognised within a sample). Grain data for each sample are included in appendix 1. The data presented all samples had appreciable De scatter. It is thought this maybe due to post-depositional disturbance for samples Shfd12031 and Shfd12032 and partial bleaching for samples Shfd12033. Whilst efforts have been made to mitigate the impact of this, ages may still incorporate some problems and should be treated with due caution. Ages presented in Tables 3 should be viewed in alongside site stratigraphy and sedimentological evidence that might provide information of depositional and post-depositional contexts within which the OSL results could be interpreted. The best estimates of ages range from $0.23 \pm 0.02 \mathrm{ka}$ (Shfd12032) to $0.94 \pm 0.08 \mathrm{ka}$ (Shfd12033).

Prof Mark D. Bateman

\section{References:}

ADAMIEC G. and AITKEN MJ. (1998). Dose-rate conversion factors update. Ancient TL 16: 37-50

AITKEN, M. J. (1998). An Introduction to Optical Dating: The dating of Quaternary sediments by the use of PhotoStimulated Luminescence. Oxford Science Publication.

BATEMAN, M.D., BOULTER, C.H. AND MURTON J.B. (2010). The source of $D_{e}$ variability in periglacial sand wedges: depositional processes v. measurement issues. Quaternary Geochronology (in press).

BATEMAN, M.D., BOULTER, C.H., CARR, A.S., FREDERICK, C.D., PETER, D., WILDER, M. (2007a). Detecting Post-depositional sediment disturbance in sandy deposits using optical luminescence. Quaternary Geochronology 2, 57-64.

BAteman, M.D., BOULTeR, C.H., CARR, A.S., FREDERICK, C.D., PETER, D. AND WILDER, M. (2007b). Preserving the palaeoenvironmental record in Drylands: Bioturbation and its significance for luminescence derived chronologies. Sediment Geology, 195, 5-19.

BATEMAN, M.D., FREDERICK, C.D., JAISWAL, M.K. AND SINGHVI, A.K. (2003). Investigations into the potential effects of pedoturbation on luminescence dating. Quaternary Science Reviews, 22, 1169-1176.

BATEMAN, M.D. \& CATT, J.A. (1996). An absolute chronology for the raised beach deposits at Sewerby, E. Yorkshire, UK. Journal of Quaternary Science, 11, 389-395.

GALBRAITH, R.F. and GREEN, P.F. (1990). Estimating the component ages in a finite mixture. Radiation Measurements, 17,197-206.

MARSH RE, PRESTWICH WV, RINK WJ, BRENNAN BJ. (2002). Monte Carlo determinations of the beta dose rate to tooth enamel. Radiation Measurements 35: 609-616

MURRAY, A.S. \& WINTLE, A.G. (2000). Luminescence dating of quartz using an improved single-aliquot regenerativedose protocol. Radiation Measurements 32, 57-73.

MURRAY AS, WINTLE AG. (2003). The single aliquot regenerative dose protocol: potential for improvements in reliability. Radiation Measurements 37: 377-381. 
OLLEY, J.M., PIETSCH T., ROBERTS, R.G. (2004). Optical dating of Holocene sediments from a variety of geomorphic settings using single grains of quartz. Geomorphology 60, 337-358.

PRESCOTT, J.R. \& HUTTON, J.T. (1994). Cosmic ray contributions to dose rates for luminescence and ESR dating: large depths and long-term time variations. Radiation Measurements, 2/3, 497-500.

ROBERTS, R.G. GALBRAITH, R.F., YOSHIDA, H., LASLETT, G.M. \& OLLEY, J.M. (2000). Distinguishing dose populations in sediment mixtures: a test of single-grain optical dating procedures using mixtures of laboratory-dosed quartz. Radiation Measurements 32, 459-465. 


\section{Appendix 1}

\section{Single grain data and plots for the $41 \mathrm{FN} 175$, East Texas.}

Sample specific data including:-

- $\quad$ list of De's derived from aliquots

- $\quad$ calculated statics for De distribution (Skewness, kurtosis and sorting)

- $\quad$ calculated means based on a range of statistical models including Finite Mixture Modelling (FMM)

- $\quad$ histogram plot of distribution of De within a sample

- $\quad$ probability density plot (curve) with ranked De data (black points) and probability mean (uppermost red point). 
Field Code:

Lab Code:

Aliquot Size:

\begin{tabular}{|c|c|}
\hline Aliquot & Palaeodose (Gy) \\
\hline 1 & 1.113 \\
\hline 2 & 0.127 \\
\hline 3 & 1.719 \\
\hline 4 & -0.044 \\
\hline 5 & 0.083 \\
\hline 6 & 1.035 \\
\hline 7 & 0.166 \\
\hline 8 & 0.147 \\
\hline 9 & 0.693 \\
\hline 10 & 0.254 \\
\hline 11 & -0.103 \\
\hline 12 & 0.381 \\
\hline 13 & 0.093 \\
\hline 14 & 0.376 \\
\hline 15 & 0.303 \\
\hline 16 & 0.249 \\
\hline 17 & 0.083 \\
\hline 18 & 0.273 \\
\hline 19 & 0.215 \\
\hline 20 & -0.029 \\
\hline 21 & 0.156 \\
\hline 22 & 0.034 \\
\hline 23 & 0.093 \\
\hline 24 & 0.396 \\
\hline 25 & 0.171 \\
\hline 26 & 0.059 \\
\hline 27 & 0.903 \\
\hline 28 & -0.005 \\
\hline 29 & -0.098 \\
\hline 30 & 0.728 \\
\hline 31 & 3.995 \\
\hline 32 & 0.112 \\
\hline 33 & 0.044 \\
\hline 34 & 0.772 \\
\hline 35 & 0.640 \\
\hline 36 & 1.431 \\
\hline 37 & 0.635 \\
\hline
\end{tabular}

\section{Site: $\quad 41$ FN 175 \\ East Texas}

Shfd12031

single grain

$\begin{array}{lrcc}\text { error } & \text { Aliquot } & \text { Palaeodose (Gy) } & \text { error } \\ 0.195 & 38 & 1.162 & 0.643 \\ 0.366 & 39 & 0.313 & 0.299 \\ 0.415 & 40 & 1.275 & 0.565 \\ 0.604 & 41 & 1.616 & 0.132 \\ 0.309 & 42 & 0.083 & 0.077 \\ 0.181 & 43 & 0.322 & 0.256 \\ 0.119 & 44 & -0.039 & 0.228 \\ 0.195 & 45 & 0.147 & 0.550 \\ 0.511 & 46 & -0.029 & 0.290 \\ 2.545 & 47 & 0.273 & 0.247 \\ 0.149 & 48 & 0.718 & 0.487 \\ 0.163 & 49 & 1.524 & 0.633 \\ 0.237 & 50 & 0.923 & 0.633 \\ 0.149 & 51 & 3.384 & 0.740 \\ 0.158 & 52 & 0.503 & 0.275 \\ 0.337 & 53 & -0.039 & 0.106 \\ 0.132 & 54 & 0.205 & 0.149\end{array}$

0.318

0.186

0.342

0.132

0.386

0.256

0.357

0.149

0.400

0.172

0.123

0.439

0.218

0.313

0.200

0.318

0.371

0.323

0.881

0.154 
Field Code:

Lab Code:

Aliquot Size:

Sample 1

Shfd12031

single grain

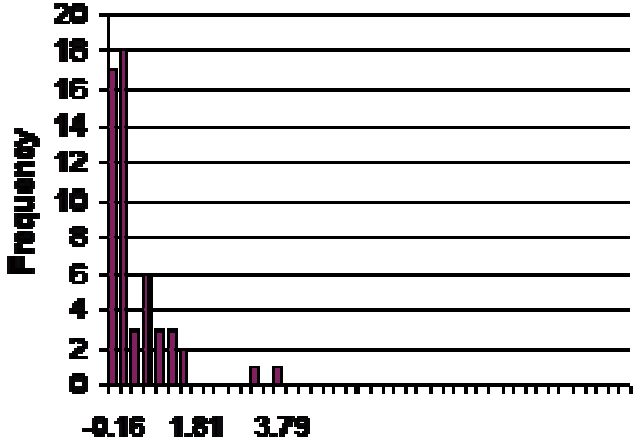

Paleodose [G]


Site:

41 FN 175

East Texas

\begin{tabular}{|ccc|}
\hline Unweighted & & \\
& All Data & Minus Outliers \\
Mean (Gy) & 0.55 & 0.32 \\
SD & 0.78 & 0.33 \\
SE & 0.11 & 0.05 \\
N & 54 & 47 \\
\hline
\end{tabular}

\begin{tabular}{|ccc|}
\hline Weighted & All Data & Minus Outliers \\
Mean (Gy) & 0.35 & 0.24 \\
SD & 0.54 & 0.34 \\
SE & 0.07 & 0.05 \\
N & 54 & 47 \\
\hline
\end{tabular}

\section{Probability}

\begin{tabular}{|c|c|c|} 
& All Data & Minus Outliers \\
Mean (Gy) & 0.29 & 0.25 \\
SD & 0.37 & 0.28 \\
SE & 0.05 & 0.04 \\
\hline N & 54 & 47 \\
\hline
\end{tabular}

\begin{tabular}{|lcc|}
\hline Central Age Model & & \\
& All Data & Minus Outliers \\
Mean (Gy) & 0.66 & 0.20 \\
SD & 0.12 & 26.48 \\
OD (all data) & $74.26 \%$ & \\
N & 54 & 47 \\
\hline
\end{tabular}

\begin{tabular}{|lcc|}
\hline & De (Gy) & error \\
Minimum & 0.00 & 0.12 \\
Maximum & 3.99 & 0.31 \\
$\mathbf{N}$ & 54 & \\
\hline
\end{tabular}

\begin{tabular}{|l|cc|}
\hline De Distribution & All Data & Minus \\
Skewness & 13.66 & 0.03 \\
Kurtosis & 8.89 & 0.26 \\
Median & 0.26 & 0.21 \\
Sorting & 0.92 & 0.59 \\
\hline
\end{tabular}

\section{Finite Mixture Modelling}

Component Mean De (Gy)

$\begin{array}{ll}1 & 0.43 \\ 2 & 1.18 \\ 3 & 3.76\end{array}$

Error

Proportion

$\begin{array}{rr}0.08 & 59 \% \\ 0.13 & 35 \% \\ 0.55 & 6 \%\end{array}$


Field Code:

Lab Code:

Aliquot Size:
Sample 2

Shfd12032

single grain
Site: 41 FN 175

\section{East}

Texas

\begin{tabular}{|c|c|c|c|c|c|}
\hline Aliquot & Palaeodose (Gy) & error & Aliquot & $\begin{array}{l}\text { Palaeodose } \\
\text { (Gy) }\end{array}$ & error \\
\hline 1 & 0.366 & 0.299 & 38 & 0.063 & 0.091 \\
\hline 2 & 0.127 & 0.328 & 39 & -0.161 & 0.177 \\
\hline 3 & 17.370 & 2.477 & 40 & 0.254 & 0.102 \\
\hline 4 & 8.126 & 1.061 & 41 & 0.591 & 0.233 \\
\hline 5 & 0.171 & 0.304 & 42 & 0.327 & 0.204 \\
\hline 6 & 0.557 & 0.424 & 43 & -0.327 & 0.405 \\
\hline 7 & 0.479 & 0.154 & 44 & 1.138 & 2.325 \\
\hline 8 & 0.713 & 0.516 & 45 & 0.303 & 0.502 \\
\hline 9 & -0.078 & 0.275 & 46 & 0.762 & 0.482 \\
\hline 10 & 0.581 & 0.313 & 47 & 0.151 & 0.190 \\
\hline 11 & 0.342 & 0.149 & 48 & -0.166 & 0.186 \\
\hline 12 & -0.205 & 0.218 & 49 & 0.298 & 0.228 \\
\hline 13 & 0.742 & 0.223 & 50 & 0.171 & 0.102 \\
\hline 14 & 0.278 & 0.163 & 51 & 1.333 & 0.328 \\
\hline 15 & 0.523 & 0.492 & 52 & 0.454 & 0.110 \\
\hline 16 & 0.640 & 0.405 & 53 & -0.024 & 0.149 \\
\hline 17 & 0.151 & 0.140 & 54 & 0.210 & 0.214 \\
\hline 18 & 0.103 & 0.251 & 55 & 0.151 & 0.652 \\
\hline 19 & 0.356 & 0.294 & 56 & 0.669 & 0.672 \\
\hline 20 & -0.024 & 0.204 & 57 & 0.288 & 0.099 \\
\hline 21 & -0.122 & 0.725 & 58 & 0.151 & 0.110 \\
\hline 22 & 0.552 & 0.357 & 59 & 0.010 & 0.158 \\
\hline 23 & -0.435 & 0.434 & 60 & 0.107 & 1.203 \\
\hline 24 & 0.278 & 0.136 & 61 & 0.518 & 0.309 \\
\hline 25 & 0.537 & 0.233 & 62 & 0.986 & 0.181 \\
\hline 26 & -0.059 & 0.318 & 63 & 0.322 & 0.439 \\
\hline 27 & 0.308 & 0.633 & 64 & 0.137 & 0.285 \\
\hline 28 & 0.024 & 0.294 & 65 & 0.723 & 0.256 \\
\hline 29 & 0.088 & 0.158 & 66 & -0.381 & 0.270 \\
\hline 30 & 0.234 & 0.647 & 67 & 0.239 & 0.190 \\
\hline 31 & 0.127 & 0.123 & 68 & -0.024 & 0.608 \\
\hline 32 & -0.273 & 0.618 & 69 & -0.161 & 0.410 \\
\hline 33 & 0.142 & 0.081 & 70 & 1.026 & 0.482 \\
\hline 34 & 0.137 & 0.214 & 71 & 0.952 & 0.565 \\
\hline 35 & 0.425 & 0.294 & 72 & 0.039 & 0.237 \\
\hline 36 & 0.313 & 0.209 & 73 & 0.132 & 0.177 \\
\hline 37 & 0.488 & 0.163 & 74 & 0.259 & 0.473 \\
\hline
\end{tabular}


Field Code:

Lab Code:

Aliquot Size:

Sample 2

Shfd12032

single grain
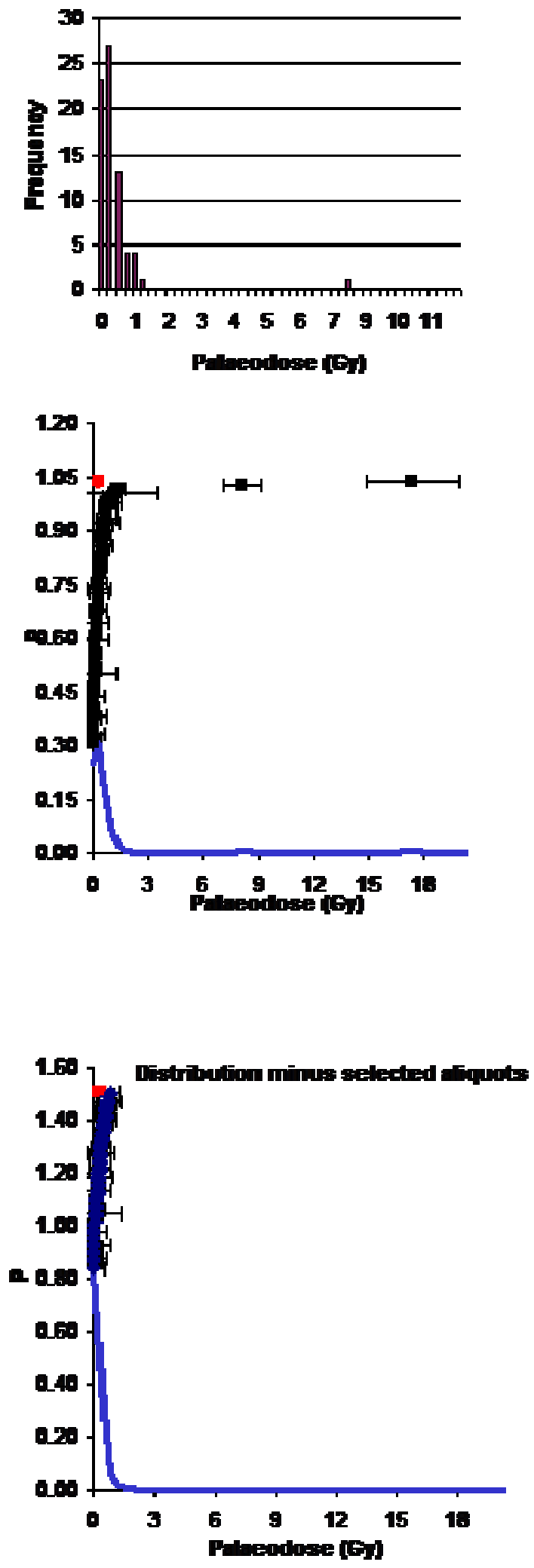

Site:

41 FN 175

E. Texas

\begin{tabular}{|ccc|}
\hline Unweighted & & \\
& All Data & Minus Outliers \\
Mean (Gy) & 0.65 & 0.26 \\
SD & 2.19 & 0.23 \\
SE & 0.25 & 0.03 \\
N & 74 & 67 \\
\hline
\end{tabular}

\begin{tabular}{|ccc|}
\hline Weighted & All Data & Minus Outliers \\
Mean (Gy) & 0.25 & 0.22 \\
SD & 0.33 & 0.24 \\
SE & 0.04 & 0.03 \\
N & 74 & 67 \\
\hline
\end{tabular}

\begin{tabular}{|c|c|c|}
\hline Probability & All Data & Minus Outliers \\
\hline Mean (Gy) & 0.30 & 0.27 \\
SD & 0.36 & 0.23 \\
SE & 0.04 & 0.03 \\
N & 74 & 67 \\
\hline
\end{tabular}

\begin{tabular}{|lcc|}
\hline \multicolumn{2}{|l|}{ Central Age Model } & \\
& All Data & Minus Outliers \\
Mean (Gy) & 0.48 & 0.09 \\
SD & 0.10 & 9.35 \\
OD (all data) & $92.58 \%$ & \\
N & 74 & 67 \\
\hline
\end{tabular}

\begin{tabular}{|lcc|}
\hline \multirow{2}{*}{ Minimum } & De (Gy) & error \\
Maximum & 0.00 & 0.61 \\
$\mathbf{N}$ & 17.37 & 2.48 \\
\hline
\end{tabular}

\begin{tabular}{|l|cc|}
\hline De & All Data & Minus \\
Distribution & 34.14 & 0.00 \\
\hline Skewness & 49.37 & -0.65 \\
Kurtosis & 0.26 & 0.21 \\
Median & 0.50 & 0.37 \\
\hline Sorting & & \\
\hline
\end{tabular}

\begin{tabular}{|c|c|c|c|}
\hline \\
\hline \multicolumn{4}{|c|}{$\begin{array}{r}\text { Finite Mixture Modelling } \\
\text { Mean De }\end{array}$} \\
\hline Component & (Gy) & Error & Proportion \\
\hline 1 & 0.40 & 0.04 & $84 \%$ \\
\hline 2 & 0.94 & 0.20 & $12 \%$ \\
\hline 3 & 11.68 & 1.68 & $4 \%$ \\
\hline 4 & & & \\
\hline
\end{tabular}

Page 11

SCIDR luminesc ence laboratory - 17/07/ 2012 
Field Code:

Lab Code:

Aliquot Size:

$\begin{array}{rcc}\text { Aliquot } & \text { Palaeodose (Gy) } & \text { error } \\ 1 & 7.423 & 0.784 \\ 2 & 3.120 & 0.381 \\ 3 & 1.802 & 0.190 \\ 4 & 1.758 & 0.223 \\ 5 & 1.797 & 0.140 \\ 6 & 16.056 & 1.852 \\ 7 & 1.255 & 0.218 \\ 8 & 1.592 & 0.218 \\ 9 & 3.042 & 0.570 \\ 10 & 5.718 & 0.589 \\ 11 & 4.429 & 0.531 \\ 12 & 2.300 & 0.261 \\ 13 & 5.601 & 0.715 \\ 14 & 2.837 & 0.405 \\ 15 & 2.012 & 0.357 \\ 16 & 5.421 & 0.497 \\ 17 & 2.095 & 0.261 \\ 18 & 1.729 & 0.132 \\ 19 & 7.916 & 0.458 \\ 20 & 2.559 & 0.429 \\ 21 & 7.916 & 0.881 \\ 22 & 3.628 & 0.487 \\ 23 & 2.476 & 0.304 \\ 24 & 2.920 & 0.570 \\ 25 & 3.467 & 0.342 \\ 26 & 4.932 & 0.940 \\ 27 & 13.717 & 1.979 \\ 28 & 4.893 & 0.677 \\ 29 & 2.681 & 0.531 \\ 30 & 6.622 & 1.159 \\ 31 & 10.353 & 0.915 \\ 32 & 1.402 & 0.190 \\ 33 & 6.226 & 0.798 \\ 34 & 1.245 & 0.209 \\ 35 & 3.789 & 0.750 \\ 36 & 3.072 & 0.390 \\ 37 & 2.754 & 0.531 \\ & & \end{array}$

Site: $\quad 41$ FN 175

East

Texas

Shfd12033

single grain

$\begin{array}{ccc}\text { Aliquot } & \text { Palaeodose (Gy) } & \text { error } \\ 38 & 6.241 & 0.915 \\ 39 & 4.390 & 0.818 \\ 40 & 8.624 & 1.032 \\ 41 & 6.246 & 0.774 \\ 42 & 7.408 & 0.677 \\ 43 & 3.570 & 0.526 \\ 44 & 3.501 & 0.662 \\ 45 & 8.233 & 0.521 \\ 46 & 13.366 & 1.686 \\ 47 & 26.795 & 1.598 \\ 48 & 5.684 & 0.759 \\ 49 & 3.550 & 0.691 \\ 50 & 4.263 & 0.565 \\ 51 & 8.097 & 1.544 \\ 52 & 20.500 & 3.448 \\ 53 & 12.501 & 1.276 \\ 54 & 1.255 & 0.177 \\ 55 & 9.874 & 1.252 \\ 56 & 9.054 & 0.935 \\ 57 & 6.983 & 0.458 \\ 58 & 4.429 & 0.497 \\ 59 & 3.350 & 0.516 \\ 60 & 7.271 & 0.550 \\ 61 & 4.361 & 0.638 \\ 62 & 3.316 & 0.521 \\ 63 & 9.137 & 1.764 \\ 64 & 9.816 & 1.764\end{array}$


Field Code:

Lab Code:

Sample 3

Shfd12033

Aliquot Size:

single grain
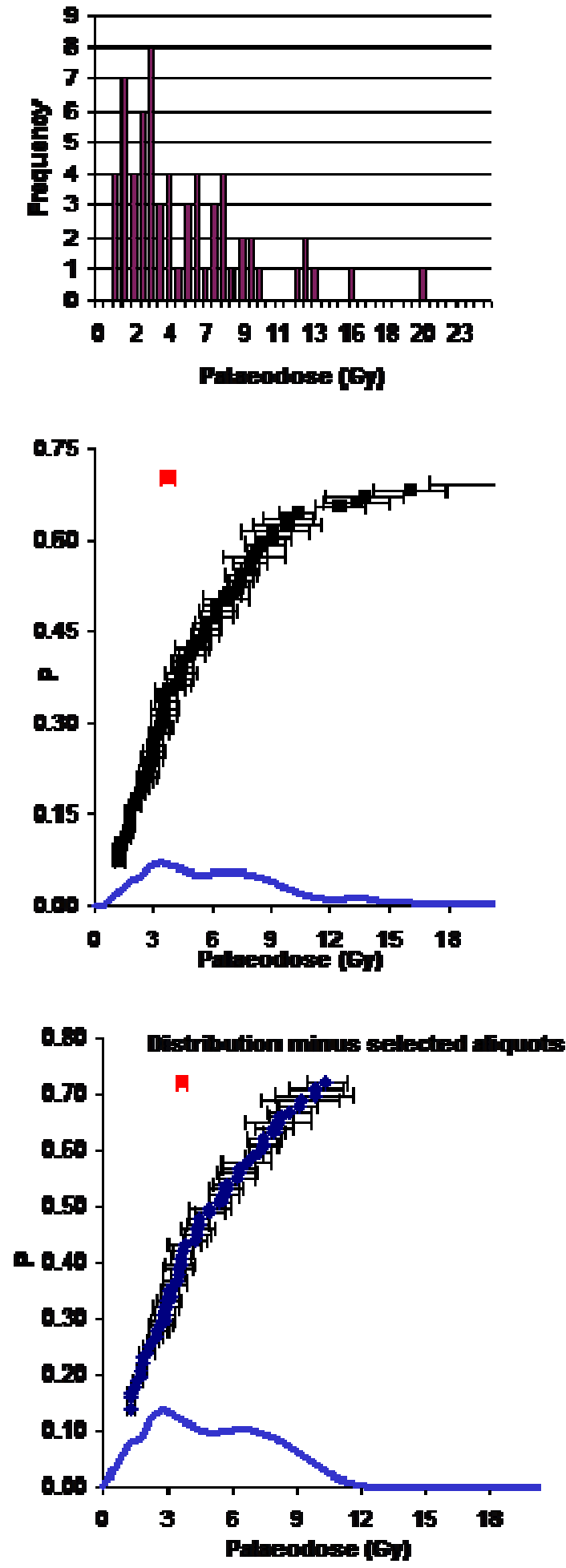

Site:

41 FN 175

E Texas

\begin{tabular}{|ccc|}
\hline & & \\
Unweighted & All Data & Minus Outliers \\
Mean (Gy) & 5.85 & 4.68 \\
SD & 4.68 & 2.58 \\
SE & 0.58 & 0.32 \\
N & 64 & 58 \\
\hline
\end{tabular}

\begin{tabular}{|ccc|}
\hline Weighted & All Data & Minus Outliers \\
Mean (Gy) & 2.57 & 2.51 \\
SD & 1.97 & 2.58 \\
SE & 0.25 & 0.34 \\
N & 64 & 58 \\
\hline
\end{tabular}

\begin{tabular}{|c|c|c|}
\hline Probability & & \\
\hline Mean (Gy) & All Data & Minus Outliers \\
\hline SD & 3.72 & 3.63 \\
SE & 2.36 & 2.10 \\
N & 0.29 & 0.28 \\
\hline
\end{tabular}

\begin{tabular}{|c|c|c|}
\hline \multicolumn{3}{|c|}{ Central Age Model } \\
\hline & All Data & Minus Outliers \\
\hline Mean (Gy) & 4.56 & 3.99 \\
\hline SD & 0.41 & 0.32 \\
\hline OD (all data) & $69.46 \%$ & $57.92 \%$ \\
\hline $\mathbf{N}$ & 64 & 58 \\
\hline
\end{tabular}

\begin{tabular}{|lcr|}
\hline & De (Gy) & error \\
Minimum & 1.25 & 0.21 \\
Maximum & 26.79 & 1.60 \\
$\mathbf{N}$ & 64 & \\
\hline
\end{tabular}

\begin{tabular}{|l|c|c|}
\hline De & & \\
Distribution & All Data & Minus \\
\hline Skewness & 3.46 & -0.12 \\
Kurtosis & 6.50 & -0.79 \\
Median & 4.41 & 4.03 \\
Sorting & 0.61 & 0.45 \\
\hline
\end{tabular}

\begin{tabular}{|rrrrr|}
\hline Finite Mixture Modelling & & & \\
Component & $\begin{array}{c}\text { Mean De } \\
\text { (Gy) }\end{array}$ & Error & \multicolumn{2}{c|}{ Proportion } \\
1 & 1.71 & & 0.12 & $19 \%$ \\
2 & 3.51 & 0.23 & $36 \%$ \\
3 & 7.36 & & 0.39 & $37 \%$ \\
4 & 17.05 & 1.59 & $9 \%$ \\
\hline
\end{tabular}

Page 13 



\section{B-3: THERMOLUMINESCENCE}





\section{LUMINESCENCE ANALYSIS OF CERAMICS FROM PANOLA COUNTY, TEXAS}

14 May 2013

James K. Feathers

Luminescence Dating Laboratory

University of Washington

Seattle, WA 98195-3412

Email: jimf@u.washington.edu

This report presents the results of luminescence analysis on four ceramic samples from site 41PN175 in Panola County, Texas. The samples were submitted by Geo-Marine, Inc., Plano, Texas. Table 1 lists the samples and provenience information. Laboratory procedures are given at the end of the report.

Table 1. Samples

\begin{tabular}{|c|c|c|c|}
\hline UW Lab \# & Provenience \# & Provenience & Burial depth (cm) \\
\hline UW2713 & 1232 & Block 4, Unit 105, Level 3A & $20-24$ \\
\hline UW2714 & 1332 & Block 4, Unit 096, Level 3B & 28 \\
\hline UW2715 & 1336 & Block 8, Unit 117, Level 6 & 29 \\
\hline UW2716 & 1714 & Block 4, Unite 086, Level 5 & 45 \\
\hline
\end{tabular}

\section{Dose rate}

The dose rate was measured on each ceramic and on an associated sediment. Dose rates were mainly determined using alpha counting and flame photometry. The beta dose rate calculated from these measurements on the ceramics was compared with the beta dose rate measured directly by beta counting. These were in statistical agreement for all samples. Moisture content was estimated as $70 \pm$ $30 \%$ of saturated value for the ceramic sherds, and $10 \pm 5$ percent for the sediments. Table 2 gives the radioactivity data and comparison of the beta dose rate calculated in the two ways mentioned earlier. Table 3 gives all dose rates for each sample. The ceramics have higher radioactivity than the sediments, which is not surprising given their clay content.

Table 2. Radionuclide concentrations

\begin{tabular}{|c|c|c|c|c|c|}
\hline \multirow[t]{2}{*}{ Sample } & \multirow{2}{*}{$\begin{array}{l}{ }^{238} U \\
(p p m)\end{array}$} & \multirow{2}{*}{$\begin{array}{l}{ }^{233} \mathrm{Th} \\
\text { (ppm) }\end{array}$} & \multirow{2}{*}{$\begin{array}{c}K \\
(\%)\end{array}$} & \multicolumn{2}{|c|}{ Beta dose rate (Gy/ka) } \\
\hline & & & & $\begin{array}{c}\text { ß- } \\
\text { counting }\end{array}$ & $\begin{array}{c}\alpha- \\
\text { counting/flame } \\
\text { photometry }\end{array}$ \\
\hline UW2713 & $3.07 \pm 0.21$ & $7.67 \pm 1.06$ & $1.13 \pm 0.09$ & $1.64 \pm 0.14$ & $1.56 \pm 0.08$ \\
\hline Sediment & $0.69 \pm 0.09$ & $3.77 \pm 0.75$ & $0.64 \pm 0.04$ & & \\
\hline UW2714 & $3.15 \pm 0.25$ & $13.27 \pm 1.46$ & $0.81 \pm 0.07$ & $1.36 \pm 0.11$ & $1.48 \pm 0.08$ \\
\hline Sediment & $1.80 \pm 0.14$ & $5.09 \pm 0.90$ & $0.68 \pm 0.04$ & & \\
\hline UW2715 & $3.82 \pm 0.22$ & $3.67 \pm 0.83$ & $1.11 \pm 0.08$ & $1.69 \pm 0.14$ & $1.55 \pm 0.08$ \\
\hline Sediment & $1.98 \pm 0.16$ & $6.33 \pm 1.02$ & $0.71 \pm 0.03$ & & \\
\hline UW2716 & $3.88 \pm 0.26$ & $8.55 \pm 1.26$ & $1.05 \pm 0.06$ & $1.67 \pm 0.14$ & $1.64 \pm 0.07$ \\
\hline Sediment & $1.62 \pm 0.14$ & $6.45 \pm 0.94$ & $0.68 \pm 0.05$ & & \\
\hline
\end{tabular}


Table 3. Dose rates $(\mathrm{Gy} / \mathrm{ka})^{*}$

\begin{tabular}{c|ccccc}
\hline Sample & alpha & beta & gamma & cosmic & total \\
\hline UW2713 & $1.87 \pm 0.25$ & $1.36 \pm 0.10$ & $0.42 \pm 0.04$ & $0.22 \pm 0.05$ & $3.88 \pm 0.28$ \\
UW2714 & $2.63 \pm 0.36$ & $1.33 \pm 0.09$ & $0.61 \pm 0.05$ & $0.22 \pm 0.04$ & $4.79 \pm 0.38$ \\
UW2715 & $1.20 \pm 0.15$ & $1.27 \pm 0.11$ & $0.66 \pm 0.06$ & $0.22 \pm 0.04$ & $3.35 \pm 0.20$ \\
UW2716 & $1.79 \pm 0.35$ & $1.41 \pm 0.10$ & $0.65 \pm 0.05$ & $0.20 \pm 0.04$ & $4.05 \pm 0.37$ \\
\hline
\end{tabular}

* Dose rates for ceramics are calculated for TL. They will be lower for OSL due to lower b-values. Also the beta dose rate is lower than that given in Table 2 due to moisture correction.

\section{Equivalent Dose}

Equivalent dose was measured for TL, OSL and IRSL has described in the appendix. TL plateaus were relatively broad for two samples, somewhat narrower for the other two (Table 4), ranging from 60 to $130^{\circ} \mathrm{C}$ in breadth. All but UW2714 showed a sensitivity change with heating. TL anomalous fading was evident in all samples. Fading g-values ranged from 4 to $9 \%$ per decade for three samples, but for UW2713 the fading rate was so high that an infinite correction was obtained, suggesting that the fading rate must have changed through time. The TL age was corrected for the other three following Huntley and Lamothe (2001).

Table 4. TL parameters

\begin{tabular}{c|cccc}
\hline Sample & Plateau $\left({ }^{\circ} \mathrm{C}\right)$ & $1^{\text {st }} / 2^{\text {nd }}$ ratio* & fit & Fading g-value ${ }^{* *}$ \\
\hline UW2713 & $250-380$ & $0.64 \pm 0.07$ & Linear & $22 \pm 4.1$ \\
UW2714 & $250-310$ & 1 & Linear & $4.0 \pm 2.6$ \\
UW2715 & $250-310$ & $0.76 \pm 0.06$ & Linear & $8.8 \pm 2.0$ \\
UW2716 & $260-370$ & $1.56 \pm 0.14$ & Linear & $8.2 \pm 5.6$ \\
\hline
\end{tabular}

*Refers to slope ratio between the first and second glow growth curves. A glow refers to luminescence as a function of temperature; a second glow comes after heating to $450^{\circ} \mathrm{C}$.

** A g-value is a rate of anomalous fading, measured as percent of signal loss per decade, where a decade is a power of 10 .

OSL/IRSL was measured on 5-7 aliquots per sample (Table 5). There was high scatter among aliquots for UW2715, as evident by over-dispersion, and it would have been high for UW2713 except that one outlier was removed. The IRSL signal on all samples was weak, 8 to 10 times less intense than the OSL signal. This is not uncommon for ceramics. IRSL stems from feldspars, which are prone to anomalous fading. A relatively large IRSL signal may suggest the OSL signal partly stems from feldspars and therefore may fade, so the weak IRSL suggests the OSL is dominated by quartz. The OSL b-value, which is a measure of alpha luminescence efficiency, is also typical of quartz. It is therefore likely the OSL signal stems mainly from quartz and does not fade. As a test of the SAR procedures, the recovered dose was nearly the same as the given dose for all samples. Equivalent dose and b-values are given in Table 6. 
Table 5. OSL/IRSL data

\begin{tabular}{|c|c|c|c|c|c|}
\hline Sample & \multicolumn{2}{|c|}{ \# aliquots* } & OSL Over-dispersion (\%) & \multicolumn{2}{c|}{ Dose Recovery (OSL) } \\
\cline { 2 - 6 } & OSL & IRSL & & $\begin{array}{c}\text { Given } \\
\text { Dose (sß) }\end{array}$ & $\begin{array}{c}\text { Recovered } \\
\text { Dose (sß) }\end{array}$ \\
\hline UW2713 & 5 & 6 & $6.9 \pm 4.7$ & 50 & $50.6 \pm 2.5$ \\
\hline UW2714 & 7 & 5 & $2.8 \pm 3.0$ & 20 & $19.6 \pm 0.8$ \\
\hline UW2715 & 6 & 1 & $30 \pm 1.2$ & 30 & $32.0 \pm 1.2$ \\
\hline UW2716 & 7 & 4 & 0 & 30 & $29.1 \pm 1.4$ \\
\hline
\end{tabular}

* Denotes number of aliquots with measurable signals.

Table 6. Equivalent dose and b-value - fine grains

\begin{tabular}{|c|c|c|c|c|c|c|}
\hline \multirow{2}{*}{ Sample } & \multicolumn{3}{|c|}{ Equivalent Dose (Gy) } & \multicolumn{3}{c|}{$b$-value $\left(G y \mu m^{2}\right)$} \\
\cline { 2 - 7 } & $T L$ & $I R S L$ & OSL & $T L$ & $I R S L$ & OSL \\
\hline UW2713 & $4.56 \pm 0.50$ & $3.11 \pm 0.32$ & $2.12 \pm 0.10$ & $2.45 \pm 0.26$ & $1.27 \pm 0.08$ & $0.66 \pm 0.03$ \\
\hline UW2714 & $2.20 \pm 0.12$ & $3.28 \pm 0.32$ & $1.38 \pm 0.03$ & $2.52 \pm 0.29$ & $1.00 \pm 0.12$ & $0.46 \pm 0.01$ \\
\hline UW2715 & $2.79 \pm 0.23$ & $8.52 \pm 0.75$ & $3.33 \pm 0.41$ & $1.80 \pm 0.14$ & $0.70 \pm 0.05$ & $0.34 \pm 0.01$ \\
\hline UW2716 & $1.75 \pm 0.20$ & $1.94 \pm 0.39$ & $1.60 \pm 0.03$ & $1.98 \pm 0.33$ & $1.28 \pm 0.14$ & $0.67 \pm 0.02$ \\
\hline
\end{tabular}

\section{Ages}

Table 7 gives the derived ages for each sample. On three of the samples, there was agreement in age between OSL and TL corrected for fading. On one even the IRSL age was in agreement. The IRSL was not reliable for the other samples because of weak signal and fading. For UW2713, the TL age was older than the OSL age $(840 \pm 160 \mathrm{AD})$. Possibly the heating was not high enough to fully reset the TL signal. It is not likely the problem is the OSL age being too young, because the weak IRSL and the bvalue typical of quartz suggested no fading of this signal.

The ages are quite varied, two in the $15^{\text {th }}$ century, one in the $12^{\text {th }}$ century and one in the $7^{\text {th }}$ century AD.

Table 7. Ages

\begin{tabular}{|c|c|c|c|c|}
\hline Sample & Age (ka) & \% error & Basis for age & Calendar date (years AD) \\
\hline UW2713 & $0.84 \pm 0.06$ & 7.5 & OSL & $1170 \pm 60$ \\
\hline UW2714 & $0.53 \pm 0.03$ & 5.5 & OSL/corrected TL & $1480 \pm 30$ \\
\hline UW2715 & $1.40 \pm 0.16$ & 11.4 & OSL/corrected TL & $610 \pm 160$ \\
\hline UW2716 & $0.56 \pm 0.03$ & 5.7 & OSL/IRSL/corrected TL & $1450 \pm 30$ \\
\hline
\end{tabular}

\section{Procedures for Thermoluminescence Analysis of Pottery}

Sample preparation -- fine grain

The sherd is broken to expose a fresh profile. Material is drilled from the center of the crosssection, more than $2 \mathrm{~mm}$ from either surface, using a tungsten carbide drill tip. The material retrieved is ground gently by an agate mortar and pestle, treated with $\mathrm{HCl}$, and then settled in acetone for 2 and 20 minutes to separate the $1-8 \mu \mathrm{m}$ fraction. This is settled onto a maximum of 72 stainless steel discs. 


\section{Glow-outs}

Thermoluminescence is measured by a Daybreak reader using a 96350 photomultiplier with a Corning 7-59 blue filter, in $\mathrm{N}_{2}$ atmosphere at $1^{\circ} \mathrm{C} / \mathrm{s}$ to $450^{\circ} \mathrm{C}$. A preheat of $240^{\circ} \mathrm{C}$ with no hold time precedes each measurement. Artificial irradiation is given with a ${ }^{241} \mathrm{Am}$ alpha source and ${ }^{90} \mathrm{Sr}$ beta source, the latter calibrated against a ${ }^{137} \mathrm{Cs}$ gamma source. Discs are stored at room temperature for at least one week after irradiation before glow out. Data are processed by Daybreak TLApplic software.

\section{Fading test}

Several discs are used to test for anomalous fading. The natural luminescence is first measured by heating to $450^{\circ} \mathrm{C}$. The discs are then given an equal alpha irradiation and stored at room temperature for varied times: $10 \mathrm{~min}, 2$ hours, 1 day, 1 week and 8 weeks. The irradiations are staggered in time so that all of the second glows are performed on the same day. The second glows are normalized by the natural signal and then compared to determine any loss of signal with time (on a log scale). If the sample shows fading and the signal versus time values can be reasonably fit to a logarithmic function, an attempt is made to correct the age following procedures recommended by Huntley and Lamothe (2001). The fading rate is calculated as the g-value, which is given in percent per decade, where decade represents a power of 10.

\section{Equivalent dose}

The equivalent dose is determined by a combination additive dose and regeneration (Aitken 1985). Additive dose involves administering incremental doses to natural material. A growth curve plotting dose against luminescence can be extrapolated to the dose axis to estimate an equivalent dose, but for pottery this estimate is usually inaccurate because of errors in extrapolation due to nonlinearity. Regeneration involves zeroing natural material by heating to $450^{\circ} \mathrm{C}$ and then rebuilding a growth curve with incremental doses. The problem here is sensitivity change caused by the heating. By constructing both curves, the regeneration curve can be used to define the extrapolated area and can be corrected for sensitivity change by comparing it with the additive dose curve. This works where the shapes of the curves differ only in scale (i.e., the sensitivity change is independent of dose). The curves are combined using the "Australian slide" method in a program developed by David Huntley of Simon Fraser University (Prescott et al. 1993). The equivalent dose is taken as the horizontal distance between the two curves after a scale adjustment for sensitivity change. Where the growth curves are not linear, they are fit to quadratic functions. Dose increments (usually five) are determined so that the maximum additive dose results in a signal about three times that of the natural and the maximum regeneration dose about five times the natural.

A plateau region is determined by calculating the equivalent dose at temperature increments between $240^{\circ}$ and $450^{\circ} \mathrm{C}$ and determining over which temperature range the values do not differ significantly. This plateau region is compared with a similar one constructed for the b-value (alpha efficiency), and the overlap defines the integrated range for final analysis.

\section{Alpha effectiveness}

Alpha efficiency is determined by comparing additive dose curves using alpha and beta irradiations. The slide program is also used in this regard, taking the scale factor (which is the ratio of the two slopes) as the b-value (Aitken 1985). 


\section{Radioactivity}

Radioactivity is measured by alpha counting in conjunction with atomic emission for ${ }^{40} \mathrm{~K}$. Samples for alpha counting are crushed in a mill to flour consistency, packed into plexiglass containers with ZnS:Ag screens, and sealed for one month before counting. The pairs technique is used to separate the $\mathrm{U}$ and Th decay series. For atomic emission measurements, samples are dissolved in $\mathrm{HF}$ and other acids and analyzed by a Jenway flame photometer. K concentrations for each sample are determined by bracketing between standards of known concentration. Conversion to ${ }^{40} \mathrm{~K}$ is by natural atomic abundance. Radioactivity is also measured, as a check, by beta counting, using a Ris $\varnothing$ low level beta GM multicounter system. About $0.5 \mathrm{~g}$ of crushed sample is placed on each of four plastic sample holders. All are counted for 24 hours. The average is converted to dose rate following B $\varnothing$ tter-Jensen and Mejdahl (1988) and compared with the beta dose rate calculated from the alpha counting and flame photometer results.

Both the sherd and an associated soil sample are measured for radioactivity. Additional soil samples are analyzed where the environment is complex, and gamma contributions determined by gradients (after Aitken 1985: appendix H). Cosmic radiation is determined after Prescott and Hutton (1988). Radioactivity concentrations are translated into dose rates following Adamiec and Aitken (1998).

\section{Moisture Contents}

Water absorption values for the sherds are determined by comparing the saturated and dried weights. For temperate climates, moisture in the pottery is taken to be $80 \pm 20$ percent of total absorption, unless otherwise indicated by the archaeologist. Again for temperate climates, soil moisture contents are taken from typical moisture retention quantities for different textured soils (Brady 1974: 196), unless otherwise measured. For drier climates, moisture values are determined in consultation with the archaeologist.

\section{Procedures for Optically Stimulated or Infrared Stimulated Luminescence of Fine-grained pottery.}

Optically stimulated luminescence (OSL) and infrared stimulated luminescence (IRSL) on finegrain $(1-8 \mu \mathrm{m})$ pottery samples are carried out on single aliquots following procedures adapted from Banerjee et al. (2001) and Roberts and Wintle (2001. Equivalent dose is determined by the singlealiquot regenerative dose (SAR) method (Murray and Wintle 2000).

The SAR method measures the natural signal and the signal from a series of regeneration doses on a single aliquot. The method uses a small test dose to monitor and correct for sensitivity changes brought about by preheating, irradiation or light stimulation. SAR consists of the following steps: 1) preheat, 2) measurement of natural signal (OSL or IRSL), L(1), 3) test dose, 4) cut heat, 5) measurement of test dose signal, $T(1), 6)$ regeneration dose, 7) preheat, 8) measurement of signal from regeneration, $L(2), 9)$ test dose, 10) cut heat, 11) measurement of test dose signal, $T(2), 12)$ repeat of steps 6 through 11 for various regeneration doses. A growth curve is constructed from the $L(i) / T(i)$ ratios and the equivalent dose is found by interpolation of $L(1) / T(1)$. Usually a zero regeneration dose and a repeated regeneration dose are employed to insure the procedure is working properly. For fine-grained ceramics, a preheat of $240^{\circ} \mathrm{C}$ for $10 \mathrm{~s}$, a test dose of $3.1 \mathrm{~Gy}$, and a cut heat of $200^{\circ} \mathrm{C}$ are currently being used, although these parameters may be modified from sample to sample. 
The luminescence, $L(i)$ and $T(i)$, is measured on a Ris $\varnothing$ TL-DA-15 automated reader by a succession of two stimulations: first $100 \mathrm{~s}$ at $60^{\circ} \mathrm{C}$ of IRSL (880nm diodes), and then $100 \mathrm{~s}$ at $125^{\circ} \mathrm{C}$ of OSL (470nm diodes). Detection is through $7.5 \mathrm{~mm}$ of Hoya U340 (ultra-violet) filters. The two stimulations are used to construct IRSL and OSL growth curves, so that two estimations of equivalent dose are available. Anomalous fading usually involves feldspars and only feldspars are sensitive to IRSL stimulation. The rationale for the IRSL stimulation is to remove most of the feldspar signal, so that the subsequent OSL (post IR blue) signal is free from anomalous fading. However, feldspar is also sensitive to blue light $(470 \mathrm{~nm})$, and it is possible that IRSL does not remove all the feldspar signal. Some preliminary tests in our laboratory have suggested that the OSL signal does not suffer from fading, but this may be sample specific. The procedure is still undergoing study.

A dose recovery test is performed by first zeroing the sample by exposure to light and then administering a known dose. The SAR protocol is then applied to see if the known dose can be obtained.

The laboratory is currently investigating using pulsed OSL to measure equivalent dose on ceramics. In pulsed mode, the stimulating light is turned off and on in a series of pulses with the luminescence only measured during the off-time. Because the time between stimulation and emission is much longer for quartz than feldspar, an appropriate pulse width can be chosen to eliminate any feldspar signal. Previous work has suggested that a $10 \mu$ s on-time and $240 \mu \mathrm{s}$ off-time for each pulse, and also using an initial infrared exposure (as in double SAR), will minimize the feldspar signal during the off-time, so that the signal stems mainly from quartz. Pulsed OSL is measured on a Risø DA-20 using similar parameters as in the double SAR. Detection is for $100 \mathrm{~s}$ total (both on- and off-time) which includes 400,000 pulses for a total on-time of 4 seconds. This procedure is currently undergoing study because it is not certain 4 seconds is sufficient exposure to deplete the signal.

Alpha efficiency will surely differ among IRSL, OSL and TL on fine-grained materials. It does differ between coarse-grained feldspar and quartz (Aitken 1985). Research is currently underway in the laboratory to determine how much b-value varies according to stimulation method. Results from several samples from different geographic locations show that OSL $b$-value is less variable and centers around 0.5. IRSL $b$-value is more variable and is higher than that for OSL. TL $b$-value tends to fall between the OSL and IRSL values. We currently are measuring the b-value for IRSL and OSL by giving an alpha dose to aliquots whose luminescence have been drained by exposure to light. An equivalent dose is determined by SAR using beta irradiation, and the beta/alpha equivalent dose ratio is taken as the $b$ value. A high OSL $b$-value is indicative that feldspars might be contributing to the signal and thus subject to anomalous fading.

\section{Age and error terms}

The age and error for both OSL and TL are calculated by a laboratory constructed spreadsheet, based on Aitken (1985). All error terms are reported at 1-sigma. The reference for ka (thousand years before present) is 2010 . 


\section{References}

Adamiec, G., and Aitken, M. J., 1998, Dose rate conversion factors: update. Ancient TL 16:37-50.

Aitken, M. J., 1985, Thermoluminescence Dating, Academic Press, London.

Banerjee, D., Murray, A. S., Bøtter-Jensen, L., and Lang, A., 2001, Equivalent dose estimation using a single aliquot of polymineral fine grains. Radiation Measurements 33:73-93.

Bøtter-Jensen, L, and Mejdahl, V., 1988, Assessment of beta dose-rate using a GM multi-counter system. Nuclear Tracks and Radiation Measurements 14:187-191.

Brady, N. C., 1974, The Nature and Properties of Soils, Macmillan, New York.

Galbraith, R. F., and Roberts, R. G., 2012. Statistical aspects of equivalent dose and error calculation and display in OSL dating: an overview and some recommendations. Quaternary Geochronology 11:1-27

Huntley, D. J., and Lamothe, M., 2001, Ubiquity of anomalous fading in K-feldspars, and measurement and correction for it in optical dating. Canadian Journal of Earth Sciences 38:1093-1106.

Murray, A. S., and Wintle, A. G., 2000, Luminescence dating of quartz using an improved single-aliquot regenerative-dose protocol. Radiation Measurements 32:57-73.

Prescott, J. R., Huntley, D. J., and Hutton, J. T., 1993, Estimation of equivalent dose in thermoluminescence dating - the Australian slide method. Ancient TL 11:1-5.

Prescott, J. R., and Hutton, J. T., 1988, Cosmic ray and gamma ray dose dosimetry for TL and ESR. Nuclear Tracks and Radiation Measurements 14:223-235.

Roberts, H. M., and Wintle, A. G., 2001, Equivalent dose determinations for polymineralic fine-grains using the SAR protocol: application to a Holocene sequence of the Chinese Loess Plateau. Quaternary Science Reviews 20:859-863.

Wintle, A. G., \& Murray, A. S., 2006, A review of quartz optically stimulated luminescence characteristics and their relevance in single-aliquot regeneration dating protocols. Radiation Measurements 41:369-391. 



\section{APPENDIX C: LITHIC MICRO USE-WEAR ANALYSIS}





\section{Macroscopic and Microscopic Analysis of Lithic Artifact Sample from 41PN175}

by Steve Tomka

On February 15, 2012, the Center for Archaeological Research (CAR) entered into a contract with Geo-Marine, to conduct use-wear analysis of a small sample of lithic artifacts recovered from site 41PN175 located in Panola County, Texas. The assemblage was derived from the data recovery efforts sponsored by the Texas Department of Transportation and conducted by staff of Geo-Marine. The goal of the project was to conduct low-powered use-wear analysis of a representative sample of artifacts, including formal tools such as projectile points and expedient specimens such as utilized flakes, recovered from the site.

The scope of work called for the examination of each tool at a range of magnification, the comparison of the artifacts to specimens with known uses to establish the manner of use of the artifacts, and when feasible, to determine the material that was worked with the tool. In addition, tools were to be examined for the presence/absence of hafting wear, and when tool failure had occurred, the specimen was to be inspected to determine whether the failure occurred during tool use, during manufacture, or following discard.

A total of 20 artifacts was provided to CAR for analysis. The specimens included four projectile points, five nonprojectile point bifaces, a retouched flake, and 10 pieces of lithic debitage. Table C-1 presents the provenience information for each of these artifacts.

\section{Macroscopic and Microscopic Use-Wear Analysis Methodology}

Typical macroscopic alterations of tools include catastrophic failure of some portion of the tool during use (i.e., projectile point breakage during impact) and the development of userelated retouch on the working edge (i.e., snap fracture of a knife due to excessive torque while in use) developed as a result of contact with the material being worked. Catastrophic tool failures are most common on functional classes that are exposed to significant forces during typical use such as piercing, cutting, scraping, and perforating. Similarly, hafted tools (e.g., projectile points, knives, scrapers, reamers, and drills) tend to be exposed to greater forces during use compared to their unhafted (hand-held) counterparts because the haft allows more pressure to be exerted on the tool compared to hand-held tool use.

Retouch derived from tool use tends to develop when a portion of the working edge is too weak and unstable to resist the forces applied against it. Such failures include the scalloping of the working edge when using an unmodified flake as a cutting tool, the removal of microflakes from the dorsal or ventral face of a flake when it is engaged in scraping a piece of bone or wood, and the detachment of microflakes from the dorsal face of a flake and generation of step fractures scars on the ventral face when the scraping motion is bidirectional rather than unidirectional. The development of such retouch continues to form 
Table C-1: Artifact Sample Provided to CAR for Micro-wear Analysis

\begin{tabular}{|r|c|c|c|l|l|}
\hline Lot No. & Block & Unit & $\begin{array}{c}\text { Subdatum / } \\
\text { Level (Depth cmbsd*) }\end{array}$ & Artifact Category & Functional Category/Task \\
\hline 608 & 2 & 64 & $2 / 4(45-48)$ & Dart point & Projectile point and knife \\
\hline 691 & 3 & 70 & $3 / 5(55-65)$ & Dart point & Projectile point and knife \\
\hline $641 \mathrm{~A}$ & 3 & 72 & $3 / 4(45-55)$ & Dart point & Projectile point and knife \\
\hline 1487 & 4 & 90 & $9 / 4(41)$ & Dart point & Projectile point and knife \\
\hline 1274 & 4 & 93 & $11 / 3$ A (35-41) & Misc. biface & Possible knife \\
\hline 629 & 3 & 70 & $3 / 3(35-45)$ & Misc. biface & Knife \\
\hline 1724 & 4 & 73 & $4 / 5(45-55)$ & Misc. biface & Manufacture failed \\
\hline 1115 & 4 & 86 & $8 / 2(15-25)$ & Misc. biface & Manufacture failed \\
\hline 545 & 3 & 69 & $3 / 2(25-35)$ & Recycled biface & Graver \\
\hline $641 B$ & 3 & 72 & $3 / 4(45-55)$ & Retouched flake & Adze and possible scraper \\
\hline 1539 & 4 & 123 & $13 / 4(25-30)$ & Utilized flake & Scraper \\
\hline 1174 & 4 & 99 & $10 / 2(25-35)$ & Utilized flake & Whittling \\
\hline 1710 & 4 & 127 & $10 / 3(35-45)$ & Utilized flake & Sawing and whittling \\
\hline 1630 & 4 & 120 & $13 / 3(35-40)$ & Utilized flake & Scraping \\
\hline 1147 & 4 & 92 & $11 / 2(25-35)$ & Utilized flake & Graver \\
\hline 1692 & 4 & 126 & $10 / 1(10-25)$ & Utilized flake & Scraper \\
\hline 1161 & 4 & 95 & $9 / 2(15-25)$ & Utilized flake & Reamer \\
\hline 713 & 4 & 78 & $4 / 4(35-45)$ & Utilized flake & Scraper \\
\hline 1175 & 4 & 99 & $10 / 2(25-35)$ & Utilized flake & Scraper (?) \\
732 & 4 & 75 & $4 / 4(35-45)$ & Utilized flake & Scraper \\
\hline
\end{tabular}

${ }^{*} \mathrm{cmbsd}=$ centimeters below subdatum

until the tool's working edge stabilizes: that is, the forces applied against it have removed all weaker portions of the tool and what remains is a working edge morphology that is sufficiently strong to withstand continued use. Typically, at this stage, the development of use-polish begins to form, and the stable edge is now exposed to prolonged contact with material being worked.

On tools that require some degree of retouch to create and/or shape the working edge, difficulty arises in determining whether damage noted on the edge of a tool results from the process of tool manufacture, tool use, or postdepositional processes. Tool manufacture can produce both step-fracturing (microcrushing of the retouched edge with a percussor or pressure-flaking tool) as well as rounding and smoothing (i.e., platform preparation). Therefore, the most reliable indicator of use-wear on retouched tools is the presence of polish.

On expedient tools representing the utilization of unmodified lithic debris to carry out a task, use-related wear is typically easier to identify since it is not masked by manufacture-related retouch. However, in some instances, expediently employed tools may be retouched once the working edge dulls, and under such circumstance, it may be impossible to correctly interpret the use-history of the tool, given that the retouch will remove the previous working 
edge. Conversely, however, expediently employed tools by definition have short use-cycles, and therefore, both the use-retouch that develops on the working edge as well as the useassociated polish is typically less extensive than on so-called formal tools that are repeatedly reused in the performance of the same or similar tasks.

In general, the analyses performed for this study are based on procedures developed and outlined by Ahler (1979), Odell $(1996,1981)$, Semenov (12964), and Vaughan (1985). The author also relied on an extensive comparative collection of use-worn tool forms as well as replicated manufacture-failed implements to discern and discriminate between manufacture and use-derived retouch.

During the macroscopic and microscopic analysis, each specimen was examined under a range of magnifications using a MEIJI Techno EMZ-13TR binocular microscope with a detached tungsten reflected light illuminator. The magnification ranged from 20X to 140X, and all included microphotographs shown were taken at 40X magnification. The field of view captured in the microphotograph is 1.2 millimeters.

Analysis began with a scan of each face of each artifact without magnification to identify macroscopic edge-wear such as microflaking, step-fracturing, and edge smoothing. The goal of this inspection was to identify and differentiate loci of edge modifications that may have resulted from manufacture, postdepositional damage, and tool use.

Microscopic analysis of each artifact began with the examination of the apparent working edge of the tool as determined during the initial macroscopic inspection. Both faces of the working edge were examined for microflake scarring and the development of use-related polish. Next, the haft element of each hafted artifact was examined for traces of wear. Finally, the body of the tool (i.e., areas away from the working edge) was examined for traces of wear that included the presence of polish on arises. Use-polish is an alteration of the surface of a tool that results in increased reflectivity of that surface. The distribution of the polish was categorized as diffuse, linear, or localized. Diffuse polish is represented by light polish that is diffused over large flat surfaces of the artifact, whereas linear polish tends to be found on arises. Finally, localized polish tends to occur on topographic microfeatures that rise above the surrounding tool surface and are repeatedly in contact with either the haft or the material being worked.

\section{Dart Points and Knives}

\section{Specimen P608}

Specimen P608 is a plano-convex hafted biface made of petrified wood. The right margin of the specimen (dorsal face up) retains a short segment of edge rounding derived from platform preparation in an attempt to remove a series of step-fractured flake scars that resulted from the poor quality of the raw material. The distal end of the blade, its tip, 
exhibits numerous arises with polish, as does the distal tip itself (Figure C-1). Both faces of the point exhibit linear polish on flake ridges as well as step-fracture terminations. Haftwear polish is present on both faces of the stem and consists of localized polish on high flake-scar ridges. Flake-scar ridges along the blade also exhibit regular linear polish consistent with knife use. The combination of use-polished surfaces and their distribution is consistent with the use of the specimens as a projectile point and cutting tool.

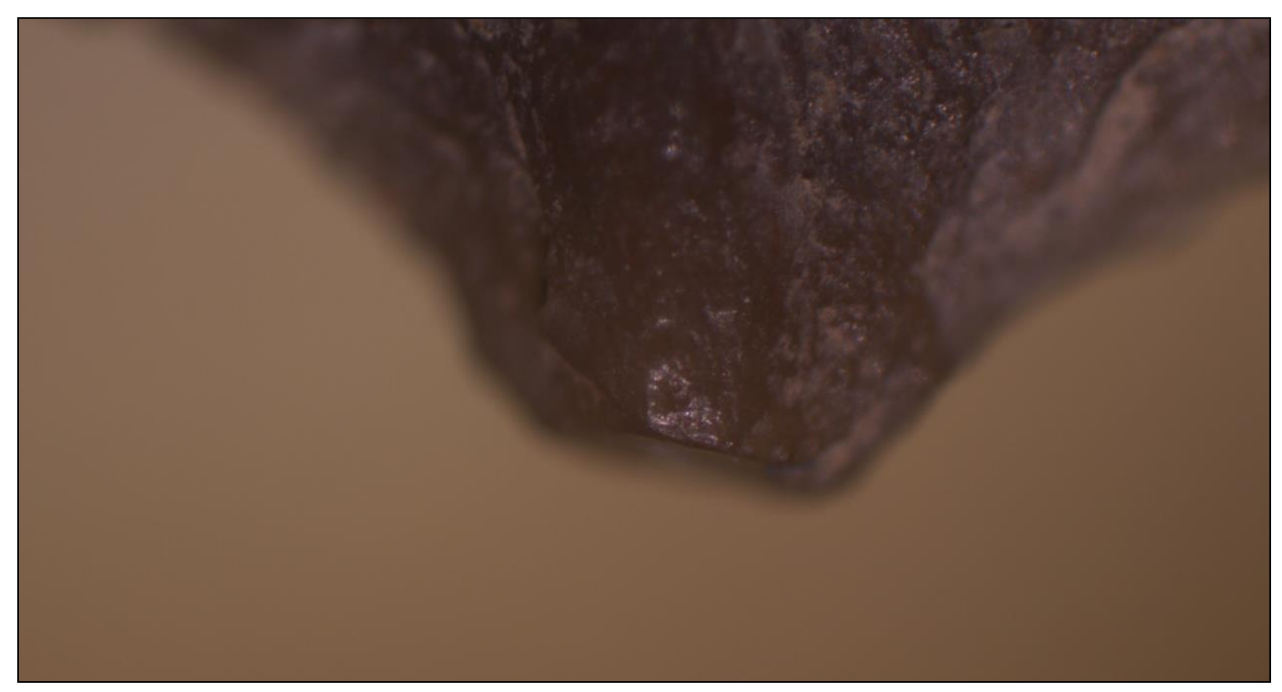

Figure C-1. Specimen P608: light use-wear polish localized on tip of tool.

\section{Specimen P691}

Specimen P691 is a proximal, hafted biface fragment broken in use. The margins of the stem as well as the base are heavily ground. The base of the blade is slightly recurved on one margin and smooth on both sides. This blade morphology is indicative of the hafting approach employed by the tool's manufacturer. Specifically, the morphology and its smoothness are suggestive of the wear pattern generated by sinew or other material that wrapped all the way up to and around the base of the blade. Light polish is present on one protrusion near the base of the blade. Haft-wear is minimal in terms of polish, suggesting perhaps that the stem was not tightly held in the shaft or foreshaft. This would be consistent with the hafting approach suggested by the lower blade polish that would draw the point back into the haft. Light polish is present on both faces of the point near the distal break (Figure C-2). This may be light use-wear created by use of the specimen as a knife.

\section{Specimen P641A}

Specimen P641A is a dart point made of fine-grained yellow chert. The specimen is complete and has a plano-convex cross section. Light polish is present throughout both faces of the specimen, suggesting engagement against soft material that came in contact with both high ridges and lower portions of the point's topography (Figure C-3). Polish is also visible on the flake-scar ridges formed by the removal of side-notching flakes of the stem. This polish is the result of hafting material being in contact with the stem of the point while 

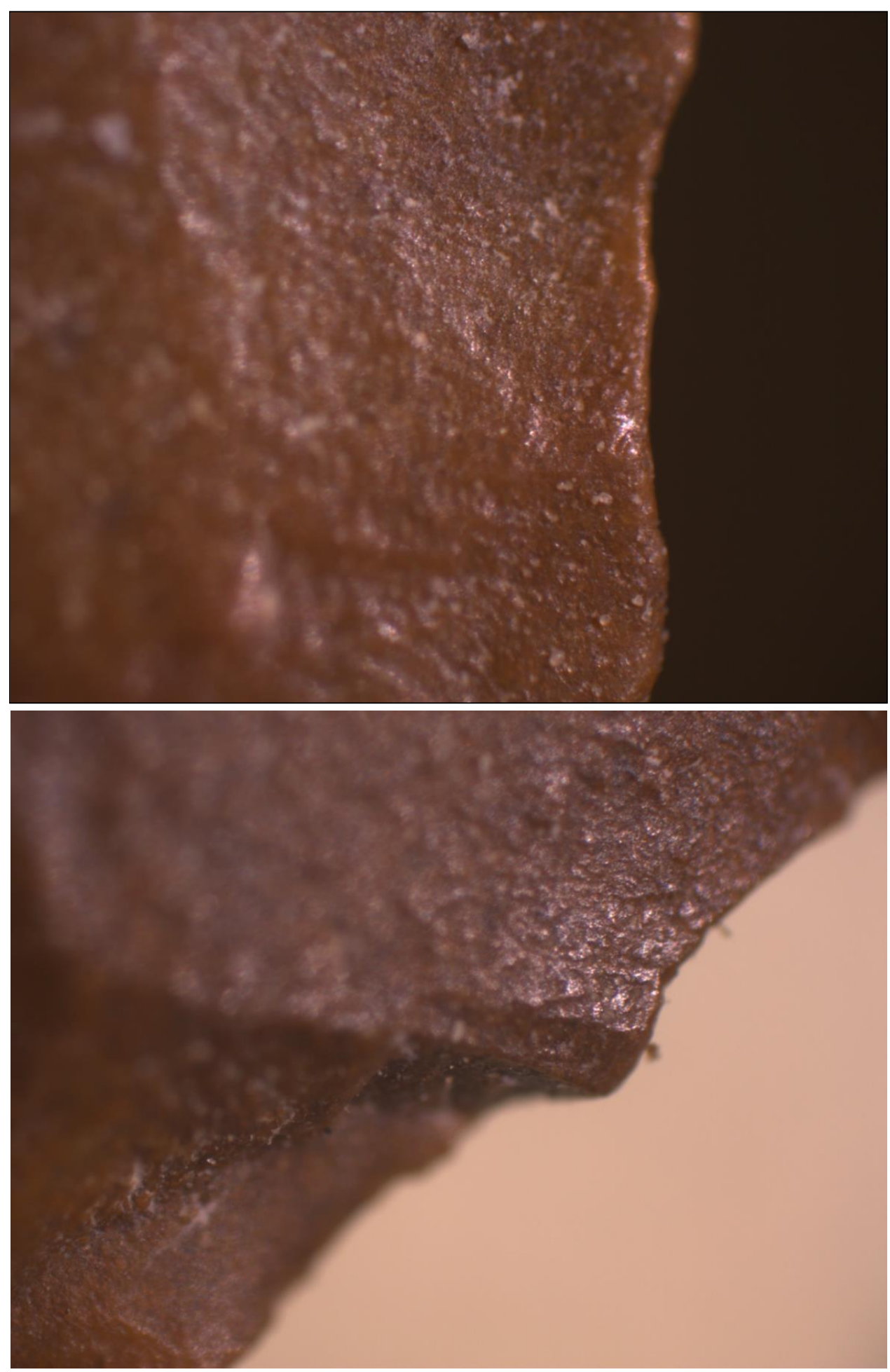

Figure C-2. Specimen P691: light use-wear polish along projectile point edge (upper and lower) 

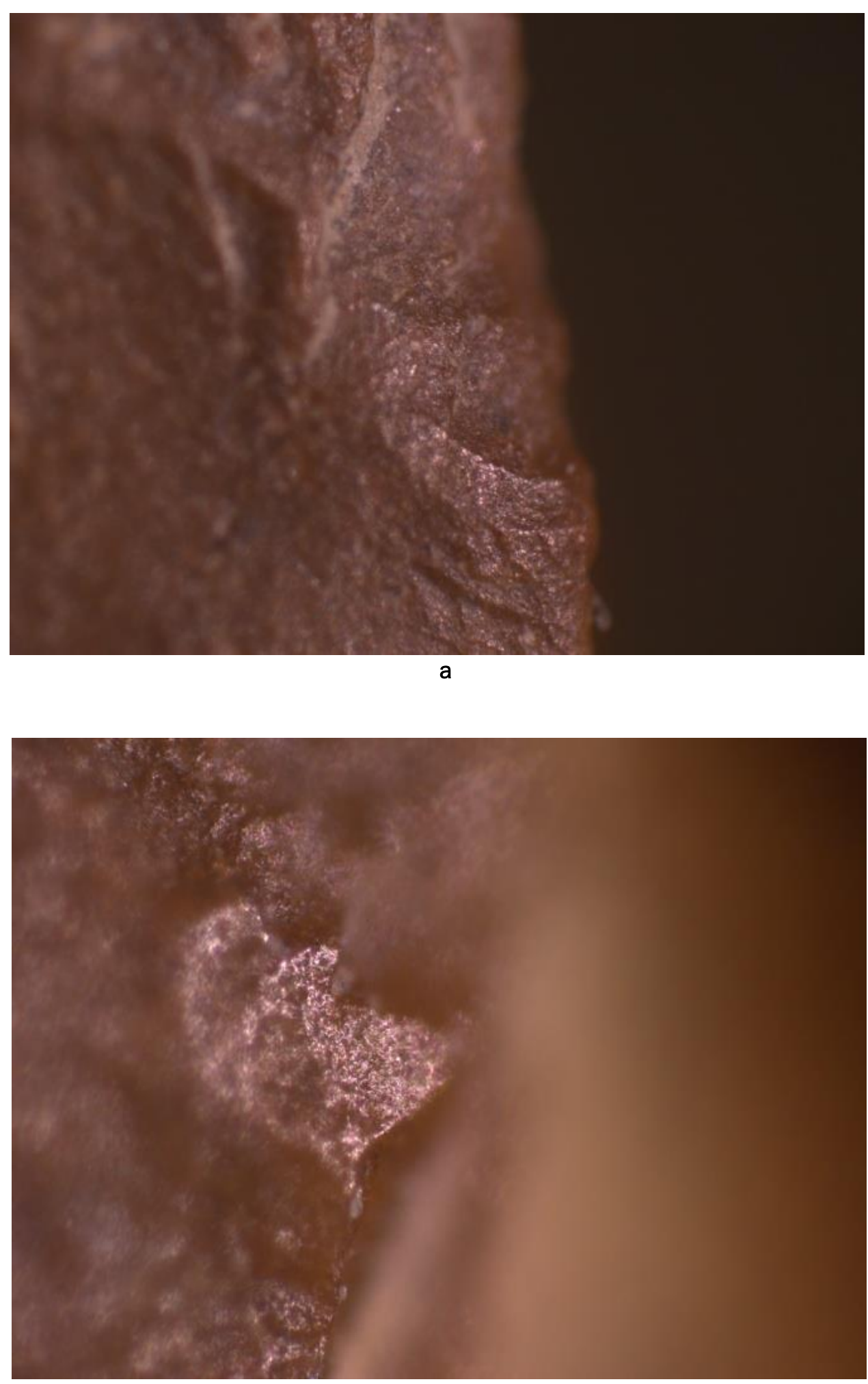

b

Figure C-3. Specimen P641A: (a) use-wear polish along bifacial edge; (b) haft-wear polish near base of blade. 
in the shaft or foreshaft of the projectile (see Figure C-3b). Light smoothing also is present on the proximal ends of the blade. This smoothing, in combination with the localized polish, is consistent with the binding agent from hafting extending onto the blade itself. The specimen is complete and exhibits polish on both faces of the blade and hafting polish on both faces of the stem.

\section{Specimen P1487}

Specimen P1487 is a complete medium-sized dart point made of chert. Both faces of the stem retain high linear polish on flake-scar ridges derived from hafting (Figure C-4). The lateral edges of the blade exhibit microflaking resulting from manufacture rather than use. The convex face of this plano-convex specimen retains some localized polish near the tip of the specimen. This polish is reminiscent of impact-derived polish resulting from contact with bone. Although the specimen seems rather poorly manufactured, the presence of haft-wear (Figure C-4 upper) does suggest that it was a fully functional point. Perhaps the quality of the manufacture says something about the context of manufacture, which appears to have been rather expedient.

\section{Miscellaneous Bifaces}

\section{Specimen P1274}

Specimen P1274 is a small, medial biface fragment made of fine-grained yellow chert. The medial fracture occurred across a coarse inclusion in the parent material. The break may have been precipitated by torque perpendicular to the long axis of the artifact. The distal tip snap break may have been the result of use-failure, and the localized polish adjacent to the break (Figure C-5) is suggestive of use-failure (i.e., contact with bone). The overall appearance of the specimen is that it is an unfinished manufacture-failed artifact. Flake scars are irregular, and the biface is not longitudinally symmetrical. However, the fact that the specimen exhibits considerable polish on one face of the artifact suggests use, as does the polish adjacent to the broken tip. Some of the polish, however, may derive from contact with the metallic surfaces of the hardware cloth typically used in screening the archaeological matrix.

\section{Specimen P629}

Specimen P629 is a silicified wood flake fragment with one bifacially flaked edge opposite a perverse break face. The two lateral margins of this specimen also are missing, and the edge opposite the bifacially shaped working edge appears to have been intentionally left unmodified to form a type of backing. Both faces of the working edge exhibit polish that occurs on high and low areas (Figure C-6). The two ends of the specimen may have been broken in use, but the tool appears to have continued in use judging from use-wear occurring on the break faces. 

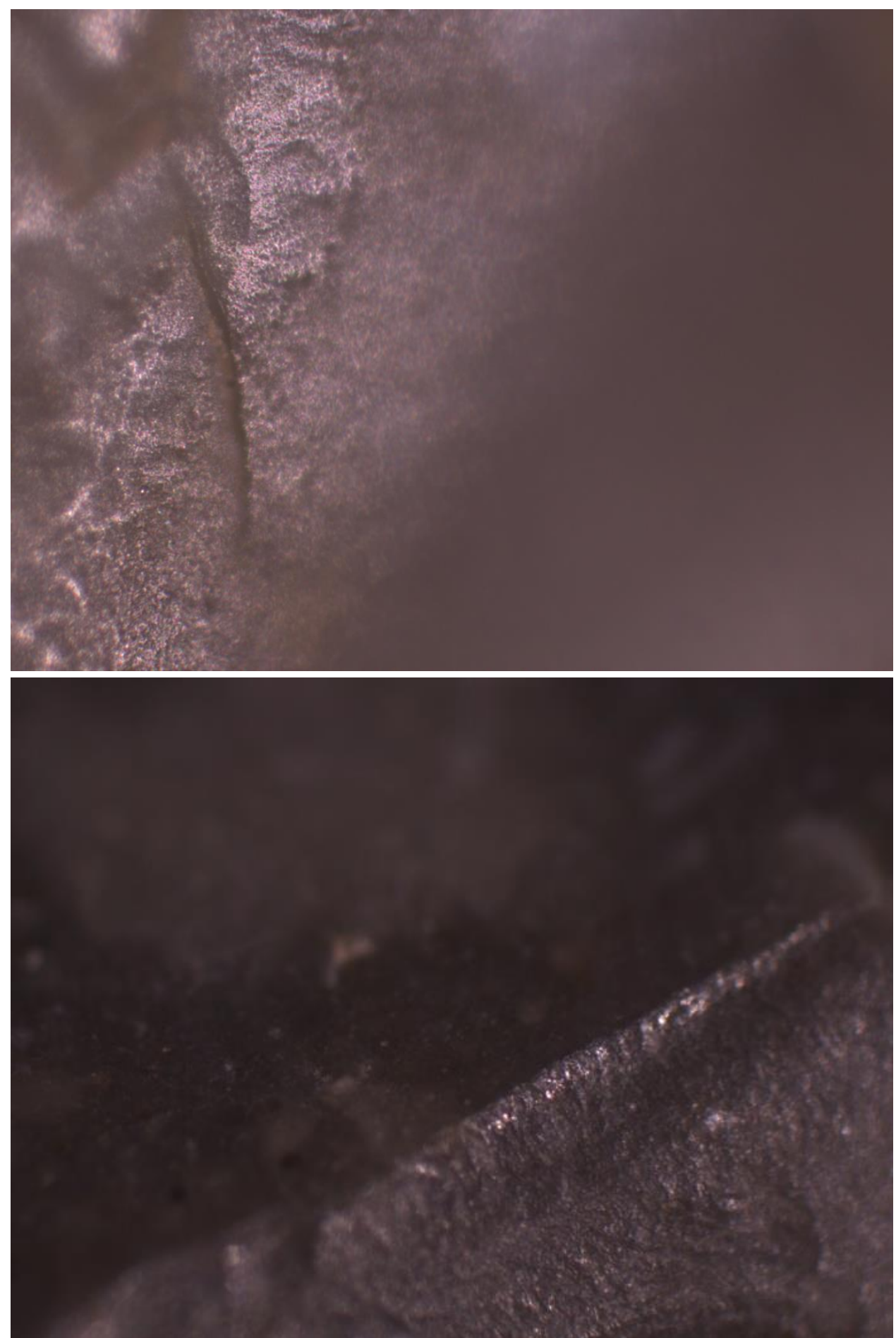

Figure C-4. Specimen P1487: (upper) hafting polish near base of blade; (lower) use-wear on longitudinal ridge. 


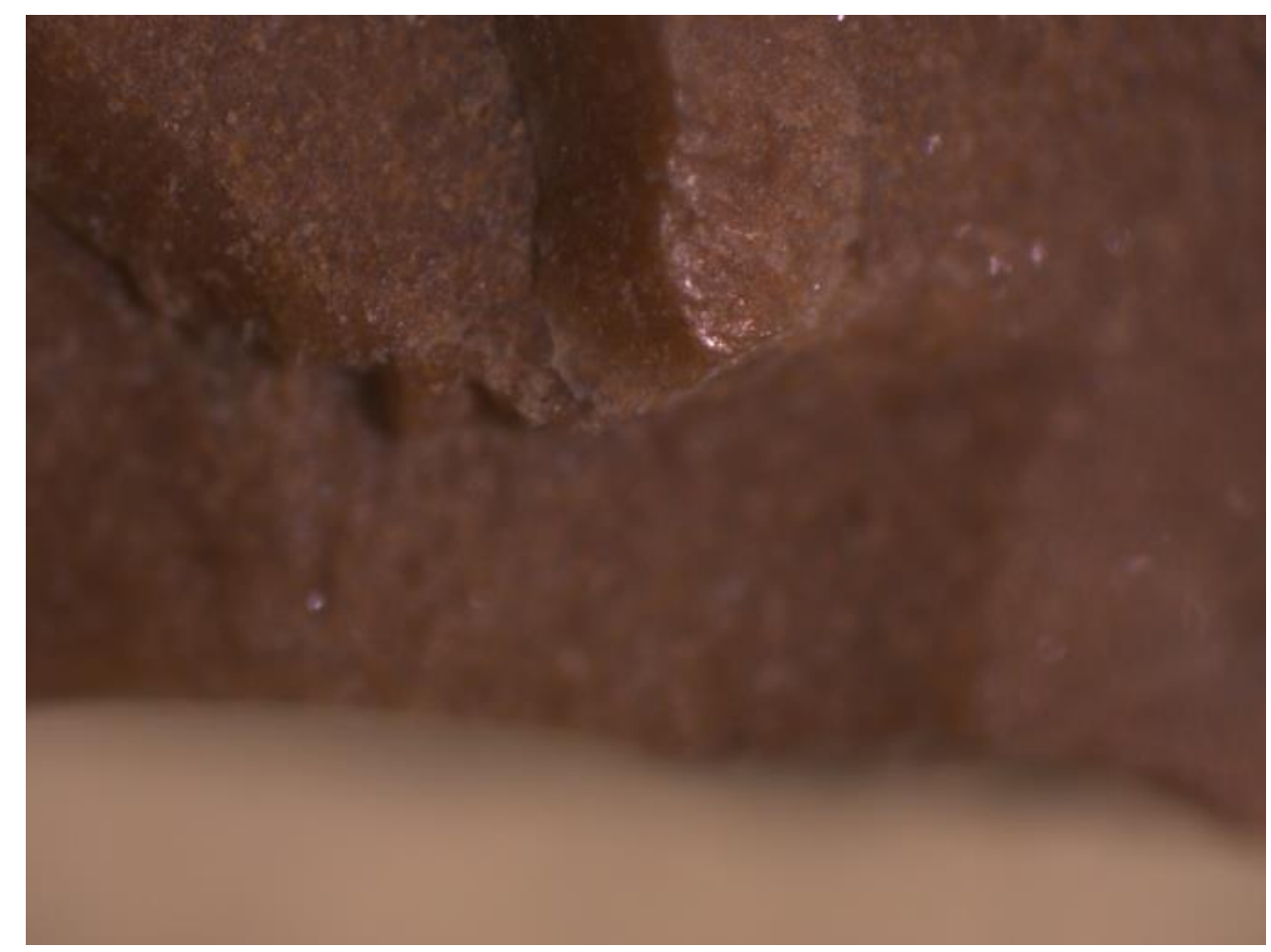

Figure C-5. Specimen P1274: localized polish on tip of flake scar ridge near tip of biface

\section{Specimen P1724}

Specimen P1724 is a biface proximal fragment. The perverse blade fracture suggests that it failed during manufacture, as do some of the irregular flake scars present on both faces of the specimen. Some localized smoothing is present on the edges of the biface, and these appear to represent remnants of platform preparation. Patches of coarse-grained inclusions are present on both faces of the fragment, and specks of orange residue on one such inclusion represents rusted metallic residue. Such residue is common on artifacts recovered from plowed fields where the plow impacts and rubs against the lithic artifact and leaves a line of metallic residue on its face. Over time, this metallic residue rusts, leaving the pattern seen on this artifact (Figure C-7).

\section{Specimen P1115 (no micrograph)}

Specimen P1115 is a proximal biface fragment with smoothing along the base and lateral edges. Smoothing derives from platform preparation rather than use. Microscopic examination of the smoothed edges of the specimen revealed no use-wear polish. Instead, the edges appear to have been smoothed by rubbing a coarse material such as a percussor along the edge. The lateral margins exhibit a substantial amount of step-fracturing, which is consistent with the grinding of the edges in the process of platform preparation. The blade break occurred near the base of the specimen, and its morphology is similar to basal thinning failures. More precisely, the break morphology indicates two unsuccessful base thinning attempts. The first basal thinning attempt did not fully break the specimen but initiated an imbedded fracture plane that weakened the blade near the base. The second attempt at basal thinning created yet another fracture plane, and the specimen broke along this plane while the force also initiated a break along the first imbedded fracture line. 

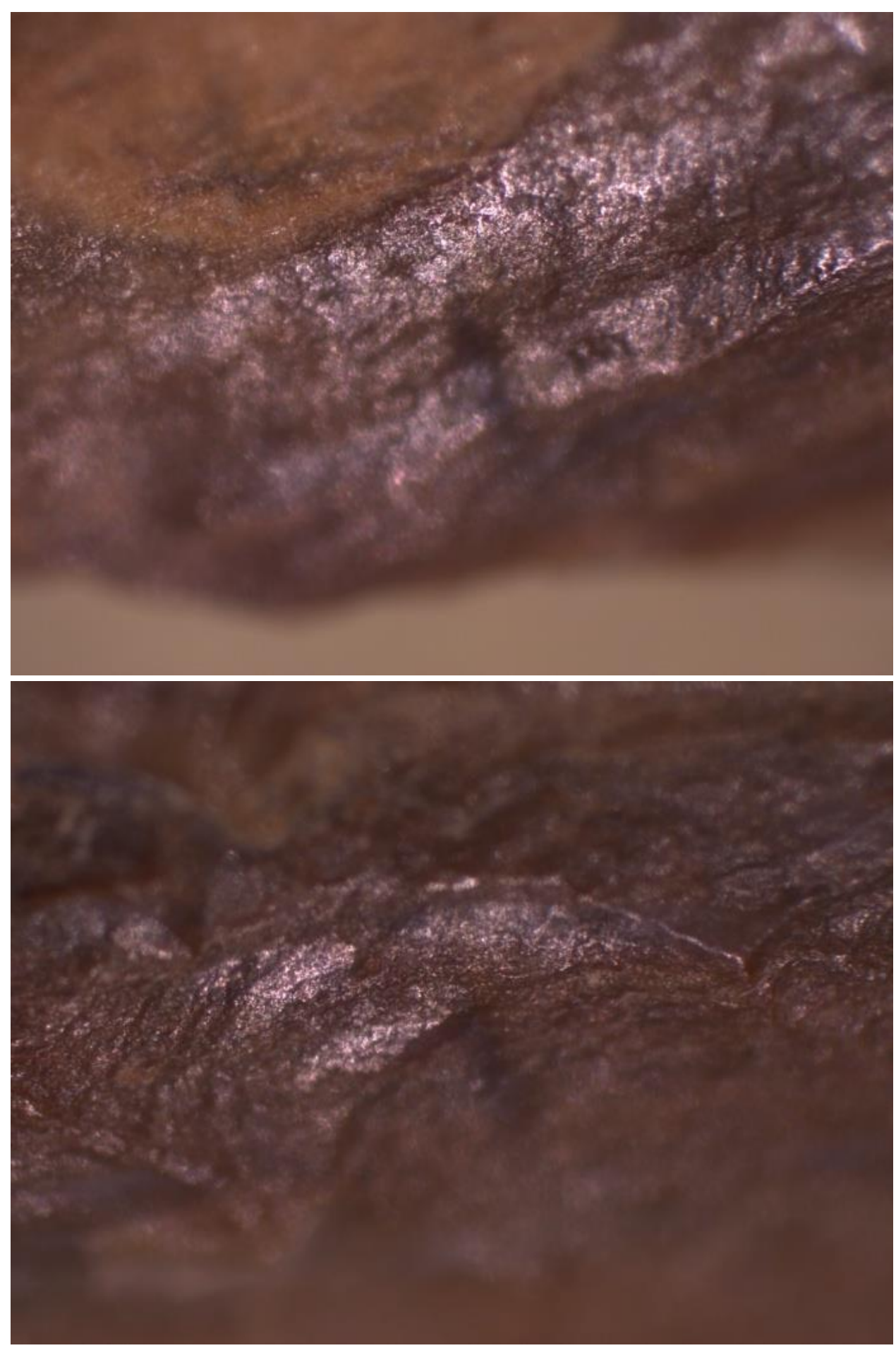

Figure C-6. Specimen P629: (upper and lower) localized use-wear polish along bifacially flaked working edge. 


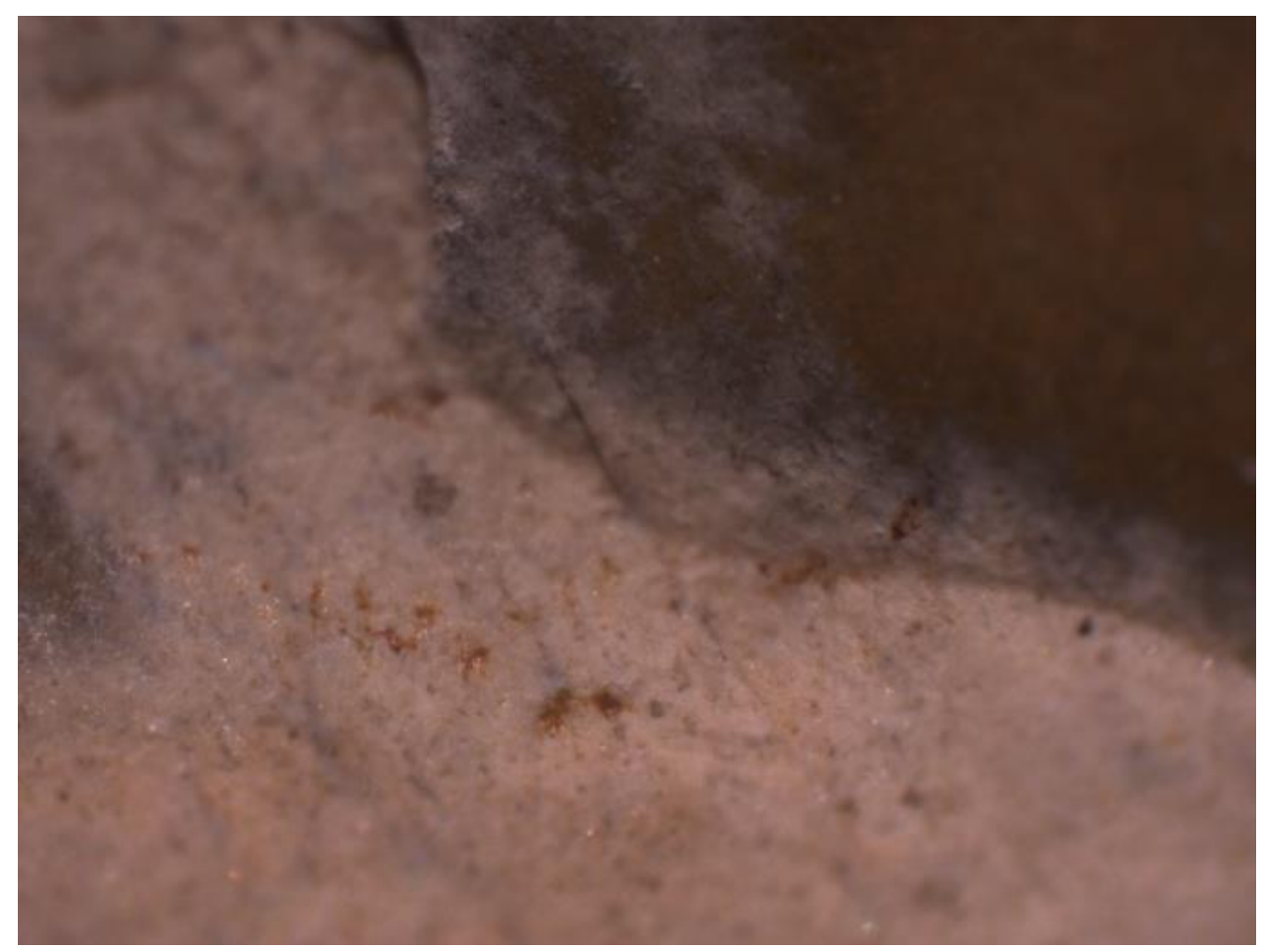

Figure C-7. Specimen P1724: rust spots on the body of specimen.

\section{Specimen P545}

Specimen P545 is a recycled projectile point medial fragment. One margin above the narrower break face has been bifacially retouched to create a concave edge. The break face adjacent to this concave edge was employed as a graver. Step-fracturing of the edges of the blade break adjacent to the graver tip is also the product of the use of the tool as a graver. Use-polish is present on the body of the biface in the vicinity of the graver tip, as well as on the tip itself (Figure C-8 upper, lower). One corner of the wider blade break also was employed as a graver tip. Subsequent to this use, the dulling of the graver bit led to a burin flake removal that also removed the entire bifacial edge. Following additional use of this break face as a graver, another attempt was made at creating a bifacial edge, and when this failed, the tool was discarded. A significant amount of heavy polish is present on both faces of the specimen near the former graver tips. The step-fracturing adjacent to the graver tips is also the result of utilization. In addition, diffuse polish is present on the entire body of the biface. Some of this polish may be the result of the original use of the tool as a knife. The well-patterned flaking of the body of the specimen, as well as its biconvex cross section, is reminiscent of late Paleoindian or Early Archaic lanceolate projectile point forms (e.g., Angostura). Recycling of failed projectile points as gravers and the subsequent rejuvenation of the working edge through burin removals is commonly seen in Late Paleoindian and Early Archaic assemblages such as the specimens noted in the Lake Limestone region. 

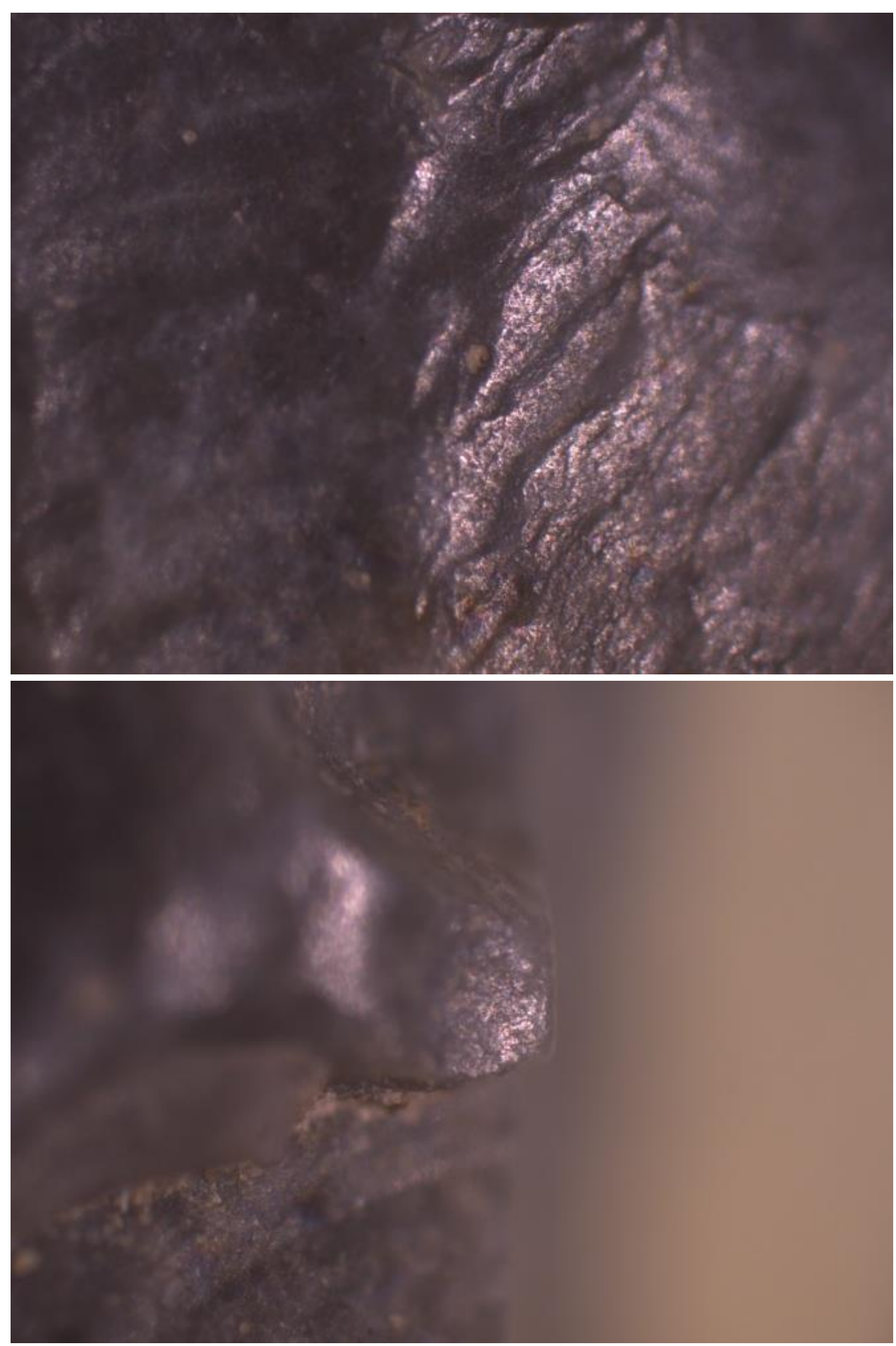

Figure C-8. Specimen P545: use-wear polish on body (upper) and leading edge (lower) of graver on recycled biface.

\section{Retouched Flake-Specimen P641b}

Specimen P641B is a marginally beveled secondary flake of silicified wood. The specimen is reminiscent of distally beveled unifaces common in South Texas during the Transitional Archaic and Late Prehistoric. Rather than having the distal end beveled, one of the lateral edges of the parent flake is beveled. It is difficult to distinguish any use-polish on the dorsal and ventral faces adjacent to the working edge because of the high natural polish of the raw 
material. Small grains of the material are missing along the edge, indicating that the tool was used in a planing or shaving motion. The rounding and microcrushing evident immediately above and behind (proximal to) the working edge derives from hafting wear (Figure C-9 upper, lower).

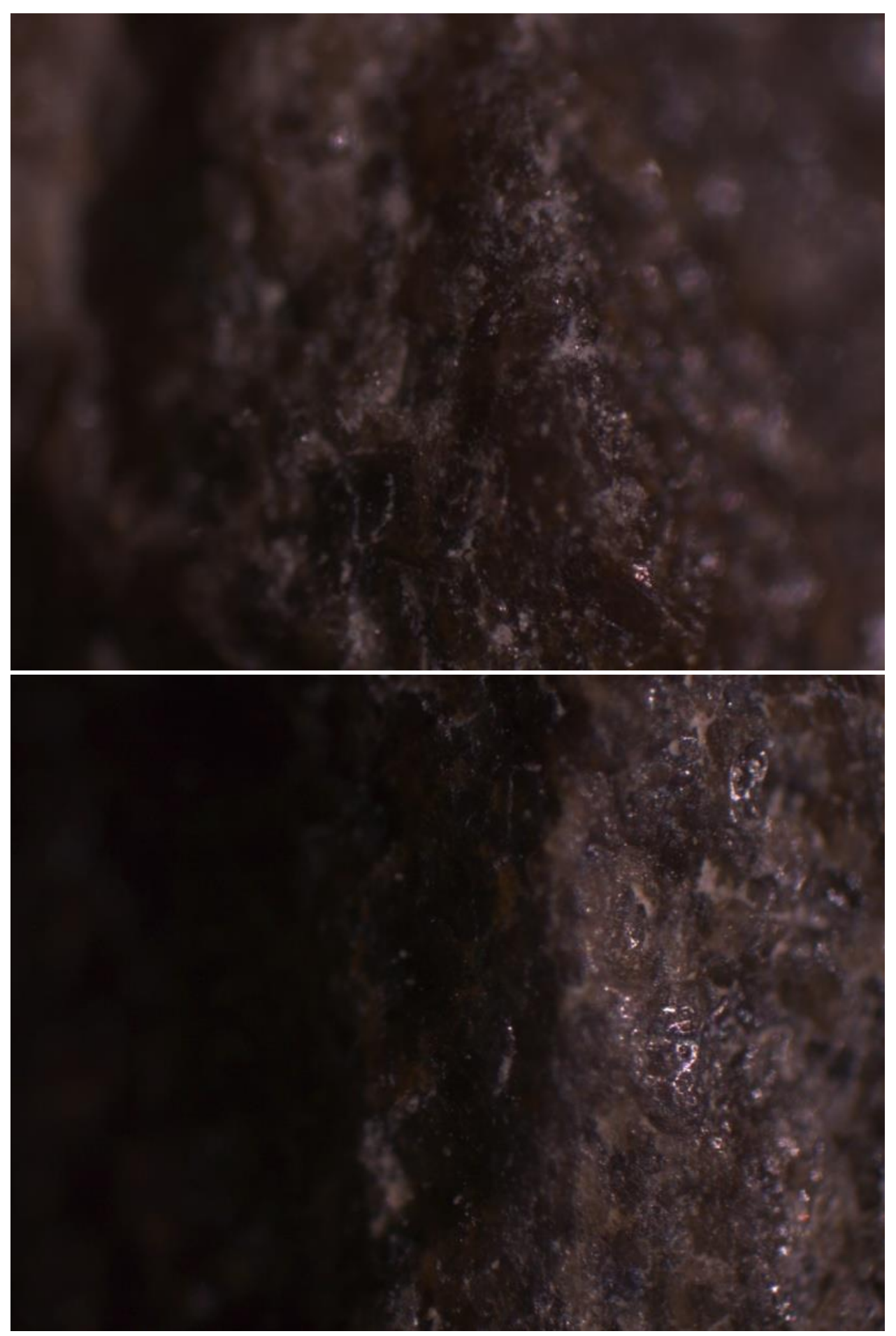

Figure C-9. Specimen P641B: (upper) haft-wear on flake-scar ridge on dorsal face; (lower) use-wear polish on dorsal leading face of tool. 


\section{Utilized Flakes}

\section{Specimen P1539}

Specimen P1539 is a secondary flake of fine-grained yellow chert with coarse-grained inclusions. A concave edge was formed by the removal of a single flake adjacent to the proximal end of the flake. A cluster of microflake scars is present on the dorsal face of the concave surface (Figure C-10 upper), and immediately underneath it-on the ventral face of the concave surface-a crushed area with step-fractured microflake scars (see Figure C-10 lower). Light polish is present on the ventral face of the flake and on the working edge. It appears that the tool represents an expediently used scraper that subsequently was exposed to some postdepositional edge damage. The light, diffuse polish may be simply from prehensile holding of the artifact while in use.

\section{Specimen P1174}

Specimen P1174 is a fine-grained red chert secondary bladelet with expedient use-wear scars along one longitudinal margin (Figure C-11 upper). The use-wear is heaviest on the dorsal face of the working edge, with flake scars being in the range of $1.0-1.5 \mathrm{~mm}$ in length. However, smaller flake scars and shallow step fractures (see Figure C-11 lower) are also present on the ventral face of the working edge, indicative of the fact that the scraping motion was bidirectional in orientation (i.e., back and forth) along the material being worked. Light polish is present on the microflake-scar ridges on the dorsal face of the working edge (see Figure C-11 upper), suggesting a soft material such as soft wood or green wood being worked with the tool. The duration of the task was very short-term, since the wear is not extensive.

\section{Specimen P1710}

Specimen P1710 is a small secondary bladelet of fine-grained quartzite. Very minute and irregular flake scars along one edge have a scalloped appearance that is consistent with the use of the flake as an expedient knife (Figure C-12). Sawing or whittling typically results in edge fracturing that is not invasive onto the edge, but rather removes entire segments of the thin and structurally weak edge. The microflake scarring scallops the edge rather than creating flake scars that are invasive onto the face of the tool. Continued use of the tool would result in the breakage of all weak portions of the edge and its stabilization at a new equilibrium between the strength of the raw material and the amount of force that is applied to the tool edge. The stabilized working edge would have a straight-line appearance rather than the sinuous look provided by the scalloping. The scalloped appearance of the tool is consistent with tool use of a short duration and relatively soft material being worked.

\section{Specimen P1630}

Specimen P1630 is a secondary flake of silicified wood. It has one concave lateral edge that has been employed in light scraping tasks. Small-less than $1 \mathrm{~mm}$ in length-microflake scars are present along the dorsal face of the working edge (Figure C-13 upper), and light 


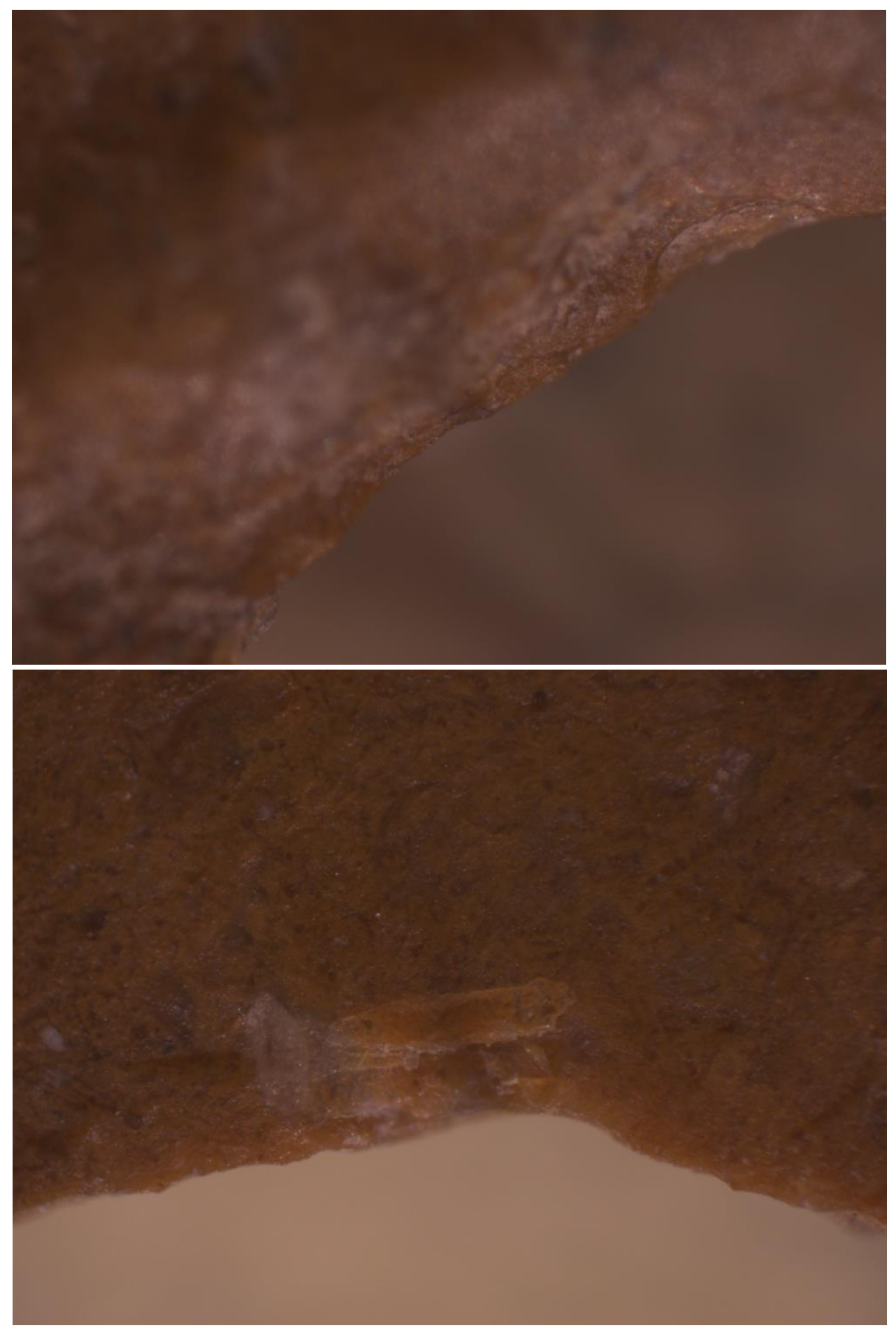

Figure C-10. Specimen P1539: (upper) step-fracturing along concave working edge; (lower) postdepositional crushing on ventral face. 

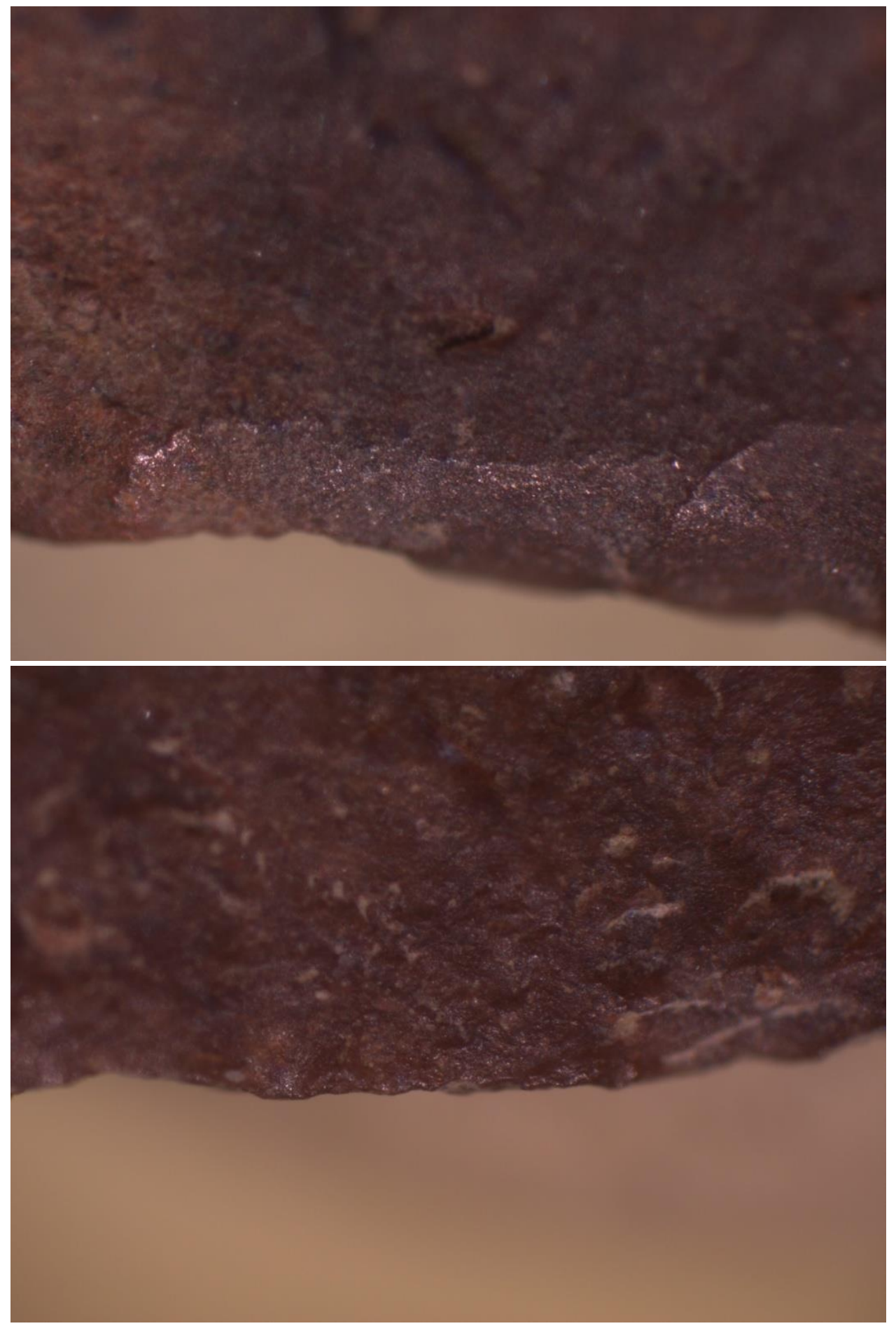

Figure C-11. Specimen P1174: (upper) microflake scars on dorsal face; (lower) step-fracturing on ventral face of working edge. 




Figure C-12. Specimen P1710: (upper and lower) scalloped working edge typical of cutting use-wear. 


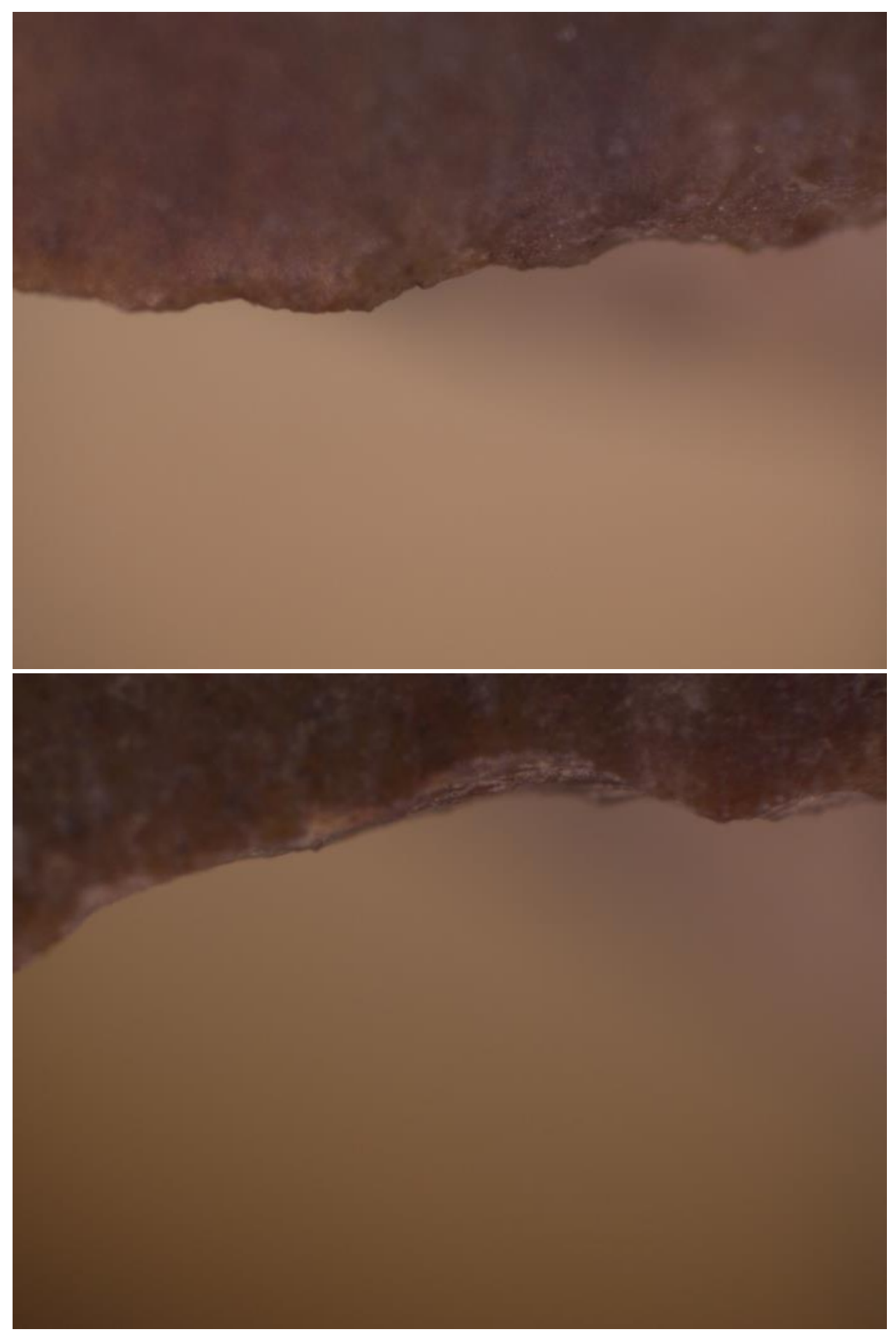

Figure C-13. Specimen P1630: (upper) microflake scarring on dorsal face of working edge; (lower) step-fracturing along working edge.

step-fracturing is noted in the flake scars (see Figure C-13 lower). Judging from the size of the scars and their small number, the tool was utilized in the performance of very light scraping tasks, and the scraping motion tended to be unidirectional rather than bidirectional as in Specimen P1174. 


\section{Specimen P1147}

Specimen P1147 is a short, fine-grained quartzite bladelet with a distal graver tip. The tip is formed by the intersection of the two lateral edges rather than being retouched into the current shape. The graver tip has two microflake scars that resulted from use and stepfracturing along one lateral edge that also derived from utilization (Figure C-14). The localized polish present on the working tip suggests that the substance that was being worked was sufficiently soft not to "grind" the naturally polished surface but hard enough to create the microflake scarring.
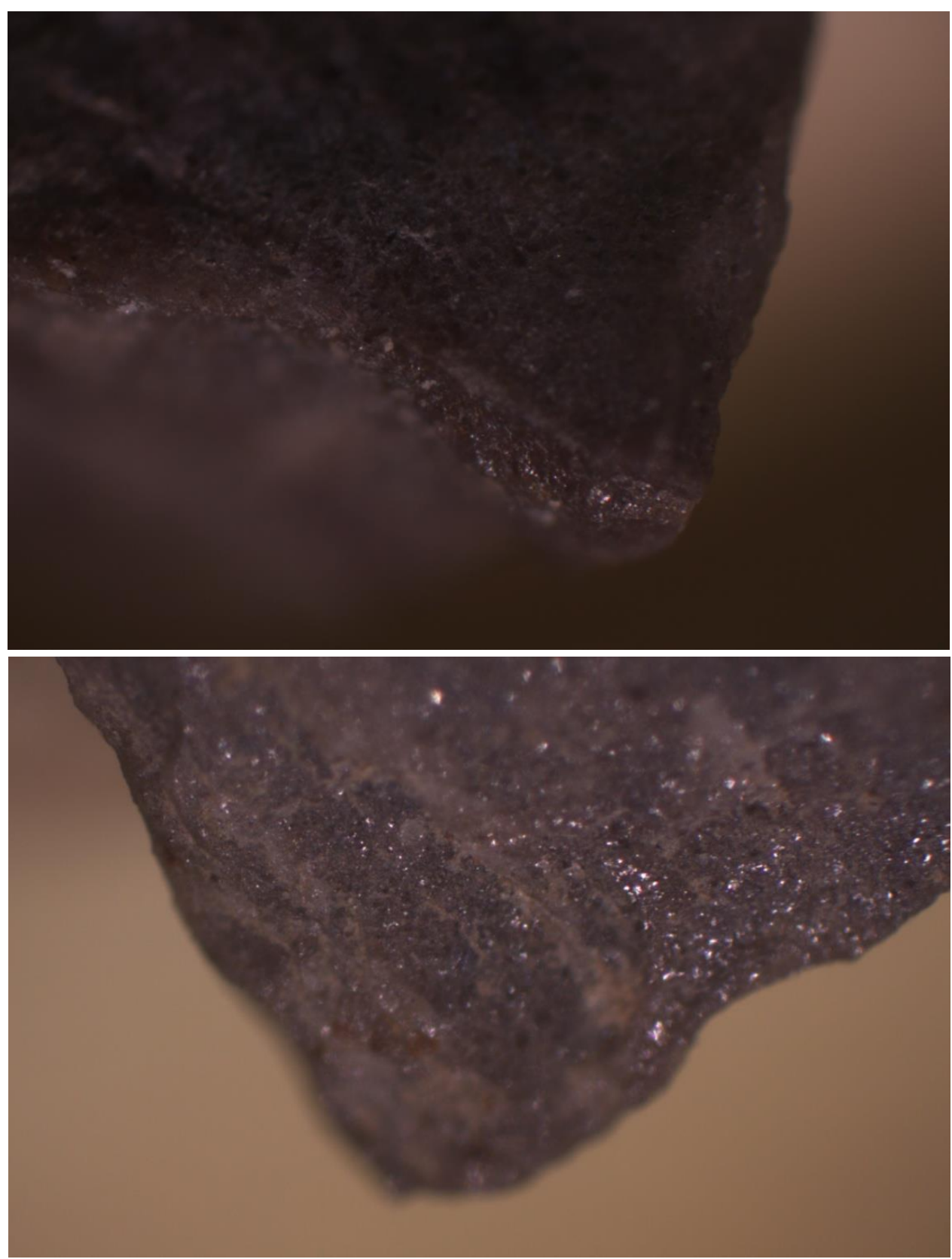

Figure C-14. Specimen P1147: (upper) crushing, and (lower) light step-fracturing of the graver tip. 


\section{Specimen P1692}

Specimen P1692 is a secondary, distal flake fragment of fine-grained chert with two areas manifesting scraper use-wear. The most extensive use-wear (Figure C-15) is noted on the distal edge of the flake fragment near the corticated flake margin. This working edge has been used sufficiently long to result in the development of light use-polish such as noted in Figure C-15. Use-wear scars are $.5-.8 \mathrm{~mm}$ in length and very patterned, indicating very uniform pressure and contact with the material being worked. More subtle use-wear is present along the break face of the flake fragment. In both instances, the utilization of the tool resulted in the development of slightly concave working edges.

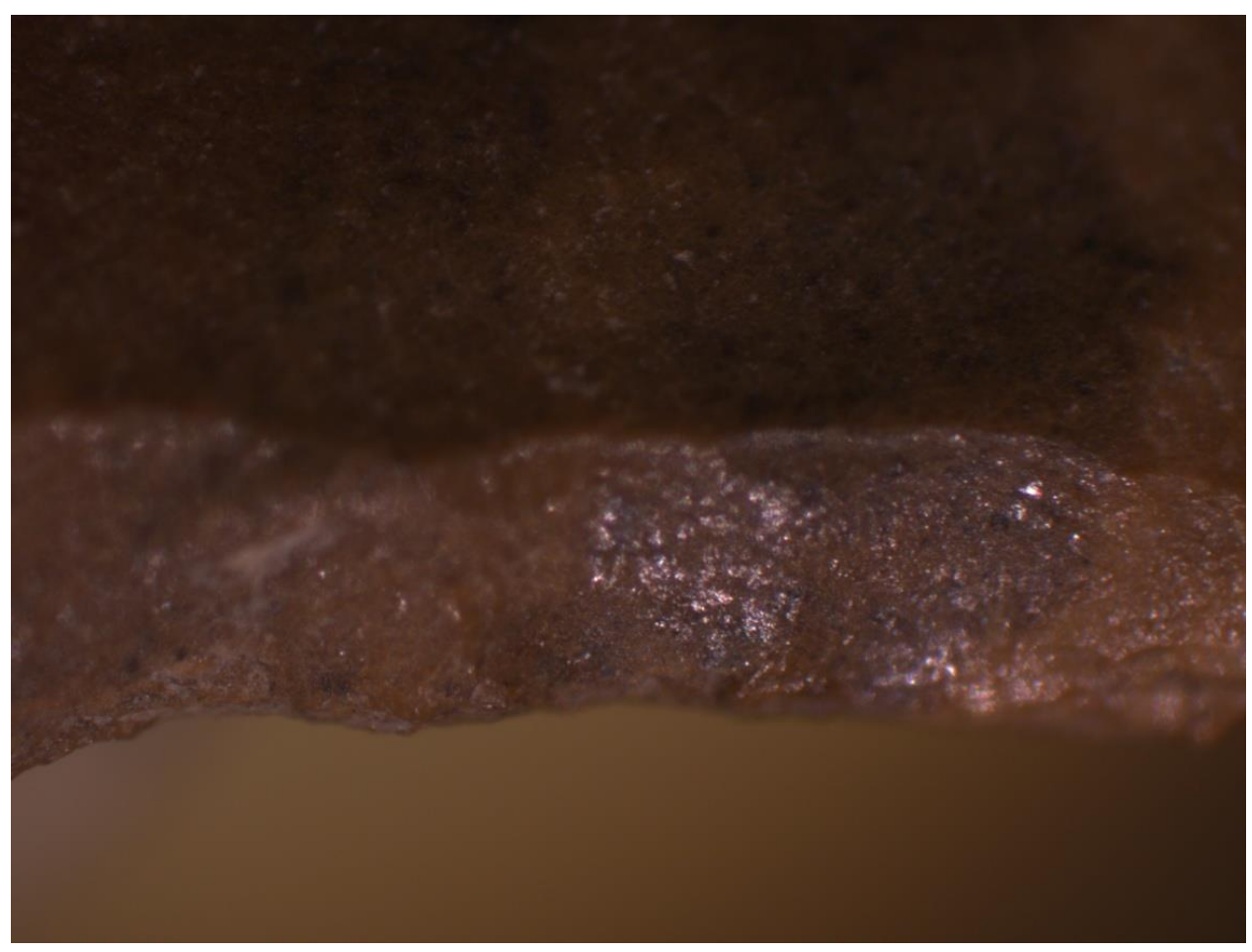

Figure C-15. Specimen P1692: microflaking and light use-polish on dorsal face of working edge.

\section{Specimen P1161}

Specimen P1161 is an irregularly shaped longitudinal proximal flake fragment with a broken distal end. The two lateral margins as well as the dorsal flake scar exhibit use-wear. The wear is manifest in the form of microflake step-fracturing and localized light polish. On the concave lateral margin, the use-wear tends to occur on the dorsal face of the edge and is reminiscent of scraping wear, although the combination of wear types and locations of usewear suggests a more complex picture.

The dorsal flake scar running the length of the specimen exhibits heavy step-fracturing and some localized light polish (Figure C-16 upper). Use-wear on the two lateral edges is manifest in the form of step-fracturing on both faces of the edge and light use-polish in some of the step-fracture scars (see Figure C-16 lower). In addition, the morphology of the 

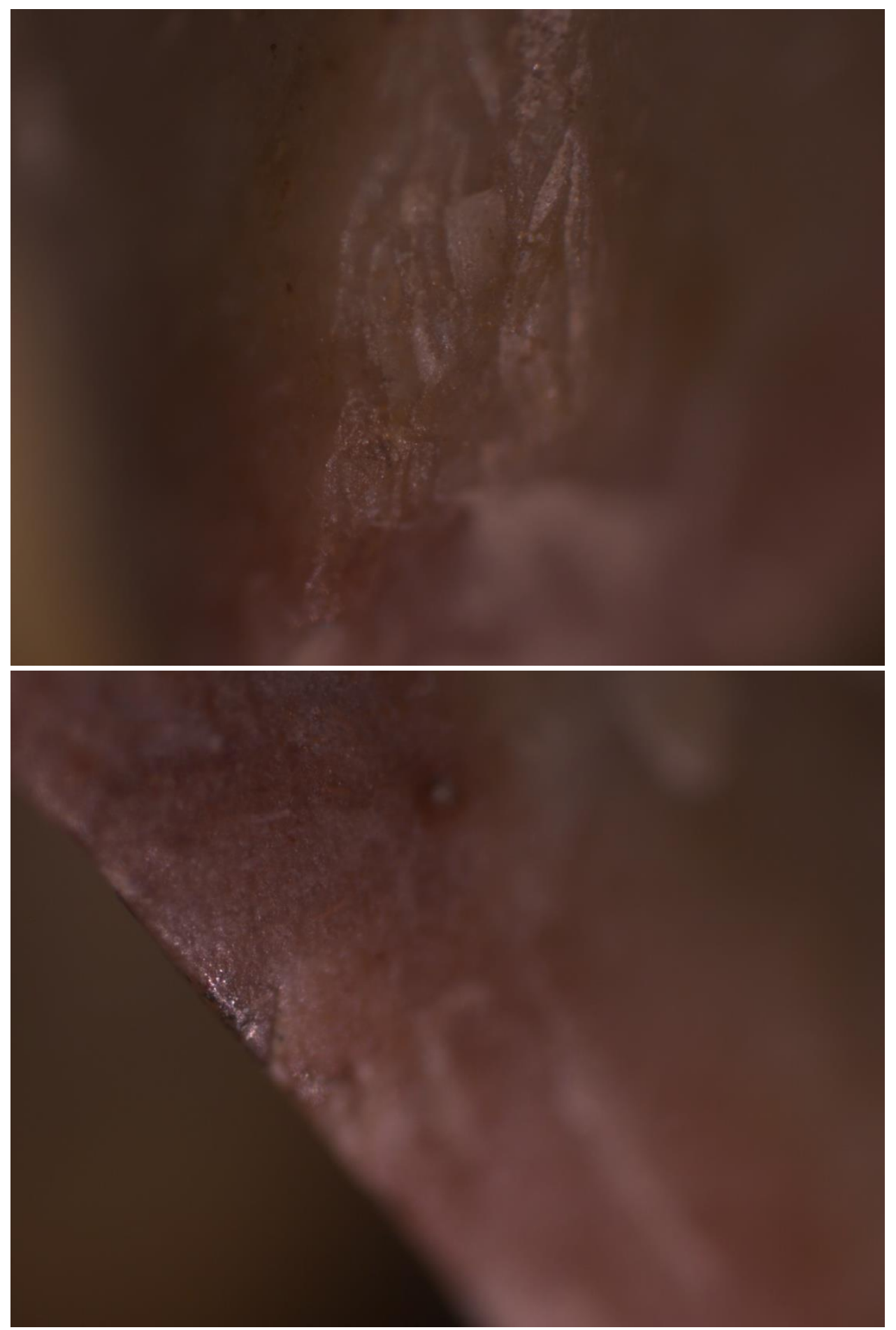

Figure C-16. Specimen P1161: (upper) crushed dorsal flake arise; (lower) light polish and step-fracture scars along one lateral edge. 
distal tip fracture is indicative of lateral torque having caused the failure of the tip. The stepfracturing seen on the dorsal ridge of the flake, the step-fracturing present on both faces of the lateral margins, and the break morphology of the tip suggest that the tool was employed as a reamer.

\section{Specimen P713}

Specimen P713 is a primary flake of fine-grained yellow chert. Use-wear is noted along one lateral edge, and postdepositional damage is present at two localized spots along the distal end. At one corner of the distal end, downward pressure from the dorsal face resulted in crushing the edge, and the same pressure resulted in the snap fracture of the opposite corner of the distal end. Highly patterned microflaking, consistent with scraper use, is present along one lateral edge (Figure $\mathrm{C}-17$ ). The edge remains rather sinuous, indicating that tool use was short-lived, and the force applied to the tool and worked material was not sufficiently great to wear down the edge to a more even alignment. Light use-polish began to develop on some flake-scar ridges.

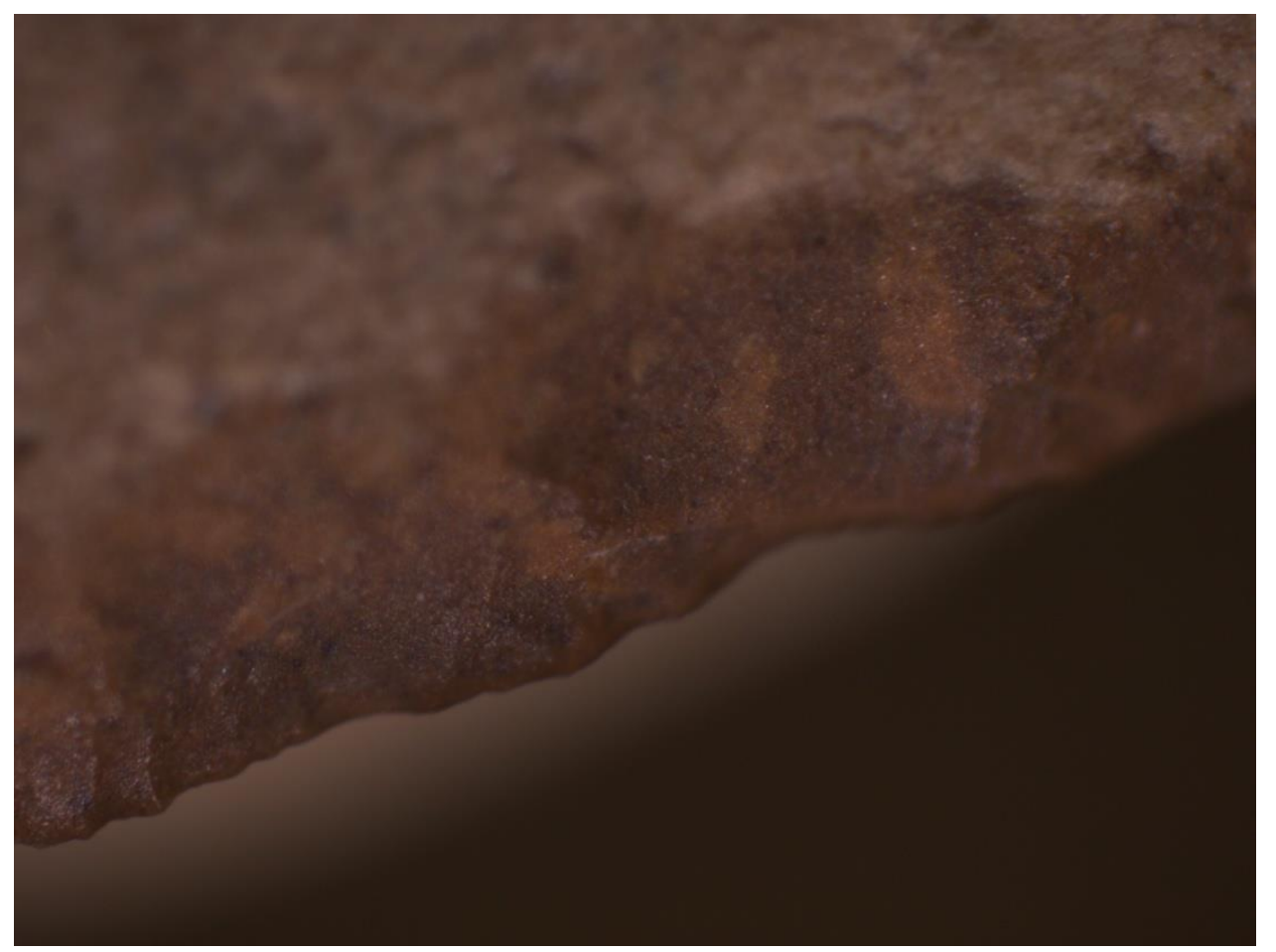

Figure C-17. Specimen P713: microflaking derived from use along dorsal face of working edge; note light use-polish on flake arises.

\section{Specimen P1175}

Specimen P1175 is a small secondary flake of yellow fine-grained chert with a semicircular working edge. Examination of the edge's dorsal and ventral surfaces does not exhibit clear signs of postdepositional alterations; therefore, it is likely that the microflaking is the result of tool use. However, no polish was noted along the edge, and the irregular nature of the concave edge (Figure C-18) suggests that the tool was used very lightly and discarded after brief use. 


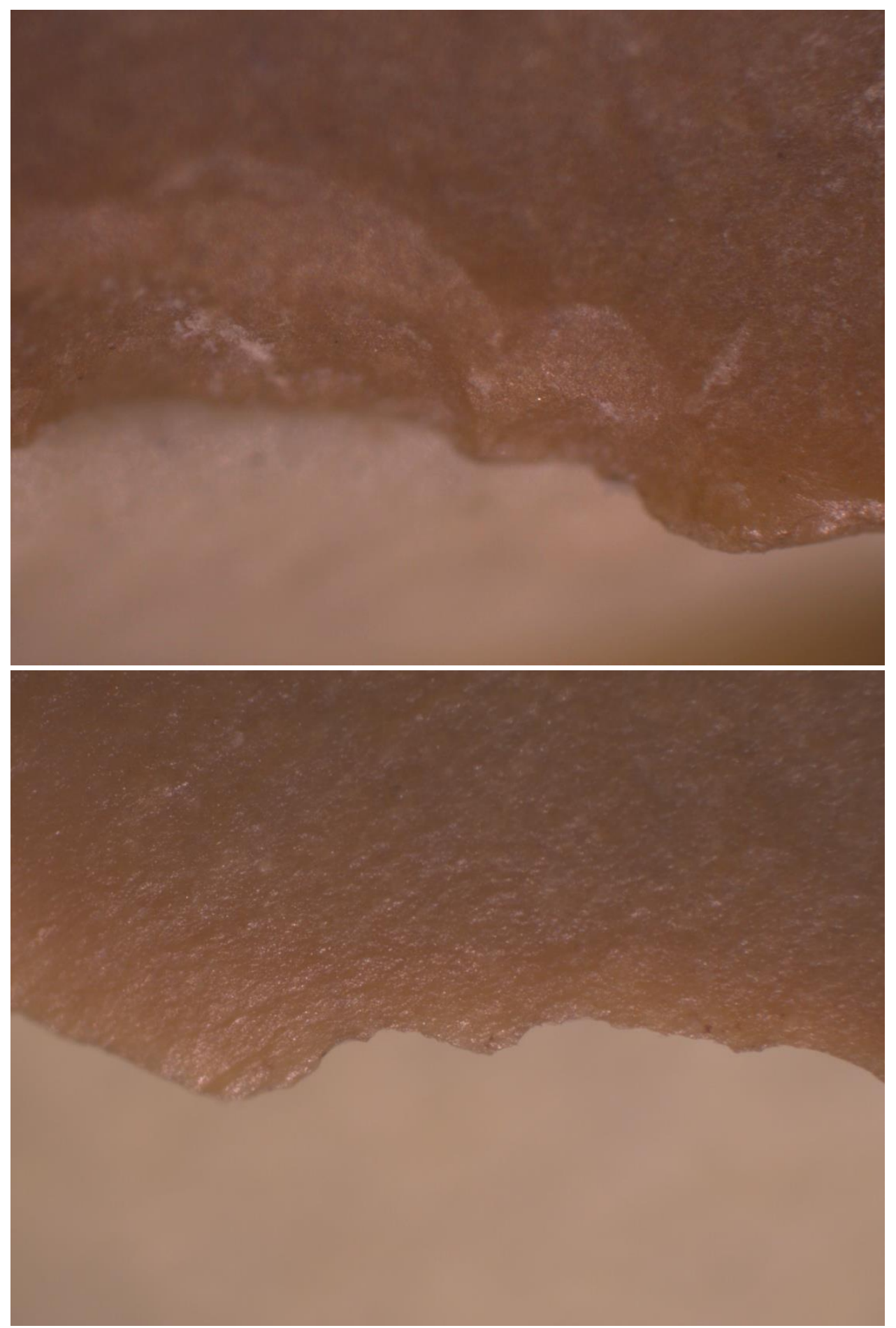

Figure C-18. Specimen P1175: (upper) microflaking on dorsal face of working edge; (lower) uneven ventral face of working edge.

\section{Specimen P732}

Specimen P732 is a small secondary flake of fine-grained chert with two lateral edges manifesting traces of use-wear. The most easily identifiable working edge has a semicircular-concave-morphology. Microflake scarring is relatively irregular, and polish is localized, light, and minimal in occurrence within the concave working edge (Figure C-19 


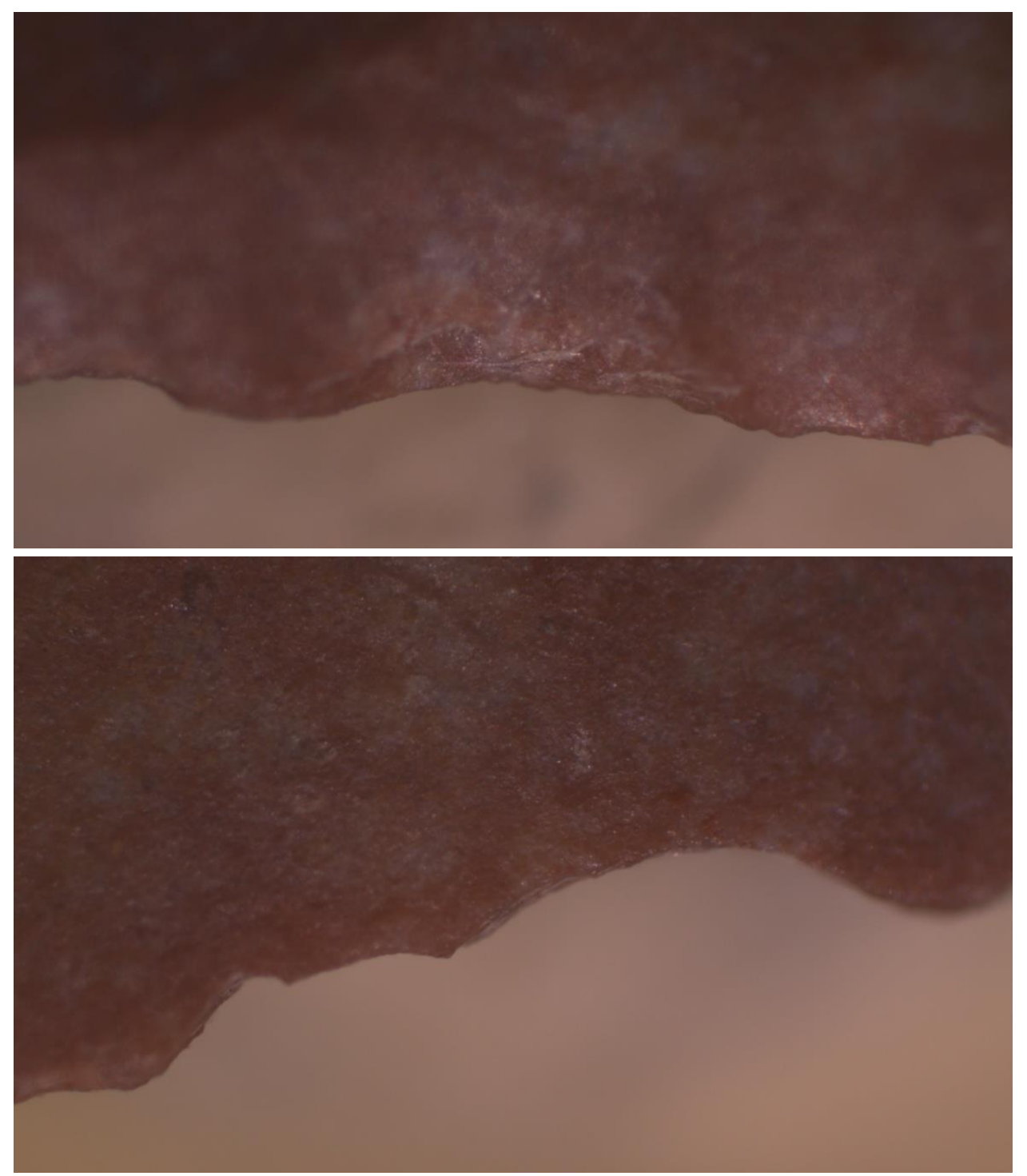

Figure C-19. Specimen P732: (upper) step-fracturing within the concave working edge; (lower) irregular sinuous working edge opposite the concave edge.

upper). The opposite flake margin has a short segment of microflaking consistent with scraping use. This working edge is very sinuous, suggestive of short-term use. Microflake scars also are present on the distal end of the flake, but they are likely the result of the original flake removal or subsequent postdepositional damage. The obtuse angle of these scars is most consistent with postdepositional alterations rather than use. No use-polish was noted on the sinuous edge or the distal end of the flake. 


\section{References Cited}

Ahler, Stanley A.

1979 Functional Analysis of Nonobsidian Chipped Stone Artifacts: Terms, Variables, Quantification. In Lithic Use-Wear Analysis, edited by Brian Hayden, pp. 301328. Academic Press, New York.

Keeley, Lawrence $\mathrm{H}$.

1980 Experimental Determination of Stone Tool Uses: A Microwear Analysis. Prehistoric Archeology and Ecology Series, Karl W. Butzer and Leslie G. Freeman, series editors. University of Chicago Press, Chicago.

Odell, George H.

1996 Stone Tools and Mobility in the Illinois Valley. International Monographs in Prehistory, Archaeological Series 10. Ann Arbor.

Semenov, S. A.

1970 Prehistoric Technology: An Experimental Study of the Oldest Tools and Artefacts from Traces of Manufacture and Wear. Translated and with a preface by M. W. Thompson. Adams and Dart, Bath, Somerset.

Vaughan, Patrick C.

1985 Use-Wear Analysis of Flaked Stone Tools. University of Arizona Press, Tucson. 



\section{APPENDIX D: GEOARCHAEOLOGICAL DATA}





\begin{tabular}{|c|c|c|c|c|c|c|c|c|c|c|c|c|c|c|c|c|}
\hline Profile & Context & Sample & $\begin{array}{c}\text { Plot Depth } \\
(\mathrm{cm})\end{array}$ & $\begin{array}{c}\text { Mean } \\
\text { (phi) }\end{array}$ & $\begin{array}{c}\text { Median } \\
\text { (phi) }\end{array}$ & $\begin{array}{c}\text { Sorting } \\
\text { (phi) }\end{array}$ & $\begin{array}{c}\text { Skewness } \\
\text { (phi) }\end{array}$ & $\begin{array}{c}\text { Kurtosis } \\
\text { (phi) }\end{array}$ & $\begin{array}{c}\text { USDA Textural } \\
\text { Class } \\
\end{array}$ & $\begin{array}{c}\text { Sand } \\
(\%) \\
\end{array}$ & $\begin{array}{l}\text { Silt } \\
(\%) \\
\end{array}$ & $\begin{array}{c}\text { Clay } \\
(\%) \\
\end{array}$ & $\begin{array}{c}\text { Xlf } \\
\left(10^{-8} \mathrm{~m}^{3} \mathrm{~kg}^{-1} \mathrm{SI}\right) \\
\end{array}$ & $\begin{array}{l}\text { Xfd } \\
(\%) \\
\end{array}$ & $\begin{array}{c}\text { LOI } \\
(\%) \\
\end{array}$ & $\begin{array}{c}\delta^{13} \mathrm{C} \\
\text { (per mil PDB) } \\
\end{array}$ \\
\hline Profile 1 & Colluvium, undif? & 1 & 2.5 & 4.07 & 3.76 & 1.81 & 0.39 & 1.22 & Sandy Loam & 55.3 & 36.58 & 8.12 & 14.7 & 8.1 & 10.1 & $\mathrm{NA}$ \\
\hline \multirow{19}{*}{ Lot 1988} & Colluvium, undif? & 2 & 7.5 & 3.91 & 3.58 & 1.82 & 0.44 & 1.42 & Sandy Loam & 60.4 & 30.86 & 8.74 & 16.9 & 3.3 & 1.5 & $\mathrm{NA}$ \\
\hline & E-Horizon & 3 & 12.5 & 3.94 & 3.61 & 1.84 & 0.46 & 1.55 & Sandy Loam & 60.3 & 30.41 & 9.29 & 22.0 & 5.6 & 0.8 & $\mathrm{NA}$ \\
\hline & E-Horizon & 4 & 17.5 & 4.50 & 3.93 & 2.16 & 0.48 & 1.28 & Loam & 51.4 & 35 & 13.6 & 24.9 & 3.4 & 0.7 & $\mathrm{NA}$ \\
\hline & E-Horizon & 5 & 22.5 & 4.89 & 4.22 & 2.35 & 0.48 & 1.14 & Loam & 44.6 & 38.5 & 16.9 & 20.8 & 7.9 & 0.6 & $\mathrm{NA}$ \\
\hline & Bw Horizon & 6 & 27.5 & 4.87 & 4.19 & 2.40 & 0.48 & 1.15 & Loam & 45.3 & 37.7 & 17 & 19.2 & 8.2 & 0.5 & $\mathrm{NA}$ \\
\hline & Bw Horizon & 7 & 32.5 & 4.85 & 4.18 & 2.40 & 0.48 & 1.10 & Loam & 45.9 & 37.1 & 17 & 21.8 & 10.4 & 0.7 & $\mathrm{NA}$ \\
\hline & Bw Horizon & 8 & 37.5 & 4.49 & 4.03 & 2.19 & 0.46 & 1.30 & Loam & 49.1 & 38.3 & 12.6 & 24.6 & 12.8 & 1.0 & $\mathrm{NA}$ \\
\hline & Bw Horizon & 9 & 42.5 & 4.85 & 4.20 & 2.34 & 0.48 & 1.13 & Loam & 45.2 & 38.3 & 16.5 & 20.3 & 8.9 & 1.0 & $\mathrm{NA}$ \\
\hline & Bw Horizon & 10 & 47.5 & 4.97 & 4.30 & 2.41 & 0.46 & 1.03 & Loam & 43.4 & 38.5 & 18.1 & 15.1 & 11.2 & 1.2 & $\mathrm{NA}$ \\
\hline & Upper Bt Horizon & 11 & 52.5 & 5.00 & 4.29 & 2.46 & 0.48 & 1.06 & Loam & 43.5 & 38 & 18.5 & 13.3 & 6.8 & 1.2 & $\mathrm{NA}$ \\
\hline & Upper Bt Horizon & 12 & 57.5 & 5.10 & 4.43 & 2.44 & 0.44 & 0.99 & Loam & 40.9 & 39.6 & 19.5 & 13.1 & 9.1 & 1.3 & NA \\
\hline & Upper Bt Horizon & 13 & 62.5 & 5.21 & 4.51 & 2.49 & 0.45 & 0.99 & Loam & 39.4 & 40.1 & 20.5 & 13.2 & 19.1 & 1.6 & $\mathrm{NA}$ \\
\hline & Upper Bt Horizon & 14 & 67.5 & 5.43 & 4.75 & 2.56 & 0.41 & 0.90 & Loam & 35.4 & 41.4 & 23.2 & 12.4 & 32.4 & 2.1 & $\mathrm{NA}$ \\
\hline & Lower Bt Horizon & 15 & 72.5 & 5.88 & 5.40 & 2.56 & 0.29 & 0.84 & Clay Loam & 26.9 & 44.5 & 28.6 & 11.5 & 5.6 & 2.7 & $\mathrm{NA}$ \\
\hline & Lower Bt Horizon & 16 & 77.5 & 6.10 & 5.63 & 2.63 & 0.27 & 0.83 & Clay Loam & 24.3 & 45 & 30.7 & 9.8 & -3.8 & 3.2 & $\mathrm{NA}$ \\
\hline & Lower Bt Horizon & 17 & 82.5 & 5.97 & 5.55 & 2.52 & 0.26 & 0.86 & Clay Loam & 23.9 & 46.9 & 29.2 & 9.7 & -3.8 & 2.9 & $\mathrm{NA}$ \\
\hline & Lower Bt Horizon & 18 & 87.5 & 5.81 & 5.26 & 2.54 & 0.33 & 0.90 & Clay Loam & 26.8 & 47.1 & 26.1 & 9.2 & -1.7 & 2.6 & $\mathrm{NA}$ \\
\hline & Lower Bt Horizon & 19 & 92.5 & 6.17 & 5.74 & 2.52 & 0.26 & 0.88 & Clay Loam & 20.8 & 49.1 & 30.1 & 10.7 & 0.4 & 2.5 & NA \\
\hline & Lower Bt Horizon & 20 & 97.5 & 5.48 & 4.82 & 2.52 & 0.41 & 0.90 & Loam & 33.8 & 42.7 & 23.5 & 9.8 & 0.4 & 2.2 & $\mathrm{NA}$ \\
\hline
\end{tabular}

\begin{tabular}{|c|c|c|c|c|c|c|c|c|c|c|c|c|c|c|c|c|}
\hline Profile & Context & Sample & $\begin{array}{c}\text { Plot Depth } \\
(\mathrm{cm}) \\
\end{array}$ & $\begin{array}{c}\text { Mean } \\
\text { (phi) } \\
\end{array}$ & $\begin{array}{c}\text { Median } \\
\text { (phi) } \\
\end{array}$ & $\begin{array}{c}\text { Sorting } \\
\text { (phi) }\end{array}$ & $\begin{array}{c}\text { Skewness } \\
\text { (phi) } \\
\end{array}$ & $\begin{array}{c}\text { Kurtosis } \\
\text { (phi) }\end{array}$ & $\begin{array}{c}\text { USDA Textural } \\
\text { Class } \\
\end{array}$ & $\begin{array}{c}\text { Sand } \\
(\%) \\
\end{array}$ & $\begin{array}{l}\text { Silt } \\
(\%) \\
\end{array}$ & $\begin{array}{c}\text { Clay } \\
(\%) \\
\end{array}$ & $\begin{array}{c}\text { Xlf } \\
\left(10^{-8} \mathrm{~m}^{3} \mathrm{~kg}^{-1} \mathrm{SI}\right) \\
\end{array}$ & $\begin{array}{c}\text { Xfd } \\
(\%) \\
\end{array}$ & $\begin{array}{c}\text { Total C } \\
(\%) \\
\end{array}$ & $\begin{array}{c}\delta^{13} \mathrm{C} \\
\text { (per mil PDB) } \\
\end{array}$ \\
\hline Profile 2 & Upper Colluvium & 1 & 2.5 & 3.99 & 3.60 & 1.86 & 0.48 & 1.39 & Sandy Loam & 59.7 & 30.98 & 9.32 & 24.7 & 32.5 & 0.59 & -26.06 \\
\hline \multirow[t]{22}{*}{ Lot 1989} & Upper Colluvium & 2 & 7.5 & 3.89 & 3.61 & 1.75 & 0.45 & 1.60 & Sandy Loam & 60.8 & 30.62 & 8.58 & 23.9 & 28.7 & 0.23 & -24.94 \\
\hline & Upper Colluvium & 3 & 12.5 & 3.62 & 3.38 & 1.59 & 0.45 & 1.66 & Sandy Loam & 68.1 & 25.14 & 6.76 & 25.5 & 26.7 & 0.37 & -25.31 \\
\hline & Lower Colluvium & 4 & 17.5 & 3.70 & 3.39 & 1.73 & 0.48 & 1.65 & Sandy Loam & 66.6 & 25.44 & 7.96 & 24.7 & 27.5 & 0.26 & -24.39 \\
\hline & Lower Colluvium & 5 & 22.5 & 3.60 & 3.34 & 1.61 & 0.46 & 1.69 & Sandy Loam & 69.1 & 23.79 & 7.11 & 28.2 & 31.6 & 0.33 & -25.66 \\
\hline & Lower Colluvium & 6 & 27.5 & 3.71 & 3.45 & 1.72 & 0.45 & 1.67 & Sandy Loam & 65.9 & 26.27 & 7.83 & 16.3 & 5.2 & 0.33 & -24.80 \\
\hline & Lower Colluvium & 7 & 32.5 & 4.25 & 3.84 & 2.06 & 0.45 & 1.39 & Sandy Loam & 53.6 & 35.2 & 11.2 & 18.7 & 10.2 & 0.25 & -24.10 \\
\hline & Paleosol & 8 & 37.5 & 4.94 & 4.39 & 2.35 & 0.41 & 1.10 & Loam & 41.6 & 41.6 & 16.8 & 20.7 & 8.5 & 0.48 & -24.38 \\
\hline & Paleosol & 9 & 42.5 & 4.72 & 4.17 & 2.29 & 0.43 & 1.14 & Loam & 46.1 & 38.8 & 15.1 & 21.5 & 8.4 & 0.49 & -24.19 \\
\hline & Paleosol & 10 & 47.5 & 4.98 & 4.40 & 2.34 & 0.42 & 1.07 & Loam & 41.2 & 41.3 & 17.5 & 24.4 & 8.4 & 0.39 & -23.88 \\
\hline & E-Horizon & 11 & 52.5 & 5.02 & 4.39 & 2.40 & 0.43 & 1.04 & Loam & 41.4 & 40.4 & 18.2 & 20.3 & 8.2 & 0.29 & -23.82 \\
\hline & E-Horizon & 12 & 57.5 & 4.89 & 4.33 & 2.33 & 0.43 & 1.15 & Loam & 42.5 & 41.2 & 16.3 & 20.8 & 6.6 & 0.17 & -24.47 \\
\hline & E-Horizon & 13 & 62.5 & 4.89 & 4.33 & 2.32 & 0.43 & 1.13 & Loam & 42.5 & 41.2 & 16.3 & 15.3 & 8.8 & 0.11 & -24.31 \\
\hline & E-Horizon & 14 & 67.5 & 4.78 & 4.24 & 2.28 & 0.43 & 1.15 & Loam & 44.3 & 40.4 & 15.3 & 11.9 & 5.7 & 0.08 & -24.23 \\
\hline & Bw Horizon & 15 & 72.5 & 5.03 & 4.39 & 2.44 & 0.44 & 1.10 & Loam & 41.5 & 40.5 & 18 & 13.2 & 0.0 & 0.08 & -22.89 \\
\hline & Bw Horizon & 16 & 77.5 & 5.21 & 4.52 & 2.52 & 0.44 & 0.97 & Loam & 39.3 & 39.9 & 20.8 & 15.2 & 2.9 & 0.09 & -23.56 \\
\hline & Bw Horizon & 17 & 82.5 & 5.18 & 4.48 & 2.51 & 0.44 & 0.98 & Loam & 40.1 & 39.4 & 20.5 & 10.7 & -6.0 & 0.16 & -23.28 \\
\hline & Bw Horizon & 18 & 87.5 & 5.23 & 4.46 & 2.54 & 0.46 & 0.94 & Loam & 40 & 38.7 & 21.3 & 10.7 & 4.8 & 0.12 & -21.48 \\
\hline & Upper Bt Horizon & 19 & 92.5 & 5.07 & 4.33 & 2.48 & 0.46 & 0.98 & Loam & 42.7 & 37.6 & 19.7 & 11.2 & 8.5 & 0.15 & -21.76 \\
\hline & Upper Bt Horizon & 20 & 97.5 & 5.11 & 4.34 & 2.50 & 0.47 & 1.00 & Loam & 42.7 & 37.5 & 19.8 & 10.2 & 7.9 & 0.14 & -21.36 \\
\hline & Upper Bt Horizon & 21 & 102.5 & 5.13 & 4.36 & 2.49 & 0.47 & 1.01 & Loam & 42 & 38.3 & 19.7 & 10.5 & 0.0 & 0.14 & -21.40 \\
\hline & Upper Bt Horizon & 22 & 107.5 & 4.92 & 4.22 & 2.42 & 0.48 & 1.10 & Loam & 45 & 37.3 & 17.7 & 10.3 & 6.4 & 0.13 & -21.44 \\
\hline & Upper Bt Horizon & 23 & 112.5 & 4.43 & 3.94 & 2.11 & 0.46 & 1.35 & Loam & 51.1 & 36.4 & 12.5 & 10.6 & 12.1 & 0.12 & -21.54 \\
\hline Profile & Context & Sample & $\begin{array}{c}\text { Plot Depth } \\
(\mathrm{cm}) \\
\end{array}$ & $\begin{array}{c}\text { Mean } \\
\text { (phi) } \\
\end{array}$ & $\begin{array}{c}\text { Median } \\
\text { (phi) } \\
\end{array}$ & $\begin{array}{c}\text { Sorting } \\
\text { (phi) } \\
\end{array}$ & $\begin{array}{c}\text { Skewness } \\
\text { (phi) } \\
\end{array}$ & $\begin{array}{c}\text { Kurtosis } \\
\text { (phi) } \\
\end{array}$ & $\begin{array}{c}\text { USDA Textural } \\
\text { Class } \\
\end{array}$ & $\begin{array}{c}\text { Sand } \\
(\%) \\
\end{array}$ & $\begin{array}{l}\text { Silt } \\
(\%) \\
\end{array}$ & $\begin{array}{r}\text { Clay } \\
(\%) \\
\end{array}$ & $\begin{array}{c}\text { Xlf } \\
\left(10^{-8} \mathrm{~m}^{3} \mathrm{~kg}^{-1} \mathrm{SI}\right) \\
\end{array}$ & $\begin{array}{c}\text { Xfd } \\
(\%) \\
\end{array}$ & $\begin{array}{c}\text { Total C } \\
(\%) \\
\end{array}$ & $\begin{array}{c}\delta^{13} \mathrm{C} \\
\text { (per mil PDB) } \\
\end{array}$ \\
\hline Profile 3 & Upper Colluvium & 1 & 1 & 4.06 & 3.66 & 1.89 & 0.45 & 1.21 & Sandy Loam & 57.2 & 33.95 & 8.85 & 16.0 & 99.0 & 2.11 & -27.58 \\
\hline \multirow[t]{22}{*}{ Lot 1990} & Upper Colluvium & 2 & 2 & 4.15 & 3.71 & 1.98 & 0.46 & 1.20 & Sandy Loam & 55.6 & 34.53 & 9.87 & 13.7 & 5.5 & 1.24 & -27.13 \\
\hline & Upper Colluvium & 3 & 3 & 4.07 & 3.58 & 2.01 & 0.50 & 1.25 & Sandy Loam & 58.5 & 31.4 & 10.1 & 14.0 & 2.0 & 0.92 & -27.18 \\
\hline & Upper Colluvium & 4 & 4 & 4.20 & 3.72 & 2.03 & 0.47 & 1.19 & Sandy Loam & 55.4 & 33.7 & 10.9 & 16.5 & 7.3 & 0.64 & -26.78 \\
\hline & Upper Colluvium & 5 & 5 & 4.03 & 3.54 & 1.99 & 0.50 & 1.27 & Sandy Loam & 59.5 & 30.4 & 10.1 & 13.3 & 10.4 & 0.58 & -26.29 \\
\hline & Upper Colluvium & 6 & 6 & 4.01 & 3.53 & 1.98 & 0.50 & 1.30 & Sandy Loam & 59.7 & 30.3 & 10 & 15.0 & 9.3 & 0.48 & -25.94 \\
\hline & Upper Colluvium & 7 & 7 & 3.87 & 3.38 & 1.93 & 0.53 & 1.35 & Sandy Loam & 63.4 & 27.49 & 9.11 & 14.1 & 16.1 & 0.42 & -26.04 \\
\hline & Upper Colluvium & 8 & 8 & 3.51 & 3.12 & 1.73 & 0.54 & 1.71 & Sandy Loam & 72.4 & 19.89 & 7.71 & 16.8 & 0.3 & 0.45 & -25.68 \\
\hline & Upper Colluvium & 9 & 9 & 3.55 & 3.14 & 1.77 & 0.54 & 1.66 & Sandy Loam & 71.4 & 20.59 & 8.01 & 13.2 & -3.4 & 0.29 & -24.59 \\
\hline & Upper Colluvium & 10 & 10 & 3.68 & 3.20 & 1.87 & 0.57 & 1.65 & Sandy Loam & 69.3 & 21.41 & 9.29 & 14.4 & 7.9 & 0.24 & -24.93 \\
\hline & Upper Colluvium & 11 & 11 & 3.43 & 3.03 & 1.79 & 0.54 & 1.76 & Sandy Loam & 73.5 & 18.42 & 8.08 & 15.9 & 9.1 & 0.48 & -25.94 \\
\hline & Upper Colluvium & 12 & 12 & 3.42 & 3.04 & 1.78 & 0.53 & 1.77 & Sandy Loam & 73.6 & 18.32 & 8.08 & 15.3 & 6.7 & 0.26 & -24.26 \\
\hline & Upper Colluvium & 13 & 13 & 3.36 & 3.05 & 1.69 & 0.50 & 1.78 & Sandy Loam & 74.3 & 18.56 & 7.14 & 17.4 & 6.2 & 0.30 & -24.09 \\
\hline & Upper Colluvium & 14 & 14 & 3.39 & 3.05 & 1.71 & 0.52 & 1.79 & Sandy Loam & 74 & 18.45 & 7.55 & 17.2 & 5.7 & 0.23 & -24.69 \\
\hline & Upper Colluvium & 15 & 15 & 3.68 & 3.39 & 1.73 & 0.48 & 1.66 & Sandy Loam & 66.5 & 25.63 & 7.87 & 19.8 & 7.1 & 0.17 & -24.35 \\
\hline & Upper Colluvium & 16 & 16 & 3.64 & 3.30 & 1.72 & 0.50 & 1.58 & Sandy Loam & 67.8 & 24.13 & 8.07 & 19.8 & 6.8 & 0.24 & -24.34 \\
\hline & Upper Colluvium & 17 & 17 & 3.69 & 3.39 & 1.74 & 0.46 & 1.57 & Sandy Loam & 65.7 & 26.59 & 7.71 & 18.7 & 8.5 & 0.21 & -24.20 \\
\hline & Upper Colluvium & 18 & 19 & 3.73 & 3.43 & 1.73 & 0.46 & 1.58 & Sandy Loam & 65.1 & 27.22 & 7.68 & 20.7 & 3.9 & 0.24 & -25.67 \\
\hline & Upper Colluvium & 19 & 20 & 3.76 & 3.48 & 1.71 & 0.45 & 1.57 & Sandy Loam & 64.1 & 28.25 & 7.65 & 17.9 & 3.9 & 0.29 & -25.35 \\
\hline & Lower Colluvium & 20 & 21 & 3.87 & 3.58 & 1.82 & 0.43 & 1.52 & Sandy Loam & 60.8 & 30.87 & 8.33 & 17.8 & 6.6 & 0.33 & -24.63 \\
\hline & Lower Colluvium & 21 & 23 & 3.62 & 3.35 & 1.72 & 0.44 & 1.61 & Sandy Loam & 67.2 & 25.6 & 7.2 & 21.0 & 7.0 & 0.15 & -24.54 \\
\hline & Lower Colluvium & 22 & 24 & 3.72 & 3.41 & 1.71 & 0.47 & 1.54 & Sandy Loam & 65.3 & 27.01 & 7.69 & 48.2 & 5.1 & 0.18 & -25.10 \\
\hline & Lower Colluvium & 23 & 25 & 3.83 & 3.51 & 1.78 & 0.46 & 1.50 & Sandy Loam & 62.6 & 28.95 & 8.45 & 17.9 & 8.4 & 0.19 & -24.23 \\
\hline
\end{tabular}




\begin{tabular}{|c|c|c|c|c|c|c|c|c|c|c|c|c|c|c|c|c|}
\hline & Lower Colluvium & 24 & 26 & 4.15 & 3.67 & 2.05 & 0.49 & 1.36 & Sandy Loam & 57.5 & 31.3 & 11.2 & 20.4 & 7.7 & 0.29 & -24.96 \\
\hline & Lower Colluvium & 25 & 28 & 4.51 & 3.96 & 2.25 & 0.46 & 1.18 & Loam & 50.6 & 35.6 & 13.8 & 23.4 & 10.6 & 0.37 & -23.46 \\
\hline & Lower Colluvium & 26 & 30 & 4.80 & 4.24 & 2.36 & 0.43 & 1.09 & Loam & 45 & 38.9 & 16.1 & 28.2 & 8.0 & 0.43 & -24.48 \\
\hline & Paleosol & 27 & 31 & 4.94 & 4.38 & 2.39 & 0.41 & 1.07 & Loam & 42.1 & 40.7 & 17.2 & 27.4 & 9.0 & 0.54 & -23.82 \\
\hline & Paleosol & 28 & 33 & 5.02 & 4.47 & 2.41 & 0.40 & 1.02 & Loam & 40.6 & 41.3 & 18.1 & 23.2 & 9.8 & 0.55 & -24.10 \\
\hline & Paleosol & 29 & 35 & 4.90 & 4.36 & 2.35 & 0.42 & 1.12 & Loam & 42.3 & 41.2 & 16.5 & 21.0 & -3.4 & 0.52 & -24.25 \\
\hline & Paleosol & 30 & 36 & 4.92 & 4.35 & 2.36 & 0.42 & 1.07 & Loam & 42.5 & 40.6 & 16.9 & 21.5 & -2.5 & 0.44 & -24.15 \\
\hline & Paleosol & 31 & 37 & 4.91 & 4.34 & 2.36 & 0.43 & 1.10 & Loam & 42.4 & 40.8 & 16.8 & 20.5 & 7.7 & 0.37 & -23.45 \\
\hline & Paleosol & 32 & 38 & 5.10 & 4.54 & 2.45 & 0.39 & 1.02 & Loam & 39.5 & 41.7 & 18.8 & 20.4 & 8.2 & 0.32 & -23.71 \\
\hline & Paleosol & 33 & 40 & 4.89 & 4.31 & 2.35 & 0.43 & 1.12 & Loam & 43 & 40.5 & 16.5 & 18.9 & 5.0 & 0.26 & -22.96 \\
\hline & Paleosol & 34 & 41 & 4.93 & 4.36 & 2.34 & 0.42 & 1.09 & Loam & 42.2 & 40.9 & 16.9 & 19.2 & 6.8 & 0.36 & -24.03 \\
\hline & Paleosol & 35 & 43 & 4.84 & 4.26 & 2.35 & 0.43 & 1.09 & Loam & 44.3 & 39.3 & 16.4 & 21.0 & 4.7 & 0.25 & -23.12 \\
\hline & Paleosol & 36 & 44 & 4.94 & 4.35 & 2.36 & 0.42 & 1.05 & Loam & 42.5 & 40.2 & 17.3 & 17.7 & 6.2 & 0.31 & -24.29 \\
\hline & E-Horizon & 37 & 45 & 4.88 & 4.30 & 2.36 & 0.43 & 1.10 & Loam & 43.6 & 39.7 & 16.7 & 20.6 & 6.0 & 0.17 & -23.78 \\
\hline & E-Horizon & 38 & 47 & 4.92 & 4.33 & 2.37 & 0.43 & 1.09 & Loam & 42.9 & 40.1 & 17 & 20.0 & 4.5 & 0.16 & -23.40 \\
\hline & E-Horizon & 39 & 49 & 4.94 & 4.33 & 2.40 & 0.44 & 1.08 & Loam & 43 & 39.6 & 17.4 & 19.0 & 2.1 & 0.12 & -23.14 \\
\hline & E-Horizon & 40 & 51 & 4.85 & 4.26 & 2.35 & 0.44 & 1.10 & Loam & 44.2 & 39.3 & 16.5 & 17.8 & 2.5 & 0.11 & -23.45 \\
\hline & E-Horizon & 41 & 53 & 4.88 & 4.28 & 2.39 & 0.43 & 1.07 & Loam & 43.9 & 39 & 17.1 & 18.4 & 8.1 & 0.12 & -23.31 \\
\hline & E-Horizon & 42 & 56 & 4.77 & 4.15 & 2.39 & 0.44 & 1.07 & Loam & 46.8 & 36.7 & 16.5 & 16.7 & 5.7 & 0.09 & -24.08 \\
\hline & E-Horizon & 43 & 59 & 4.89 & 4.28 & 2.41 & 0.43 & 1.08 & Loam & 43.9 & 38.9 & 17.2 & 18.6 & 6.6 & 0.09 & -22.96 \\
\hline Profile & Context & Sample & $\begin{array}{c}\text { Plot Depth } \\
(\mathrm{cm}) \\
\end{array}$ & $\begin{array}{c}\text { Mean } \\
\text { (phi) } \\
\end{array}$ & $\begin{array}{c}\text { Median } \\
\text { (phi) } \\
\end{array}$ & $\begin{array}{c}\text { Sorting } \\
\text { (phi) }\end{array}$ & $\begin{array}{c}\text { Skewness } \\
\text { (phi) } \\
\end{array}$ & $\begin{array}{c}\text { Kurtosis } \\
\text { (phi) } \\
\end{array}$ & $\begin{array}{c}\text { USDA Textural } \\
\text { Class } \\
\end{array}$ & $\begin{array}{c}\text { Sand } \\
(\%)\end{array}$ & $\begin{array}{l}\text { Silt } \\
(\%) \\
\end{array}$ & $\begin{array}{r}\text { Clay } \\
(\%) \\
\end{array}$ & $\begin{array}{c}\text { Xlf } \\
\left(10^{-8} \mathrm{~m}^{3} \mathrm{~kg}^{-1} \mathrm{SI}\right) \\
\end{array}$ & $\begin{array}{l}\text { Xfd } \\
(\%) \\
\end{array}$ & $\begin{array}{c}\text { Total C } \\
(\%) \\
\end{array}$ & $\begin{array}{c}\delta^{13} \mathrm{C} \\
\text { (per mil PDB) } \\
\end{array}$ \\
\hline Profile 4 & Upper Colluvium & 1 & 10 & 4.17 & 3.40 & 2.34 & 0.55 & 1.28 & Sandy Loam & 61.6 & 24.7 & 13.7 & 37.07 & 5.64 & 0.57 & -25.24 \\
\hline Lot 1991 & Upper Colluvium & 2 & 12 & 2.52 & 2.35 & 1.90 & 0.30 & 1.31 & Loamy Sand & 81.4 & 13.38 & 5.22 & 19.83 & 4.60 & 0.42 & -24.14 \\
\hline & Upper Colluvium & 3 & 14 & 3.52 & 3.23 & 2.04 & 0.38 & 1.47 & Sandy Loam & 66.5 & 24.78 & 8.72 & 18.84 & 6.09 & 0.37 & -23.92 \\
\hline & Upper Colluvium & 4 & 16 & 3.36 & 3.16 & 1.93 & 0.36 & 1.69 & Sandy Loam & 71 & 20.76 & 8.24 & 20.27 & 5.60 & 0.33 & -23.56 \\
\hline & Upper Colluvium & 5 & 18 & 3.60 & 3.38 & 1.68 & 0.44 & 1.77 & Sandy Loam & 68 & 24.47 & 7.53 & 16.03 & 4.20 & 0.29 & -23.29 \\
\hline & Upper Colluvium & 6 & 19 & 3.85 & 3.54 & 1.78 & 0.46 & 1.56 & Sandy Loam & 62 & 29.14 & 8.86 & 17.81 & 5.53 & 0.27 & -22.61 \\
\hline & Upper Colluvium & 7 & 21 & 3.75 & 3.48 & 1.79 & 0.46 & 1.72 & Sandy Loam & 64.6 & 27.13 & 8.27 & 22.26 & 6.10 & 0.20 & -23.96 \\
\hline & Upper Colluvium & 8 & 22 & 3.55 & 3.33 & 1.62 & 0.43 & 1.74 & Sandy Loam & 69.7 & 23.26 & 7.04 & 18.12 & 5.36 & 0.27 & -23.16 \\
\hline & Upper Colluvium & 9 & 23 & 3.54 & 3.34 & 1.61 & 0.43 & 1.83 & Sandy Loam & 70.2 & 22.73 & 7.07 & 19.26 & 2.03 & 0.17 & -24.44 \\
\hline & Upper Colluvium & 10 & 25 & 3.54 & 3.34 & 1.61 & 0.43 & 1.83 & Sandy Loam & 70.2 & 21.94 & 7.86 & 19.06 & 1.97 & 0.16 & -24.33 \\
\hline & Upper Colluvium & 11 & 27 & 3.16 & 3.07 & 1.01 & 0.32 & 1.47 & Loamy Sand & 82.7 & 13.34 & 3.96 & 15.47 & 5.12 & 0.12 & -23.89 \\
\hline & Upper Colluvium & 12 & 28 & 3.11 & 2.99 & 1.04 & 0.40 & 1.68 & Loamy Sand & 84.4 & 11.43 & 4.17 & 14.09 & 6.81 & 0.09 & -23.83 \\
\hline & Upper Colluvium & 13 & 29 & 3.47 & 3.37 & 1.37 & 0.36 & 1.81 & Sandy Loam & 72.4 & 21.95 & 5.65 & 13.54 & 1.79 & 0.13 & -23.87 \\
\hline & Lower Colluvium & 14 & 31 & 4.08 & 3.75 & 1.87 & 0.46 & 1.57 & Sandy Loam & 56.5 & 39.33 & 4.17 & 18.52 & 4.43 & 0.18 & -23.81 \\
\hline & Lower Colluvium & 15 & 32 & 4.26 & 3.99 & 1.87 & 0.40 & 1.55 & Loam & 49.9 & 39.9 & 10.2 & 15.41 & 1.36 & 0.31 & -23.61 \\
\hline & Lower Colluvium & 16 & 33 & 4.24 & 4.03 & 1.96 & 0.34 & 1.49 & Loam & 48.9 & 40.7 & 10.4 & 15.24 & 7.99 & 0.35 & -23.35 \\
\hline & Lower Colluvium & 17 & 34 & 4.07 & 4.07 & 1.81 & 0.19 & 1.74 & Loam & 47.2 & 44.76 & 8.04 & 15.12 & 9.18 & 0.25 & -24.96 \\
\hline & Lower Colluvium & 18 & 35 & 3.35 & 3.28 & 1.84 & 0.23 & 1.61 & Sandy Loam & 69.5 & 23.94 & 6.56 & 16.19 & 8.84 & 0.12 & -23.58 \\
\hline & Lower Colluvium & 19 & 36 & 2.95 & 2.99 & 1.89 & 0.17 & 1.25 & Sandy Loam & 73.9 & 20.62 & 5.48 & 16.67 & 6.99 & 0.12 & -23.72 \\
\hline & Lower Colluvium & 20 & 38 & 4.29 & 3.90 & 2.23 & 0.37 & 1.27 & Loam & 51.7 & 36.2 & 12.1 & 17.27 & 8.74 & 0.27 & -23.66 \\
\hline & Lower Colluvium & 21 & 39 & 4.61 & 4.25 & 2.31 & 0.33 & 1.20 & Loam & 45 & 41 & 14 & 16.95 & 7.16 & 0.41 & -24.18 \\
\hline & Lower Colluvium & 22 & 40 & 4.57 & 4.14 & 2.24 & 0.39 & 1.26 & Loam & 46.9 & 39.4 & 13.7 & 16.40 & 8.05 & 0.37 & -23.20 \\
\hline & Lower Colluvium & 23 & 41 & 3.98 & 3.62 & 2.03 & 0.40 & 1.46 & Sandy Loam & 58.7 & 31.1 & 10.2 & 15.98 & 6.54 & 0.39 & -23.94 \\
\hline & Lower Colluvium & 24 & 42 & 4.12 & 3.79 & 2.02 & 0.40 & 1.41 & Sandy Loam & 54.6 & 35 & 10.4 & 14.99 & 5.34 & 0.29 & -24.75 \\
\hline & Lower Colluvium & 25 & 43 & 3.30 & 3.09 & 1.87 & 0.33 & 1.63 & Sandy Loam & 72.6 & 20.47 & 6.93 & 20.05 & 6.42 & 0.22 & -24.52 \\
\hline & Lower Colluvium & 26 & 45 & 3.37 & 3.18 & 1.57 & 0.42 & 1.91 & Sandy Loam & 75 & 18.54 & 6.46 & 15.87 & 8.39 & 0.15 & -23.98 \\
\hline & Lower Colluvium & 27 & 46 & 2.83 & 2.75 & 1.64 & 0.25 & 1.47 & Loamy Sand & 80.4 & 14.87 & 4.73 & 17.78 & 8.44 & 0.14 & -23.64 \\
\hline & Lower Colluvium & 28 & 47 & 3.40 & 3.20 & 1.73 & 0.35 & 1.50 & Sandy Loam & 69.7 & 24.01 & 6.29 & 15.46 & 6.61 & 0.13 & -23.69 \\
\hline & Lower Colluvium & 29 & 49 & 3.10 & 2.86 & 1.57 & 0.40 & 1.63 & Sandy Loam & 78 & 16.77 & 5.23 & 16.51 & 5.73 & 0.15 & -24.17 \\
\hline & Lower Colluvium & 30 & 51 & 3.19 & 3.01 & 1.66 & 0.34 & 1.58 & Sandy Loam & 75.3 & 19.18 & 5.52 & 18.37 & 6.49 & 0.14 & -23.92 \\
\hline & Lower Colluvium & 31 & 52 & 3.17 & 3.03 & 1.62 & 0.30 & 1.58 & Sandy Loam & 75.9 & 18.77 & 5.33 & 15.19 & 8.90 & 0.16 & -24.82 \\
\hline & Lower Colluvium & 32 & 54 & 3.16 & 3.06 & 1.96 & 0.25 & 1.40 & Sandy Loam & 71.4 & 22.38 & 6.22 & 16.47 & 8.29 & 0.19 & -24.24 \\
\hline & Lower Colluvium & 33 & 55 & 2.64 & 2.69 & 1.70 & 0.10 & 1.07 & Loamy Sand & 80.6 & 15.41 & 3.99 & 14.29 & 8.57 & 0.15 & -23.69 \\
\hline & Lower Colluvium & 34 & 57 & 2.92 & 2.92 & 1.72 & 0.17 & 1.40 & Sandy Loam & 77.4 & 17.78 & 4.82 & 17.35 & 7.45 & 0.20 & -24.54 \\
\hline & Lower Colluvium & 35 & 58 & 4.09 & 3.73 & 1.88 & 0.45 & 1.53 & Sandy Loam & 57.2 & 31.4 & 11.4 & 16.36 & 6.13 & 0.21 & -24.50 \\
\hline & Lower Colluvium & 36 & 60 & 4.30 & 3.72 & 2.04 & 0.51 & 1.40 & Sandy Loam & 57.2 & 29.6 & 13.2 & 14.92 & 4.35 & 0.22 & -24.73 \\
\hline & Lower Colluvium & 37 & 61 & 4.30 & 3.72 & 2.06 & 0.51 & 1.39 & Sandy Loam & 57 & 29.3 & 13.7 & 17.07 & 2.79 & 0.24 & -24.43 \\
\hline & Lower Colluvium & 38 & 63 & 4.31 & 3.69 & 2.08 & 0.52 & 1.41 & Sandy Loam & 58 & 28.2 & 13.8 & 17.70 & 4.08 & 0.20 & -24.49 \\
\hline & Lower Colluvium & 39 & 64 & 4.32 & 3.70 & 2.09 & 0.52 & 1.42 & Sandy Loam & 57.7 & 28.4 & 13.9 & 15.85 & 3.31 & 0.19 & -24.22 \\
\hline & Lower Colluvium & 40 & 66 & 4.61 & 3.78 & 2.28 & 0.55 & 1.34 & Sandy Loam & 55.3 & 28.7 & 16 & 16.93 & 3.47 & 0.19 & -24.16 \\
\hline & Lower Colluvium & 41 & 68 & 4.14 & 3.69 & 1.94 & 0.49 & 1.53 & Sandy Loam & 58.2 & 29.4 & 12.4 & 17.39 & 0.66 & 0.22 & -24.42 \\
\hline & Paleosol & 42 & 71 & 2.60 & 2.51 & 1.83 & 0.21 & 1.08 & Loamy Sand & 79.2 & 16.52 & 4.28 & 16.00 & 0.00 & 0.23 & -24.47 \\
\hline & Paleosol & 43 & 73 & 2.93 & 2.85 & 1.95 & 0.24 & 1.28 & Sandy Loam & 74.3 & 19.99 & 5.71 & 15.71 & 0.39 & 0.29 & -24.78 \\
\hline & Paleosol & 44 & 75 & 2.72 & 2.53 & 2.05 & 0.30 & 1.20 & Loamy Sand & 76.6 & 17.5 & 5.9 & 17.39 & 0.34 & 0.52 & -26.51 \\
\hline & Paleosol & 45 & 76 & 2.83 & 2.53 & 2.18 & 0.35 & 1.20 & Sandy Loam & 74.6 & 18.43 & 6.97 & 19.43 & 0.96 & 0.35 & -24.13 \\
\hline & Paleosol & 46 & 77 & 2.69 & 2.38 & 2.18 & 0.36 & 1.21 & Loamy Sand & 76.3 & 17.05 & 6.65 & 19.99 & 6.29 & 0.26 & -23.64 \\
\hline & Paleosol & 47 & 79 & 3.26 & 2.97 & 2.26 & 0.35 & 1.25 & Sandy Loam & 68.5 & 22.89 & 8.61 & 20.99 & 8.43 & 0.28 & -23.65 \\
\hline & Paleosol & 48 & 80 & 2.97 & 2.67 & 2.22 & 0.35 & 1.21 & Sandy Loam & 72.6 & 19.81 & 7.59 & 20.86 & 8.73 & 0.53 & -25.79 \\
\hline & Paleosol & 49 & 82 & 3.05 & 2.69 & 2.24 & 0.40 & 1.23 & Sandy Loam & 71.9 & 19.74 & 8.36 & 20.35 & 3.84 & 0.33 & -24.29 \\
\hline & E-Horizon & 50 & 84 & 2.86 & 2.50 & 2.17 & 0.41 & 1.23 & Sandy Loam & 74.5 & 18.12 & 7.38 & 21.74 & 6.76 & 0.20 & -23.45 \\
\hline & E-Horizon & 51 & 85 & 3.10 & 2.75 & 2.28 & 0.38 & 1.22 & Sandy Loam & 70.9 & 20.59 & 8.51 & 21.05 & 11.24 & 0.19 & -23.01 \\
\hline & E-Horizon & 52 & 86 & 3.09 & 2.69 & 2.29 & 0.40 & 1.22 & Sandy Loam & 71.3 & 20.06 & 8.64 & 20.73 & 10.24 & 0.16 & -23.26 \\
\hline
\end{tabular}




\begin{tabular}{|c|c|c|c|c|c|c|c|c|c|c|c|c|c|c|c|}
\hline E-Horizon & 53 & 87 & 3.39 & 3.01 & 2.35 & 0.38 & 1.22 & Sandy Loam & 67.1 & 23.05 & 9.85 & 20.22 & 7.07 & 0.15 & -23.15 \\
\hline E-Horizon & 54 & 89 & 2.88 & 2.46 & 2.26 & 0.43 & 1.23 & Sandy Loam & 74 & 17.88 & 8.12 & 22.54 & 8.93 & 0.16 & -23.41 \\
\hline E-Horizon & 55 & 90 & 2.57 & 2.12 & 2.22 & 0.44 & 1.26 & Sandy Loam & 77.6 & 15.2 & 7.2 & 20.11 & 9.09 & 0.14 & -22.76 \\
\hline E-Horizon & 56 & 92 & 3.23 & 2.75 & 2.42 & 0.41 & 1.18 & Sandy Loam & 69.1 & 20.99 & 9.91 & 17.39 & 7.17 & 0.13 & -23.41 \\
\hline E-Horizon & 57 & 94 & 2.96 & 2.52 & 2.33 & 0.42 & 1.24 & Sandy Loam & 72.8 & 18.56 & 8.64 & 16.85 & 6.85 & 0.13 & -23.00 \\
\hline E-Horizon & 58 & 96 & 3.44 & 3.01 & 2.42 & 0.39 & 1.22 & Sandy Loam & 66.6 & 22.9 & 10.5 & 17.26 & -0.29 & 0.11 & -22.28 \\
\hline E-Horizon & 59 & 98 & 3.48 & 2.96 & 2.43 & 0.45 & 1.23 & Sandy Loam & 67 & 21.8 & 11.2 & 16.05 & -3.23 & 0.11 & -21.81 \\
\hline E-Horizon & 60 & 100 & 3.35 & 2.71 & 2.47 & 0.51 & 1.25 & Sandy Loam & 69.2 & 19.4 & 11.4 & 16.07 & -3.61 & 0.21 & -21.92 \\
\hline E-Horizon & 61 & 102 & 2.66 & 2.12 & 2.22 & 0.53 & 1.38 & Sandy Loam & 77.7 & 13.99 & 8.31 & 14.77 & 6.60 & 0.19 & -21.75 \\
\hline Upper Bt Horizon & 63 & 106 & 3.45 & 2.79 & 2.49 & 0.50 & 1.24 & Sandy Loam & 68.5 & 19.3 & 12.2 & 16.18 & 10.36 & 0.27 & -21.95 \\
\hline Upper Bt Horizon & 64 & 108 & 3.00 & 2.32 & 2.32 & 0.58 & 1.34 & Sandy Loam & 74.2 & 15.3 & 10.5 & 14.78 & 7.59 & 0.28 & -21.48 \\
\hline Upper Bt Horizon & 65 & 110 & 2.88 & 2.23 & 2.29 & 0.57 & 1.35 & Sandy Loam & 75.3 & 14.99 & 9.71 & 15.36 & 6.57 & 0.27 & -21.99 \\
\hline
\end{tabular}



APPENDIX E: DROUGHT HISTORY 



\begin{tabular}{|c|c|c|c|c|c|c|c|c|c|c|c|c|c|c|c|c|}
\hline No. & Trinomial & Digitized Polygon From & Acres & Site Classification & $\begin{array}{l}\text { Clusters of } 2 \Sigma \text { range or single } 2 \Sigma \text { (\# of } \\
\text { dates in parenthesis) }\end{array}$ & $\begin{array}{l}1200- \\
1249 \\
\end{array}$ & $\begin{array}{r}1250- \\
1299\end{array}$ & $\begin{array}{l}1300- \\
1349\end{array}$ & $\begin{array}{l}1350- \\
1399\end{array}$ & $\begin{array}{l}1400- \\
1449\end{array}$ & $\begin{array}{l}1450- \\
1499\end{array}$ & $\begin{array}{l}1500 \\
1549\end{array}$ & $\begin{array}{l}1550- \\
1599\end{array}$ & $\begin{array}{l}1600 \\
1649\end{array}$ & $\begin{array}{l}1650- \\
1680\end{array}$ & Period \\
\hline 1 & 41LR297 & THC Atlas & 5.91728 & LARGE & $1440(1)$ & & & & & $\mathrm{x}$ & & & & & & Middle/Late \\
\hline 2 & 41LR039 & THC Site Form (bounding elipse) & 1.616069 & SMALL & 1305(1) & & & $\mathrm{x}$ & & & & & & & & Middle \\
\hline 3 & 41RR016 & THC Atlas & 450.998944 & LARGE & $1306-1307(2) ; 1480(1) ; 1522(1)$ & & & $\mathrm{x}$ & & & $x$ & $\mathrm{x}$ & & & & Middle/Late \\
\hline 4 & 41RR204 & THC Atlas & 2.554404 & SMALL & 1350(1); 1532(1) & & & & $\mathrm{x}$ & & & $\mathrm{x}$ & & & & Middle/Late \\
\hline 5 & 41RR011 & THC Atlas & 2.963723 & SMALL & $1360-1391(2) ; 1464-1474(2)$ & & & & $\mathrm{x}$ & & $x$ & & & & & Middle/Late \\
\hline 6 & 41RR077 & THC Atlas & 8.503536 & LARGE & 1361-1372(2); 1629-1634(2) & & & & $x$ & & & & & $x$ & & Middle/Late \\
\hline 7 & 41BW171 & Perttula (2005) JNTA No. 22 & 0.222347 & SMALL & 1239(1); 1300(1); $1400(1)$ & $x$ & & $x$ & & $x$ & & & & & & Middle/Late \\
\hline 8 & 41BW003 & THC Atlas & 80.454759 & LARGE & $1230(1) ; 1336(1) ; 1560-1565(2)$ & $x$ & & $\mathrm{x}$ & & & & & $\mathrm{x}$ & & & Middle/Late \\
\hline 9 & 41BW226 & THC Atlas & 0.57671 & SMALL & 1265 & & $x$ & & & & & & & & & Middle \\
\hline 10 & 41DT011 & Fields et al (1994) & 0.617627 & SMALL & 1229(1);1362(1) & $\mathrm{x}$ & & & $x$ & & & & & & & Middle \\
\hline 11 & 41DT052 & Doehner and Larson (1975) & 1.308559 & SMALL & $1323(1) ; 1628(1)$ & & & $x$ & & & & & & $\mathrm{x}$ & & Middle/Late \\
\hline 12 & 41DT050 & Gadus et al (1992) & 2.964603 & SMALL & $1337(1)$ & & & $\mathrm{x}$ & & & & & & & & Middle \\
\hline 13 & 41HP175 & Fields et al (1993) & 0.395281 & SMALL & $\begin{array}{l}\text { 1226(1);1346-1374(2); } 1462- \\
1472(4) ; 1534-1537(3)\end{array}$ & $\mathrm{x}$ & & $\mathrm{x}$ & $\mathrm{x}$ & & $\mathrm{x}$ & $\mathrm{x}$ & & & & Middle/Late \\
\hline 14 & 41HP159 & Jurney and Bohlin (1993) & 0.216169 & SMALL & $1376(1)$ & & & & $\mathrm{x}$ & & & & & & & Middle \\
\hline 15 & 41HP102 & Jurney and Bohlin (1993) $\square$ & 12.970148 & LARGE & 1248(1); 1457(1) & $\mathrm{x}$ & & & & & $\mathrm{x}$ & & & & & Middle/Late \\
\hline 16 & 41HP116 & THC Site Form (bounding box) & 0.138348 & SMALL & $1451(1)$ & & & & & & $\mathrm{x}$ & & & & & Late \\
\hline 17 & 41HP106 & THC Atlas & 23.74104 & LARGE & $1250-1354(6) ; 1643(1)$ & & $\mathrm{x}$ & $\mathrm{x}$ & $\mathrm{x}$ & & & & & $\mathrm{x}$ & & Middle/Late \\
\hline 18 & 41HP237 & THC Atlas & 0.307874 & SMALL & $1530(1) ; 1595(1)$ & & & & & & & $\mathrm{x}$ & $\mathrm{x}$ & & & Late \\
\hline 19 & 41TT769 & Perttula et al (2010) Caddo Archaeology Journal Vol. 20 & 0.148229 & SMALL & 1285(1);1525(1);1635(1) & & $\mathrm{x}$ & & & & & $\mathrm{x}$ & & $\mathrm{x}$ & & Middle/Late \\
\hline 20 & 41TT406 & THC Site Form (bounding elipse) & 0.229708 & SMALL & $1472(1)$ & & & & & & $\mathrm{x}$ & & & & & Late \\
\hline 21 & 41TT653 & THC Site Form (bounding elipse) & 0.041294 & SMALL & 1332(1);1365-1373(2);1511-1582(14) & & & $\mathrm{x}$ & $\mathrm{x}$ & & & $\mathrm{x}$ & $\mathrm{x}$ & & & Middle/Late \\
\hline 22 & 41MX005 & THC Site Form (bounding box) & 0.185286 & SMALL & $1470-1479(2)$ & & & & & & $\mathrm{x}$ & & & & & Late \\
\hline 23 & 41BW553 & THC Atlas & 10.406341 & LARGE & 1391(1); 1488(1) & & & & $\mathrm{x}$ & & $\mathrm{x}$ & & & & & Middle/Late \\
\hline 24 & 41CS151 & THC Atlas & 10.263643 & LARGE & 1476(1); 1521(1) & & & & & & $\mathrm{x}$ & $\mathrm{x}$ & & & & Late \\
\hline 25 & $41 \mathrm{CS} 150$ & THC Site Form (map) & 1.985682 & SMALL & 1354(1) & & & & $\mathrm{x}$ & & & & & & & Middle \\
\hline 26 & $41 \mathrm{CS} 001$ & THC Atlas & 6.328549 & LARGE & 1364(1) & & & & $\mathrm{x}$ & & & & & & & Middle \\
\hline 27 & $41 \mathrm{CS} 014$ & THC Atlas & 8.186623 & LARGE & 1316(1); 1444(1) & & & $\mathrm{x}$ & & $\mathrm{x}$ & & & & & & Middle/Late \\
\hline 28 & $41 \mathrm{CS} 085$ & THC Site Form (bounding box) & 0.01112 & SMALL & 1292(1) & & $x$ & & & & & & & & & Middle \\
\hline 29 & $41 T T 373$ & THC Site Form (bounding elipse) & 0.414097 & SMALL & 1479(1) & & & & & & $x$ & & & & & Late \\
\hline 30 & 41TT372 & THC Atlas & 4.829297 & LARGE & 1343-1348(2); 1641(1) & & & $\mathrm{x}$ & & & & & & $\mathrm{x}$ & & Middle/Late \\
\hline 31 & 41TT550 & THC Atlas & 0.602887 & SMALL & 1228(1);1524-1558(4);1626-1633(2) & $\mathrm{x}$ & & & & & & $\mathrm{x}$ & $\mathrm{x}$ & $\mathrm{x}$ & & Middle/Late \\
\hline 32 & 41TT865 & Perttula et al (2003) Caddoan Archaeology Journal & 0.592915 & SMALL & $1538(1)$ & & & & & & & $\mathrm{x}$ & & & & Late \\
\hline 33 & 41TT672 & THC Atlas & 9.362097 & LARGE & 1521(1) & & & & & & & $\mathrm{x}$ & & & & Late \\
\hline & & & & & $1427-1447$ ( 6); 1529-1577(13); 1653- & & & & & & & & & & & \\
\hline 34 & 41TT852 & THC Atlas & 24.669809 & LARGE & $1661(6)$ & & & & & $\mathrm{x}$ & & $x$ & $\mathrm{x}$ & & $\mathrm{x}$ & Late \\
\hline 35 & $41 T T 853$ & THC Atlas & 1.979072 & SMALL & 1518-1572(14); 1646-1660(3) & & & & & & & $\mathrm{x}$ & $\mathrm{x}$ & $\mathrm{x}$ & $\mathrm{x}$ & Late \\
\hline 36 & 41TT851 & THC Site Form (bounding box) & 22.234276 & LARGE & $\begin{array}{l}\text { 1213; 1234-1260; 1324-1381; 1423- } \\
1430 ; 1522-1577\end{array}$ & $\mathrm{x}$ & $\mathrm{x}$ & $x$ & $\mathrm{x}$ & $\mathrm{x}$ & & $\mathrm{x}$ & $\mathrm{x}$ & & & Middle/Late \\
\hline 37 & 41TT011 & THC Atlas & 12.467003 & LARGE & 1385(1) & & & & $\mathrm{x}$ & & & & & & & Middle \\
\hline 38 & 41FK107 & THC Site Form (bounding box) & 2.470474 & SMALL & $1350(1)$ & & & & $\mathrm{x}$ & & & & & & & Middle \\
\hline 39 & $41 \mathrm{CP} 230$ & Nelson \& Perttula (2003) JNTA No. 17 & 1.493692 & SMALL & 1528(1); 1632(1) & & & & & & & $\mathrm{x}$ & & $\mathrm{x}$ & & Late \\
\hline 40 & 41CР335 & THC Site Form (bounding box) & 0.494095 & SMALL & 1535 & & & & & & & $\mathrm{x}$ & & & & Late \\
\hline 41 & 41СР313 & THC Site Form (bounding box) & 0.395276 & SMALL & 1275(1);1558(1) & & $\mathrm{x}$ & & & & & & $\mathrm{x}$ & & & Middle/Late \\
\hline & & & & & $1305(1) ; 1345-1380(6) ; 1442-$ & & & & & & & & & & & \\
\hline 42 & 41СР304 & Perttula (2005) & 9.881901 & LARGE & 1469(4);1518-1566(17) & & & $\mathrm{x}$ & $\mathrm{x}$ & $x$ & $\mathrm{x}$ & $\mathrm{x}$ & $\mathrm{x}$ & & & Middle/Late \\
\hline
\end{tabular}




\begin{tabular}{|c|c|c|c|c|c|c|c|c|c|c|c|c|c|c|c|c|}
\hline No. & Trinomial & Digitized Polygon From & Acres & Site Classification & $\begin{array}{l}\text { Clusters of } 2 \Sigma \text { range or single } 2 \Sigma \text { (\# of } \\
\text { dates in parenthesis) }\end{array}$ & $\begin{array}{l}1200- \\
1249\end{array}$ & $\begin{array}{l}1250- \\
1299\end{array}$ & $\begin{array}{l}1300- \\
1349\end{array}$ & $\begin{array}{r}1350- \\
1399\end{array}$ & \begin{tabular}{|l|}
$1400-$ \\
1449
\end{tabular} \mid & \begin{tabular}{|l|}
$1450-$ \\
1499
\end{tabular} & \begin{tabular}{|l|}
$1500-$ \\
1549
\end{tabular} & $\begin{array}{l}1550- \\
1599\end{array}$ & $\begin{array}{l}1600 \\
1649\end{array}$ & $\begin{array}{l}1650- \\
1680\end{array}$ & Period \\
\hline 43 & $41 \mathrm{CP} 316$ & THC Site Form (bounding box) & 0.481743 & SMALL & $1523-1555(2)$ & & & & & & & $x$ & $x$ & & & Late \\
\hline 44 & 41CP245 & Perttula \& Nelson (2006) JNTA No. 24 & 1.185828 & SMALL & 1360 & & & & $\mathrm{x}$ & & & & & & & Middle \\
\hline 45 & 41CP408 & THC Site Form (bounding box) & 0.494087 & SMALL & $1330-1380(4) ; 1430(1)$ & & & $\mathrm{x}$ & $\mathrm{x}$ & $\mathrm{x}$ & & & & & & Middle/Late \\
\hline 46 & 41CP088 & THC Atlas & 1.187663 & SMALL & 1550-1590(3); 1660(2) & & & & & & & & $\mathrm{x}$ & & $\mathrm{x}$ & Late \\
\hline 47 & 41CP005 & & NA & NA & 1542 & & & & & & & $\mathrm{x}$ & & & & Late \\
\hline 48 & 41CP010 & THC Atlas & 17.349207 & LARGE & $1390 ; 1540$ & & & & $\mathrm{x}$ & & & $\mathrm{x}$ & & & & Middle/Late \\
\hline 49 & 41UR279 & Perttula et al (2004) JNTA No. 19 & 0.540886 & SMALL & $1455(1)$ & & & & & & $\mathrm{x}$ & & & & & Late \\
\hline 50 & 41CP220 & THC Site Form (bounding box) & 3.705668 & SMALL & 1440(1); 1520(1) & & & & & $\mathrm{x}$ & & $\mathrm{x}$ & & & & Late \\
\hline 51 & 41CP071 & Perttula \& Nelson (2004) FNTA No. 5 & 8.893609 & LARGE & $\begin{array}{l}1325-1333(2) ; 1383-1385(2) ; 1528- \\
1545(4)\end{array}$ & & & $\mathrm{x}$ & $\mathrm{x}$ & & & $x$ & & & & Middle/Late \\
\hline 52 & 41UR129 & THC Site Form (map) & 0.101959 & SMALL & 1509(1) & & & & & & & $\mathrm{x}$ & & & & Late \\
\hline 53 & 41UR118 & THC Atlas & 5.831377 & LARGE & $1515-1530(2) ; 1636-1640(2)$ & & & & & & & $\mathrm{x}$ & & $x$ & & Late \\
\hline 54 & 41UR133 & THC Atlas & 11.281084 & LARGE & 1430(1); 1520-1565(5); 1640 (1) & & & & & $\mathrm{x}$ & & $\mathrm{x}$ & $x$ & $x$ & & Late \\
\hline 55 & 41UR011 & THC Site Form (bounding elipse) & 0.065191 & SMALL & $1465(1)$ & & & & & & $\mathrm{x}$ & & & & & Late \\
\hline 56 & 41UR144 & Turner (1993) NNTA No. 2 & 0.134394 & SMALL & $1550-1565(2) ; 1633(1)$ & & & & & & & & $x$ & $\mathrm{x}$ & & Late \\
\hline 57 & 41UR010 & & NA & NA & $1463(1) ; 1608-1632(3)$ & & & & & & $\mathrm{x}$ & & & $\mathrm{x}$ & & Late \\
\hline 58 & 41UR315 & THC Site Form (map) & 2.133598 & SMALL & 1545-1555(2) & & & & & & & $\mathrm{x}$ & $\mathrm{x}$ & & & Late \\
\hline 59 & 41WD109 & Bruseth and Perttula (1981) & 0.919266 & SMALL & 1234(1); 1301(1); 1312(1) & $\mathrm{x}$ & & $\mathrm{x}$ & & & & & & & & Middle \\
\hline 60 & 41WD482 & THC Atlas & 2.987248 & SMALL & 1462(1) & & & & & & $\mathrm{x}$ & & & & & Late \\
\hline 61 & 41WD060 & & NA & NA & 1220 (1); $1350(1) ; 1530(1)$ & $\mathrm{x}$ & & & $\mathrm{x}$ & & & $\mathrm{x}$ & & & & Middle/Late \\
\hline 62 & 41WD244 & & NA & NA & $1560(1)$ & & & & & & & & $\mathrm{x}$ & & & Late \\
\hline 63 & 41WD529 & THC Atlas & 35.460071 & LARGE & $1466(1)$ & & & & & & $\mathrm{x}$ & & & & & Late \\
\hline 64 & 41WD055 & THC Site Form (map) & 0.06528 & SMALL & 1320-1333(5); 1365-1366(2) & & & $\mathrm{x}$ & $\mathrm{x}$ & & & & & & & Middle \\
\hline 65 & 41SM054 & & NA & NA & $1375(1)$ & & & & $\mathrm{x}$ & & & & & & & Middle \\
\hline 66 & 41WD046 & Perttula et al (1993) NNTA No. 1 & 25.374678 & LARGE & 1376(1) & & & & $\mathrm{x}$ & & & & & & & Middle \\
\hline 67 & 41SM055 & THC Atlas & 27.832209 & LARGE & 1338(1) & & & $\mathrm{x}$ & & & & & & & & Middle \\
\hline 68 & 41SM195 & Walters (2003), JNTA No. 18 & 1.702837 & SMALL & 1378(1) & & & & $\mathrm{x}$ & & & & & & & Middle \\
\hline 69 & 41SM193 & Walters et al (1998), JNTA No. 11 & 1.852858 & SMALL & 1369(1); 1518(1) & & & & $\mathrm{x}$ & & & $\mathrm{x}$ & & & & Middle/Late \\
\hline 70 & 41SM056 & Walters (2009), JNTA No. 31 & 0.266812 & SMALL & $1360(1)$ & & & & $\mathrm{x}$ & & & & & & & Middle \\
\hline 71 & 41SM325 & Walters (2008) Caddo Archeology Journal Vol. 17 & 0.494095 & SMALL & $1295-1300(2) ; 1345-1365(2) ; 1435(1)$ & & $\mathrm{x}$ & $\mathrm{x}$ & $\mathrm{x}$ & $\mathrm{x}$ & & & & & & Middle/Late \\
\hline 72 & 41GG069 & THC Site Form (map) & 4.517011 & LARGE & $1365(1) ; 1403(1)$ & & & & $\mathrm{x}$ & $\mathrm{x}$ & & & & & & Middle/Late \\
\hline 73 & 41RK468 & THC Atlas & 1.223778 & SMALL & $1425(1)$ & & & & & $\mathrm{x}$ & & & & & & Late \\
\hline 74 & 41RK243 & THC Site Form (bounding box) & 2.309916 & SMALL & $1321(1)$ & & & $\mathrm{x}$ & & & & & & & & Middle \\
\hline 75 & 41RK342 & THC Site Form (bounding box) & 0.074115 & SMALL & $1357(1)$ & & & & $\mathrm{x}$ & & & & & & & Middle \\
\hline 76 & 41RK214 & THC Atlas & 1.880838 & SMALL & $1205(1) ; 1278-1391(24) ; 1524(1)$ & $\mathrm{x}$ & $\mathrm{x}$ & $\mathrm{x}$ & $\mathrm{x}$ & & & $\mathrm{x}$ & & & & Middle/Late \\
\hline 77 & 41HS573 & THC Atlas & 8.708801 & LARGE & $1555(1)$ & & & & & & & & $\mathrm{x}$ & & & Late \\
\hline 78 & 41HS574 & THC Atlas & 4.637456 & LARGE & 1330(1) & & & $\mathrm{x}$ & & & & & & & & Middle \\
\hline 79 & 41HS015 & THC Atlas & 20.849091 & LARGE & $\begin{array}{l}\text { 1305(1); 1350-1395(15); 1401(1); 1443- } \\
\text { 1574(71);1647-1667(9) }\end{array}$ & & & $\mathrm{x}$ & $\mathrm{x}$ & $\mathrm{x}$ & $\mathrm{x}$ & $\mathrm{x}$ & $\mathrm{x}$ & $\mathrm{x}$ & $\mathrm{x}$ & Middle/Late \\
\hline 80 & 41HS846 & THC Atlas & 7.571291 & LARGE & $1340-1355(2)$ & & & $\mathrm{x}$ & $\mathrm{x}$ & & & & & & & Middle \\
\hline 81 & $41 \mathrm{HS} 843$ & THC Site Form (bounding elipse) & 0.14733 & SMALL & 1555(1) & & & & & & & & $\mathrm{x}$ & & & Late \\
\hline 82 & 41HS231 & THC Atlas & 11.443515 & LARGE & $1225-1250(2) ; 1335(2) ; 1440(1)$ & $\mathrm{x}$ & $\mathrm{x}$ & $\mathrm{x}$ & & $\mathrm{x}$ & & & & & & Middle/Late \\
\hline 83 & 41HS588 & THC Atlas & 12.1912 & LARGE & $\begin{array}{l}\text { 1355-1380(4);1435-1455(2); 1540- } \\
\text { 1560(3) }\end{array}$ & & & & $\mathrm{x}$ & $\mathrm{x}$ & $\mathrm{x}$ & $x$ & $x$ & & & Middle/Late \\
\hline 84 & 41RK562 & THC Atlas & 2.71457 & SMALL & $1360(1)$ & & & & $x$ & & & & & & & Middle \\
\hline
\end{tabular}




\begin{tabular}{|c|c|c|c|c|c|c|c|c|c|c|c|c|c|c|c|c|}
\hline No. & Trinomial & Digitized Polygon From & Acres & Site Classification & $\begin{array}{l}\text { Clusters of } 2 \Sigma \text { range or single } 2 \Sigma \text { (\# of } \\
\text { dates in parenthesis) }\end{array}$ & $\begin{array}{l}1200- \\
1249\end{array}$ & $\begin{array}{l}1250- \\
1299\end{array}$ & $\begin{array}{l}1300- \\
1349\end{array}$ & $\begin{array}{l}1350- \\
1399\end{array}$ & $\begin{array}{l}1400- \\
1449\end{array}$ & $\begin{array}{l}1450- \\
1499\end{array}$ & $\begin{array}{l}1500- \\
1549\end{array}$ & $\begin{array}{l}1550- \\
1599\end{array}$ & $\begin{array}{l}1600 \\
1649\end{array}$ & $\begin{array}{l}1650- \\
1680\end{array}$ & Period \\
\hline 85 & 41RK557 & THC Atlas & 15.905288 & LARGE & $1216(1) ; 1360(1) ; 1443(1) ; 1563(1)$ & $\mathrm{x}$ & & & $\mathrm{x}$ & $\mathrm{x}$ & & & $\mathrm{x}$ & & & Middle/Late \\
\hline 86 & 41RK558 & THC Atlas & 10.200721 & LARGE & 1395(1);1563(1) & & & & $\mathrm{x}$ & & & & $\mathrm{x}$ & & & Middle/Late \\
\hline 87 & 41PN149 & & NA & NA & $1547(1)$ & & & & & & & $\mathrm{x}$ & & & & Late \\
\hline 88 & 41PN175 & THC Atlas & 0.545261 & SMALL & $1540(1)$ & & & & & & & $\mathrm{x}$ & & & & Late \\
\hline 89 & 41HE245 & & NA & NA & $1473(1)$ & & & & & & $\mathrm{x}$ & & & & & Late \\
\hline 90 & 41SM404 & THC Atlas & 0.967102 & SMALL & 1265(1); 1330(1); 1435(1) & & $\mathrm{x}$ & $\mathrm{x}$ & & $\mathrm{x}$ & & & & & & Middle/Late \\
\hline 91 & $41 \mathrm{SM} 300$ & THC Atlas & 14.243882 & LARGE & $1520(1)$ & & & & & & & $\mathrm{x}$ & & & & Late \\
\hline 92 & $41 S M 273$ & THC Site Form (map) & 5.748609 & LARGE & $1350-1360(2)$ & & & & $\mathrm{x}$ & & & & & & & Middle \\
\hline 93 & 41HE139 & THC Site Form (map) & 10.888167 & LARGE & $1440(1)$ & & & & & $\mathrm{x}$ & & & & & & Late \\
\hline 94 & 41HE343 & THC Site Form (bounding box) & 0.691716 & SMALL & $1540(2)$ & & & & & & & $\mathrm{x}$ & & & & Late \\
\hline 95 & 41HE08O & & NA & NA & 1331(1) & & & $\mathrm{x}$ & & & & & & & & Middle \\
\hline 96 & 41AN067 & Wilson, Perttula \& Walters (2012) JNTA No. 38 & 1.210514 & SMALL & 1542 & & & & & & & $\mathrm{x}$ & & & & Late \\
\hline 97 & 41CE354 & THC Atlas & 16.845481 & LARGE & $1240 ; 1340 ; 1540-1570$ & $\mathrm{x}$ & & $\mathrm{x}$ & & & & $\mathrm{x}$ & $\mathrm{x}$ & & & Middle/Late \\
\hline 98 & 41CE015 & & NA & NA & 1550 & & & & & & & & $\mathrm{x}$ & & & Late \\
\hline 99 & 41ANO38 & & NA & NA & $\begin{array}{l}\text { 1325-1375(12); 1425-1440(4); 1550(1); } \\
\text { 1655(1) }\end{array}$ & & & $\mathrm{x}$ & $\mathrm{x}$ & $x$ & & & $\mathrm{x}$ & & $\mathrm{x}$ & Middle/Late \\
\hline 100 & 41ANO21 & & NA & NA & 1565 & & & & & & & & $\mathrm{x}$ & & & Late \\
\hline 101 & 41ANO32 & THC Site Form (bounding box) & 0.592905 & SMALL & 1550 & & & & & & & & $\mathrm{x}$ & & & Late \\
\hline 102 & 41ANO34 & THC Site Form (bounding box) & 0.002038 & SMALL & 1535 & & & & & & & $\mathrm{x}$ & & & & Late \\
\hline 103 & 41RK170 & THC Atlas & 9.696205 & LARGE & $1315-1360(5) ; 1530(1)$ & & & $\mathrm{x}$ & $\mathrm{x}$ & & & $\mathrm{x}$ & & & & Middle/Late \\
\hline 104 & 41CE299 & THC Atlas & 7.450434 & LARGE & $1518(1) ; 1550(1)$ & & & & & & & $\mathrm{x}$ & $\mathrm{x}$ & & & Late \\
\hline 105 & 41AN051 & THC Atlas & 93.943745 & LARGE & 1235(1); 1350(1) & $\mathrm{x}$ & & & $\mathrm{x}$ & & & & & & & Middle \\
\hline 106 & 41AN087 & THC Atlas & 17.765672 & LARGE & $1462(1) ; 1540(2)$ & & & & & & $\mathrm{x}$ & $\mathrm{x}$ & & & & Late \\
\hline 107 & 41CE019 & THC Atlas & 222.537888 & LARGE & $\begin{array}{l}\text { 1202-1236(17); 1272-1335(11); } 1535 \\
\text { (1)_55 Early Caddo dates; } 28 \text { Formative }\end{array}$ & $\mathrm{x}$ & $\mathrm{x}$ & $\mathrm{x}$ & & & & $x$ & & & & Middle/Late \\
\hline 108 & 41HO15O & THC Site Form (map) & 21.3745 & LARGE & $1543(1)$ & & & & & & & $\mathrm{x}$ & & & & Late \\
\hline 109 & 41HO216 & Perttula and Nelson (2006) & 0.617624 & SMALL & $1245(1)$ & $\mathrm{x}$ & & & & & & & & & & Middle \\
\hline 110 & 41HO214 & Perttula and Nelson (2006) & 1.086771 & SMALL & $1555(1)$ & & & & & & & & $\mathrm{x}$ & & & Late \\
\hline 111 & 41NA044 & THC Site Form (bounding elipse) & 0.857976 & SMALL & $1346(1) ; 1488(1)$ & & & $\mathrm{x}$ & & & $\mathrm{x}$ & & & & & Middle/Late \\
\hline 112 & 41NA049 & THC Atlas & 48.359185 & LARGE & 1287(1);1350-1356(2) & & $\mathrm{x}$ & & $\mathrm{x}$ & & & & & & & Middle \\
\hline 113 & 41NA280 & Perttula (2000) & 0.864657 & SMALL & $1540-1555(3)$ & & & & & & & $\mathrm{x}$ & $\mathrm{x}$ & & & Late \\
\hline 114 & 41NA248 & Perttula (2000) & 0.44468 & SMALL & $1340-1350(2)$ & & & $\mathrm{x}$ & $\mathrm{x}$ & & & & & & & Middle \\
\hline 115 & 41NA285 & Perttula (2008) & 0.296454 & SMALL & $1265(1) ; 1350(1)$ & & $\mathrm{x}$ & & $\mathrm{x}$ & & & & & & & Middle \\
\hline 116 & 41NA231 & Perttula (2008) & 1185815 & SMAH & $1240-1250(2) ; 1295(1) ; 1340-1390(6) ;$ & $x$ & $x$ & $x$ & $x$ & & & $x$ & & & & Middle/l ate \\
\hline 117 & 41NA242 & Perttula (2008) & $\begin{array}{l}1.1003150 \\
1.383451\end{array}$ & SMALL & $1315(1) ; 1345-1370(7) ; 1520-1545(2)$ & & & $\hat{x}$ & $\hat{x}$ & & & $\hat{x}$ & & & & Middle/Late \\
\hline 118 & 41NA235 & Perttula (2008) & 0.148227 & SMALL & 1380(3);1430(1);1535(1) & & & & $\mathrm{x}$ & $\mathrm{x}$ & & $\mathrm{x}$ & & & & Middle/Late \\
\hline 119 & 41SY092 & THC Atlas & 48.80288 & LARGE & 1346(1);1391-1396(2);1476(1) & & & $\mathrm{x}$ & $\mathrm{x}$ & & $\mathrm{x}$ & & & & & Middle/Late \\
\hline 120 & 41SA123 & THC Site Form (bounding elipse) & 1.66013 & SMALL & $1474(1)$ & & & & & & $\mathrm{x}$ & & & & & Late \\
\hline Totals & & & & & & 17 & 14 & 36 & 47 & 20 & 23 & 46 & 29 & 13 & 5 & \\
\hline
\end{tabular}



The following is a synopsis of drought/ moisture regimes grouped in 50 year periods for Grid Point 194 (Figure E-1).

The 50 year period from A.D. 1200 to 1249, a 5-year period of moderate to extreme drought begins in A.D. 1198 and continues through A.D. 1202. It also had two single year droughts, one 2-year period of drought, three 3-year drought periods, and one 5-year period of drought. During this 50 year period there were no single or multiple year periods of increased moisture within the region. This was the only instance where there was no wetter than normal period during the period of study.

The 50 year period from A.D. 1250 to 1299 had had two single year droughts, one 2-year period of drought, two 3-year drought periods, and one 5-year period of drought. It had four single year periods of wetter than normal, and a single 3-year period of wetter than normal. The 50 year period from A.D. 1300 to 1349 had seven single year droughts and one 2-year period of drought. The moisture for this period had four single year periods of wetter than normal, a single 2-year period of drought, and one 3-year period of drought.

The 50 year period from A.D. 1349 to 1399 had five single year droughts, two 2-year droughts, and a single 3-year period of drought. The years of 1352, 1360, 1369, 1370, and 1374 are classified as severe or extreme droughts ranging from -3.806 to -5.26 coupled to a 3 year moderate drought occurring from 1365 to 1367 . The period also had six single years of increased moisture and one 3-year period of increased moisture.

The 50 year period from A.D. 1400 to 1449 had seven single year droughts and one 3-year period of drought. The period also had seven single years of increased moisture.

The 50 year period from A.D. 1450 to 1499 had four single year droughts, three 2-year periods of drought and one 5-year period of drought. A period of drought begins in 1350 with a severe drought followed by a reprieve of moisture until 1454 when there is 5-year period of severe to extreme drought that last until 1458 followed by single year droughts in 1460 , and 1463 . This period of drought is followed by two almost consecutive 2-year periods of drought that occur in 1468-1469 and 1472-1473 before the return of a normal or wetter than normal cycle of moisture. The period as whole had two single years of increased moisture and two 2-year period of wetter than normal periods.

The 50 year period from A.D. 1500 to 1549 had three single year droughts and four 2-year periods of droughts. Each of these drought periods are followed by a multiyear period of consecutive normal or wetter than normal moisture. The period had a total of five single year periods of increased moisture. 
The 50 year period from A.D. 1550 to 1599 had five single year droughts and three 2-year periods of drought. A 2-year period of drought begins in 1570-1571, followed by a one year reprieve followed by a single year of severe drought. At the end of the century, a 2-year drought begins in 1593 followed by a two year reprieve followed by a two year period of severe drought 1597-1598, and a single year of moderate drought in 1601. The 50 year period had a total of four single year periods, and two 2-year periods of increased moisture.

The 50 year period from A.D. 1600 to 1649 had 4 single year droughts, two 2-year droughts and one 3-year period of drought. It had 3 single years of increased moisture, one 3-year, and one 4-year period of increased moisture. The 4-year period of increased moisture from 1603 to1606 was bracketed by two single year drought followed by an 11-year period of normal to increased moisture followed by a 3-year period of increased moisture occurring from1620 to 1622.

The 50 year period from A.D. 1650 to 1699 had 6 single year droughts, and two 2-year periods of drought. The period from 1681 to 1682 experienced moderate to severe drought followed by a normal year followed by a 2-year period of moderate drought. It had three single years of increased moisture, and two 2-year periods of increased moisture. 


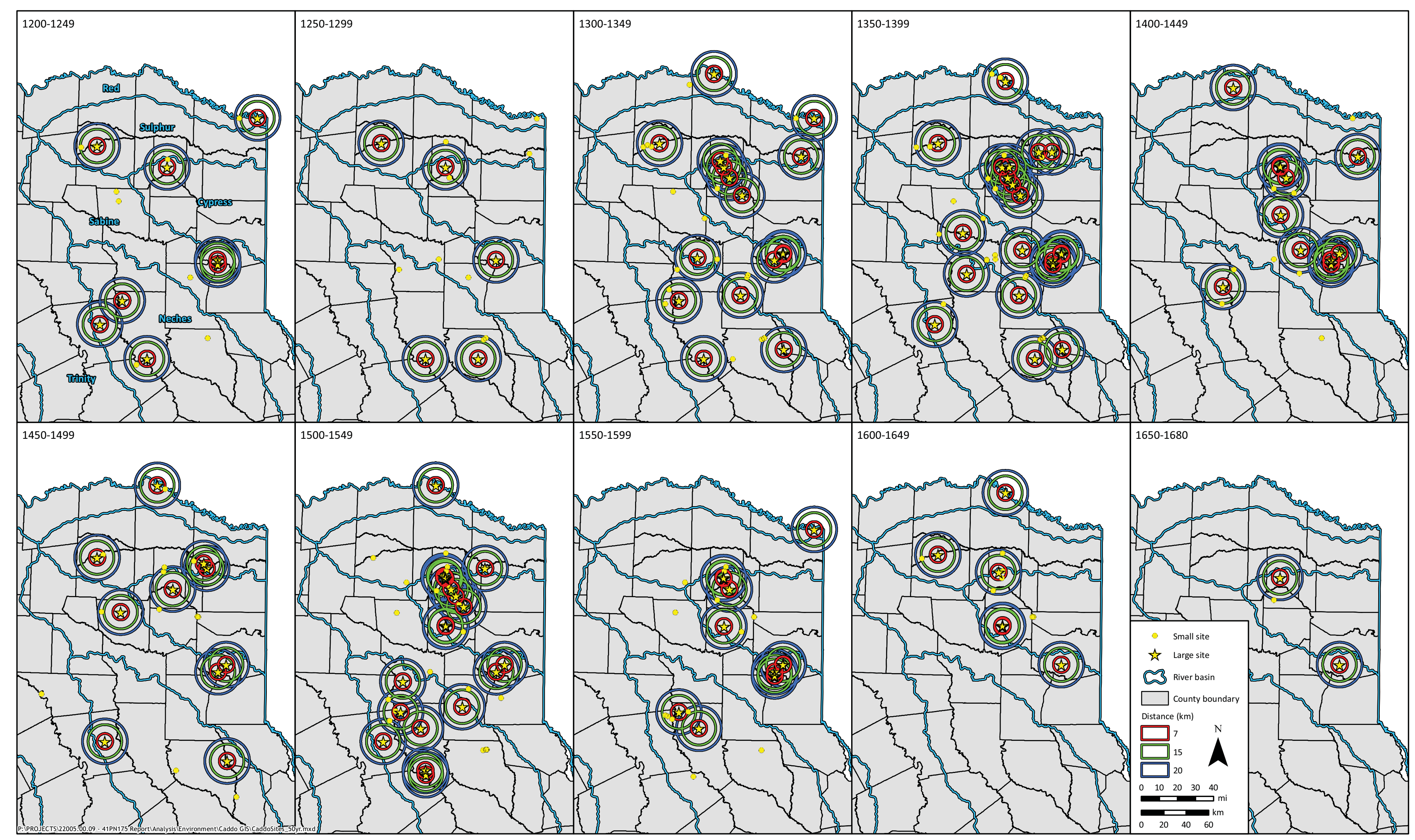

Figure E-1. Drought/moisture regimes grouped in 50 year periods for Grid Point 194 

APPENDIX F: NEUTRON ACTIVATION ANALYSIS 

Table F-1: Descriptive Information and Compositional Group Assignments

\begin{tabular}{|c|c|c|c|c|}
\hline MURR ANID & Lot. Artifact No. & Prov No. & $\begin{array}{l}\text { Caddo Ref Group } \\
\text { Association }\end{array}$ & Ware Desig. \\
\hline GMI028 & 414.1 & 533 & Region $7 / 8 / 9$ core & Fine? \\
\hline GMI029 & 514.23 & 1724 & Region 9 Group 3 & \\
\hline GMI030 & 647.1 & 789 & Region $7 / 8 / 9$ core & \\
\hline GMI031 & 836.27 & 1482 & Region 9 Group 3 & \\
\hline GMI032 & 1105.4 & 1241 & Region 9 Group 3 & \\
\hline GMI033 & 1121.6 & 1203 & Region 9 Group 3 & \\
\hline GMI034 & 1174.14 & 931 & Region $7 / 8 / 9$ core & \\
\hline GMI035 & 1191.1 & 960 & Region $7 / 8 / 9$ core & Fine? \\
\hline GMI036 & 1558.4 & 1600 & Region $7 / 8 / 9$ core & \\
\hline GMI037 & 1766.9 & 2002 & Region 9 Group 3 & Fine? \\
\hline GMI038 & TKP-1 & & Region 9 Group 3 & \\
\hline GMI039 & TKP-2 & & Region 9 Group 3 & \\
\hline GMI040 & TKP-3 & & Region 9 Group 3 & \\
\hline GMI041 & TKP-4 & & Region 9 Group 3 & \\
\hline GMI042 & TKP-5 & & Region 9 Group 3 & \\
\hline GMI043 & GEO-1 & Quaternary Alluvium & Clay & \\
\hline GMI044 & GEO-2 & Fluvatile Terrace & Clay & \\
\hline GMI045 & GEO-3 & Carrizo Sands & Clay & \\
\hline GMI046 & GEO-4 & Weches Formation & Clay & \\
\hline GMI047 & GEO-5 & Queen City Sand & Clay & \\
\hline GMI048 & GEO-6 & Reklaw Formation & Clay & \\
\hline GMI049 & GEO-7 & 41PN175 subsoil & Clay & \\
\hline GMI050 & GEO-8 & 41PN175 subsoil & Clay & \\
\hline GMI051 & GEO-9 & 41PN175 subsoil & Clay & \\
\hline
\end{tabular}


$m \infty \infty$ m

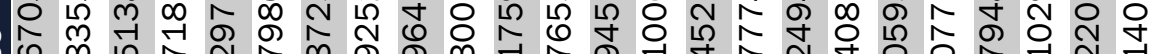



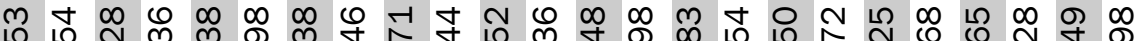

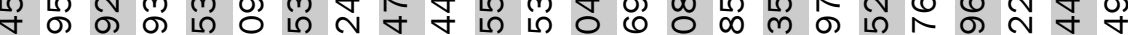
ฟ ம

$N$ 기 공

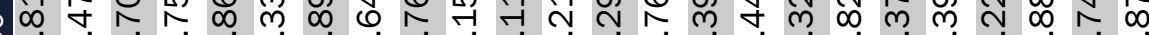
ન 아 윅 악

ம

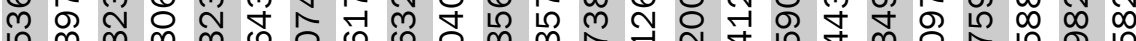
ம

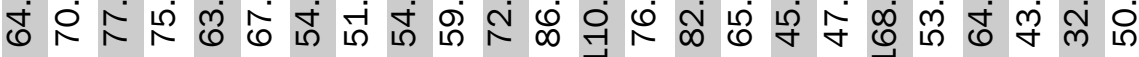

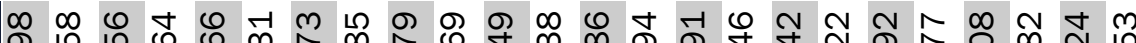
○ 冓

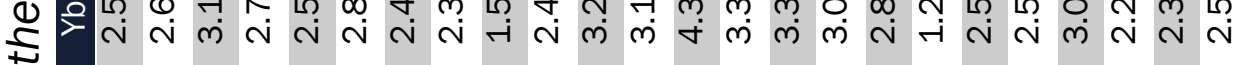

¿艹 ᄂ

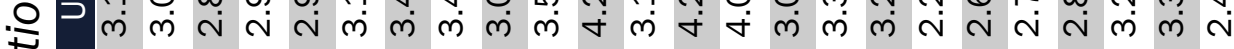

은

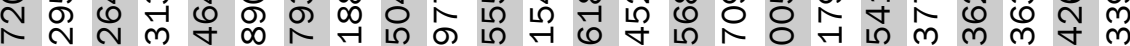

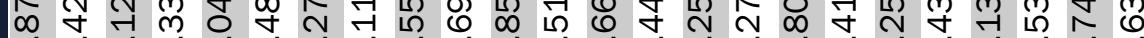
ठெ

ত্ $\infty$ t

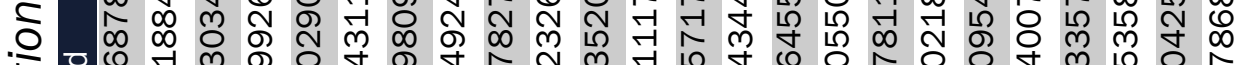

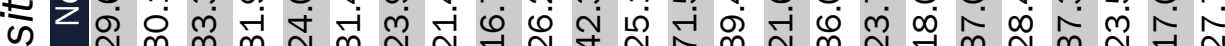

○े

ह้ U $=\hat{m}$ 守导

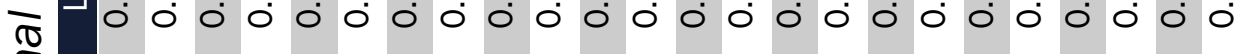

$\stackrel{\frac{1}{ \pm}}{ \pm}$

\ m 0 o m o ठ ब

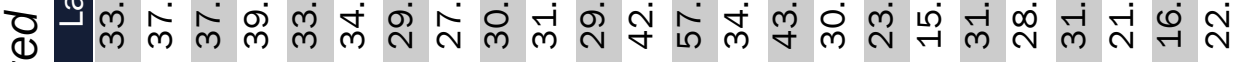

$\stackrel{\vartheta}{\supset}$

궁 ত $\Sigma$ ட

ì



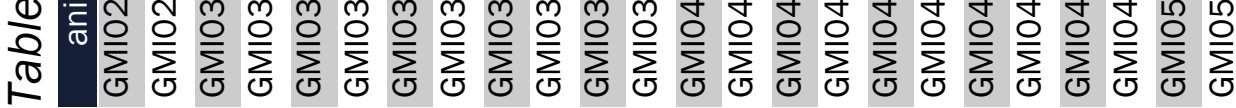




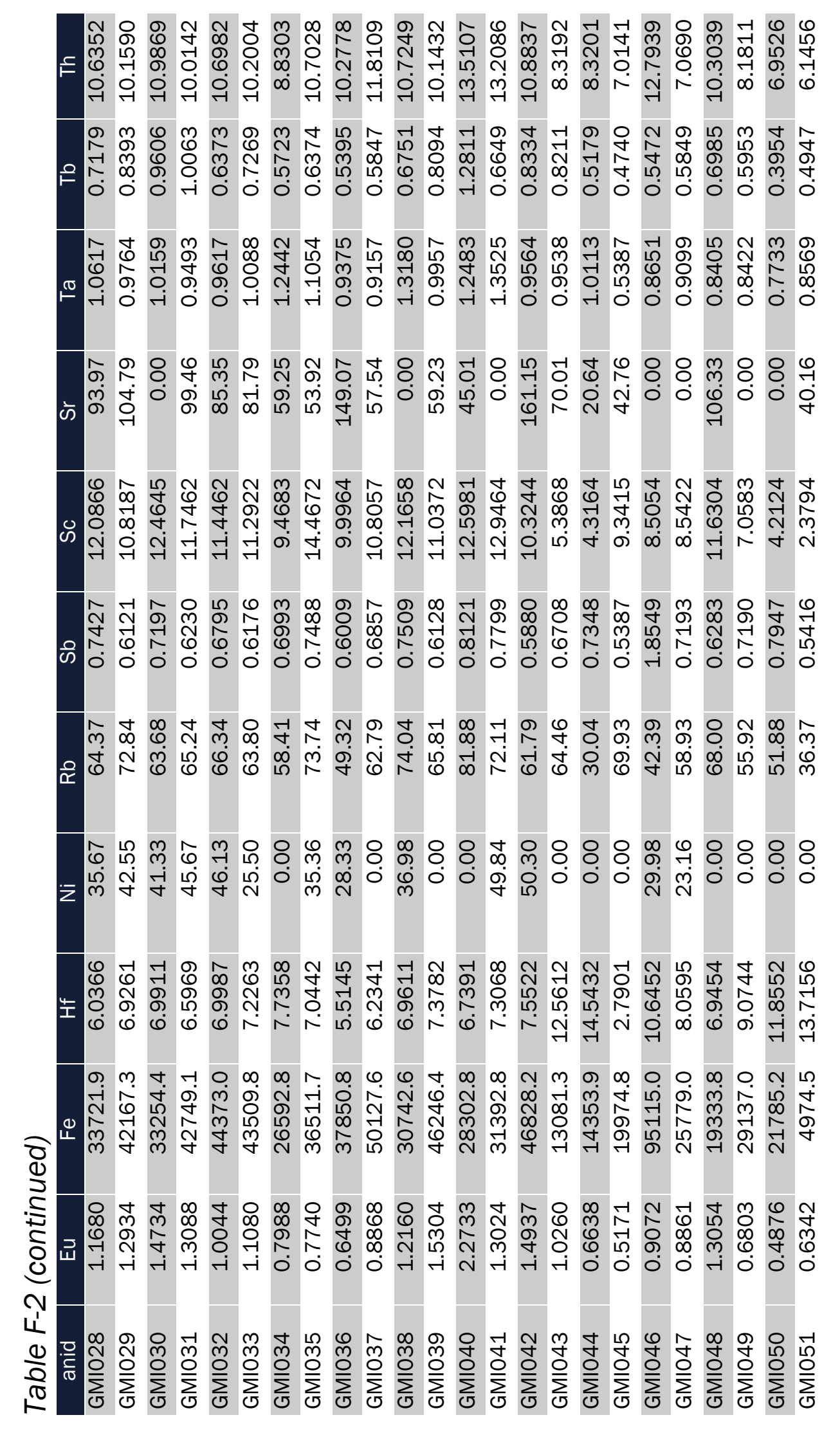


$\stackrel{\infty}{0}$ ᄀ ஸें

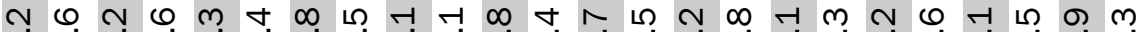
ஸ் ஸ் ர் -

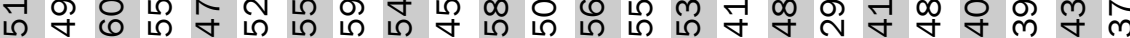

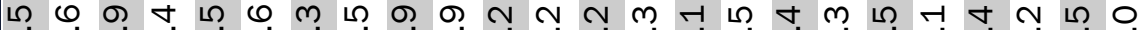

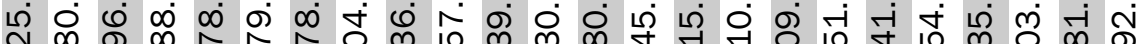

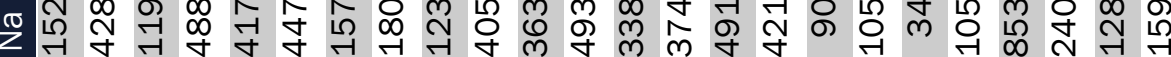

กิ の 0 m 0 m 0 . ᄀํㄱ

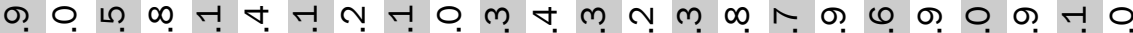

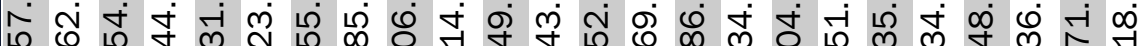
L

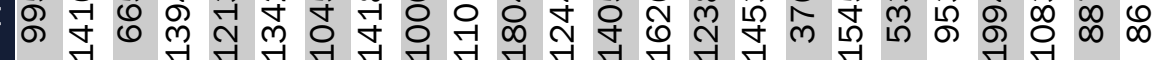

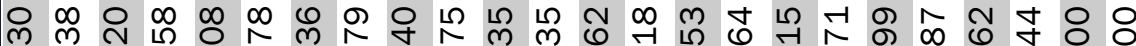

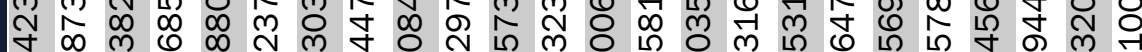

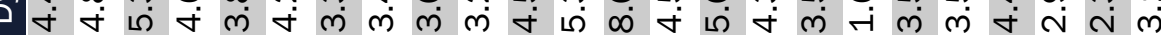

$0 . \forall$ t

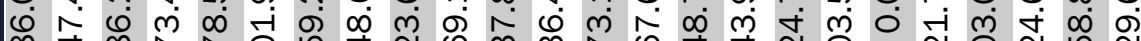
চ্

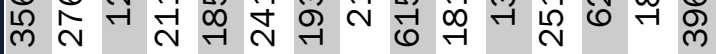

$\infty$ A 0 C ம்

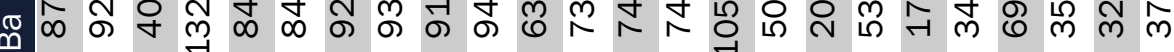

o a ப் ற்

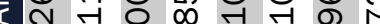



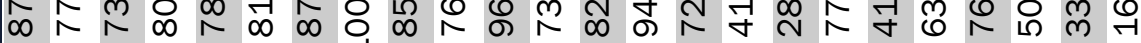

암 స $\widehat{\partial}$

$\stackrel{5}{\frac{1}{2}}$

$\infty$ ㄱ வ ம் ठิ

ऽ

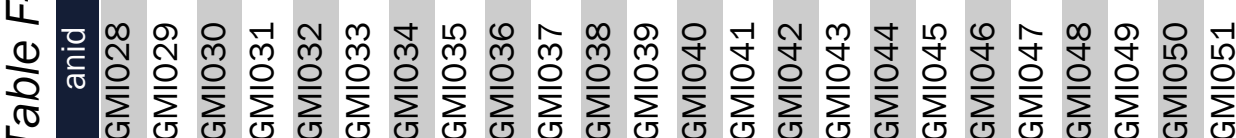

